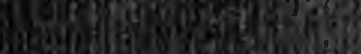
whe

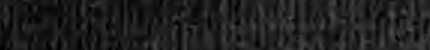
jolingonghth

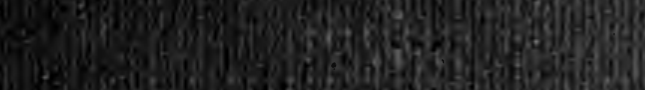

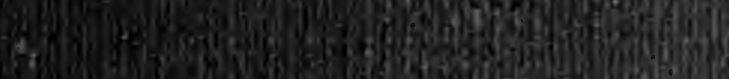

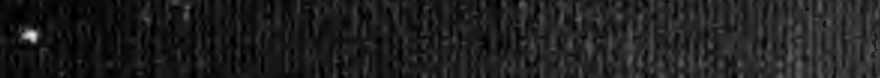

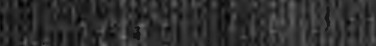

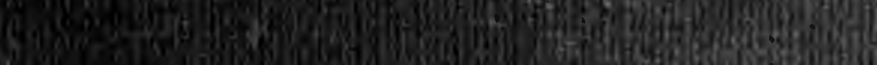

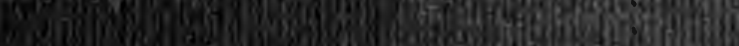

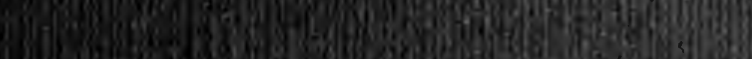

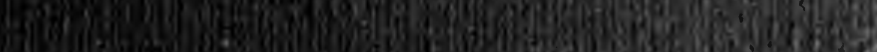
0.5

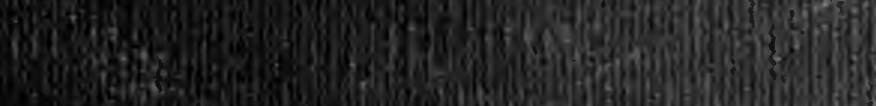

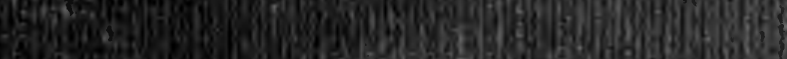

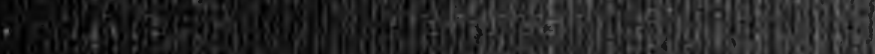

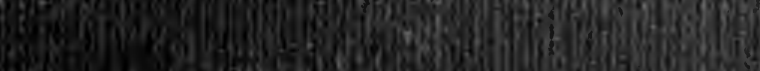

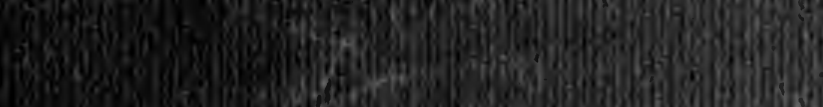

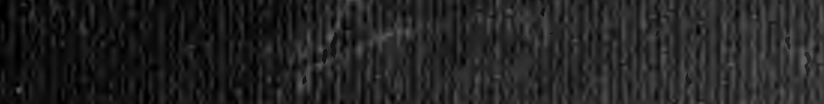
90.

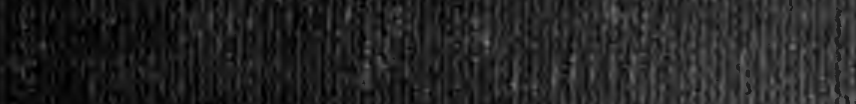
10.8. 18.

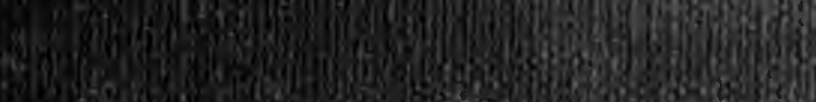
6:

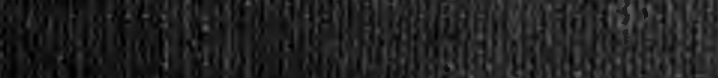

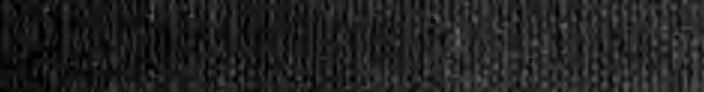
X.5.

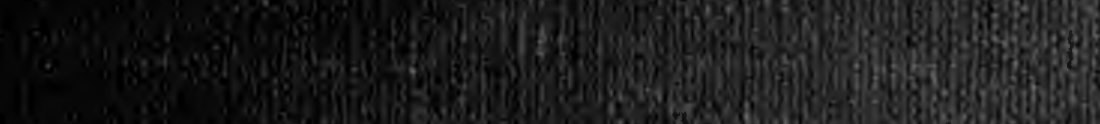
4.4. ing Qhes

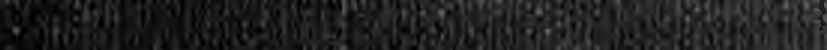

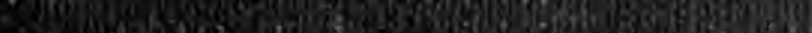
on now Rekikn

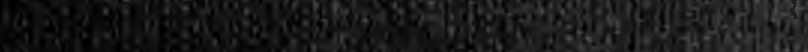
Nowa

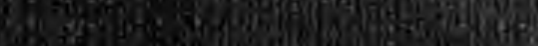
a s, los.

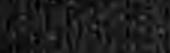




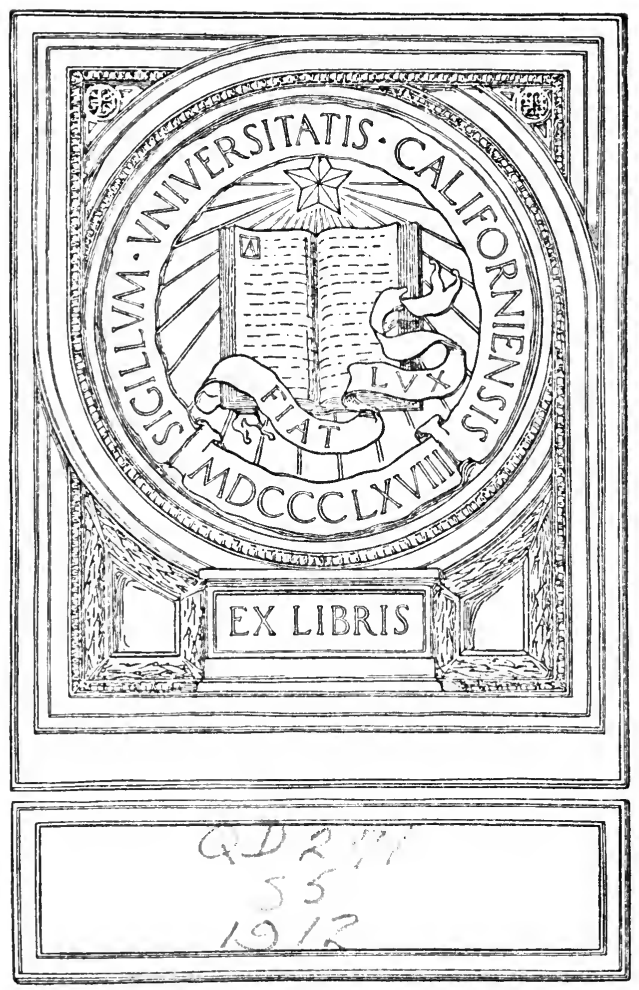



s.t.

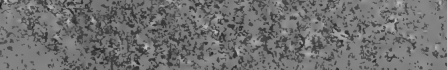

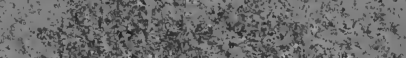

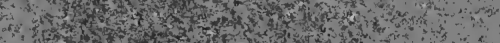

4 .

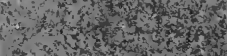

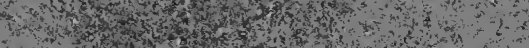

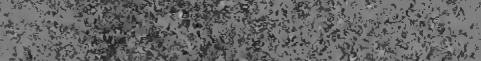

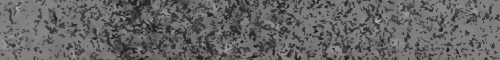

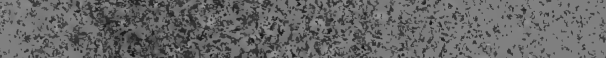

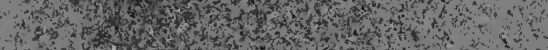

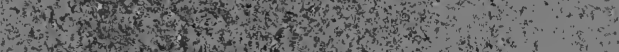

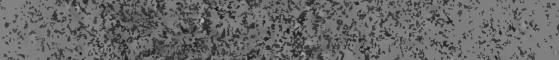

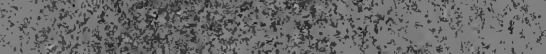

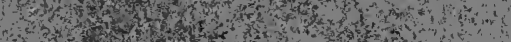

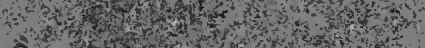

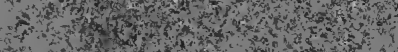

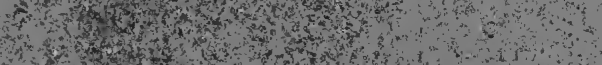

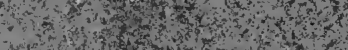

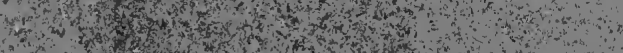

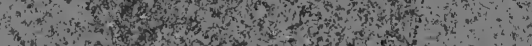

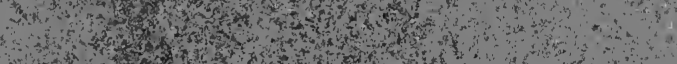

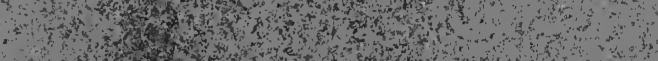

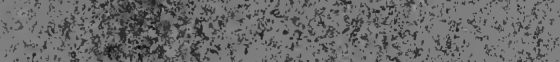

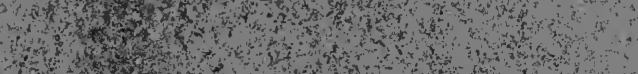

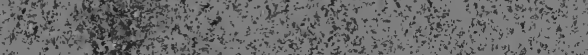

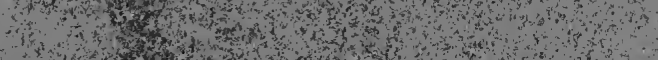

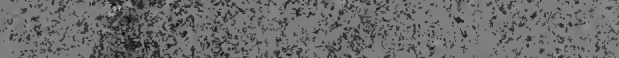

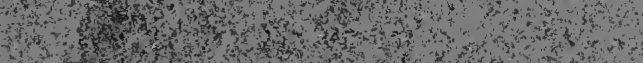

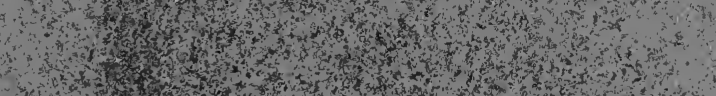

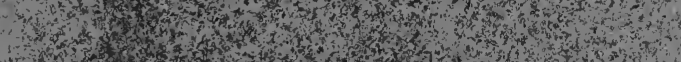

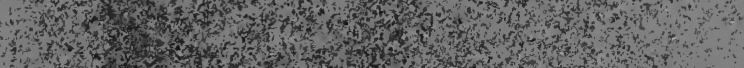

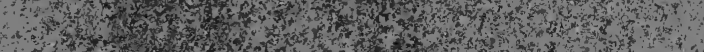

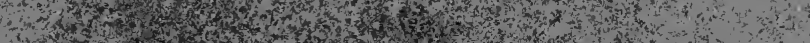

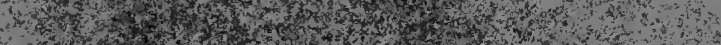

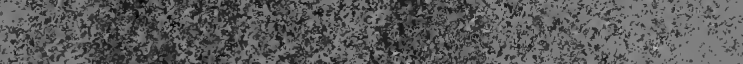

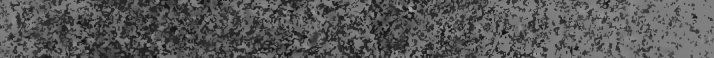

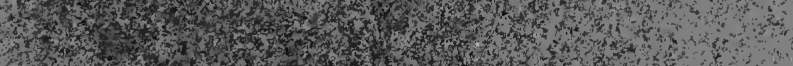

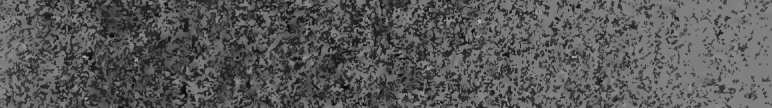

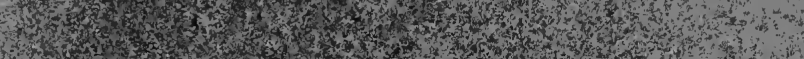

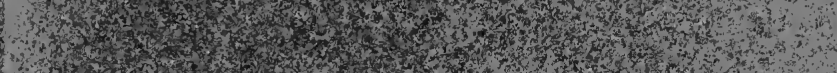

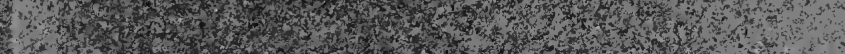

(3)

>

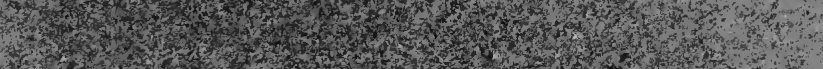

3.

1.

3
H.

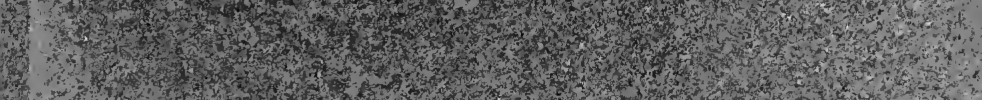

3

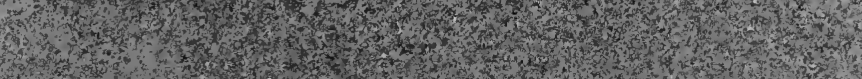

1. 


METHODS OF ORGANIC ANALYSIS 


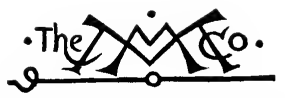

THE MACMILLAN COMPANY

NEW YORK - BOSTON - CHICAGO

DALLAS - SAN FRANCISCO

MACMILLAN \& CO., LIMITED

LONDON - BOMBAY - CALCUTTA

MELBOURNE

THE MACMillan CO. OF CANADA, Ltd. TORONTO 


\section{METHODS OF}

\section{ORGANIC ANALYSIS}

BY

HENRY C. SHERMAN, Рh.D.

PROFESSOR OF FOOD CHEMISTRY IN COLUMBIA UNIVERSITY AUTHOR OF "CHEMISTRY OF FOOD AND NUTRITION"

SECOND EDITION

REWRITTEN AND ENLARGED

Xerow Hark

THE MACMILLAN COMPANY

1912

All rights reserved 
Copyright, 1905, BY HENRY C. SHERMAN.

CoPYRIGHT, 1912, BY THE MACMILLAN COMPANY.

Set up and electrotyped. Published June, xqr2.

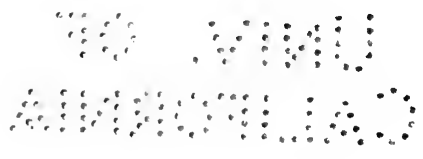




\section{PREFACE TO SECOND EDITION}

IN rewriting this work after six years of constant use in the classroom and laboratory, the author has endeavored to keep in mind the needs both of students and of practising chemists. Methods which are commonly used as exercises for beginners (such, for example, as the determination of alcohol by the distillation method) are fully described with detailed explanatory and precautionary notes, while those methods which are apt to be used only by advanced students or professional chemists are given more concisely. At the end of each chapter will be found, first a list of reference books arranged alphabetically by authors, and then a chronological list of journal articles, bulletins, etc., particularly of the last few years. The abbreviations are those used by the American Chemical Society in the publication of Chemical Abstracts.

The scope of the work has been somewhat extended, the new matter including a chapter on solid and liquid fuels, and sections on industrial alcohol, drying oils, crude petroleum, the new international methods of glycerin analysis, and quantitative methods for the testing of enzymes. The discussions of aldehydes, sugars, proteins, and food preservatives are also much fuller than in the first edition.

These additions, and the rewriting of the original text to embody recent advances and certain changes of arrangement which have been found advantageous from the standpoint of teaching, make the present edition practically a new work.

The text and references are designed to cover, along with the directions for laboratory work, so much at least of the technology of the various topics considered as is involved in a proper appreciation of the purposes of the analyses and the significance of the analytical results. 
The writer takes pleasure in acknowledging his indebtedness to Dr. C. A. Browne, Chemist-in-charge of the New York Sugar Trade Laboratory, and Mr. T. T. Gray, Chief Chemist of the Tide-Water Oil Company, for criticism of the sections relating to their specialties, and to his colleague, Dr. J. M. Nelson, for many helpful suggestions.

JANUARY, 1912.

H. C. S. 


\section{PREFACE TO FIRST EDITION}

THE purpose of this work is to give a connected introductory training in organic analysis, especially as applied to plant and animal substances and their manufactured products. No attempt is made to touch upon all important branches of this subject, but representative topics are treated in considerable detail with reference both to analytical methods and to the interpretation of results.

The greater part of the book is devoted to quantitative methods for food materials and related substances. Standard works of reference and the publications of the Association of Official Agricultural Chemists have been freely used. The nomenclature adopted in these publications has been followed as closely as possible. As a rule, footnotes show the original sources of statements or methods included in the text, while general or additional references are given at the end of each chapter. The references have been carefully selected and are believed to be sufficient to put the reader in touch/with the most important literature.

The descriptions of methods were written primarily for the use of third-year students in the School of Chemistry, Columbia University, and therefore presuppose a knowledge of inorganic quantitative analysis, elementary organic chemistry, and general physics.

The writer takes pleasure in acknowledging his indebtedness to Professor Edmund H. Miller for helpful advice and suggestions throughout the work, and to Mr. Roland H. Williams for assistance in testing methods and in the revision of parts of the manuscript.

New YoRk, July 1, 1905.

H. C. S. 



\section{CONTENTS}

INTRODUCTION PAGR

\section{CHAPTER I}

\section{Alcohols}

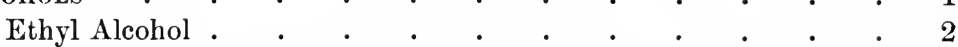

Detection and Identification of Ethyl Alcohol . . . 5

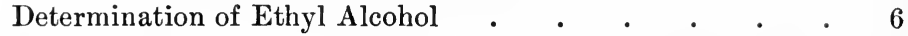

Determination and Identification of Small Amounts of Alcohol 23

Detection and Determination of Methyl Alcohol . • . 24

Determination of Amyl Alcohols or Fusel Oil . $\quad$ • 28

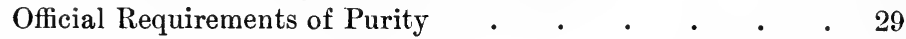

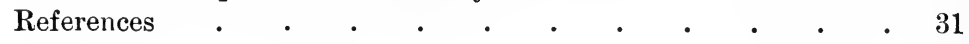

\section{CHAPTER II}

Aldehydes . . . . . . . . . . . . . 34

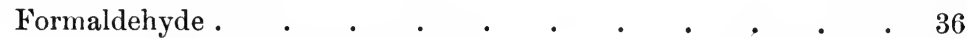

Detection and Identification . . . . . . . . $\quad . \quad 38$

Quantitative Determination . . . . . . . 40

Benzaldehyde . . . . . . . . . . . . . . 46

Vanillin . . . . . . . . . . . . . . . . .

References . . . . . . . . . . . .

\section{CHAPTER III}

Carbohydrates-General Methods

Occurrence and Relations

Solubilities

Reactions with Acids

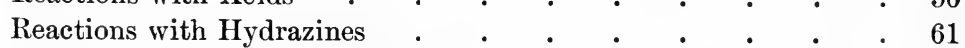

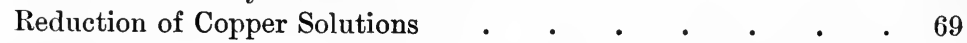

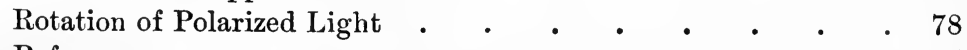

References 
CHAPTER IV

Special Methods of Sugar Analysis . . . . . . . 87

Analysis of Raw Sugar . . . . . . . . . . . . $\quad . \quad 87$

Polariscopic Examination $\quad . \quad$. $\quad . \quad$. $\quad . \quad$. $\quad$. 87

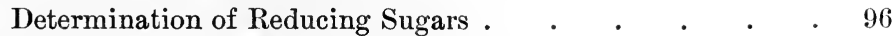

Determination of Moisture and Ash $\quad$. $\quad$. $\quad . \quad$. $\quad . \quad 97$

Determination of Sucrose in Beets and Cane . . . . . . $\quad .98$

Density and Purity of Sugar Solutions . . . . . . . 100

Identification and Analysis of "Unknown" Sugars . . . . 101

References . . . . . . . . . . . 103

\section{CHAPTER V}

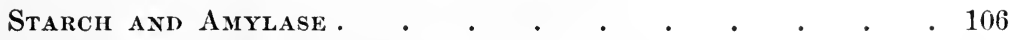

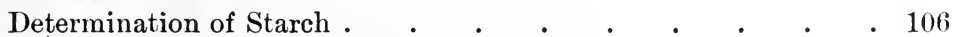

Diastatic Power of Amylases . $\quad . \quad$. $\quad . \quad$. $\quad . \quad$. $\quad .113$

References . . . . . . . . . . . 121

CHAPTER VI

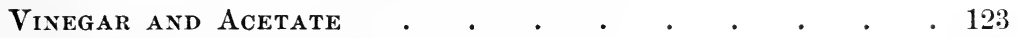

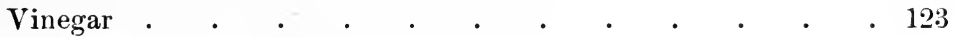

Determination of Source . . . . . . . . . 124

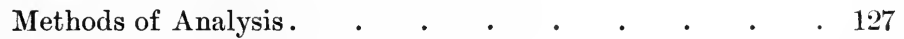

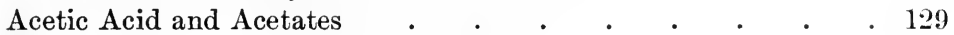

References . . . . . . . . . . . . 131

CHAPTER VII

Fatty Acids .

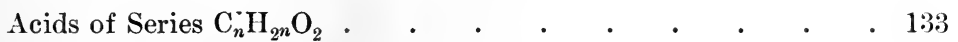

Acids of Series $\mathrm{C}_{n} \mathrm{H}_{2 n-2} \mathrm{O}_{2}$.

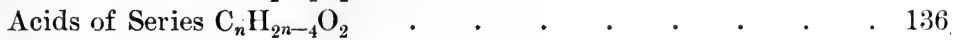

Acids of Series $\mathrm{C}_{n} \mathrm{H}_{2 n-6} \mathrm{O}_{2} \quad$.

Acids of Series $\mathrm{C}_{n} \mathrm{H}_{2 n-8} \mathrm{O}_{2}$.

Saturated Hydroxy Acids $\quad . \quad$. $\quad . \quad$. . . . . . . 137

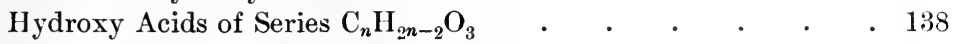

References . . . . . . . . . . . . 139

CHAPTER VIII

Oils, Fats, and Waxes-General Methods . • . . 140

Classification . ." . . . . . . . . . 141

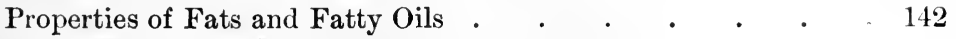


Analytical Methods

$\cdot \cdot \cdot \cdot \cdot \cdot 143$

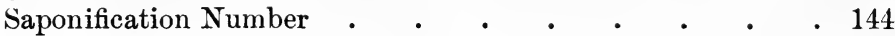

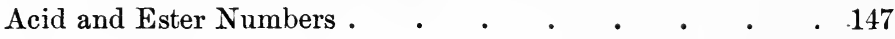

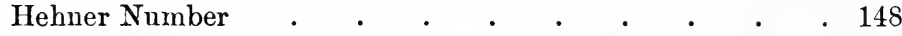

Reichert-Meissl Number . . . . . . . . . 148

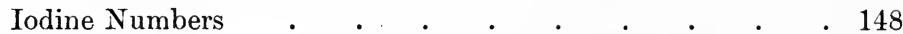

Maumené Number - Specific Temperature Reaction . . 157

Acetyl Number. . . . . . . . . . . . 160

Specific Gravity . . . . . . . . . . 162

Index of Refraction . . . . . . . . . . . . 164

Alcohols of Fats and Waxes - Unsaponifiable Matter . . . 169

References . . . . . . . . . . . 172

\section{CHAPTER IX}

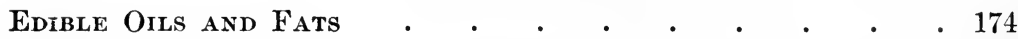

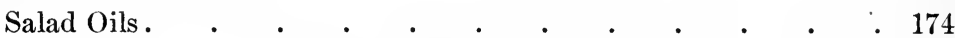

Analytical Properties of Olive Oil . . . . . . . 175

Detection of Adulterants . . . . . . . . . . 177

Butter

Determination of Water, Fat, Curd, Ash . . . . 186

Examination of Butter Fat . . . . . . . . 188

References . . . . . . . . . . . 199

\section{CHAPTER X}

DRYing OILS . . . . . . . . . . • . . 203

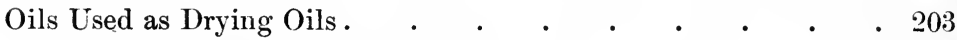

Analytical Properties of Linseed Oil $\quad . \quad$. $\quad . \quad$. $\quad$. $\quad 205$

Adulterants and Methods of Detection . . . . . . . 206

Oils Alteied by Age or Oxidation . . . . . . . . 210

"Unknown" Oils and Mixtures . . . . . . . . . 213

References . . . . . . . . . . . . 214

\section{CHAPTER XI}

Petroleum and Lubricating Oils . . . . . . . 218

Examination of Crude Petroleum . . . . . . . . 218

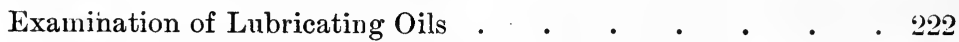

Determination of Constituents $\quad . \quad$. $\quad . \quad$. $\quad . \quad 223$

Viscosity . . . . . . . . . . . . . . 226

Acidity . . . . . . . . . . . . . . 231

Cold Test and Chilling Point or Cloud Test . • . . 232

Flashing and Burning Points . . . . . . . 233

Additional Determinations $\quad . \quad$. $\quad . \quad$. $\quad . \quad$. 234

Examination of Lubricating Greases . . . . . . . 235

References . . . . . . . . . . . . 236 


\section{CHAPTER XII}

\section{Fuels}

Determination of Calorific Power . $\quad$. $\quad . \quad$. $\quad . \quad$. $\quad$. 239

Chemical Composition and Calorific Power of Organic Compounds 245

Fuel Oils and Gasoline . . . . . . . . . 247

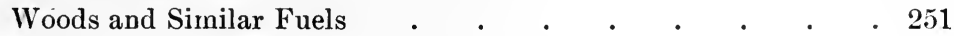

Coal . . . . . . . . . . . . . . 254

Ultimate Composition and Calorific Power . . . . 254

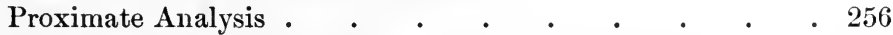

Relation of Proximate Composition to Calorific Power . . 257

References . . . . . . . . . . . 261

\section{CHAPTER XIII}

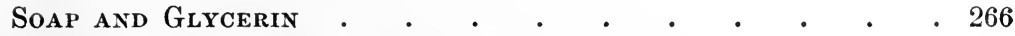

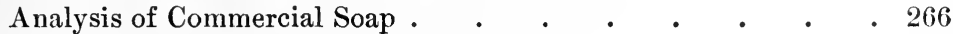

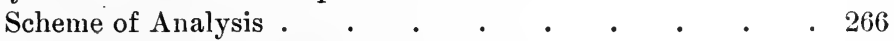

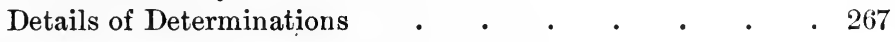

Glycerol . • . . . . • • • • • • 275

Analysis of Crude Glycerin • • • • • • . 276

References . . . . . . . . . . . . .

\section{CHAPTER XIV}

Nitrogen, Sulphur, and Phosphorus : . • • . . . 288

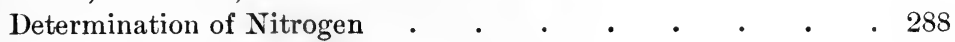

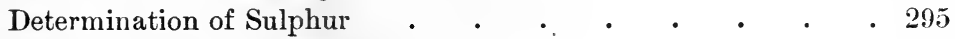

Determination of Phosphorus . . . . . . . . . . 303

References . . . . . . . . . . . . . 306

\section{CHAPTER XV}

Proteins and Proteases . . . . . . . . . . . 308

Analytical Reactions of Proteins . . . . . . . . . 313

Color Reactions . . . . . . . . . . . . . 313

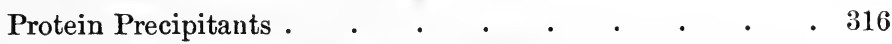

Separation of Proteins from Simpler Nitrogen Compounds and

from each other . . . . . . . . . . . . 322

Proteases or Proteolytic Enzymes . . . . . . . . . 323

References . • . . . • . . . . . . 329 


\section{CHAPTER XVI}

Grain Products

Preparation of Samples

Methods of Analysis

Interpretation of Results .

References

PAGE

334

334

335

343

346

\section{CHAPTER XVII}

Milk

Sampling and Preservation of Samples . . . . . . . 352

Preliminary or Partial Examination . . . . . . . 353

Determination of Fat, Proteins, Milk Sugar, and Ash . . 356

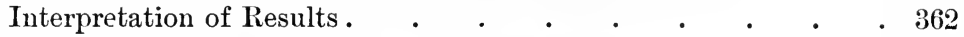

Examination of Milk Serum for Added Water . . . . $\quad 364$

Chemical Preservatives . . . . . . . . . 365

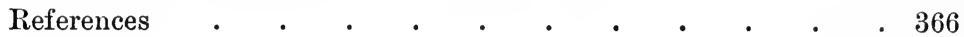

\section{CHAPTER XVIII}

Food Preservatives . . . . . . . . . . . . . . . . 369

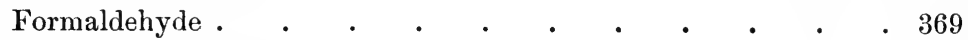

Hydrogen Peroxide . $\quad . \quad$. $\quad . \quad$. $\quad . \quad$. $\quad . \quad 372$

Boric Acid and Borates ..$\quad$. $\quad . \quad$. $\quad . \quad$. $\quad . \quad 373$

Fluorides. . . . . . . . . 375

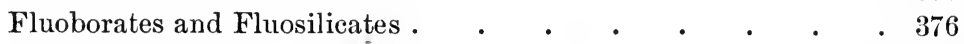

Sulphurous Acid . . . . . . . . . . . . .

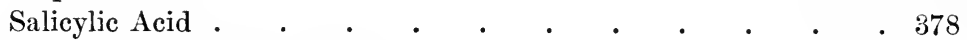

Benzoic Acid and Benzoates . . . . . . . . . . . 385

Saccharin . . . . . . . . . . . . 388

Beta-Naphthol . . . . . . . . . . . . . . .

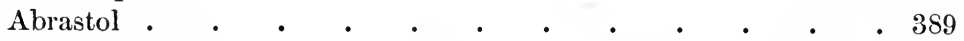

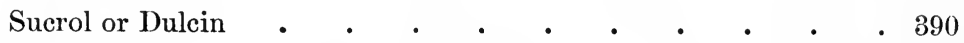

References • • • • • • • • • • • 391 
ing 


\section{INTRODUCTION}

Ultimate organic analysis is the determination of the elements composing any organic substance. Proximate organic analysis is the determination of the compounds present in a mixture or of the radicles present in a compound.

Both ultimate and proximate analysis are often required in the examination of organic materials. In the case of a complex mixture, however, proximate analysis is frequently directed to the determination of the principal groups of related compounds rather than of each individual compound present. Thus in the analysis of a food it may sometimes suffice to determine the percentage of moisture and of each of the groups of substances represented by the terms proteins, fats, carbohydrates, and ash constituents.

In the analysis of ordinary animal and vegetable substances it is usually difficult to make a clear distinction between organic and inorganic constituents, because in many cases the inorganic compounds found in the ash are formed during combustion, the bases having existed in combination with organic acids or with proteins, while the acid radicles may also have existed in organic combination or may have been formed by the oxidation of the carbon, sulphur, and phosphorus of the organic matter.

The chapters which follow are devoted chiefly to methods of proximate analysis. The determination of carbon and hydrogen by means of the combustion furnace is doubtless already familiar to most users of this book and is therefore omitted, but methods for determining nitrogen, sulphur, and phosphorus will be found in Chapter XIV. 
The sequence of topics is in the main that usually followed in textbooks of organic chemistry, but exceptions are made in some cases in order to bring together subjects which are closely related in practical interest. 


\section{METHODS OF}

\section{ORGANIC ANALYSIS}





\section{METHODS OF ORGANIC ANALYSIS}

\section{CHAPTER I}

\section{Alcohols}

Alcohols are neutral hydroxyl derivatives capable of reacting with acids to form esters, and the most characteristic alcohol reactions are those involving the replacement of the hydroxyl by an acid radicle. For detailed discussions of the analytical behavior of the hydroxyl radicle and characteristic reactions of the different groups of alcohols, the reader is referred to the works of Meyer, ${ }^{1}$ and of Meyer and Tingle, ${ }^{1}$ while Mulliken's tables ${ }^{1}$ may be followed in the systematic identification of individual pure preparations.

Of the analytical methods involving reactions of the hydroxyl group, the more important are those in which the acetyl or benzoyl ester is formed. The preparation of the dinitrobenzoate serves for the identification of ethyl alcohol, and glycerol may be determined quantitatively by acetylation. Often, however, esterification is either not quantitative or is less convenient or less delicate than other methods of determination. Thus the monatomic alcohols containing less than four carbon atoms mix freely with water and are not readily separated from it, but may be determined in aqueous solution either by physical methods or by the behavior of the alcohols on oxidation.

The present chapter will deal with ethyl alcohol and the detection and determination of a few of its more important homologues. Glycerol will be considered in connection with soap in a later chapter. For more extended discussions of the analytical chemistry of the alcohols the works cited at the end of the chapter may be consulted.

1 The titles of these and other works of reference will be found at the end of the chapter. 


\section{ETHYL ALCOHOL}

Pure ethyl alcohol is a colorless, mobile liquid of characteristic penetrating odor and "hot" pungent taste, boiling at about $78.4^{\circ}$ and showing at $15.56^{\circ}\left(60^{\circ} \mathrm{F}\right.$.), compared with water at the same temperature, a specific gravity of 0.79387 (Bureau of Standards). It mixes with water in all proportions and is only with great difficulty obtained in the anhydrous or "absolute" state. According to Allen the presence of as small proportion as 0.5 per cent of water in alcohol is indicated by the pink color assumed by the liquid on introducing a crystal of potassium permanganate. The so-called "absolute alcohol" used in analytical operations ordinarily contains from 0.2 to 1 per cent of water.

Alcohol is of great importance in organic analysis not only as a constituent to be determined but also as a solvent in analytical operations. It dissolves many organic substances which are not soluble in water, but inorganic compounds insoluble in water are usually also insoluble in alcohol. As a rule chlorides, bromides, iodides, and acetates are soluble in fairly strong alcohol, while carbonates, borates, sulphates, phosphates, oxalates, and tartrates are only very sparingly soluble.

Three strengths of alcohol are recognized in the U.S. Pharmacopœia of 1905 :

"Alcohol" containing about 92.3 per cent by weight or about 94.9 per cent by volume of actual ethyl alcohol and about 7.7 per cent by weight of water; specific gravity about 0.816 at $60^{\circ} \mathrm{F}$.

"Absolute Alcohol" containing not more than 1 per cent by weight of water; specific gravity not higher than 0.798 at $60^{\circ} \mathrm{F}$.

"Diluted Alcohol" made by mixing equal volumes of "alcohol" and water and containing about 41.5 per cent by weight or 48.9 per cent by volume of actual ethyl alcohol; specific gravity about 0.936 at $60^{\circ} \mathrm{F}$.

On mixing alcohol with water a considerable evolution of heat takes place and the volume of the mixture after cooling 
is less than the sum of the volumes of the alcohol and water mixed. This contraction is not uniformly proportional to the amount of alcohol in the mixture. Hence in mixtures of water and alcohol the relation between the percentages of alcohol "by volume" and "by weight" varies somewhat with the strength of the solutions. These variations together with the differences in the density of the supposedly absolute alcohol used as standard by the various observers account for the small discrepancies found on comparing the commonly used tables showing the relation between density and percentage.

In the United States the tables principally used are (1) those of the U. S. Bureau of Standards recalculated from the determinations made by Mendelejeff and (2) those based on Squibb's determinations, which have been very generally used in this country and incorporated in the methods of the Association of Official Agricultural Chemists and the U. S. Pharmacopœia.

The official chemists have under consideration the question of substituting the tables of the Bureau of Standards for those now official. The two systems differ chiefly in the conditions taken as standard, the final results when properly calculated being very nearly the same, especially for solutions containing less than 25 per cent of alcohol. The methods and data given in this chapter provide for the use of either system, Table I (beyond) being from the Bureau of Standards, and Table II condensed from the tables of the Official Agricultural Chemists and of the U. S. Pharmacopœia.

The table of Morley (J.Am. Chem. Soc., 26, 1185-1193), also based on the observations of Mendelejeff, gives the specific gravity of alcohol for each integral percentage by weight and for each degree of the hydrogen thermometer from $15^{\circ}$ to $22^{\circ} \mathrm{C}$.

For revenue purposes, both in the United States and Great Britain, the strength of alcoholic liquors is expressed in terms of "proof spirit," but the term has different meanings in the two countries.

American proof spirit is defined by Section 3249 of the Revised Statutes of the United States as follows: "Proof spirit shall be held to be that alcoholic liquor which contains one half 
its volume of alcohol of a specific gravity of seven thousand nine hundred and thirty-nine ten-thousandths at $60^{\circ} \mathrm{F}$." It is therefore practically 50 per cent by volume or about 42.5 per cent by weight.

British proof spirit is defined by Parliament as having such a density that at $57^{\circ} \mathrm{F}$. thirteen volumes shall weigh the same as twelve volumes of water at the same temperature. This corresponds to about 49.2 per cent by weight.

"Rectified spirit" of the British Pharmacopœia has 84 per cent by weight and British "methylated spirit" consists of nine parts of rectified spirit to one part of commercial " wood spirit" or "wood naphtha," the latter containing, according to the observations of Thorpe and Holmes, from 72 to 80 per cent of methyl alcohol by volume.

\section{Denatured Alcohol}

A statute of the United States of June 7, 1906, provides that domestic alcohol may be withdrawn from bond for'use in the arts and industries, and for fuel, light, and power, without the payment of an internal revenue tax, on condition that it shall have been denatured by the admixture of some material which destroys its character as a beverage and renders it unfit for liquid medicinal purposes.

The regular formula for denaturing alcohol is as follows: To 100 parts by volume of ethyl alcohol (not less than 90 per cent strength) add 10 parts of approved methyl (wood) alcohol and 0.5 part of approved benzine. Such alcohol is classed as completely denatured and becomes a regular article of commerce. The wood alcohol used in denaturing must have a specific gravity not above 0.830 at $60^{\circ} \mathrm{F}$. (corresponding, if impurities be neglected, to about 88 per cent strength, or to $91^{\circ}$ on Tralles scale) and meet several other requirements which include maximum and minimum limits for acetone and bromine-absorbing constituents and a maximum limit for esters.

The Bureau of Internal Revenue of the Treasury Department establishes the detailed regulations governing both the regular method of denaturing and the special formula permitted in the 
several classes of industries in which tax-free alcohol may be used, but for which the alcohol denatured by the regular formula would not be suitable. These formulæ and specifications are given in Regulation No. 30 of the U. S. Internal Revenue, together with the supplementary regulations and the series of "Treasury Decisions."

\section{Detection and Identification of Ethyl Alcohol}

\section{Lieben's Iodoform Test}

The "iodoform test," while not distinctive, is often useful. It may be carried out as follows : To $10 \mathrm{cc}$. of the clear liquid to be tested, add 5 or 6 drops of 10 per cent solution of sodium or potassium hydroxide, heat to about $50^{\circ} \mathrm{C}$., and add drop by drop with constant shaking a saturated solution of iodine in aqueous potassium iodide until the liquid becomes just permanently yellowish brown, then carefully decolorize by adding more of the hydroxide solution, avoiding excess. If alcohol were present, iodoform gradually separates out as a yellow or yellowish white crystalline deposit. Even when very little iodoform is produced its odor can usually be recognized. While the test is quite delicate, the appearance of a precipitate of iodoform does not prove the presence of ethyl alcohol, since it may result from various other compounds, especially acetone, aldehydes, and the propyl and butyl alcohols. If the original liquid contained carbohydrate or organic acid, it should be neutralized, distilled, and the first portion of the distillate used for the test.

\section{Ethyl Dinitrobenzoate Test}

As a specific test for ethyl alcohol Mulliken ${ }^{1}$ recommends the preparation of Ethyl 3, 5-Dinitrobenzoate as follows :

Heat together gently in a three-inch test tube held over a small flame, $0.15 \mathrm{gm}$. of 3,5-dinitrobenzoic acid and $0.20 \mathrm{gm}$. of phosphorus pentachloride. When signs of chemical action are seen, remove the heat for a few seconds. Then heat again,

1 Identification of Pure Organic Compounds, Vol. I, p. 168. 
boiling the liquefied mixture very gently for one minute. Pour out on a very small watchglass and allow to solidify. As soon as solidification occurs, remove the liquid phosphorus oxychloride, with which the crystalline mass is impregnated, by rubbing the latter between two small pieces of porous tile. Place the powder in a dry five- or six-inch test tube. Allow four drops of the alcohol (which must contain not more than about 10 per cent of water) to fall upon it, and then stopper the tube tightly without delay. Immerse the lower part of the test tube in water having a temperature of $75^{\circ}-85^{\circ}$. Shake gently, and continue the heating for ten minutes.

To purify the ester produced in the reaction, crush with a stirring rod any hard lumps which may form when the mixture cools and boil gently with 15 cc. of methyl alcohol (2:1) until all is dissolved, or for a minute or two. Filter boiling hot if the solution is not clear. Cool, shake and filter. Wash with 3 cc. cold methyl alcohol $(2: 1)$. Recrystallize from 9 cc. of boiling methyl alcohol (2:1). Wash with 2 cc. of the same solvent. Spread out the product on a piece of tile. Allow to become air-dry, and determine the melting point.

Ethyl 3, 5-Dinitrobenzoate, the product of this test, crystallizes in white needles melting at $92^{\circ}-93^{\circ}$ (uncorr.).

The corresponding derivatives of methyl, propyl, butyl, and isobutyl alcohols melt at $107.5^{\circ}$ (uncorr.), $73^{\circ}-73.5^{\circ}$ (uncorr.), $64^{\circ}$ (uncorr.), and $83^{\circ}-83.5^{\circ}$ (uncorr.), respectively.

\section{Other Methods}

The methods recommended by Bacon as being especially adapted to the detection and determination of such small amounts of alcohol as may be present as the result of incipient spoilage in food material are described later in this chapter.

\section{Determination of Ethyl Alcohol}

As already stated, the complete separation of ethyl alcohol from water is very difficult. The quantitative determination of the alcohol is therefore carried out in water solution and is usually accomplished by one of the following methods: from 
(1) the density, (2) the index of refraction, (3) the boiling point of the solution, or (4) by quantitative oxidation of the alcohol to acetic acid by heating in acid solution with potassium dichromate.

The first and second methods are seriously influenced by the presence of any substance other than the alcohol and water and are ordinarily applied only after distillation; the third method is less influenced by dissolved solids, and for approximate work may be applied directly to ordinary. alcoholic liquors, but is not so accurate as the specific gravity method; the fourth method is applicable only in the absence of any other substance capable of reacting with the acid-dichromate mixture, and is not readily adaptable to the determination of more than small amounts.

Generally the most accurate and satisfactory method is to separate the alcohol from substances other than water by distillation and find the amount of alcohol in the distillate by a careful determination of its specific gravity.

Preparations containing chloroform, ether, or essential oils may be treated as follows: ${ }^{1}$ Dilute $25 \mathrm{cc}$. of the sample with water to about $100 \mathrm{ec}$. in a separatory funnel, add sodium chloride to saturation and then 50 to $80 \mathrm{cc}$. of light petroleum distillate (boiling below $60^{\circ}$ ). Shake vigorously for five minutes, allow to stand for half an hour, draw off the lower layer into another separatory funnel, wash again in the same way with a small amount of petroleum ether, and then draw off into a distillation flask. Unite the petroleum ether layers, wash with two successive portions each 25 cc. of water saturated with salt, adding these washings to the main solution. The alcohol remains dissolved in the aqueous salt solution, which (after neutralizing if necessary) may be distilled as described below.

Carbon dioxide if present in large amount should be removed as well as possible in advance by shaking the liquor in a large flask at room temperature and avoiding the froth when taking the portions for analysis.

1 Thorpe and Holmes : J. Chem. Soc., 1903, 83, 314. 
Unless the appearance or odor of the sample suggests the presence of carbon dioxide or of chloroform, ether, or essential oil, it is usually permissible to proceed with the determination as described below without subjecting the sample to any of the above preliminary treatment.

\section{Specific Gravity Method}

In the case of a fermented liquor or other sample containing less than 25 per cent of alcohol (Note 1), dry and weigh a 100 cc. graduated flask, fill to the mark with the sample and weigh (Note 2); transfer to a distilling flask of about 300 cc. capacity, rinsing the measuring flask with about $50 \mathrm{cc}$. of water, bringing the total volume in the distilling flask to about 150 cc. Drop into the solution a small piece of delicate litmus paper, or better a few milligrams of solid phenolphthalein, and neutralize with a dilute solution of sodium or potassium hydroxide in order that no volatile acid may be distilled with the alcohol (Note 3). Connect the distilling flask with a wellcooled condenser and distill, collecting the distillate in a $100 \mathrm{cc}$. flask, preferably the one used in measuring the sample. The distillate should not be unnecessarily exposed to the air but should be conducted well into the receiving flask. If the condenser is vertical or nearly so, it is only necessary to support the flask in such a position that the condenser tube projects into the neck of the flask, otherwise an adapter may be used (Note 4). The apparatus may be arranged as shown in Fig. 1.

When the distillate amounts to nearly $100 \mathrm{cc}$. remove the receiver; and if the alcohol is to be reported in percentage by volume, the temperature should be brought to that at which the sample was measured out for analysis and water then added to bring the distillate exactly to $100 \mathrm{cc}$., but if the results are to be reported by weight, this adjustment is not important. See that the flask is clean and dry outside, weigh and subtract the weight of the empty flask as found at the beginning of the experiment. Mix the distillate well by shaking, and by means of a pyknometer (Note 5) determine carefully its specific 
gravity to the fifth decimal place, with special attention to the accurate control of the temperature, which should be governed according to the table which is to be used in finding the percentage of alcohol from the specific gravity (Note 6). Find in Table 1 the percentage of alcohol by weight, or in Table 2

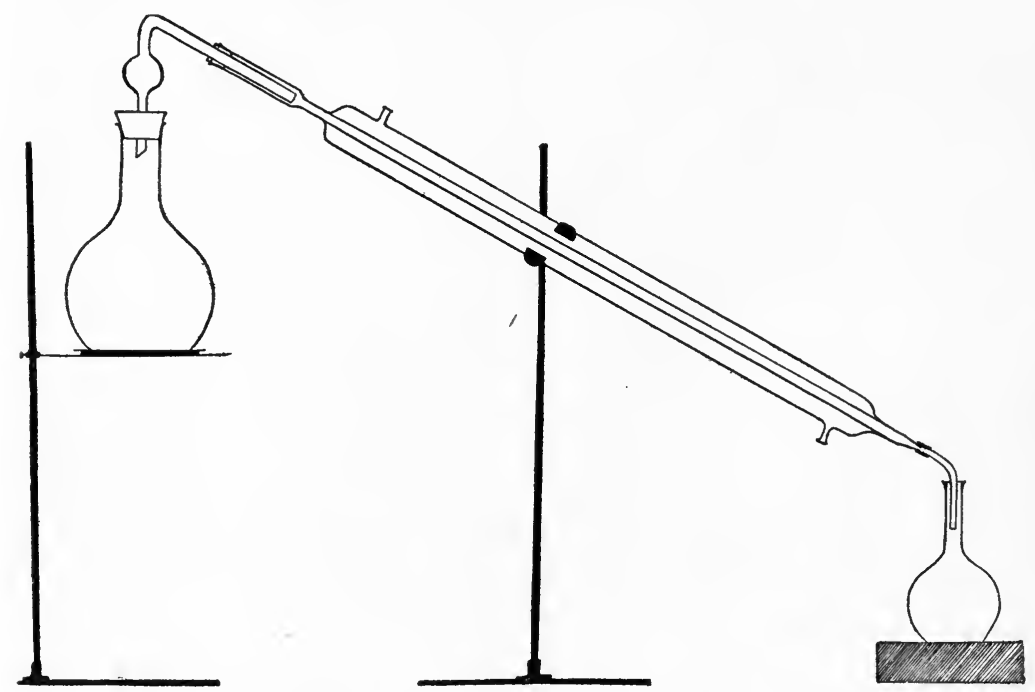

- Fig. 1. - Arrangement of distillation apparatus for alcohol determination.

the percentages by weight and by volume, corresponding to this specific gravity.

The percentage by weight of alcohol in the distillate, multiplied by the weight of the latter, shows the actual weight of alcohol distilled over, and this divided by the weight of sample taken gives the percentage by weight of alcohol in the liquor. The percentage by volume is of course the same for the original sample as for the distillate, if both are measured in the same flask at the same temperature.

Note 1. - If the sample contains more than 25 per cent of alcohol, a proportionately smaller amount than 100 cc. should be taken for the alcohol determination. Of cordials, $50 \mathrm{cc}$., and of distilled liquors or commercial alcohol, 25 cc., will usually be a suitable amount. If results are to be expressed in 
percentage by weight, the weight as well as the volume of the sample taken must of course be known.

Note 2. - If the specific gravity of the sample. is known, or if the results of the analysis are to be expressed only in percentage by volume or grams per $100 \mathrm{cc}$., the portion desired for the determination may be measured by means of a pipette. If, however, the same $100 \mathrm{cc}$. flask is used for measuring the sample and for the distillate and each is weighed as well as measured, the accuracy of the result whether expressed in percentage by weight or by volume will be independent of the accuracy of calibration of this measuring flask.

Note 3. - Most alcoholic beverages, unless they have undergone acetic fermentation, contain too little volatile acid to affect materially the result of the alcohol determination. It is, however, not safe to neglect the neutralization unless volatile acids are known to be absent.

Note 4. - A suitable arrangement for this distillation is shown in Fig. 1. The bulb in the connecting tube serves to prevent the mechanical carrying over of spray, and permits the distillation to be carried on at a fairly rapid rate, provided a sufficient stream of cold water flows through the condenser to thoroughly chill the distillate. If a distilling flask having side tube on the neck is used, the distillation should be conducted more slowly, in order to avoid danger from spray, and if possible a flask should be selected which has the exit tube high on the neck. If it is desired to expel the alcohol quickly, the solution may be saturated with salt before distilling.

Note 5. - On account of the importance of exact control of temperature, it is desirable to use either a thin-walled SprengelOstwald pyknometer, which may be hung in water of the desired temperature for adjustment, or a pyknometer bottle of the type carrying, a thermometer in the ground glass stopper and having a capillary side tube for overflow and adjustment. In either case the pyknometer is calibrated by weighing first empty and dry, then filled with recently boiled distilled water of known temperature. When using the pyknometer, care must be exercised to avoid warming it by contact with the hand. 
The Ostwald pyknometer (Fig. 2) has one arm ( $A$ ) of uniform bore, while the other arm carries a pipette body $(B)$ and ends in a capillary tip $(C)$. It is filled by attaching a small rubber tube at $C$ and drawing the liquid through $A$ until the entire pyknometer is full, when it is suspended in water at the desired temperature, and when this is reached the adjustment is made by touching the point $C$ with filter paper until just enough liquid is removed to bring the meniscus in $A$ to the graduation mark $M$; then cap the tips (if

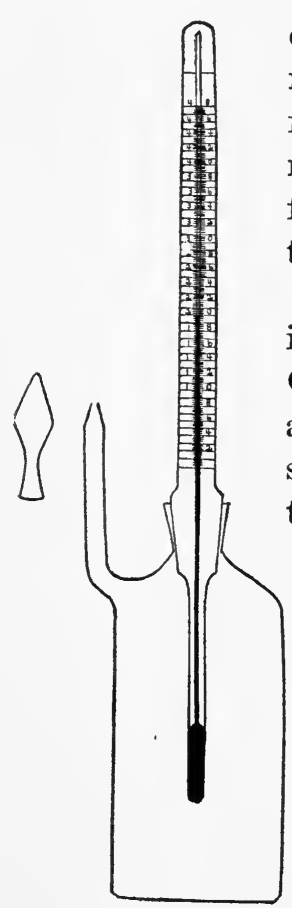

Fig. 3. - Specific gravity bottle with thermometer in stopper.

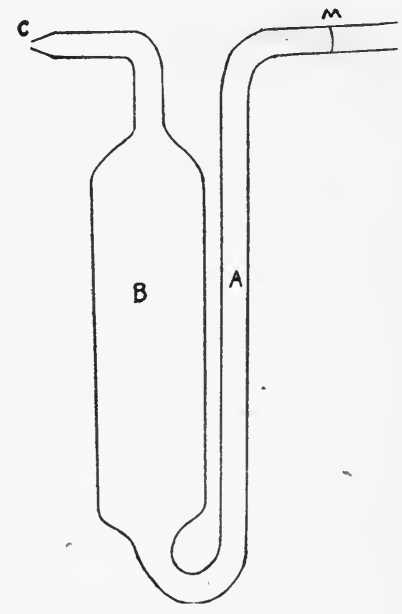

Frg. 2. - The Ostwald pyknometer. from the hook over the balance pan, and weigh.

The specific gravity bottle of the form shown in Fig. 3, while not so good in principle as the Ostwald pyknometer, is more widely used in analytical laboratories. The thermometer of such a bottle should (unless it bears a "control stamp") be tested, by comparison with a thermometer known to be accurate, at the temperature at which it is to be used. This pyknometer should always be filled either at or slightly below the temperature at which the weighing is to be made. Fill to about the middle of the ground portion of the neck so that when the stopper is inserted the liquid will overflow not only around the stopper, but also through the side tube. The stopper should be inserted snugly but without unnecessary force. As 
soon as the stopper is in place, wipe off the tip of the side tube, place the pyknometer in water (or air) maintained at the desired temperature, and notice whether any further overflow occurs. When the reading of the thermometer in the pyknometer and the position of the surface of the liquid at the tip of the side tube remain constant, the contents are evidently at the same temperature with the surrounding water or air. The glass cap is now placed over the tip, the pyknometer carefully wiped with a clean, dry cloth, weighed, and the weight of the empty pyknometer deducted.

Note 6. - The pyknometer, having been weighed with water as just described, is either dried or repeatedly rinsed with small portions of the distillate and then filled with the latter at a little below the standard temperature for the alcohol table which is to be used, then allowed to warm slowly up to the exact temperature desired and at exactly this temperature the volume is carefully adjusted, the pyknometer capped, wiped, and weighed as described in Note 5 .

To use Table 1, correct the weighings of water and of distillate by adding 0.00106 gram to each gram of water or distillate which the pyknometer apparently contains. (This corrects for the buoyant effect of the air when weighings are made with brass weights in air of average humidity at room temperature.) To simplify calculations adopt either $20^{\circ}$ or $25^{\circ}$ as the temperature for adjusting the pyknometer both with water and with the distillate. Divide the corrected weight of distillate by the corrected weight of water, thus obtaining the specific gravity at $\frac{20}{2} 0$ or $\frac{25}{25}$. Divide this by the density of water at $20^{\circ}$ $(0.998234)$ or $25^{\circ}(0.997077)$ as the case may be to obtain the density of the distillate at $\frac{20}{4}$ or $\frac{25}{4}$, from which by interpolation in Table 1 find the percentage by weight of alcohol in the distillate to the second decimal place.

If for any reason the temperature of adjustment has been between $20^{\circ}$ and $25^{\circ}$, the density of water at the known temperature of adjustment can be found from Table 3 below and the corrected weight of water calculated thus to $4^{\circ}$ can be used in finding the density of distillate for the temperature of adjustment. 
Table 1.-Density of Mixtures of Ethyl Alcohol and Water (Bureau of Standards)

\begin{tabular}{|c|c|c|c|c|c|c|c|}
\hline $\begin{array}{l}\text { Per cent } \\
\text { alcohol } \\
\text { by } \\
\text { weight }\end{array}$ & $D_{4}^{251}$ & $D_{\frac{20}{4}}^{20}$ & $D \frac{25}{4}$ & $\begin{array}{c}\text { Per cent } \\
\text { alcohol } \\
\text { by } \\
\text { weight }\end{array}$ & $\mathrm{D}_{\frac{16}{4}}$ & $D_{\frac{2 O}{4}}$ & $D_{\frac{25}{4}}$ \\
\hline $\begin{array}{l}0 \\
1 \\
2 \\
3 \\
4 .\end{array}$ & $\begin{array}{r}0.99913 \\
.99725 \\
.99543 \\
.99366 \\
.99197\end{array}$ & $\begin{array}{r}0.99824 \\
.99636 \\
.99453 \\
.99274 \\
.99102\end{array}$ & $\begin{array}{r}0.99708 \\
.99521 \\
.99338 \\
.99159 \\
.98984\end{array}$ & $\begin{array}{l}50 \\
51 \\
52 \\
53 \\
54\end{array}$ & $\begin{array}{r}0.91757 \\
.91566 \\
.91344 \\
.91120 \\
.90895\end{array}$ & $\begin{array}{r}0.91386 \\
.91164 \\
.90940 \\
.90715 \\
.90488\end{array}$ & $\begin{array}{r}0.90983 \\
.90758 \\
.90533 \\
.90307 \\
.90079\end{array}$ \\
\hline $\begin{array}{l}5 \\
6 \\
7 \\
8 \\
9\end{array}$ & $\begin{array}{l}.99033 \\
.98577 \\
.95726 \\
.98581 \\
.98442\end{array}$ & $\begin{array}{l}.98936 \\
.98776 \\
.98620 \\
.98470 \\
.98325\end{array}$ & $\begin{array}{l}.98815 \\
.98651 \\
.98491 \\
.98336 \\
.98185\end{array}$ & $\begin{array}{l}55 \\
56 \\
57 \\
58 \\
59\end{array}$ & $\begin{array}{l}.90670 \\
.90443 \\
.90215 \\
.89987 \\
.89758\end{array}$ & $\begin{array}{l}.90262 \\
.90034 \\
.89805 \\
.89576 \\
.69346\end{array}$ & $\begin{array}{l}.89851 \\
.89622 \\
.89392 \\
.89162 \\
.88931\end{array}$ \\
\hline $\begin{array}{l}10 \\
11 \\
12 \\
13 \\
14\end{array}$ & $\begin{array}{l}.98307 \\
.98176 \\
.98049 \\
.97925 \\
.97803\end{array}$ & $\begin{array}{l}.98185 \\
.95047 \\
.97913 \\
.97781 \\
.97651\end{array}$ & $\begin{array}{l}.98038 \\
.97893 \\
.97752 \\
.97612 \\
.97474\end{array}$ & $\begin{array}{l}60 \\
61 \\
62 \\
63 \\
64\end{array}$ & $\begin{array}{l}.89528 \\
.89297 \\
.89066 \\
.88 \$ 34 \\
.88601\end{array}$ & $\begin{array}{l}.89115 \\
.88853 \\
.88651 \\
.88418 \\
.88185\end{array}$ & $\begin{array}{l}.88700 \\
.88467 \\
.88234 \\
.88000 \\
.87766\end{array}$ \\
\hline $\begin{array}{l}15 \\
16 \\
17 \\
18 \\
19\end{array}$ & $\begin{array}{l}.97683 \\
.97563 \\
.97444 \\
.97324 \\
.97203\end{array}$ & $\begin{array}{l}.97522 \\
.97393 \\
.97264 \\
.97134 \\
.97003\end{array}$ & $\begin{array}{l}.97336 \\
.97199 \\
.97061 \\
.96922 \\
.96782\end{array}$ & $\begin{array}{l}65 \\
66 \\
67 \\
68 \\
69\end{array}$ & $\begin{array}{l}.85368 \\
.88134 \\
.87899 \\
.87664 \\
.87428\end{array}$ & $\begin{array}{l}.87950 \\
.87716 \\
.87480 \\
.87244 \\
.87008\end{array}$ & $\begin{array}{l}.87530 \\
.87295 \\
.87058 \\
.86821 \\
.86583\end{array}$ \\
\hline $\begin{array}{l}20 \\
21 \\
22 \\
23 \\
24\end{array}$ & $\begin{array}{l}.97080 \\
.96956 \\
.96829 \\
.96699 \\
.96566\end{array}$ & $\begin{array}{l}.96870 \\
.96736 \\
.96599 \\
.96459 \\
.96317\end{array}$ & $\begin{array}{l}.96640 \\
.96497 \\
.96352 \\
.96203 \\
.96052\end{array}$ & $\begin{array}{l}70 \\
71 \\
72 \\
73 \\
74\end{array}$ & $\begin{array}{l}.87192 \\
.86954 \\
.86716 \\
.86477 \\
.86237\end{array}$ & $\begin{array}{l}.86770 \\
.86532 \\
.86292 \\
.86052 \\
.85812\end{array}$ & $\begin{array}{l}.86344 \\
.86105 \\
.85864 \\
.85622 \\
.85380\end{array}$ \\
\hline $\begin{array}{l}25 \\
26 \\
27 \\
28 \\
29\end{array}$ & $\begin{array}{l}.96430 \\
.96289 \\
.96145 \\
.95997 \\
.95845\end{array}$ & $\begin{array}{l}.96171 \\
.96021 \\
.95568 \\
.95711 \\
.95550\end{array}$ & $\begin{array}{l}.95597 \\
.95739 \\
.95577 \\
.95412 \\
.95244\end{array}$ & $\begin{array}{l}75 \\
76 \\
77 \\
78 \\
79\end{array}$ & $\begin{array}{l}.85997 \\
.85755 \\
.85513 \\
.85270 \\
.85026\end{array}$ & $\begin{array}{l}.85570 \\
.85328 \\
.85084 \\
.84840 \\
.84595\end{array}$ & $\begin{array}{l}.85137 \\
.84893 \\
.84648 \\
.84403 \\
.84157\end{array}$ \\
\hline $\begin{array}{l}30 \\
31 \\
32 \\
33 \\
34\end{array}$ & $\begin{array}{r}.95688 \\
.95526 \\
. .95360 \\
.95191 \\
.95017\end{array}$ & $\begin{array}{l}.95385 \\
.95215 \\
.95042 \\
.94865 \\
.94684\end{array}$ & $\begin{array}{l}.95071 \\
.94594 \\
.94713 \\
.94529 \\
.94342\end{array}$ & $\begin{array}{l}80 \\
81 \\
82 \\
83 \\
84\end{array}$ & $\begin{array}{l}.84751 \\
.84534 \\
.84286 \\
.84037 \\
.83786\end{array}$ & $\begin{array}{l}.84349 \\
.84101 \\
.83852 \\
.83602 \\
.83350\end{array}$ & $\begin{array}{l}.83909 \\
.83660 \\
.83410 \\
.83159 \\
.82906\end{array}$ \\
\hline $\begin{array}{l}35 \\
36 \\
37 \\
38 \\
39\end{array}$ & $\begin{array}{l}.94839 \\
.94657 \\
.94471 \\
.94282 \\
.94089\end{array}$ & $\begin{array}{l}.94499 \\
.94311 \\
.94119 \\
.93924 \\
.93725\end{array}$ & $\begin{array}{l}.94152 \\
.93957 \\
.93760 \\
.93560 \\
.93356\end{array}$ & $\begin{array}{l}85 \\
86 \\
87 \\
88 \\
89\end{array}$ & $\begin{array}{l}.83534 \\
.83279 \\
.83022 \\
.82762 \\
.82500\end{array}$ & $\begin{array}{l}.83097 \\
.82542 \\
.82583 \\
.82323 \\
.82060\end{array}$ & $\begin{array}{l}. \$ 2652 \\
.82396 \\
.82137 \\
.81876 \\
.81613\end{array}$ \\
\hline $\begin{array}{l}40 \\
41 \\
42 \\
43 \\
44\end{array}$ & $\begin{array}{l}.93893 \\
.93694 \\
.93491 \\
.93286 \\
.93078\end{array}$ & $\begin{array}{l}.93524 \\
.93320 \\
.93113 \\
.92904 \\
.92693\end{array}$ & $\begin{array}{l}.93151 \\
.92943 \\
.92732 \\
.92519 \\
.92305\end{array}$ & $\begin{array}{l}90 \\
91 \\
92 \\
93 \\
94\end{array}$ & $\begin{array}{l}.82235 \\
.81966 \\
.81694 \\
.81418 \\
.81138\end{array}$ & $\begin{array}{l}.81795 \\
.81527 \\
.81255 \\
.80979 \\
.80700\end{array}$ & $\begin{array}{l}.81348 \\
.81080 \\
.80809 \\
.80534 \\
.80256\end{array}$ \\
\hline $\begin{array}{l}45 \\
46 \\
47 \\
48 \\
49\end{array}$ & $\begin{array}{l}.92868 \\
.92655 \\
.92441 \\
.92225 \\
.92006\end{array}$ & $\begin{array}{l}.92480 \\
.92264 \\
.92047 \\
.91 \$ 28 \\
.91608\end{array}$ & $\begin{array}{l}.92088 \\
.91870 \\
.91650 \\
.91429 \\
.91207\end{array}$ & $\begin{array}{l}95 \\
96 \\
97 \\
98 \\
99\end{array}$ & $\begin{array}{l}.80854 \\
.80564 \\
.80271 \\
.79972 \\
.79668\end{array}$ & $\begin{array}{r}.80417 \\
.80129 \\
.79838 \\
.79541 \\
.79240\end{array}$ & $\begin{array}{l}.79974 \\
.79689 \\
.79400 \\
.79106 \\
.78809\end{array}$ \\
\hline 50 & .91787 & .91386 & $.909 \$ 3$ & 100 & .79358 & $.7 \$ 933$ & .78507 \\
\hline
\end{tabular}

${ }^{1} \mathrm{D}_{\frac{4}{4}}^{15}=$ Density at $15^{\circ} \mathrm{C}$. referred to water at $4^{\circ} \mathrm{C}$. 
Suppose this is found to be 0.98123 at $\frac{23}{4}$; by comparing the figures in Table 1 for $\frac{20}{4}$ and $\frac{25}{4}$ at about this density it will be seen that the decrease of density in alcohol of this strength is about 0.000294 for each $1^{\circ} \mathrm{C}$. between $20^{\circ}$ and $25^{\circ}$. Hence by adding $3 \times 0.000294$ to 0.98123 (the density at $\frac{23}{4}$ ) we obtain 0.98211 as the density at $\frac{20}{4}$, which is found from Table 1 to correspond to 9.81 per cent of alcohol by weight. The interpolation for temperature is apt to introduce a slight error, for which reason as well as to simplify calculation it is preferable to adjust the pyknometer always at $20^{\circ}$ or $25^{\circ}$ if practicable.

To use Table 2 find the weight of distillate which the pyknometer holds at $15.56^{\circ}$ and divide by the weight of water which it holds at the same temperature. In this case the weighings are not corrected for displaced air (since this correction was apparently not made in determining the data from which the table was constructed), but it is necessary to exercise great care in the control of the temperature, since $15.56^{\circ}$ is much below the usual working temperature of the laboratory. The water content of the pyknometer may be observed at a higher temperature and calculated to $15.56^{\circ}$ from the data in Table 3 ; but since with rising temperature alcohol expands at an uneven rate the adjustment of the pyknometer with the distillate must be at or near the standard temperature, though if only a few degrees from standard the temperature may be taken, the weight divided by the weight of water at $15.56^{\circ}$ to find the "apparent specific gravity," then correct this by the following empirical formula given by Allen, ${ }^{1}$ in which $D$ is the specific gravity at $15.56^{\circ}, D^{\prime}$ the "apparent specific gravity," and $d$ the difference (in degrees centigrade) between $15.56^{\circ}$ and the temperature at which $D^{\prime}$ was determined:

$$
D=D^{\prime} \pm d\left(0.00014+\frac{1-D^{\prime}}{150}\right)
$$

The correction is added to or subtracted from $D^{\prime}$ according as the pyknometer was filled and adjusted at a temperature above or below the standard.

${ }^{1}$ Commercial Organic Analysis, Vol. I (3d Ed.) p. 93. 
Table 2.-Percentage of Alcohol by Weight and by Volume

[Recalculated from the determinations of Gilpin, Drinkwater, and Squibb, by EDgar Richards.]

\begin{tabular}{|c|c|c|c|c|c|c|c|c|}
\hline 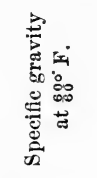 & 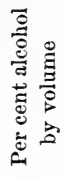 & 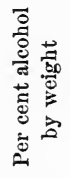 & 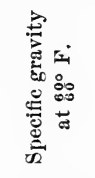 & 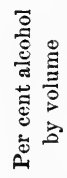 & 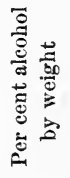 & 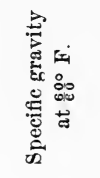 & 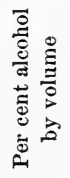 & 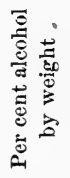 \\
\hline 1.00000 & 0.00 & 0.00 & 0.99281 & 5.00 & 4.00 & 0.98660 & 10.00 & 8.04 \\
\hline 0.99984 & .10 & .08 & 268 & .10 & .08 & 649 & .10 & .12 \\
\hline 968 & .20 & .16 & 255 & .20 & .16 & 637 & .20 & .20 \\
\hline 953 & .30 & .24 & 241 & .30 & .24 & 626 & .30 & .29 \\
\hline 937 & .40 & .32 & 228 & .40 & .32 & 614 & .40 & .37 \\
\hline .99923 & 0.50 & 0.40 & .99215 & 5.50 & 4.40 & .98603 & 10.50 & 8.45 \\
\hline 907 & .60 & .48 & 202 & .60 & .48 & 592 & .60 & .53 \\
\hline 892 & .70 & .56 & 189 & .70 & .56 & 580 & .70 & .61 \\
\hline 877 & .80 & .64 & 175 & .80 & .64 & 569 & .80 & .70 \\
\hline 861 & .90 & .71 & 162 & .90 & .72 & 557 & .90 & .78 \\
\hline .99849 & 1.00 & 0.79 & .99149 & 6.00 & 4.80 & .98546 & 11.00 & 8.86 \\
\hline 834 & .10 & .87 & 136 & .10 & .88 & 535 & .10 & .94 \\
\hline 819 & .20 & .95 & 123 & .20 & .96 & 524 & .20 & 9.02 \\
\hline 805 & .30 & 1.03 & 111 & .30 & 5.05 & 513 & .30 & .11 \\
\hline 790 & .40 & .11 & 098 & .40 & .13 & 502 & .40 & .19 \\
\hline .99775 & 1.50 & 1.19 & .99085 & 6.50 & 5.21 & .98491 & 11.50 & 9.27 \\
\hline 760 & .60 & .27 & 072 & .60 & .29 & 479 & .60 & .35 \\
\hline 745 & .70 & .35 & 059 & .70 & .37 & 468 & .70 & .43 \\
\hline 731 & .80 & .43 & 047 & .80 & .45 & 457 & .80 & .51 \\
\hline 716 & .90 & .51 & 034 & .90 & .53 & 446 & .90 & .59 \\
\hline .99701 & 2.00 & 1.59 & .99021 & 7.00 & 5.61 & .98435 & 12.00 & 9.67 \\
\hline 687 & .10 & .67 & 009 & .10 & .69 & 424 & .10 & .75 \\
\hline 672 & .20 & .75 & .98996 & .20 & .77 & 413 & .20 & .83 \\
\hline 658 & .30 & .83 & 984 & .30 & .86 & 402 & .30 & .92 \\
\hline 643 & .40 & .91 & 971 & .40 & .94 & 391 & .40 & 10.00 \\
\hline .99629 & 2.50 & 1.99 & .98959 & 7.50 & 6.02 & .98381 & 12.50 & 10.08 \\
\hline 615 & .60 & 2.07 & 947 & .60 & .10 & 370 & .60 & .16 \\
\hline 600 & .70 & .15 & 934 & .70 & .18 & 359 & .70 & .24 \\
\hline 586 & .80 & .23 & 922 & .80 & .26 & 348 & .80 & .33 \\
\hline 571 & .90 & .31 & 909 & .90 & .34 & 337 & .90 & .41 \\
\hline .99557 & 3.00 & 2.39 & .98897 & 8.00 & 6.42 & .98326 . & 13.00 & 10.49 \\
\hline 543 & .10 & .47 & 885 & .10 & .50 & 315 & .10 & .57 \\
\hline 529 & .20 & .55 & 873 & .20 & .58 & 305 & .20 & .65 \\
\hline 515 & .30 & .64 & 861 & .30 & .67 & 294 & .30 & .74 \\
\hline 501 & .40 & .72 & 849 & .40 & .75 & 283 & .40 & .82 \\
\hline .99487 & 3.50 & 2.80 & .98837 & 8.50 & 6.83 & .98273 & 13.50 & 10.90 \\
\hline 473 & .60 & .88 & 825 & .60 & .91 & 262 & .60 & .98 \\
\hline 459 & .70 & .96 & 813 & .70 & .99 & 251 & .70 & 11.06 \\
\hline 445 & .80 & 3.04 & 801 & .80 & 7.07 & 240 & .80 & .15 \\
\hline 431 & .90 & .12 & 789 & .90 & .15 & 230 & .90 & .23 \\
\hline .99417 & 4.00 & 3.20 & .98777 & 9.00 & 7.23 & .98219 & 14.00 & 11.31 \\
\hline 403 & .10 & .28 & 765 & .10 & .31 & 209 & .10 & .39 \\
\hline 390 & .20 & .36 & 754 & 20 & .39 & 198 & .20 & .47 \\
\hline 376 & .30 & .44 & 742 & .30 & .48 & 188 & .30 & .56 \\
\hline 363 & .40 & .52 & 730 & .40 & .56 & 177 & .40 & .64 \\
\hline .99349 & 4.50 & 3.60 & .98719 & 9.50 & 7.64 & .98167 & 14.50 & 11.72 \\
\hline 335 & .60 & .68 & 707 & .60 & .72 & 156 & .60 & .80 \\
\hline 322 & .70 & .76 & 695 & .70 & .80 & 146 & .70 & .88 \\
\hline 308 & .80 & .84 & 683 & .80 & .88 & 135 & .80 & .97 \\
\hline 295 & .90 & .92 & 672 & .90 & .96 & 125 & .90 & 12.05 \\
\hline
\end{tabular}


Percentage of Alcohol by Weight and by Volume. - Continued.

[Recalculated from the determinations of Gilpin, Drinkwater, and Squibb, by Edgar Richards.]

\begin{tabular}{|c|c|c|c|c|c|c|c|c|}
\hline 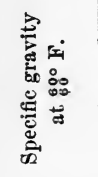 & 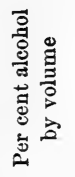 & 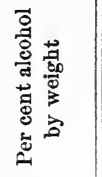 & 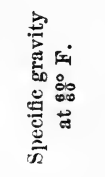 & 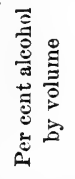 & 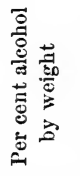 & 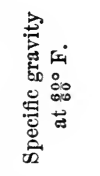 & 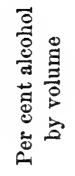 & 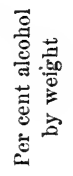 \\
\hline 0.98114 & 15.00 & 12.13 & 0.97608 & 20.00 & 16.26 & 0.97097 & 25.00 & 20.43 \\
\hline 104 & .10 & .21 & 598 & .10 & .34 & 086 & .10 & .51 \\
\hline 093 & .20 & .29 & 588 & .20 & .42 & 076 & .20 & .60 \\
\hline 083 & .30 & .38 & 578 & .30 & .51 & 065 & .30 & .68 \\
\hline 073 & .40 & .46 & 568 & .40 & .59 & 055 & .40 & .77 \\
\hline .98063 & 15.50 & 12.54 & .97558 & 20.50 & 16.67 & .97044 & 25.50 & 20.85 \\
\hline 052 & .60 & .62 & 547 & .60 & .75 & 033 & .60 & .93 \\
\hline 042 & .70 & .70 & 537 & .70 & .84 & 023 & .70 & 21.02 \\
\hline 032 & .80 & .79 & 527 & .80 & .92 & 012 & .80 & .10 \\
\hline 021 & .90 & .87 & 517 & .90 & 17.01 & 001 & .90 & .19 \\
\hline .98011 & 16.00 & 12.95 & .97507 & 21.00 & 17.09 & .96991 & 26.00 & 21.27 \\
\hline 001 & .10 & 13.03 & 497 & .10 & .17 & 980 & .10 & .35 \\
\hline .97991 & .20 & .12 & 487 & .20 & .26 & 969 & .20 & .44 \\
\hline 980 & .30 & .20 & 477 & .30 & .34 & 959 & .30 & .52 \\
\hline 970 & .40 & .29 & 467 & .40 & .43 & 949 & .40 & .61 \\
\hline .97960 & 16.50 & 13.37 & .97457 & 21.50 & 17.51 & .96937 & 26.50 & 21.69 \\
\hline 950 & .60 & .45 & 446 & .60 & .59 & 926 & .60 & .77 \\
\hline 940 & .70 & .53 & 436 & .70 & .67 & 915 & .70 & .86 \\
\hline 929 & .80 & .62 & 426 & .80 & .76 & 905 & .80 & .94 \\
\hline 919 & .90 & .70 & 416 & .90 & .84 & 894 & .90 & 22.03 \\
\hline .97909 & 17.00 & 13.78 & .97406 & 22.00 & 17.92 & .96883 & 27.00 & 22.11 \\
\hline 899 & .10 & .86 & 396 & .10 & 18.00 & 872 & .10 & .20 \\
\hline 889 & .20 & .94 & 386 & .20 & .09 & 861 & .20 & .28 \\
\hline 879 & .30 & 14.03 & 375 & .30 & .17 & 850 & .30 & .37 \\
\hline 869 & .40 & .11 & 365 & .40 & .26 & 839 & .40 & .45 \\
\hline .97859 & 17.50 & 14.19 & .97355 & 22.50 & 18.34 & .96828 & 27.50 & 22.54 \\
\hline 848 & .60 & .27 & 345 & .60 & .42 & 816 & .60 & .62 \\
\hline 838 & .70 & .35 & 335 & .70 & .51 & 805 & .70 & .71 \\
\hline 828 & .80 & .44 & 324 & .80 & .59 & 794 & .80 & .79 \\
\hline 818 & .90 & .52 & 314 & .90 & .68 & 783 & .90 & .88 \\
\hline .97808 & 18.00 & 14.60 & .97304 & 23.00 & 18.76 & .96772 & 28.00 & 22.96 \\
\hline 798 & .10 & .68 & 294 & .10 & .84 & 761 & .10 & 23.04 \\
\hline 788 & .20 & .77 & 283 & .20 & .92 & 749 & .20 & .13 \\
\hline 778 & .30 & .85 & 273 & .30 & 19.01 & 738 & .30 & .21 \\
\hline 768 & .40 & .94 & 263 & .40 & .09 & 726 & .40 & .30 \\
\hline .97758 & 18.50 & 15.02 & .97253 & 23.50 & 19.17 & .96715 & 28.50 & 23.38 \\
\hline 748 & .60 & .10 & 242 & .60 & .25 & 704 & .60 & .47 \\
\hline 738 & .70 & .18 & 232 & .70 & .34 & 692 & .70 & .55 \\
\hline 728 & .80 & .27 & 222 & .80 & .42 & 681 & .80 & .64 \\
\hline 718 & .90 & .38 & 211 & .90 & .51 & 669 & .90 & .72 \\
\hline .97708 & 19.00 & 15.43 & .97201 & 24.00 & 19.59 & .96658 & 29.00 & 23.81 \\
\hline 698 & .10 & .51 & 191 & .10 & .67 & 646 & .10 & .89 \\
\hline 688 & .20 & .59 & 180 & .20 & .76 & 635 & .20 & .98 \\
\hline 678 & .30 & .68 & 170 & .30 & .84 & 623 & .30 & 24.06 \\
\hline 668 & .40 & .76 & 159 & .40 & .93 & 611 & .40 & .15 \\
\hline .97658 & 19.50 & 15.84 & .97149 & 24.50 & 20.01 & .96600 & 29.50 & 24.23 \\
\hline 648 & .60 & .93 & 139 & .60 & .09 & 587 & .60 & .32 \\
\hline 638 & .70 & 16.01 & 1.28 & .70 & .18 & 576 & .70 & .40 \\
\hline 628 & .80 & .09 & 118 & .80 & .26 & 564 & .80 & .49 \\
\hline 618 & .90 & .18 & 107 & .90 & .35 & 553 & .90 & .57 \\
\hline
\end{tabular}


In the writer's laboratory this formula has given fairly satisfactory results when applied to temperatures differing but little from the standard. The actual weighing of the pyknometer must always be made at a temperature above the "dew point," but a pyknometer which provides for expansion of its contents without loss may be filled and adjusted at the standard temperature and then allowed to stand until it reaches room temperature before weighing.

Table 3. - Density of Pure Water free from Air ${ }^{1}$

\begin{tabular}{c|c|c|c}
\hline \hline Temperature & Density & Temperature & Density \\
\cline { 2 - 3 } $4^{\circ} \mathrm{C}$. & 1.0000000 & $21^{\circ} \mathrm{C}$. & 0.9980233 \\
15 & 0.9991266 & 22 & 0.9978019 \\
15.56 & 0.9990415 & 23 & 0.9975702 \\
16 & 0.9989705 & 24 & 0.9973286 \\
17 & 0.9988029 & 25 & 0.9970770 \\
18 & 0.9986244 & 26 & 0.9968158 \\
19 & 0.9984347 & 27 & 0.9965451 \\
20 & 0.9982343 & 28 & 0.9962652 \\
\hline \hline
\end{tabular}

\section{Refractometer Method}

In solutions containing only alcohol and water, such as the distillates obtained in the specific gravity method above, described, the percentage of alcohol can be found from the index of refraction as well as from the specific gravity. The recently introduced "immersion refractometer" is the most convenient form of apparatus for this purpose. Figure 4 shows the apparatus complete in position for an observation, while Fig. 5 shows the positions of the principal parts.

The liquid to be examined is placed in a small beaker surrounded by water of the required temperature, usually $17.5^{\circ}$ or $20^{\circ}$, and the refractometer is suspended from the wire frame

1 According to Chappius (Bureau international des Poids et Mesures, Travaux et Mémoires XIII, 1907). The data given above are taken from the table published by the Bureau of Standards, which shows density for each tenth of a degree from 0 to $41^{\circ} \mathrm{C}$., referred to water at $4^{\circ} \mathrm{C}$. as unity. 
in such a position that the prism is immersed in the liquid to be observed. By means of a mirror the light from a window is reflected through the glass bottom of the water bath and upward through the refractometer. On looking downward through the ocular $O c$ and the telescope of the refractometer

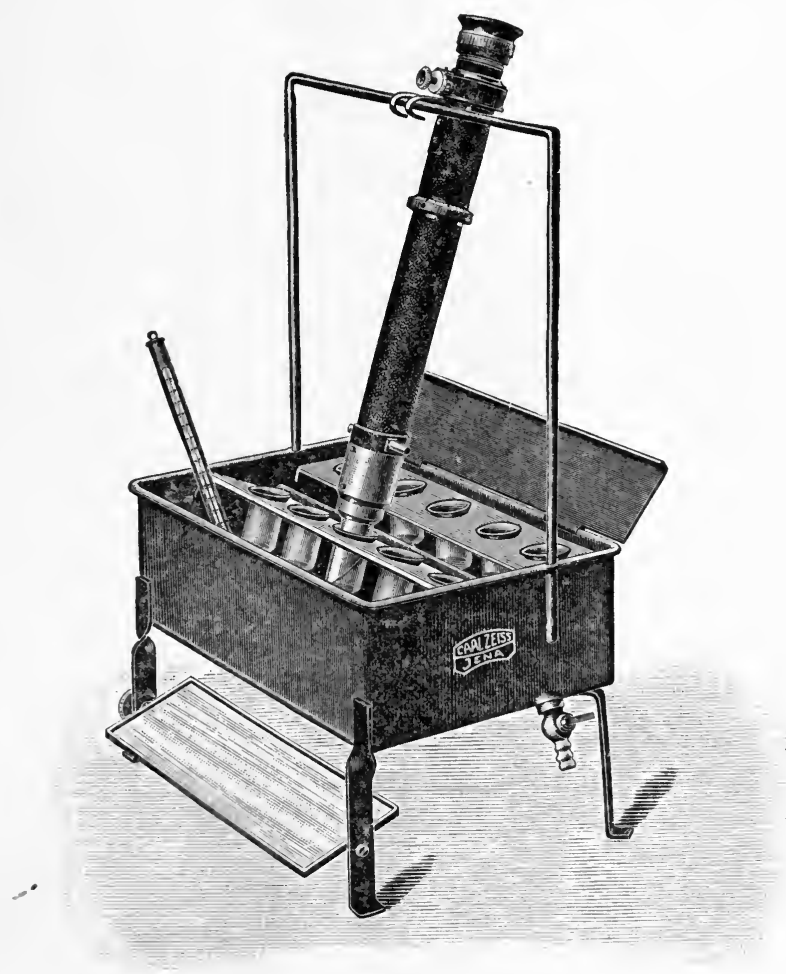

Fig. 4. - General view of immersion refractometer.

(Courtesy of Eimer and Amend.)

one observes the border line of total reflection, the upper part of the field of vision being light and the lower part shaded. A scale, marked in degrees of arbitrary but known and constant value, extends from top to bottom of the field of vision, and the position of the border of the shadow upon this scale indicates the index of refraction. The index of refraction cor- 
responding to each degree of the immersion refractometer scale is shown in Table 4.

In using this refractometer, after everything is in position it should be allowed to stand ten minutes before taking observations in order to insure uniformity of temperature. The

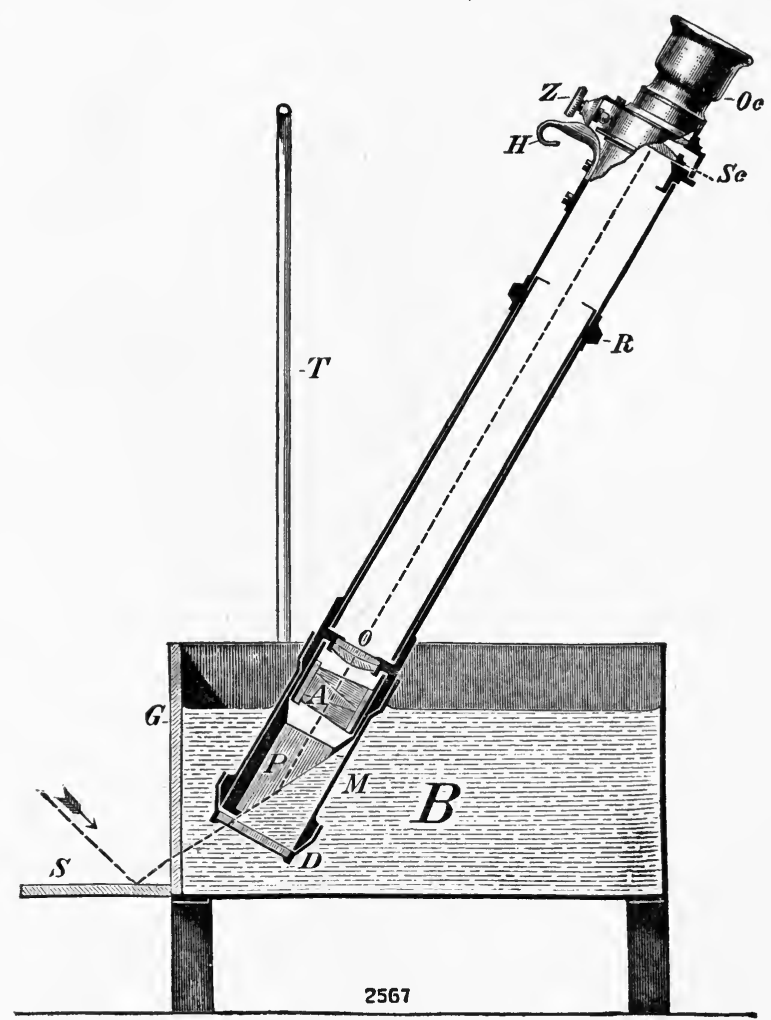

FIG. 5. - Sectional view of immersion refractometer.

(Courtesy of Eimer and Amend.)

adjustment of the instrument should first be tested by taking reading on distilled water. The index at $R$ should stand at 5 and the ocular $O c$ should be focused so that the edge of the shadow is clearly marked. If when the micrometer screw $Z$ stands at zero the line lies between two of the scale degrees, its 
Table 4.- Index of Refraction for each Degree on Scale of THE IMmersion Refractometer

\begin{tabular}{|c|c|c|c|c|c|}
\hline Scale reading & Index of refraction & Scale reading & Index of refraction & Scale reading & Index of refraction \\
\hline-5 & 1.32539 & 32 & 1.33972 & 69 & 1.35352 \\
\hline-4 & 1.32578 & 33 & 1.34010 & 70 & 1.35388 \\
\hline-3 & 1.32618 & 34 & 1.34048 & 71 & 1.35425 \\
\hline-2 & 1.32657 & 35 & 1.34086 & 72 & 1.35461 \\
\hline-1 & 1.32696 & 36 & 1.34124 & 73 & 1.35497 \\
\hline 0 & 1.32736 & 37 & 1.34162 & 74 & 1.35533 \\
\hline 1 & 1.32775 & 38 & 1.34199 & 75 & 1.35569 \\
\hline 2 & 1.32814 & 39 & 1.34237 & 76 & 1.35606 \\
\hline 3 & 1.32854 & 40 & 1.34275 & 77 & 1.35642 \\
\hline 4 & 1.32893 & 41 & 1.34313 & 78 & 1.35678 \\
\hline 5 & 1.32932 & 42 & 1.34350 & 79 & 1.35714 \\
\hline 6 & 1.32971 & 43 & 1.34388 & 80 & 1.35750 \\
\hline 7 & 1.33010 & 44 & 1.34426 & 81 & 1.35786 \\
\hline 8 & 1.33049 & 45 & 1.34463 & 82 & 1.35822 \\
\hline 9 & 1.33087 & 46 & 1.34500 & 83 & 1.35858 \\
\hline 10 & 1.33126 & 47 & 1.34537 & 84 & 1.35894 \\
\hline 11 & 1.33165 & 48 & 1.34575 & 85 & 1.35930 \\
\hline 12 & 1.33204 & 49 & 1.34612 & 86 & 1.35966 \\
\hline 13 & 1.33242 & 50 & 1.34650 & 87 & 1.36002 \\
\hline 14 & 1.33281 & 51 & 1.34687 & 88 & 1.36038 \\
\hline 15 & 1.33320 & 52 & 1.34724 & 89 & 1.36074 \\
\hline 16 & 1.33358 & 53 & 1.34761 & 90 & 1.36109 \\
\hline 17 & 1.33397 & 54 & 1.34798 & 91 & 1.36145 \\
\hline 18 & 1.33435 & 55 & 1.34836 & 92 & 1.36181 \\
\hline 19 & 1.33474 & 56 & 1.34873 & 93 & 1.36217 \\
\hline 20 & 1.33513 & 57 & 1.34910 & 94 & 1.36252 \\
\hline 21 & 1.33551 & 58 & 1.34947 & 95 & 1.36287 \\
\hline 22 & 1.33590 & 59 & 1.34984 & 96 & 1.36323 \\
\hline 23 & 1.33628 & 60 & 1.35021 & 97 & 1.36359 \\
\hline 24 & 1.33667 & 61 & 1.35058 & 98 & 1.36394 \\
\hline 25 & 1.33705 & 62 & 1.35095 & 99 & 1.36429 \\
\hline 26 & 1.33743 & 63 & 1.35132 & 100 & 1.36464 \\
\hline 27 & 1.33781 & 64 & 1.35169 & 101 & 1.36500 \\
\hline 28 & 1.33820 & 65 & $1.35205 \tilde{5}$ & 102 & 1.36535 \\
\hline 29 & 1.33858 & 66 & 1.35242 & 103 & - 1.36570 \\
\hline 30 & 1.33896 & 67 & 1.35279 & 104 & 1.36605 \\
\hline 31 & 1.33934 & 68 & 1.35316 & 105 & 1.36640 \\
\hline
\end{tabular}


position may be estimated in tenths of a degree by the eye or the micrometer screw $Z$ may be turned until the line of the shadow comes exactly to a scale division. The latter then indicates the whole degrees and the tenths are read from the scale on the micrometer screw. This method is usually more accurate than estimating the tenths by the eye alone, and has the advantage that one may quickly turn the micrometer screw back to zero and repeat the operation as often as desired, finally averaging the readings. With water the reading, depending upon the temperature, should be as follows:

\begin{tabular}{l|c|c|c|c|c|c|c|c|c|c|c|c}
\hline \hline Temperature C. & $17.5^{\circ}$ & $20^{\circ}$ & $21^{\circ}$ & $22^{\circ}$ & $23^{\circ}$ & $24^{\circ}$ & $25^{\circ}$ & $26^{\circ}$ & $27^{\circ}$ & $28^{\circ}$ & $29^{\circ}$ & $30^{\circ}$ \\
Scale reading & 15.0 & 14.5 & 14.25 & 14.0 & 13.75 & 13.5 & 13.25 & 13.0 & 12.7 & 12.4 & 12.1 & 11.8 \\
\hline
\end{tabular}

Having thus tested the adjustment of the instrument by readings in water, the readings in the alcoholic solution are taken in the same way. The percentages by weight of alcohol corresponding to the scale readings at $17.5^{\circ}$ as found by Ackermann and Steinmann ${ }^{1}$ are as follows:

Tarle 5. - Percentages by Weight of Alcohol Corresponding to Scale Readings of Zeiss Immersion Refractometer at $17.5^{\circ} \mathrm{C}$. (Ackermann and Steinmann)

\begin{tabular}{c|c|c|c}
\hline \hline Scale reading & Alcohol per cent & Scale reading & Alcohol per cent \\
\cline { 2 - 3 } 15.0 & 0.00 & 19.5 & 2.80 \\
15.5 & 0.32 & 20.0 & 3.10 \\
16.0 & 0.64 & 20.5 & 3.38 \\
16.5 & 0.95 & 21.0 & 3.67 \\
17.0 & 1.25 & 21.5 & 3.96 \\
17.5 & 1.57 & 22.0 & 4.22 \\
18.0 & 1.87 & 22.5 & 4.49 \\
18.5 & 2.19 & 23.0 & 4.76 \\
19.0 & 2.49 & 23.5 & 5.02 \\
\hline \hline
\end{tabular}

For tables extending to higher percentages of alcohol (sometimes expressed as percentage by volume or grams per $100 \mathrm{cc}$.$) ,$ 1 Ztschr. f. der gesamte Brauwesen, 28 (1905). 
see papers by Wagner, Wagner and Schultze, and Doroshevski and Dvorzhanchik among references given at the end of this chapter. The table given by the last-named authors is particularly noteworthy, since it gives the actual index of refraction for alternate percentages of alcohol from 0 to 100 and for the temperatures $17.5^{\circ}, 20^{\circ}, 22^{\circ}, 24^{\circ} \mathrm{C}$.

For a fuller description of the immersion refractometer see Leach's Food Inspection and Analysis (latest edition) or the circulars furnished by the manufacturer of the instrument.

\section{Boiling Point Method}

In mixtures of alcohol and water containing no appreciable amount of other volatile substances and only small quantities of dissolved solids, the difference between the boiling point of the mixture and that of pure water under the same conditions gives a measure of the percentage of alcohol present. For the rapid determination of alcohol on this principle, several forms of ebullioscope have been devised. The liquid to be tested is boiled under a reflux condenser while a thermometer bulb is fixed just above the surface of the liquid so as to be entirely surrounded by the vapor. The more common technical forms, such as those of Pohl and Kappeller, have scales reading percentage of alcohol instead of thermometer scales. Water is first boiled in the apparatus and the scale adjusted so that the mercury stands at zero. If then the water be removed and the sample introduced and brought to boiling under the same barometric conditions, the point reached by the mercury column shows the amount of alcohol present.

Wiley $^{1}$ uses a delicate differential thermometer with an apparatus similar to that employed for the determination of molecular weights by the boiling point method. Up to five per cent of alcohol, the depression of the boiling point is said to be so regular that the results are entirely satisfactory for practical work. In the ebullioscopes bearing scales graduated in terms of alcohol, the variations in the boiling point curve at

${ }^{1}$ J. Am. Chem. Soc., 1896, 18, 1063. 
the higher percentages are, of course, allowed for. The boiling point method is very rapid and gives results sufficiently accurate for many purposes. Reference may be made to Vaubel ${ }^{1}$ for a general discussion of methods based on the determination of the boiling point and to Freyer ${ }^{2}$ for experimental results on the influence of dissolved solids in the ebullioscopic determination of alcohol.

\section{Oxidation Method}

Under suitable conditions ethyl alcohol can be quantitatively oxidized to acetic acid by means of potassium dichromate in the presence of sulphuric acid. The amount of alcohol can then be ascertained either by determining the amount of dichromate reduced ${ }^{3}$ or by distilling and titrating the acetic acid formed. ${ }^{4}$ The conditions of oxidation must be carefully regulated, and as a rule the method is used only for the determination of very small amounts of alcohol, the specific gravity method being preferable for the examination of any but very dilute solutions. The alcohol must of course be separated by distillation from any other oxidizable matter before the oxidation method can be applied. A comparison of the results obtained by oxidation with those shown by the specific gravity method may be useful in demonstrating the presence of homologous alcohols.

\section{Determination and Identification of Small Amounts of Alcohol}

This subject has been studied by Bacon ${ }^{5}$ with special reference to its application in demonstrating alcoholic fermentation in food products. Bacon recommends that the alcohol be concentrated by distillation after addition of salt (which as noted above results in the alcohol being removed with a smaller

${ }^{1}$ Quantitative Bestimmung organischer Verbindungen. Berlin, 1902.

${ }^{2} Z$. angew. Chem., 1896,654 .

${ }^{3}$ Hehner: Analyst, 1887, 12, 25. Benedict and Norris : J. Am. Chem. Soc., 1898, 20, 293.

${ }^{4}$ Dupre: J. Chem. Soc., 1867, 20, 495.

${ }^{5}$ U. S. Dept. Agriculture, Bureau of Chemistry, Circular No. 74. 
amount of water) after which the distillate is examined as to density and index of refraction as well as by chemical tests to 'demonstrate the presence of alcohol. In a typical experiment given by Bacon, 1000 cc. of a 0.1 per cent solution of alcohol were three fourths saturated with salt, $150 \mathrm{cc}$. distilled off, this again three fourths saturated with salt and $25 \mathrm{cc}$. distilled off. This distillate showed at $17.5^{\circ}$ a refractometer reading of 21.3 corresponding to 3.84 per cent of alcohol in the distillate or 0.096 per cent in the original sample, a recovery of 96 per cent of the amount present.

Bacon considers that if the refractometer reading and the specific gravity indicate the same percentage of alcohol it is almost a certainty that it is ethyl alcohol which is present, and that the substance under examination contains the percentage of ethyl alcohol equivalent to these constants. As a further demonstration Bacon recommends that the solution containing the alcohol be treated with a slight excess of paranitrobenzoyl chloride and an equivalent amount of sodium hydroxide and the mixture shaken until the odor of the acid chloride disappears, when the crystalline ester (readily identified by its melting point of $57^{\circ} \mathrm{C.}^{1}$ ) may be collected and weighed. The yield is stated to be 70 to 90 per cent when working with small quantities of alcohol. If the paranitrobenzoyl chloride is not available, Bacon suggests benzoyl chloride, which when used in the same way yields the benzoic acid ethyl ester (ethyl benzoate), which may be weighed (yield said to be nearly quantitative) and identified by its odor and its boiling point, $212^{\circ}$ C., using for the determination of the latter the method of Smith and Menzies ${ }^{2}$; and that in addition the iodoform reaction be applied since the only benzoic ester having an odor similar to ethyl benzoate is the methyl ester, and methyl alcohol does not give the iodoform reaction.

Detection and Determination of Methyl Alcohol

The great difference in price between denatured alcohol and alcohol which has been subject to internal revenue tax some-

${ }^{1}$ Ber., 1905, 38, 620.

2 J. Am. Chem. Soc., 1910, 32, 897. 
times results in the substitution of denatured alcohol in cases where only ethyl alcohol should be used. This is detected by demonstrating the presence of methyl alcohol, but since denatured alcohol contains only one part of methyl to ten parts of ethyl alcohol, it is evident that methods to be useful for this purpose must be applicable to the detection of relatively small amounts of methyl alcohol in the presence of relatively large amounts of ethyl alcohol.

Many methods have been proposed for this purpose. Those which probably have been most generally used are based upon the oxidation of methyl alcohol to formaldehyde and the detection of the latter by one of the methods described in the next chapter. In the methods of Mulliken and Scudder ${ }^{1}$ and of the U.S. Pharmacopœia (Edition of 1905, p. 34) the oxidation of methyl alcohol to formaldehyde is accomplished by means of a copper spiral which is heated until covered with oxide and then plunged into the liquid, the copper being reduced and the alcohol partially oxidized. Vorisek ${ }^{2}$ prefers to oxidize by means of chromic acid; and Bacon (loc. cit.) recommends that 5 to 8 grams of chromic acid be added to $100 \mathrm{cc}$. of the aqueous methyl alcohol in a $200 \mathrm{cc}$. distilling flask and the first $10 \mathrm{cc}$. of the distillate be tested for formaldehyde. The results of such oxidation methods must be interpreted with caution, since several observers have reported formaldehyde among the products of oxidation of ethyl alcohol.

The Association of Official Agricultural Chemists have provisionally adopted the methods of Trillat and of Riche and Bardy. Trillat's method ${ }^{3}$ is based upon the observation that the products of oxidation of ethyl and methyl alcohol combine with dimethyl aniline to form bases which differ in their color reactions. The method of Riche and Rardy ${ }^{4}$ depends upon the formation of methyl aniline violet.

1 Am. Chem. J., 1900, 24, 444.

2 J. Soc. Chem. Ind., 1909, 28, 823.

${ }^{3}$ Compt. rend., 1898, 127, 232. Ann. chim. anal., 1899, 4, 42. J. pharm. chim., 1899, 9, 372. Analyst, 24, 13, 211, 212. Leach's Food Inspection and Analysis, $2 d$ Ed., p. 750. U.S. Dept. Agr., Bur. Chem., Bul. 107, p. 99.

${ }^{4}$ Compt. rend., 1875, 80, 1076. Allen's Commercial Organic Analysis, 4th Ed., Vol. I, p. 98. Leach's Food Inspection and Analysis, 2d Ed., p. 751. U.S. Dept. Agr., Bur. Chem., Bul. 107, p. 99. 
The Method of Leach and Lythgoe ${ }^{1}$ depends upon the fact that although ethyl and methyl alcohol in solutions have very similar densities, they differ considerably in their indices of refraction. This difference in properties is utilized both in detecting the presence of methyl alcohol and in estimating its amount as follows :

Submit the alcoholic distillate obtained in the determination of alcohol to refraction with the immersion refractometer at exactly $20^{\circ} \mathrm{C}$. and note the reading. If on reference to the table the refraction shows the percentage of alcohol agreeing with that obtained from the specific gravity in the regular manner, it may safely be assumed that no methyl alcohol is present. If, however, there is an appreciable amount of methyl alcohol, the low refractometer reading will at once indicate the fact.

Addition of methyl to ethyl alcohol decreases the refraction in direct proportion to the amount present; hence the quantitative calculation is readily made by interpolation in the table, using the figures for pure ethyl and methyl alcohol of the same alcoholic strength as the sample.

Example: Suppose the distillate from a vanilla extract made up to the original volume of the measured portion taken for the alcohol determination has a specific gravity of 0.97350 , corresponding to 18.38 per cent alcohol by weight, and has a refraction of 35.8 on the immersion refractometer at $20^{\circ}$. By interpolation in the refractometer table the readings of ethyl and methyl alcohol corresponding to 18.38 per cent alcohol are 47.2 and 25.4 , respectively, the difference being 21.8 ; 47.2$35.8=11.4 ;(11.4 \div 21.8) 100=52.3$, showing that 52.3 per cent of the alcohol present is methyl.

The Method of Thorpe and Holmes ${ }^{2}$ is probably the best for the quantitative analysis of mixtures of ethyl and methyl alcohols in cases in which the immersion refractometer is not available. It depends upon the oxidation of the mixture of

${ }^{1}$ J. Am. Chem. Soc., 1905, 27, 964. U. S. Dept. Agr., Bur. Chem., Cir. 29 and Bul. 107.

${ }^{2} J$. Chem. Soc., 1904, 85, 1. 
Table 6. - Scale Readings on Zeiss Immersion Refractometer at $20^{\circ}$ C., corresponding to each Per cent by Weight of Ethyl and Methyl Alcohols (Leach and Lythgoe)

\begin{tabular}{|c|c|c|c|c|c|c|c|c|c|c|c|}
\hline \multirow{2}{*}{ 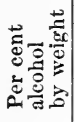 } & \multicolumn{2}{|c|}{ Scale readings } & \multirow{2}{*}{ 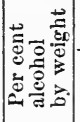 } & \multicolumn{2}{|c|}{ Scale readings } & \multirow{2}{*}{ 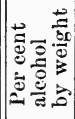 } & \multicolumn{2}{|c|}{ Scale readings } & \multirow{2}{*}{ 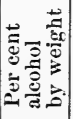 } & \multicolumn{2}{|c|}{ Scale readings } \\
\hline & $\begin{array}{l}\text { Methyl } \\
\text { alcohol }\end{array}$ & $\begin{array}{c}\text { Ethyl } \\
\text { alcohol }\end{array}$ & & $\begin{array}{l}\text { Methyl } \\
\text { alcohol }\end{array}$ & $\begin{array}{c}\text { Ethyl } \\
\text { alcohol }\end{array}$ & & $\begin{array}{l}\text { Methyl } \\
\text { alcohol }\end{array}$ & $\begin{array}{c}\text { Ethyl } \\
\text { alcohol }\end{array}$ & & & $\begin{array}{l}\text { Ethyl } \\
\text { alcohol }\end{array}$ \\
\hline 0 & 14.5 & 14.5 & 26 & 30.3 & 61.9 & 51 & 39.7 & 91.1 & 76 & 29.0 & 101.0 \\
\hline 1 & 14.8 & 16.0 & 27 & 30.9 & 63.7 & 52 & 39.6 & 91.8 & 77 & 28.3 & 100.9 \\
\hline 2 & 15.4 & 17.6 & 28 & & & 53 & & 2.4 & 78 & 27.6 & 100.9 \\
\hline 3 & 16.0 & 19.1 & 29 & 2 & 67.2 & 54 & & & 79 & 26.8 & 100.8 \\
\hline 4 & 16.6 & 20.7 & 30 & & & 55 & & 93.6 & 80 & 26.0 & 100.7 \\
\hline 5 & 17.2 & 22.3 & 31 & & 70.4 & 56 & 39.2 & 94.1 & 81 & 25.1 & 100.6 \\
\hline 6 & 17.8 & 24.1 & 32 & & 71.7 & 57 & 39.0 & 94.7 & 82 & 24.3 & 100.5 \\
\hline 7 & 18.4 & 25.9 & 33 & & & 58 & & & 83 & 23.6 & 100.4 \\
\hline 8 & & 27.8 & 34 & & & 59 & & & 84 & & 100.3 \\
\hline 9 & 19.6 & 29.6 & 35 & 35.8 & 75.8 & 60 & 37.9 & 96.2 & 85 & 21.8 & 100.1 \\
\hline 10 & 20.2 & 31.4 & 36 & 36.3 & 76.9 & 61 & 37.5 & 96.7 & 86 & 20.8 & 99.8 \\
\hline 11 & 20.8 & 33.2 & 37 & 36.8 & 78.0 & 62 & 37.0 & 97.1 & 87 & 19.7 & 99.5 \\
\hline 12 & & & 38 & & & 63 & & 97.5 & 88 & 18.6 & 99.2 \\
\hline 13 & 22.0 & 36.9 & 39 & 37.7 & 80.2 & 64 & 36.0 & 98.0 & 89 & 17.3 & 98.9 \\
\hline 14 & 22.6 & 38.7 & 40 & 38.1 & 81.3 & 65 & 35.5 & 98.3 & 90 & 16.1 & 98.6 \\
\hline 15 & 23.2 & 40 & 41 & & 82.3 & 66 & & 98.7 & 91 & 14.9 & 98.3 \\
\hline 16 & 23.9 & 42.5 & 42 & 38 & 83.3 & 67 & 34.5 & 99.1 & 92 & 13.7 & 97.8 \\
\hline 17 & 24.5 & 44.5 & 43 & 39.2 & 84.2 & 68 & 34.0 & 99.4 & 93 & 12.4 & 97.2 \\
\hline 18 & 25.2 & 46.5 & 44 & 39.3 & 85.2 & 69 & 33.5 & 99.7 & 94 & 11.0 & 96.4 \\
\hline 19 & 25.8 & 48.5 & 45 & 39.4 & 86.2 & 70 & 33.0 & 100.0 & 95 & 9.6 & 95.7 \\
\hline 20 & 26.5 & 50.5 & 46 & 39.5 & 87.0 & 71 & 32.3 & 100.2 & 96 & 8.2 & 94.9 \\
\hline 21 & 27.1 & 52.4 & 47 & 39.6 & 87.8 & 72 & 31.7 & 100.4 & 97 & 6.7 & 94.0 \\
\hline 22 & 27.8 & 54.3 & 48 & 39.7 & 88.7 & 73 & 31.1 & 100.6 & 98 & 5.1 & 93.0 \\
\hline 23 & 28.4 & 56.3 & 49 & 39.8 & 89.5 & 74 & 30.4 & 100.8 & 99 & 3.5 & 92.0 \\
\hline 24 & 29.1 & 58.2 & 50 & 39.8 & 90.3 & 75 & 29.7 & 101.0 & 100 & 2.0 & 91.0 \\
\hline 25 & 29.7 & 60.1 & & & & & & & & & \\
\hline
\end{tabular}

ethyl and methyl alcohols under such conditions that the former is converted into acetic acid while the latter is completely oxidized to carbon dioxide and water. The total amount of alcohols (estimated as ethyl alcohol) having been 
determined by distillation and specific gravity, a part of the distillate is mixed with water in such proportions that $50 \mathrm{cc}$. of the mixture shall contain not more than 1 gram of methyl alcohol nor more than 4 grams of ethyl and methyl alcohols together. Fifty cubic centimeters of this mixture are introduced into a 300 -cc. flask having a tight stopper and fitted with a funnel and side tube, 20 grams of potassium dichromate and $80 \mathrm{cc}$. of dilute sulphuric acid (1:4) added, and the mixture allowed to remain for 18 hours. A further quantity of 10 grams of potassium dichromate and $50 \mathrm{cc}$. of sulphuric acid mixed with an equal volume of water are now added, and the contents of the flask heated to the boiling point for about 10 minutes, the evolved carbon dioxide being. swept out of the apparatus by a current of air and collected in weighed sodalime tubes. Under these conditions each gram of ethyl alcohol was found to yield about 0.01 gram of carbon dioxide. The remaining carbon dioxide found is calculated as being derived from the complete oxidation of methyl aclohol.

\section{Determination of Amyl Alcohols or Fusel Oil}

The amyl alcohols are the principal constituents of fusel oil, and most of the methods proposed for the determination of fusel oil in distilled liquors are essentially attempts to estimate the amyl alcohols. In order to separate the amyl alcohols from the relatively large amounts of ethyl alcohol ordinarily present, advantage is taken of the fact that the former are much more soluble in chloroform or carbon tetrachloride than is the latter, so that on shaking a small amount of chloroform or carbon tetrachloride with a distillate containing ethyl and amyl alcohols practically all of the amyl alcohols and only a little of the ethyl alcohol is extracted by the chloroform or the carbon tetrachloride.

The amount of amyl alcohols or of fusel oil is usually estimated either:

1. By extracting under fixed conditions with an accurately known volume of chloroform and estimating the fusel oil from 
the increase in volume of the chloroform layer (Roese's method).

2. By extracting with carbon tetrachloride, oxidizing the alcohols of the extracted fusel oil to the corresponding acids by means of potassium dichromate, and distilling and titrating the acids thus formed (Allen-Marquardt method).

Both of these methods have been adopted provisionally by the Association of Official Agricultural Chemists and are given in full on pp. 97-98, Bul. 107 (Revised), Bureau of Chemistry, U. S. Dept. Agriculture.

\section{Official Requirements as to Purity}

\section{Ethyl Alcohol}

The U. S. Pharmacopœia in addition to the requirements above given and a test which is supposed to show methyl alcohol if more than two per cent is present, prescribes the following tests of purity for alcohol:

It should not affect the color of blue or red litmus paper previously moistened with water.

If $50 \mathrm{cc}$. of alcohol be evaporated in a clean vessel, no color or weighable residue should remain.

If $10 \mathrm{cc}$. of alcohol be mixed with 5 cc. of water and 1 cc. of glycerin, and the mixture allowed to evaporate spontaneously from a piece of clean, odorless blotting paper, no foreign odor should become perceptible when the last traces of the alcohol leave the paper (absence of fusel oil constituents).

If 25 cc. be allowed to evaporate spontaneously in a porcelain evaporating dish, carefully protected from dust, until the surface of the dish is barely moist, no red or brown color should be produced upon the addition of a few drops of colorless, concentrated sulphuric acid (absence of amyl alcohol, or non-volatile carbonizable, organic impurities, etc.).

If $10 \mathrm{cc}$. of alcohol be mixed in a test tube with 5 cc. of potassium hydroxide test solution, the liquid should not at once assume a yellow color (absence of aldehyde or oak tannin).

If $20 \mathrm{cc}$. of alcohol be shaken in a clean glass-stoppered vial with $1 \mathrm{cc}$. of silver nitrate test solution, the mixture should not become more than faintly opalescent, nor acquire more than a faint brownish tint when exposed for six hours to diffused daylight (limit of organic impurities, amyl alcohol, aldehyde, etc.). 


\section{Methyl Alcohol}

The following requirements have been established by the Bureau of Internal Revenue for methyl alcohol to be used in denaturing grain alcohol:

The methyl alcohol submitted must be partially purified wood alcohol obtained by the destructive distillation of wood. It must conform to the following analytical requirements :

1. Color. - This shall not be darker than that produced by a freshly prepared solution of 2 cc. of tenth-normal iodine diluted to $1000 \mathrm{cc}$. with distilled water.

2. Specific Gravity. - It must have a specific gravity of not more than 0.830 at $60^{\circ} \mathrm{F}$. $\left(15.56^{\circ} \mathrm{C}\right.$.), corresponding to $91^{\circ}$ of Tralles' scale.

3. Boiling Point. - One hundred cubic centimeters slowly heated in a flask under conditions as described below must give a distillate of not less than 90 cc. at a temperature not exceeding $75^{\circ} \mathrm{C}$. at the normal pressure of the barometer $(760 \mathrm{~mm}$.). One hundred cc. of wood spirit are rum into a shortnecked copper flask of about 180-200 cc. capacity and the flask placed on an asbestos plate having a circular opening of $30 \mathrm{~mm}$. diameter. In the neck of this flask is fitted a fractionating tube $12 \mathrm{~mm}$. wide and $170 \mathrm{~mm}$. long, with a bulb just 1 centimeter below the side tube which is connected with a Liebig's condenser having a water jacket not less than $400 \mathrm{~mm}$. long. In the upper opening of the fractionating tube is placed a standardized thermometer, so adjusted that its mercury bulb comes in the center of the bulb. The distillation is conducted in such a manner that 5 cc. pass over in one minute. The distillate is run into a graduated cylinder, and when the temperature of $75^{\circ} \mathrm{C}$. has been reached at the normal barometric pressure of $760 \mathrm{~mm}$. at least 90 cc. shall have been collected.

Should the barometer vary from $760 \mathrm{~mm}$. during the distillation, $1^{\circ} \mathrm{C}$. shall be allowed for every variation of $30 \mathrm{~mm}$. For example, at $770 \mathrm{~mm}$. $90 \mathrm{cc}$. should have distilled at $75.3^{\circ} \mathrm{C}$., and at $750 \mathrm{~mm}$. $90 \mathrm{cc}$. should have distilled at $74.7^{\circ} \mathrm{C}$.

4. Miscibility with Water. - It must give a clear or only slightly opalescent solution when mixed with twice its volume of water.

5. Acetone Content. - It must contain not more than 25 nor less than 15 grams per $100 \mathrm{cc}$. of acetone and other substances estimated as acetone when tested by the following method (Messinger).

One cubic centimeter of a mixture of $10 \mathrm{cc}$. wood alcohol with $90 \mathrm{cc}$. of water is treated with $10 \mathrm{cc}$. of double normal soda solution. Then $50 \mathrm{cc}$. of tenth-normal iodine solution are added while shaking, and the mixture made acid with dilute sulphuric acid three minutes after the addition of the iodine. The excess of iodine is titrated back with tenth-normal sodium thiosulphate solution, using a few drops of starch solution for an indicator. 
From 15.5 to 25.8 cc. of tenth-normal iodine solution should be used by the spirit. The solution should be kept at a temperature between $15^{\circ}$ and $20^{\circ} \mathrm{C}$.

Calculation :

$x=$ grams of acetone in $100 \mathrm{cc}$. of spirit.

$y=$ number of cubic centimeters of tenth-normal iodine solution required. $N=$ volume of spirit taken for titration.

Then $x=\frac{y \times 0.096672}{N}$.

6. Esters. - It should contain not more than 5 grams of esters per $100 \mathrm{cc}$. of spirit, calculated as methyl acetate and determined as follows:

Five cubic centimeters of wood spirit are run into a flask and $10 \mathrm{cc}$. normal sodium hydroxide free from carbonates are added, and the flask connected with a return condenser and boiled for two hours. Instead of digesting at boiling temperature the flask may be allowed to stand overnight at room temperature and then heated on a steam bath for thirty minutes with an ordinary tube condenser. The liquid after digestion is cooled and titrated with normal sulphuric acid, using phenolphthalein as an indicator.

$\left.\begin{array}{c}\text { Methyl acetate, in grams } \\ \text { per } 100 \text { cc. of spirit }\end{array}\right\}=\frac{.074 \times \text { cc. of normal soda required } \times 100}{\text { cc. of spirit taken }}$.

7. Bromine Absorption. - It must contain a sufficient quantity of impurities derived from the wood so that not more than 25 cc. nor less than 15 cc. shall be required to decolorize a standard solution containing 0.5 gram of bromine, as follows:

The standard bromine solution is made by dissolving 12.406 grams of potassium bromide and 3.481 grams of potassium bromate (which is of tested purity and has been dried for two hours at $100^{\circ} \mathrm{C}$.) in a liter of water. Fifty cubic centimeters of the standard solution containing 0.5 gram of bromine are placed in a glass-stoppered flask having a capacity of about 200 cc. This is acidified by the addition of 10 cc. of diluted sulphuric acid $(1: 4)$, and the whole shaken and allowed to stand a few minutes. The wood alcohol is then allowed to flow slowly into the mixture, drop by drop, from a burette until the color is entirely discharged. The temperature of the mixture should be $20^{\circ} \mathrm{C}$.

In addition to the above requirements the methyl alcohol must be of such a character as to render the ethyl alcohol with which it is mixed unfit for use as a beverage.

\section{REFERENCES}

Allen: Commercial Organic Analysis, Vol. 1.

Leach : Food Inspection and Analysis.

LUNGE: Chemisch-technische Untersuchungsmethoden, Bd. III. 
MeYer: Analyse und Konstitutionsermittelung organischer Verbindungen. Meyer-Tingle: Determination of Radicles in Carbon Compounds.

Mulliken: Identification of Pure Organic Compounds, Vol. I.

Schмidт: Ausführliches Lehrbuch der pharmaceutischen Chemie, Bd. II, Abth. I.

U. S. Dept. Agriculture, Bureau of Chemistry, Bul. 107 (Revised). Official and Provisional Methods of Analysis.

U. S. Dept. Commerce and Labor, Bureau of Standards, Circular No. 19, Standard Density and Volumetric Tables.

U. S. Pharmacopœia.

VAubeL: Die physikalischen und chemischen Methoden der quantitativen Bestimmung organischer Verbindungen.

Young: Fractional Distillation.

1905. Leach and Lythgoe: The Detection and Determination of Ethyl and Methyl Alcohols in Mixtures by the Immersion Refractometer. J. Am. Chem. Soc., 27, 964.

Schidrowitz and KaYe: The Determination of Higher Alcohols in Spirits. Analyst, 30, 190.

WinkLer: (Preparation of Ethyl Alcohol). Ber., 38,3612; Analyst, 31, 76 .

1906. ScudDer and Riggs: The Detection of Methyl Alcohol. J. Am. Chem. Soc., 28, 1202.

Tolman and Trescotт: Methods for the Determination of Esters, Aldehydes, and Furfural in Alcoholic Liquors. J. Am. Chem. Soc., 28, 1619.

1907. Fleischer and Frank : (Rapid Estimation of Alcohol and Ether in their Mixtures). Chem. Ztg., 31, 665 .

Schidrowitz: The Estimation of Higher Alcohols ("Fusel Oil ") in Distilled Liquors. J. Am. Chem. Soc., 29, 561.

U.S. Internal Revenue Regulations, No. 30-Revised. Concerning Denatured Alcohol, Central Denaturing Bonded Warehouses, and Industrial Distilleries.

WAgner and Schultze: (Estimation of Ethyl Alcohol with the Zeiss Immersion Refractometer). Z. anal. Chem., 46, 508.

1908. Andrews: The Refractive Indices of Alcohol-Water Mixtures. J. Am. Chem. Soc., 30, 353.

Crampton and Tolman: A Study of the Changes taking Place in Whiskey stored in Wood. J. Am. Chem. Soc., 30, 98.

Doroshevskil and Dvorzhanchik: Index of Refraction of Mixtures of Alcohol and Water. J. Russ. Phys.-Chem. Soc., 40,101; Chem. Abs., 2, 2181. 
DudLey: Notes on the Roese Method for the Determination of Fusel Oil, and a Comparison of the Results by the Allen-Marquardt Method. J. Am. Chem. Soc., 30, 1271.

Hinkel: The Detection of Small Quantities of Methyl Alcohol in the Presence of Ethyl Alcohol. Analyst, 33, 417.

1909. Plücker: Preparation of Pure Ethyl Alcohol. Z. Nahr. Genussm., 17, 454 .

Tolman and Hillyer: Methods of Analysis of Distilled Spirits. U. S. Dept. Agriculture, Bur. Chem., Bul. 122, p. 206.

Vorickк: Detection of Methyl Alcohol in Ethyl Alcohol. J. Soc. Chem. Ind., 28, 823.

1910. Wiley: Manufacture of Denatured Alcohol. U. S. Dept. Agriculture, Bur. Chem., Bul. 130.

1911. BAcon: Detection and Determination of Small Quantities of Ethyl and Methyl Alcohol and of Formic Acid. U. S. Dept. Agriculture, Bur. Chem., Cir. No. 74.

GoRE: An Electrically Controlled Constant Temperature Water Bath for the Immersion Refractometer. J. Ind. Eng. Chem., 3, 506 . 


\section{CHAPTER II}

\section{Aldehydes}

THE most important methods for the detection and determination of aldehydes are based upon reactions of oxidation, of condensation, and of direct addition. In this chapter the analytical application of these reactions will be illustrated by methods for formaldehyde, benzaldehyde, and vanillin.

The readiness with which aldehydes undergo oxidation gives them the property of reducing ammoniacal silver solution, which is the basis of one of the most delicate qualitative tests for this group of compounds. The test may be carried out as follows: ${ }^{1}$

Mix, in a test tube previously cleaned with hot sodium hydroxide solution, $1 \mathrm{cc}$. of ammoniacal silver nitrate solution (containing one part of silver nitrate in ten parts of ammonium hydroxide of $0.923 \mathrm{sp} . \mathrm{gr}$ ) and $1 \mathrm{cc}$. of ten per cent sodium hydroxide solution. Shake the mixture in the tube and then allow two or three drops of the solution to be tested to flow slowly down the moistened glass surface into the reagent. Shake and allow to stand cold for five minutes. Aldehydes (and a few other compounds including some of the polyatomic alcohols) cause the production of a dark brown or black precipitate or mirror of metallic silver. This reaction is given by all of the ordinary aldehydes of the fatty series, including the aldose carbohydrates, but not by all aromatic aldehydes.

The ammoniacal silver solution and the sodium hydroxide must not be mixed in advance and must always be kept cool, as a dangerously explosive precipitate is apt to form on warming

1 Noyes and Mulliken : Identification and Class Reactions of Organic Substances. Mulliken : Identification of Pure Organic Compounds, Vol. I., p. 22. 
or on long standing. The use of a mixture of sodium hydroxide and ammoniacal silver nitrate (Tollens' aldehyde reagent) makes the test more delicate than when the ammoniacal silver solution is used alone.

Alkaline solutions of other metals are reduced by many aldehydes, especially on boiling, and many quantitative methods for individual aldehydes are based upon the determination of the amount of metal reduced.

Condensation reactions, especially with phenylhydrazine, hydroxylamine, and phenols, are often used for the detection and sometimes for the determination of aldehydes. A general discussion of such methods will be found in the works of Vaube and of Meyer. Several special methods will be described in this and the two following chapters.

Of the addition reactions of aldehydes, that with bisulphite is of especially wide application. On shaking a liquid aldehyde or a concentrated solution of aldehyde in water or ether, with an equal volume of strong sodium bisulphite solution, addition takes place with the formation of the saturated compound $\mathrm{RCH}(\mathrm{OH}) \mathrm{SO}_{3} \mathrm{Na}$ which usually separates as a white crystalline precipitate. Ketones containing the $\mathrm{CH}_{3} \mathrm{CO}$ group also give the reaction. A negative result is not conclusive, as the addition product may be too soluble to appear as a precipitate.

According to Ripper, ${ }^{1}$ the bisulphite reaction can be utilized for the determination of any aldehyde soluble in water or which can be brought into solution by a small amount of alcohol. A one half per cent solution of the aldehyde is mixed with twice its volume of a solution of potassium bisulphite of known strength (about 12 grams per liter), and after 15 minutes the excess of bisulphite is determined by titration with iodine. Ripper applied this method with satisfactory results to solutions of formaldehyde, acetaldehyde, benzaldehyde, and vanillin.

A similar addition reaction gives rise to the well-known and delicate "fuchsin test" for aldehydes. This test, as developed by Mulliken, ${ }^{2}$ is as follows:

1 Monatsh. Chem., 1900, 21, 1079.

2 Identification of Pure Organic Compounds, Vol. I, p. 15. 
To prepare the fuchsin aldehyde reagent, dissolve 0.2 gram of rosanilin, or, if the free base cannot be obtained, of the hydrochloride or acetate, in $10 \mathrm{cc}$. of a freshly prepared, cold, saturated aqueous solution of sulphur dioxide. Allow the solution to stand until all signs of pink disappear and it becomes colorless or pale yellow. This will require several hours. Then dilute with water to $200 \mathrm{cc}$. and preserve for use in a tightly stoppered bottle.

To 5 cc. of this reagent add 0.05 gram, or one drop, of the substance to be tested (if pure, or a few drops if in solution). If the substance is a liquid, or dissolves in the reagent, allow to stand two minutes and observe the color. If the substance does not dissolve, shake gently for two minutes and then observe the color. / The appearance of a distinct pink, red, purple, or blue coloration indicates the presence of an aldehyde. ${ }^{r}$ The test to be of value must be applied under carefully regulated conditions. The reagent is reddened by alkalies or alkaline salts of weak acids, by heating or by long exposure to air at ordinary temperature. In general, the test as here described distinguishes aldehydes other than carbohydrates from the latter and from ketones. A few acetals show the reaction through being partially hydrolyzed to aldehydes under the conditions of the test. Acetone and some other soluble ketones prepared by destructive distillation gradually redden the reagent if added to it in large quantity or if allowed to remain in contact for a number of minutes; but this is thought to be due chiefly, if not wholly, to the presence of traces of aldehydes or acetals (Mulliken).

This reaction serves for the detection of minute quantities of aldehydes present as impurities in commercial alcohol, and for the colorimetric estimation of aldehydes in distilled liquors. ${ }^{1}$

\section{FORMALDEHYDE}

Formaldehyde gas, produced by the partial oxidation of methyl alcohol, is freely soluble in water and is most com-

1 Medicus: Forschungsber. über Lebensmittel, 1895, 1, 299. Bul. 107, Bur. Chem., U. S. Dept. Agriculture. Tolman and Trescott : J. Am. Chem. Soc., 1906, 28, 1619. 
monly handled as a 35 to 40 per cent aqueous solution. Such solutions are often designated formalin, formol, or formal. More dilute solutions are sometimes sold as food preservatives under fanciful or misleading names.

In dilute aqueous solution, formaldehyde exists in the "monomolecular" state, as $\mathrm{CH}_{2} \mathrm{O}$. Such solutions do not change if kept at ordinary temperature in closed vessels. When an aqueous solution is concentrated either by spontaneous evaporation or by heating, a white flocculent deposit appears. If the solution is then separated from the deposit, it is found to contain condensed or polymerized formaldehyde. ${ }^{1}$ The material which deposits from a concentrated aqueous solution of formaldehyde has, after drying, the composition $\left(\mathrm{CH}_{2} \mathrm{O}\right)_{6} \cdot \mathrm{H}_{2} \mathrm{O}$ to $\left(\mathrm{CH}_{2} \mathrm{O}\right)_{8} \cdot \mathrm{H}_{2} \mathrm{O}^{2}$ It is amorphous, soluble in warm water, and has an odor resembling that of formaldehyde. The paraformaldehyde of commerce consists essentially of this material.

Metaformaldehyde (oxymethylene, "trioxymethylene"), $\left(\mathrm{CH}_{2} \mathrm{O}\right)_{x}$, may also be formed by evaporation of formaldehyde solutions. By prolonged digestion at ordinary temperature or by heating for a short time at $130^{\circ}-150^{\circ}$ with a large excess of water, metaformaldehyde passes into solution and into the "mono-molecular" form. Polymeric modifications of formaldehyde in aqueous solution resemble closely the original substance in its behavior toward reagents, so that, as measured by the ordinary methods, a solution does not lose strength by the partial polymerization of the formaldehyde so long as all remains in solution.

Commercial solutions of formaldehyde commonly contain methyl alcohol and may contain small amounts of any of the impurities of commercial wood spirit. Solutions of the usual strength, from 35 to 40 per cent., should have specific gravities of about 1.08 to 1.11 at $15^{\circ}$, lower figures ordina-

1 Tollens and Mayer: Ber., 1888, 21, 1571, 3503. Kraut, Eschweiler, and Grossmann : Ann. Chem., 1890, 258, 103.

${ }^{2}$ Losekann : Chem. Ztg., 1890, 14, 1408. Delephine: Compt. rend., 1897, 124, 1525. Beilstein: Organische Chemie, Erganzbd., I., 467. 
rily indicating the presence of excessive amounts of methyl alcohol.1

The methods given in this chapter for the detection and determination of formaldehyde refer especially to the examination of commercial solutions containing only such impurities as ordinarily occur in crude preparations of formaldehyde, or substances which might be used with formaldehyde in preservative mixtures. The examination of food products for formaldehyde will be discussed in connection with other food preservatives in a subsequent chapter.

If a solution to be examined contains dissolved solids which interfere with the direct application of the tests as described, it can be acidified with a small excess of phosphoric or sulphuric acid, distilled, and the test applied to the distillate. The latter, however, will never contain all of the formaldehyde, since some is always polymerized and left as paraformaldehyde in the distilling flask.

\section{Detection AND IDENTIFICATION}

\section{Resorcin Test ${ }^{2}$}

Mix one drop of a 1 per cent aqueous solution of resorcin with 1 cc. of a dilute aqueous solution (preferably about 0.2 per cent) of the aldehyde. Allow the mixture to flow gently down the side of an inclined test-tube containing 3-5 cc. of pure concentrated sulphuric acid (or incline the test tube containing the mixture and pour in the acid). Impart a gentle rotary motion to the liquids by cautiously swaying the lower end of the tube through a circle about a decimeter in diameter, in such a manner as not to cause the disappearance of the two layers. If formaldehyde is present, a red ring slightly tinged with violet will soon appear. Above this ring a light flocculent precip-

1 On the determination of methyl alcohol in formaldehyde solutions see Duyk : Ann. chim. anal., 1901, 6, 407 ; J. Chem. Soc., 1902, 82, ii, 110. Stritrar: Z. anal. Chem., 1904, 43, 401. Gnehm and Kaufler: Z. angew. Chem., 1904, 17, 673; 1905, 18, 93. Bamberger: Ibid., 1904, 17, 1246.

${ }^{2}$ Mulliken and Scudder: Am. Chem. J., 1900, 24, 451. Mulliken : Identification of Pure Organic Compounds, Vol. I, p. 24. 
itate, at first nearly white on its upper surface and red-violet beneath, but soon changing to flocks that are red throughout, will be seen suspended in the aqueous upper layer.

This reaction is very satisfactory for solutions containing one part of formaldehyde in 100 to 5000 parts of solution and can be detected to a dilution of $1: 100,000$. A similar reaction is obtained if phenol is used instead of resorcin.

\section{Gallic Acid Test ${ }^{1}$}

Mix 0.2 cc. of a saturated solution of gallic acid in pure ethyl alcohol, with 1 to $2 \mathrm{cc}$. of the solution to be tested, and introduce a layer of concentrated sulphuric acid, as in the resorcin test. In the presence of formaldehyde, a green zone appears at the line of contact of the two liquids. This gradually changes to a pure blue ring, which, in the case of pure aqueous solutions of formaldehyde, can be detected without difficulty at a dilution of $1: 500,000$. If the solution tested contains as much as one part of formaldehyde in 20,000, a yellowish color appears immediately at the line of contact of the two liquids. This quickly turns green, and the blue color develops both above and below the green zone. If other substances which give color reactions are also present, the upper layer will vary in color, but the green and lower blue ring will still appear beneath (Mulliken and Scudder). On swaying the tube, or allowing it to stand for some time, the blue color spreads throughout the zone and a pure blue ring is usually obtained. The color is quite permanent and apparently quite characteristic, no other substance having been noted as giving the blue ring. Acetaldehyde tested in the same way gives a reddish brown coloration.

\section{Hydrochloric Acid and Casein Test ${ }^{2}$}

Mix 5 cc. of the solution to be tested with 5 cc. of pure milk in a porcelain casserole, add $10 \mathrm{cc}$. of concentrated hydrochloric

1 Barbier and Jandrier: Ann. chim. anal., 1, 325 ; Abs. Analyst, 1896, 21, 295. Mulliken and Scudder: Am. Chem. J., 1900, 24, 444.

${ }^{2}$ Leach : Ann. Rept. Mass. State Board of Health, 1897, 558; 1899, 699. 
acid containing 0.002 gram of ferric chloride, and heat slowly over a free flame nearly to boiling, meanwhile giving the casserole a rotary motion to break up the curd. A violet coloration indicates formaldehyde. According to Leach, various aldehydes give color reactions under this treatment, but formaldehyde alone shows the unmistakable violet coloration. This test is especially useful for the detection of formaldehyde in milk, and will be more fully discussed in that connection.

\section{Methylene-di- $\beta$-naphthol Test}

Since formaldehyde is frequently sold under other names, its identification by some method independent of the above color reactions may be a matter of importance. In such cases the following test given by Mulliken ${ }^{1}$ will be useful.

Place in a test tube 3 drops of a 30 to 40 per cent, or 10 drops of a 10 per cent, solution of the formaldehyde, 3 cc. of dilute alcohol (1:2), 0.04 to 0.06 gram $\beta$-naphthol, and 3 to 5 drops of concentrated hydrochloric acid. Boil gently until the liquid becomes filled with an abundant precipitate of small white needles. Filter while hot. Wash with 1 cc. of dilute alcohol $(1: 2)$. Boil the precipitate with 4 cc. of dilute alcohol (1:1). (It is not necessary that all should dissolve.) Cool and filter off the precipitate. Wash with 1 cc. of dilute alcohol $(1: 1)$. Dry on porous tile in a warm place and determine the melting point.

Methylene-di- $\beta$-naphthol, the product, forms white needles, which, when the temperature in the neighborhood of the melting point is raised at the rate of $1^{\circ}$ in 15 seconds, begin to turn brown at $180^{\circ}$. It melts with decomposition to a red-brown liquid at $189^{\circ}-192^{\circ}$ (uncorr.).

\section{Determination by Oxidation}

\section{Iodimetric Method ${ }^{2}$}

This method depends upon the oxidation of formaldehyde to formic acid by means of iodine in alkaline solution. Two atoms

1 Identification of Pure Organic Compounds, Vol. I, p. 24.

2 Romijn : Z. anal. Chem., 1897, 36, 18. Williams: J. Am. Chem. Soc., 1905, 27, 596 . 
of iodine oxidize one molecule of formaldehyde, and the excess of iodine is liberated by acidulation and determined by titration with sodium thiosulphate.

$$
\mathrm{CH}_{2} \mathrm{O}+\mathrm{I}_{2}+\mathrm{H}_{2} \mathrm{O}=\mathrm{CH}_{2} \mathrm{O}_{2}+2 \mathrm{HI} \text {. }
$$

Reagents. - Standard solutions of iodine and sodium thiosulphate, preferably about tenth-normal. Approximately normal solutions of sodium hydroxide and hydrochloric acid.

Determination. - Dilute a weighed portion of the sample with a known quantity of water so as to obtain a solution containing 0.5 to 1 per cent of actual formaldehyde. Mix $10 \mathrm{cc}$. of this solution with $25 \mathrm{cc}$. normal sodium hydroxide and add from a burette 50 to $75 \mathrm{cc}$. of tenth-normal iodine solution or enough to assure an excess of iodine as shown by the permanent yellow color of the solution. Shake or stir thoroughly and after ten minutes add 35 cc. normal hydrochloric acid and titrate with sodium thiosulphate in the usual way, using starch solution as indicator. At the same time determine the strength of the iodine in terms of thiosulphate solution and from the amount of iodine consumed in oxidizing the formaldehyde calculate the weight of the latter in the $10 \mathrm{cc}$. taken for the determination.

Notes. - Under the conditions given the oxidation of formaldehyde is rapid and complete but the method is applicable only in the absence of all other substances capable of consuming iodine under these conditions. Other aldehydes, acetone, and alcohol cause high results, the latter probably through absorbing iodine with the formation of iodoform.

In the absence of interfering compounds, the method is very satisfactory, even for solutions containing only 0.1 per cent of formaldehyde. Variations in the excess of iodine added have no appreciable influence upon the results.

\section{Hydrogen Peroxide Method ${ }^{1}$}

In this method, formaldehyde is oxidized to formic acid by means of hydrogen peroxide in the presence of a known amount of alkali.

$$
\mathrm{CH}_{2} \mathrm{O}+\mathrm{H}_{2} \mathrm{O}_{2}=\mathrm{CH}_{2} \mathrm{O}_{2}+\mathrm{H}_{2} \mathrm{O} \text {. }
$$

${ }^{1}$ Blank and Finkenbeiner : Ber., 1898, 31, 2979. Haywood and Smith: $J$. Am. Chem. Soc., 1905, 27, 1183. Bul. 107, Bur. Chem., U. S. Dept. Agriculture. 
The excess of alkali, over that required to combine with the formic acid produced, is determined by titration.

The method is here described as used by the Association of Official Agricultural Chemists.

Reagents. - Normal solutions of sodium hydroxide and sulphuric acid. Neutral ${ }^{1} 3$ per cent solution of hydrogen peroxide. Solution of purified litmus as indicator.

Determination. - Measure $50 \mathrm{cc}$. of normal sodium hydroxide into a 500-cc. Erlenmeyer flask, add $50 \mathrm{cc}$. hydrogen peroxide solution, then 3 grams of the formaldehyde solution. Place a funnel in the neck of the flask and stand it on a steam bath for 5 minutes, shaking occasionally during this time. Remove,

- wash funnel with water, cool to room temperature, and titrate excess of alkali with normal acid, using litmus as indicator. Each molecule of sodium hydroxide which has been consumed (deducting the amount required to neutralize any free acid which the peroxide solution or the original solution of formaldehyde may have contained) represents one molecule of formaldehyde oxidized to formic acid.

Notes. - The use of exactly 3 grams of formaldehyde is not essential, but the exact weight must of course be known. It is convenient to use a weighing bottle containing a small pipette, measure out about $3 \mathrm{cc}$. and obtain the weight by difference.

Acetaldehyde is partially oxidized under the same conditions. Its presence, therefore, causes high results, but not so high as by the iodimetric method. The results are not influenced by the presence of paraldehyde, acetone, or ethyl or methyl alcohol. Commercial formalin containing only traces of acetone or acetaldehyde should show the same percentage of formaldehyde by the peroxide as by the iodimetric method.

\section{Determination by Condensation Reactions}

Several of the condensation reactions of formaldehyde have been utilized for its quantitative determination. One of the

1 If all available peroxide is acid, the acidity must be determined by titration, using litmus as indicator, and allowed for in calculating the amount of alkali consumed in the formaldehyde determination. 
oldest and best-known methods is based upon the fact that formaldehyde and ammonia when mixed in not too dilute solution condense to form hexamethylene tetramine :

$$
6 \mathrm{CH}_{2} \mathrm{O}+4 \mathrm{NH}_{4} \mathrm{OH}=\mathrm{N}_{4}\left(\mathrm{CH}_{2}\right)_{6}+10 \mathrm{H}_{2} \mathrm{O} \text {. }
$$

If a known amount of ammonia is used, the determination of the excess shows the amount of formaldehyde originally present.

\section{Legler's Ammonia Method ${ }^{1}$}

Weigh about 1.5 grams of the solution containing 30 to 40 per cent formaldehyde, or an equivalent amount of a more dilute solution, into a 250-cc. glass-stoppered flask or bottle. Add 100 cc. of fifth-normal ammonia solution; stopper tightly at once; mix and allow to stand overnight at room temperature. Standing for two or three days does no harm, provided the stopper fits so tightly as to prevent any loss of ammonia. Finally, add a very small amount of rosolic acid as indicator and titrate the excess of ammonia with standard sulphuric acid. Calculate the quantity of formaldehyde originally present from the amount of ammonia consumed in condensing with it according to the equation given above.

Notes and Precautions. - In order to prevent loss of ammonia during the determination, the flask or bottle must be tightly closed, the stopper being coated with vaseline if necessary. For the same reason the excess of ammonia should be titrated quickly after opening the flask. Normal or half-normal ammonia is commonly recommended for this method, but the fifth-normal solution is less likely to lose strength and has been found by Williams to give as complete reactions as the stronger solutions. In titrating the excess of ammonia the end reaction is usually unsatisfactory, especially when the solution is highly colored by the indicator. Two drops of a freshly prepared 0.1 per cent solution of rosolic acid have been found sufficient.

The results are not affected by the presence of acetone,

${ }^{1}$ Legler: Ber., 1883, 16, 1333. Smith : J. Am. Chem. Soc., 1903, 25, 1028. Williams : loc. cit. 
methyl or ethyl alcohol, paraldehyde, or benzaldehyde. Acetaldehyde reacts with ammonia and thus causes high results if present in the formaldehyde solution.

This method was formerly much used in analysis of commercial formalin, but on account of the tendency toward low results it is now generally displaced by the hydrogen peroxide method.

\section{Determination by Addition Reactions}

The general addition reaction of aldehydes with bisulphites has been used quantitatively by Ripper, as already noted. For the determination of formaldehyde, however, the reaction with potassium cyanide has been found especially useful.

\section{Potassium. Cyanide Method ${ }^{1}$}

On mixing aqueous solutions of formaldehyde and potassium cyanide an addition product is formed, which, according to Romijn, is probably the potassium compound of oxyacetonitril :

$$
\mathrm{CH}_{2} \mathrm{O}+\mathrm{KCN}=\mathrm{CH}_{2} \mathrm{OK} \cdot \mathrm{CN} \text {. }
$$

The addition product reduces silver nitrate in alkaline solution, but has no effect in the presence of an excess of nitric acid. If, therefore, the formaldehyde to be tested be mixed with a known solution of potassium cyanide, the latter being in excess, and the mixture added to a standard solution of silver nitrate acidulated with nitric acid, only the excess of potassium cyanide reacts with the silver nitrate. 'The amount of formaldehyde originally present is shown by the quantity of potassium cyanide consumed in the formation of the addition product. The details of the method as here given are nearly identical with those originally recommended by Romijn.

Reagents. - Tenth-normal solutions of silver nitrate and ammonium thiocyanate. A solution of potassium cyanide 6.2 grams per liter. Saturated solution of ferric ammonium sulphate. Nitric acid $1.32 \mathrm{sp}$. gr. (50 per cent). cit.

${ }^{1}$ Romijn : Z. anal. Chem., 1897, 36, 18. Smith : loc. cit. Williams : loc. 
Determination. - (1) Measure 15 cc. tenth-normal silver nitrate into a 100 -cc. flask, add 6 to 8 drops of the nitric acid and $10 \mathrm{cc}$. of the cyanide solution; shake, dilute to the mark, mix thoroughly, and filter through a dry paper. Titrate $50 \mathrm{cc}$. of the filtrate with tenth-normal ammonium thiocyanate, using 5 cc. of the ferric solution as indicator. The strength of the silver and of the thiocyanate solutions being known, this titration shows the strength of the cyanide.

(2) Dilute the sample until it contains about 1 per cent of formaldehyde, mix $10 \mathrm{cc}$. of this dilute solution with $35 \mathrm{cc}$. of the cyanide solution, and rinse the mixture into another portion of $15 \mathrm{cc}$. tenth-normal silver nitrate, acidulated with 6 to 8 drops of the nitric acid and contained in a 100-cc. flask; shake and determine the excess of silver by means of thiocyanate in the same way as before. Twice the difference between the two titrations (since only half the liquid was used in each case) represents the amount of cyanide consumed by the formaldehyde. If the thiocyanate solution is exactly tenth-normal, twice the difference (in cubic centimeters) between the two titrations, multiplied by $0.0030 \Omega 2$, gives the weight of formaldehyde (in grams) in the portion taken for the determination.

Notes. - This method is applicable to very dilute solutions of formaldehyde, larger volumes being used in place of the $10 \mathrm{cc}$. called for in the above directions. Smith obtained accurate results upon a solution containing 0.01 per cent.

Ethyl and methyl alcohols, acetone, benzaldehyde, and paraldehyde do not interfere. Acetaldehyde causes high results if allowed to stand for some time in contact with the cyanide solution, but if the formaldehyde solution is added to the cyanide and, after mixing, poured at once into the silver nitrate solution, the presence of acetaldehyde does not influence the results. With commercially pure solutions of formaldehyde in water Romijn and Smith obtained concordant results by the iodimetric and cyanide methods. Williams obtained concordant results by the ammonia and the cyanide methods, which were slightly lower than those obtained by the oxidation methods. 


\section{BENZALDEHYDE}

Many methods have been advanced for the determination of benzaldehyde. Among these the methods based on reactions with phenylhydrazine and its derivatives, and with neutral sulphite, are worthy of special notice in that they are fairly accurate and serve to illustrate the analytical application of fairly general aldehyde reactions.

\section{Phenylhydrazine Method}

By simple condensation benzaldehyde and phenylhydrazine yield an insoluble derivative which may be collected, washed, dried, and weighed.

$\mathrm{C}_{6} \mathrm{H}_{5} \mathrm{CHO}+\mathrm{C}_{6} \mathrm{H}_{5} \mathrm{NH} \cdot \mathrm{NH}_{2}=\mathrm{C}_{6} \mathrm{H}_{5} \mathrm{CH}: \mathrm{N} \cdot \mathrm{NH} \cdot \mathrm{C}_{6} \mathrm{H}_{5}+\mathrm{H}_{2} \mathrm{O}$.

This method, as developed by Denis and Dunbar ${ }^{1}$ and adopted by the Association of Official Agricultural Chemists, ${ }^{2}$ is as follows :

Determination of Benzaldehyde in Almond Extract (Denis and Dunbar)

Reagent. - Add 1.5 cc. of glacial acetic acid to $20 \mathrm{cc}$. of water and mix with $1 \mathrm{cc}$. of phenylhydrazine.

Manipulation.-Measure out two portions of $10 \mathrm{cc}$. each of the extract in $300 \mathrm{cc}$. Erlenmeyer flasks and add $10 \mathrm{cc}$. of the reagent to one flask and $15 \mathrm{cc}$. to the other. Allow to stand in a dark place overnight, add 200 cc. of water, and filter on a weighed Gooch crucible having a thin felt of asbestos. Wash first with cold water, finally with $10 \mathrm{cc}$. of 10 per cent alcohol, and dry for three hours in a vacuum oven at $70^{\circ} \mathrm{C}$., or to constant weight over sulphuric acid. If the duplicate results do not agree, repeat the determination, using a larger quantity of the reagent.

\section{Neutral Sulphite Method}

Benzaldehyde, in common with many other aldehydes, reacts with neutral sodium sulphite in such a way that there results

1 J. Ind. Eng. Chem., 1907, 1, 256.

2 U. S. Dept. Agr., Bur. Chem., Bul. 137, pp. 74, 121. 
the aldehyde-bisulphite addition product and sodium hydroxide, thus:

$\mathrm{C}_{6} \mathrm{H}_{5} \mathrm{CHO}+\mathrm{Na}_{2} \mathrm{SO}_{3}+\mathrm{H}_{2} \mathrm{O}=\mathrm{C}_{6} \mathrm{H}_{5} \mathrm{CH}(\mathrm{OH}) \mathrm{SO}_{3} \mathrm{Na}+\mathrm{NaOH}$.

The sodium hydroxide formed is titrated and furnishes a measure of the amount of benzaldehyde which was present.

The details of this method as adopted in the U.S. Pharmacopœia are as follows :

\section{Assay of Benzaldehgde (U. S. Pharmacopoia)}

Introduce into a 150 -cc. flask $10 \mathrm{cc}$. purified kerosene, note the exact weight, add 12 drops of benzaldehyde, and again note the weight; add 20 cc. of water, 6 drops of phenolphthalein solution, and neutralize exactly by the addition of tenth-normal sodium hydroxide, shaking thoroughly. Then add from a burette, gradually, a solution of sodium sulphite ( 1 in 5), alternating with half-normal hydrochloric acid from a second burette, until $10 \mathrm{cc}$. of the sodium sulphite solution have been added, and enough half-normal hydrochloric acid to maintain the neutrality of the mixture; after adding a few drops of phenolphthalein solution and shaking the flask frequently, allow it to stand two hours to insure a permanent condition of neutrality, and then note the volume of half-normal acid used. Carry out a blank test identical with the foregoing except that the benzaldehyde is omitted and note the amount of acid consumed.

From the difference in volume of the half-normal acid used in the two cases, calculate the amount of benzaldehyde which reacted with sulphite according to the equation given above.

For discussion of the application of the neutral sulphite method to other aldehydes see the papers of Burgess ${ }^{1}$ and Sadtler ${ }^{2}$.

When this method is applied to aldehydes which have an ethylene linkage, there may occur a further reaction with addition of bisulphite at this point as well as at the carbonyl group and a correspondingly increased liberation of sodium hydroxide. 


\section{VANILLIN}

In the case of vanillin, the sulphite method appears to be inapplicable, and in this laboratory the bisulphite method has given results somewhat too low. ${ }^{1}$ Better results have been obtained by the alkalimetric method of Welmans, ${ }^{2}$ and by the Hanus ${ }^{3}$ method of condensation with p. bromphenylhydrazine:

$$
\begin{aligned}
& \mathrm{C}_{6} \mathrm{H}_{3}(\mathrm{OH})\left(\mathrm{OCH}_{3}\right) \mathrm{CHO}+\mathrm{C}_{6} \mathrm{H}_{4}(\mathrm{Br}) \mathrm{NH} \cdot \mathrm{NH}_{2}= \\
& \mathrm{C}_{6} \mathrm{H}_{3}(\mathrm{OH})\left(\mathrm{OCH}_{3}\right) \mathrm{CH}: \mathrm{NH} \cdot \mathrm{NHC}_{6} \mathrm{H}_{4} \mathrm{Br}+\mathrm{H}_{2} \mathrm{O} .
\end{aligned}
$$

Twenty-five cubic centimeters of a water solution containing 0.5 to 1 per cent of vanillin are treated with $75 \mathrm{cc}$. of a hot water solution containing 0.5 to 0.75 gram of p. bromphenylhydrazine. At the conclusion of the precipitation the mixed liquid should be at about $50^{\circ}$; the precipitate is allowed to stand 5 hours, filtered on a Gooch crucible, washed with hot water till washings show no precipitation nor distinct coloration with silver nitrate, dried at $95^{\circ}-100^{\circ}$ to constant weight, and weighed. With pure vanillin solutions this method gave nearly theoretical results. ${ }^{1}$

\section{REFERENCES}

\section{I}

Allen : Commercial Organic Analysis.

LUNGE: Chemisch-technische Untersuchungsmethoden.

MeYer : Analyse und Konstitutiousermittelung organischer Verbindungen. Mulliken : Identification of Pure Organic Compounds, Vol. I.

VAUBEL: Quantitative Bestimmung organischer Verbindungen.

\section{II}

1904. Burgess : Estimation of Aldehydes and Ketones in Essential Oils. Analyst, 29, 78.

1905. Hanus: Ueber eine quantitative Bestimmung des Vanillins. $Z$. Nahr. Genussm., 10, 585.

Haywood and Sмiтh: A Study of the Hydrogen Peroxide Method of Determining Formaldehyde. J. Am. Chem. Soc., 27, 1183.

1 Determinations by B. G. Feinberg, not yet published.

2 Pharm. Ztg., 1898, 34, 634 ; Vaubel, II, 88.

${ }^{3}$ Z. Nahr. Genussm., 3, 531 ; 10, 585. 
SadtLer: A Fuller Study of the Neutral Sulphite Method for Determining Some Aldehydes and Ketones in Essential Oils. $J$. Am. Chem. Soc., 27, 1321.

Williams : A Study of Methods for the Determination of Formaldehyde. J. Am. Chem. Soc., 27, 596.

Winton and BaIley: The Determination of Vanillin, Coumarin, and Acetanilid in Vanilla Extract. J. Am. Chem. Soc., 27, 719.

1906. Chase: A Method for the Determination of Citral in Lemon Oils and Extracts. J. Am. Chem. Soc., 28, 1472.

1907. DoвY: (Comparison of Methods for Determination of Formaldehyde). $Z$. angew. Chem., 20, 353.

1908. Woodman and Lyford: The Colorimetric Estimation of Benzaldehyde in Almond Extracts. J. Am. Chem. Soc., 30, 1607.

1909. Denis and Dunbar: Determination of Benzaldehyde in Almond Flavoring Extract. J. Ind. Eng. Chem., 1, 256. 


\section{CHAPTER III}

\section{Carbohydrates - General Methods}

THE carbohydrates include the simple sugars (monosaccharides) and the substances which can be converted into simple sugars by hydrolysis. The monosaccharides are aldehyde alcohols or ketone alcohols, each molecule containing a carbonyl group, either as such or in tautomeric form, and several hydroxyl groups, one of the latter being adjacent to the carbonyl group.

The purpose of this chapter is to outline the more important general methods and analytical properties of the following carbohydrates:

Monosaccharides: Hexoses - Dextrose (d. glucose), Levulose (d. fructose), Galactose, Mannose; Pentoses-Xylose, Arabinose.

Disaccharides: Sucrose, Lactose, Maltose.

Trisaccharide: Raffinose.

Polysaccharides: Starch, Dextrin, Glycogen, Galactan, Cellulose, Pentosans.

\section{OCCURRENCE AND RELATIONS}

Monosaccharides (glucoses, glycoses, monoses) have the composition $\left(\mathrm{CH}_{2} \mathrm{O}\right)_{x}{ }^{1}$ and are called tetroses, pentoses, hexoses, etc., according to the number of carbon atoms in the molecule. Only pentoses and hexoses are of sufficient practical importance to call for consideration in connection with ordinary methods of analysis. The pentoses do not occur free in nature but

1 This statement does not apply to the methyl derivatives now frequently classified as monosaccharides. 
are met by the analyst as products of the hydrolysis of the pentosans. The hexoses include all of the monosaccharides of present commercial importance and all whose biological relations have been thoroughly studied.

Dextrose (d. glucose, grape sugar, starch sugar, diabetic sugar, ordinary glucose) is widely distributed in nature, occurring especially in fruits and plant juices, often mixed with other sugars. It is a normal constituent of blood and is the form of carbohydrate ordinarily found in the urine in diabetes or glycosuria. With the exception of the pentosans and galactan, all of the di-, tri-, and polysaccharides mentioned above yield dextrose on hydrolysis.

Levulose (d. fructose, fruit sugar) occurs with dextrose in plant juices and especially in fruits and honey. It is also a product of the hydrolysis of sucrose and of raffinose.

Galactose does not occur free; but as a product of hydrolysis of lactose, raffinose, and the galactans it is of considerable analytical importance.

Mannose also is not found free, but has been detected among the products of hydrolysis of the insoluble carbohydrate matter of a number of thick-walled vegetables tissues, nut shells, etc., and of several Japanese vegetables.

The disaccharides considered here are all hexo-bioses $\left(\mathrm{C}_{12} \mathrm{H}_{22} \mathrm{O}_{11}\right)$.

Sucrose (saccharose, cane sugar) is widely distributed in the vegetable kingdom, being found in considerable quantity, generally mixed with dextrose and levulose, in the fruits and juices of many plants. The most important sources of sucrose are the sugar beet, the sugar and sorghum canes, and the sugar maple. A molecule of sucrose yields on hydrolysis one molecule each of dextrose and levulose. The hydrolysis of sucrose is often called "inversion" and the resulting mixture of equal parts dextrose and levulose is known as "invert sugar."

Lactose (lactobiose, milk sugar) occurs in the milk of most mammals, constituting usually from 4 to 7 per cent of the fresh secretion. Lactose crystallizes with one molecule of water which it retains on drying at room temperature over 
sulphuric acid or on heating in the air at $100^{\circ}$, but loses at about $130^{\circ}$. A molecule of lactose yields on hydrolysis one molecule each of dextrose and galactose.

Maltose (malt sugar) is formed from starch by the action of diastatic enzymes and is therefore an important constituent of germinating cereals, malt, malt extract, and beer wort. It is also formed as an intermediate product when starch is hydrolyzed to dextrose by boiling with dilute mineral acids, as in the manufacture of commercial glucose. Maltose crystallizes with one molecule of water, which it loses on heating in the air at $100^{\circ}$. Each molecule of maltose yields two molecules of dextrose on hydrolysis.

The only trisaccharide of practical importance is raffinose $\left(\mathrm{C}_{18} \mathrm{H}_{32} \mathrm{O}_{16}\right)$, also called meletriose and formerly melitose or gossypose. It occurs in cotton seed and in small quantity in the germs of various other seeds including wheat and barley. Sugar beets, especially if unhealthy or injured, sometimes contain raffinose in sufficient quantity to affect the refining process. Raffinose crystallizes with five molecules of water in needles or slender prisms and has a marked influence upon the crystallization of the cane sugar present. ${ }^{1}$ Raffinose loses its water of crystallization at $100^{\circ}$. On hydrolysis it yields one molecule each of dextrose, levulose, and galactose. Partial hydrolysis results in the formation of levulose and the disaccharide, melibiose.

Starch $\left(\mathrm{C}_{6} \mathrm{H}_{10} \mathrm{O}_{5}\right)_{x}$ is the most important of the polysaccharides, being the principal form of carbohydrate in grains and most other edible seeds, as well as in potatoes and other tubers. It is the main product of the assimilation process and the principal reserve carbohydrate of most green plants. Commercially it is of great importance as a constituent of foods, as the source of dextrin, maltose, and commercial glucose, and as the principal raw material of many of the fermentation industries. Starch çonstitutes over one half of the solid matter of all ordinary cereals and about three fourths of the total solids in potatoes. Starch granules of different plants vary in size

1 Stone and Baird: J. Am. Chem. Soc., 1897, 19, 116. 
and structure so that in most cases the source of a starch which has not been altered by heat, ferments, or chemical reagents can be determined by microscopical examination. All starches yield dextrose only, as the final product of complete hydrolysis.

Dextrins, $\left(\mathrm{C}_{6} \mathrm{H}_{10} \mathrm{O}_{5}\right)_{x}$ or $\left(\mathrm{C}_{6} \mathrm{H}_{10} \mathrm{O}_{5}\right)_{x} \cdot \mathrm{H}_{2} \mathrm{O}$, are formed from starch by the action of enzymes, acids, or heat. Small amounts of dextrin are found in normal, and larger amounts in germinating, cereals. Malt diastase acting upon starch in fairly concentrated solution yields usually about one part of dextrin to four of maltose. During acid hydrolysis, dextrin is formed as an intermediate product between soluble starch and maltose. Commercial dextrin, the principal constituent of "British gum," is obtained by heating starch, either alone or with a small amount of dilute acid.

Glycogen, $\left(\mathrm{C}_{6} \mathrm{H}_{10} \mathrm{O}_{5}\right)_{x}$ or perhaps $\left(\mathrm{C}_{6} \mathrm{H}_{10} \mathrm{O}_{5}\right)_{x} \cdot \mathrm{H}_{2} \mathrm{O}$, is the principal carbohydrate of the animal organism, being found in small quantity in the muscles and more abundantly in the liver of all well-nourished animals. It is a white amorphous powder intermediate in properties between starch and dextrin and is sometimes called animal starch. The determination of glycogen is often important in physiological investigations and is sometimes useful in distinguishing horseflesh from beef, the latter containing usually less than 0.7 per cent of glycogen, the former often two or three times this amount. On complete hydrolysis glycogen yields only dextrose.

Galactans, amorphous polysaccharides yielding galactose on hydrolysis, occur in small quantity in many plants and in relative abundance in the seeds of the legumes where they largely replace starch as reserve carbohydrate. Since the galactans are readily hydrolyzed by hot dilute acids and are digested by some of the diastatic enzymes, it is probable that galactan has been reported as starch in many analyses.

Cellulose occurs in the cell walls of all vegetable tissues. The term is sometimes applied to the whole of the fiber which is unattacked by boiling dilute acids and alkalies, but should be restricted to that constituent of the fiber which is of a true carbohydrate nature. "Normal" cellulose, such as is derived 
from cotton and flax fibers, yields dextrose on hydrolysis. A few celluloses have been found to yield mannose or a pentose (probably xylose) in addition to dextrose (Tollens).

Pentosans, anhydrides of arabinose and xylose, are the principal constituents of the vegetable gums, araban occurring especially in the soluble gums such as cherry gum and gum arabic, xylan in the so-called wood gum of fibrous tissues such as wood, straw, vegetables, and the outer portion of the cereal grains. The wheat grain, for example, contains 3 to 5 per cent of pentosan, which in the milling process is largely left in the bran. The so-called patent flour obtained from the interior of the grain contains hardly any pentosan, while the breakfast cereals and the so-called entire wheat and graham flours have usually about as much as the original grain.

\section{SOLUBILITIES}

\section{IN WATER}

Of the carbohydrates mentioned above, all except the polysaccharides are crystallizable compounds dissolving in water to form clear solutions. Milk sugar dissolves in six parts of water at ordinary temperature; all of the other members are more freely soluble. Among the polysaccharides, dextrin, glycogen, and some of the galactans and pentosans are soluble; starch, cellulose, some of the galactans, and most of the pentosans of ordinary food materials are insoluble in cold water. Glycogen gives a strongly opalescent solution, which is not cleared by repeated filtration but loses its opalescence on the addition of a little potassium hydroxide or acetic acid. On heating with water, starch grains swell and finally gelatinize with the formation of " starch paste." Different starches vary considerably in the temperature at which they gelatinize and in the physical properties of the paste produced. Thin starch pastes can be filtered through paper, but almost always leave some gelatinous residue upon the filter. Pastes containing only a few hundredths of one per cent of starch become clear on boiling and can be filtered without loss. Water-soluble starch 
can be prepared ${ }^{1}$ by chemical treatment and is sometimes found in natural products, for example in immature grains.

Cellulose and the ordinary pentosans of foods and fibers are insoluble in water and not gelatinized by boiling.

\section{In Alcohol and Ether}

Levulose is soluble in 5 parts of cold absolute alcohol and is somewhat soluble in mixtures of ether and strong alcohol. The other monosaccharides are sparingly soluble in cold alcohol, insoluble in ether, and practically insoluble in the alcohol-ether mixture. Dextrose is much more readily soluble in hot than in cold alcohol; 100 parts of 90 per cent alcohol dissolve about 2 parts dextrose at $18^{\circ}$, about 22 parts at boiling temperature.

The di-, tri-, and polysaccharides are insoluble in ether. Diand trisaccharides are less soluble in alcohol than is dextrose. Lactose is practically insoluble in alcohol, even when the latter is diluted to 60 per cent.

Sucrose is much more readily soluble in diluted than in concentrated alcohol. According to Scheibler :

100 parts 90 per cent alcohol dissolve 0.9 parts at $14^{\circ} ; 2.3$ parts at $40^{\circ}$

100 parts 80 per cent alcohol dissolve 6.6 parts at $14^{\circ} ; 13.3$ parts at $40^{\circ}$

100 parts 70 per cent alcohol dissolve 18.8 parts at $14^{\circ} ; 31.4$ parts at $40^{\circ}$

100 parts 60 per cent alcohol dissolve 33.9 parts at $14^{\circ} ; 49.9$ parts at $40^{\circ}$

100 parts 50 per cent alcohol dissolve 47.1 parts at $14^{\circ} ; 63.4$ parts at $40^{\circ}$

Sucrose dissolves in about 80 parts of boiling absolute alcohol.

All of the polysaccharides are insoluble in alcohol. Those which are soluble in water can be precipitated from their aqueous solutions by the addition of strong alcohol.

\section{In Acids AND Alkalies}

Among the carbohydrates which are insoluble in water, separations can sometimes be made by the use of acid or alkaline solutions.

Cellulose is soluble in concentrated sulphuric acid, but in-

${ }^{1}$ Lintner: J. prakt. Chem., 1886, [2] 34, 381. Wroblewski : Z. physiol. Chem., 1898, 24, 173. Vaubel : II., 500. 
soluble in any ordinary aqueous solution of acid or alkali. It dissolves in Schweitzer's reagent (aqueous ammonia saturated with cupric hydroxide) to a viscous solution, from which it is precipitated by neutralization with acid.

Starch is insoluble in Schweitzer's reagent or in solutions of ammonia. Treated with dilute aqueous solutions of sodium or potassium hydroxide, starch swells, gelatinizes, and becomes soluble. It can be completely precipitated from such a solution by neutralizing with acetic acid and adding strong alcohol. Starch is not affected by dilute solutions of alkalies in strong alcohol. By diluted solutions of strong acids starch is first dissolved, then hydrolyzed. Some weak organic acids dissolve starch with little if any hydrolysis. Boiling water containing 1 per cent of salicylic acid dissolves starch to an opalescent solution which filters much more readily than a corresponding starch paste made with water alone.

The pentosans of foods and fibers - so-called wood gums consisting mainly of xylan - are insoluble in dilute ammonia or in Schweitzer's reagent, largely soluble in dilute aqueous solutions of sodium or potassium hydroxide and in cold dilute acids. From such solutions the pentosan is precipitated by alcohol. Boiling dilute mineral acids dissolve and hydrolyze pentosans almost as readily as starch. Hence pentosans have frequently been reported as starch when the latter has been estimated by direct hydrolysis with acid and determination of the resulting glucose.

\section{REACTIONS WITH ACIDS}

All carbohydrates on treatment with moderately strong hydrochloric or sulphuric acid yield furfural, which is probably the cause of the color reaction with $\alpha$-naphthol described below. Hexacarbohydrates form only small amounts of furfural and relatively large amounts of levulinic acid. Pentacarbohydrates yield large amounts of furfural and no levulinic acid. In all cases, however, there is more or less formation of "humus" and other by-products.

The following is widely used as a general qualitative test for carbohydrates. 


\section{Molisch's $\alpha$-NAphthol Reaction 1}

Mix 2 to 3 ec. of the very dilute solution to be tested with 2 or 3 drops of a 15 per cent solution of $\alpha$-naphthol in alcohol or chloroform, incline the tube, and pour in carefully 2 to $3 \mathrm{cc}$. of pure concentrated sulphuric acid. In the presence of carbohydrate, a violet zone appears quickly and spreads by diffusion. If the solution tested contains more than a few milligrams of carbohydrate, it quickly blackens and on dilution with water gives a dull violet precipitate.

A similar test with thymol gives a crimson or carmine-red solution which soon becomes turbid.

\section{Furfural Test for Pentoses and Pentosans}

Place the substance to be tested in an Erlenmeyer flask, add hydrochlóric acid (1.06 sp. gr.), and boil. Lay over the mouth of the flask a small filter paper moistened with a solution of anilin acetate. ${ }^{2}$ If the vapor escaping from the flask contains more than traces of furfural, a bright red coloration appears. A test with sucrose or pure starch will show the amount of color to be expected from hexacarbohydrates.

If the reaction obtained from the unknown substance is much stronger than from sucrose or starch, the presence of pentoses or pentosans is indicated. A few other substances such as glycuronic acid and oxycellulose have been found to yield considerable amounts of furfural; but these rarely occur in sufficient quantities to require consideration.

\section{Determination of Pentoses and Pentosans}

Under carefully regulated conditions the yield of furfural from either xylose or arabinose, or from the corresponding anhydride, is nearly constant. If the furfural is distilled and collected, its amount can be estimated by adding anilin acetate

1 Molisch : Monatsh. Chem., 1886, 7, 198. Udranszky : Z. physiol. Chem., 1888, 12, 355, 377. Tollens : Handbuch der Kohlenhydrate, II, 101. Mulliken : Identification of Pure Organic Compounds, I, 26.

2 Prepared by mixing equal volumes of anilin and 50 per cent acetic acid. 
and comparing the red color with that shown by furfural solutions of known strength. ${ }^{1}$ This method is delicate, but gives only approximate results. Unless the quantity is very small, it should be determined gravimetrically.

Furfural forms sparingly soluble condensation products with a number of bases and phenols. Phenylhydrazine and phloroglucin have been principally used as precipitants. ${ }^{2}$ The Association of Official Agricultural Chemists has adopted provisionally the phloroglucin method. ${ }^{3}$

\section{Levulinic Acid Reaction of Hexacarbohydrates}

According to Wehmer and Tollens, ${ }^{4}$ all hexacarbohydrates yield levulinic acid in sufficient quantity for identification when treated as follows :

Heat 5 to 20 grams of the substance with $100 \mathrm{cc}$. of hydrochloric acid (1.10 sp. gr.) for 18 hours on a boiling water bath; filter the solution, shake with ether to extract the levulinic acid, and convert the latter into the zinc or silver salt for identification. As this method is purely qualitative and is not often used in ordinary analytical work, reference must be made to the original paper for details of manipulation.

\section{Oxidation by Nitric Acid}

By heating with moderately strong nitric acid, xylose and arabinose are oxidized, each yielding a trioxyglutaric acid, $\mathrm{COOH}(\mathrm{CHOH})_{3} \mathrm{COOH}$, while aldoses of the hexose group yield the corresponding acids, $\mathrm{COOH}(\mathrm{CHOH})_{4} \mathrm{COOH}$, sac-

1 This colorimetric method is also used for the estimation of furfural in distilled liquors.

${ }^{2}$ Barbituric acid has recently been recommended as preferable to phenylhydrazine and phloroglucin as a precipitant for furfural. Unger: Dissertation, Munich, 1904. Jäger and Unger: Ber., 1902, 35, 4440.

${ }^{3}$ Bull. 107, Bur. Chem., U. S. Dept. Agriculture. For a review and discussion of the methods of determining the pentosans and the practical applications of the results see Kröber, Rimbach, and Tollens, Z. angew. Chem., 1902, 15, 477, 508. Also Fraps : Am. Chem. J., 1901, 25, 501.

${ }^{4}$ Ann. Chem., 1887, 243, 314. 
charic, mannosaccharic, and mucic acids being obtained respectively from dextrose, mannose, and galactose.

All of these oxidation products are freely soluble except mucic acid, which is practically insoluble in water or dilute nitric acid. Since mucic acid is produced in fairly constant proportion, its insolubility affords a means for the approximate determination of galactose or any substance which yields galactose on hydrolysis.

\section{Mucic Acid Method for Galactose, Lactose, Raffinose, and Galactans. ${ }^{1}$}

Weigh 1 to 3 grams of substance according to the amount of mucic acid expected; remove fat if necessary by washing with ether; transfer to a beaker about $5.5 \mathrm{~cm}$. in diameter and $7 \mathrm{~cm}$. deep; add $60 \mathrm{cc}$. of nitric acid of $1.15 \mathrm{sp}$. gr. and evaporate the solution to exactly one third its volume on a water bath at a temperature of $94^{\circ}$ to $96^{\circ}$. After standing 24 hours, add 10 cc. of water to the precipitate and allow it to stand another 24 hours. The mucic acid has now crystallized, and, unless contaminated with insoluble residue from the sample, it can be transferred to a weighed filter, washed with $30 \mathrm{cc}$. of water, then with alcohol and ether, dried at $100^{\circ}$, and weighed.

In case other insoluble substances are present, return the filter and mucic acid, after washing with water, to the beaker; warm 15 minutes with a mixture of 1 part strong ammonia, 1 part ammonium carbonate, and 19 parts of water : filter and wash; evaporate filtrate to dryness over a water bath; add 5 cc. nitric acid of $1.15 \mathrm{sp}$. gr.; stir thoroughly and allow to stand for 30 minutes. Collect the mucic acid on a weighed filter, wash with 10 to $15 \mathrm{cc}$. of water, then with $60 \mathrm{cc}$. of alcohol and a number of times with ether; dry at $100^{\circ}$ and weigh.

When these directions are strictly followed, galactose yields about three fourths its weight of mucic acid. The weight of

1 Tollens and Rischbiet: Ber., 1885, 18, 2616, Creydt : Ibid., 1886, 19, 3115. Bull. 107, Bur. Chem., U. S. Dept. Agriculture. 
mucic acid obtained from lactose, raffinose, or galactan is calculated às three fourths the weight of galactose which could be obtained by hydrolysis. The yield of mucic acid is, however, considerably influenced by the details of manipulation. In carrying out the method comparative determinations with pure milk sugar (both alone and mixed in known proportions with the sample under examination) should always be made, as substances may be present which prevent the crystallization of the mucic acid. 1

\section{Hydrolysis by Dilute Acids}

Monosaccharides, as the name implies, cannot be hydrolyzed to simpler sugars. As a rule, they are unaffected by dilute acids except on prolonged heating, when they are gradually attacked, yielding in part decomposition products like those produced by stronger acids, and undergoing a partial "reversion" with the formation of di- or polysaccharides. Dextrose, for example, when heated too long with dilute hydrochloric or sulphuric acid, is changed partially to "isomaltose" and to dextrin-like anhydrides, such as "gallisin," the unfermentable constituent of crude commercial glucose. Levulose decomposes much more readily than dextrose on heating with dilute acids.

Disaccharides differ considerably in the readiness with which they are hydrolyzed by acids. Sucrose is very easily hydrolyzed, a 20 per cent solution being completely changed to invert sugar by mixing with one tenth its volume of concentrated hydrochloric acid (making about 3 per cent of actual acid in the mixture) and warming to $68^{\circ}$ at such a rate as to require 15 minutes' heating. ${ }^{2}$

Maltose is less easily hydrolyzed than sucrose, a 2 per cent solution in 2 to 3 per cent hydrochloric acid requiring 30 to 40

1 Herzfeld : Z. Vereins Rübenzucker Ind., 1890, 40, 265 ; Abs. Chem. Ztg., 1890, 14, Rep., 108. Stone and Baird: J. Am. Chem. Soc., 1897, 19, 119.

2 Clerget's method, described by Wiley : Agricultural Analysis, Vol. III., pp. 105-107. According to Bornträger and to Samelson (Z. angew. Chem., 1892, $334 ; 1893,690 ; 1894,267,351)$, the sucrose in such a mixture is completely hydrolyzed by standing overnight at room temperature. 
minutes' boiling, or 2 to 3 hours' heating on a water bath, for complete hydrolysis to dextrose. ${ }^{1}$ Lactose is also less readily hydrolyzed than sucrose, the difference being especially marked in the case of weak acid or low temperature.

Raffinose is hydrolyzed by dilute mineral acids, slowly in the cold, much more rapidly on boiling, requiring in either case more vigorous treatment than does sucrose.

Dextrin, glycogen, and starch are hydrolyzed to dextrose under the same conditions as maltose, but require somewhat longer heating. Pentosans and galactans (at least the more common forms) are hydrolyzed by acids almost as readily as starch. Normal cellulose is not hydrolyzed by boiling dilute acids. Hemicellulose is a term commonly applied to the carbohydrate matter in the cell walls of plants, more resistant to enzymes than starch, but dissolved and hydrolyzed on boiling with dilute mineral acids. The hemicellulose of many of the common food plants consists largely of pentosans.

\section{REACTIONS WITH HYDRAZINES}

All of the monosaccharides, and maltose and lactose among the disaccharides, have the carbonyl group (as such or in the tautomeric form), and therefore on treatment with hydrazines yield hydrazones or osazones. The hydrazines chiefly used are phenylhydrazine, para-bromphenylhydrazine, methylphenylhydrazine $\left(\mathrm{C}_{6} \mathrm{H}_{5} \mathrm{~N}\left(\mathrm{CH}_{3}\right) \cdot \mathrm{NH}_{2}\right)$, diphenylhydrazine, and naphthylphenylhydrazine.

Phenylhydrazine has been most commonly employed, and its behavior with the different sugars has been most fully investigated. The analytical application of the phenylhydrazine reaction is discussed below.

The other hydrazines are used largely for confirmatory tests and also for the purpose of distinguishing between sugars whose behavior with phenylhydrazine is not sufficiently

1 It is sometimes stated that maltose cannot be made to yield more than about 98 per cent of the theoretical amount of dextrose, the acid always beginning to attack the latter before the hydrolysis of the former is quite complete. See notes on the determination of starch. 
different to permit of satisfactory identification by that reagent alone.

Mannose is distinguished from the other sugars by forming with phenylhydrazine an insoluble hydrazone, and this reaction has been applied quantitatively by Bourquelot and Herissey. ${ }^{1}$

The phenylhydrazones of the other sugars are soluble, but when heated with an excess of phenylhydrazine react to form osazones. ${ }^{2}$

To form the osazone, ${ }^{2}$ dissolve one part of the sugar, two of pure phenylhydrazine hydrochloride, and three of crystallized sodium acetate in 20 parts of water in a test tube; filter if not clear ; cork' loosely to avoid evaporation and place the tube in boiling water.

Under these conditions the maximum yield of osazone is usually obtained by warming the solution in the water bath for one to two hours and then allowing it to cool. The osazone thus obtained is ordinarily a yellow iridescent precipitate, more or less distinctly crystalline according to the purity and concentration of the solution. To purify it, filter on a small paper, wash with a little cold water, dissolve in the smallest possible amount of boiling 50 per cent alcohol, and filter hot. The osazone which separates from the alcohol solution may, if desired, be further recrystallized from alcohol or from pyridine.

Dextrose and levulose yield the same osazone, glucosazone, which crystallizes in needles melting at $204^{\circ}-205^{\circ}$ when heated at such a rate that the melting point is reached in three to four minutes. Galactosazone crystallizes in needles which melt at $193^{\circ}$; maltosazone, in independent needles or tables melting at $206^{\circ}$; lactosazone, in masses of microscopic prisms melting at $200^{\circ}$. Xylosazone and arabinosazone melt at about $160^{\circ}$.

A conclusive method of ascertaining whether an osazone is that of a pentose, a hexose, or a disaccharide is to determine the percentage of nitrogen. In the case of a mixture, the hex-

${ }^{1}$ J. Pharm. Chim., 1899, [6], 10, 206. See also Pellet : Bul. Assoc. Chim. Sucr. Distill., 1900-01, 18, 70̄8; Abs. Z. Nahr. Genussm., 1902, 5, 74.

${ }^{2}$ Fischer : Ber., 1884, 17, 579; 1887, 20, $821 ; 1888,21,1805,2631$; 1889, 22, $87 ; 1890,23,2117$. 
osazone can be freed from osazones of the other two groups by washing with hot water. Maltosazone is soluble in about 75 parts, xylosazone in about 50 parts, and lactosazone in 80 to 90 parts, of boiling water, while the pure hexosazones are nearly insoluble.

Since the melting points of osazones are not very sharply defined and may be appreciably below the figures just given if the osazones are not entirely pure or are not heated at a sufficiently rapid rate, an entirely conclusive identification of a sugar by means of phenylhydrazine alone is usually not to be expected, but by similar reactions with some of the derivatives of phenylhydrazine above mentioned a characteristic hydrazone or osazone may be obtained.

Thus Neuberg ${ }^{1}$ identified arabinose by its diphenylhydrazone and distinguished between glucose and fructose by the difference in their behavior toward methylphenylhydrazine; Tollens and Maurenbrecker ${ }^{2}$ also separate arabinose from xylose by the preparation of the diphenylhydrazone.

$\mathrm{Kahl}^{3}$ found that parabrombenzylhydrazide condenses to form an insoluble hydrazone with glucose, galactose, mannose, or arabinose; not with levulose, maltose, or lactose; and to only a slight extent with xylose. Kendall and Sherman ${ }^{4}$ worked out a method by which this reaction can be made to serve for the identification of any one of the four reacting sugar's, but the usefulness of this method is restricted by the fact that the preparation of the reagent is troublesome and time-consuming.

Neuberg ${ }^{5}$ made use of the differences in optical activity of the osazones as a means of identification. Dissolving $0.2 \mathrm{gram}$ osazone in $4 \mathrm{cc}$. pyridine plus $6 \mathrm{cc}$. absolute alcohol and examining the solution in a $100-\mathrm{mm}$. tube in the circular-scale polariscope (see discussion of polariscope methods beyond), he obtained the following results:

1 Ber., 33, 2243 ; 35, 959.

2 Ber., 38, 500.
${ }^{3}$ Dissertation, Freiburg, 1904.

4 J. Am. Chem. Soc., 1908, 30, 1451.

5 Ber., 1899, 32, 3384 


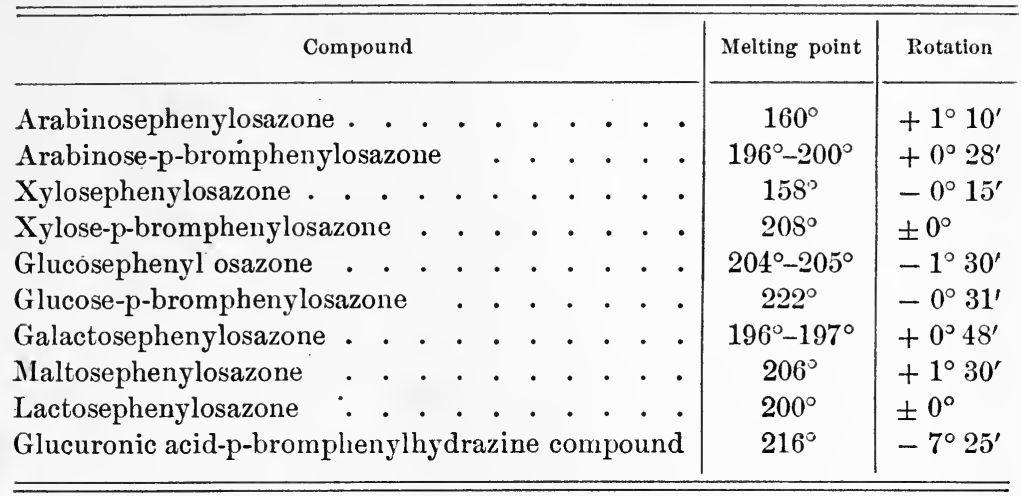

Only rarely are the rotatory powers of the osazones of assistance in analytical work, since the differences are small and difficult to observe.

By the application of the different hydrazines as reagents, and the study of the products obtained with reference to solubility, crystalline form, melting point, etc., it is possible to identify the individual sugars even in such mixtures as are obtained by the hydrolysis of plant tissues or in complex artificial mixtures of carbohydrates which do not occur together in nature. For a full account of such methods see the works of Abderhalden, Browne, Lippmann, Oppenheimer, and Tollens.

If, however, the problem is to identify a pure sugar or a simple mixture of sugars, it will usually be more convenient to apply the osazone reaction in the manner described below and depend upon other methods to complete the identification as described later.

\section{Analytical Application of the Osazone Reaction}

Maquenne ${ }^{1}$ found that the reducing sugars when treated in a uniform way with phenylhydrazine showed considerable differences both in the yield of osazone and in the time required for the appearance of the osazone precipitate. Mulliken ${ }^{2}$ has

1 Compt. rend., 112, 799.

2 Identification of Pure Organic Compounds, Vol. I. 
studied these differences in rapidity of osazone formation and makes use of them to an important extent in his scheme, for the identification of pure sugars. According to Mulliken, 0.1 gram sugar, 0.2 gram pure phenylhydrazine hydrochloride, 0.3 gram sodium acetate, and 2 cc. water are mixed in a small test tube, corked loosely to prevent evaporation, and heated in boiling water. If the tube is occasionally shaken without removing it from the boiling water, the osazone precipitate usually separates out quite suddenly so that duplicate experiments usually give results that agree within half a minute. Under these conditions the time required for the appearance of the osazone in the hot solution is given by Mulliken as follows: Fructose, two minutes; sorbinose, three and one half minutes; glucose, four to five minutes; xylose, seven minutes; rhamnose, nine minutes; arabinose, ten minutes; galactose, fifteen to nineteen minutes. Sucrose, after about thirty minutes' heating, is sufficiently hydrolyzed to yield a slight precipitate of glucosazone. Maltose and lactose give no precipitate in the hot solution even when the heating is continued for two hours.

Sherman and Williams ${ }^{1}$ followed closely the conditions adopted by Mulliken except that, for greater convenience of manipulation, twice the quantities were used throughout. Having confirmed the results above given for glucose, fructose, sucrose, maltose, and lactose at the standard dilution, they determined the times required for the osazone precipitation with smaller amounts of glucose or fructose in pure solution and also when different amounts of other sugars were present at the same time. Every test was carried out as has been described, using 0.4 gram phenylhydrazine hydrochloride, 0.6 gram sodium acetate, and $4 \mathrm{cc}$. water, ${ }^{2}$ so that the only variable factor was the amount of sugar or sugars present. The tabular statements which follow show the time of heating required for the appearance of an osazone precipitate in each case.

1 J. Am. Chem. Soc., 1906, 28, 629.

2 If the solution at this point is not clear, it is filtered through a dry paper before heating. 
Pure Solutions of Glucose, Fructose, Invert Sugar, or Sucrose

\begin{tabular}{l|c|c|c|c}
\hline \hline \multirow{2}{*}{$\begin{array}{c}\text { Weight of sugar } \\
\text { taken }\end{array}$} & $\begin{array}{c}\text { Glucose } \\
\text { Minutes }\end{array}$ & $\begin{array}{c}\text { Fructose } \\
\text { Minutes }\end{array}$ & $\begin{array}{c}\text { Invert sugar } \\
\text { Minutes }\end{array}$ & $\begin{array}{c}\text { Sucrose } \\
\text { Minutes }\end{array}$ \\
\cline { 2 - 5 } 0.2 & $4-5$ & $1 \frac{1}{4}-1 \frac{1}{2}$ & $1 \frac{1}{2}-1 \frac{2}{3}$ & $\frac{31}{35}$ \\
0.1 & 5 & $1 \frac{3}{4}-2$ & 2 & 78 \\
0.05 & $6 \frac{1}{2}$ & $2 \frac{1}{2}$ & 3 & No ppt. \\
0.01 & 17 & $5 \frac{1}{2}$ & $6-6 \frac{1}{2}$ & \\
0.005 & 34 & 10 & 14 & \\
0.0025 & 65 & 17 & & \\
\hline \hline
\end{tabular}

With less than 0.005 gram glucose or 0.0025 gram fructose, the amount of osazone separating in the hot solution was small and the time of its appearance doubtful.

Influence of Maltose on Glucose

\begin{tabular}{|c|c|c|c|c|c|}
\hline \multirow{2}{*}{$\begin{array}{l}\text { Weight of } \\
\text { glucose } \\
\text { Gram }\end{array}$} & \multicolumn{4}{|c|}{ Weight of maltose } & \multirow{2}{*}{$\begin{array}{c}\text { In absence } \\
\text { of } \\
\text { maltose }\end{array}$} \\
\hline & $0.2 \mathrm{gram}$ & 0.1 gram & 0.05 gram & $0.01 \mathrm{gram}$ & \\
\hline 0.01 & No ppt. & $40 \mathrm{~min}$. & $30 \mathrm{~min}$. & 22 min. & 17 min. \\
\hline
\end{tabular}

Influence of Lactose on Glucose

Weight of glucose Gram

0.01 0.02

Weight of lactose

\begin{tabular}{cccc}
\hline 0.2 gram & $0.1 \mathrm{gram}$ & $0.05 \mathrm{gram}$ & $0.01 \mathrm{gram}$ \\
No ppt. & $50 \mathrm{~min}$. & $32 \mathrm{~min}$. & $25 \mathrm{~min}$. \\
\hline $5-48 \mathrm{~min}$. & & &
\end{tabular}

In absence of lactose

$17 \mathrm{~min}$. 12-13 $\mathrm{min}$.

It is evident that both maltose and lactose interfere seriously with the formation and precipitation of glucosazone and that the influence of lactose is greater than that of maltose. Thus a mixture of 0.01 gram glucose and 0.1 gram lactose required ten minutes' longer heating than a parallel mixture with 0.1 gram maltose, and when the quantities of glucose, lactose, and maltose are doubled (the amounts of reagents and the volume of the solution remaining the same) the lactose mixture required twenty minutes' longer heating than the maltose mixture.

\section{Influence of Sucrose on Glucose}

$\begin{gathered}\text { Weight of } \\ \text { glucose } \\ \text { Gram }\end{gathered}$
0.005
0.01

\begin{tabular}{|c|c|c|c|}
\hline \multicolumn{4}{|c|}{ Weight of sucrose } \\
\hline 0.2 gram & 0.1 gram & 0.05 gram & $0.01 \mathrm{gram}$ \\
\hline $\begin{array}{l}15-17 \mathrm{~min} . \\
14-16 \mathrm{~min} .\end{array}$ & $\begin{array}{c}15-17 \mathrm{~min} . \\
16 \mathrm{~min} .\end{array}$ & $\begin{array}{l}22 \mathrm{~min} . \\
17 \mathrm{~min} .\end{array}$ & $\begin{array}{l}30 \mathrm{~min} . \\
17 \mathrm{~min} .\end{array}$ \\
\hline
\end{tabular}

In absence of 33-39 min. $17 \mathrm{~min}$. 
Influence of Maltose on Fructose

Weight of
fructose fructose

0.01

Weight of fructose Gram 0.01

\begin{tabular}{|c|c|c|c|c|}
\hline \multicolumn{4}{|c|}{ Weight of maltose } & \multirow{2}{*}{$\begin{array}{c}\text { In absence } \\
\text { of } \\
\text { maltose }\end{array}$} \\
\hline $0.2 \mathrm{gram}$ & 0.1 gram & 0.05 gram & 0.01 gram & \\
\hline $7-8 \mathrm{~min}$. & $5 \frac{1}{2}-6$ min. & $5 \frac{1}{2}-5 \frac{3}{4} \mathrm{~min}$ & $5 \frac{1}{2} \mathrm{~min}$. & $5 \frac{1}{2} \mathrm{~min}$ \\
\hline
\end{tabular}

Influence of Lactose on Fructose

\begin{tabular}{|c|c|c|c|c|}
\hline \multicolumn{4}{|c|}{ Weight of lactose } & \multirow{2}{*}{$\begin{array}{c}\text { In absence } \\
\text { of } \\
\text { lactose }\end{array}$} \\
\hline 0.2 gram & 0.1 gram & 0.05 gram & $0.01 \mathrm{gram}$ & \\
\hline $9 \frac{1}{2}-10 \mathrm{~min}$. & $7 \frac{3}{4} \mathrm{~min}$ & $6 \frac{3}{4} \min$. & $6 \mathrm{~min}$. & $5 \frac{1}{2} \min$. \\
\hline
\end{tabular}

Comparing these results with the corresponding figures for glucose, it will be seen that the interference of maltose and lactose is less marked with fructose than with glucose. In both cases, however, the appearance of the osazone precipitate is retarded distinctly by maltose and to a greater extent by lactose.

\section{InflufNeE of Sucrose on Fructose}

Weight of
fructose

Gram

\begin{tabular}{ccccc}
\multicolumn{4}{c}{ Weight of sucrose } & In absence \\
of \\
0.2 gram & $0.1 \mathrm{gram}$ & $0.05 \mathrm{gram}$ & $0.01 \mathrm{gram}$ & \\
$8 \frac{1}{2} \mathrm{~min}$. & $8 \frac{3}{4} \mathrm{~min}$. & $9 \frac{1}{3} \mathrm{~min}$. & $9 \frac{1}{4} \mathrm{~min}$. & $9 \frac{1}{2} \mathrm{~min}$.
\end{tabular}

0.005

$8 \frac{3}{4} \min$.
Here the effect of the sucrose was not so noticeable.

Interpretation of time relations in applying the osazone test to sugar mixtures or to samples in which the concentration of the reacting sugar is not known or is different from that of the conventional method, should be based on a careful study of the above data.

The delicacy of the test in the presence of dextrin (which is known to retard the formation of the osazone), and of substances other than carbohydrates, has not been studied in detail, except in the case of certain constituents of urine. ${ }^{1}$ Hence in applying the osazone test to an unknown solution, it is well to make'at the same time two check experiments, one with a mixture of carbohydrates corresponding to that which the unknown solution is believed to contain, the other with a portion

1 The influence of other substances likely to be present, upon the osazone test for dextrose in urine has been studied by Hirschl : Z. physiol. Chem., 1890, 14, 377. Jaffé : Ibid., 22, 532. Neuberg: Ibid., 1900, 20, 274. Neumann-Wender : Pharmac. Post., 26, 573, 614 (Vaubel ; l. c., II, 309). 
of the unknown solution to which has been added a very small amount of dextrose.

The yield of osazone has been studied especially by Maquenne, ${ }^{1}$ Laves, ${ }^{2}$ Fischer, ${ }^{3}$ Lintner and Kröber, ${ }^{4}$ Raimann, ${ }^{5}$ and Davis and Ling. ${ }^{6}$ In each of these cases the osazone was formed by heating for one or two hours, in a more dilute solution than that above given, with a considerable excess of a slightly acid solution of phenylhydrazine acetate. The precipitate of osazone can then be filtered, washed with water, and weighed. According to Laves the following corrections should be applied for the amount of glucosazone left in solution or dissolved by washing :

100 parts boiling water dissolve . . . . . . . . 0.01 part osazone 100 parts water at $20^{\circ}$ dissolve . . . . . . . 0.0042 part osazone 100 parts 2 per cent acetic acid at $20^{\circ}$ dissolve . . . 0.007 part osazone 100 parts 3 per cent acetic acid at $20^{\circ}$ dissolve . . . 0.0145 part osazone 100 parts 4 per cent acetic acid at $20^{\circ}$ dissolve . . . 0.022 part osazone 100 parts 5 per cent acetic acid at $20^{\circ}$ dissolve . . . 0.031 part osazone 100 parts 10 per cent alcohol slightly acidulated dissolve at $20^{\circ}$

Corresponding corrections have not been worked out for other osazones, as the attempts to apply the osazone method quantitatively have been mainly with the object of determining dextrose or dextrose and levulose. In comparative tests carried out in exactly the same manner, the weight of osazone obtained is proportional to the amount of sugar originally present, but slight differences of manipulation affect the yield to such an extent that the osazone precipitation cannot yet be regarded as a satisfactory quantitative method.

Williams ${ }^{7}$ has recently studied the conditions affecting the yield of glucosazone from pure glucose solutions. It was found best to allow one hour of heating for the formation of the

1 Compt. rend., 1891, 112, 799 ; Z. anal. Chem., 1894, 33, 226.

2 Archiv. der Pharm., 1893, 231, 366. Vaubel's Bestimmung organischer Verbindungen, II, 307, 311, 312.

${ }^{8}$ Ber., 1895, 28, 1437.

${ }^{4}$ Z. f. d. ges. Brauwesen, 1895, 18, 153; Z. anal. Chem., 1896, 35, 95 ; Analyst, 1897, 20, 167.

5 Z. anal. Chem., 1901, 40, 390.

6 J. Chem. Soc., 1904, 85, 24.

7 Data not yet published. 
osazone, since longer heating, although increasing the yield, gave a less pure product. A solution of phenylhydrazine acetate made by dissolving the free base in acetic acid gave as good results as the reagent made by mixing phenylhydrazine hydrochloride with sodium acetate and was found more convenient. The amounts of phenylhydrazine and acetic acid greatly influenced the yield of osazone. In a series of experiments in which 0.2 gram pure dextrose in 20 cc. water was heated with varying amounts of reagents for one hour, then washed with 100 cc. water, dried and weighed, it was found that maximum yields, about 60 per cent of the theoretical, were obtained by using 2 grams of phenylhydrazine in 8 cc. of 50 per cent acetic acid, 2.4 grams phenylhydrazine in 9.2 cc. of 50 per cent acetic acid, or 3.2 grams of phenylhydrazine in 6.4 cc. of 50 per cent acetic acid. Thus while a large excess of phenylhydrazine was always essential to high yields, the amount required for a maximum yield depended upon the ratio between the phenylhydrazine and the acetic acid, as well as upon that between the reagents and the sugar.

\section{REDUCTION OF COPPER SOLUTIONS}

The same sugars which react with phenylhydrazine have the power of reducing certain metallic salts, especially salts of copper, silver, and mercury in alkaline solution. This "reducing power," due to the susceptibility of the carbonyl sugars to oxidation, can be utilized for the detection and determination of these sugars.

When for example an alkaline cupric solution, such as Fehling's, is boiled with one of these "reducing" sugars, the copper is reduced to the cuprous state and the sugar is attacked in two ways: (1) oxidation and (2) decomposition by the alkali. Tartronic acid is often cited as a typical oxidation product formed by the action of boiling alkaline copper solution upon dextrose, but in practice less than half as much copper is reduced as would correspond to a complete oxidation of glucose to tartronic acid, because the boiling alkali so largely decomposes the glucose into other than oxidation products. 
In order that the amount of copper reduced may indicate quantitatively the amount of reducing sugar, it is necessary to control the decomposing action of the alkali. This is usually accomplished by prescribing the amount and final dilution of the alkaline solution and either (1) adjusting the sugar solution so as to find the amount which in measured volume will exactly reduce the copper as described below for Fehling's volumetric method, or (2) treating a fixed amount of the reagent with a prescribed volume of the reducing sugar solution of a strength insufficient to reduce all the copper and collecting and weighing the copper reduced as in Defren's gravimetric method described later.

\section{Fehling's Voldmetric Method}

Reagents. - (1) Copper solution: Dissolve 34.64 grams of pure crystallized copper sulphate in water, and dilute to exactly 500 c.c.

(2) Alkaline tartrate solution: Dissolve 175 grams of pure sodium potassium tartrate and 50 grams of pure sodium hydroxide in water, and dilute to 500 c.c. This reagent does not keep well unless carefully protected from the air (Note 1).

Determination. - Measure accurately into a small flask or casserole or a deep porcelain dish, 5 cc. of each of the above solutions, making 10 cc. of the "mixed Fehling reagent." Add 40 cc. of water, mix, and boil. To the boiling liquid, add from a burette a solution which contains not over 1 per cent of the reducing sugar to be determined, boiling two minutes after each addition of sugar until the blue color is entirely discharged, showing that all of the copper has been reduced. This test indicates approximately the amount of reducing sugar in the sample. Now adjust the strength of the sugar solution, if necessary, so that about $20 \mathrm{cc}$. will be required to reduce $10 \mathrm{cc}$. of the mixed Fehling reagent. Repeat the test, adding the calculated amount of sugar solution at once to the boiling copper solution; regulate the heating so that the mixture will again begin to boil about one minute 
after the addition of the sugar; note the exact time that actual boiling commences and continue to boil for just two minutes, then remove the flame and at once test the liquid for unreduced copper (Note 2).

Repeat the test, using more or less of the sugar solution depending upon the presence or absence of an excess of copper in the preceding experiment until two amounts of sugar solution are found which differ by only 0.1 or 0.2 cc., one giving complete reduction and the other leaving a small amount of copper in the cupric state. The mean of these two amounts is taken as the volume of solution required for complete reduction of the copper reagent.

Note 1. - All of the reagents used must be the purest obtainable, and the two constituents of the "mixed Fehling reagent" must be kept separate instead of being made up in one solution as was formerly done. If the solutions are not fresh, make a blank test in a casserole, boiling as above without the addition of any sugar, allow to stand for a few minutes, then decant off the liquid and notice whether any cuprous oxide has been precipitated. Finally wipe out the casserole with a small piece of filter paper and examine the latter, which may show traces of cuprous oxide not visible in the presence of the blue solution. If the liquid shows any change of color in this blank test, or if the slightest trace of cuprous oxide is found, the alkaline tartrate solution must be rejected and another blank test made with a freshly prepared solution.

Note 2. - So long as the solution shows a distinct blue color it is unnecessary to apply other tests for cupric copper. When the test is made in a flask, the blue color is best seen by holding level with the eye and looking horizontally through the meniscus, but on account of the presence of the cuprous oxide the disappearance of the blue color is not alone a safe criterion. When the blue color is no longer apparent, test for cupric copper by one of the following methods. It is well for each analyst to try all three methods and select the one which he finds most satisfactory for the conditions of his work. 
Whichever test is used it is important to work quickly lest reduced copper be oxidized by contact with the air.

Ferrocyanide Test. - Quickly filter a portion of the liquid through two or three thickness of paper, repeating the filtration if necessary, observe carefully that the filtrate is free from any trace of cuprous oxide, then acidulate with acetic acid and add a few drops of a dilute solution of potassium ferrocyanide when if copper is present a red-brown coloration or precipitate will appear.

Watts and Tempany suggest as a modification of this test that the liquid instead of filtering be poured upon a small pad consisting of several layers of filter paper, and the bottom paper (which should be wet with the solution but free from cuprous oxide) be removed, acidulated with acetic acid, and then tested with ferrocyanide.

Starch-iodide Test. ${ }^{1}$ - As soon as the sugar solution and the copper reagent have been boiled together for the required two minutes, add a drop or two of the solution (which need not be filtered from cuprous oxide) to a considerable excess of cold acidulated starch-iodide solution, when, if cupric copper is present, a blue or purple coloration is obtained.

The starch-iodide solution is prepared as follows: Boil 0.02 gram starch with 15 to 20 cc. of water, cool, add 4 to 5 grams of potassium iodide and dilute to $50 \mathrm{cc}$. A fresh solution must be prepared each day. When the test is to be made, pour about 1 cc. of this starch-iodide solution into a test tube, add two or three drops of acetic acid and then immediately a drop or two of the solution to be tested.

Thiocyanate Test. ${ }^{2}$ - This requires a solution of ferrous thiocyanate which may be prepared as follows: Dissolve 1 gram ferrous sulphate and 1.5 gram ammonium thiocyanate in $10 \mathrm{cc}$. water at about $45^{\circ}$ to $50^{\circ} \mathrm{C}$., cool immediately, add $2 \frac{1}{2}$ cc. concentrated hydrochloric acid and a trace of zinc dust to decolorize the solution.

1 Harrison: Pharm. J., 1903, 170 (Sutton's Volumetric Analysis, 9th Ed., p. 312.)

${ }^{2}$ Ling and Rendle: Analyst, 1905, 30, 183. Ling and Jones : Ibid., 1908, 33, 160. 
Place a drop of the freshly prepared ferrous thiocyanate solution on a porcelain plate and add a drop of the solution to be tested (which need not be filtered), when, if cupric copper is present, the well-known red color of ferric thiocyanate will be produced.

Calculation and Verification of Results. - In calculating the results it is commonly assumed that $10 \mathrm{cc}$. of the mixed Fehling reagent require for reduction under the above conditions:

0.0500 gram of anhydrous dextrose, levulose, or invert sugar. 0.0678 gram of dry crystallized lactose $\left(\mathrm{C}_{12} \mathrm{H}_{22} \mathrm{O}_{11} \cdot \mathrm{H}_{2} \mathrm{O}\right)$. 0.0807 gram of anhydrous maltose.

That these factors are not absolute, but are dependent upon exact uniformity of conditions has already been explained. To secure the greatest accuracy, therefore, the result obtained should be verified by a check experiment, carried out under the exact conditions of the analysis, with a known solution of pure sugar of the kind actually determined.

It is sometimes convenient to express the reducing power of some other sugar or of a mixture in terms of the reducing power of dextrose, the latter being taken as 100 .

Thus, if 0.0678 gram lactose or 0.0807 gram maltose has the same reducing power as 0.05 gram dextrose, then on the basis of dextrose $=100$, the reducing power of lactose is $\frac{.05}{.0678} \times 100$ $=74$, and that of maltose is $\frac{.05}{.0807} \times 100=62$.

According to Soxhlet, levulose and galactose have distinctly less reducing power than dextrose. It is quite commonly assumed, however, that these three monosaccharides have the same reducing power, but that invert sugar often fails to show its full effect because of the decomposing action of the acid used for inversion. Xylose and arabinose reduce Fehling's solution somewhat more strongly than does dextrose. 


\section{Defren's Gravimetric Method ${ }^{1}$}

This method is based on that of O'Sullivan ${ }^{2}$ and provides a uniform procedure for the determination of dextrose, maltose, or lactose.

Reagents. - (1) Dissolve 34.64 grams of copper sulphate in water, add $0.5 \mathrm{cc}$. strong sulphuric acid, and dilute to $500 \mathrm{cc}$.

(2) Dissolve 178 grams of sodium potassium tartrate and 50 grams of sodium hydroxide in water and dilute to $500 \mathrm{cc}$.

Determination. - Mix $15 \mathrm{cc}$. of each of the above reagents in an Erlenmeyer flask having a capacity of 250 to 300 cc., dilute with $50 \mathrm{cc}$. of freshly boiled distilled water, and place the flask in a boiling water bath for five minutes; then add 25 cc., accurately measured from a burette or pipette, of a solution containing approximately 0.5 per cent of the sugar to be determined and allow the mixture to stand in the boiling water bath for fifteen minutes. Remove the flask from the bath and filter at once (using moderate suction) through asbestos prepared as described below (Note 1); wash the cuprous oxide with boiling distilled water until the filtrate is no longer alkaline. The cuprous oxide can now be (1) washed with alcohol and then with ether, dried in a boiling water oven for 20 minutes, and weighed (Note 2); (2) ignited and weighed as cupric oxide, as recommended by Defren ; or (3) dissolved in nitric acid and the copper determined by electrolysis or by any other reliable method, in which case it will not be necessary to use asbestos especially prepared by boiling with acid and alkali. From the weight of copper or cuprous oxide determined, calculate the equivalent amount of cupric oxide and find the corresponding weight of reducing sugar from Defren's table (Note 3).

Note 1. - The filtrate from the cuprous oxide must always be distinctly blue, showing that a sufficient excess of Fehling solution was used, otherwise the determination must be repeated, using a more dilute solution of the reducing sugar. If the copper reduced is to be weighed as cuprous or cupric oxide on the asbestos filter, the latter must be especially prepared in

${ }^{1}$ J. Amer. Chem. Soc., 1896, 18, 749.

${ }^{2}$ J. Chem. Soc., 1876, 30, 130. 
order that it shall lose no weight when treated with the hot alkaline Fehling solution. Asbestos of good quality is boiled with nitric acid (1.05 to $1.10 \mathrm{sp}$. gr.), washed with water, then boiled with 25 per cent sodium hydroxide, washed, and the treatment with acid and alkali repeated. The prepared asbestos is used to make a tight felt about 1 centimeter thick in a Gooch crucible. When the crucible has been prepared for use and weighed, it should be tested by running through it a "blank" of hot alkaline Fehling solution and washing with water as in a regular determination. The loss of weight should not exceed one half milligram. After each determination the precipitate is dissolved in nitric acid, and the crucible washed, ignited, and reweighed. If a loss of over one milligram is found, the determination should be rejected and the filter treated alternately with acid and alkali until it ceases to lose in weight.

Note 2. - The method of weighing the cuprous oxide as such is convenient, and when working with fairly puresugar mixtures is accurate, but impure material, such as crude raw sugar, molasses, or malt extract, is liable to leave some organic matter with the cuprous oxide on the filter so that for the most accurate results with such materials, one should ignite to cupric oxide or dissolve and determine copper.

Note 3. - Defren determined the amount of copper reduced by fifteen to twenty known solutions each of dextrose, maltose, and lactose, with the following results:

$$
\begin{aligned}
& \text { Dextrose }=(0.4400+0.000037 W) W . \\
& \text { Maltose }=(0.7215+0.000061 W) W \\
& \text { Lactose }=(0.6270+0.000053 W) W .
\end{aligned}
$$

In which $W$ is the weight of cupric oxide obtained, the values of $W$ varying from 30 to 320 milligrams.

From these formulæ it is apparent that the reducing power of each of the sugars increases slightly with the concentration; hence, to find the amount of reducing sugar corresponding to any given weight of copper, one must use, not a simple factor, but a formula or (more conveniently) a table. 
Table 7 is condensed from that given in Defren's paper (l.c.), in which the weights of sugars corresponding to each milligram of cupric oxide are stated. In using either table the weight of copper or cupric oxide should be taken to one tenth milligram and the corresponding weight of reducing sugar found by the method of proportional parts.

Table 7.-Defren's Table for Dextrose, Mattose, and Lactose

\begin{tabular}{r|c|c|c||c|c|c|c}
\hline \hline $\begin{array}{c}\text { Cupric } \\
\text { Oxide } \\
\text { Mgms. }\end{array}$ & $\begin{array}{c}\text { Dextrose } \\
\text { Mgms. }\end{array}$ & $\begin{array}{c}\text { Maltose } \\
\text { Mgms. }\end{array}$ & $\begin{array}{c}\text { Lactose } \\
\text { Mgms. }\end{array}$ & $\begin{array}{c}\text { Cupric } \\
\text { Oxide } \\
\text { Mgms. }\end{array}$ & $\begin{array}{c}\text { Dextrose } \\
\text { Mgms. }\end{array}$ & $\begin{array}{l}\text { Maltose } \\
\text { Mgms. }\end{array}$ & $\begin{array}{l}\text { Lactose } \\
\text { Mgms. }\end{array}$ \\
\cline { 2 - 6 } 30 & 13.2 & 21.7 & 18.8 & 180 & 80.4 & 131.8 & 114.6 \\
40 & 17.6 & 29.0 & 25.2 & 190 & 84.9 & 139.1 & 121.0 \\
50 & 22.1 & 36.2 & 31.5 & 200 & 89.5 & 146.6 & 127.5 \\
60 & 26.5 & 43.5 & 37.8 & 210 & 94.0 & 154.1 & 134.1 \\
70 & 30.9 & 50.8 & 44.1 & 220 & 98.6 & 161.5 & 140.6 \\
80 & 35.4 & 58.1 & 50.5 & 230 & 103.2 & 169.1 & 147.0 \\
90 & 39.9 & 65.5 & 56.8 & 240 & 107.7 & 176.6 & 153.5 \\
100 & 44.4 & 72.8 & 63.2 & 250 & 112.3 & 184.1 & 160.0 \\
110 & 48.9 & 80.1 & 69.5 & 260 & 116.9 & 191.6 & 166.5 \\
120 & 53.3 & 87.4 & 75.9 & 270 & 121.4 & 199.2 & 173.0 \\
130 & 57.8 & 94.8 & 82.4 & 280 & 126.1 & 206.8 & 179.6 \\
140 & 62.2 & 102.1 & 88.7 & 290 & 130.7 & 214.3 & 186.2 \\
150 & 66.8 & 109.5 & 95.2 & 300 & 135.3 & 221.9 & 192.8 \\
160 & 71.3 & 116.9 & 101.7 & 310 & 139.9 & 229.6 & 199.3 \\
170 & 75.8 & 124.4 & 108.2 & 320 & 144.5 & 237.2 & 205.9 \\
\hline \hline
\end{tabular}

On account of the difference in experimental conditions the relative reducing powers of dextrose, maltose, and lactose do not show exactly the same ratios for this method as for the volumetric method described above.

\section{Barfoed's Cupric Acetate Method ${ }^{1}$}

For distinguishing dextrose from maltose Barfoed used a slightly acid solution of cupric acetate, which is reduced by the

1 Barfoed : Z. anal. Chem., 1873, 12, 27. Müller and Hagen : Arch. ges. Physiol. (Pflüger), 22, 325. Lieben : Z. Vereins Rübenzucker-Industrie, 1884, 34, 857 ; Wiley, Agricultural Analysis, Vol. III, p. 291. 
former and not by the latter. Lieben attempted to determine dextrose quantitatively in the presence of maltose by means of such solutions. This test, like the formation of the osazone, is not of much quantitative value unless for comparisons under identical conditions, but is often useful as a qualitative method for distinguishing between dextrose (or other monosaccharide) and those disaccharides which also reduce Fehling's solution.

Reagent. - Dissolve 45 grams of neutral crystallized cupric acetate in $900 \mathrm{cc}$. of water, filter if necessary; add $1.2 \mathrm{cc}$. of 50 per cent acetic acid and dilute to a liter. A portion of this reagent heated in a water bath must show no change.

Test. - To 5 cc. of this reagent in a test tube add 5 cc. of the solution to be tested and place in a boiling water bath for $3 \frac{1}{2}$ minutes; examine for cuprous oxide, viewing the tube against a black background in a good light. If no evidence of reduction is found, allow the tube to stand at room temperature for 5 or 10 minutes and examine again. For uniform results the different tubes used in making the tests should be of nearly the same diameter and thickness of wall. It is sometimes advantageous to pour out the liquid at the end of the test, leaving any cuprous oxide as far as possible adhering to the bottom of the test tube, and examine again.

It has been found ${ }^{1}$ that the test, when carefully carried out in this manner was efficient for the detection of 0.0004 gram of glucose, either alone or in the presence of maltose, lactose, or sucrose up to 0.02 gram.

Reduction due to disaccharide occurs if too much either of sugar or of acid be present, or if the heating be too prolonged.

In order to effect complete destruction of the glucose, so that the filtrate might be utilized in testing for maltose or lactose, it was necessary to limit the amount to about 0.002 gram of glucose to 5 cc. of the reagent.

The test requires very careful regulation as to details of manipulation and amount of sugar tested, but under such restrictions is capable of greater usefulness than has generally been appreciated.

1 Hinkel and Sherman: J. Am. Chem. Soc., 29, 1744. 
On account of the difficulty of securing an exact degree of acidity in the cupric acetate solution, each chemist should demonstrate the efficiency of his reagent, as well as verify his manipulation, by check experiments upon known sugar solutions covering the probable range of composition of the unknown solutions to be tested.

\section{Other Copper Reduction Methods}

Until recently the methods based upon copper reduction were for the most part worked out for the different reducing sugars individually, with the result that the methods and tables were applicable only to individual sugars. The method of Defren unifies the determination of dextrose, lactose, and maltose. The table of Allihn given beyond (Chapter V), while devised only for dextrose, has been so widely used that some workers have preferred to use the same procedure and table for other reducing sugars with a factor to convert the results from apparent glucose to the sugar sought. The destruction of reducing sugar by the decomposing action of the alkali noted above as a source of error in working with the ordinary Fehling solution, is still greater in Allihn's method, which employs a more strongly alkaline solution. To avoid this the caustic alkali may be replaced by a weaker alkali such as carbonate (Soldani, Sidersky, Ost, Benedict) or ammonia (Allein and Gaud). Others have advocated the addition of ammonia (Pavy) or cyanide (Gerard) to the usual Fehling solution in order to hold in solution the reduced copper so that the point of complete reduction may be directly observed by the disappearance of the blue color.

These methods possess obvious points of advantage, but as yet have not come into such general use as the Fehling, Allihn, and Defren methods.

\section{ROTATION OF POLARIZED LIGHT}

Solutions of the natural carbohydrates have the property of rotating the plane of polarized light. 'This rotating power 
is also shown by most other natural substances containing one or more asymmetric carbon atoms in the molecule.

\section{Preparation of Solutions for Polarization}

Solutions for polarization must be clear and free from all impurities having an influence upon polarized light. Among the optically active substances most likely to be met in natural products are organic acids (especially tartaric), pectin bodies, gums, resins, coloring matters, glucosides, alkaloids, and proteins. In most cases the interfering substances can be precipitated and the solution clarified by adding, first, basic lead acetate, and then a cream of aluminium hydroxide. It is sometimes advantageous to add a small amount of tannin before clarifying. Tannin combines with many of the substances mentioned, either precipitating them or forming compounds more easily precipitated by the basic acetate. In case the solution, after being treated with clarifying agents, made up to definite volume and filtered, is too highly colored for examination in the polariscope, it may be poured slowly through a small amount of pure dry animal charcoal on a paper filter. Since the charcoal absorbs a small amount of the sugar, the first portions of the filtrate must be rejected. The technical details of clarifying sugar solutions for polarization will be discussed in the next chapter. A solution of pure sugar or mixture of pure sugars should not require the use of clarifying agents, but should be filtered to remove dust particles.

Reducing sugars when first dissolved often show multirotation due to the spacial arrangement of the tautomeric form of the carbonyl group, for explanation of which reference may be made to the latest edition of Hollemann's Organic Chemistry, or to Armstrong's Simple Carbohydrates and Glucosides. The "normal" rotation is established by allowing the solution to stand for some hours, by boiling, or by the addition of about 0.1 per cent of ammonia. Solutions containing ammonia darken on standing and should therefore be polarized as soon as prepared. Sucrose does not show multirotation. 


\section{Determination of Angular Rotation}

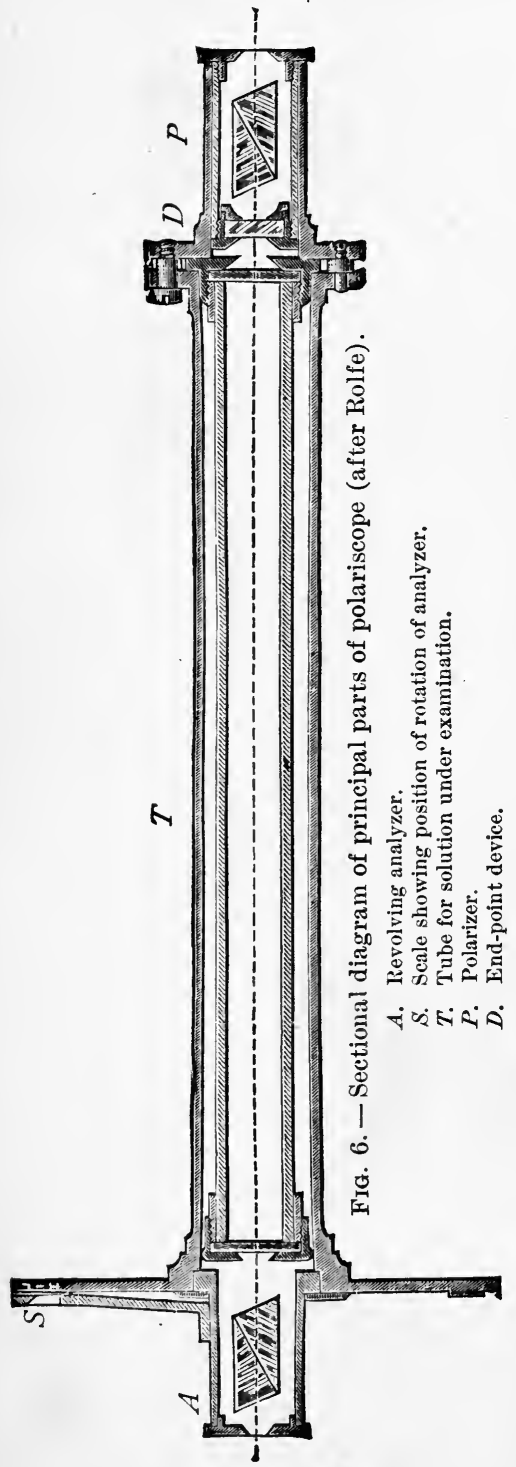

The standard instrument for the direct measurement of angular rotation is the Laurent polariscope or one of its modifications. This has a circular scale graduated in angular degrees. The principal parts of an instrument of this type are indicated in the diagram, Fig. 6. With this polariscope the rotation produced by the active substance is found by turning the analyzing prism, which is fixed in position in regard to the scale but rotates on its axis. The zero point is found with the polariscope empty and is the point at which the crystal axis of the analyzing prism is at right angles to the plane of polarization, and the field of vision is uniformly lighted. At either side of the zero point the field is unequally lighted, as shown in Fig. 7, which indicates the appearance of the field of vision on approaching, on reaching, and after passing, the zero point. The upper figures of the diagram represent the field of a "halfshade," and the lower figures those of a "triple-field" instrument. If now an active" substance is placed as in the tube $T$ between the polarizing and analyzing prisms, the latter must be rotated until it stands at right 
angles to the new plane, when the appearance of the field will be the same as that previously observed at the zero point. The position of the scale now shows directly the number of angular degrees through which the plane of polarization has been
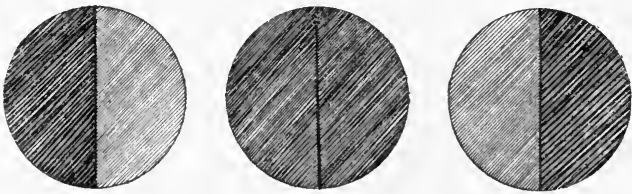
rotated by the active substance. With this polariscope sodium light is used.

For the examination of sugar solutions in technical
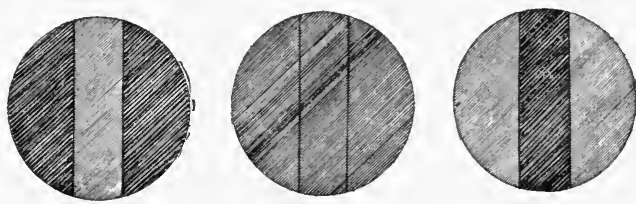

FIG. 7.-Diagram of zero point in reading polariscope. analysis the "saccharimeter" with the Ventzke scale is commonly used. This instrument, which is made in several forms, is different from the Laurent polariscope in construction and in that white light is used; and the rotation, instead of being measured directly, is "compensated" by means of quartz wedges, the thickness of quartz required to neutralize the

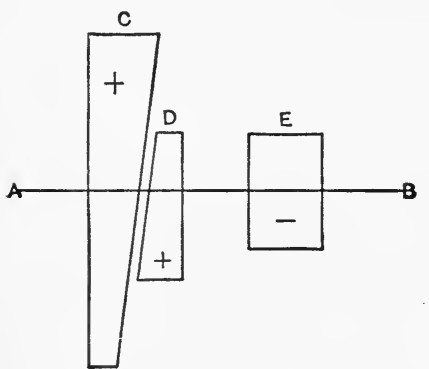
effect of the active substance giving a measure of the rotation produced by the latter.

Figure 8 shows diagrammatically the working of the quartz compensator. $A B$ represents the line of vision, $C$ and $D$ the wedges of dextrorotatory quartz, and $E$ a section of levorotatory quartz. At the Fig. 8. - Diagram of quartz com- zero point the combined thicknesses pensator (after Rolfe). of $C$ and $D$ are equal to the thickness of $E$. When a tube of (dextrorotatory) sugar solution is placed in the instrument between the polarizer and the compensating device, the wedges are moved so as to bring less dextrorotatory quartz into the line of vision until the levorotatory effect of $E$ balances the combined effects of $C, D$, and the 
sugar solution. A scale attached to the wedge shows the amount of "compensation" required to balance the rotation produced by the sugar and thus affords a measure of the rotatory power of the sugar. Instruments of this type are usually fitted with the Ventzke scale. One degree on the Ventzke scale is taken as equivalent to 0.3468 degree of angular rotation. This method of measuring the rotation by means of quartz with white light illumination is applicable only to such active bodies as have practically the same rotation dispersion as quartz. This is true of aqueous sugar solutions, which can therefore be examined by either of the instruments mentioned.

A recent form of instrument of the "saccharimeter" type is shown in Fig. 9.

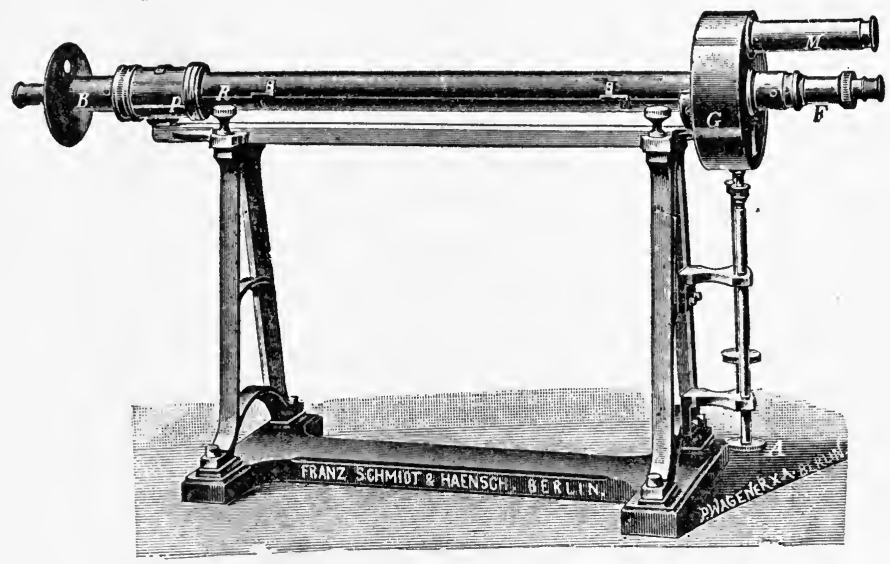

FIG. 9. - Recent form of saccharimeter. (Courtesy of Eimer and Amend.)

\section{Measure of Rotating Power-Specific Rotation}

The first organic substances to be studied by means of polarized light were liquids, or solutions of known density. In order to reduce the observations to a common basis for comparison Biot introduced the following formula:

$$
[\alpha]=\frac{a}{l \times d},
$$

in which $[\alpha]=$ the "specific rotating power"; 
$a=$ the observed angular degrees of rotation ;

$l=$ the length in decimeters of the column of liquid traversed by the polarized light;

$d=$ the density of the liquid.

On extending the calculation to solids in solution the above formula becomes

$$
[\alpha]=\frac{100 a}{l \times d \times p},
$$

in which $p=$ the percentage of solid in the solution.

But since $d \times p$ equals the number of grams of solid in $100 \mathrm{cc}$. of solution, it is simpler to write the formula

$$
[\alpha]=\frac{100 a}{l \times c},
$$

in which $c=$ concentration, i.e. the number of grams of dissolved solid in $100 \mathrm{cc}$. of the solution.

The value of the specific rotating power $[\alpha]$ is, therefore, the number of angular degrees through which a ray of polarized light would be rotated in traversing one decimeter of a solution of which each cubic centimeter contained one gram of the active substance.

The angular rotation is always directly proportional to the length of the column of liquid through which the light passes. It always depends upon the wave length of the light ray employed. By measuring the rotation for different rays the rotation dispersion of the substance is determined. Sodium light corresponding to the Fraunhofer line $D$ is commonly used in determining the rotating power. In many cases the rotating power is appreciably influenced by temperature. The latter should therefore be stated in giving the value of $[\alpha]$. Thus $[\alpha]_{D}{ }^{20}$ indicates the rotating power as measured with $D$ light at $20^{\circ}$. The concentration of the solution has usually an appreciable influence upon the value of $[\alpha]$, as is shown by the detailed formulæ for rotating power of the more important carbohydrates given below. 


\section{Rotatory Power of Pure Sugars}

To determine the rotatory power of a pure sugar dissolve 10 grams in about $80 \mathrm{cc}$. of water in a 100 -cc. flask, see that the solution is at room temperature, and note this temperature, which should preferably be $20^{\circ} \mathrm{C}$., fill to the holding mark, mix the solution well, and filter through a dry paper into the polariscope tube. The first portions of the filtrate should be used for rinsing the tube and then rejected. Finally fill, cover, and cap the tube, place it in the polariscope, and observe the rotation which it produces either directly in angular degrees or by multiplying the observed Ventzke degrees by 0.3468 , then calculate the specific rotatory power as explained above. The details and precautions regarding the handling of the solution and use of polariscope which are given in the next chapter should be carefully studied in this connection.

Following are the detailed formulæ for six of the more important sugars, showing corrections for concentration and temperature or for concentration at some fixed temperature:

Dextrose ('Tollens) ${ }^{1}$

$$
[\alpha]_{D}^{17}={ }^{2} 52.50+0.018796 p+0.0005168 p^{2} .
$$

Lievulose (Jungfleish and Grimbert) ${ }^{3}$

$$
[\alpha]_{D}{ }^{t}=-[101.38-0.56 t+0.108(c-10)] .
$$

Galactose (Meissl) ${ }^{4}$

$$
[\alpha]_{D}{ }^{t}=83.883+0.0785 p-0.209 t .
$$

Sucrose (Tollens) ${ }^{5}$

$$
[\alpha]_{D}{ }^{20}=66.386+0.015035 p-0.0003986 p^{2} .
$$

Lactose (Schmöger) ${ }^{6}$

$$
[\alpha]_{D}^{20}=52.53 \text { (constant for } c=2.4 \text { to } 40 \text { ). }
$$

Maltose (Meissl) ${ }^{7}$

$$
[\alpha]_{p}{ }^{t}=140.375-0.01837 p-0.095 t .
$$

1 Ber., 1876, 9, 487, 1531; 1884, 17, 2234.

2 Unless otherwise stated the rotation is to the right $(+)$; levorotation is indicated by $(-)$.

4 J.prakt. Chem., 1880, [2], 22, 97.

3 Compt. rend., 1888, 107, 390.

6 Ber., 1880, 13, 1922.

5 Ber., 1877, 10, 1403.

7 J.prakt. chem., 1882, [2], 25, 114. 
At ordinary concentrations and laboratory temperatures, the values for $[\alpha]_{D}$ are approximately:

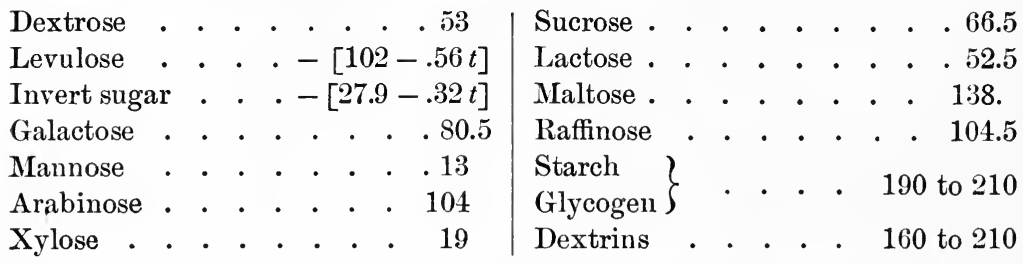

In order to avoid confusing the determination of specific rotatory power with the conventional polariscope examination of "raw sugar" described in the next chapter, it is well for the student at this point to determine the specific rotatory power of a sample of sugar of known purity.

\section{REFERENCES}

I

AbDerhalden: Handbuch der Biochemisches Arbeitsmethoden.

Allex : Commercial Organic Analysis.

Browne: Handbook of Sugar Analysis.

Hoppe-Seyler: Physiologisch und Pathologisch-Chemischen Analyse.

LANDoLt: Optical Rotation of Organic Substances.

LipPMANN: Chemie der Zuckerarten.

Maquenne: Les Sucres.

Mryer and JacoBson : Organische Chemie.

Mullikex: Identification of Pure Organic Compounds.

OpPenheimer: Handbuch der Biochemie.

Rolfe: The Polariscope in the Chemical Laboratory.

Tollens : Chemie der Kohlenhydrate.

Vauber: Bestimmung Organischer Verbindungen.

WILEY : Agricultural Analysis, Vol. III.

\section{II}

1905. Ling and Rende: The Volumetric Determination of Reducing Sugars. Analyst, 30, 182.

1906. Browne: The Analysis of Sugar Mixtures. J. Am. Chem. Soc., 28, 439.

Munson and Walker: Unification of Reducing Sugar Methods. $J$. Am. Chem. Soc., 28, 663. See also Walker, same, 29, 541; 34, 202. 
1907. BANG : (Determination of Reducing Sugars by means of Hydroxylamine). Biochem. Z., 2, 271; Chem. Abs., 1, 590.

BAtes: A Quartz Compensating Polariscope with Adjustable Sensibility. U. S. Bur. Standards, Bul. 4. Also Z. Ver. Zuckerind., 58, 105, 821; Chem. Abs., 2, 1219, 3165.

Benedict: The Detection and Estimation of Reducing Sugars. J. Biol. Chem., 3, 101.

Browne and Halligan: Report on Sugar. U. S. Dept. Agr., Bur. Chem., Bul. 105, p. 116.

Hinkel and Sherman: Experiments on Barfoed's Acid Cupric Acetate Solution as a Means of Distinguishing Glucose from Maltose, Lactose, and Sucrose. J. Am. Chem. Soc., 29, 1744.

Hudson: Action of Acids and Bases on the Mutarotation of Glucose. J. Am. Chem. Soc., 29, 1571.

Mathews and McGuigax: The Oxidation of Sugars by Cupric Acetic Acid Mixtures. Proc. Soc. Exp. Biol. Med., 1907; Chem. Abs., 1, 1780.

NeF : Behavior of Sugars toward Fehling Solution as well as toward other Oxidizing Agents. Ann. Chem., 357, 214; Chem. Abs., 2, 799.

1908. Bunzes: The Rate of Oxidation of Sugars in an Acid Medium. Am. J. Physiol., 21, 23.

Browne: Determination of Reducing Sugars from Weight of Cuprous Oxide. Intern. Sugar J., 10, 537; Chem. Abs., 3, 385.

Kendall and Sherman: The Detection and Identification of Certain Reducing Sugars by Condensation with p. Brombenzylhydrazide. J. Am. Chem. Soc., 30, 1451.

LiNg and Jones : (Accuracy of Determination of Reducing Sugars). Analyst, 33, 160.

Meisenheimer : Behavior of Glucose, Fructose, and Galactose toward dilute Sodium Hydroxide. Ber., 41, 1009.

1910. Herstern: (Historical Sketch of Fehling's Solution). J. Am. Chem. Soc., 32, 779.

Hudsox: Mutarotation. J. Am. Chem. Soc., 32, 889.

NeF : Behavior of Sugars toward Caustic Alkalies. Ann. Chem., 376, 1.

Schliephacke: Mutarotation of Maltose. Ann. Chem., 397, 164.

1911. BANG: Preparation of Copper Solutions for Sugar Titration. Biochem. Z., 32, 443.

Benedict : A Method for the Estimation of Reducing Sugars. $J$. Biol. Chem., 9, 57.

Fischer : (Determination of Rotatory Power on Small Amounts of Material). Ber., 44, 129.

1912. Kendall: A New Method for the Determination of the Reducing Sugars. J. Am. Chem. Soc., 34, 317. 


\section{CHAPTER IV}

\section{Special Methods of Sugar Analysis}

\section{ANALYSIS OF RAW SUGAR}

\section{Polariscopic Examination}

A SPECIAL room of even temperature which can be darkened when desired, or a dark screen which can be placed around the instrument on the laboratory table, should be provided for the polariscopic examination. For illumination of the white-light saccharimeter, a triple flame or an Argand burner with incandescent mantle is used. In taking readings the polariscope must be brought only near enough to the burner to secure a good illumination of the field, never within less than eight inches. As soon as the reading is taken the polariscope should be turned away or the flame lowered, in order to avoid any possible warming of the instrument.

Determination of the Zero Point. - The trough of the saccharimeter being empty, set the scale within a few degrees of zero and focus the eyepiece so that the field of vision is clear and the perpendicular line or band dividing it is perfectly distinct. Now rotate the milled head so as to move the zero point of the scale toward that of the vernier. When the neutral point (the true zero point for the instrument as it stands) is reached, the appearance of the entire field is uniform. Approach the zero point first from one side then from the other, taking the reading each time as soon as the entire field appears uniform, until successive readings do not differ by more than $0.2^{\circ}$ on the Ventzke sugar scale. The average of six to ten such readings is taken as the zero point in the subsequent work of 
the day, unless the instrument should be jarred or moved, in which case the zero point should be redetermined.

Test with Pure Sugar. - Weigh $26^{1}$ grams of pure sucrose, dissolve in water in an accurately calibrated 100 -cc. flask, fill to the mark at $20^{\circ}$ to $22^{\circ}$, and mix thoroughly by shaking, holding the flask in such a way as not to warm the solution. The latter should have a density very nearly 1.10 and should rotate the plane of polarized light $34.68^{\circ}$ to the right, giving a reading of $100.0^{\circ}$ on the Ventzke scale.

Rinse the $200-\mathrm{mm}$. tube several times with the solution, then fill (by pouring through a dry filter, rejecting the first portions of the filtrate) until the curved surface of the liquid projects above the open end of the tube; see that all air bubbles have risen to the surface, and then slide on the cover-glass horizontally in such a manner that the excess of liquid is carried over the side, leaving the cover glass exactly closing the tube, with no air bubbles beneath it and none of the liquid upon its upper surface. The cover glass being in position, the tube is closed by screwing on the cap. The latter should be only tight enough to prevent leakage, as any considerable pressure on the glass plate may cause it to become optically active. Look through the tube lengthwise to be sure that the cover glasses are clean and dry and the contents of the tube perfectly clear.

Place the tube in the trough of the saccharimeter or polariscope and take readings as in setting the zero point. Unless the ends of the polariscope tube are ground absolutely parallel, the position of the tube in the instrument may influence slightly the length of column of sugar solution in the line of vision.

1 The value of the Ventzke scale was originally fixed by means of pure sucrose solutions of $1.100 \mathrm{sp}$. gr. at $17.5^{\circ}$ and it was found that $100 \mathrm{cc}$. of such a solution contains 26.048 grams of sucrose (weighed in air with brass weights). For many years, however, it has been the custom of instrument makers to calibrate the Ventzke scale by means of solutions containing 26.048 grams of sucrose in 100 Mohr's cubic centimeters. A solution of very nearly the same strength is obtained by dissolving 26 grams and completing the volume at $20^{\circ}$ to 100 metric cubic centimeters (the volume occupied by 100 grams of water at $4^{\circ}$ weighed in vacuo). The latter proportions have been adopted by the International Commission for Unifying Methods of Sugar Analysis. 
In order to be sure that the length whose rotation is measured is the true length of the polariscope tube, take two or four readings, then turn the tube two thirds over in the trough of the instrument, and after taking two or four readings in this position turn the tube again two thirds over in the same direction for the final two or four readings. After a little practice it will probably be found sufficient to take one reading approaching the end point from the right and one from the left in each of three positions of the tube, or six readings in all. The average reading (corrected for zero point) should not differ from $100.0^{\circ}$ by more than $0.2^{\circ}$ on the Ventzke scale, nor from $34^{\circ} 41^{\prime}$ on the Laurent polariscope, by more than $5^{\prime}$.

Polarization of the Raw Sugar. - Mix the sample, weigh out 26 grams, and dissolve in 60 to $80 \mathrm{cc}$. of water in a $100 \mathrm{cc}$. flask. When the sugar is entirely dissolved, add from 1 to $5 \mathrm{cc}$. (according to the nature of the sample; only very dark sugars should require more than 2 cc.) of a solution of basic lead acetate of about $1.25 \mathrm{sp}$. gr. $^{1}$ A decided excess of basic acetate should be carefully avoided. This may be done by adding the lead solution a few drops at a time, shaking after each addition, and stopping as soon as another drop of the solution produces no further precipitate.

After the lead acetate has been added and mixed, add twice its volume of "alumina cream." 2 This assists in the clarification, precipitates the excess of lead, and facilitates filtration. A moderate excess of alumina cream does no harm. With high grade sugars the use of alumina cream alone may be sufficient for clarification. Make up to volume with distilled

1 This may be prepared by dissolving the solid basic salt or by boiling an excess of litharge with a strong solution of neutral lead acetate.

2 Prepared as follows: Shake powdered commercial alum with water at ordinary temperature until a saturated solution is obtained. Set aside a little of the solution, and to the residue add ammonia, little by little, stirring between additions, until the mixture is alkaline to litmus paper. Then drop in additions of the portion left aside, until the mixture is just acid to litmus paper. By this procedure a cream of aluminium hydroxide is obtained suspended in a solution of ammonium sulphate. This sulphate is advantageous when added after the basic acetate, since it precipitates whatever excess of lead may be present. 
water, ${ }^{1}$ shake well, and then pour the whole solution, or as much as practicable, on a dry filter. Reject the first 20 or 30 cc. of filtrate and then polarize the remainder as already described for the pure sugar solution. The average reading of the Ventzke scale, corrected for the deviation of the zero point, is reported as "polarization."

Notes and Precautions. - Care must be taken to avoid the following errors due to manipulation : (1) change in moisture content of sample during weighing, (2) change in volume of solution due to fluctuations of temperature, (3) imperfect mixing of solution after diluting to volume in the graduated flask, (4) evaporation during filtration, (5) too great compression of cover glasses in closing the polariscope tube.

Clarification is very important. All proteins are levorotatory and must be entirely removed. The solution must be free from turbidity, but not necessarily free from color. Only so much of the clarifying agents should be used as is necessary to free the solution from optically active impurities and from turbidity. Any excess increases the error due to the presence of precipitate when the solution is diluted to volume. The volume occupied by the precipitate varies between 0.05 and $1.0 \mathrm{cc}$. for ordinary raw sugars. It can be determined by Scheiber's method of double dilution, ${ }^{2}$ in which a duplicate determination is made in a flask of twice the volume, or by determining the weight and density of the precipitate as recommended by Sachs. ${ }^{3}$ The latter method is preferred by Wiechmann ${ }^{4}$ and Horne, ${ }^{5}$ each of whom determined the volume of the precipitate for a number of raw sugars from different localities. Horne has found (loc. cit.) that the error can be almost entirely avoided by clarifying with anhydrous basic acetate after the sugar solution has been diluted to volume, and this method of clarification, after extended investigation

1 If frothing interferes at this point, add two or three drops of ether.

2 Z. Vereins. Rübenzucker Industrie, 1875, 25, 1054.

${ }^{3}$ Ibid., 1880, 50, 229. See also the paper by Horne.

4 Ibid., 1903, [n. f.] 40, 498; Abs. J. Chem. Soc., 1903, 84, ii, 699.

5 J. Am. Chem. Soc., 1904, 26, 186. 
and discussion, has now been adopted by the International Commission on a parity with the usual method. According to Browne, the amount of dry acetate used should not exceed 0.5 gram.

\section{Regulations of the International Commission}

The following resolutions, ${ }^{1}$ adopted by the International Commission for the Unification of Sugar Analysis, in 1900, have since been generally accepted.

1. In general, all sugar tests shall be made at $20^{\circ} \mathrm{C.}{ }^{2}$

2. The graduation of the saccharimeter shall be made at $20^{\circ}$ C. Twenty-six grams of pure sugar, dissolved in water, and the volume made up to 100 metric cubic centimeters, or during the period of transition 26.048 grams of pure sugar in 100 Mohr cubic centimeters, all weighings to be made in air with brass weights, the completion of the volume and the polarizations to be made at $20^{\circ}$ on an instrument graduated at $20^{\circ}$, should give an indication of 100 on the scale of the saccharimeter. For countries where temperatures are usually higher than $20^{\circ}$, it is permissible that saccharimeters be graduated at $30^{\circ}$, or any other suitable temperature, under the conditions specified above, providing that the analysis of the sugar be made at the same temperature - that is, that the volume be completed and the polarization made at the temperature specified.

3. Preparation of pure sugar: Purest commercial sugar is to be further purified in the following manner: A hot saturated aqueous solution is prepared and the sugar precipitated with absolute ethyl alcohol; the sugar is carefully spun in a

1 U. S. Dept. Agriculture, Bur. Chem., Bull. 73, p. 58. J. Am. Chem. Soc., 1901, 23, 59 .

2 In polarizing pure sucrose with quartz wedge saccharimeter there is a falling off in polarization of about $0.03^{\circ}$ Ventzke for each degree C. that the temperature rises above $20^{\circ}$. Crude sugars, however, may contain enough levulose so that their polariscope readings are increased by a rise of temperature. Browne has clearly shown that temperature errors cannot be compensated by corrections based on pure sucrose, but must be avoided by actually polarizing at or near $20^{\circ} \mathrm{C}$. 
small centrifugal machine and washed in the latter with absolute alcohol. The sugar thus obtained is redissolved in water, the saturated solution again precipitated with alcohol and washed as above. The product of the second crop of crystals is dried between blotting paper and preserved in glass vessels for use. The moisture still contained in the sugar is determined and taken into account when weighing the sugar which is to be used.

The committee further decided that central stations shall be designated in each country which are to be charged with the preparation and distribution of chemically pure sugar. Wherever this arrangement is not feasible, quartz plates, the values of which have been determined by means of chemically pure sugar, shall serve for the control of the saccharimeters.

The committee further decided that the above control of quartz plates by means of chemically pure sugar should, as a rule, apply only to the central stations which are to test the correctness of saccharimeters ; for those who execute commercial analyses, the repeated control of the instruments is to be accomplished, now as before, by quartz plates.

4. In effecting the polarization of substances containing sugar, half-shaded instruments, or triple field, only are to be employed.

5. During the observation the apparatus must be in a fixed position and so far removed from the source of light that the polarizing nicol is not warmed.

6. Sources of light may be gas, triple burner with metallic cylinder, lens, and reflector; gas lamp with Auer (Welsbach) burner; electric lamp; petroleum duplex lamp; sodium light. Several readings are to be made and the mean thereof taken, but any one reading must not be neglected.

7. In making a polarization the whole normal weight for 100 cubic centimeters is to be used, or a multiple thereof for any corresponding volume.

8. As clarifying and decolorizing reagents there may be used : (a) subacetate of lead, ( 3 parts by weight of acetate of lead, one part by weight of oxide of lead, 10 parts by weight 
of water); (b) alumina cream; (c) concentrated solution of alum. Boneblack and decolorizing agents are to be excluded.

9. After bringing the solution exactly to the mark, at the proper temperature, and after wiping out the neck of the flask with filter paper, all of the well-shaken clarified sugar solution is poured upon a dry rapidly acting filter. The first portions of the filtrate are to be rejected and the rest, which must be perfectly clear, used for polarization.

\section{Use of Dichromate Solution as Light Filter}

As explained in the last chapter the use of white light in polarizing sugar solutions with the quartz compensator is based upon the observation that the rotation dispersion is the same for sugar solutions as for quartz. While. this is practically true, it is not absolutely correct at high rotations, even with sucrose solutions, since the dispersive powers of sucrose and quartz are slightly different at the blue end of the spectrum. This difference results in a slight disparity of tint which may give rise to apparent differences of rotation when the same sugar solution is read by different observers or even by the same observer with white light from different sources. Browne, ${ }^{1}$ confirming and extending the earlier work of Schönrock, finds that this source of error is by no means negligible even when working with ordinary. sugar solutions, and should always be guarded against by passing the light through a solution of dichromate to absorb the blue and violet rays which cause the greatest amount of rotation dispersion. Saccharimeters of recent manufacture have a short tube or cell through which the light passes after entering the instrument and before reaching the solution. This tube if $3 \mathrm{~cm}$. long should be filled with a three per cent solution of potassium dichromate; if $1.5 \mathrm{~cm}$., with a 6 per cent solution. The German Reichsanstalt and the U. S. Bureau of Standards now use such dichromate cells in testing quartz-wedge saccharimeters. Before using a new instrument or one which has been out of use for a time, the dichromate cell should be examined

${ }^{1}$ U. S. Dept. Agr., Bur. Chem., Bul. 122, p. 221. 
to see that it is properly filled. In class-work this will be done by the instructor rather than the individual student.

In working with substances such as commercial glucose, malt products, and dextrins (whose rotation dispersion is greater than that of cane sugar), Browne finds it desirable to double the usual strength of the dichromate solution.

\section{Relation of Polarization to Percentage of Sucrose}

In the absence of other active substances, the reading of the Ventzke scale as obtained above shows the percentage of sucrose in the sample. In commercial raw sugar the only other sugars likely to be present are dextrose and levulose. If the total amount of these reducing sugars, as shown by Fehling's solution, is small, the value of the sample is sufficiently indicated by the polarization, the invert sugar, and the amounts of moisture and ash.

Since, however, the "reducing sugar" contains dextrose and levulose in unknown proportions, the determination of "reducing power" does not give the necessary data for estimating the percentage of sucrose in the sample. The percentage of sucrose can, however, be determined in the presence of dextrose and levulose by observing the change in rotation produced by hydrolysis. This is unaffected by the presence of monosaccharides, since the latter cannot be hydrolyzed.

The following method is based on this principle.

\section{Clerget's Method for Sucrose}

A pure sucrose solution polarizing $100^{\circ}$ on the Ventzke scale at a temperature of $20^{\circ}$, will, after hydrolysis, polarize about $-33^{\circ}$ on the same scale at the same temperature. It is this change of rotation from right to left which gives rise to the terms "inversion" for hydrolysis, and "invert sugar" for the mixture of equal parts of dextrose and levulose produced. Since the levorotatory power of levulose, and hence of invert sugar, decreases rapidly with increase of temperature, the reading $-33^{\circ}$ would be found only at $20^{\circ}$. An increase of $1^{\circ}$ in temper- 
ature causes a decrease of practically 0.5 degree Ventzke in the levorotation of the invert sugar, and at about $87^{\circ}$ the reading becomes zero. The change in rotation produced by hydrolysis in pure sucrose solution is, therefore, about $143-0.5 t$, in which $t$ is the temperature at which the readings are taken.

To estimate the sucrose in an unknown sample, dissolve 26 grams, clarify if necessary, dilute to $100 \mathrm{cc}$., filter, and polarize as usual at $20^{\circ}$. The remainder of the solution is freed from lead if necessary by treating with anhydrous sodium carbonate or sulphate, or with potassium oxalate, followed by filtration through dry paper. To $50 \mathrm{cc}$. of the solution add $25 \mathrm{cc}$. of water and then add, in small portions with constant shaking, 5 cc. of concentrated hydrochloric acid (1.196 specific gravity). Place the flask in a bath of water kept at $70^{\circ}$. The temperature of the solution in the flask should reach $67^{\circ}$ to $69^{\circ}$ in $2 \frac{1}{2}$ to 3 minutes and is then maintained at a temperature as nearly $69^{\circ}$ as possible for 7 to $7 \frac{1}{2}$ minutes, making a total time of heating of 10 minutes; or the solution after adding the acid may be set aside at a temperature of $20^{\circ}$ to $24^{\circ} \mathrm{C}$. for 24 hours. The sucrose having been thus hydrolyzed, cool the solution, if necessary, to $20^{\circ} \mathrm{C}$. (or to room temperature), dilute to $100 \mathrm{cc}$, mix the solution well and polarize, preferably in a waterjacketed $200-\mathrm{mm}$. tube with a side tube through which the exact temperature of the solution at the time of polarization may be determined. Since $50 \mathrm{cc}$. of the sugar solution was diluted for hydrolysis and finally made up to $100 \mathrm{cc}$., this second reading must be doubled to obtain the so-called "invert polarization." The difference between the first polarization and the "invert" polarization, multiplied by 100 and divided by the difference which pure sucrose would show under the same conditions, gives the percentage of sucrose; or

$$
s=\frac{100(P-I)}{142.66-0.5 t}
$$

in which $s=$ percentage of sucrose, $P$ the original polarization, $I$ the "invert" polarization, and $t$ the temperature in degrees centigrade. 
The method is applicable to the determination of sucrose in the presence of any other sugar, or mixture of sugars, which is not measurably affected by the prescribed treatment with acid.

\section{Determination of Reducing Sugar}

Usually the reducing power of the raw sugar is determined volumetrically, as described in the preceding chapter, the results being calculated as percentage of invert sugar. For the determination dissolve 5 grams of the sample if dark, or 10 grams if light, dilute to 100 cc., and filter through dry paper. If the preliminary test shows the solution to be too strong or too weak, prepare a fresh solution for the final determination. When the volumetric method is carefully carried out, the results are sufficiently exact for all ordinary work. ${ }^{1}$ For the determination of very small amounts of invert sugar the method introduced by Herzfeld, ${ }^{2}$ and recommended by the International Commission, ${ }^{3}$ is probably the most accurate.

\section{Herzfeld's Gravimetric Method}

Dissolve 20 grams of sample in $80 \mathrm{cc}$. of water, clarify with basic lead acetate, and precipitate the excess of lead by sodium carbonate (avoiding more than a slight excess) or by neutral potassium oxalate; ${ }^{4}$ dilute to $100 \mathrm{cc}$, mix thoroughly, and filter through dry paper. The filtrate must be perfectly clear. In a beaker of $250 \mathrm{cc}$. capacity, place $50 \mathrm{cc}$. of the undiluted "mixed Fehling reagent" (see Fehling's volumetric method) and $50 \mathrm{cc}$. of the clarified sugar solution; heat at such a rate that the mixture boils in about four minutes, and boil for exactly two minutes. Add $100 \mathrm{cc}$. of cold, recently boiled, distilled water, filter immediately, and find the amount of copper

1 If a gravimetric method is preferred, use that of Meissl and Hiller, as adopted by the Official Agricultural Chemists: Bul. 107, Bur. Chem., U. S. Dept. Agriculture.

${ }^{2} Z$. Vereins Rübenzucker Industrie, 1886, 6.

${ }^{3} \mathrm{~J}$. Am. Chem. Soc., 1901, 23, 64.

4 Sawyer: J. Am. Chem. Soc., 1904, 26, 1631. 
reduced in one of the ways described under Defren's method in the preceding chapter. The following table shows the corresponding percentage of invert sugar.

Table 8. - Herzfeld's Table for the Determination of Invert

Sugar in Materials containing 1 per Cent or less of Invert Sugar and a High Percentage of Sucrose

\begin{tabular}{c|c||c|c||c|c}
\hline $\begin{array}{c}\text { Copper reduced } \\
\text { by 10 grams } \\
\text { of material } \\
\text { Milligrams }\end{array}$ & $\begin{array}{c}\text { Invert sugar } \\
\text { Per cent }\end{array}$ & $\begin{array}{c}\text { Copper reduced } \\
\text { by 10 grams } \\
\text { of material } \\
\text { Milligrams }\end{array}$ & $\begin{array}{c}\text { Invert sugar } \\
\text { Per cent }\end{array}$ & $\begin{array}{c}\text { Copper reduced } \\
\text { by 10 grams } \\
\text { of material } \\
\text { Milligrams }\end{array}$ & $\begin{array}{c}\text { invert sugar } \\
\text { Per cent }\end{array}$ \\
\cline { 2 - 6 } 50 & 0.05 & 120 & 0.40 & 190 & 0.79 \\
55 & 0.07 & 125 & 0.43 & 195 & 0.82 \\
60 & 0.09 & 130 & 0.45 & 200 & 0.85 \\
65 & 0.11 & 135 & 0.48 & 205 & 0.88 \\
70 & 0.14 & 140 & 0.51 & 210 & 0.90 \\
75 & 0.16 & 145 & 0.53 & 215 & 0.93 \\
80 & 0.19 & 150 & 0.56 & 220 & 0.96 \\
85 & 0.21 & 155 & 0.59 & 225 & 0.99 \\
90 & 0.24 & 160 & 0.62 & 230 & 1.02 \\
95 & 0.27 & 165 & 0.65 & 235 & 1.05 \\
100 & 0.30 & 170 & 0.68 & 240 & 1.07 \\
105 & 0.32 & 175 & 0.71 & 245 & 1.10 \\
110 & 0.35 & 180 & 0.74 & & \\
115 & 0.38 & 185 & 0.76 & & \\
\hline \hline
\end{tabular}

\section{Determination of Moisture and Ash}

Dry about 2 grams of sample in a flat-bottomed platinum dish in the boiling water oven until the loss in weight on heating for one hour does not exceed 0.10 per cent. A perfectly constant weight cannot be expected if invert sugar is present, since levulose is slowly decomposed by heating at $100^{\circ}$ in the air. Calculate the loss of weight as moisture.

Moisten the residue with a few drops of concentrated sulphuric acid and burn to whiteness. Weigh, and report the result as "sulphated ash." The true ash is considered by some as nine tenths and by others as four fifths of the "sulphated ash." 


\section{Official Methods of Testing Sugars}

Methods of the Association of Official Agricultural Chemists : Bul. 107, Bur. Chem., U. S. Dept. Agriculture.

Regulations governing the sampling and classification of imported sugars and molasses: Treasury Department Document No. 2470.

German official methods: Z. Nahr. Genussm., 1903, 6, 1059; Z. anal. Chem., 1903, 42 (Appendix to Nos. 9 and 10).

\section{DETERMINATION OF SUCROSE IN BEETS AND CANE}

Sucrose is the only sugar found in appreciable quantity in the fresh juice of healthy sugar beets. Sugar cane juice contains a small amount of reducing sugar which is usually considered to be invert sugar, but which, as Browne has shown, may contain dextrose and levulose in varying proportions. In the routine testing of beet and cane juices, the polarization of a properly clarified sample is considered as showing the amount of sucrose present.

Beets after being thoroughly cleaned (the loss of weight in cleaning being determined and reported as " tare ") are reduced to a fine pulp by rasping or grinding. This pulp is then treated in one of three ways: (1) separating the insoluble matter by pressure and polarizing the juice, (2) extracting with alcohol, (3) extracting with water. The first method introduces errors from imperfect separation of the juice, the second is longer and more expensive than the third, which is now generally used. If, however, the pulp be treated with water and filled to volume in a graduated flask, as in the analysis of raw sugar, it is very difficult to expel the air bubbles entirely from the mixture of pulp and water. The following method, described by Sachs, ${ }^{1}$ avoids this inconvenience and has been found satisfactory for rapid work: Weigh 26 grams of the pulp in a beaker and add $177 \mathrm{cc}$. of water containing $5 \mathrm{cc}$. of the usual solution of basic lead acetate; shake or stir for three minutes; filter, and polarize the filtrate. Twice

1 Paper read before the Second International Congress of Applied Chemistry, Paris, 1896 ; quoted by Hiltner and Thatcher, J. Am. Chem. Soc., 1901, 23, 300. 
the reading of the Ventzke scale is approximately the percentage of sucrose in the sample. This method assumes that 26 grams of beet pulp contain $23 \mathrm{cc}$. of juice, making the total volume of liquid in the mixture $200 \mathrm{cc}$. Many German sugar chemists hold that extraction with water gives high results because the beet contains substances other than sucrose which are soluble in water and rotate polarized light to the right. According to Sachs, however, these substances are pectin-like bodies and are completely precipitated by basic lead acetate. Trowbridge ${ }^{1}$ also found that the results obtained by digestion in water were practically identical with those of the alcohol extraction method.

In sampling sugar cane for analysis, the canes are usually cut into thin oblique chips, "cosettes," by means of rotating knives. These coseties may be further reduced by passing through a sausage cutter or a special shredding machine. It is not feasible, however, to reduce the cane to as fine a pulp as is obtained from sugar beets. For this reason more time and a higher temperature must be used to insure complete extraction of the sucrose. According to Wiley, 26 grams of the chips or pulp are weighed into a flask graduated at 102.6 cc., this graduation being based on the assumption that the cane contains ten per cent of insoluble matter of a specific gravity about equal to that of the solution. The flask is nearly filled with water, and warmed on a water bath for one hour with frequent shaking; then filled a little above the mark and warmed for ten minutes longer with frequent shaking. The flask is now cooled, the volume adjusted if necessary, and the liquid filtered through dry paper. Fifty cubic centimeters of filtrate are collected in a 55-cc. flask, clarified with basic acetate, filled to the mark, shaken, filtered, and the filtrate polarized in a $220-\mathrm{mm}$. tube.

For further information on the analysis of sugar beets and sugar cane, the following books and papers may be consulted:

1 J. Am. Chem. Soc., 1901, 23, 300.

2 Agricultural Análysis, Vol. III, p. 238. 
Wiley: Agricultural Analysis, Vol. III, Part III.

Spencer: Handbook for Beet Sugar Chemists. Handbook for Sugar Manufacturers.

Lippmann and Pulvermacher: Lunge's Chemisch-technische Untersuchungsmethoden.

Frühling: Anleitung zur Untersuchung für die Zuckerindustrie.

Trowbridge: Notes on Sugar Beets, J. Am. Chem. Soc., 1901, 23, 216.

Hiltner and Thatcher: An improved Method for the Rapid Estimation of Sugar in Beets, J. Am. Chem. Soc., 1901, 23, 299, 863.

\section{DENSITY AND PURITY OF SUGAR SOLUTIONS}

The relations between density and percentage in pure sugar solutions have been very carefully determined, and hydrometers are specially made for sugar testing which are graduated to give at a standard temperature (usually $17.5^{\circ}$ C.) a direct reading of the per cent of sugar present in the solution. These are usually known as Brix (sometimes as Balling) hydrometers or "spindles." Brix hydrometers often have thermometers attached, and sometimes carry scales for reading temperature corrections directly from the instrument. Detailed tables for temperature corrections of Brix hydrometers and for conversion of specific gravity in Brix reading (percentage of sucrose) or the reverse are given in technical works on sugar analysis such as those of Rolfe and Spencer.

Beet and cane juices, although containing other solids than sucrose, are often tested with the Brix hydrometer and the reading taken as an approximate measure of the total solids present.

The polarization multiplied by 100 and divided by the Brix reading is sometimes called the "quotient of purity" of the juice. This of course is not exactly the percentage of sucrose in the solids, because in impure solutions the polarization may not be an exact measure of the sucrose, and the Brix hydrometer reading is not an exact measure of the percentage of total solids ; it is, 
however, an indication of purity which is of much value in controlling the operations of the sugar house.

In recent years the immersion refractometer has been considerably used in sugar house control. Tables have been constructed showing the percentages of sugar corresponding to each degree on the refractometer scale, and, with the aid of these, refractometer readings of sugar solutions or of cane or beet juices are readily translated into terms comparable with the Brix hydrometer readings, though of course any given impurity may not indicate exactly the same percentage of sugar by refraction that it would by specific gravity.

\section{IDENTIFICATION AND ANALYSIS OF “UNKNOWN" SUGARS}

Given an "unknown" sugar in an approximately pure state or an unknown mixture of two or three such sugars, one should find little difficulty in making out an indentification and analysis from the methods and data which have been given in this and the preceding chapter.

It is well to begin by determining the specific rotatory power and the reducing power (calculating the latter in percentage of the reducing power of pure dextrose as explained in the last chapter) and by performing an osazone test under the standard conditions, noting the time of appearance and the abundance of the osazone precipitate, if any appears in the hot solution, and whether any further precipitation of osazone occurs as the solution cools. Keeping in mind the influence of quantity of reacting sugar and of other sugars present on the formation and precipitation of the osazone and comparing the result obtained in the osazone test with the rotatory and reducing powers, one can usually judge from these data the sugars likely to be present in the sample. ${ }^{1}$

1 This judgment may be aided by certain superficial observations, e.g. if the 10 grams taken for the determination of rotatory power are difficultly soluble in 60 cc. of water the presence of considerable lactose may be suspected; while if the solution readily undergoes fermentation with yeast, it is probable that glucose, levulose, or maltose is present, though it is to be remembered that ordinary yeast also acts on sucrose, first hydrolyzing it to dextrose and levulose and then fer- 
If only two sugars are present in the mixture, the two quantitative determinations already made will suffice for the calculation of the percentage of each. For example, if the mixture consists of dextrose and lactose, then

$$
\begin{aligned}
100 \alpha & =53 d+52.5 l, \\
K & =d+.74 l,
\end{aligned}
$$

in which $\alpha$ is the specific rotatory power of the mixture, $K$ the cupric reducing power of the mixture (calculated in percentage of the reducing power of an equal weight of pure dextrose as explained before), $d$ the percentage of dextrose, and $l$ the percentage of lactose. Such a pair of simultaneous equations, one expressing rotatory and the other reducing power, will serve for any two of the common sugars at least one of which reduces Fehling's solution.

If three sugars are present in the mixture, it is evident that a third quantitative determination (to give the data for a third equation) will be required. If sucrose is one of the three, it may be determined by the Clerget method, even in the presence of maltose or lactose, with at least approximately correct results, since the acid treatment prescribed for the hydrolysis of the sucrose does not hydrolyze maltose nor lactose to any great extent. The analysis of a mixture of three sugars other than sucrose is less easy and will usually be less accurate. Levulose, if present, may be determined by the decrease of its levorotatory power on polarizing at a known high temperature; lactose, galactose, or raffinose may be estimated from the yield of mucic acid; maltose may be quantitatively hydrolyzed as described in the next chapter. In some cases it may be feasible to determine the moisture, the ash, and any organic constituents other than the sugars, subtract the sum of the percentages of all these impurities from 100, and take the remainder as the sum of the percentages of the different

menting these. [In laboratories where pure cultures of different yeasts are available the difference of behavior of different yeasts toward the sugars can be made use of for analytical purposes, but in most chemical laboratories such pure cultures are not at hand.] 
sugars present; but, as a rule, this should be resorted to only for purposes of verification, and not as a means of obtaining one of the original simultaneous equations.

\section{REFERENCES}

Allen : Commercial Organic Analysis, Vol. I.

Browne: Handbook of Sugar Analysis.

FrüHıING : Anleitung zür Untersuchung für die Zuckerindustrie.

Landolt (transl. by Long): Optical Rotation of Organic Substances.

LEACH : Food Inspection and Analysis.

Lippuann: Chemie der Zuckerarten.

LuNGE : Chemisch-technische Untersuchungsmethoden.

Rolfe: The Polariscope in the Chemical Laboratory.

Spencer : Handbook for Beet Sugar Chemists.

Handbook for Sugar Manufacturers (Cane Sugar).

Wiley : Agricultural Analysis.

\section{Periodicals devoted to Sugar}

American Sugar Industry and Beet Sugar Gazette.

Bulletin de l'Association des Chimistes de Sucrerie et de Distillation.

Centralblatt für die Zuckerindustrie.

Die Deutsche Zuckerindustrie.

International Sugar Journal.

Louisiana Sugar Planter and Sugar Manufacturer.

Oesterreichisch-Ungarische Zeitschrift für Zuckerindustrie.

Neue Zeitschrift für Rübenzuckerindustrie.

Zeitschrift des Vereins der deutschen Zuckerindustrie.

Zeitschrift für Zuckerindustrie in Böhmen.

\section{II}

1895. Weber and McPherson: On the Determination of Cane Sugar in the Presence of Commercial Glucose. J. Am. Chem. Soc., 17, 312.

1896. WILEY : On the Estimation of Levulose in Honeys and Other Substances. J. Am. Chem. Soc., 18, 81 .

1902. Boydex: On the Quantitative Separation of Maltose and Lactose. J. Am. Chem. Soc., 24, 993.

1903. Leacil: The Determination of Commercial Glucose in Molasses, Syrups, and Honey. J. Am. Chem. Soc., 25, 982.

1904. Harrison: Determination of Sucrose and Lactose in Condensed Milk. Analyst, 29, 248. 
Hortvet: The Chemical Composition of Maple Syrup and Maple Sugar, Methods of Analysis, and Detection of Adulteration. $J$. Am. Chem. Soc., 26, 1523.

LAXA : (Determination of Sucrose and Lactose in Milk Chocolate). Z. Nahr. Genussm., 7, 471.

1905. BAKER and Dick: The Detection and Estimation of Small Quantities of Maltose in the Presence of Dextrose: Analyst, 30, 79.

Pfyl and Linne: Hydrolysis of Sucrose, Maltose, Lactose and Raffinose. Z. Nahr. Genussm., 10, 104.

SAwyer: The Commercial Analysis of Cane Molasses. J. Am. Chem. Soc., 27, 691.

1906. Davoll: An Accurate Commercial Method for the Analysis of Sugar Beets. J. Am. Chem. Soc., 28, 1606.

Horne : Chemical Control of Cane Sugar Factories. School of Mines Quarterly, 27, 128.

Tolman and Sмiтh: Estimation of Sugars by Means of the Refractometer. J. Am. Chem. Soc., 28, 1476.

Winton and Kreider: Determination of the "Lead Number" in Maple Syrup and Maple Sugar. J. Am. Chem. Soc., 28, 1204.

1907. Andrim and Staner: (Use of Hydrochloric Acid and Urea in Direct Polarization for Clerget Method). Z. Zuckerind. Böhmen, 31, 417 ; Chem. Abs., 1, 1912.

BAtes and BLAKE: Influence of Basic Lead Acetate on the Rotation of Sucrose in Water Solution. J. Am. Chem. Soc., 29, 286.

Horne: Dry Lead Clarification. J. Am. Chem. Soc., 29, 926.

Konig and Hörmann: Separation of Carbohydrates by Pure Yeasts. Z. Nahr. Genussm., 13, 113.

U. S. Treasury, Document No. 2470: Regulations Governing the Weighing, Taring, Sampling, Classification, and Polarization of Imported Sugars and Molasses.

1908. Browne: Chemical Analysis and Composition of American Honeys. Bul. 110, Bur. Chem., U. S. Dept. Agriculture.

Browne and Halligain: Report on Sugar and Molasses Methods. U. S. Dept. Agriculture, Bur. Chem., Bul. 116, p. 68.

Ling and Rende: Determination of Sucrose and Invert Sugar in Mixtures. Analyst, 33, 167.

Sx: Apparatus for Polarizing at $87^{\circ}$. J. Am. Chem. Soc., 30, 1790.

U. S. Dept. Agriculture, Bur. Chem., Bul. 107 (Revised) : Official and Provisional Methods of Analysis.

1909. Browne: Use of Temperature Corrections in the Polarization of Raw Sugars and other Products upon Quartz Wedge Saccharimeters. J. Ind. Eng. Chem., 1, 567.

Unification of Saccharimeter Observations. U. S. Dept. Agr., Bur. Chem., Bul. 122, p. 221. 
Report of the New York Sugar Trade Laboratory. Z. Ver. Zuckerind., 59, II, 297.

Herzfeld : Estimation of Sugar in the Sugar Beet. Z. Ver. Zuckerind., 59, 627. Chem. Abs., 3, 2249.

Horne: Clarification with Dry Lead Subacetate in the Analysis of Raw Sugars. Z. Ver. Zuckerind., 59, 639; Chem. Abs., 3, 2393.

1910. Baker and Hulton: The Estimation of Lactose in the Presence of the Commonly Occurring Sugars. Analyst, 35, 512.

Browne: The Normal Weight of Dextrose. J. Ind. Eng. Chem., 2, 526.

Browne and Bryan: Lead Clarification in Sugar Analysis. Z. Ver. Zuckerind., 59, 922 ; Chem. Abs., 4, 258.

Hudson: The Quantitative Determination of Cane Sugar by the Use of Invertase. J. Ind. Eng. Chem., 2, 143.

Ling, Eynon, and Lane: Solution Densities of Dextrose, Levulose, and Maltose. J. Soc. Chem. Ind., 28, 730.

ReEs : Optically Active Non-Sugars of the Sugar Beet: J. Ind. Eng. Chem., 2, 323.

Spencer: The Milling of Cane in Sugar Manufacture Control. $J$. Ind. Eng. Chem., 2, 253.

Witte: Examination of Honey. Z. Nahr. Genussm., 21, 305.

1911. Andrlik and Stanek: Determination of Solids, Coęfficient of Purity, Loss of Polarization during Saturation, and Amount of Non-Sugar removed during Clarification in Diffusion and Saturated Juices.

Z. Zuckerind. Böhmen, 35, 257; Chem. Abs., 5, 2751.

Bryan: Analyses of Sugar Beets (with Methods and Bibliography).

U. S. Dept. Agriculture, Bur. Chem., Bul. 146.

Dubors : (Determination of Sucrose and Lactose in Milk Chocolate). U. S. Dept. Agr., Bur. Chem., Bul. 137, p. 98.

Kerser: Contribution to the Chemistry of Honey, with Special Reference to Differences from Artificial Wares. Arb. kais. Gesundh., 30, 637; Chem. Abs., 5, 538.

Pellet : Determination of Sucrose in Cane Molasses. Intern. Sugar J., 13, 206 ; Chem. Abs., 5, 2752.

Schöхrock : Refractive Index of Sugar Solutions. Z. Ver. Zuckerind., 61, 421; Chem. Abs., 5, 2575. 


\section{CHAPTER V}

\section{Starch and Amylase}

\section{THE DETERMINATION OF STARCH}

\section{Method of Direct Acid Hydrolysis}

THIs method is accurate for the determination of starch in such samples as contain no other substance insoluble in water and capable of yielding reducing sugar on heating with dilute acid. It is now well known that such interfering substances, especially the pentosans, are generally found in vegetable tissues, and that the results obtained by direct acid hydrolysis are usually too high. This is, however, the method which has been commonly used until recently, and for comparisons with earlier work determinations by direct hydrolysis are still frequently required.

\section{Conversion of Starch to Dextrose 1}

Weigh 2 to 5 grams and, if much fat is present, wash with four or five successive portions each $10 \mathrm{cc}$. of ether; reject the filtrate, allow the adhering ether to evaporate from the residue, and wash with $150 \mathrm{cc}$. of 10 per cent alcohol to remove soluble carbohydrates. Wash the residue into a 250 cc. flask with $200 \mathrm{cc}$. of water, add $20 \mathrm{cc}$. of hydrochloric acid of $1.125 \mathrm{sp} . \mathrm{gr}$., and heat in a boiling water bath with a reflex condenser for two and one half hours. Cool to room temperature, nearly neutralize with sodium hydroxide, dilute to $250 \mathrm{cc}$., filter, and determine dextrose in a portion of the filtrate, using either Defren's method, already described, or Allihn's method, as follows :

1 Sachsse, Chem. Centrbl., 1877, 732 ; Bul. 107, Bur. Chem., U. S. Dept. Agriculture. 


\section{Allihn's Method for the Determination of Dextrose ${ }^{1}$}

Reagents. - (1) 34.64 grams of crystallized copper sulphate dissolved in water and diluted to $500 \mathrm{cc}$.

(2) 173 grams of sodium potassium tartrate and 125 grams of potassium hydroxide dissolved in water and diluted to $500 \mathrm{cc}$.

Determination. - Place 30 cc. of the copper solution, 30 cc. of the alkaline tartrate solution and $60 \mathrm{cc}$. of water in a beaker or casserole and heat to boiling. To the boiling liquid add 25 cc. of the dextrose solution; note the time at which the mixture begins to boil and continue the boiling for exactly two minutes. Filter at once and obtain the weight of copper in the precipitated cuprous oxide by any of the methods suggested in connection with Defren's process described in Chapter III, or as follows: Collect the cuprous oxide on an asbestos filter in a Gooch crucible; wash the precipitate (including any which may adhere to the beaker and which need not be transferred to the filter) thoroughly with hot water, transfer the asbestos and adhering oxide from the crucible to the beaker. Dissolve the oxide still remaining in the crucible by means of 1 to $2 \mathrm{cc}$. of concentrated nitric acid, adding the acid from a pipette and receiving the solution in the beaker containing the asbestos and the main part of the precipitate. Rinse the crucible with a jet of water, allowing the rinsings to flow into the beaker. Heat the contents of the beaker until all copper is in solution; filter, wash thoroughly, dilute the filtrate to 100 to 150 cc., add one drop of concentrated sulphuric acid and determine copper by electrolysis. Find the corresponding weight of dextrose from Allihn's table.

Notes. - The conditions described must be observed carefully. The dextrose solution added to the copper reagent must be free from turbidity and only faintly acid. The rapid addition of this cold solution stops the boiling of the reagent, and it is well to have the heat so regulated that the mixture will boil again in about two minutes; then, after exactly two minutes of actual

1 J. prakt. Chem., 1880, 22, 46 ; Bul. 107, loc. cit. 
Table 9. - Allihn's Table for the Determination of Dextrose

\begin{tabular}{|c|c|c|c|c|c|c|c|c|c|}
\hline $\begin{array}{c}\text { Milli- } \\
\text { grams } \\
\text { of } \\
\text { copper }\end{array}$ & $\begin{array}{l}\text { Milli- } \\
\text { grams } \\
\text { of dex- } \\
\text { trose }\end{array}$ & $\begin{array}{c}\text { Milli- } \\
\text { grams } \\
\text { of } \\
\text { copper }\end{array}$ & $\begin{array}{l}\text { Milli- } \\
\text { grams } \\
\text { of dex- } \\
\text { Irose }\end{array}$ & $\begin{array}{l}\text { Milli- } \\
\text { grams } \\
\text { of } \\
\text { copper }\end{array}$ & $\begin{array}{l}\text { Milli- } \\
\text { grams } \\
\text { of dex- } \\
\text { trose }\end{array}$ & $\begin{array}{l}\text { Milli- } \\
\text { grams } \\
\text { of } \\
\text { copper }\end{array}$ & $\begin{array}{l}\text { Milli- } \\
\text { grams } \\
\text { of dex- } \\
\text { trose }\end{array}$ & $\begin{array}{l}\text { Milli- } \\
\text { grams } \\
\text { of } \\
\text { copper }\end{array}$ & $\begin{array}{l}\text { Milli- } \\
\text { grams } \\
\text { of dex- } \\
\text { trose }\end{array}$ \\
\hline 10 & 6.1 & 67 & 34.3 & 124 & 63.1 & 181 & 92.6 & 238 & 122.8 \\
\hline 11 . & 6.6 & 68 & 34.8 & 125 & 63.7 & 182 & 93.1 & 239 & 123.4 \\
\hline 12 & 7.1 & 69 & 35.3 & 126 & 64.2 & 183 & 93.7 & 240 & 123.9 \\
\hline 13 & 7.6 & 70 & 35.8 & 127 & 64.7 & 184 & 94.2 & 241 & 124.4 \\
\hline 14 & 8.1 & 71 & 36.3 & 128 & 65.2 & 185 & 94.7 & 242 & 125.0 \\
\hline 15 & 8.6 & 72 & 36.8 & 129 & 65.7 & 186 & 95.2 & 243 & 125.5 \\
\hline 16 & 9.0 & 73 & 37.3 & 130 & 66.2 & 187 & 95.7 & 244 & 126.0 \\
\hline 17 & 9.5 & 74 & 37.8 & 131 & 66.7 & 188 & 96.3 & 245 & 126.6 \\
\hline 18 & 10.0 & 75 & 38.3 & 132 & 67.2 & 189 & 96.8 & 246 & 127.1 \\
\hline 19 & 10.5 & 76 & 38.8 & 133 & 67.7 & 190 & 97.3 & 247 & 127.6 \\
\hline 20 & 11.0 & 77 & 39.3 & 134 & 68.2 & 191 & 97.8 & 248 & 128.1 \\
\hline 21 & 11.5 & 78 & 39.8 & 135 & 68.8 & 192 & 98.4 & 249 & 128.7 \\
\hline 22 & 12.0 & 79 & 40.3 & 136 & 69.3 & 193 & 98.9 & 250 & 129.2 \\
\hline 23 & 12.5 & 80 & 408 & 137 & 69.8 & 194 & 99.4 & 251 & 129.7 \\
\hline 24 & 13.0 & 81 & 41.3 & 138 & 70.3 & 195 & 100.0 & 252 & 130.3 \\
\hline 25 & 13.5 & 82 & 41.8 & 139 & 70.8 & 196 & 100.5 & 253 & 130.8 \\
\hline 26 & 14.0 & 83 & 42.3 & 140 & 71.3 & 197 & 101.0 & 254 & 131.4 \\
\hline 27 & 14.5 & 84 & 42.8 & 141 & 71.8 & 198 & 101.5 & 255 & 131.9 \\
\hline 28 & 15.0 & 85 & 43.4 & 142 & 72.3 & 199 & 102.0 & 256 & 132.4 \\
\hline 29 & 15.5 & 86 & 43.9 & 143 & 72.9 & 200 & 102.6 & 257 & 133.0 \\
\hline 30 & 16.0 & 87 & 44.4 & 144 & 73.4 & 201 & 103.1 & 258 & 133.5 \\
\hline 31 & 16.5 & 88 & 44.9 & 145 & 73.9 & 202 & 103.7 & 259 & 134.1 \\
\hline 32 & 17.0 & 89 & 45.4 & 146 & 74.4 & 203 & 104.2 & 260 & 134.6 \\
\hline 33 & 17.5 & 90 & 45.9 & 147 & 74.9 & 204 & 104.7 & 261 & 135.1 \\
\hline 34 & 18.0 & 91 & 46.4 & 148 & 75.5 & 205 & 105.3 & 262 & 135.7 \\
\hline 35 & 18.5 & 92 & 46.9 & 149 & 76.0 & 206 & 105.8 & 263 & 136.2 \\
\hline 36 & 18.9 & 93 & 47.4 & 150 & 76.5 & 207 & 106.3 & 264 & 136.8 \\
\hline 37 & 19.4 & 94 & 47.9 & 151 & 77.0 & 208 & 106.8 & 265 & 137.3 \\
\hline 38 & 19.9 & 95 & 48.4 & 152 & 77.5 & 209 & 107.4 & 266 & 137.8 \\
\hline 39 & 20.4 & 96 & $\begin{array}{l}48.9 \\
48.9\end{array}$ & 153 & 78.1 & 210 & 107.9 & 267 & 138.4 \\
\hline 40 & 20.9 & 97 & 49.4 & 154 & 78.6 & 211 & 108.4 & 268 & 138.9 \\
\hline 41 & 21.4 & 98 & 49.9 & 155 & 79.1 & 212 & 109.0 & 269 & 139.5 \\
\hline 42 & 21.9 & 99 & 50.4 & 156 & 79.6 & 213 & 109.5 & 270 & 140.0 \\
\hline 43 & 22.4 & 100 & 50.9 & 157 & 80.1 & 214 & 110.0 & 271 & 140.6 \\
\hline 44 & 22.9 & 101 & 51.4 & 158 & 80.7 & 215 & 110.6 & 272 & 141.1 \\
\hline 45 & 23.4 & 102 & 51.9 & 159 & 81.2 & 216 & 111.1 & 273 & 141.7 \\
\hline 46 & 23.9 & 103 & 52.4 & 160 & 81.7 & 217 & 111.6 & 274 & 142.2 \\
\hline 47 & 24.4 & 104 & 52.9 & 161 & 82.2 & 218 & 112.1 & 275 & 142.8 \\
\hline 48 & 24.9 & 105 & 53.5 & 162 & 82.7 & 219 & 112.7 & 276 & 143.3 \\
\hline 49 & 25.4 & 106 & 54.0 & 163 & 83.3 & 220 & 113.2 & 277 & 143.9 \\
\hline 50 & 25.9 & 107 & 54.5 & 164 & 83.8 & 221 & 113.7 & 278 & 144.4 \\
\hline 51 & 26.4 & 108 & 55.0 & 165 & 84.3 & 222 & 114.3 & 279 & 145.0 \\
\hline 52 & 26.9 & 109 & 55.5 & 166 & 84.8 & 223 & 114.8 & 280 & 145.5 \\
\hline 53 & 27.4 & 110 & 56.0 & 167 & 85.3 & 224 & 115.3 & 281 & 146.1 \\
\hline 54 & 27.9 & 111 & 56.5 & 168 & 85.9 & 225 & 115.9 & 282 & 146.6 \\
\hline 55 & 28.4 & 112 & 57.0 & 169 & 86.4 & 226 & 116.4 & 283 & 147.2 \\
\hline 56 & 28.8 & 113 & 57.5 & 170 & 86.9 & 227 & 116.9 & 284 & 147.7 \\
\hline 57 & 29.3 & 114 & 58.0 & 171 & 87.4 & 228 & 117.4 & 285 & 148.3 \\
\hline 58 & 29.8 & 115 & 58.6 & 172 & 87.9 & 229 & 118.0 & 286 & 148.8 \\
\hline 59 & 30.3 & 116 & 59.1 & 173 & 88.5 & 230 & 118.5 & 287 & 149.4 \\
\hline 60 & 30.8 & 117 & 59.6 & 174 & 89.0 & 231 & 119.0 & 288 & 149.9 \\
\hline 61 & 31.3 & 118 & 60.1 & 175 & 89.5 & 232 & 119.6 & 289 & 150.5 \\
\hline 62 & 31.8 & 119 & 60.6 & 176 & 90.0 & 233 & 120.1 & 290 & 151.0 \\
\hline 63 & 32.3 & 120 & 61.1 & 177 & 90.5 & 234 & 120.7 & 291 & 151.6 \\
\hline 64 & 32.8 & 121 & 61.6 & 178 & 91.1 & 235 & 121.2 & 292 & 152.1 \\
\hline 65 & 33.3 & 122 & 62.1 & 179 & 91.6 & 236 & 121.7, & 293 & 152.7 \\
\hline 66 & 33.8 & 123 & 62.6 & 180 & 92.1 & 237 & 122.3 & 294 & 153.2 \\
\hline
\end{tabular}


Allihn's Table for the Determination of Dextrose-Continued

\begin{tabular}{|c|c|c|c|c|c|c|c|c|c|}
\hline $\begin{array}{l}\text { Milli- } \\
\text { grams } \\
\text { of } \\
\text { copper }\end{array}$ & $\begin{array}{c}\text { Milli- } \\
\text { grams } \\
\text { of dex- } \\
\text { trose }\end{array}$ & $\begin{array}{c}\text { Milli- } \\
\text { grams } \\
\text { of } \\
\text { copper }\end{array}$ & $\begin{array}{l}\text { Milli- } \\
\text { grams } \\
\text { of dex- } \\
\text { trose }\end{array}$ & $\begin{array}{l}\text { Milli- } \\
\text { grams } \\
\text { of } \\
\text { copper }\end{array}$ & $\begin{array}{l}\text { Milli- } \\
\text { grams } \\
\text { of dex- } \\
\text { trose }\end{array}$ & $\begin{array}{l}\text { Milli- } \\
\text { grams } \\
\text { of } \\
\text { copper }\end{array}$ & $\begin{array}{l}\text { Milli- } \\
\text { grams } \\
\text { of dex- } \\
\text { trose }\end{array}$ & $\begin{array}{l}\text { Milli- } \\
\text { grams } \\
\text { of } \\
\text { copper }\end{array}$ & $\begin{array}{l}\text { Milli- } \\
\text { grams } \\
\text { of dex- } \\
\text { trose }\end{array}$ \\
\hline 295 & 153.8 & 329 & 172.5 & 363 & 191.7 & 397 & 211.2 & 431 & 231.0 \\
\hline 296 & 1504.3 & 330 & 173.1 & 364 & 192.3 & 398 & 211.7 & 432 & 231.6 \\
\hline 297 & 154.9 & 331 & 173.7 & 365 & 192.9 & 399 & 212.3 & 433 & 232.2 \\
\hline 298 & 155.4 & 332 & 174.2 & 366 & 193.4 & 400 & 212.9 & 434 & 232.8 \\
\hline 299 & 156.0 & 333 & 174.8 & 367 & 194.0 & 401 & 213.5 & 435 & 233.4 \\
\hline 300 & 156.5 & 334 & 175.3 & 368 & 194.6 & 402 & 214.1 & 436 & 233.9 \\
\hline 301 & 157.1 & $3: 35$ & 175.9 & 369 & 195.1 & 403 & 214.6 & 437 & 234.5 \\
\hline 302 & 157.6 & 336 & 176.5 & 370 & 195.7 & 404 & 215.2 & 438 & 235.1 \\
\hline 303 & 158.2 & 337 & 177.0 & 371 & 196.3 & 405 & 215.8 & 439 & 235.7 \\
\hline 304 & 158.7 & 338 & 177.6 & 372 & 196.8 & 406 & 216.4 & 440 & 236.3 \\
\hline 305 & 159.3 & 339 & 178.1 & 373 & 197.4 & 407 & 217.0 & 441 & 236.9 \\
\hline 306 & 159.8 & 340 & 178.7 & 374 & 198.0 & 408 & 217.5 & 442 & 237.5 \\
\hline 307 & 160.4 & 341 & 179.3 & 375 & 198.6 & 409 & 218.1 & 443 & 238.1 \\
\hline 308 & 160.9 & 342 & 179.8 & 376 & 199.1 & 410 & 218.7 & 444 & 238.7 \\
\hline 309 & 161.5 & 343 & 180.4 & 377 & 199.7 & 411 & 219.3 & 445 & 239.3 \\
\hline 310 & 162.0 & 344 & 180.9 & 378 & 200.3 & 412 & 219.9 & 446 & 239.8 \\
\hline 311 & 162.6 & 345 & 181.5 & 379 & 200.8 & 413 & 220.4 & 447 & 240.4 \\
\hline 312 & 163.1 & $3 \pm 6$ & 182.1 & 380 & 201.4 & 414 & 221.0 & 448 & 241.0 \\
\hline 313 & 163.7 & $3 \pm 7$ & 182.6 & 381 & 202.0 & 415 & 221.6 & 449 & $2+1.6$ \\
\hline 314 & 164.2 & 348 & 183.2 & 382 & 202.5 & 416 & 222.2 & 450 & 242.2 \\
\hline 315 & 164.8 & 349 & 183.7 & 383 & 203.1 & 417 & 222.8 & 451 & 242.8 \\
\hline 316 & 165.3 & 350 & 184.3 & 384 & 203.7 & 418 & 223.3 & 452 & 243.4 \\
\hline 317 & 165.9 & 351 & 184.9 & 385 & 204.3 & 419 & 223.9 & 453 & 244.0 \\
\hline 318 & 166.4 & 352 & 185.4 & 386 & 204.8 & 420 & 224.5 & 454 & 244.6 \\
\hline 319 & 167.0 & 353 & 186.0 & 387 & 205.4 & 421 & 225.1 & 455 & 245.2 \\
\hline 320 & 167.5 & 354 & 186.6 & 388 & 206.0 & $42 \cdot 2$ & 225.7 & 456 & 245.7 \\
\hline 321 & 168.1 & 355 & 187.2 & 389 & 206.5 & 423 & 226.3 & 457 & 246.3 \\
\hline $32 \%$ & 168.6 & 356 & 187.7 & 390 & 207.1 & 424 & 226.9 & 458 & 246.9 \\
\hline 323 & 169.2 & 357 & 188.3 & 391 & 207.7 & 425 & 227.5 & 459 & 247.5 \\
\hline 324 & 169.7 & 358 & 188.9 & 392 & 208.3 & 426 & 228.0 & 460 & 248.1 \\
\hline 325 & 170.3 & 359 & 189.4 & 393 & 208.8 & 427 & 228.6 & 461 & 248.7 \\
\hline 326 & 170.9 & 360 & 190.0 & 394 & 209.4 & 428 & 229.2 & 462 & 249.3 \\
\hline 327 & 171.4 & 361 & 190.6 & 395 & 210.0 & 429 & 229.8 & 463 & 249.9 \\
\hline 328 & 172.0 & 362 & 191.1 & 396 & 210.6 & 430 & 230.4 & & \\
\hline
\end{tabular}

boiling, remove the flame and filter the solution at once. The cuprous oxide is very apt to run through the filter. To prevent this, after making the asbestos filter as usual, pour on it some very fine asbestos suspended in water, so as to form a tight layer on the top of the felt. On the assumption that the starch is quantitatively hydrolyzed to dextrose, the weight of the latter multiplied by 0.9 gives the corresponding weight of starch. For detailed studies of the hydrolysis of starch by acids, see the papers of Rolfe ${ }^{1}$ and of Noyes. ${ }^{2}$ According to several

1 J. Am. Chem. Soc., 1896, 18, $869 ;$ 1897, 19, 261; 1903, 25, 1003, 1015.

2 Ibid., 1904, 26, 266. 
investigators, ${ }^{1}$ the weight of dextrose should be multiplied by a higher factor ( 0.917 to 0.941$)$ rather than 0.9 , to find the corresponding weight of starch.

\section{Method of Digestion with Diastase or Saliva}

If starch is gelatinized by boiling with water and then treated with malt diastase or saliva, it can be converted into maltose and dextrin and these separated by filtration from the insoluble residue containing the pentosans and other substances which cause the results by the preceding method to be too high.

Determination. - Extract 2.5 to 5 grams of sample with five successive portions each $10 \mathrm{cc}$. of ether, decanting the washings through a hardened filter; wash with $150 \mathrm{cc}$. of 10 per cent alcohol $;^{2}$ transfer the residue to a beaker with 50 to $100 \mathrm{cc}$. of water; heat gradually to boiling, stirring constantly to prevent bumping or the formation of lumps. Cool to $55^{\circ}$ for diastase, or to $38^{\circ}$ for saliva; add the solution containing the enzyme and keep the mixture within two degrees of the stated temperature until a drop, removed and tested on a porcelain plate, no longer shows a reaction for starch on mixing with a drop of dilute solution of iodine in aqueous potassium iodide. Now heat the solution again to boiling in order to gelatinize any starch granules which may remain; test the solution, and if starch is found, cool to the proper temperature; add more of the enzyme and digest as before. Continue this treatment until the solution gives no starch reaction after boiling, or until a careful microscopic examination shows that the insoluble residue is entirely free from starch. Dilute to 250 cc., mix thoroughly and pour on a dry fluted filter. Transfer 150 cc. of the filtrate to a 250 -cc. flask; add $15 \mathrm{cc}$. of hydrochloric acid of $1.125 \mathrm{sp}$. gr., attach the flask to a reflux condenser and heat in

${ }^{1}$ Salomon : Z. anal. Chem., 1883, 22, 593. Soxhlet: Wochenschr. für Brauer., 1885, 193. Vaubel, II, 455. Sostegni : Chem. Zentrbl., 1887, 58, 896. Lintner and Düll : Z. angew. Chem., 1891, 537. Ost : Chem. Ztg., 1895, 19, 1502. Rössing: Z. öffentl. Chem., 1904, 10,61 ; Abs. J. Chem. Soc., 1904, 86, ii, 298. Noyes : J. Am. Chem. Soc., 1904, 26, 280.

2 This extraction can often be omitted, since for many purposes it is unnecessary to distinguish between starch and soluble carbohydrates. 
a boiling water bath for two and one half hours, or boil gently on a hot plate or sand bath for 35 to 45 minutes. Complete the determination as described in the preceding method.

The determination should be carried through without interruption. If this is impossible, care must be taken to avoid alcoholic or lactic fermentation. After the digestion with the enzyme is finished, but not before, salicylic acid may be added as a preservative. It has been recommended that a trace of fluoride be added at the start to retard lactic fermentation while the digestion with the enzyme is taking place.

When only a few determinations are to be made, freshly collected saliva can conveniently be used, as this is free from carbohydrate. If commercial diastase or an infusion of malt ${ }^{1}$ is used, the amount added must be noted and a correction applied for the carbohydrate thus introduced. This is found by heating a quantity of the diastase or infusion with acid and determining the resulting dextrose as in the starch determination.

\section{Comparison of Results}

The diastase method, carefully carried out, is believed to yield practically correct results. As already explained, the results obtained by direct acid hydrolysis are usually higher owing to the presence of other substances which yield reducing sugars. A comparison of the results of the two methods is of considerable interest, both because many of the recorded determinations of starch were made by the acid method and because the determination of copper-reducing substance obtained by direct hydrolysis is sometimes used as a means of detecting adulterants in spices. The table below shows the results of comparison of the two methods on a variety of substances. Many of the results of Winton and associates are averaged from the comparative examination of several samples. The other results were obtained by the writer, only one sample of each kind being examined.

${ }^{1}$ An active malt infusion can be prepared by digesting 10 grams of fresh, finely-ground malt, overnight at room temperature, with $200 \mathrm{cc}$. of water or 10 per cent alcohol. 
Table 10. - Results by Diastase and by Direct Acid Hydrolysis

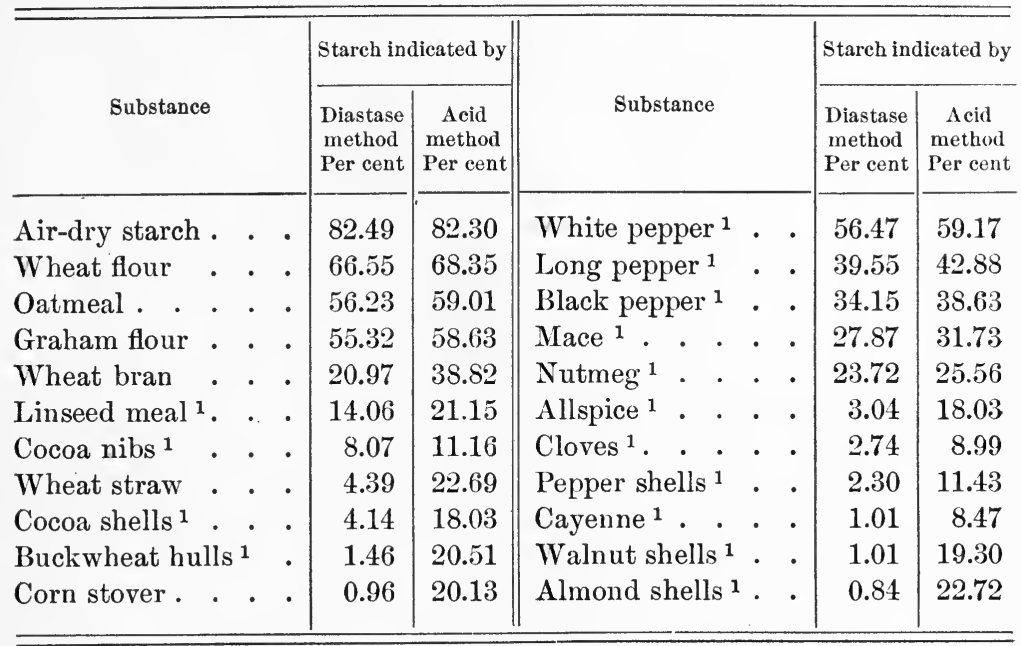

\section{Other Methods}

For the determination of starch in a particular sort of material, it is sometimes feasible to obtain accurate results by methods shorter than those above described. Thus, the starch in cereals is sometimes determined by dissolving in acid and taking the rotatory power of the solution, and the starch content of potatoes may be estimated approximately from their specific gravity. Papers describing these methods are included among the references at the end of this chapter.

Starchy materials are sometimes added as "fillers" to sausages and other forms of chopped meat. This adulteration is easily detected by the iodine reaction, which, however, must be carefully interpreted since a small amount of starch may legitimately be present from the spices added in the manufacture. The quantitative determination is complicated by the fact that meat appears to contain some substance which interferes with the separation of the cuprous oxide reduced in applying the usual

1 Results by Winton and associates, compiled from Leach's Food Inspection and Analysis. 
method. Advantage is therefore taken of the insolubility of starch in alcoholic, and its solubility in aqueous, potassium hy. droxide. The method of Mayrhofer as modified by Bigelow and adopted by the Association of Official Agricultural Chemists is as follows: ${ }^{1}$

Treat from 10 to 20 grams of the sample under examination (depending upon the amount of starch indicated by the iodine reaction) in a porcelain dish or casserole with $50 \mathrm{cc}$. of an 8 per cent solution of potassium hydroxide and heat the mixture on the water bath until the meat is entirely dissolved. Add an equal volume of 95 per cent alcohol, mix thoroughly, filter the mixture through an asbestos filter, and wash twice with a hot 4 per cent solution of potassium hydroxide in 50 per cent alcohol. Then wash with 50 per cent alcohol until a small portion of the filtrate does not become turbid upon the addition of acid. Return the precipitate and filter to the original vessel and dissolve the precipitate with the aid of heat in $60 \mathrm{cc}$. of a normal solution of potassium hydroxide: In the case of sausage, with a high starch content, a somewhat larger volume of alkali may be required. Acidify the filtrate strongly with acetic acid, dilute to a definite volume, mix thoroughly by shaking, filter through a fluted paper, and precipitate the starch from an aliquot part of the filtrate by means of an equal volume of 95 per cent alcohol. Transfer the precipitate to a weighed filter, wash thoroughly with 50 per cent alcohol, with absolute alcohol, and finally with ether, dry to a constant weight at the temperature of boiling water, and weigh.

\section{DIASTATIC POWER OF AMYLASES}

Of interest in connection with the determination of starch are the methods for the determination of the activity or power of the starch-splitting enzymes (amylases) or of substances containing such enzymes. Such methods are of growing importance, not only in scientific investigations, but also in industrial analysis.

i Bul. 107, Bur. Chem., U. S. Dept. Agriculture. 
Malt extracts are being purchased by bakers on the basis of diastatic power, and the diastatic power of malt is important in the alcohol industry when it is desired to convert as large an amount of foreign starch, by the use of as little malt, as possible. In the past, malt has been so freely used in the alcohol industry in America that its diastatic power has been a matter of comparative indifference, but it is to be expected that in the production of industrial alcohol more economical methods will be employed, and the proportion of malt used be governed by its diastatic power. Already, efforts are being made to develop cheaper sources of amylase for industrial use.

In the field of food and drug inspection, there is also a demand for methods for the determination of diastatic power as a means of measuring the activity of the various starch-digesting preparations which are offered in the food and drug trade as aids to digestion or as means of predigesting starchy food.

Many methods of determining diastatic power have been proposed, but most of these have been tested for only a single amylase or only a narrow range of conditions. There are two main types of methods, based respectively upon the measurement of the amount of reducing sugar produced by the amylase or the measurement of the conversion of a given amount of starch into hydrolytic products.

Evidently the result obtained by either type of method will depend upon the conditions under which it is applied, and further it may be expressed in a number of ways, for example, the amount of change produced by a given amount of enzyme in a given time, the amount of enzyme required to produce a given change in a given time, or the time required for a given amount of enzyme to produce a given change.

Three typical methods are outlined below. Whatever method be used, the observant worker will find that the results may be influenced by many conditions which cannot be entered into fully here. The chemist who is not already familiar with the difficulties of work of this nature should not rely upon the outlines here given, but should also study carefully the original papers cited at the end of the chapter. 


\section{Lintner's Method ANd Scale ${ }^{1}$}

Make a paste of two grams of purified, ${ }^{2}$ dry, soluble starch in a little cold neutral distilled water and pour into 50 or $60 \mathrm{cc}$. of boiling water. Boil for a minute or two after the starch is dissolved, then cool and dilute to $100 \mathrm{cc}$. in a graduated flask. Place $10 \mathrm{cc}$. of this starch solution in each of a series of ten test tubes and place the tubes in a water bath kept at $21^{\circ} \mathrm{C}$.

Weigh out 50 to 100 milligrams of diastase and after first mixing with 4 or 5 cc. of water dilute to 100 or 200 cc. according to the strength of the enzyme. Then add to each of the starch tubes known quantities of enzyme solution in increasing amounts (or if the diastatic power of malt is to be determined, add to each tube a different amount of malt extract prepared as below), e.g. $0.1,0.2,0.3,0.4,0.5$ cc., etc. After digesting for one hour at $21^{\circ} \mathrm{C}$., stop the action by the addition of 5 cc. of Fehling solution to each tube, place the tubes in boiling water for 10 minutes and then by means of the potassium ferrocyanide test (or one of the other tests described in Chapter III) examine to determine the first tube in which the copper is all reduced, i.e. the smallest amount of enzyme or extract which has produced sufficient maltose to reduce 5 cc. of Fehling solution. Lintner prepared a sample of diastase of which $0.12 \mathrm{mg}$. produced under these conditions the maltose necessary to reduce the $5 \mathrm{cc}$. of Fehling solution. This preparation was rated as having a diastatic power of 100 and the diastatic powers of other preparations were calculated as inversely proportional to the amount of sample required to produce this fixed amount of reducing sugar. Thus, if $0.3 \mathrm{mg}$. were required

$$
\begin{gathered}
0.3: 0.12:: 100: X \\
X=40,
\end{gathered}
$$

the diastatic power of the preparation tested (Lintner Scale).

In applying this method to malt, digest 25 grams of the dried, ground sample with $500 \mathrm{cc}$. of water and use portions of the

1 J. prakt. Chem., [2], 34, 378.

2 The preparation of soluble starch for this purpose is discussed fully by Ford: J. Soc. Chem. Ind., 23, 414. 
clear filtered extract, as described for the diastatic solution. Then if $V=$ volume of extract, required to give complete reduction of the Fehling solution,

\section{$V: 0.1:: 100:$ diastatic power of malt (Lintner Scale).}

If in such a test cupric copper be found in the tube representing $0.2 \mathrm{cc}$. and not in that which had $0.3 \mathrm{cc}$. of the extract, it is evident that the true value of $V$ lies either at $0.3 \mathrm{cc}$. or between $0.2 \mathrm{cc}$. and $0.3 \mathrm{cc}$. and can be found accurately only by repeating the test with small increments of volume between these limits.

Note that the values on Lintner's scale for malt are about 40 times as high as on his scale for enzyme preparations.

\section{Wohlgemuth's Method and Scale 1}

Place in each of a series of test tubes diminishing amounts of saliva or enzyme solution to be tested. Introduce into each tube 5 cc. of a 1 per cent solution of soluble starch and place each tube immediately in a bath of ice water. When all are prepared, transfer the tubes to a water bath at $40^{\circ}$ for 30 minutes.

At the end of this time transfer again immediately to the ice water bath to stop the action.

Dilute the contents of each tube to within $\frac{1}{2}$ inch from the top with water. Add one drop of a tenth-normal solution of iodine and shake well. One usually obtains colors from blue through violet, red, and yellow to colorless indicative of starch, erythrodextrin, achroödextrin, etc. Taking the tube where the blue or violet has entirely disappeared, giving place either to red or orange-red color as judged by Mulliken's Color Standard Sheet C, note the amount of enzyme solution in this tube and calculate the power of the enzyme as the number of cubic centimeters of 1 per cent starch solution which is digested to this stage in a given time by $1 \mathrm{cc}$. of enzyme solution.

Thus if 0.2 gram (or cubic centimeter) of the substance tested completely digests $5 \mathrm{cc}$. of starch solution in 30 minutes

1 Biochem. Zeitschrift, 9, 1. 
at $40^{\circ}$, then 1 gram or 1 cc. of that solution will be able to digest 250 cc. of 1 per cent starch solution, or its power is then stated :

$$
D_{30}^{400}=250 \text { (Wohlgemuth Scale). }
$$

Here the time of reaction is brought into the final expression of diastatic power so that the method may be applied to substances whose power is very slight by allowing them to act a long time.

Wohlgemuth has used the method principally in testing physiological fluids, in which case the results are expressions of relative volumes. When the substance tested is a solid, the results are calculated in the same way, cubic centimeter and gram being considered practically interchangeable.

\section{New Method and Scale ${ }^{1}$}

Prepare 400 cc. of 2 per cent soluble starch solution. Weigh a portion (depending upon its activity) of the material to be tested, dissolve, and dilute to volume (Note 1). By means of a $1 \mathrm{cc}$. Mohr's pipette accurately calibrated in hundredths, measure into four 200 -cc. Erlenmeyer flasks such volumes of the enzyme solution as will contain suitable quantities of amylase (Note 2). Then, noting accurately the time at which the starch and enzyme solutions are mixed, pour into each flask $100 \mathrm{cc}$. of the starch solution which has been previously warmed to $40^{\circ}$ (Note 3 ). Place the flasks immediately in a water or air bath, the temperature of which is maintained at exactly $40^{\circ}$ throughout the entire time of reaction and allow to digest for 30 minutes (Note 4). At the expiration of the thirty minutes stop the action immediately by adding to each flask 50 cc. of Fehling solution (Note 5), and immersing the flasks in a large bath of boiling water for 15 minutes. See that the water in the bath is kept boiling and that it stands above the level of the contents of any of the flasks. ${ }^{2}$ At the end of this heating filter quickly on weighed

I Sherman, Kendall and Clark: J. Am. Chem. Soc., 32, 1082.

2 The flasks may be weighted with lead rings to prevent upsetting. 
Gooch crucibles having thick felts of specially prepared asbestos; wash the cuprous oxide thoroughly with hot water, then with alcohol and ether; dry for at least 20 minutes in a boiling water oven and weigh. Use for the following calculation that one of the four determinations which shows the highest weight of cuprous oxide not exceeding $300 \mathrm{mg}$. when corrected for the reducing power of the soluble starch.

Correct the weight of cuprous oxide found for the reducing power of the soluble starch by subtracting from it the weight obtained in a "blank" test in which the starch solution is treated directly with the Fehling reagent, heated, etc., exactly as in a regular determination.

From this corrected weight of cuprous oxide find the corresponding value of $K$ in the following table, and divide this value for $K$ by the weight in milligrams of the substance under examination (Note 6 ).

Table 11. - Values for $K$ from Cuprous Oxide found

\begin{tabular}{c|c||c|c||c|c||c|r}
\hline \hline $\begin{array}{c}\text { Cuprous } \\
\text { oxide } \\
\text { Mgms. }\end{array}$ & $\boldsymbol{K}$ & $\begin{array}{c}\text { Cuprous } \\
\text { oxide } \\
\text { Ngms. }\end{array}$ & $K$ & $\begin{array}{c}\text { Cuprous } \\
\text { oxide } \\
\text { Mgms. }\end{array}$ & $K$ & $\begin{array}{c}\text { Cuprous } \\
\text { oxide } \\
\text { Mgms. }\end{array}$ & $\boldsymbol{K}$ \\
\hline 30 & 9.1 & 100 & 31.2 & 170 & 54.1 & 240 & 78.3 \\
40 & 12.2 & 110 & 34.4 & 180 & 57.5 & 250 & 81.8 \\
50 & 15.3 & 120 & 37.6 & 190 & 60.9 & 260 & 85.4 \\
60 & 18.4 & 130 & 40.9 & 200 & 64.3 & 270 & 89.0 \\
70 & 21.6 & 140 & 44.2 & 210 & 67.8 & 280 & 92.6 \\
80 & 24.8 & 150 & 47.5 & 220 & 71.3 & 290 & 96.3 \\
90 & 28.0 & 160 & 50.8 & 230 & 74.8 & 300 & 100.0 \\
\hline \hline
\end{tabular}

Example. - Suppose a flask which had received a solution corresponding to $1.2 \mathrm{mgm}$. of substance shows $284.2 \mathrm{mgm}$. cuprous oxide and the "blank" for the soluble starch is 60.2 mgm. Then find the value of $K$ corresponding to $284.2-$ 60.2 or $224 \mathrm{mgm}$. cuprous oxide. This is 72.7 , which when divided by 1.2 (the weight of substance acting) gives a diastatic power of 66 (new scale). 
Note 1. - In making the solutions of starch and of enzyme the purest obtainable water should be used. To this. water should be added the necessary "activating agents" to enable the enzyme to exert its normal action. In testing pancreatic amylase $300 \mathrm{mg}$. sodium chloride and $7 \mathrm{cc}$. fiftieth-molar disodium phosphate are added to $100 \mathrm{cc}$. of water used as solvent both in making up the starch solution and for dissolving the enzyme.

Note 2. - The amount of sample required will vary greatly with the nature of the material. In testing malts a volume of extract corresponding to several milligrams of sample may be required; in testing commercial diastatic preparations, from a fraction of a milligram to a few milligrams; in testing highly purified preparations a volume of solution corresponding to only a few hundredths of a milligram of the substance may be required.

Note 3. - A temperature of $40^{\circ} \mathrm{C}$. is suitable for enzymes of animal origin, and as those of vegetable origin have heretofore been tested at various temperatures from $21^{\circ}$ to $60^{\circ} \mathrm{C}$. it seems very desirable that a uniform temperature of $40^{\circ}$ be adopted for all testing of amylases, notwithstanding the fact that they will usually be found to work more rapidly at a higher temperature. In general, results obtained by different observers working at different temperatures cannot properly be compared.

Note 4. - The regulation of temperature is very important, as a difference of $1^{\circ} \mathrm{C}$. (throughout the time of digestion) under the optimum conditions here described for pancreatic amylase affects the result about 10 per cent. It is well to stand the flasks in a water bath containing a thermometer and place the whole in an air bath. The importance of accurate regulation of the time is obvious. Should the time of action of the enzyme be other than 30 minutes, the result need not be rejected, but the value found for $K$ below must be multiplied by 30 and divided by the actual time in minutes, before dividing by the weight of enzyme to find the diastatic power.

Note 5. - Fehling solution as described under Fehling's or Defren's method in Chapter III is used. The addition of this 
to each solution should be accurately timed so that the time of action of the enzyme on the starch shall be exactly known in each case.

Note 6. - As is usual in such cases the rate of amylolytic action decreases slightly as the conversion of starch into maltose proceeds, so that weights of maltose or of cuprous oxide cannot be taken as showing directly the true diastatic power, but if the velocity curve of the amylolytic action be plotted with time as abscissas and yield of reducing sugar as ordinates a scale may be established which will permit of an expression of true diastatic power based upon the weight of cuprous oxide obtained, as above described. If $300 \mathrm{mg}$. of cuprous oxide be taken as 100 on such a scale, the value on this scale of any lesser weight of cuprous oxide is obtained by expressing the abscissa corresponding to such weight as percentage of the abscissa for 300 mgm. cuprous oxide. The values thus obtained are given as "values for $K$ " in the table.

\section{UNIFICATION OF METHODS FOR DETERMINING AND expressing Diastatic Power}

The data at present available are not sufficient to warrant the proposal of any one method as being the most accurate and the most logical for all cases. It remains to be determined whether the power of forming reducing sugars (saccharifying power) and the power of converting starch into products which do not give a blue color with iodine ("liquefying" power) run parallel as between amylases of different origins. If not, it may well be that the use for which the amylase is intended will influence the choice of method. When the conditions governing the saccharifying power of the different amylases, and the products of their action, have been more fully determined, it is hoped that a simple mode of expressing diastatic power in terms of a ratio of the weight of active substance to starch converted or to reducing sugar formed under standard conditions may be found feasible for general use.

In the meantime if those having occasion to determine and 
record diastatic powers will use one of the methods above described, the comparison of results obtained by different workers will be greatly facilitated and all results will thereby gain in value.

\section{REFERENCES}

Abderhalden: Biochemisches Arbeitsmethoden.

Allen: Commercial Organic Analysis.

Brows: Laboratory Studies for Brewing Students.

EFfront: Enzymes and their Applications.

LUNGE: Chemisch-technisch Untersuchungsmethoden.

Oppenhermer: Die Fermente und ihre Wirkungen.

Post : Chemisch-technische Analyse.

VAUBeL: Quantitative Bestimmung Organischer Verbindungen.

Wiley: Agricultural Analysis.

\section{II}

\section{(On the Determination of Starch)}

1888. Winton: J. Anal. Appl. Chem., 2, 153.

1895. Ost : Chem. Ztg., 19, 1501.

1896. Shermax: School of Mines Quarterly, 17, 356.

1898. Wiley and Krug : J. Am. Chem. Soc., 20, 253, 266.

1904. Witte: Z. Nahr. Genussm., 7, 65.

1907. Lintner : Z. Nahr. Genussm., 14, 205, and Z. Ges. Brauw., 30, 109.

Parow and Neumann: $Z$. Spiritusind., 30, 561.

1908. Dubors: (Cocoa products). U. S. Dept. Agriculture, Bur. Chem., Bul. 122, p. 214.

Heide: (Potatoes). Chem. Ztg., 31, 398.

1909. ThorNe and JeFFers: J. Soc. Chem. Ind., 28, 731.

\section{(On Amylases and the Determination of Diastatic Power)}

1880. KJeldahl : (First systematic study of method). Dingler's polyt. J., 235, 379, 452 .

1886. Lintwer: (Preparation of amylase and method for diastatic power). J. prakt. Chem., [2], 34, 378.

1895-96. Osborne: (Malt Amylase). J. Am. Chem. Soc., 17, 587 ; 18, 536. 1896. Sykes and Mitchell: (Method). Analyst, 21, 122.

1898. Francis: (Method). Bulletin of Pharmacy, 12, 52.

Takamine: (Method). J. Soc. Chem. Ind., 17, 118, 437. 
1904. ForD: (Details of method and preparation of soluble starch). $J$. Soc. Chem. Ind., 23, 414.

1907. Wohlgemuth : (Method). Biochem. Z., 9, 1.

1908. Johnson: (Method). J. Am. Chem. Soc., 30, 798.

Jones: (Discussion of Lintner Method). J. Inst. Brew., 14, 13; Chem. Abs., 2, 1592.

Lintner and Wirth: (Method). Z. ges. Brauw., 31, 421; Chem. $A b s ., 2,3257$.

1910. Sherman, Kendall, and Clark: (Methods). J. Am. Chem. Soc., 32, 1073.

Kendall and Sherman: (Pancreatic Amylase). J. Am. Cihem. Soc., 32, 1087.

1911. Sherman and Schlesinger: (Pancreatic Amylase). J. Am. Chem. Soc., 33, 1195. 


\section{CHAPTER VI \\ Vinegar and Acetate}

VINEGAR

THE principal varieties of vinegar have been defined and standardized by the U. S. Department of Agriculture as follows :

Vinegar, cider vinegar, apple vinegar, is the product made by the alcoholic and subsequent acetous fermentations of the juice of apples, is levorotatory, and contains not less than 4 grams of acetic acid, not less than 1.6 grams of apple solids, of which not more than 50 per cent are reducing sugars, and not less than 0.25 gram of apple ash in $100 \mathrm{cc} .\left(20^{\circ} \mathrm{C}\right.$. $)$; and the watersoluble ash from $100 \mathrm{cc} .\left(20^{\circ} \mathrm{C}\right.$.) of the vinegar contains not less than $10 \mathrm{mg}$. of phosphoric acid $\left(\mathrm{P}_{2} \mathrm{O}_{5}\right)$, and requires not less than $30 \mathrm{cc}$. of decinormal acid to neutralize its alkalinity.

Wine vinegar, grape vinegar, is the product made by the alcoholic and subsequent acetous fermentations of the juice of grapes, and contains in $100 \mathrm{cc}$. $\left(20^{\circ} \mathrm{C}\right.$.) not less than 4 grams of acetic acid, not less than 1.0 gram of grape solids, and not less than 0.13 gram of grape ash.

Malt vinegar is the product made by the alcoholic and subsequent acetous fermentations, without distillation, of an infusion of barley malt, or cereals whose starch has been converted by malt, is dextrorotatory, and contains in $100 \mathrm{cc} .\left(20^{\circ} \mathrm{C}\right.$. $)$ not less than 4 grams of acetic acid, not less than 2 grams of solids, and not less than 0.2 gram of ash; and the water soluble ash from $100 \mathrm{cc} .\left(20^{\circ} \mathrm{C}\right.$.) of the vinegar contains not less than $9 \mathrm{mg}$. 
of phosphoric acid $\left(\mathrm{P}_{2} \mathrm{O}_{5}\right)$, and requires not less than 4 cc. of decinormal acid to neutralize its alkalinity.

Sugar vinegar is the product made by the alcoholic and subsequent acetous fermentations of solutions of sugar, syrup, molasses, or refiner's syrup, and contains in $100 \mathrm{cc} .\left(20^{\circ} \mathrm{C}\right.$.) not less than 4 grams of acetic acid.

Glucose vinegar is the product made by the alcoholic and subsequent acetous fermentations of solutions of starch sugar or glucose, is dextrorotatory, and contains in $100 \mathrm{cc} .\left(20^{\circ} \mathrm{C}\right.$.) not less than 4 grams of acetic acid.

Spirit vinegar, distilled vinegar, grain vinegar, is the product made by the acetous fermentation of dilute distilled alcohol and contains in $100 \mathrm{cc}$. $\left(20^{\circ} \mathrm{C}\right.$.) not less than 4 grams of acetic acid.

Vinegar of any of these varieties may contain as an adulterant, or substitute, acetic acid made by refining the product of the dry distillation of wood and which is sometimes known as wood vinegar. This wood vinegar or pyroligneous acid if not well refined may be recognized by the tarry taste and odor and identified chemically by a color reaction for furfural (see qualitative test for pentoses, Chapter III). When well refined, the dilute solution of commercial acetic acid cannot be recognized in vinegar by any characteristic of its own, but only by its effect in lowering the amounts of solids, ash, etc., which characterize the pure vinegars of the different varieties.

\section{Determination of Source}

The principal vinegar of the United States is cider vinegar, although considerable quantities of malt vinegar are also used. The substitutes are made mainly of spirit vinegar, sugarhouse or glucose wastes, and wood vinegar from acetate of lime. Cheap apple jelly is sometimes added to the substitutes to give them the color, flavor, and body of cider vinegar.

Cider vinegar contains from 1.2 to 8 per cent of solids, average about 2.5 per cent; malt vinegar 1.75 to 6 , average about 3 per cent; spirit vinegar rarely over 0.75 per cent, average about 0.30 per cent. 
The total ash in spirit and wood vinegars rarely exceeds 0.1 per cent. In fruit and malt vinegars it rarely falls below 0.2 , cider vinegar averaging about 0.35 per cent.

The alkalinity of the ash, expressed as above, is, according to Frear, for cider vinegar 26 to 65 , average 39 ; for malt vinegar, 5.5 ; for spirit vinegar, 1.1 .

The flame reaction of the solids is said to be always that of potash in the case of cider vinegar, while spirit, sugar, and glucose vinegars and any which have been artificially colored show the sodium flame (Davenport-Frear).

The optical activity of vinegar often indicates the source. Pure cider vinegar, after clarification with basic lead acetate, is levorotatory, a $200-\mathrm{mm}$. tube giving usually a reading of $-0.5^{\circ}$ to $-1.4^{\circ}$ on the Ventzke scale. According to Browne, levulose is the only sugar present in properly fermented cider vinegar, the sucrose and dextrose having both disappeared in the alcoholic fermentation. Wine vinegar is also slightly levorotatory. Vinegar from sugarhouse wastes is dextrorotatory before, and levorotatory after, hydrolysis. Glucose vinegar shows dextrorotation both before and after hydrolysis. Artificial vinegars can, of course, be made levorotatory by the addition of apple juice or cider.

It has been supposed that the presence of malic acid should be one of the characteristics of genuine cider vinegar, but the work of Van Slyke and others has shown that malic acid of apple juice is largely, if not wholly, destroyed during the alcoholic and acetous fermentations, so that tests for or determinations of malic acid are of little if any value in the examination of vinegar as to its source.

Balcom finds that determination of the nonsugar solids by subtracting the total sugar from the total solids is an important aid in the judgment of vinegars. Table 12 and the explanations which follow are based on Balcom's report published in $1910 .{ }^{1}$

1 U. S. Dept. Agriculture, Bur. Chem., Bul. 132. 
Table 12. - Comparison of Analyses of Vinegars of Known Characters with Commercial Samples and with Average Data (Balcom).

[Grams per $100 \mathrm{cc}$.

\begin{tabular}{|c|c|c|c|c|c|c|c|c|c|c|c|c|}
\hline \multirow{2}{*}{\multicolumn{2}{|c|}{ No. }} & \multirow{2}{*}{$\begin{array}{c}\text { Total } \\
\text { acid } \\
\text { as } \\
\text { acetic }\end{array}$} & \multirow{2}{*}{$\begin{array}{l}\text { Total } \\
\text { solids }\end{array}$} & \multirow{2}{*}{$\begin{array}{l}\text { Non- } \\
\text { sugar } \\
\text { solids }\end{array}$} & \multirow{2}{*}{$\begin{array}{l}\text { Reduc- } \\
\text { ing } \\
\text { sugars } \\
\text { in } \\
\text { solids }\end{array}$} & \multirow{2}{*}{$\begin{array}{c}\text { Total } \\
\text { ash }\end{array}$} & \multirow{2}{*}{$\begin{array}{c}\text { Alka- } \\
\text { linity } \\
\text { of } \\
\text { water- } \\
\text { soluble } \\
\text { ash }\end{array}$} & \multirow{2}{*}{$\begin{array}{l}\text { Ash } \\
\text { in } \\
\text { non- } \\
\text { sugar } \\
\text { solids }\end{array}$} & \multicolumn{3}{|c|}{$\begin{array}{l}\text { Phosphoric acid } \\
\qquad\left(\mathrm{P}_{2} \mathrm{O}_{5}\right)\end{array}$} & \multirow{2}{*}{$\begin{array}{l}\text { Polari- } \\
\text { zation } \\
\text { (direct) }\end{array}$} \\
\hline & & & & & & & & & $\begin{array}{c}\text { Solu- } \\
\text { ble }\end{array}$ & $\begin{array}{c}\text { Insolu- } \\
\text { ble }\end{array}$ & Total & \\
\hline \multirow{3}{*}{$1^{1}$} & & 4.94 & 2.54 & 1.90 & $\begin{array}{c}\text { Per ct. } \\
19.6\end{array}$ & 0.367 & $\begin{array}{c}c c . \\
35.7\end{array}$ & $\begin{array}{c}\text { Per ct. } \\
18.8\end{array}$ & 17.3 & 12.0 & 29.3 & $\begin{array}{c}V \\
-1.46\end{array}$ \\
\hline & . & 7.96 & 4.52 & 2.89 & 45.0 & 0.52 & 56.0 & 26.5 & 39.9 & 32.0 & 64.2 & -3.6 \\
\hline & & 3.29 & 1.37 & 1.26 & 5.6 & 0.20 & 21.5 & 11.2 & 6.7 & 4.3 & 15.1 & -0.2 \\
\hline 2 & . & 4.65 & 2.40 & 1.51 & 37.2 & 0.32 & 31.9 & 21.2 & 12.4 & 10.5 & 22.9 & -1.3 \\
\hline 3 & . & 4.31 & 0.18 & 0.16 & 11.1 & 0.016 & 1.5 & 10.0 & 0.2 & 1.5 & 1.7 & +0.6 \\
\hline 4 & . & 4.51 & 1.27 & 0.80 & 37.0 & 0.20 & 17.5 & 25.0 & 5.5 & 7.4 & 12.9 & -0.3 \\
\hline 5 & . & 4.72 & 2.15 & 1.05 & 51.2 & 0.28 & 37.0 & 26.7 & 8.7 & 10.4 & 19.1 & - \\
\hline 6 & . & 4.46 & 2.11 & 0.91 & 56.9 & 0.29 & 33.0 & 31.9 & 11.5 & 10.8 & 22.3 & -1.0 \\
\hline 7 & . & 4.66 & 2.09 & 1.73 & 17.0 & 0.56 & 11.1 & 32.4 & 1.8 & 15.2 & 17.0 & \pm 0.0 \\
\hline
\end{tabular}

No. 1 is the normal basis for comparison established by the compilation of about 100 analyses of cider vinegars of known purity. These analyses were made by different chemists and represent vinegars from different parts of the country, although the greater number were of vinegars made from apples grown in New England.

No. 2 was a blend of about 50 commercial samples which from analysis were passed as pure, but some of which were regarded as not above suspicion.

No. 3 was an uncolored spirit vinegar. (Commercial spirit vinegars are frequently colored with caramel.)

No. 4 was a mixture of equal volumes of Nos. 2 and 3. Note the differences in analytical data between this sample and No. 2 or No. 1.

No. 5 was a known mixture of cider vinegar, spirit vinegar, and boiled cider, the latter bringing up the total solids, etc. Note the low value of the nonsugar solids considered in connection with the abnormally high percentage of sugar in the total solids.

1 Average, maximum, and mininum data on about 100 vinegar samples. 
No. 6 was a commercial sample whose analysis, as will be seen, shows it to have been of the same character as No. 5 . The analysis of No. 6 is given by Balcom as typical of a large number of vinegars found on the market at the present time and which are regarded as mixtures of cider vinegar and dilute acetic acid, the latter, perhaps, in the form of spirit vinegar, to which has been added some foreign material high in sugar. Whether this material is unfermented apple juice, boiled cider, apple jelly, or some other material is difficult, if not impossible, to determine with certainty. In most cases it is believed to be either unfermented apple juice or boiled cider.

No. 7 is a sugar vinegar made from New Orleans molasses and is supposed to be fairly representative of this kind of vinegar which is being introduced to an increasing extent to take the place of the cheap artificially colored spirit vinegar. It will be noted that the analytical data of the sugar vinegar show considerable resemblance to that of a cider vinegar, the most noticeable differences being a relatively higher ash or lower alkalinity of the water-soluble ash, low water-soluble phosphoric acid, and usually, though not necessarily, a difference in behavior toward polarized light. The latter property will, of course, depend upon the nature of the residual sugar, as determined by the composition of the sugar syrup or molasses fermented.

\section{Methods of Analysis}

A thorough examination of vinegar should include determinations of specific gravity, total solids or extract, total and soluble ash, alkalinity and phosphoric acid of the ash, sugars, polarization, total and volatile acidity, and alcohol. Free mineral acids should be determined if present.

Methods for all of these determinations have been adopted by the Association of Official Agricultural Chemists. The following directions for the more important determinations are in accordance with the official methods. It is not necessary to repeat here the methods for alcohol, sugars, and for polarization. 


\section{Total Solids or Extract}

Evaporate $10 \mathrm{cc}$. nearly to dryness in a weighed platinum dish of $50 \mathrm{~mm}$. diameter on a steam bath, dry for two and one half hours in a boiling water oven, cool thoroughly in a desiccator and then weigh quickly.

\section{Total Ash}

Char the extract thoroughly at a low red heat, leach with water, burn the insoluble residue to whiteness, add the water solution, evaporate and heat to low redness, cool in a desiccator and weigh.

\section{Solubility and Alkalinity of Ash ${ }^{1}$}

Evaporate 25 cc. to dryness, burn, cool, and weigh; extract the ash repeatedly on an ash-free filter; dry and ignite the filter and residue, and weigh as insoluble ash. Titrate the filtrate, using methyl orange as indicator, and calculate the number of cubic centimeters of tenth-normal acid which would be required to neutralize the corresponding filtrate from 100 cc. of the vinegar.

\section{Total Acidity}

Dilute $10 \mathrm{cc}$. in a beaker until the solution appears very light-colored against a white background, add phenolphthalein and titrate with standard sodium hydroxide. If only the total acidity is determined, the result is expressed as acetic acid.

\section{Volatile Acids}

Heat 15 cc. of the vinegar to boiling in a flask, adding a little tannin to check foaming, if necessary; lower the flame and distill with steam until the distillate no longer contains acid. Titrate the distillate with standard sodium hydroxide and calculate as acetic acid.

The difference between the total acidity and that due to volatile acids gives a measure of the fixed acids and, in the case of cider vinegar, is calculated as malic acid.

1 Smith's method modified by Frear: J. Am. Chem. Soc., 18ะ8, 20, 5. 


\section{Detection of Free Mineral Acid}

Dilute 5 cc. of the vinegar with 5 to $10 \mathrm{cc}$. water to reduce the acidity to about 2 per cent of acetic acid, add four or five drops of an aqueous solution of methyl violet (one part of "methyl violet 2 B" - No. 56 of Bayer Farbenfabrik, Elberfeld - in 10,000 parts of water). Mineral acids change the blue violet color to a blue green or green.

\section{Determination of Free Mineral Acid-Hehner's Method}

To a weighed quantity of the sample, add a measured amount (more than sufficient to neutralize all mineral acid present) of tenth-normal alkali; evaporate to dryness, incinerate, and titrate the ash with tenth-normal acid, using methyl orange as indicator. The difference between the volume of alkali added and that of acid required represents the equivalent of the free mineral acid in the sample.

\section{ACETIC ACID AND ACETATE}

Pure acetic acid is a colorless liquid of 1.056 specific gravity, miscible in all proportions with water, alcohol, and ether. When nearly anhydrous it solidifies at about $16^{\circ}$ - hence the term "glacial" as applied to very strong acetic acid. The pure acid boils without decomposition at $119^{\circ}$. From dilute aqueous solutions it distills readily with steam at the temperature of boiling water. The principal source of commercial acetic acid is the crude acetate of lime made by neutralizing the acid obtained in the dry distillation of wood. For the determination of the volatile acid obtainable from commercial acetate the following method can be used.

\section{Determination of Acetic Acid in Calciom Acetate}

Arrange a round-bottomed flask of $300 \mathrm{cc}$. capacity in such a way that it can be inclined at an angle of about $60^{\circ}$ from the perpendicular and connected with a nearly vertical condenser, while another tube passing through the stopper of the flask provides for the continuous introduction of water, drop by 
drop, during the distillation. The flow of water can be controlled by means of a screw pinchcock or a small dropping funnel.

Weigh 2 grams of the ground sample, ${ }^{1}$ transfer to the flask, and add $15 \mathrm{cc}$. of 50 per cent phosphoric acid and $25 \mathrm{cc}$. of water, being sure that the water washes down any of the sample or acid which may have adhered to the neck of the flask. Connect the flask with the condenser and distill, collecting the distillate in a receiver containing $50 \mathrm{cc}$. of water to prevent loss due to evaporation of acetic acid. During the distillation keep the volume of liquid nearly constant at $40 \mathrm{cc}$. by admitting water free from carbon dioxide, in such a way that the drops fall upon the inner surface of the neck of the flask and not directly into the boiling liquid. Continue the distillation until the distillate is no longer acid. This usually requires about one and one half hours. Titrate the distillate with freshly standardized sodium hydroxide solution, using phenolphthalein as indicator. Calculate the total acidity as percentage of acetic acid in the sample.

Notes. - It has been found that small amounts of phosphoric acid are frequently carried over mechanically if the acetic acid is removed by a current of steam or if the distillation is conducted in an upright flask, especially when drops of water are allowed to fall directly into the boiling acid mixture. The directions for arrangement of apparatus are intended to avoid this source of error.

The phosphoric acid used must not contain nitric or any other volatile acid. The large excess recommended dissolves the calcium phosphate formed and thus prevents bumping. Oxalic may be substituted for phosphoric acid, and the calcium oxalate removed by filtration before distilling. This method, however, is longer and no more accurate than the phosphoric acid method as described. Sulphuric acid cannot be used, as it would be partially reduced to sulphurous acid by the tarry matter present in the crude acetate.

${ }^{1}$ In sampling, grinding, and weighing portions for analysis, special care must be taken to avoid changes in moisture content. 
The distillate obtained as described should contain only a minute amount of carbonic acid. It is frequently recommended that the distillate be caught in standard alkali nearly sufficient to neutralize all the volatile acid expected. In this case the distillate would contain all carbonic acid liberated in the distillation. The distillate containing some free acetic acid should therefore be boiled under a reflux condenser to expel carbonic acid before making the final titration. Errors may also be caused by variation in the amount of carbonate in the alkali used for titration. Hence this must be freshly standardized, using phenolphthalein as indicator, under the same conditions of temperature and removal of carbon dioxide as exist in the titration of the distillate.

The volatile organic acid, though calculated as acetic, always contains some formic, propionic, and butyric acids. In addition to these Scheuer found small amounts of valerianic, caproic, heptylic, caprylic, and nonylic acids. In view of the danger of phosphoric acid being carried over, it is advisable, after titrating the distillate, to add nitric acid, evaporate to $25 \mathrm{cc}$., and test for phosphoric acid by adding ammonium nitrate and molybdate solution.

The distillation method has now almost entirely replaced the indirect methods formerly used. There is, however, no general agreement as to the details of manipulation. A full description of the method as used in the laboratory of the General Chemical Company is given by Grosvenor.

\section{REFERENCES}

I

Allen : Commercial Organic Analysis.

Brannt: Treatise of the Manufacture of Vinegar.

LEACH : Food Inspection and Analysis.

LUNGE: Chemisch-technische Untersuchungsmethoden.

Post : Chemisch-technische Analyse.

Sutton: Volumetric Analysis.

U. S. Dept. Agriculture, Bur. Chem., Bul. 107. 
1899. Crampton and Simons: Detection of Caramel in Spirits and Vinegar. J. Am. Chem. Soc., 21, 16.

1900. Doolittle and Hess: Cider Vinegar, its Solids and Ash. J. Am. Chem. Soc., 22, 218.

1901. Browne: The Apple and Some of its Products. J. Am. Chem. Soc., 23, 869.

1902. Frear: Vinegar. U. S. Dept. Agriculture, Bur. Chem., Bul. 65.

ScheUER: Analyse von Graukalk (Calcium Acetate). Dissertation. Munich.

1903. Browne: Effects of Fermentation upon the Composition of Cider and Vinegar. J. Am. Chem. Soc., 25, 16.

1904. Grosvenor: Analysis of Commercial Acetate of Lime. J. Soc. Chem. Ind., 23, 530.

LeACH and Lxtugoe: Cider Vinegar and Suggested Standards of Purity. J. Am. Chem. Soc., 26, 375.

Stillwell : Acetic Acid (Determination) in Acetate of Lime. $J$. Soc. Chem. Ind., 23, 305.

VAN Slyke: A Study of the Chemistry of Home-made Cider Vinegar. Bul. 258, New York State Agricultural Experiment Station.

1907. Dubors: The Fuller's Earth Test for Caramel in Vinegar. J. Am. Chem. Soc., 29, 75.

RatclifF: The Composition of English Fermentation Vinegars. Analyst, 32, 85.

Woodman and Shingler: The Composition of American Malt Vinegar. Technology Quarterly, 19, 404.

1909. Barker and Russel: The Composition of Cider. Analyst, 34, 125.

Gladding: The Analysis of Commercial Acetate of Lime. J. Ind. Eng. Chem., 1, 250.

1910-11. Balcom: Reports on Vinegar. U. S. Dept. Agriculture, Bur. Chem., Bul. 132, p. 93, and Bul. 137, p. 57.

1911. Mотт : Cider Vinegar. J. Ind. Eng. Chem., 3, 747.

Ross: Determination of Glycerol in Vinegar and Characteristic Vinegar Ratios. U. S. Dept. Agriculture, Bur. Chem., Bul. 137, p, 61 .

U. S. Dept. Agriculture, Office of the Secretary, Notice of Judgment No. 1159. Adulteration and Misbranding of Vinegar. 


\section{CHAPTER VII}

\section{Fatty Acids}

WITH a few possible exceptions the fatty acids are all monobasic and those of the common fats all contain an even number of carbon atoms in the molecule. Fatty acids containing odd numbers of carbon atoms are not widely distributed in nature, being usually characteristic of some particular fat or small group of fats. The following statement of analytical properties is based mainly upon the data given by Lewkowitsch.

\section{Acids of the Series $\mathrm{C}_{n} \mathrm{H}_{2 n} \mathrm{O}_{2}$}

Butyric acid, $\mathrm{C}_{4} \mathrm{H}_{8} \mathrm{O}_{2}$, occurs ${ }^{1}$ in butter and in very small quantity in a few other fats. It is a mobile liquid, mixing in all proportions with water, alcohol, and ether. It boils without decomposition at $162^{\circ}$ and is readily volatile with steam. Specific gravity at $20^{\circ}, 0.959$.

Caproic acid, $\mathrm{C}_{6} \mathrm{H}_{12} \mathrm{O}_{2}$, occurs in butter and coconut fat. It is a rather oily liquid of unpleasant odor, not miscible with water, but somewhat soluble in it, and volatile with steam. Boiling point about $200^{\circ}$. Specific gravity at $20^{\circ}, 0.924$ :

Caprylic acid, $\mathrm{C}_{8} \mathrm{H}_{16} \mathrm{O}_{2}$, occurs in coconut oil, butter, and human fat. In the cold it crystallizes in leaflets which melt at $16.5^{\circ}$. It boils at $237^{\circ}$ and is volatile with steam. One part dissolves. in 400 parts of boiling water, from which it separates almost completely on cooling. It is readily soluble in alcohol and ether. Specific gravity at $20^{\circ}, 0.910$.

Capric acid, $\mathrm{C}_{10} \mathrm{H}_{20} \mathrm{O}_{2}$, has been found chiefly in coconut oil, butter, and the fat of the spice bush, Lindera benzoin. It melts

1 These statements refer to the occurrence of the acids as esters rather than in the free state. 
at $31.3^{\circ}$, boils at $270^{\circ}$, and distills with steam. Soluble in about 1000 parts of boiling water; almost insoluble in cold water; soluble in alcohol and ether.

Lauric acid, $\mathrm{C}_{12} \mathrm{H}_{24} \mathrm{O}_{2}$, occurs abundantly in the fat of the seeds of Lindera benzoin, and in smaller proportions in coconut fat, palm oil, laurel oil, butter, and the nut oil of the California bay tree. Lauric acid melts at $43.6^{\circ}$ and cannot be distilled at atmospheric pressure without decomposition. It is practically insoluble in cold water, slightly soluble in boiling water, and appreciably volatile with steam. Specific gravity at $20^{\circ}, 0.883$.

Myristic acid, $\mathrm{C}_{14} \mathrm{H}_{28} \mathrm{O}_{2}$, is found in nutmeg butter, dika fat, butter, coconut oil, lard, and many other fats, as well as in spermaceti and wool wax. It crystallizes in leaflets which melt at $53.8^{\circ}$. Myristic acid is insoluble in water and only very slightly volatile with steam. It is not readily soluble in cold alcohol.

Palmitic acid, $\mathrm{C}_{16} \mathrm{H}_{32} \mathrm{O}_{2}$, occurs in nearly all solid fats and non-drying oils as well as in several waxes including spermaceti and beeswax. It melts at $62.6^{\circ}$ and solidifies on cooling to a scaly crystalline mass. It is insoluble in water and not readily soluble in cold 95 per cent alcohol, but dissolves in about 10 parts of cold absolute alcohol.

Stearic acid, $\mathrm{C}_{18} \mathrm{H}_{36} \mathrm{O}_{2}$, is found in most fats and occurs most abundantly in those having high melting points. It crystallizes in leaflets which melt at $69.3^{\circ}$. It is less soluble in alcohol than palmitic acid, requiring about 40 parts of absolute alcohol in the cold. It dissolves readily in ether, benzol, carbon bisulphide, or hot alcohol.

Arachidic acid, $\mathrm{C}_{20} \mathrm{H}_{40} \mathrm{O}_{2}$, occurs in arachis (peanut) oil, and has been obtained in very small quantities from several other fats. Arachidic acid is distinguished from all its lower homologues by its insolubility in cold alcohol. It dissolves freely in hot alcohol, but on cooling separates almost completely as needles or crystalline scales, which melt at $77^{\circ}$ to $78^{\circ}$.

Behenic acid, $\mathrm{C}_{22} \mathrm{H}_{44} \mathrm{O}_{2}$, lignoceric acid, $\mathrm{C}_{24} \mathrm{H}_{48} \mathrm{O}_{2}$, and an isomer of the latter, carnaiubic acid, occur respectively in oil of ben, arachis oil, and carnaüba wax. 
Cerotic acid, $\mathrm{C}_{26} \mathrm{H}_{52} \mathrm{O}_{2}$, and melissic acid, $\mathrm{C}_{30} \mathrm{H}_{60} \mathrm{O}_{2}$, are found in the free state in beeswax.

The change in properties of these acids, as the molecular weight increases, is noticeably regular. The melting and boiling points rise while the specific gravities and solubilities decrease. In general the properties of the glycerides and other esters vary in the same way as those of the free acids.

With the exception of caproic, all of the acids of this series, occurring in natural fats, are believed to be of the "normal" (straight chain) structure.

\section{Acids of the Series $\mathrm{C}_{n} \mathrm{H}_{2 n-2} \mathrm{O}_{2}$}

These acids are unsaturated. Each molecule contains one ethylene linkage or double bond, and is, therefore, capable of taking up by direct addition two atoms of halogen or one molecule of hydrobromic acid to form a saturated compound. Careful oxidation in the presence of moisture results in the formation of the corresponding dihydroxy compounds. The unsaturated acids have, as a rule, lower melting points than the saturated acids containing the same number of carbon atoms, and are therefore found more largely in oils and soft fats. Only the more important members of the series will be considered here.

Hypogceic acid, $\mathrm{C}_{16} \mathrm{H}_{30} \mathrm{O}_{2}$, obtained from arachis (peanut) oil, melts at $33^{\circ}$, but is converted by the action of nitrous acid into the isomeric gaidic acid which melts at $39^{\circ}$. Phycetoleic acid, isomeric with hypogæic, is obtained from sperm oil and seal oil. It melts at $30^{\circ}$ and is not changed by nitrous acid.

Oleic acid, $\mathrm{C}_{18} \mathrm{H}_{34} \mathrm{C}_{2}$, occurs in nearly all fats and fatty oils. It is an oily liquid which solidifies at $4^{\circ}$ and melts at $14^{\circ}$. Nitrous acid converts oleic acid into the isomeric elaidic acid, which is a crystalline solid melting at $44.5^{\circ}$ (Lewkowitsch).

Erucic acid, $\mathrm{C}_{22} \mathrm{H}_{42} \mathrm{O}_{2}$, found in rape seed and mustard seed oils, melts at $33^{\circ}$ to $34^{\circ}$. By the action of nitrous or sulphurous acid it can be converted into brassidic acid, melting at $65^{\circ}$. This change, however, takes place less readily than the corresponding conversion of oleic acid into elaidic acid. 
The gradual change in properties with increasing molecular weight, noticed in the saturated acids, is not apparent in this series, doubtless because the known acids of the series differ as regards the position of the double bond and are therefore not strictly similar in constitution. The property of changing to solid isomers under the influence of nitrous acid is characteristic of the acids of this series and furnishes a means of testing for them in fatty oils. Any of the latter which contain large amounts of these acids become solid or semisolid on treatment with nitrous acid. This is known as the elaidin test, and is sometimes useful in the preliminary examination of oils, but has been shown to be less reliable than was formerly supposed.

An important characteristic of the unsaturated acids is the solubility of the lead soaps in ether. Muter's method for the separation of saturated and unsaturated fatty acids is to precipitate the mixture of acids as lead soaps and wash with ether. While this method is not strictly quantitative, it is probably the best which has been devised for the purpose.

\section{Acids of the Series $\mathrm{C}_{n} \mathrm{H}_{2^{n-4}} \mathrm{O}_{2}$}

The acids of this series unite with four atoms of bromine or iodine and absorb oxygen on exposure to the air.

Linoleic acid, $\mathrm{C}_{18} \mathrm{H}_{32} \mathrm{O}_{2}$, is the only important member of the series. It is widely distributed, occurring most abundantly in the "drying oils," so called because they are oxidized to solids on exposure to air. While especially characteristic of the drying and semidrying oils, linoleic acid is also found in nondrying oils and in small quantity in some of the solid fats. It absorbs oxygen from the air so readily as to interfere seriously with ordinary methods of purification and examination. Its physical properties are therefore not well known, but it has been shown to have a higher specific gravity than oleic acid and a much lower melting point since it does not solidify at $-18^{\circ}$. Linoleic acid is not changed by nitrous acid. Lead linoleate is soluble in ether. 


\section{Acids of the Series $\mathrm{C}_{n} \mathrm{H}_{2^{n-6}} \mathrm{O}_{2}$}

Linolenic acid, $\mathrm{C}_{18} \mathrm{H}_{30} \mathrm{O}_{2}$, is the only member of this series which has been obtained in the free state. It is a colorless oil which absorbs oxygen very rapidly from the air, at the same time becoming dark brown. The purest preparations of the acid which have been described absorbed 242 to 245 per cent of iodine. The pure acid should absorb six atoms or 274 per cent of iodine. Linolenic acid has not been solidified. Its specific gravity is greater than that of linoleic acid. The lead soap is soluble in ether. Linolenic acid occurs in considerable quantity in linseed oil. By oxidizing linseed oil acids with alkaline permanganate, Hazura obtained two acids of the formula $\mathrm{C}_{18} \mathrm{H}_{30}(\mathrm{OH})_{6} \mathrm{O}_{2}$, linusic and isolinusic acids, and hence inferred the presence of an isolinolenic acid in linseed oil. Jecoric acid, isomeric with linolenic acid, is reported by Fahrion ${ }^{1}$ as existing in sardine oil. The highly unsaturated acids of fish and marine animal oils are in general isomeric, rather than identical, with those of linseed oil.

\section{Acids of the Series $\mathrm{C}_{n} \mathrm{H}_{2 n-8} \mathrm{O}_{2}$}

Five acids have been reported as belonging to this series. The only one which need be mentioned here is clupanodonic acid, $\mathrm{C}_{18} \mathrm{H}_{28} \mathrm{O}_{2}$, occurring in Japanese sardine oil, herring oil, and whale and turtle oils.

\section{Saturated Hydroxy-Acids}

- When any of the unsaturated acids occurring in vegetable oils is carefully oxidized in alkaline solution by means of potassium permanganate, hydroxyl is added until the molecule becomes saturated. Thus oleic acid yields dihydroxystearic acid, $\mathrm{C}_{18} \mathrm{H}_{34}(\mathrm{OH})_{2} \mathrm{O}_{2}$, linoleic acid yields sativic acid, $\mathrm{C}_{18} \mathrm{H}_{32}(\mathrm{OH})_{4} \mathrm{O}_{2}$, and linolenic acid yields linusic acid, $\mathrm{C}_{18} \mathrm{H}_{30}(\mathrm{OH})_{6} \mathrm{O}_{2}$. Similar changes in the unsaturated acids may take place when their glycerides are exposed to the air. Small amounts of saturated hydroxy-acids are, therefore, likely

1 Chem. Ztg., 1893, 17, 521. 
to be present in fatty oils which have been kept for some time in partially filled bottles or otherwise exposed to atmospheric oxidation. Only two acids of this series have as yet been found in nature. Juillard ${ }^{1}$ found about 1 per cent of dihydroxystearic acid in castor oil and Browne ${ }^{2}$ the same quantity in butter fat. A dihydroxy-acid of the formula, $\mathrm{C}_{30} \mathrm{H}_{60} \mathrm{O}_{4}$, is said to occur in wool wax $^{3}$ and is called lanocerinic or lanoceric acid.

\section{Hydroxy-Acid of the Series $\mathrm{C}_{n} \mathrm{H}_{2 n-2} \mathrm{O}_{3}$}

Ricinoleic acid, $\mathrm{C}_{18} \mathrm{H}_{34} \mathrm{O}_{3}$, the only important member of this series, occurs in large quantity in castor oil. It is a very viscous liquid of much higher specific gravity than oleic or linoleic acid. According to Juillard ${ }^{4}$ the pure acid melts at $4^{\circ}-5^{\circ}$, and is miscible with alcohol and ether in all proportions. Ricinoleic acid is dextrorotatory. The position of the asynmetric carbon atom, as well as of the double bond, is shown by the following formula: ${ }^{5}$

$$
\mathrm{C}_{6} \mathrm{H}_{13} \cdot \mathrm{CH}(\mathrm{OH}) \cdot \mathrm{CH}_{2} \cdot \mathrm{CH}: \mathrm{CH} \cdot\left(\mathrm{CH}_{2}\right)_{7} \cdot \mathrm{COOH} \text {. }
$$

Ricinoleic resembles oleic acid in its chemical reactions. It absorbs two atoms of bromine or iodine. By treatment with permanganate in alkaline solution two hydroxyl radicles are added. By treatment with nitrous acid, ricinoleic acid is converted into the solid isomer, ricinelaidic acid. Lead ricinoleate is easily soluble in ether.

\section{Separation of Fatty Acids}

For the analytical separation of saturated from unsaturated fatty acids, Muter's method, based on the solubility of the lead soaps of the latter in ether, is generally used. In order to determine the constituents of a mixture of homologous acids

1 Bull. Soc. Chim., 1895, [3], 13, 238.

${ }^{2}$ J. Am. Chem. Soc., 1899, 21, 817.

3 Darmstaedter and Lifschütz: Ber., 1896, 29, 1476.

4 Bull. Soc. Chim., 1895, [3], 13, 240.

${ }^{5}$ Goldsobel : Ber., 1894, 27, 3121. Kasansky : J. prakt. Chem., 1900, 62, 363. 
it is usually necessary to fraction the mixture repeatedly either by distillation, precipitation, or crystallization until each fraction contains only two acids. The proportions of the two constituents can then be found by determining the mean molecular weight of the mixture. This is usually done in a manner similar to the determination of the saponification number or saponification equivalent as described in the next chapter.

\section{REFERENCES}

Allex : Commercial Organic Analysis.

Benedikt and Ulzer: Analyse der Fette und Wachsarten.

Leathes : The Fats.

Lewnowitsch: Chemical Technology and Analysis of the Oils, Fats, and Waxes.

Oppenheimer: Handbuch der Biochemie.

Ulzer and Klimont: Allgemeine und Physiologische Chemie der Fette.

II

1899. Browne: (Separation of the Acids of Butter Fat). J. Am. Chem. Soc., 21, 807.

1906. Haller : (Separation of Fatty Acids). Compt. rend., 143, 657.

1908. Dunham: Carnaübic Acid from Beef Kidneys. Proc. Soc. Exper. Biol. Med., 5, 58.

1909. Erdmand, Bedford and Raspe: Constitution of Linolenic Acid. Ber., 42, 1334.

HartLey: Nature of the Fat contained in the Liver, Kidney, and Heart. J. Physiol., 38, 353.

Rallett : (On Linoleic and Linolenic Acids). Z . physiol. Chem., 62, 410, 422.

Tolman: A Study of the Fatty Acids of Fish Oils. J. Ind. Eng. Chem., 1, 340.

1910. Rosauer: The Manufacture and Examination of Technical Oleic Acid. Chem. Rev. Fette-Harz-Ind., 18, 28, 43 ; Chem. Abs., 5, $1683,1846$.

1911. Holland : Purification of Insoluble Fatty Acids. J. Ind. Eng. Chem., 3, 171. 


\section{CHAPTER VIII}

\section{Oils, Fats, and Waxes-General Methods}

ALL the glycerides of fatty acids are known as fats. As a matter of convenience those fats which are liquid at ordinary temperatures are commonly called fatty oils. Fats and fatty oils, therefore, consist of a definite group of compounds, the glyceryl esters of the fatty acids. In any given natural fat a number of glycerides may be found, as is evident from the statements regarding sources of the various fatty acids in the last chapter.

Waxes are esters of fatty acids with monatomic alcohols of high molecular weight. Most waxes are solid at ordinary temperatures and the term is sometimes applied to other solids of similar physical properties, solid hydrocarbons, for example, being frequently called mineral waxes. Among the esters found in some of the common waxes are: cetyl palmitate in spermaceti; myricyl palmitate in beeswax; dodecatyl oleate in sperm oil.

The term "oil" has no strict chemical significance, being applied not only to liquid fats, but also to substances, such as mineral and essential oils, which are similar in some physical properties but entirely different in constitution. The mineral oils are conveniently treated in connection with the fatty oils in works on technical analysis, because on account of their similar physical properties they may for certain uses be mixed with, or substituted for, the fatty oils and are sometimes found as adulterants of the latter. The same is true of some of the essential oils, notably rosin oil and turpentine.

The methods given in this chapter are described and dis- 
cussed with special reference to the analysis of solid and liquid fats. In most cases, however, they are also applicable to waxes, non-fatty oils, and technical mixtures.

\section{CLASSIFICATION}

Lewkowitsch classifies chiefly according to capacity for absorbing iodine. "Drying" oils show high iodine absorption; "non-drying" oils, low; "semidrying" oils, intermediate.

In Allen's Commercial Organic Analysis the solid and liquid fats and waxes are classified in twelve groups essentially as follows :

I. Olive oil group - vegetable fatty oils consisting chiefly of olein, with smaller amounts of palmitin, stearin, and perhaps other saturated glycerides, and containing also, at least in some cases, linolein or similarly unsaturated glycerides in small amount.

II. Rape oil group - fatty oils from the seeds of the cruciferoe, characterized by containing erucin.

III. Cottonseed oil group (semidrying oils) - consisting largely of olein and linolein with small quantities of palmitin and stearin and traces of linolenin.

IV. Linseed oil group (drying oils) - differing in composition from group III in containing larger proportions of linolein and linolenin.

V. Castor oil group - in some at least of which (e.g. castor and grapeseed oils) ricinolein predominates.

VI. Cacao butter group - vegetable fats consisting mainly of olein, stearin, palmitin, and myristin, with perhaps small amounts of linolein and of the glycerides of saturated acids of lower molecular weight than myristic.

VII. Coconut'oil group - vegetable fats containing less olein than those of group VI and more of the glycerides of the saturated fatty acids of low molecular weight, laurin, etc.

VIII. Lard oil group - animal fatty oils consisting mainly of olein with smaller amounts of stearin and palmitin and probably some linolein (or isomeric glyceride).

IX. Tallow group - animal fats consisting in most cases chiefly of olein, stearin, and palmitin with perhaps a little of linolein 
(or an isomer). Butter differs from the other members of the group in containing butyrin, laurin, and myristin with smaller amounts of caproin, caprylin, and caprin, and very little stearin.

X. Whale (and fish) oil group - fatty oils of marine animals, including fish, consisting largely of glycerides of acids isomeric with linoleic and linolenic.

XI. Sperm oil group - liquid waxes.

XII. Spermaceti group - solid waxes of both animal and vegetable origin.

\section{PROPERTIES OF FATS AND FATTY OILS}

Refined fats and fatty oils are usually light yellow to colorless. Vegetable oils are sometimes tinged green by the presence of chlorophyll. Crude oils are often reddish or even dark brown. The characteristic colors, odors, and flavors of natural fats are due to small quantities of substances other than glycerides, and therefore become less perceptible the more thoroughly the oil is refined. It is believed that all of the natural glycerides except butyrin, if obtained absolutely pure, would be colorless, tasteless, and odorless.

The natural glycerides are all lighter than water and insoluble in it. They can take up a very small amount of water, which is given off as steam on heating. The quantity of water which can be held by a fatty oil without causing turbidity is, however, negligible, so that for practical purposes the fatty oils may be considered as immiscible with water.

They dissolve readily in ether, carbon bisulphide, chloroform, carbon tetrachloride, and benzol, and mix with each other in all proportions. With the exception of castor oil and a few other oils characterized by a large proportion of hydroxy-acids, they are sparingly soluble in alcohol or acetic acid, but dissolve readily in petroleum ether and mix in all proportions with mineral oils. Castor oil is readily soluble in alcohol or acetic acid and not readily miscible with petroleum ether or mineral oils.

The natural fats do not distill without decomposition. When decomposed by heating they give off acrolein, which is readily recognized by its characteristic irritating odor. 
A simple triglyceride is one in which the three acid radicals are of the same kind. A glyceryl ester containing the radicals of two or three different fatty acids is known as a mixed glyceride. Both simple and mixed glycerides have been isolated from natural fats, and certain physical differences in fats which contain practically the same acids are now attributed to the presence of mixed glycerides. For discussions of simple and mixed triglycerides, see Lewkowitsch's Oils, Fats, and Waxes, Chapter I, and recent papers by Hansen, ${ }^{1}$ Holde, ${ }^{2}$ and Kreis and Hafner. ${ }^{3}$

\section{ANALYTICAL METHODS}

The object of an ordinary fat or oil analysis is not so much to separate individual constituents as to determine certain chemical and physical properties which are fairly constant for each variety when pure, and are therefore frequently called analytical constants. All solid and liquid fats being essentially mixtures of triglycerides, any differences in chemical and physical properties (except such physical variations as are due to mixed glycerides) must be attributed mainly to the presence of different fatty acids, or of the same acids in different proportions.

The principal differences to be expected are: (1) In the mean molecular weight of the acids present or the relative proportions of acids of high and those of low molecular weight; (2) in the relative number of "double bonds" depending upon the proportions of acids of the stearic, oleic, linoleic, and linolenic types; (3) in the proportion of hydroxy-acids present.

Some of the analytical "constants" express direct measures of one of these three properties. Others, especially the physical constants, are influenced by variation in any of these three directions and therefore express no one chemical property, but a resultant of all. The "constants" most used may be grouped on this principle as follows :

${ }^{1}$ Ueber das Vorkommen gemischter Fettesaüre-Glyceride in theirischen Fette. Dissertation, Rostock, 1902 ; Arch. Hygiene, 1902, 42, 1.

2 Ber., 1902, 35, 4306.

${ }^{3}$ Ber., 1903, 36, 1123, 2766 ; Z. Nahr. Genussm., 1904, 7, 641. 
1. (a) Measuring the mean molecular weight. - Saponificition or Koettstorfer number. (b) Measuring the proportion of acids of high or of low molecular weight. - Hehner number, Reichert-Meissl number.

2. (a) Measuring the proportion of unsaturated acids (number of "double bonds"). - Hübl number and other halogen absorption numbers. (b) Depending mainly upon the proportion of unsaturated acids. - Thermal reactions with bromine or sulphuric acid, Maumené number.

3. Measuring the hydroxyl radical and therefore, depending mainly upon the presence of hydroxy-acids. - Acetyl number.

4. Influenced by all of the above properties. - Specific gravity, index of refraction, melting point, "titer test," viscosity, solubilities.

\section{The Saponification or Koettstorfer Number ${ }^{1}$}

The saponification or Koettstorfer number is the number of milligrams of potassium hydroxide consumed in the complete saponification of one gram of the fat or wax; or, in other words, it is ten times the percentage of potassium hydroxide required to neutralize the total fatty acids ir the sample, whether free or in the form of esters.

Reagents. - 1. Standard solution of hydrochloric acid preferably half normal.

2. Alcoholic potash solution containing 40 grams of potassium hydroxide per liter of purified 95 per cent alcohol. In the preparation of this solution the best available potassium hydroxide (purified by alcohol) should be dissolved in alcohol which has been purified by redistillation over caustic alkali. The solution must be clear when used (filter if necessary).

3. As indicator a 1 per cent solution of phenolphthalein in purified 95 per cent alcohol.

Determination. - Weigh 4 to 5 grams of the fat or oil in a 250cc. Erlenmeyer flask, add 50 cc. of the alcoholic potash solution,

${ }^{1}$ Koettstorfer : Z. anal. chem., 1879, 18, 199, 431. Reprinted in Ephraim's Originalarbeiten über Analyse der Nahrungsmittel, Leipzig, 1895. 
connect with a reflux condenser and boil for thirty minutes, or until the oil is completely saponified, so that the liquid in the flask appears homogeneous and clear. At the same time measure $50 \mathrm{cc}$. of the alcoholic potash solution into an empty flask of the same size and shape, connect with a similar reflux condenser and boil for the same length of time, and treat in all respects in exactly the same way as in the case of the solution containing the sample. Cool the flasks and titrate each with the standard hydrochloric acid, using $1 \mathrm{cc}$. of the phenolphthalein solution as indicator. The difference between the titrations gives a measure of the potassium hydroxide consumed in saponifying the sample.

Notes. - When ordinary alcohol is used for the potassium hydroxide solution, the latter rapidly turns brownish so that the final titration is difficult. Alcohol which has been treated with potassium hydroxide, allowed to stand for one to two weeks, and then redistilled gives a much more permanent solution. According to Gill, scarcely any darkening of the solution occurs if it is kept under an atmosphere of hydrogen. Great care must be exercised to measure exactly the same quantity of the alkaline solution into each flask, and to treat the blank solution in exactly the same way as that containing the sample, so that any loss of alkalinity due to absorption of carbon dioxide from the air, or to the possible action of the alkali on the solvent, may be the same in each case. If sulphuric acid were used in place of hydrochloric for the final titration, a precipitate of potassium sulphate would be formed in the alcoholic solution, thus impairing the delicacy of the end reaction.

In order to avoid the changes which may occur in the alkali solution on boiling, Henriques ${ }^{1}$ recommends that the saponification be conducted in the cold. From 3 to 4 grams of oil are mixed with $25 \mathrm{cc}$. of petroleum ether and 25 cc. of normal alcoholic potash and allowed to stand overnight at room temperature, when the saponitication is said to be complete. A blank test with the same amounts of petroleum ether and alcoholic potash should be made alongside.

1 Z. angew. Chem., 1895, 721; 1896, 221, 423. 


\section{The Saponification Equivalent}

The results obtained, as described above, are sometimes expressed in terms of the saponification equivalent. This is the weight of fat which reacts with the molecular weight of sodium or potassium hydroxide; or, in other words, the number of grams of fat which would be saponified by one liter of normal alkali. Comparing this with the definition of the saponification (or Koettstorfer) number, it will be seen that these two values express the same property in reciprocal terms and that the product of the two values is always equal to the number of milligrams of potassium hydroxide in a liter of normal solution, viz. 56,108 .

Hence

$$
\begin{aligned}
\text { Saponification equivalent } & =\frac{56108}{\text { saponification number }} \\
\text { Saponification number } & =\frac{56108}{\text { saponification equivalent }}
\end{aligned}
$$

For a sample consisting entirely of triglycerides the saponification equivalent would be exactly one third of the mean molecular weight of these glycerides, and the mean molecular weight of the fatty acids would be $\left(\mathrm{C}_{3} \mathrm{H}_{2} \div 3\right)$ or 12.67 units lower than the saponification equivalent.

Table 13. - Saponification Data of Some Pure Esters

\begin{tabular}{|c|c|c|c|c|c|c|c|c|c|c|c|c|c|}
\hline & & & & Ester & & & & & & & & $\begin{array}{l}\text { Saponification } \\
\text { number }\end{array}$ & $\begin{array}{l}\text { Saponification } \\
\text { equivalent }\end{array}$ \\
\hline Butyrin & . & . & . & . & . & . & - & . & . & - & . & 557.7 & 100.7 \\
\hline Laurin . & . & . & . & . & . & . & . & . & . & . & • & 263.8 & 212.9 \\
\hline Palmitin & . & . & . & . & . & . & . & . & . & . & . & 208.8 & 268.9 \\
\hline Stearin . & . & . & . & . & . & . & . & . & . & . & . & 189.1 & 297.0 \\
\hline Olein . & . & . & . & . & . & . & . & . & . & . & . & 190.4 & 294.9 \\
\hline Erucin . & . & . & . & . & . & . & . & & . & . & & 160.0 & 351.0 \\
\hline Linolein & . & . & . & . & . & . & . & & . & . & $\boldsymbol{\theta}^{\circ}$ & 191.7 & 292.9 \\
\hline Linolenin & . & . & . & . & . & . & . & $\bullet$ & . & . & . & 193.0 & 290.9 \\
\hline Ricinolein & & & . & . & . & . & . & & . & - & & 180.6 & 310.9 \\
\hline Dodecatyl & & eate $^{1}$ & 1. & & . & . & . & • & . & . & & 124.5 & 450.5 \\
\hline
\end{tabular}
(Data are given to nearest 0.1 only)

1 Constituent of sperin oil. 
The natural fats, being mixtures of glycerides, rarely show such distinct differences in their saponification numbers and equivalents as the above data might suggest. The saponification number is, however, a useful aid in detection of glycerides of acids below $\mathrm{C}_{16}$ or above $\mathrm{C}_{18}$, of unsaponifiable oils, or of waxes.

\section{Acid ANd Ester Numbers}

The acid number of a fat or wax is the number of milligrams of potassium hydroxide required to neutralize the free fatty acids in one gram of substance. The ester number is the difference between the saponification number and the acid number and therefore shows the amount of alkali consumed in the saponification of esters.

To determine the acid number, shake 4 to 5 grams of the sample with $50 \mathrm{cc}$. of carefully neutralized warm alcohol and titrate with tenth-normal or half-normal alkali, using phenolphthalein as indicator. As the oil itself mixes but slightly with alcohol, it is necessary toward the end of the titration to shake thoroughly after each addition of alkali to secure complete extraction of the fatty acid from the oily layer. The alcohol should be neutralized immediately before using.

Note. - The acidity of a sample of oil or fat is not always expressed as the "acid number." Frequently it is recorded in terms of the equivalent percentage of free oleic acid, "acidity as oleic," and sometimes as "degrees of acidity," which indicates the number of cubic centimeters of normal caustic alkali required to neutralize the free acids in 100 grams of the fat. This latter form of expressing acidity is not common and should not be encouraged. The expressing of acidity sometimes as acid number and sometimes as percentage of oleic acid is not seriously inconvenient if it be kept in mind that in any given case the acid number is almost exactly twice the percentage of free acid calculated as oleic.

For titrating dark fats the use of Alkali Blue $6 \mathrm{~B}$ as indicator in place of phenolphthalein has been recommended. About 2 cc. of a 2 per cent alcoholic solution are used. 


\section{The Hehner Number}

The Hehner number is the percentage of insoluble fatty acids obtainable from a fat.

As the determination is ordinarily made, the unsaponifiable matter present in the fat is weighed with the insoluble acids. The great majority of fats and fatty oils have Hehner numbers between 94.5 and 96 . Butter fat has a lower Hehner number and the determination of this "constant" is of value chiefly in testing the purity of butter. The detailed description of the process will therefore be given in the section on butter analysis.

\section{The Reichert-Meissl Number}

The Reichert-Meissl number is the number of cubic centimeters of tenth-normal caustic alkali required to neutralize the soluble volatile acids obtained from 5 grams of a fat by the Reichert distillation process.

This number serves as a comparative measure of the acids of low molecular weight. Its principal use is in the examination of butter fat and the detailed description will be given in that connection. The Reichert number is about one half the Reichert-Meissl number, Reichert having originally recommended the use of 2.5 grams of fat. Among the common oils and fats the Reichert-Meissl numbers are usually less than 1.0. Butter fat has a high Reichert-Meissl number and as this determination is used principally in the examination of butter, it will be described in the section on butter analysis.

\section{The IODINe oR HüBL NuMBeR}

The iodine or Hübl number is the percentage of iodine (or of iodine chloride or bromide expressed in terms of iodine) absorbed by the sample.

This number gives a quantitative measure of the unsaturated fatty acids (or of the "number of double bonds") in a fat or wax.

Mills, Snodgrass, and Akitt were probably the first to make 
systematic use of the halogen absorbing power in fat analysis. In their experiments ${ }^{1}$ the oil or fat to be tested was dissolved in carbon tetrachloride and titrated with a standard solution of bromine in carbon tetrachloride as long as the bromine was absorbed. At about the same time, Hübl published ${ }^{2}$ a method based upon the use of iodine in an alcoholic solution of mercuric chloride which was so carefully worked out in all of its details that the original form of the process is still used in many laboratories in preference to any of the modifications which have been proposed. Recently, however, the $\mathrm{Wijs}^{3}$ and the Hanus ${ }^{4}$ modifications have been largely used, and it is probable that they will gradually replace the Hübl process. These three methods will be given here. The bromine absorption method as developed by McIlhiney ${ }^{5}$ can be used in place of the iodine methods, but is more especially adapted to the examination of linseed oil for rosin oil or rosin, and will be referred to in that connection.

\section{Method of Hübl}

Reagents. - 1. Iodine solution. Dissolve 26 grams of pure iodine in 500 cc. of 95 per cent alcohol. Dissolve 30 grams of mercuric chloride in $500 \mathrm{cc}$. of alcohol of the same strength. Mix the two solutions at least 12 hours before using. As this solution is expensive and does not keep well, no more than three days' supply should be made at one time.

2. Standard solution of sodium thiosulphate. Dissolve 24 grams of the crystallized salt in a liter of water, allow to stand at least 24 hours, and then determine the strength of the solution in terms of iodine. While any of the well-known methods may be used, it is convenient to standardize the thiosulphate solution as follows:

Weigh 3.8694 grams of pure dry potassium dichromate, dissolve in water and dilute to $1000 \mathrm{cc}$. In a well-filled tightly stoppered bottle, this standard solution of dichromate can be

1 J. Soc. Chem. Ind., 1883, 2, 435; 1884, 3, 366.

${ }^{2}$ Dingl. polyt. J., 1884, 253, 281. Reprinted by Ephraim, loc. cit.

${ }^{3}$ Ber., 1898, 31, 750.

4 Z. Nahr.-Genussin., 1901, 4, 913.

5 J. Am. Chem. Soc., 1884, 16, 245 ; 1899, 21, 1084; 1902, 24, 1109. 
kept indefinitely without deterioration. Each cubic centimeter of this solution is equivalent to 0.01 gram iodine. Mix 25 cc. of a 15 per cent potassium iodide solution with 5 cc. of hydrochloric acid, add $50 \mathrm{cc}$. of the dichromate solution, and titrate the liberated iodine by means of the thiosulphate solution, observing procedure and precautions described below. Calculate the amount of iodine consumed by each cubic centimeter of the thiosulphate solution. As a precaution the strength of either the dichromate or the thiosulphate solution should also be determined by an independent method.

3. An approximately 15 per cent solution of pure potassium iodide in cold, recently boiled, distilled water.

4. Freshly prepared starch solution, 1 part starch to 200 parts of water, for use as indicator.

5. Pure chloroform.

6. Cold, recently boiled, distilled water.

Determination. - Thoroughly clean and dry two or more thin Erlenmeyer flasks, of the form made for this purpose, having

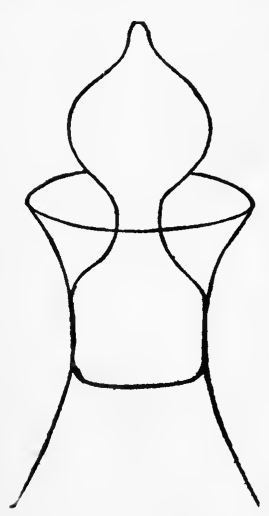
accurately ground glass stoppers and flaring mouths which form a gutter between the stopper and the lip, as shown in Fig. 10. Into one of these flasks weigh accurately about 0.25 gram of the sample, and add $10 \mathrm{cc}$. of chloroform. When the sample has completely dissolved, add 50 cc. of the mixed iodine solution, stopper carefully, fill the gutter around the stopper with potassium iodide solution to guard against loss of iodine, shake gently, and allow the flask to stand in a cool dark closet for three hours. In another clean dry flask of Fig. 10. - Flask for the same size and form make a blank deterdetermination of iodine number.

\section{f} mination, using the same amounts of chloroform, iodine solution, and potassium iodide. Allow the two flasks to stand side by side for the same length of time. When the absorption is complete, lift the stopper in such a way that its lower surface will be washed by the iodide solution from the gutter; add 100 cc. of cold, recently boiled, 
distilled water and $20 \mathrm{cc}$. more of the potassium iodide solution, washing down the sides of the flask with the latter. In case a red precipitate of mercuric iodide appears, add more potassium iodide until the precipitate is dissolved. Titrate the excess of iodine at once by means of the standard thiosulphate solution. The latter may be run in rapidly until the iodine is nearly consumed and the solution is only light yellow; then add 2 cc. of the starch solution and finish the titration carefully but without delay. As the end point is approached, stopper the flask quickly after each addition of thiosulphate and shake vigorously to insure thorough and rapid mixing of the contents. The end point should be sharper than in standardizing since in this case there is no green color due to chromium and the solution passes at once from blue to nearly colorless. The difference between the volume of thiosulphate solution required for the blank test and that required for the solution containing the sample gives a measure of the iodine absorbed by the latter. Calculate the iodine absorbed in terms of percentage of the original weight of sample.

Notes. - Most of the difficulties which are met in using this method are due to impure reagents or failure to observe carefully the conditions worked out by Hübl. Probably the most important sources of error are: (1) The use of impure chloroform or water containing dissolved air causing a liberation of iodine from the potassium iodide, or the iodine addition product of the oil; (2) loss of iodine from the use of vessel with imperfectly fitting stopper or from titrating at too high temperature or with too much exposure to air; (3) deterioration of the iodine solutions due chiefly to impurities in the alcohol or too high temperature; (4) insufficient excess of iodine over that absorbed; (5) variations in the length of time allowed for the reaction.

The small amount of oil needed for this determination can conveniently be drawn from near the center of the sample bottle by means of clean glass tubing of about $2 \mathrm{~mm}$. internal diameter. Use a piece of thin tubing like a pipette, wiping it free from oil on the outside and allowing it to deliver drop by drop into the 
weighed flask which should then be stoppered and reweighed at once. If more than one sample of oil is to be tested, delays can be avoided by having a number of pieces of the tubing cleaned and dried in advance so that each can be rejected after using it for one sample.

The best results are obtained by using such proportions of oil and of iodine solution as to have present from two to three times the amount of iodine which will be absorbed. The form of vessel to be used for the test is, of course, immaterial so long as loss of iodine is avoided. Some prefer to weigh the sample on a small watch glass and place the latter with the sample in a wide-mouth glass stoppered bottle. A blank test must be made with each determination, or if several samples are treated at once, there should be at least two or three blanks.

For discussion of the theory of the action of Hübl's solution see Lewkowitsch's Oils, Fats, and Waxes, or Gill's Oil Analysis.

\section{Method of Wijs}

Wijs found that a solution of iodine monochloride in glacial acetic acid acts in the same manner as Hübl's solution, but more quickly. Moreover, the reagent is much more stable than that of Hübl, so that the same solution can be used for several months and blank tests are not so frequently required.

Reagents. - 1. Glacial acetic acid. This must contain not over 0.5 per cent of water and no impurity capable of reducing potassium dichromate. Test by adding a small amount of a solution of dichromate in sulphuric acid and warming on a water bath; no green tinge should appear.

2. Iodine chloride solution. Dissolve 12.5 to 13 grams of iodine in a liter of the glacial acetic acid, warming gently to aid solution, if necessary. Cool, withdraw 25 cc., add potassium iodide solution, and determine the iodine content by titration with standard thiosulphate; into the remainder of the solution pass a current of chlorine until the deep iodine color changes to an oránge yellow, showing that the conversion of iodine to iodine monochloride is complete. A portion of the 
solution titrated with thiosulphate should now show twice the original halogen content. In case an excess of chlorine is found, a weighed amount of iodine equivalent to this excess should be dissolved in the solution.

3. Standard solution of sodium thiosulphate, as in the method of Hübl.

4. Pure chloroform, potassium iodide, starch solution, and distilled water, as described under Hübl's method.

Determination. - The determination is carried out in exactly the manner described for the Hübl method except that $10 \mathrm{cc}$. of the potassium iodide solution are sufficient and a much shorter time is required for the reaction. With non-drying oils the addition of iodine chloride is usually complete in 15 minutes. Ordinarily the solution should be titrated after standing one half hour.

Notes. - As the solution of iodine chloride in acetic acid is more viscous and has a higher coefficient of expansion than ordinary aqueous solutions, the portions added must be measured as carefully as possible, allowing a uniform time for the burette to drain, and observing that the temperature does not vary more than three or four degrees. With these precautions it is not always necessary to make a blank test with each determination after a sufficient number of such tests have been made to show that the solution is not changing appreciably in strength.

\section{Method of Hanus}

Hanus suggested that Wijs' method be modified by the use of iodine bromide in place of iodine chloride. As an excess of iodine does no harm, the preparation of the reagent is simpler than in the preceding method.

The details of this method as adopted by the Association of Official Agricultural Chemists in 1905 are essentially as follows :

Reagents. - 1. Iodine solution. - (a) Dissolve 13.2 grams iodine in 1000 cc. glacial acetic (99.5 per cent) acid (showing no reduction with bichromate and sulphuric acid), add enough 
bromine to double the halogen content determined by titration; 3 cc. of bromine is about the proper amount. The iodine may be dissolved by the aid of heat, but the solution should be cold when bromine is added.

2. Decinormal sodium thiosulphate solution. - Dissolve 24.8 grams of chemically pure sodium thiosulphate, freshly pulverized as finely as possible, and dried between filter or blotting paper and dilute with water to 1 liter at the temperature at which the titrations are to be made. [This solution is to be standardized when used as described below.]

3. Starch paste. - One gram of starch is boiled in $200 \mathrm{cc}$. of distilled water for 10 minutes and cooled to room temperature.

4. Solution of potassium iodide. - One hundred and fifty grams of potassium iodide are dissolved in water and made up to 1 liter.

5. Decinormal potassium dichromate. - Dissolve 4.9066 grams of chemically pure potassium dichromate in distilled water, and make the volume up to 1 liter at the temperature at which the titrations are to be made. The dichromate solution should be checked against pure iron.

Determination. - (1) Standardizing the sodium thiosulphate solution. - Place $20 \mathrm{cc}$. of the potassium dichromate solution, to which has been added $10 \mathrm{cc}$. of the solution of potassium iodide, in a glass-stoppered flask. Add to this 5 cc. of strong hydrochloric acid. Allow the solution of sodium thiosulphate to flow slowly into the flask until the yellow color of the liquid has almost disappeared. Add a few drops of the starch paste, and with constant shaking continue to add the sodium thiosulphate solution until the blue color just disappears.

(2) Weighing the sample. - Weigh about one half gram of fat, 0.25 gram of salad oil, or $0.10-0.20$ gram of drying oil on a small watch crystal and introduce the watch crystal into a widemouth 16-ounce bottle with ground-glass stopper [or weigh the portion in a glass-stoppered Erlenmeyer flask as described under the Hübl method above].

(3) Absorption of iodine. - The fat or oil in the bottle is 
dissolved in $10 \mathrm{cc}$. of chloroform. After complete solution has taken place, $25 \mathrm{cc}$. of the iodine solution are added. Allow to stand, with occasional shaking, for thirty minutes. The excess of iodine should be at least 60 per cent of the amount added.

(4) Titration of the unabsorbed iodine. - Add $10 \mathrm{cc}$. of the potassium iodide solution and shake thoroughly, then add $\mathbf{1 0 0}$ cc. of distilled water to the contents of the bottle. Titrate the excess of iodine with the sodium thiosulphate solution, which is added gradually, with constant shaking, until the yellow color of the solution has almost disappeared. Add a few drops of starch paste, and continue the titration until the blue color has entirely disappeared. Toward the end of the reaction stopper the bottle and shake violently, so that any iodine remaining in solution in the chloroform may be taken up by the potassium iodide solution.

(5) Setting the value of iodine solution by blank determinations. - At the time of adding the iodine solution to the fat run the same amount into each of two bottles of the same size as those used for the determination and carry these "blanks" through exactly the same manipulation as in the determination upon the fat. These blank experiments must be made each time the iodine solution is used.

Notes. - Great care must be taken that the temperature of the solution does not change during the time of the operation, as acetic acid has a very high coefficient of expansion, and a slight change of temperature makes an appreciable difference in the strength of the solution. Where any great number of determinations are to be made, blanks should be measured out at short intervals. This precaution applies as well to the use of the Hübl solution, as the coefficient of the expansion of alcohol is large.

When the potassium iodide is added, the solution should be thoroughly mixed before the addition of water.

The acetic acid must be full strength and pure in order to obtain a solution which will keep well.

This method has several advantages over that of Hübl, the most important being that the reagent keeps better and acts more rapidly so that there is less danger of discrepancies from sec- 
ondary reactions. In general the apparatus and manipulations described under the Hübl method apply also here and should be carefully noted. In this case the flasks may be sealed by pouring a little glacial acetic acid into the gutter instead of the potassium iodide described under the Hübl method, or since the time of standing is here only 30 minutes an exceptionally well-ground stopper may not require any liquid seal to prevent the escape of iodine.

\section{Comparison of the Hïbl, Wijs, and Hanus Methods}

Wijs based his modification on the belief that iodine chloride is the active substance in Hübl's solution and that the more stable solution of iodine chloride in acetic acid should give the same theoretical results with greater certainty. That higher results were often obtained by his method Wijs attributed to the deterioration or incomplete action of the Hiibl solution as ordinarily prepared. Lewkowitsch ${ }^{1}$ confirms this, stating that, when the Hübl method is carried out under the best conditions, the results of the two methods agree.

Tolman and Munson, ${ }^{2}$ however, in an extended comparison of the three methods almost invariably obtained higher results by the Wijs than by the Hübl method. Hanus' method gave intermediate results, usually agreeing quite closely with the Hübl numbers. Similar results on a smaller number of samples had previously been published by $\mathrm{Hunt}^{3}$ and have since been obtained by W. G. Tice. ${ }^{4}$ For oils having iodine numbers below 100, the three methods can be used interchangeably, as the differences in results will rarely exceed 1.5 units, which is only about one tenth of the variation which occurs among pure oils of the same species. In applying the Wijs solution to oils having much higher iodine numbers it is to be remembered that results as much as 10 units in excess of these given by Hübl's solution will sometimes be obtained.

The method of Hübl is "official" in the United States, but

1 Analyst, 1899, 24, 259.

2 J. Amer. Chem. Soc., 1903, 25, 244.

${ }^{3}$ J. Soc. Chem. Ind., 1902, 21, 454.

${ }^{4}$ In the Havemeyer Laboratory, Columbia University. 
that of Hanus has been adopted by the Association of Official Agricultural Chemists as an optional method for the examination of edible oils and fats. The method of Wijs is more commonly used in England and is recommended in preference to the Hanus method by Lewkowitsch ${ }^{1}$ and Archbutt, ${ }^{2}$ the latter pointing out especially that the Wijs solution gives approximately the theoretical iodine number for turpentine (which is often mixed with drying oils) while that of Hanus gives much lower results.

In comparing analytical results with the numbers found in "tables of constants" it should be remembered that the latter are based in part upon results obtained (not always under the best conditions) by Hübl's method, and in part upon later results determined according to Wijs. Such tables tend to show a greater range than would be found by the use of either method alone, this being especially true of the oils having high iodine numbers.

\section{Maumené Number - Specific Temperature Reaction}

Long before the introduction of any of the halogen absorption methods, Maumené ${ }^{3}$ tested olive oil for adulterants such as poppyseed oil by mixing fixed volumes of oil with strong sulphuric acid and observing the rise in temperature which is much greater with seed oils than with olive. The rise in temperature, expressed in degrees C., which occurs on mixing $10 \mathrm{cc}$. of strong sulphuric acid with 50 grams of oil is commonly known as the Maumené number. Thomson and Ballantyne ${ }^{4}$ pointed out the discrepancies which may be introduced through variations in the strength of the acid used, and showed that they can be largely avoided by comparing the rise observed in the case of an oil with that shown by water under the same conditions, the latter being taken as 100. The result thus obtained is known as the specific temperature reaction or specific Maumené number. Thus if 50 grams of water mixed with $10 \mathrm{cc}$. of sulphuric acid showed a rise of $40^{\circ}$ and 50 grams of an oil under the same con-

1 Oils, Fats, and Waxes (4th Ed.), I., 323.

2 J. Soc. Chem. Ind., 1904, 23, 306.

${ }^{3}$ Compt. rend., 1852, 35, 572.

4 J. Soc. Chem. Ind., 1891, 10, 234. 
ditions a rise of $36^{\circ}$, the specific Maumené number of the oil would be 90 .

With fresh fatty oils the rise of temperature depends mainly upon the presence of glycerides of the unsaturated acids, and the Maumené and Hübl numbers are therefore roughly proportional. This relation, however, is not sufficient to permit of the determination of the Maumené in place of the iodine number except for rough work or as a preliminary test. The greatest value of this temperature reaction lies in the fact that as oils become altered by absorption of oxygen from the air, the Maumené numbers increase while the iodine numbers decrease. The significance of this relation is more fully explained in Chapter X.

Apparatus and Reagents. - A deep beaker of 150 tỏ 200 cc. capacity should be used for this test and should be so jacketed as to prevent a rapid loss of heat. This is done by placing it in a larger beaker or a metal cup and filling the space with asbestos or other dry porous material. If a new nest of beakers is at hand, the test can be made in one of the smaller beakers using the remainder of the nest with the packing intact as a jacket. The insulation should be sufficient to prevent the outer vessel from becoming perceptibly warm when a test is made. The only reagent required is strong sulphuric acid which can be added either from a burette or (more satisfactorily) from a 10-cc. pipette. For observing the rise in temperature use a thermometer graduated in degrees on which the readings can be taken with accuracy to 0.2 degree.

Determination. - The oils to be tested, the acid and apparatus to be used, and the distilled water which is to serve for comparison must all have the same initial temperature, which should be between $20^{\circ}$ and $24^{\circ}$. This will usually be the case if they have stood side by side at room temperature for several hours. Measure $50 \mathrm{cc}$. of water into the beaker, introduce the thermometer, and note the temperature; add $10 \mathrm{cc}$. of the acid, stirring thoroughly with the thermometer so that the solution is kept well mixed and the temperature rises steadily. At intervals of a few seconds bring the bulb of the thermometer 
to the center of the solution and observe the temperature. Record the highest reading found. When the mixture in the beaker has reached its maximum temperature, it should be rejected at once to avoid unnecessary warming of the apparatus, since the initial temperature must be restored before beginning another test. After the rise with water has been found by duplicate determinations which do not differ by more than $0.4^{\circ}$, dry the beaker thoroughly, weigh into it 50 grams of oil (within 0.05 gram), and treat with $10 \mathrm{cc}$. of acid in exactly the same manner. The mixture often becomes very viscous, requiring vigorous stirring to insure a uniform temperature. In reporting results give the actual rise observed with oil and with water as well as the specific temperature reaction.

Notes. - By the use of a pipette having a fine outlet requiring 30 to 60 seconds for the $10 \mathrm{cc}$. of acid to flow into the beaker, more thorough mixing becomes possible and better results are secured. In order to avoid trouble in cleaning the beaker it should be emptied and wiped with dry cotton waste or porous paper while still warm. The acid used must be protected from unnecessary exposure to air, as it readily absorbs moisture to an extent sufficient to cause an appreciably lower temperature reaction. In testing edible and lubricating oils the strongest available sulphuric acid should be used. In the case of drying oils and fish or other marine animal oils, the addition of such strong acid to the undiluted oil causes violent frothing and decomposition. This may be avoided ${ }^{1}$ by the use of sulphuric acid of such strength as to give a rise of $35^{\circ}$ when added to water under the conditions to be used in testing the oil. An important modification of this test has been proposed by Mitchell. ${ }^{2}$

In mixtures, especially of fatty with mineral oils, this temperature reaction is not to be relied upon as an additive property. Sherman, Danziger, and Kohnstamm ${ }^{3}$ found that mixtures of

${ }^{1}$ Sherman, Danziger, and Kohnstamm: J. Am. Chem. Soc., 1902, 24, 266.

2 Analyst, 1901, 26, 169. See also Sherman and Falk: J. Am. Chem. Soc., 1903, 25, 713 .

${ }^{3}$ J. Am. Chem. Soc., 24, 269. 
various fatty oils with mineral oil gave results much higher than would correspond to the known constituents of the mixture. Similar results were afterward published by Suzzi, ${ }^{1}$ and later Richter ${ }^{2}$ experimented with mixtures of fatty oils and reported that the addition of 5 to 10 per cent of sunflower, rape, or peanut oil depressed the specific temperature reaction of olive oil, and other mixtures of olive and rape or olive and peanut oils gave results constantly lower than the calculated values, while with mixtures of olive and sunflower oils containing 20 per cent or more of the latter the results found were sometimes above and sometimes below the calculated values, the differences being never greater than 5 units.

The question was then reinvestigated by Boynton and Sherman, ${ }^{3}$ using an acid which gave with water a rise of $44^{\circ} \mathrm{C}$. and carrying out the tests as described above. In a series of tests upon 40 samples ( 7 individual oils and 33 mixtures) it was found that the specific temperature reactions of mixtures of fatty oils did not as a rule differ greatly from the values ealculated on the assumption that this is an additive property. Where differences were found, the observed temperature reaction of the mixture of two fatty oils was always lower than the calculated value. On the other hand, in mixtures of fatty and mineral oils the values found were invariably higher than those calculated, the difference between the theoretical and the observed values apparently depending more upon the proportion of mineral oil than upon the nature of the fatty oil present.

More recently, Kessler and Mathiason ${ }^{4}$ have also reported that the Maumené test is not an additive property.

\section{The Acetyl Number}

The acetyl number is the number of milligrams of potassium hydroxide required to neutralize the acetic acid obtained by saponification of one gram of the acetylated fat or wax.

${ }^{1}$ Boll. chim. farm., 44, 301; Chem. Zentrbl., 1905, II, 80.

${ }^{2} Z$. angew. Chem., 1907, 1605.

${ }^{3}$ School of Mines Quarterly, 31, 64.

${ }^{4}$ J. Ind. Eng. Chem., 3, 66. 
It indicates the proportion of hydroxyl groups in the original substance. If the latter consists essentially of triglycerides, the acetyl number serves as a measure of the hydroxy-acids present. Free alcohols, if present, increase the acetyl numbers. Lewkowitsch finds that free fatty acids of the stearic series may also react in such a way as to increase the apparent acetyl numbers, and therefore prefers to acetylate the original fat or wax rather than to work with the mixed fatty acids as was previously recommended by Benedikt and Ulzer. ${ }^{1}$

The method of Lewkowitsch is essentially as follows: ${ }^{2}$

Boil 10 grams of the substance with twice its weight of acetic anhydride in a round-bottomed flask under a reflux condenser for two hours; pour the resulting mixture into a large beaker containing $500 \mathrm{cc}$. of hot water and boil for half an hour, passing a slow current of carbon dioxide through the solution to prevent bumping. Allow the mixture to separate into two layers, siphon off the water, and boil the oily layer with three successive portions of fresh water, the last of which should not react acid to litmus paper.

All free acetic acid having been removed, the acetylated fat is carefully separated from water and further dried by filtering through anhydrous paper in a drying oven.

Weigh 2 to 5 grams of the acetylated fat and saponify with a measured volume of standard alcoholic potash as in the determination of the saponification number; evaporate nearly to dryness to expel the alcohol, dissolve the soap in water, and add an amount of standard sulphuric acid exactly equivalent to the alkali used for saponification. Warm gently until the fatty acids separate as a layer at the top. Filter through wet paper, wash the fatty acids with boiling water until the filtrate is no longer acid, and titrate the filtrate and washings with tenthnormal alkali, using phenolphthalein as indicator. Deduct the amount of alkali required to neutralize any soluble fatty acids in the original substance and calculate the acetic acid found, in terms of acetyl number, as defined above.

Instead of filtering and washing with water, the acetic acid 1 Monatsh. Chem., 1887, 8, 40.

2J. Soc. Chem. Ind., 1897, 16, 503. 
can be separated from the fatty acids by distilling with steam, but this requires considerably more time than the filtration process.

In the examination of waxes the acetyl numbers show the proportional amounts of free alcohols. Fats and fatty oils contain free alcohols (cholesterol, phytosterol, etc.), but in very small amounts only, so that the acetyl numbers are low except in oils which contain hydroxy-acids (Chapter VII). The acetyl number of castor oil is about 150 . Other oils rarely show acetyl numbers higher than 10 or 15 , unless they have been exposed to atmospheric oxidation, in which case hydroxyacids may have been formed from the unsaturated acids originally present.

\section{SPECIFIC GRAvity}

The specific gravities of the glycerides depend upon the fatty acids which they contain. Comparing acids of the same homologous series the specific gravity was found to decrease with increasing molecular weight; while between corresponding acids of different series it increases with the number of double bonds and of hydroxyl groups.

Fresh specimens of the same kind vary but little in specific gravity, and the determination of this property is often useful in differentiating oils, fats, and waxes, and in testing them for adulterations. 'The determination can be made either by means of a very clelicate special hydrometer, by the Westphal balance (Fig. 11), or by one of the methods described in Chapter I. On account of the high coefficient of expansion of oils it is important that the temperature at which the specific gravity is taken be accurately known. This is conveniently accomplished by means of a hydrometer having a thermometer scale in the stem, or by using the thermometer sinker with the Westphal balance.

Directions for the use of the Westphal balance are usually supplied with the instrument. It should always be placed upon a firm level table and very carefully adjusted before using. In setting up the instrument be sure that the leveling screw is 
directly beneath the arm which supports the beam. Place the latter in position and hang the sinker. Compare the height of the beam with that of the cylinder which is to contain the oil, and lengthen or shorten the standard if necessary, then carefully adjust the balance by means of the leveling screw until the point projecting from the end of the beam stands exactly opposite the fixed point at the left. Nearly fill the cylinder with the oil at about $14^{\circ}$. Lift the sinker, place the cylinder under

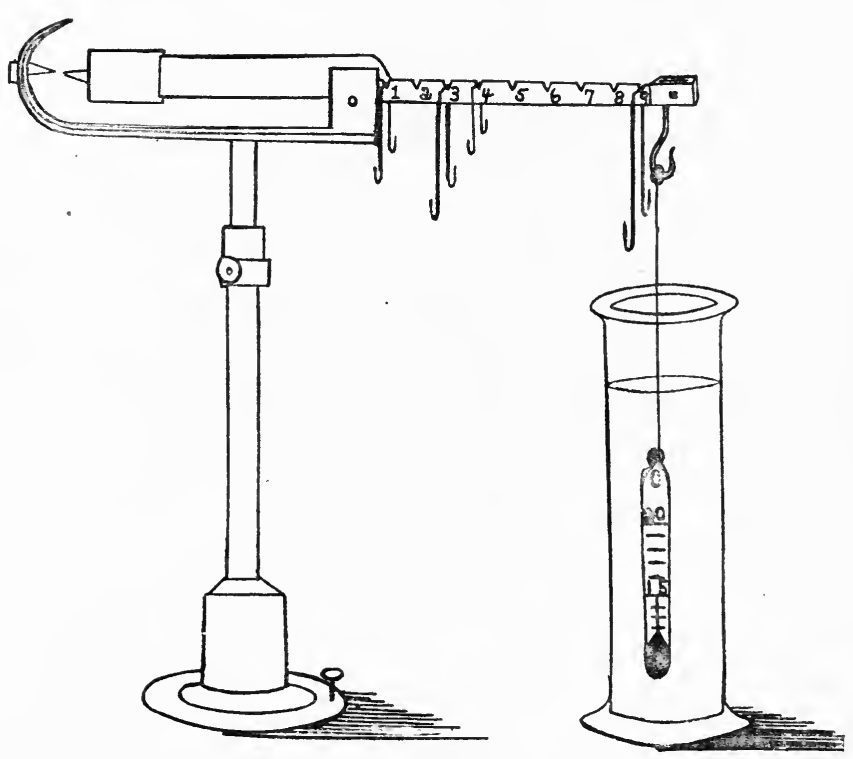

FIG. 11. - The Westphal balance.

the end of the beam, and replace the sinker so that it hangs freely in the oil. The specific gravity is now found by placing weights on the beam until the balance is restored. Make the final adjustment of the smallest weight when the thermometer in the sinker shows the desired temperature - for oils, $15.5^{\circ}$. The specific gravity is read directly from the position of the weights on the beam. If the largest weight is at 9 , the second at 3 , the third at 1 , and the fourth at 4 , the specific gravity of the oil is 0.9314 . The readings should be made to four decimal places (i.e. all four sizes of weights should be used) and the 
error of determination should not exceed 0.0005. By using the same sinker on the analytical balance or by weighing the oil in a pyknometer, more accurate results can be obtained. The Westphal balance, is, however, sufficiently delicate for routine work if carefully used. Care must be taken that the sinker is always suspended at the same depth in the liquid under examination. The graduation of the beam should be tested to see that the spaces are all of equal length, and the results should occasionally be verified by comparative determinations with the pykometer. In working with very viscous oils it is advantageous to use a sinker of high specific gravity. ${ }^{1}$

If a delicate form of Westphal balance is not at hand or if more accurate results are desired, the pyknometer will be found well suited to this work.

For calculating data obtained at higher temperatures to the standard temperature of $15.5^{\circ} \mathrm{C}$., Wright ${ }^{2}$ gives the following factors : $16^{\circ}, 1.00035 ; 17^{\circ}, 1.00106 ; 18^{\circ}, 1.00177 ; 19,1.00248$; $20^{\circ}, 1.00319 ; 21^{\circ}, 1.00391 ; 22^{\circ}, 1.00462 ; 23^{\circ}, 1.00534 ; 24^{\circ}$, $1.00605 ; 25^{\circ}, 1.00677$. Since the different oils vary somewhat in coefficient of expansion it is better to work at the standard temperature than to depend upon factors for correcting results.

For the determination of specific gravity at temperatures considerably above that of the laboratory it is convenient to use an Ostwald pyknometer. This is filled with the sample, suspended in water at the required temperature, and adjusted, then removed, dried on the outside, cooled to room temperature, and weighed. For a detailed description of the standardization of pyknometers at high temperatures consult any standard work on physicochemical measurements or Bulletin 107, Bureau of Chemistry, U. S. Department of Agriculture.

\section{INDEX OF REFRACTION}

The index of refraction, like the specific gravity, increases with the proportion of unsaturated, or of hydroxy, acids. Un-

1 McGill : Analyst, 1896, 21, 156.

${ }^{2} J$. Soc. Chem. Ind., 1907, 26, 513. Allen's Commercial Organic Analysis, Fourth Edition, Vol. II, p. 51. 
like the specific gravity, the index of refraction increases with the molecular weight in the homologous series of saturated acids, so that the presence of the fatty acids of low molecular weight in butter causes this fat to have a lower index of refraction than other animal fats, while its specific gravity is higher. In almost all of the fatty oils the index of refraction varies with the specific gravity, so that in routine work it is not necessary to make both determinations, but either may be used to confirm the inferences drawn from the other determinations. ${ }^{1}$

The index of refraction has usually been determined by means of the Abbe or the Pulfrich refractometer or the "butyro-refractometer" of Zeiss. The latter has an arbitrary scale of known value whose readings may be converted into actual index of refraction by the use of the table on page 166 .

The construction of the butyro-refractometer is shown in Fig. 12. To take the index of refraction of an oil, tilt the instrument until the glass plate on the inner surface of $B$ is horizontal, place a few drops of the oil upon it, then close it against $A$ and clamp in position by turning $\boldsymbol{F}^{\prime}$.

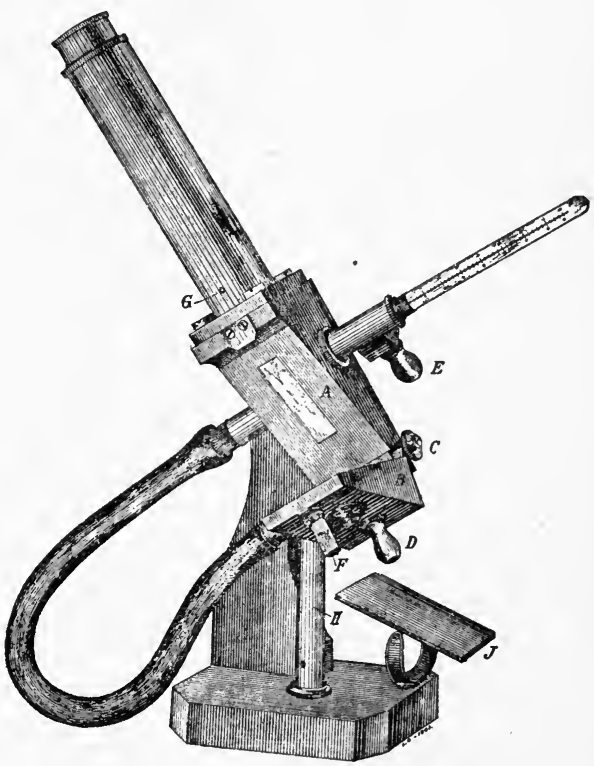

FIG. 12. - The Zeiss butyro-refractometer.

The space between $A$ and $B$ is now filled with oil, and by looking through the telescope and tilting the mirror $J$ until the scale is well illuminated, one obtains a measure of the index of refraction of the oil from the position of the shadow upon

1 For a very full discussion of the relation of index of refraction to specific gravity in oils and fats see Proctor: J. Soc. Chem. Ind., 1898, 17, 1021. 
the scale. Detailed directions for the care and adjustment of the instrument are furnished by the manufacturers and may also be found in Leach's Food Inspection and Analysis.

Table 14. - Index of Refraction corresponding to Scale Reading of Butyro-Refractometer

\begin{tabular}{|c|c|c|c|c|c|c|c|}
\hline $\begin{array}{l}\text { Scale } \\
\text { reading }\end{array}$ & $\begin{array}{l}\text { Index of } \\
\text { refraction }\end{array}$ & $\begin{array}{l}\text { Scale } \\
\text { reading }\end{array}$ & $\begin{array}{l}\text { Index of } \\
\text { refraction }\end{array}$ & $\begin{array}{l}\text { Scale } \\
\text { reading }\end{array}$ & $\begin{array}{l}\text { Index of } \\
\text { refraction }\end{array}$ & $\begin{array}{l}\text { Scale } \\
\text { reading }\end{array}$ & $\begin{array}{l}\text { Index of } \\
\text { refraction }\end{array}$ \\
\hline 0 & 1.4220 & 26 & 1.4423 & 51 & 1.4600 & 76 & 1.4760 \\
\hline 1 & 1.4228 & 27 & 1.4430 & 52 & 1.4607 & 77 & 1.4766 \\
\hline 2 & 1.4236 & 28 & 1.4438 & 53 & 1.4613 & 78 & 1.4772 \\
\hline 3 & 1.4244 & 29 & 1.4445 & 54 & 1.4620 & 79 & 1.4778 \\
\hline 4 & 1.4252 & 30 & 1.4452 & 55 & 1.4626 & 80 & 1.4783 \\
\hline 5 & 1.4260 & 31 & 1.4460 & 56 & 1.4633 & 81 & 1.4789 \\
\hline 6 & 1.4268 & 32 & 1.4467 & 57 & 1.4640 & 82 & 1.4795 \\
\hline 7 & 1.4276 & 33 & 1.4474 & 58 & 1.4646 & 83 & 1.4801 \\
\hline 8 & 1.4284 & 34 & 1.4481 & 59 & 1.4653 & 84 & 1.4807 \\
\hline 9 & 1.4292 & 35 & 1.4488 & 60 & 1.4659 & 85 & 1.4812 \\
\hline 10 & 1.4300 & 36 & 1.4495 & 61 & 1.4666 & 86 & 1.4818 \\
\hline 11 & 1.4308 & 37 & 1.4502 & 62 & 1.4672 & 87 & 1.4824 \\
\hline 12 & 1.4316 & 38 & 1.4510 & 63 & 1.4679 & 88 & 1.4829 \\
\hline 13 & 1.4324 & 39 & 1.4517 & 64 & 1.4685 & 89 & 1.4835 \\
\hline 14 & 1.4331 & 40 & 1.4524 & 65 & 1.4691 & 90 & 1.4840 \\
\hline 15 & 1.4339 & 41 & 1.4531 & 66 & 1.4698 & 91 & 1.4846 \\
\hline 16 & 1.4347 & 42 & 1.4538 & 67 & 1.4704 & 92 & 1.4851 \\
\hline 17 & 1.4354 & 43 & 1.4545 & 68 & 1.4710 & 93 & 1.4857 \\
\hline 18 & 1.4362 & 44 & 1.4552 & 69 & 1.4717 & 94 & 1.4862 \\
\hline 19 & 1.4370 & 45 & 1.4559 & 70 & 1.4723 & 95 & 1.4868 \\
\hline 20 & 1.4377 & 46 & 1.4566 & 71 & 1.4729 & 96 & 1.4873 \\
\hline 21 & 1.4385 & 47 & 1.4573 & 72 & 1.4736 & 97 & 1.4879 \\
\hline 22 & 1.4392 & 48 & 1.4580 & 73 & 1.4742 & 98 & 1.4884 \\
\hline 23 & 1.4400 & 49 & 1.4587 & 74 & 1.4748 & 99 & 1.4890 \\
\hline 24 & 1.4408 & 50 & 1.4593 & 75 & 1.4754 & 100 & 1.4895 \\
\hline 25 & 1.4415 & & & & & & \\
\hline
\end{tabular}

Since the butyro-refractometer is constructed to cover only such a range in the index of refraction as is likely to be met in work with fats and oils, it is possible to make more delicate readings with this instrument than with the Abbe refrac- 
tometer. It is preferable, however, to express all results in terms of the index of refraction, especially as some substances frequently mixed with oils and fats are too highly refractive to be examined in the butyro-refractometer.

The index of refraction decreases with rising temperature. The rate of change is nearly constant for the common oils and fats, a rise of $1^{\circ}$ causing a diminution of 0.000365 in the index of refraction. ${ }^{1}$

\section{Melting And Solidifying Points-Titer Test}

The melting point of a fat, or of the mixed fatty acids obtained from it, increases with the mean molecular weight among acids of the saturated series, while in mixtures of acids of the saturated and unsaturated series (or their glycerides) the melting point becomes lower as the proportion of unsaturated compounds increases. The melting point of a solid fat is best determined by heating a thin disk of the substance suspended in a mixture of water and alcohol as described in Chapter IX. 'This method being applicable only to substances practically insoluble in alcohol, the melting point of a mixture of fatty acids or of a wax may be taken as follows : ${ }^{2}$

Draw up the melted fatty acids (or the wax) into a very thin-walled capillary tube 3 to $5 \mathrm{~cm}$. long according to the length of the bulb of the thermometer to be used. Seal one end of the tube and allow the substance to cool on ice for 12 to 15 hours. Attach to the bulb of a delicate thermometer graduated to $0.2^{\circ}$, immerse in water, and heat very slowly. The temperature at which the substance becomes transparent is taken as the melting point.

Natural fats sometimes show changeable or "double" melting points. Grün and Schacht ${ }^{3}$ account for this as due to the presence of mixed glycerides capable of existence in two

1 Proctor: J. Soc. Chem. Ind., 1898, 17, 1023. Tolman and Munson: J. Am. Chem. Soc., 1902, 24, 754.

2 U. S. Dept. Agriculture, Bur. Chem., Bul. 107.

${ }^{3}$ Ber., 1907, 40, 1778; Allen (4), II, 52. 
isomeric forms of different melting points, one of which forms may be gradually changing into the other.

The so-called "titer test" is the determination of the solidifying point of the mixed fatty acids.

\section{VIScosity}

The presence of glycerides of hydroxy-acids, whether natural, as in castor oil, or artificial, as in the "blown" oils of commerce, gives a very high viscosity compared with fatty oils in which hydroxy-acids are absent. Among the latter, viscosities vary as a rule with the melting points of the mixed fatty acids. While the viscosity may aid in the identification of fats and oils in certain cases, its determination is especially important in the examination of lubricating oils and will, therefore, be discussed in that connection.

\section{Heat of Combustion}

The heat of combustion of fats and fatty oils is a property as nearly constant as the specific gravity to which it stands. in approximately inverse proportion, being lowered by the presence of acids of low molecular weight, highly unsaturated acids, or acids containing hydroxyl groups. The heats of combustion of butter-fat, drying oils, castor oil, and blown oils are, therefore, lower than those of body fats and such fatty oils as olive, almond, peanut, or rape-seed. Waxes and hydrocarbons have higher heats of combustion than the glycerides. The same is true of the alcohols of very high molecular weight, such as cholesterol and phytosterol of which the so-called unsaponifiable matter of fats is chiefly composed. With a suitable oxygen calorimeter ${ }^{1}$ the heat of combustion can be very accurately determined and the results are often useful in verifying the conclusions drawn from other determinations. For fuller discussions see J. Am. Chem. Soc., 1896, 18, 178; 1901, 23, 164; 1902, 24, 348; 1903, 25, 659 .

1 For discussion of calorimeters and determination of heat of combustion see Chapter XII. 


\section{ALCOHOLS OF FATS AND WAXES}

\section{(UNSAPONIFIABLE MATTER)}

Under the term " unsaponifiable matter" are included all substances found in fats and waxes which are insoluble in water and do not combine with caustic alkali to form soluble soaps. In a commercially pure fat or wax the "unsaponifiable matter" consists chiefly of one or more alcohols of high molecular weight, the more important of which are mentioned below.

The common waxes yield 50 to 55 per cent of insoluble alcohol, but the total amount of unsaponifiable matter in fats is much lower, being usually less than 1 per cent in animal and less than 2 per cent in vegetable fats.

\section{Alcohols of the Series $\mathrm{C}_{n} \mathrm{H}_{2^{n+2}} \mathrm{O}$}

Cetyl alcohol, $\mathrm{C}_{16} \mathrm{H}_{34} \mathrm{O}$, occurs as palmitate in spermaceti. It is a tasteless and odorless solid, insoluble in water, but soluble in alcohol and very easily soluble in ether or benzene. Melting point $50^{\circ}$; specific gravity at $99^{\circ}, 0.7837$.

Octodecyl alcohol, $\mathrm{C}_{18} \mathrm{H}_{38} \mathrm{O}$, also occurs as ester in spermaceti. It is similar in properties to cetyl alcohol. Melting point $59^{\circ}$; specific gravity at $99^{\circ}, 0.7849$.

Myricyl alcohol, $\mathrm{C}_{30} \mathrm{H}_{62} \mathrm{O}$, occurs as palmitate in beeswax and both free and combined in carnaüba wax. It melts at $85^{\circ}$ to $88^{\circ}$ and is nearly insoluble in cold, but readily soluble in hot, alcohol.

Other alcohols of this series are known, but need not be considered here. From the examples given it will be seen that the melting points rise and the alcohols become less soluble with increasing molecular weight. The specific gravity, however, does not decrease as in the case of homologous fatty acids.

\section{Cholesterol and Related Alcohols}

Cholesterol, $\mathrm{C}_{26} \mathrm{H}_{44} \mathrm{O}$, is the characteristic constituent of the unsaponifiable matter of animal fats. It is only sparingly soluble in cold dilute alcohol or cold petroleum ether, but 


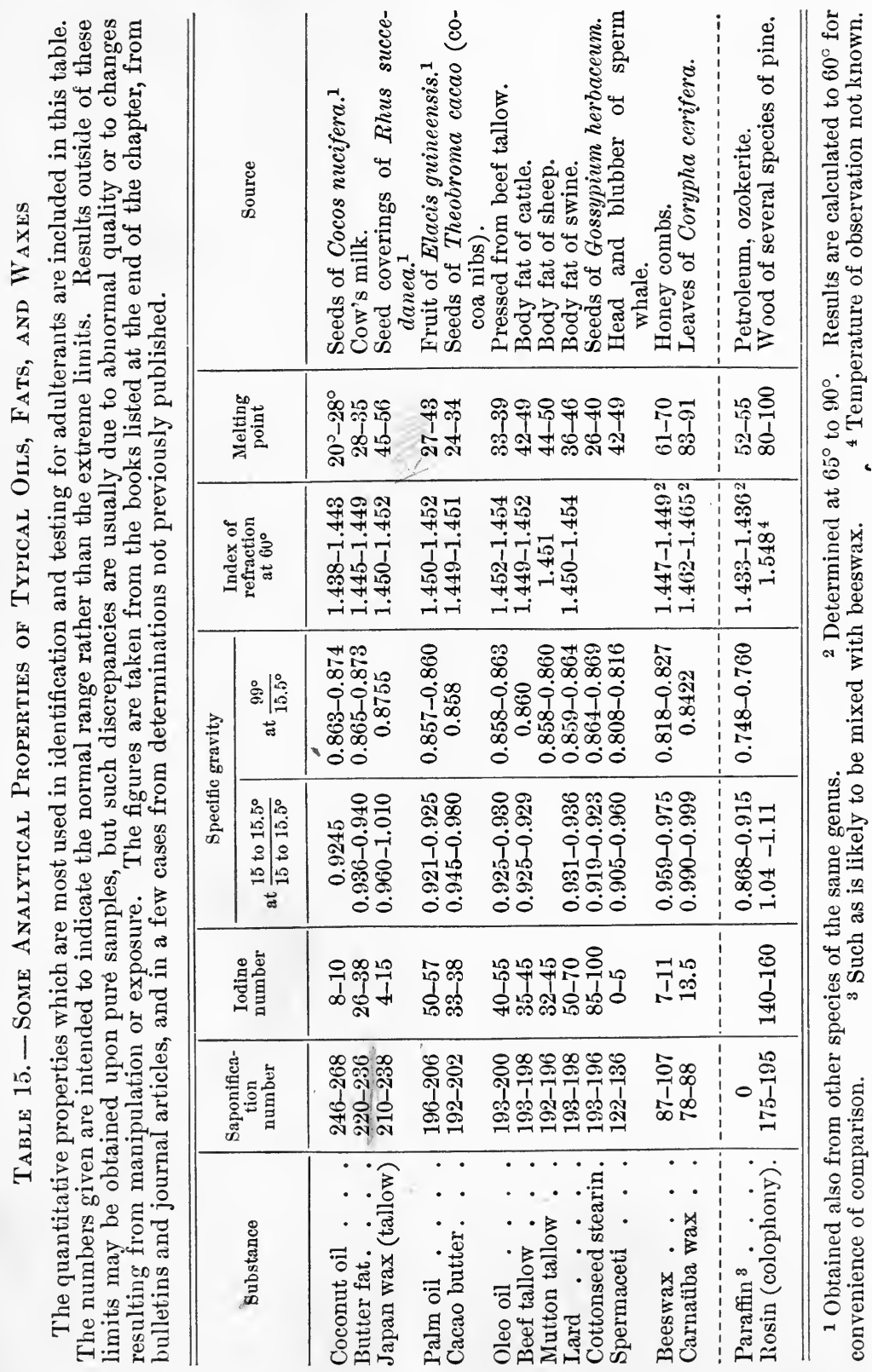




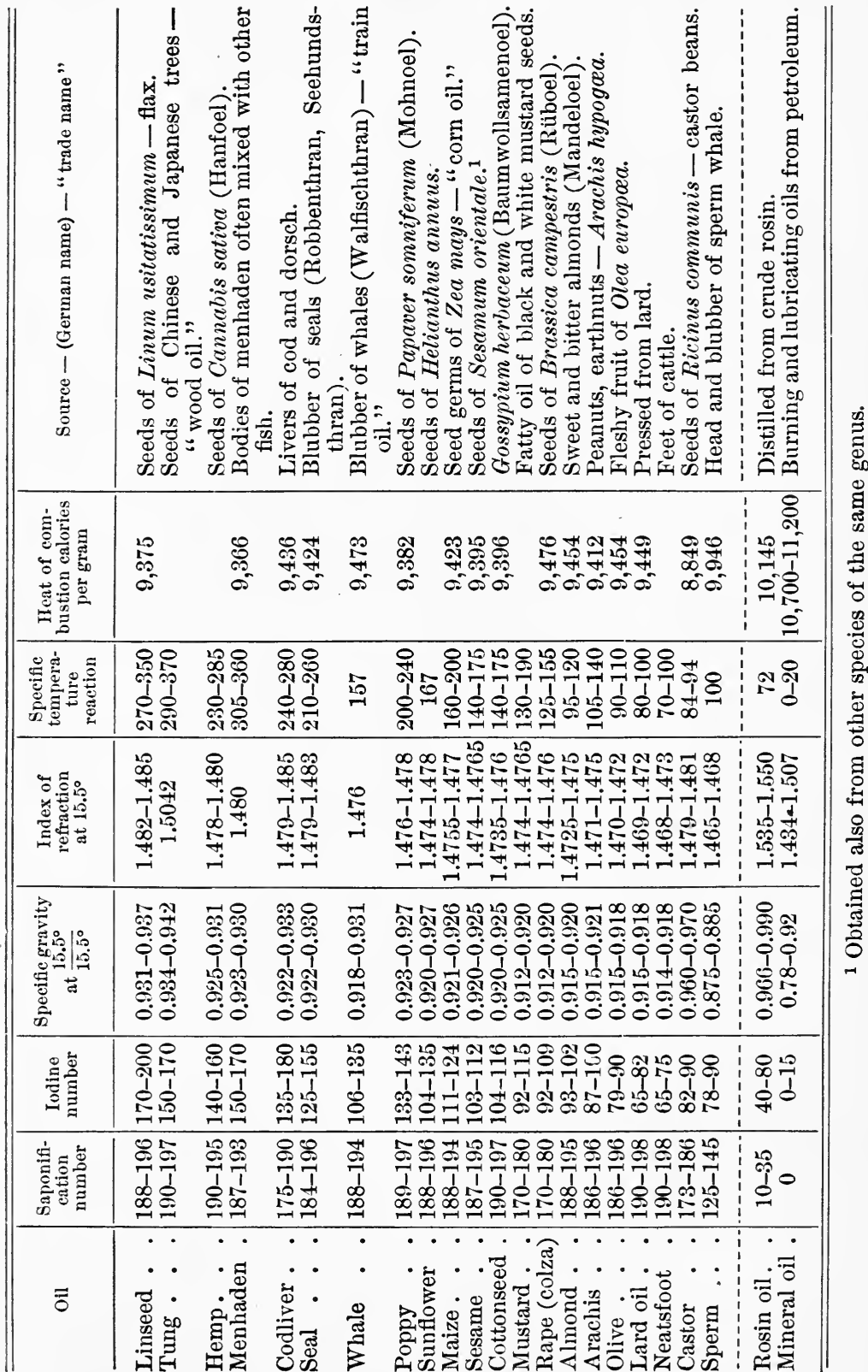


dissolves readily in ether, chloroform, carbon bisulphide, fatty oils, turpentine, or hot strong alcohol.

Phytosterol and sitosterol are alcohols, probably isomeric with cholesterol and resembling it closely in physical properties, which occur in vegetable fats, the latter being found especially in the oils of the cereal grains. The identification of one of these alcohols may, therefore, aid in determining the origin of an oil or fat or in the detection of vegetable fats present as adulterants in the more expensive fats of animal origin, as will be explained in the next chapter.

\section{REFERENCES}

\section{I}

Alder-Wright and Mitchell: Animal and Vegetable Fixed Oils, Fats, Butters, and Waxes.

Allex: Commercial Organic Analysis, Vol. II.

Benedikt and Ulzer: Analyse der Fette und Wachsarten.

GilL: Short Handbook of Oil Analysis.

Hefter: Technologie der Fette und Oele.

Hopkins : Oil-Chemists' Handbook.

LEACH : Food Inspection and Analysis.

Lewkowitsch : Chemical Technology and Analysis of Oils, Fats, and Waxes.

Laboratory Companion to Fat and Oil Industries.

LUNGE: Chemisch-technische Untersuchungsmethoden.

Ubielonde: Handbuch der Chemie und Technologie der Oele und Fette. Wright : Analysis of Oils and Allied Substances.

\section{II}

1902. Hunt : A Comparison of Methods used to determine Iodine Values of Oils. J. Soc. Chem. Ind., 21, 454.

Tolman and Munson: Refractive Indices of Salad Oils. J. Am. Chem. Soc., 24, 754.

1903. Lythgoe: Zeiss Butyro-refractometer Readings of Edible Oils and Fats. Technology Quarterly, 16, 222.

Tolman and Munson: Iodine Absorption of Oils and Fats. J. Am. Chem. Soc., 25, 244.

1905. Lythgoe: Refraction Indices of Oils. J. Am. Chem. Soc., 27, 887.

1906. Dunlap: Preparation of Aldehyde-free Ethyl Alcohol for use in Oil and Fat Analysis. J. Am. Chem. Soc., 28, 395.

Haller : Alcoholysis of Fatty Substances. Compt. rend., 143, 657, 803. 
Radcliff: Analytical Constants of Carnaüba Wax. J. Soc. Chem. Ind., 25, 158.

Schneider and Blumenfeld: Characteristics of Certain Animal Fats. Chem. Ztg., 30, 53.

Thompson and Dunlop: (Revision of Iodine Numbers). Analyst, 31, 281.

1907. Berg: Examination of Beeswax. Chem. Ztg., 31, 337.

Doss: Index of Refraction of Fats and Fatty Acids. Z. Nahr. Genussm., 13, 257.

Louis and Sauvage: New Characteristic Constant of Oils. Compt. rend., 145, 183.

Meyer: Determination of Unsaponifiable Matter. Chem. Ztg., 31, 423.

Rakusin: Optical Properties of Animal Fats. Chem. Ztg., 30, 1247.

Richmond: Temperature Corrections of the Zeiss Butyro-refractometer. Analyst, 32, 44.

Richter: Maumené Test and Iodine Number of Certain Oils. $Z$. angew. Chem., 37, 1605.

Schicht and Halpern: Estimation of Unsaponifiable Matters in Fats. Chem. Ztg., 31, 279.

Twitchell: A Reagent in the Chemistry of Fats. J. Am. Chem. Soc., 29, 566.

1908. Berg: Analytical Chemistry of Beeswax. Chem. Ztg., 32, 777.

Fahrion: Progress in Fat Analysis in 1907. Z. angew. Chem., 1908, 1125.

Halla: Preparation of Alcoholic Potassium Hydroxide. Chem. Ztg. 32, 890 .

INGLE: Notes on Wijs Solution. J. Soc. Chem. Ind., 27, 314.

1909. Bartlett and Sherman : Effect of Excess of Reagent and Time of Reaction in the Determination of Iodine Numbers of Fatty Oils. School of Mines Quarterly, 31, 55.

Bömer : Mixed Glycerides of Palmitic and Stearic Acids. Z. Nahr. Genussm., 17, 353.

Boynton and Suerman: A Comparison of the Calculated and Determined Values for the Specific Temperature Reactions of Oil Mixtures with Sulphuric Acid. School of Mines Quarterly, 31, 64.

Freudich: Analytical Methods in Stearin Manufacture. Chem. Rev. Fette-Harz.-Ind., 15, 224, 246, 277; Chem. Abs., 3, 253.

1911. Committee Report: (Methods for Moisture, Volatile Matter, Suspended Impurities, Free Fatty Acids, Unsaponifiable Matter, Metallic Soaps, and Titer Test in Fats and Fatty Oils). J. Ind. Eng. Chem., 3, 50.

Fahrion: Fat Analysis and Fat Chemistry in 1910. Z Z. angew. Chem., 24, 241. 


\section{CHAPTER IX}

\section{Edible Oils and Fats}

\section{SALAD OILS}

Most salad oils are sold as olive oil. The principal substitutes and adulterants are cottonseed, arachis (peanut), sesame, maize, poppyseed, and lard oils. Both quantitative and qualitative methods must be used in any thorough examination of an oil for adulterants. As a rule an oil should be pronounced adulterated only when quantitative determinations yield results which could not be obtained from a pure oil of normal character. Qualitative tests are usually required to show which of several possible adulterants is present. As yet certain color reactions are indispensable for this purpose, but in using these tests and interpreting the results, it must be remembered that they depend upon the presence of constituents which may be removed or destroyed by improved methods of refining, and that olive oil which has been altered by long exposure to air in loosely stoppered or partially filled vessels, or oil containing a small amount of some accidental impurity, may give a reaction which cannot be distinguished from that of the adulterant sought. The results of even the best of the color reactions must therefore be interpreted with great caution and must usually be regarded as of much less significance than the quantitative numbers.

On the other hand, a good grade of arachis oil, or a carefully prepared mixture of lard oil with one of the seed oils, can be added to olive oil in large proportion without affecting the ordinary constants to such an extent as to pass the limits which can fairly be regarded as normal. It is necessary, therefore, not only to compare each number found with the established 
limits for pure oil, but also to view the quantitative results in their relations to each other and to the indications of the qualitative tests.

While the system to be followed and the number of tests required will naturally vary in different laboratories, the following may be recommended. Determine accurately the specific gravity or the index of refraction, the iodine number, and saponification number. If an abundance of the sample is at hand, determine the specific temperature reaction and the acidity. Apply the nitric acid test, Halphen's test, one or more of the color reactions for sesame oil, and examine for arachidic acid by Renard's method. Finally, if the importance of the sample justifies the time acquired, separate and examine the unsaponifiable matter and the mixed fatty acids and determine the viscosity of the soap solution by Abraham's method described below.

\section{Analytical Properties of Olive Oil}

The numbers included in the table of "constants" already given are intended to cover the range of normal variations in oils found in the American markets. Müntz, Durand, and Milliau ${ }^{1}$ examined samples from Africa, Spain, Portugal, Greece, Turkey, and the Levant without finding any significant variation in the specific gravity, iodine number, or temperature reaction. According to Milliau, Bertainchand, and Malet, ${ }^{2}$ however, Tunis oils have high specific gravities, the average of 49 samples being 0.9183 . Tolman and Munson have recently examined a large number of olive oils many of which were of known origin. The following are taken from their results: ${ }^{3}$

The average iodine number of the oils from California is therefore higher than that of the French and Italian oils and, as might be expected, the higher iodine number is accompanied

${ }^{1}$ Bulletin du Ministêre de l'Agriculture, 1895. Quoted from Bul. 77, Bur. Chem., U. S. Dept. Agriculture.

${ }^{2}$ Bulletin de l'Agriculture et Commerce de Tunis. Quoted from Bull. 77, loc. cit.

${ }^{3}$ Bul. 77, Bur. Chem., U. S. Dept. Agriculture. 


\section{Table 16. - Avalytical Properties of Olive Oils}

\begin{tabular}{|c|c|c|c|c|c|c|}
\hline \multicolumn{2}{|l|}{ Description } & 㝕葛 & 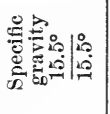 & 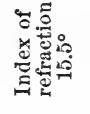 & 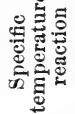 & 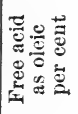 \\
\hline \multirow{3}{*}{$\begin{array}{l}\text { California oils of known origin } \\
\text { (42 samples). }\end{array}$} & Max. & 89.8 & 0.9180 & 1.4718 & 109.7 & $8.21^{1}$ \\
\hline & Min. & 78.5 & 0.9162 & 1.4703 & 94.5 & 0.20 \\
\hline & Avg. & 85.3 & 0.9170 & 1.4713 & 101.8 & 1.20 \\
\hline \multirow{3}{*}{$\begin{array}{l}\text { Italian oils of known origin } \\
\text { (17 samples). }\end{array}$} & Max. & 86.1 & 0.9180 & 1.4713 & 104.7 & 2.79 \\
\hline & Min. & 79.2 & 0.9155 & 1.4705 & 95.6 & 0.57 \\
\hline & Avg. & 81.6 & 0.9163 & 1.4709 & 99.1 & 1.11 \\
\hline \multirow{3}{*}{$\begin{array}{l}\text { Italian oils (commercial) not found } \\
\text { adulterated ( } 57 \text { samples). }{ }^{2}\end{array}$} & Max. & 84.5 & 0.9179 & 1.4712 & 108.4 & 5.30 \\
\hline & Min. & 77.5 & $0.91 \check{0} 0$ & 1.4701 & 88.4 & 0.72 \\
\hline & Avg. & 80.9 & 0.9161 & 1.4706 & 97.8 & 2.42 \\
\hline \multirow{3}{*}{$\begin{array}{l}\text { French oils (commercial) not } \\
\text { found adulterated ( } 60 \text { samples). }\end{array}$} & Max. & 85.0 & $0.9183^{3}$ & 1.4713 & 114.4 & 3.63 \\
\hline & Min. & 79.0 & 0.9150 & 1.4699 & 90.4 & 0.45 \\
\hline & Avg. & 81.3 & 0.9166 & 1.4708 & 100.1 & 1.59 \\
\hline
\end{tabular}

by a higher specific gravity, refractive index, and temperature reaction. Although individual samples will show slight variations, the same general relation is found on comparing oils which differ in iodine numbers though obtained from the same locality. Thus taking at random, from among the California oils of known origin examined by Tolman and Munson, 10 samples with high and 10 with low iodine numbers the following average figures were found:

\begin{tabular}{|c|c|c|c|c|c|c|}
\hline & & & Iodine number & $\begin{array}{c}\text { Specific gravity } \\
\frac{15.5^{\circ}}{15.5^{\circ}}\end{array}$ & $\begin{array}{l}\text { Index of } \\
\text { refraction } \\
15.5^{\circ}\end{array}$ & $\begin{array}{l}\text { Specific } \\
\text { temperature } \\
\text { reaction }\end{array}$ \\
\hline First group & . & . & 88.44 & 0.9172 & 1.4716 & 105.7 \\
\hline Second group & . & . . & 83.12 & 0.9166 & 1.4711 & 98.6 \\
\hline
\end{tabular}

1 Two samples with larger amounts of free acids were found, but were excluded from average as being unfit for use as salad oils.

${ }^{2}$ Omitting one sample containing 15.25 per cent of free acid and having a specific gravity of 0.9134 .

${ }^{3}$ Omitting one sample having an abnormally high specific gravity (0.9196) which may have been due to exposure. 
The normal relations of the constants to each other and the changes which may occur as the result of age or exposure 1 must always be taken into consideration when interpreting the results of an analysis. For records of individual samples of olive oil showing iodine numbers of 90.5 to 94.7 see Allen's Commercial Organic Analysis, Fourth Edition, II, 113.

The standard adopted by the United States Department of Agriculture is as follows :

Olive oil is the oil obtained from the sound, mature fruit of the cultivated olive tree (Olea europcea L.) and subjected to the usual refining processes; is free from rancidity; has a refractive index $\left(25^{\circ} \mathrm{C}\right.$.) not less than 1.4660 and not exceeding 1.4680 ; and an iodine number not less than 79 and not exceeding 90 .

\section{Detection of Cottonseed OrL}

The presence of cottonseed oil in olive oil raises the specific gravity, iodine number, and temperature reaction and lowers the viscosity of the soap solution. A mixture of cottonseed and lard oils may, however, be added to olive oil in large quantity without greatly affecting any but the last of these "constants." The qualitative tests for cottonseed oil are therefore of considerable importance.

\section{Halphen's Reaction ${ }^{2}$}

Dissolve 1 part of sulphur in 100 parts of carbon bisulphide and mix the solution with an equal volume of amyl alcohol.

Mix equal volumes, 2 to $3 \mathrm{cc}$. each, of the reagent and the oil to be tested and heat the test tube containing the mixture gently at first until violent boiling ceases, then in a bath of boiling saturated solution of common salt. Heat for 2 hours unless a color develops sooner. If cottonseed oil is present, the solution turns orange or red.

This is probably the most sensitive and characteristic test for

1 See section on this subject beyond.

${ }^{2}$ Halphen : Ann. chim. anal., 1898, 3, 9 ; Analyst., 1898, 23, 131 ; Bul. 107. Bur. Chem., U. S. Dept. Agriculture. 
cottonseed oil and the least liable to give unsatisfactory results in the hands of an inexperienced person. The presence of 1 or 2 per cent of unchanged cottonseed oil in olive oil is detected without difficulty. A reaction is often obtained ${ }^{\mathbf{1}}$ with lard or lard oil or even with butter fat ${ }^{2}$ from animals which have been fed upon cottonseed meal. Copac (or kapok) oil, which is closely related to cottonseed oil, gives the same reaction. When heated at $250^{\circ}$ for 10 to 20 minutes, cottonseed oil loses the property of giving this reaction. No pure olive oil has yet been known to give a similar coloration. Hence a positive result is considered conclusive, but a negative result is not. The substance to which the reaction is due cannot be removed by treatment with animal charcoal ${ }^{3}$ and is supposed to be an unsaturated acid which combines with sulphur giving a red compound. 4

\section{Nitric Acid Test}

Cottonseed oil shaken at room temperature with an equal volume of nitric acid, of 1.37 to 1.38 specific gravity, gives a brown coloration, sometimes only on standing overnight. Other seed oils give similar reactions. Normal olive oil under the same treatment shows no change of color.

The test is not so delicate as that of Halphen, but is applicable to cottonseed oil which has been heated until it no longer colors the Halphen reagent. "It may also be of value in determining whether a weak test with the latter reagent is due to a small amount of unheated cottonseed oil or to a larger amount which has been heated sufficiently to weaken the Halphen reaction (Tolman and Munson). According to Lewkowitsch this reaction cannot be relied upon to detect less than 10 to 20 per cent of American cottonseed oil in olive oil. Tolman and Munson consider the test much more delicate. A positive re-

${ }^{1}$ Soltsein : Z. öffentl. Chem., 1901, 7, 140.

${ }^{2}$ Wauters: Bull. Assoc. Belg. Chem., 13, 404 ; J. Soc. Chem. Ind., 1900, 19, 172.

${ }^{3}$ Utz : Chem. Rev. Fett.-Harz-Ind., 1902, 9, 125 ; Gill's Oil Analysis, p. 73.

${ }^{4}$ Raikow : Chem. Ztg., 1900, 24, 562, 583 ; 1902, 26, 10 . See also Halphen: Bull. Soc. Chim., 1905, [3], 33, 108. 
sult should always be confirmed by finding a high iodine number or by proving the presence of some other oil having a very low iodine number; for pure olive oil if much altered as the result of age and exposure will sometimes give a reaction which cannot be distinguished from that of cottonseed oil.

\section{Detection of Arachis (Peanut) Oil}

Arachis oil has usually a higher specific gravity and iodine number and practically always a higher temperature reaction than olive oil. The specific temperature reaction of arachis oil with concentrated sulphuric acid is usually 40 to 60 units higher than the iodine number of the same sample, whereas olive oil usually shows a difference of less than 20 and very rarely of more than 25 units between the iodine and the specific Maumené numbers. The presence of peanut oil in olive oil greatly diminishes the viscosity of the soap solution obtained on saponification. Any of these changes, however, might be due to other adulterants. Arachis oil is shown conclusively by isolating and identifying arachidic acid. This can be done with approximately quantitative results by Tolman's modification of Renard's method.

\section{Renard-Tolman Test for Arachidic Acid ${ }^{1}$}

Weigh 20 grams of oil in an Erlenmeyer flask. Saponify with alcoholic potash, neutralize exactly with dilute acetic acid, using phenolphthalein as indicator, and wash the solution into a 500 -cc. flask containing a boiling mixture of $100 \mathrm{cc}$. of water and $120 \mathrm{cc}$. of a 20 per cent lead acetate solution. Boil one minute and then cool by immersing the flask in water, occasionally giving it a whirling motion to cause the precipitated lead soaps to stick to the sides of the flask. After thorough cooling, pour off the water containing the excess of lead acetate and wash the soap with cold water and then with 90 per cent alcohol. After pouring off the alcohol as completely as

${ }^{1}$ Renard : Compt. rend., 1871, 73, 1330.

Tolman: U. S. Dept. Agriculture, Bur. Chem., Bul. 81, p. 64. See also Archbutt: J. Soc. Chem. Ind., 1898, 17, 1124. 
possible, add 200 cc. of ether, cork the flask and allow to stand until the soap is disintegrated, then connect with a reflux condenser, heat gently to boiling, and boil for 5 minutes on a safety water bath or an electric heater. Cool to $15^{\circ}$ and allow to stand overnight.

Filter, wash the residue thoroughly with ether, and then transfer it from the filter to the flask by means of a stream of hot water acidified with hydrochloric acid. Add an excess of dilute hydrochloric acid and $200 \mathrm{cc}$. of hot water and heat until the fatty acids separate as a clear oily layer. Nearly fill the flask with hot water and allow to stand at room temperature until the layer of fatty acids has completely separated and solidified. Remove and drain the cake of fatty acids, wash again with hot water, then dissolve in 100 cc. of boiling alcohol, 90 per cent by volume. Cool the solution to $15^{\circ}$, shaking frequently, and allow it to stand as long as any acid continues to crystallize out, or overnight, at a temperature not above $20^{\circ}$. Filter, wash the crystals twice with $10 \mathrm{cc}$. of 90 per cent alcohol, noting the total volume of filtrate and washings, and then with alcohol, 70 per cent by volume (in which the crystals are practically insoluble). Dissolve the crystals by means of hot absolute alcohol in a weighed dish, evaporate, dry, and weigh. To this weight add 0.0045 gram for each $10 \mathrm{cc}$. of 90 per cent alcohol in the filtrate and washings if the temperature of filtration was $20^{\circ}$; or 0.0025 gram for each $10 \mathrm{cc}$. if the temperature was $15^{\circ}$.

The melting point of arachidic acid obtained in this way is $71^{\circ}$ to $73^{\circ}$. According to Tolman and Munson ${ }^{1}$ the determination of the melting point must not be neglected since cottonseed and lard oils have been found to give crystals resembling arachidic acid in appearance, but having a lower melting point. Tolman finds that from 5 to 10 per cent of the oil can be detected by this method. On the usual assumption that the oil yields 5 per cent of the acid, ${ }^{2}$ each centigram found as described

1 U. S. Dept. Agriculture, Bur. Chem., Bul. 77, p. 35.

2 This is probably more nearly a maximum than an average yield. Tolman and Munson (loc. cit.) obtained from 3.41 to 4.24 per cent. 
above (using 20 grams of oil) indicates 1 per cent of arachis (peanut) oil in the sample.

Archbutt (Allen's Commercial Organic Analysis, 4th Ed., II, 99-100) recommends the following method of Bellier ${ }^{1}$ as giving satisfactory qualitative results.

Solutions. - (1) Alcoholic potassium hydroxide, made by dissolving 8.5 grams pure potassium hydroxide in 70 per cent alcohol and making up to $100 \mathrm{cc}$.

(2) Acetic acid of such strength that 1.5 cc. will exactly neutralize $5 \mathrm{cc}$. of the potash solution (about 28-29 per cent of actual acetic acid).

Test. - Weigh 1 gram of sample into a dry test tube, add 5 cc. of the potash solution, and boil gently, avoiding evaporation, over a free flame until saponification is complete, probably a little over 2 minutes; then add 1.5 cc. acetic acid, or just sufficient to neutralize the alkali, mix well, cool rapidly in water at $17^{\circ}$ to $19^{\circ}$, and let stand at this temperature for at least 30 minutes, shaking occasionally; then add 50 cc. 70 per cent alcohol containing 1 per cent by volume of hydrochloric acid of $1.16 \mathrm{sp}$. gr., shake well, and again place in the cold water for 1 hour. In the absence of arachis oil the liquid should remain clear or become merely opalescent, while if the sample contained more than 10 per cent of arachis oil a flocculent, crystalline precipitate is obtained.

\section{Detection of Sesame OIL}

Sesame oil affects the usually determined constants in the same way as cottonseed oil and to practically the same extent. The color reactions are usually considered quite characteristic.

\section{Baudouin's Test}

Dissolve 0.1 gram of sugar in $10 \mathrm{cc}$. of hydrochloric acid of 1.18 to 1.20 specific gravity and add $20 \mathrm{cc}$. of the oil. Shake thoroughly in a test tube for one minute and allow to stand. The water solution separates quickly and shows a distinct red 
or crimson color if the sample contains 1 per cent or more of sesame oil. The active reagent is probably furfural formed by the action of the acid upon the sugar.

Villivecchia's modification consists in shaking $10 \mathrm{cc}$. of the oil with $10 \mathrm{cc}$. of hydrochloric acid (1.20 sp. gr.) to which has been added 0.1 cc. of a 2 per cent solution of furfural in 95 per cent alcohol.

Olive oils of known purity have usually been found to give only a slight pink color, but sometimes the reddening of the water solution is so pronounced as to cause confusion with that produced by a small amount of sesame oil. Check experiments therefore should always be made. If much sesame oil is present, the red color should be perceptible in the oily layer as well as in the water solution.

\section{Tocher's Test}

Dissolve 1 gram of pyrogallol in 15 cc. of concentrated hydrochloric acid. Shake this solution with $15 \mathrm{cc}$. of oil in a separatory funnel and allow to stand for 1 or 2 minutes. Draw off the aqueous solution and boil for 5 minutes. The presence of sesame oil is indicated if the solution after boiling appears red by transmitted and blue by reflected light.

This test has not been so generally used nor so thoroughly studied as the preceding. The Association of Official Agricultural Chemists authorize the use of either Baudouin's, Villivecchia's, or Tocher's test for the detection of sesame oil in edible oils and fats.

\section{Detection of Maize, Poppyseed, and Lard Oils}

Each of these oils has a characteristic odor or taste, the odor of lard oil being intensified by heating. These properties, however, cannot be relied upon, as the substances to which they are due can be almost entirely eliminated in the refining process. The effect of maize or poppy oil in raising the iodine number would be very noticeable, but might be neutralized by the addition of a somewhat greater quantity of lard oil. The difference between the iodine number and the specific tem- 
perature reaction would be appreciably greater in such a mixture than in pure olive oil (compare detection of arachis oil). Maize and poppyseed oils react with nitric acid, giving brown colors similar to that produced by cottonseed oil. Lard oil often gives the same reaction and might be indicated by the melting point of the fatty acids (those of lard oil having a relatively high melting point, $33^{\circ}$ to $38^{\circ}$ according to Tolman and Munson) or by the character of the unsaponifiable matter - phytosteryl acetate test. ${ }^{1}$ (See references at the end of this chapter.)

All of these oils (as well as most others) yield soap solutions of much lower viscosity than those obtained from pure olive oils. $^{2}$ The determination and significance of this property has been studied in some detail ${ }^{3}$ and the method has been found capable of giving valuable results, especially if the conditions worked out by Abraham are carefully observed. For a full discussion of these conditions the original paper must be consulted. The essential features of the process are as follows :

\section{Abraham's Modification of Blasdale's Viscosity Test}

The saponification number having been determined, weigh 3 grams of oil in an accurately graduated 100-cc. flask, add 2 cc. of alcohol and an amount of standard potash solution sufficient to saponify the oil and leave an excess of 0.625 gram of potassium hydroxide. Close the flask with a stopper having a Kroonig valve and saponify on a water bath. After saponification expel the alcohol by warming and allowing air freed from carbon dioxide to pass through the flask, while a partial vacuum is maintained by means of a suction pump. In this way the alcohol is entirely removed in 5 to 10 minutes. Evaporation should not be carried to complete dryness. Without allowing the flask to cool, add $50 \mathrm{cc}$. of hot water, rotate gently

1 Bömer : Z. Nahr.-Genussm., 1901, 4, 1091.

2 Blasdale : J. Am. Chem. Soc., 1895, 17, 937. 977.

${ }^{3}$ Abraham : Ibid., 1903, 25, 968. Sherman and Abraham : Ibid., 1903, 25, 
until a homogeneous solution of the soap is obtained, cool to $20^{\circ}$, fill to the mark with distilled water, and mix well by shaking or by repeatedly pouring the solution from one flask to another. Determine the viscosity of the solution in an Ostwald viscosimeter immersed in water kept at $20^{\circ}$. Care must be taken to avoid the introduction of air bubbles into the viscosimeter and to maintain the exact temperature. Repeat the readings until five or more concordant results are obtained. The viscosimeter is standardized by means of distilled water, and it is advisable to select for this work an instrument in which the time of flow of water is about 100 seconds. Successive readings of a soap solution should then agree within 2 seconds.

The viscosity is calculated as follows:

where

$$
v_{1}=100\left(\frac{s_{1}}{t}\right) t_{1}
$$

$v_{1}=$ the viscosity number.

$t_{1}=$ the time of flow (in seconds).

$s_{1}=$ specific gravity of the solution.

$t=$ time of flow of distilled water.

The viscosity numbers obtained by this method were:

\begin{tabular}{|c|c|c|c|c|c|c|}
\hline live oil of known purity ( & $9 \mathrm{sam}$ & nples) & & & & \\
\hline Olive oil of doubtful purity & $(4 \mathrm{sa}$ & amples) & & . & & $145.8-165.8$ \\
\hline Lard oil (5 samples) . & & & . & & & $122.9-135.0$ \\
\hline Arachis (peanut) oil (1 sam & aple) & & & & & 126.6 \\
\hline Cottonseed oil (3 samples) & & & & & & $126.6-127.9$ \\
\hline Rapeseed oil (3 samples) & & & & & & $124.7-125.7$ \\
\hline sample) . & & . & & & & 139.2 \\
\hline & . & & & & & 126.6 \\
\hline Poppyseed oil (1 sample) & & & & & & 123.9 \\
\hline
\end{tabular}

Mixtures of olive and lard oils gave viscosity numbers agreeing closely with those obtained by calculation, but cottonseed or arachis oil when added to olive oil lowered the viscosity to a much greater extent than would have been predicted, indicating that the viscosity number is a more useful means of detecting adulteration than appears from a comparison of 
the results obtained on testing the olive oil and its adulterants separately.

The usefulness of the method for testing isolated samples is limited by the fact that comparable results can be obtained only under strictly uniform conditions, the viscosity of the soap solution being greatly influenced by slight variations in strength, alkalinity, or temperature, while the terms in which the results are expressed will naturally vary with the form of viscosimeter used; but in cases of sufficient importance to justify the time required to arrange the apparatus and make comparative determinations, the "viscosity number" will be found an important factor in the examination of olive oil for adulterants.

\section{BUTTER}

Butter is officially defined ${ }^{1}$ as " the product made by gathering in any manner the fat of fresh or ripened cream into a mass which also contains a small portion of the other milk constituents, with or without salt." Standard butter contains not less than 82.5 per cent of butter fat, having a Reichert-Meissl number not less than 24 and a specific gravity not less than 0.905 at $40^{\circ} / 40^{\circ}$. By acts of Congress approved August 2, 1886, and May 9, 1902, butter may also contain additional coloring matter.

Butter may fail to meet the requirements of the official standard either because of a deficiency in the percentage of fat or because of the presence of foreign fat or butter fat of abnormal character, though the sample may contain nothing which can be regarded as unwholesome. Butter analysis therefore includes (1) the examination of the whole butter, (2) the examination of the butter fat.

The methods of examining butter fat will be given fully below, but for convenience the determination of water, fat, curd, and ash will be described first. The point at which tests for preservatives can conveniently be made will be indicated, but the detection of foreign colors will not be considered, as these are not regarded as adulterants.

${ }^{1}$ Circular No 19, Office of the Secretary, U. S. Dept. Agriculture. 
Determination of Water, Fat, Curd, and Ash

Especial care must be taken in sampling butter, since the water, salt, and curd are often unevenly distributed and an attempt to mix by stirring is apt to result in squeezing out drops of brine. If a large quantity is to be sampled, a butter trier should be used, and the portions thus drawn united until a working sample of about 500 grams is obtained.

Melt the sample at the lowest possible temperature in a widemouthed glass-stoppered bottle, shake violently to insure a homogeneous mixture, and continue the shaking while cooling the sample until it is thoroughly solidified. Great care is necessary here to prevent a separation of water and fat.

Thoroughly clean and dry a lipped dish or beaker having a flat bottom of at least 20 square centimeters. Weigh the dish, introduce 1.5 to 2 grams of butter, and reweigh quickly to avoid evaporation. Dry to constant weight in a boiling water oven. The loss is water. Treat the dry residue with petroleum ether or benzine, transfer it to a weighed Gooch crucible having a felt of ignited asbestos, and wash with the solvent until all fat is removed. Dry the crucible and residue to constant weight in a water oven or an air bath not above $110^{\circ}$. The material dissolved by the petroleum ether or benzine is fat. Burn the curd at a temperature below a red heat and weigh the crucible containing the ash.

Notes. - The method given is essentially that of the Association of Official Agricultural Chemists. ${ }^{1}$ If preferred the butter can be dried on clean dry sand or asbestos. When the latter is not used, it is important that the butter form only a very thin layer on the bottom of the dish or beaker ; otherwise the water sinks into the melted fat and is only very slowly expelled at the temperature of the boiling water oven. For convenience in transferring and washing the residue with petroleum ether the latter should be used in a small wash bottle having a ground glass stopper. Instead of drying in a dish and transferring the residue to a crucible, the butter may. be weighed directly in a

1 Bul. 107, Bur. Chem., U. S. Dept. Agriculture. 
crucible two thirds filled with fibrous asbestos, dried to constant weight, and then extracted. ${ }^{1} \quad$ A device for facilitating this drying by passing a current of air through the heated crucible has been described by Bird. ${ }^{2}$ References to a number of other quick methods for moisture in butter will be found at the end of the chapter.

Great care must be taken to burn the curd at the lowest temperature possible in order to avoid loss of chlorine (see Chapter XVII). Any small amount of milk sugar which the butter might contain would be counted as curd in this analysis. The percentage of salt can be found by determining chlorine in the ash, or by repeatedly washing the butter with hot water in a separatory funnel and titrating the combined washings with a standard solution of silver nitrate. Good butter usually contains 10 to 14 per cent water, 84 to 87 per cent fat, 0.5 to 1.5 per cent curd, 2.0 to 4.0 per cent ash if salted; if unsalted, 0.25 to 0.5 per cent. The water content of butter has often been limited by legal or trade standards to 16 per cent. This is considerably above the present average for creamery butter, as shown by an investigation made by the United States Department of Agriculture in 1902.3 Of 800 samples from 400 creameries in 18 states, the average water content was 11.78 per cent; 85 per cent of the samples contained between 10 and 14 per cent; the extreme limits were 7.20 and 17.62 per cent. The amount of salt added to butter varies greatly with the demands of different markets, but over 5 per cent would be excessive unless the butter were intended for export to a tropical country. An excessive amount of curd indicates careless manufacture or fraudulent increase of weight and is likely to injure the keeping qualities of the butter.

1 Richards and Woodman: Air, Water, and Food (2d Ed.), 201.

${ }^{2} \mathrm{~J}$. Am. Chem. Soc., 1905, 27, 818.

${ }^{3}$ Circular No. 39, Bureau of Animal Industry ; Analyst, 1903, 28, 184. 


\section{Examination of Butter Fat}

\section{Preparation}

Melt 100 grams or more of the butter and allow it to stand at $45^{\circ}$ to $55^{\circ}$ until the water and salt settle to the bottom. ${ }^{1}$ Pour off the melted fat by decantation and filter it through a dry paper in a funnel heated by a water jacket or supported in a drying oven kept at about $60^{\circ}$. The filtered fat, which must be free from turbidity, is received in a wide-mouthed bottle and kept stoppered in a cool place until analyzed.

\section{Reichert-Meissl or Reichert-Wollny Number ${ }^{2}$}

This number is a comparative measure of the proportion of volatile acids, and is the most important basis for deciding the purity of butter fat. Often the presence or absence of foreign fat in butter, or the proportion of butter fat in oleomargarine, is inferred from this number alone. In the interest of uniformity of results official chemists have sought to describe the method in such detail as to eliminate variations due to manipulation.

Reagents. - 1. Caustic soda solution, made by dissolving sodium hydroxide (nearly free from carbonate) in an equal weight of distilled water.

2. Alcohol, 92 to 95 per cent, containing no appreciable amount of volatile acid, either free or combined.

3. Dilute sulphuric acid, made by mixing pure concentrated sulphuric acid with four times its volume of water.

4. An accurately standardized approximately tenth-normal solution of barium (or sodium) hydroxide.

5. A 1 per cent solution of phenolphthalein in alcohol.

Determination. - Thoroughly clean and dry a flask of 250 to

1 This water solution can be tested for preservatives, of which boric acid and borax are most likely to be found in butter. For methods see Chapter XVIII.

${ }^{2}$ Reichert: Z. anal. Chem., 1879, 18, 69. Meissl: Dingler's polytech. Journ., 1879, 233, 229. Wollny : Milch Ztg., 1887, 16, 609; Analyst, 1887, 12, 203, $235 ; 1888,13,8,38$. These papers are reprinted in Ephraim's Original Arbẻiten über Analyse der Nahrungsmittel. 
300 cc. capacity Weigh the flask, thoroughly mix the melted fat, introduce 5.6 to $5.8 \mathrm{cc}$. measured at about $50^{\circ}$, allow the flask and fat to cool for 15 to 20 minutes, and reweigh (or, if convenient, weigh exactly 5 grams of fat into the flask). Add $10 \mathrm{cc}$. of the alcohol and $2 \mathrm{cc}$. of the caustic soda solution, attach the flask to a reflux condenser, and boil on a water bath or electric heater for at least half an hour to insure complete saponification. ${ }^{1}$

Evaporate the alcohol by heating the flask in a steam bath, shaking occasionally to avoid danger of loss from frothing and to facilitate the removal of the alcohol. Add $132 \mathrm{cc}$. of recently boiled distilled water, warm at $60^{\circ}$ to $70^{\circ}$ until the soap is completely dissolved, add $8 \mathrm{cc}$. of the dilute sulphuric acid and a few pieces of pumice stone, re-stopper the flask or connect it with a condenser, and warm without boiling until the fatty acids separate as a clear layer. Distill ${ }^{2}$ through a glass condenser, collecting the distillate in a flask accurately graduated at $110 \mathrm{cc}$. The distillation should be so regulated that $110 \mathrm{cc}$. will be collected in from 28 to 32 minutes. Mix the distillate, filter through dry paper, and titrate $100 \mathrm{cc}$. of the filtrate, using 0.5 cc. of the phenolphthalein solution as indicator, until the red color remains apparently unchanged for 2 minutes. Increase the burette reading by one tenth (on account of the $10 \mathrm{cc}$. of distillate not titrated), and calculate the number of cubic centimeters of tenth-normal alkali which would have been required if exactly 5 grams of fat were taken for the determination. This is the Reichert-Meissl number.

Notes. - The number thus found does not represent the total volatile acids present. The yield is fairly uniform if the given conditions of dilution and distillation are maintained. Wollny submitted this method to an exhaustive examination and pointed out the following sources of error : (1) absorption of

1 The saponification can also be accomplished by heating in a closed flask, using either aqueous or alcoholic alkali, or by means of the glycerol-soda solution proposed by Leffmann and Beam : Analyst, 1891, 16, 153.

2 In ordinary work a form of apparatus similar to that shown in Fig. 1, Chapter I, may be used. 
carbon dioxide during saponification, (2) formation of esters during saponification, (3) formation of esters during distillation, (4) coherence of fatty acids during distillation, resulting in holding back some of the volatile acid, (5) variations in the proportion of volatile acid carried over, due to differences in size and shape of distillation apparatus. In order to avoid discrepancies from these and other causes he published an elaborately detailed system of manipulation and precautions. It has been shown that Wollny greatly overestimated the probable errors of the method as previously carried out and that some of his precautions are unnecessary; but as in the main they tend toward greater uniformity of results, they have been adopted with slight modifications by the Association of Official Agricultural Chemists, whose methods ${ }^{1}$ are usually accepted as standard in the United States and should be followed exactly in any determination which is likely to be made the basis of legal action.

In Great Britain, a joint committee representing the Government Laboratory and the Society of Public Analysts has adopted the method essentially as described above with the following specifications for the apparatus to be employed: ${ }^{2}$ Flask used for saponification and distillation: capacity, $300 \mathrm{cc}$; length of neck, 7 to $8 \mathrm{~cm}$.; width of neck, $2 \mathrm{~cm}$. The flask is connected with the condenser by means of a bent glass tube $7 \mathrm{~mm}$. wide, so placed that the bend is $15 \mathrm{~cm}$. above the top of the cork. At a distance of $5 \mathrm{~cm}$. above the cork is a bulb $5 \mathrm{~cm}$. in diameter. The flask is supported on a circular piece of asbestos $12 \mathrm{~cm}$. in diameter, having a hole $5 \mathrm{~cm}$. in diameter in the center, so that the bottom of the flask is heated by a free flame during the distillation. The British committee further prescribed that blank determinations be made and the volume of alkali found necessary to neutralize the distillate (which volume should not exceed 0.3 cc.) be deducted in calculating the results of each determination. The number so obtained is called the ReichertWollny number.

1 Bul. 107, Bur. Chem., U. S. Dept. Agriculture.

${ }^{2}$ Analyst, 1900, 25, 309. 
The Reichert-Meissl or Reichert-Wollny number of butter fat is usually between 24 and 34 ; that of coconut fat, between 6 and 8 ; of other edible fats and oils, usually less than 1.

\section{Specific Gravity}

The specific gravity of butter fat has often been determined either at $100^{\circ}$ or at $37.8^{\circ}\left(100^{\circ} \mathrm{F}\right.$.). The standard recently established for the United States prescribes a minimum specific gravity at $40^{\circ}$, water at the same temperature being taken as unity. Either a specific gravity flask or an Ostwald pyknometer can be used conveniently for the determination, the pyknometer being filled and adjusted while surrounded by water kept at the required temperature, then removed from the water bath, wiped dry on the outside, allowed to cool to the temperature of the balance, and weighed.

\section{Saponification Number}

This is determined as described in Chapter VIII. Since the normal saponification numbers of butter fat are only about 15 per cent in excess of those of the fats commonly used as adulterants, the determination in order to be of much value must be very accurately made.

\section{Insoluble Fatty Acids - Hehner Number}

Reagents. - 1. The alcoholic potash solution used in the determination of the saponification number.

2. Alcohol, about 95 per cent by volume, which leaves no appreciable residue upon evaporation.

Determination. - Saponify 4 grams of butter fat with $50 \mathrm{cc}$. of the alcoholic potash solution, evaporate to a paste to expel alcohol, dissolve the soap in about $400 \mathrm{cc}$. of hot water in a weighed beaker, add hydrochloric acid in excess of the amount required to neutralize the potash used, and heat nearly to boiling with occasional stirring until the fatty acids have collected in a clear layer on the surface. Cool thoroughly, pour the solution through a filter, and wash the cake with cold water with- 
out removing it from the beaker. Stir up the fatty acids in the beaker with another portion of hot water (200 to 300 cc.), cool thoroughly, filter, and wash again. Repeat this treatment three times. After a final thorough washing with cold water, put the beaker containing the fatty acids beneath the funnel and dissolve any fatty acids which the filter may contain by washing with hot 95 per cent alcohol, allowing the washings to run into the beaker. Evaporate off the alcohol and dry the beaker containing the fatty acids to constant weight in a boiling water oven.

Notes. - This is the modification of Hehner's method adopted by the Association of Official Agricultural Chemists ${ }^{1}$ and by the chemists of the Government Laboratory, London. ${ }^{2}$ The original method, ${ }^{3}$ which is still largely used, involves washing the melted fatty acids with hot water on a paper filter. The results thus obtained are usually 1 to 2 per cent lower than those by the official method. In the hot filtration method there is danger of washing some of the melted fatty acid through the paper.

\section{Iodine Number}

The determination of the iodine number has been fully described in Chapter VIII. As butter fat absorbs only 26 to 38 per cent of its weight of iodine, a gram of sample can be used for each determination. According to Patrick, ${ }^{4}$ butter fat shows iodine numbers about 1 unit higher by the Hanus than by the Hübl method.

\section{Melting Point - Wiley's Method ${ }^{5}$}

Apparatus and Reagents. - 1. An accurate thermometer reading to 0.1 degree.

1 Bul. 107, Bur. Chem., U. S. Dept. Agriculture.

2 Thorpe: J. Chem. Soc., 1904, 85, 248.

${ }^{3}$ Hehner and Angell : Butter, its Composition and Adulterations. London, 1874. Hehner : Z. anal. Chem., 1877, 16, 145. Ephraim, loc. cit.

${ }^{4}$ U. S. Dept. Agriculture, Bur. Chem., Bul. 81, p. 49.

5 Wiley's Agricultural Analysis, Vol. III ; Bul. 107, Bur. Chem., U. S. Dept. Agriculture. 
2. A tall beaker nearly filled with water and arranged to be heated gradually with constant stirring from bottom to top.

3. A wide test tube suspended in the water in the beaker and nearly filled with water and alcohol as follows: Half fill the tube with hot recently boiled distilled water, then pour a nearly equal volume of hot recently boiled alcohol into the tube, carefully floating the alcohol on the water with as little mixing of the liquids as possible.

Determination. - Allow a drop of the melted butter fat to fall upon a smooth piece of ice floating in recently boiled distilled water. A thin disk of fat about $1 \mathrm{~cm}$. in diameter should be obtained. Remove the disk from the ice by forcing the latter below the water when the fat will come to the surface whence it is removed by means of a steel spatula or knife blade and dropped into the tube containing the water and alcohol. The disk sinks to the point where the density of the alcoholwater mixture is equal to its own. It must not touch the side of the tube. Suspend the thermometer so that the bulb hangs in the tube exactly level with the disk of fat. Gradually heat the beaker, keeping the water well stirred. After the disk begins to shrivel, indicating that the temperature is within a few degrees of the melting point, the heat must be applied very carefully. The temperature at which the fat becomes a sphere is taken as the melting point. Repeat the determination twice, heating the bath at such a rate that 8 to 10 minutes are required to raise the temperature through the last 2 degrees. The second and third determinations should agree within $0.2^{\circ}$.

Notes. - The special advantage of this method is that it avoids the discrepancies caused by the adherence of the melting fat to solid surfaces, which in most other methods makes it difficult to determine the exact point of fusion. It is important to secure a very thin disk of fat for the determination, to avoid all adherence of air bubbles, and to secure uniform heating of the thermometer bulb and the disk by occasionally swaying the former around the latter as the temperature approaches the 
melting point. By using hot recently boiled water and alcohol in preparing the test tube for the determination the danger of air bubbles is avoided.

\section{Additional Determinations}

The index of refraction and Crismer's test ${ }^{1}$ based upon the critical temperature of solution in alcohol are considerably used in the examination of butter fat. They are especially useful where rapid "sorting tests" are required, as in food inspection laboratories, where only the suspected samples are submitted to complete examination.

The phytosteryl acetate test ${ }^{2}$ is occasionally employed as a means of detecting the presence of vegetable fat, but requires too much time and skill for routine use and is liable to give misleading results in the hands of an inexperienced person.

The chief vegetable fats in use as butter substitutes are cottonseed oil, the methods for which have already been described, and coconut oil, whose detection is discussed below. (See also the references at the end of this chapter.)

Acidity of butter fat is sometimes determined and interpreted as a measure of the rancidity, although the odor and taste which cause a butter to be regarded as rancid are more largely due to aldehydes and other decomposition products than to free fatty acids. On the assumption that acidity can serve as a measure of rancidity, the term degree of rancidity is sometimes used as synonymous with degree of acidity, i.e. to show the number of cubic centimeters of normal alkali required to neutralize the free acid in 100 grams of fat. One "degree" is, therefore, equivalent to an acid number of 0.56 or to 0.28 per cent of free oleic acid.

1 Crismer: Bull. Assoc. belge. Chim., 1895, 9, 71; 1896, 10, 312; Abs. Analyst., 1895, 20, 209 ; 1897, 22, 71. See also Weiss: Pharm. Ztg., 41, 268 ; Chem. Centrbl., 1896, I, 1212. Asboth: Chem. Ztg., 1896, 20, 685. Browne: J. Am. Chem. Soc., 1899, 21, 990.

2 Bömer : Z. Nahr.-Genussm., 1901, 4, 865, 1070 ; 1902, 5, 1018. Juckenack and Pasternack : Ibid., 1904, 7, 193. Gill and Tufts : J. Am. Chem. Soc., 25, 251, 254, 498. Tolman: J. Am. Chem. Soc., 27, 589. Lewkowitsch: Oils, Fats, and Waxes, 4th Ed., p. 473. 


\section{Composition of Butter Fat}

Browne analyzed the mixture of fatty acids from a sample having a rather low iodine number (29.28) with the following results : 1

\begin{tabular}{|c|c|c|c|c|c|c|c|c|c|c|c|c|c|c|}
\hline & \multicolumn{12}{|c|}{ Acids } & \multirow{2}{*}{$\begin{array}{c}\begin{array}{c}\text { Percentage of acid } \\
\text { in fat }\end{array} \\
32.50\end{array}$} & \multirow{2}{*}{$\begin{array}{c}\begin{array}{c}\text { Corresponding per- } \\
\text { centage of tri- } \\
\text { glyceride }\end{array} \\
33.95\end{array}$} \\
\hline Oleic . . & . & . & . & - & - & . & - & . & - & - & . & • & & \\
\hline Dioxystearic & & . & . & . & . & . & . & . & . & - & . & - & 1.00 & 1.04 \\
\hline Stearic . & - & . & - & . & . & - & . & . & - & . & . & . & 1.83 & 1.91 \\
\hline Palmitic & . & . & . & . & . & - & . & . & - & - & . & - & 38.61 & 40.51 \\
\hline Myristic & . & . & . & . & . & . & . & . & . & . & . & - & 9.89 & 10.44 \\
\hline Lauric . & . & . & . & . & . & . & . & . & . & . & . & . & 2.57 & 2.73 \\
\hline Capric . & . & . & . & . & . & - & . & . & • & ${ }^{\circ}$ & . & . & 0.32 & 0.34 \\
\hline Caprylic & . & . & . & . & . & . & . & . & - & • & . & - & 0.49 & 0.53 \\
\hline Caproic. & . & . & . & . & . & • & . & - & - & • & . & - & 2.09 & 2.32 \\
\hline Butyric . . & . & . & . & . & . & . & . & . & - & - & . & . & 5.45 & 6.23 \\
\hline Total & . & . & . & . & . & . & . & . & . & . & . & . & $\overline{94.75}$ & $\overline{100.00}$ \\
\hline
\end{tabular}

This calculation neglects the unsaponifiable matter, which according to Browne amounts to only about 0.1 per cent.

The composition of butter fat is, however, quite variable, as will be seen from the range in analytical properties.

\section{Variations And Relations of Analytical Properties}

The Reichert-Meissl or Reichert-IVollny number is much the most important of the data obtained in the examination of butter fat. The proportion of volatile acids tends to decrease as the period of lactation advances. The estimated normal range for the other important properties has been given in the table at the end of Chapter VIII.

Any of these properties may be influenced by the feeding or health of the animal and occasionally vary much beyond the usually accepted "normal" limits, as is shown by the following data collected by Browne: ${ }^{2}$

\footnotetext{
1 J. Am. Chem. Soc., 1899, 21, 823.

2 J. Am. Chem. Soc., 1899, 21, 632.
} 


\begin{tabular}{|c|c|c|}
\hline & $\begin{array}{l}\text { General } \\
\text { limits }\end{array}$ & Extreme limits \\
\hline Reichert-Meissl number & $20-33$ & $11.2[$ Morse $]-41[$ Nilson $]$ \\
\hline Saponification number . & $220-236$ & $216[$ Samelson $]-245[$ Fischer $]$ \\
\hline Iodine number . . . & $26-38$ & 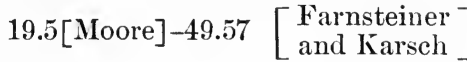 \\
\hline
\end{tabular}

The results of analyses of 357 authentic samples of butter fat collected from various parts of Great Britain and examined at the Government Laboratory, London, have been arranged by Thorpe ${ }^{1}$ according to the Reichert-Wollny numbers and averaged by groups to show the relations between the principal physical and chemical properties of pure butter fat.

The following table shows the averages for each group of samples. The first line, for example', gives the average Reichert-Wollny number for all samples in which this number lay between 22.00 and 22.99, with the average of the same samples for each of the other determinations. The second line shows the averages for all samples having Reichert-Wollny numbers from 23.00 to 23.99 , etc.

Table 17. - Relation of Physical and Chemical Properties of Butter fat (Thorpe)

\begin{tabular}{c|c|c|c|c|c|c}
\hline \hline $\begin{array}{c}\text { Number } \\
\text { of } \\
\text { samples }\end{array}$ & $\begin{array}{c}\text { Reichert- } \\
\text { Wollny } \\
\text { number }\end{array}$ & $\begin{array}{c}\text { Specific } \\
\text { gravity } \\
\frac{37.8^{\circ}}{37.5^{\circ}}\end{array}$ & $\begin{array}{c}\text { Butyro- } \\
\text { refractometer } \\
\text { reading } \\
\text { at } 45^{\circ}\end{array}$ & $\begin{array}{c}\text { Saponification } \\
\text { number }\end{array}$ & $\begin{array}{c}\text { Mean } \\
\text { Insoluble } \\
\text { fatty acids } \\
\text { per cent }\end{array}$ & $\begin{array}{c}\text { Mecular } \\
\text { molecular } \\
\text { weight of } \\
\text { insoluble } \\
\text { acids }\end{array}$ \\
\cline { 3 - 6 } 7 & 22.5 & 0.9101 & 42.0 & 219.9 & 90.1 & 266.9 \\
17 & 23.5 & 0.9104 & 41.5 & 221.6 & 89.7 & 265.5 \\
15 & 24.5 & 0.9108 & 41.5 & 223.5 & 89.4 & 265.0 \\
27 & 25.5 & 0.9110 & 41.3 & 223.6 & 89.3 & 264.2 \\
37 & 26.5 & 0.9113 & 41.0 & 225.6 & 88.9 & 261.9 \\
51 & 27.5 & 0.9114 & 40.6 & 227.0 & 88.7 & 261.7 \\
78 & 28.8 & 0.9118 & 40.1 & 228.6 & 88.4 & 260.9 \\
56 & 29.5 & 0.9120 & 40.1 & 230.2 & 88.3 & 259.6 \\
41 & 30.5 & 0.9123 & 39.9 & 231.7 & 87.9 & 260.1 \\
18 & 31.3 & 0.9125 & 39.7 & 232.5 & 87.9 & 258.0 \\
10 & 32.6 & 0.9130 & 39.4 & 232.8 & 87.7 & 257.8 \\
\hline \hline
\end{tabular}

1 J. Chem. Soc., 1904, 85, 248. 
In order to show to what extent increase of volatile acids takes place at the expense of oleic acid, the iodine numbers of 50 of the above samples were determined. Arranging these in groups of 20 and 30 , respectively, according to the ReichertWollny values, the following average figures were obtained :

\begin{tabular}{|c|c|c|c|c|c|c|c|}
\hline & & & $\begin{array}{l}\text { Reichert- } \\
\text { Wollny } \\
\text { number }\end{array}$ & $\begin{array}{l}\text { Iodine } \\
\text { number }\end{array}$ & $\begin{array}{l}\text { Oleic acid } \\
\text { per cent }\end{array}$ & $\begin{array}{l}\text { Insoluble } \\
\text { acid } \\
\text { per cent }\end{array}$ & $\begin{array}{l}\text { Mean molecular } \\
\text { weight of } \\
\text { insoluble acids }\end{array}$ \\
\hline First group. & . & . & 24.2 & 40.0 & 44.4 & 89.6 & 264.6 \\
\hline Second group . & . & . . . & 30.8 & 32.4 & 36.0 & 88.1 & 259.8 \\
\hline
\end{tabular}

\section{Detection of Oleomargarine}

Butter substitutes or "artificial butters," unless sold under special names indicating their origin, are collectively termed "oleomargarine" in America or "margarine" in England. The oleomargarine made in America ${ }^{1}$ consists chiefly of refined

1 The materials used in the manufacture of oleomargarine in the United States during the fiscal year ending June 30, 1899 (Senate Document No. 168, 57th Congress, 1st Session), were as follows :

\section{Table 18. - Materials used in Manufacture of Oleomargarine, 1899}

\begin{tabular}{|c|c|c|c|c|c|c|c|c|c|c|c|c|}
\hline \multicolumn{9}{|c|}{ Material } & \multirow{2}{*}{$\begin{array}{c}\text { Quantity } \\
\text { Pounds } \\
31,297,251\end{array}$} & \multirow{2}{*}{$\begin{array}{c}\begin{array}{c}\text { Percentage } \\
\text { of the } \\
\text { whole }\end{array} \\
34.27\end{array}$} & \multirow{2}{*}{$\begin{array}{c}\begin{array}{c}\text { Value } \\
\text { per } \\
\text { pound }\end{array} \\
\text { Cents } \\
8\end{array}$} & \multirow{2}{*}{$\begin{array}{c}\text { Total value } \\
\$ 2,503,780.08\end{array}$} \\
\hline Neutral & laro & & . & 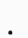 & . & . & . & . & & & & \\
\hline Oleo oil & & & & & . & . & 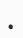 & & $24,491,769$ & 26.82 & 9 & $2,144,917.69$ \\
\hline Cottons & ed & & . & . & . & . & . & . & $4,357,514$ & 4.77 & 6 & $522,025.08$ \\
\hline "Butte & oil & & & & - & - & & . & $4,342,904$ & 4.76 & 6 & $260,520.00$ \\
\hline Sesame & & . & - & . & . & . & - & . & 486,310 & .53 & 10 & $4,863.10$ \\
\hline Coloring & $\mathrm{me}$ & tte & & 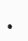 & . & . & 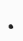 & . & 148,970 & .16 & 20 & $29,296.00$ \\
\hline Sugar & . & . & - & & . & - & • & . & 110,161 & .12 & 4 & $4,406.50$ \\
\hline Glycerir & & . & & & . & & • & . & 8,963 & .01 & 10 & 896.30 \\
\hline Stearin & • & • & & & . & - & . & . & 5,890 & .007 & 8 & 459.60 \\
\hline Glucose & . & . & & $\bullet^{\circ}$ & . & - & • & . & 2,550 & .003 & 3 & 76.50 \\
\hline Milk . & • & - & • & - & . & - & . & . & $14,200,576$ & 15.55 & 1 & $142,005.76$ \\
\hline Salt . & - & . & 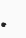 & • & . & - & - & . & $6,773,670$ & 7.42 & 1 & $67,726.70$ \\
\hline Butter & - & - & • & - & . & . & . & . & $1,568,319$ & 1.72 & 20 & $313,663.80$ \\
\hline Cream & . & - & $\bullet^{-}$ & & . & - & • & . & $3,527,410$ & 3.86 & 5 & $176,370.50$ \\
\hline \multicolumn{3}{|c|}{ Total } & • & . & . & . & . & . & $91,322,260$ & 100 & - & $\$ 6,171,007.61$ \\
\hline
\end{tabular}


lard, "oleo oil" (the soft part of beef fat), and cottonseed oil, often mixed with a small amount of butter and almost always churned with milk or cream. Palm oil and sesame oil are known to be used to some extent and other semi-drying and non-drying oils are probably utilized in some factories.

Hence, in comparison with any of the common constituents of oleomargarine, butter fat is characterized by its high proportion of soluble volatile acids, together with low percentages of oleic and stearic acids. The presence of oleomargarine in butter fat, therefore, lowers the Reichert-Meissl and saponification numbers and the specific gravity, while it raises the percentage of insoluble acids and either the melting point or the iodine number or both.

Several European governments require that sesame oil be added to oleomargarine in order to faciliate its detection when mixed with, or substituted for, butter. Similarly, the addition of butter to oleomargarine is sometimes forbidden or restricted in order to prevent the production of mixtures too closely resembling genuine butter. The essential features of the oleomargarine laws of the principal countries are given in Lewkowitsch's Oils, Fats, and Waxes.

\section{Detection of Coconut Fat}

Since coconut fat consists largely of glycerides of saturated acids of low molecular weight, it could be added in considerable quantity to butter fat of average composition without causing the latter to vary beyond the normal limits in any of the important analytical properties. A comparison of the ReichertMeissl and saponification numbers, however, would lead to the detection of this adulteration since the former number is higher and the latter lower in butter than in coconut fat. In pure

"Butter oil" is commonly stated to be a special brand of cottonseed oil ; but the high federal tax laid upon artificially colored oleomargarine by the law of May 9, 1902, practically prohibits the use of coloring matters employed before that date and has led to the introduction of "butter oils" containing palm oil which is naturally highly colored (Crampton and Simons: J. Am. Chem. Soc, 1905, 27, 270). 
butter fat the value of the factor [Saponification number $(200+$ Reichert-Meissl number) $]$ varies from 3.4 to -4.1 ; in pure coconut fat it varies from 47 to 50.7 .1

Another method of showing the presence of coconut oil is to determine the volume of tenth-normal alkali required to neutralize the insoluble volatile acids from 5 grams of fat. Under the conditions described by Polenske ${ }^{2}$ the results thus obtained are approximately quantitative, the percentage of insoluble volatile acids (mainly lauric acid) being much higher in coconut fat than in butter.

\section{REFERENCES}

\section{I}

(See book references at end of preceding chapter.)

\section{II}

1887-89. Wrley: Butter and Lard, Parts 1 and 4, Bul. 13, Bur. Chem., U.S. Dept. Agriculture.

1897. Sadtler: Arachis (Peanut) Oil. Am. J. Pharm., 69,490; Analyst, 22, 284.

1898. Archiutt : Estimation of Arachidic Acid. J. Soc. Chem. Ind., 17, 1124.

Hopkins: Maize Oil. J. Am. Chem. Soc., 20, 948.

Tortelli and Ruggeri: Detection of Cottonseed Oil. $Z$. angew. Chem., 1898, 464.

1898-99. Bömer: Phytosterol Test. Z. Nahr. Genussm., 1, 21, 81, 532; 2, 46,705 .

1899. Bellier: Color Reactions for Sesame Oil. Ann. de Chim. Anal., 4, 217; Analyst, 25, 50.

Browne: Butter Fat. J. Am. Chem Soc., 21, 632, 823, 975.

Cochras: Butter and Butter Adulterants. J. Franklin Inst., 147, 85.

1900. Bellifer: Detection of Arachis Oil. Bull. Soc. Chim., [3], 23, 358.

Estcourt: Butters from Various Countries Compared. Analyst, $25,113$.

Oilar: Halphen Test. Am. Chem. J., 24, 355.

Williams: Maize Oil. Analyst, 25, 146.

1 Juckenack and Posternack: Z. Nahr. Genussm., 1904, 7, 193.

${ }^{2}$ Polenske : Ibid., 1904, 7, 273. See also, Lewkowitsch's Oils, Fats, and Waxes. Muntz and Coudon: Ann. d. Inst. Agron., 1904; Analyst, 1905, 30, 155. Hesse : Milchwirthschaftl. Centrb?., 1905, 1, 13 ; Chen. Centrbl., 1905, I, 566 . 
1900-01. Vulte and Gibson: Maize Oil. J. Am. Chem. Soc., 22, 453; 23, 1. 1901. Holm, Krarup, and Peterson: Refraction, Iodine Number, and Volatile Acid Content of Butter. Z. Nahr. Genussm. 4, 746.

Pfrrin : Separation of Arachidic Acid. Monat. scientif., [4], 15, 320; Z. Nahr. Genussm., 4, 986 .

Siegfeld : Judgment of Butter by Reichert-Meissl Number. $Z$. Nahr. Genussm., 4, 433.

Weems and Grettenberg: Analytical Characters of Different Cottonseed Oils. Proc. Iowa Acad. Sci., 1901; Z. Nahr. Genussm., 1902, 5, 465.

1901-02. Böмеr: Phytosterol Test. Z. Nahr. Genussm., 4, 865, 1070; 5, 1018. 1902. Behrend and Wolfs: Butter Fats from Individual Cows. Z. Nahr. Genussm., 5, 689.

Fulmer: Halphen's Test. J. Am. Chem. Soc., 24, 1148.

LAXA: Change of Butter Fat by Microörganisms. Arch. Hyg., 41, 119.

Ritter: Phytosterol. Z. physiol. Chem., 34, 430, 461; 35, 550.

1902. Tolman and Munson: Olive Oil and its Substitutes. Bul. 77, Bur. Chem., U. S. Dept. Agriculture.

1903. Crampton: Composition of Process or Renovated Butter. J. Am. Chem. Soc., 25, 358.

Gill and Tufrs: (Cholesterol, Phytosterol; Sytosterol.) J. Am. Chem. Soc., 25, 251, 254, 498.

Kreis: Detection of Sesame Oil. Chem. Ztg., 27, 1030.

Swavivg: Influence of Feeding Cottonseed and Sesame Meal on the Properties of Butter Fat. Z Z. Nahr. Genussm., 6, 97.

Tolman and Munson: (Analysis of Salad Oils). J. Am. Chem. Soc., 25, 954 .

WiJs: (Iodine Numbers of Edible Oils). Z. Nahr. Genussm., 6, 692. 1904. Grossmann and Meinhard: Dutch Butter. Z. Nahr. Genussm., 8, 237.

Kraus: (Conditions Affecting the Keeping Qualities of Butter). Arb. kaiserl. Gesundheitsamte, 22, 235; Z. Nahr. Genussm., 9, 286.

1905. Arnold : (Analysis of Food Fats). Z. Nahr. Genussm., 10, 201.

Crampton and Srmons: Detection of Palm Oil when used as a Coloring Material in Oils and Fats. J. Am. Chem. Soc., 27, 270.

Fischer and Peyan: (Detection of Cottonseed Oil). Z. Nahr. Genussm., 9, 81.

Lythgoe: Refractive Index of Codliver Oil. J. Am. Chem. Soc., 27, 887.

PARry: Codliver Oil Standards. Chemist and Druggist, 46, 491; Analyst, 30, 208.

Polenske: (Analysis of Lard and Butter). Arb. kaiserl. Gesundheitsamte, 22, 557, 576; Analyst, 31, 46. 
1905. Tolman: Phytosteryl Acetate Test for Examination of Lard from Cottonseed-meal-fed Hogs. J. Am. Chem. Soc., 27, 589.

Wesson and Lane: Quantitative Analysis of Lard. J. Soc. Chem. Ind., 24, 714.

1906. Aschmann and Arend: Determination of Water in Butter and Other Fats. Chem. Ztg., 30, 953.

Farnsteiner: (Examination of Lard). Z. Nahr. Genussm., 11, 1; Analyst, 31, 72.

HARris : Estimation of Coconut Oil in Butter Fat. Analyst, 31, 353. Patrick : Rapid Determination of Water in Butter. J. Am. Chem. Soc., 28, 1611.

Rideal and Harrison: On the Polenske Method for Coconut Oil in Butter. Analyst, 31, 254.

Tolman: American Codliver Oils. J. Am. Chem. Soc., 28, 388.

Walker: Coconut Oil. Philippine J. Sci., 1, 117; Analyst, 31, 165. 1907. Amberger: Influence of Food on Composition of Butter Fat. $Z$. Nahr. Genussm., 13, 614.

Arch вutт: Tunisian and Algerian Olive Oils. J. Soc. Chem. Ind., 26, 453 .

Bellier : Analysis of Butter (New Method). Ann. de Chim. Anal., 11, 412 ; Analyst, 32, 22.

Hanus: (Detection of Coconut Oil). Z. Nahr. Genussm., 13, 18.

Henseval and Huvart: Contribution to the Study of Fish Liver

Oil. Chem. Rev. Fette-Harz-Ind., 14, 191; Chem. Als., 1, 2751.

Hrnks: Detection of Coconut Oil in Butter. Analyst, 32, 160.

Hodgson: Detection of Coconut Oil in Butter. Chem. News, 95, 121.

JeAN: Examination of Butter. Rev. gen. Chim., 10, 253; Chem. Abs., 1, 2617.

Kunn and Bengen : Cause of Halphen Reaction. Z. Nahr. Genussm., 12,145 .

KuhN and HalepaAp: Critical Study of Welman's Reaction. $Z$. Nahr. Genussm., 12, 449.

McPherson and Ruth : Corn Oil - Its Possibilities as an Adulterant in Lard and its Detection. J. Am. Chem. Soc., 29, 921.

Patrick: Rapid Determination of Water in Butter. J. Am. Chem. Soc., 29, 1126.

SifGreld : (Detailed Study of Polenske Number). Chem. Ztg., 32, 511 . - Influence of Feed on Butter Fat. Z. Nahr. Genussm., 13, 513.

Smith : Application of Arachidic Acid Test to Solid Fats. J. Am. Chem. Soc., 29, 1756.

Soltsein: Detection of Tallow and Lard in Presence of Each Other. Chem. Rev. Fette-Harz-Ind., 13, 240; Chem. Abs., 1, 109. Windaus: Separation of Cholesterol and Phytosterol. Chem. Ztg., 30, 1011. 
1908. Connelison: Detection of Synthetic Color in Butter. J.Am. Chem. Soc., 30, 1478.

Emery: Detection of Beef Fat in Lard. U. S. Dept. Agr., Bur. Animal Ind., Cir. 132; Chem. Abs., 2, 2461.

Farrington: Determination of Water in Butter. Wis. Agl. Expt. Station, Bul. 154 ; Chem. Abs., 2, 870.

Fulmer and Manchester: Effect of Heat on Cottonseed Oil Constants. J. Am. Chem. Soc., 30, 1477.

Fritsche: (Polenske Number of Dutch Butter). Z. Nahr. Genussm., 15, 193.

Hanus and Stehl: The Ethyl Ester Number, A New Method for Coconut Fat. Z. Nahr. Genussm., 15, 576.

KreIs: Influence of Rancidity on Baudouin Reaction. Chem. Abs., 2, 2165,2166 .

Wagner and Chement: Cottonseed Oil. Z. Nahr. Genussm., 16, 145.

1909. Köнnig and Schluckebier: Influence of Fat in Feed upon Fat of Pigs with Special Regard to Phytosterol. Z. Nahr. Genussm., 15, 641 .

Tatrock and Thomson: The Value of the Polenske Method. $J$. Soc. Chem. Ind., 28, 69.

Mruliau : (Reactions of Kapok and Baobab Oils). Matières grasses, 2, 1545 ; Chem. Abs., 4, 969 .

1910. Bull and SAether: Can One Determine the Nature of the Vegetable Oil on Sardines? Chem. Ztg., 34, 733.

Grbbs and Agcaolli: Lard from Wild and Domestic Philippine Hogs and the Changes Effected by Feeding Copra Cake. Philippine. J. Sci., 5A, 33; Chem. Abs., 4, 2748.

HARE: Some Effects of Feeds upon the Properties of Lards. $J$. Ind. Eng. Chem., 2, 264.

Lindsay et al: (Effect of Feed on Butter Fat). Mass. Agl. Expt. Sta. Report, 1908, 66-110; Chem. Abs., 4, 1774.

1911. Arnold : Determination of Coconut Oil in Edible Fats. Z. Nahr. Genussm., 21, 587.

Marcille: Olive Oils from Tunis. Ann. falsif., 3, 372; Chem. Abs., 5, 732.

Revis and Bolton: Methods of Estimating Coconut Oil and Butter in Butter and Margarine. Analyst, 36, 333.

Richardson: Coconut Oil of High Iodine Value. J. Ind. Eng. Chem., 3, 574 .

Stennock: Modification of Wiley's Method for Melting Point of Fats. J. Ind. Eng. Chem., 2, 480.

Tortelli and Fortini: (Detection of Rape Oil in Salad Oils). Ann. falsif., 4, 139; Chem. Abs., 5, 1947. 


\section{CHAPTER X}

\section{Drying 0ils}

Several oils of both vegetable and animal origin (among the latter more particularly the fish oils) contain glycerides of highly unsaturated fatty acids which on exposure in thin layers to air readily absorb oxygen and become converted into solids. This property gives rise to the term "drying" oils. A few of the more prominent drying oils may be listed as follows :

\section{Vegetable Drying Oils}

Linseed oil, pressed from the seeds of the flax plant, produced in large quantities in North and South America, Russia, and India, is the most important of the drying oils and is extensively used for direct application as a protective coating to wood and metal, and in the manufacture of paints and varnishes, oilcloths, linoleum, printing inks, rubber substitutes, etc. Lewkowitsch estimates that there were produced in 1907, $1,200,000$ tons of linseed in Argentina; 646,275 tons in the United States ; 450,000 tons in Russia; and 419,900 tons in India. Linseed yields in general about 35 per cent of its weight of oil.

The seed are usually crushed between rollers, then heated to about $160^{\circ} \mathrm{F}$. and pressed while warm. The oil so obtained is yellowish brown or brownish yellow and somewhat turbid from traces of moisture and mucilaginous material, which impurities, however, gradually settle out when the oil is stored. Oil which has been thus purified by long standing is sometimes called "tanked oil." A more rapid method of refining is to 
treat the oil with 1 to 2 per cent of sulphuric acid. This produces a charred mass which carries down with it the greater part of the impurities.

Boiled linseed oils are made by heating linseed oil with a "drier," formerly to temperatures of $210^{\circ}$ to $260^{\circ} \mathrm{C}$., now, according to Lewkowitsch, to a temperature of about $150^{\circ} \mathrm{C}$. The extent to which the analytical characters are changed depends upon the details of the process and the extent to which the oil is exposed to air.

Tung oil, also called Chinese or Japanese wood oil, is pressed from the nuts of the tung tree. According to Ennis the use of this oil in the United States is steadily increasing and experiments are being made with the cultivation of the tree in California. At present the oil comes chiefly from China, where the natives roast and crush the nuts and press from them about 40 per cent of oil, which is only about three fourths of the amount present. According to Lewkowitsch the oil is also produced in Madagascar under the name of "Bakoly oil." Tung oil has a characteristic persistent odor which is not easily removed by refining. It is used largely for oiling wood and waterproofing paper. Lewkowitsch states that while many patents have been taken out for the substitution of tung oil for linseed oil in manufactures, little progress has yet been made in this direction.

Walnut oil and poppyseed oil, while they have not such pronounced drying properties as linseed and tung oils, have the advantage of yielding almost colorless films which are not likely to crack. These oils are therefore especially adapted to use in white or delicately colored paints for artists.

Maize oil has not sufficient drying property to be useful as a paint oil but "dries" well enough to permit of its use in putty. It is also used like linseed oil in making rubber substitute. It has probably been used to some extent as an adulterant of linseed oil.

Soy $(\operatorname{soj} a, \operatorname{soy} a)$ bean oil is used to some extent in admixture with linseed oil. 


\section{Fish OILS}

In recent years the fish oil industry has been much altered through the introduction of steam trawlers and the prompt rendering of the oil, which results in a product largely free from the dark color and rank odor formerly regarded as characteristic of fish oils.

Menhaden oil is obtained from the bodies of the menhaden, a fish somewhat larger than a herring, which abounds in the Atlantic especially off the coast of New Jersey.

Toch ${ }^{1}$ states that menhaden is the best fish oil for use in paint and that the winter-bleached variety is to be preferred. This should be fairly pale in color, with an iodine number of 150 or over, and with little or no fishy odor. Such an oil, Toch finds, may be mixed with linseed oil even up to 75 per cent of the mixture with good results when used for exterior painting where its odor is not noticeable. In fact paint made from such a fish oil is said to be more resistant to heat than a linseed oil paint and therefore preferable for smoke stacks, boiler fronts, etc. It is also said to be more resistant to the salt air of the seacoast. Menhaden oil has also been used in place of linseed oil in the manufacture of enamel leather and of printing inks.

Japanese sardine oil, sardine oil, and herring oil have some drying property and are used to some extent as partial substitutes for, or adulterants of, linseed oil.

\section{LINSEED OIL}

\section{Analytical Properties of Linseed Orl}

Commercial linseed oil is usually designated by the region of its origin. It varies considerably, the variation being due mainly to the presence of foreign seeds in the linseed at the time of pressing. Hempseed is practically always present, sometimes in very small proportions but often to the extent of 5 per cent, or more, of the weight of seed. The drying properties of lin- 
seed oil are better the purer the seeds from which it is pressed. The iodine number and the drying power of the oil decrease as the proportion of hempseed increases. Hence a linseed oil containing much hemp oil would be shown to be of inferior quality by its low iodine number, but could not be pronounced adulterated so long as this did not fall below 170. The maximum iodine number of linseed oil is difficult to fix, since the results obtained by the method of Wijs often exceed the Huibl numbers, but since no other common oil has a higher iodine number than linseed, the maximum limit is of little practical importance in the detection of adulterations.

The usual range of the more important analytical constants of linseed oil has been given in the table at the end of Chapter VIII. The interpretation of "constants," their relations to each other, and their use in the detection of adulterations having been discussed in some detail in connection with the examination of salad oils, it will be sufficient in this case to mention briefly the principal adulterants with means for the detection of each, and describe the hexabromide test which distinguishes linseed from practically all other oils.

\section{Adulterants AND Methods of Detection}

\section{Mineral Oil}

Mineral oil would greatly lower the iodine number, temperature reaction, and saponification number. Whenever a low saponification number is found, the unsaponifiable matter should be separated and examined. Any mineral oil which is not volatile at $100^{\circ}$ can be separated quantitatively, dried, and weighed. Volatile mineral oil can be distilled by means of a current of steam, separated from water in the distillate, and measured or weighed. In case turpentine were present, as in some so-called "boiled" oils, it would be distilled with steam and would separate from water in the distillate in the same way. The optical rotatory power of turpentine affords an easy means of distinguishing it from benzine or other volatile mineral oil. 


\section{Rosin and Rosin Oil}

Rosin dissolved in linseed oil raises the specific gravity and index of refraction while the saponification and iodine numbers are appreciably decreased only when large amounts of rosin are added. Presence of rosin greatly increases the acid number, which in pure linseed oil is usually less than 7. Rosin acids can be separated and determined by Twitchell's method as described under soap analysis beyond.

Rosin oil in linseed oil would raise the specific gravity and greatly lower the saponification and iodine numbers. Rosin oil is a mixture of substances many of which are unsaponifiable, so that its presence in linseed oil would increase the amount of unsaponifiable matter.

Either rosin or rosin oil can be detected by the LiebermannStorch color reaction or by determining the bromine substitution number.

Liebermann-Storch Reaction. - Shake 2 cc. of the oil with 5 cc. of acetic anhydride, warming gently. Allow to cool, draw off the anhydride, and test by adding one drop of sulphuric acid $(1: 1)$. A violet color (not permanent) is produced in the presence of rosin or rosin oil. Cholesterol, which might be found in linseed oil if fish oil were present as an adulterant, gives a similar color reaction.

Bromine Substitution Number (McIlhiney). - Fatty oils take up bromine by direct addition, little or no substitution taking place. With rosin and rosin oil much the greater part of the bromine is taken up by substitution, a molecule of hydrobromic acid being formed for each molecule of bromine which disappears. The hydrobromic acid thus affords a means of measuring the amount of substitution. It is determined by adding an excess of potassium iodate and titrating the liberated iodine. The same apparatus can be used as in the determination of the iodine number and the manipulation is similar. From 0.2 to 0.3 gram of the drying oil is dissolved in $10 \mathrm{cc}$. of carbon tetrachloride and $20 \mathrm{cc}$. of a one third normal solution of bromine in carbon tetrachloride is added. After two minutes potassium iodide is 
added and the excess of halogen titrated by means of thiosulphate as in the determination of the iodine number. This shows the total amount of bromine which has disappeared. As soon as this titration is finished, add 5 cc. of a 2 per cent solution of potassium iodate and titrate the iodine set free from the iodate by the action of the free halogen acids, according to the reaction:

$$
6 \mathrm{HI}+\mathrm{KIO}_{3}=3 \mathrm{I}_{2}+\mathrm{KI}+3 \mathrm{H}_{2} \mathrm{O} \text {. }
$$

The bromine thus found is equal in amount to that which has combined with the sample by substitution. For further details of manipulation the reader must be referred to the original papers. $^{1}$ According to McIlhiney the bromine substitution number of raw or boiled linseed oil is always less than 7, while rosin oil gives numbers from 40 to 100 and rosin from 65 to 80 .

\section{Maize Oil}

The presence of maize oil in linseed oil lowers the specific gravity, index of refraction, iodine number, and temperature reaction. The amount which can be added without carrying these numbers below the normal limits of variation will depend upon the quality of the linseed oil in the mixture. Since the maize oil used as an adulterant of linseed would probably not be highly refined, the characteristic odor and taste would aid in its detection.

\section{Cottonseed Oil}

The "constants" of linseed oil would be lowered by cottonseed in the same way as by maize oil and to a somewhat greater extent. If the cottonseed oil had not been heated, its presence would be detected by the Halphen test as described under salad oils.

\section{Fish Oils}

Menhaden and other fish oils are often used as adulterants, and are difficult to detect with certainty since their "constants" are frequently within the limits found for pure linseed oil. Their presence is often indicated by the odor, but this cannot be relied upon, as the difference in odor between refined men-

1 McIlhiney : J. Am. Chem. Soc., 1894, 16, 275; 1899, 21, 1084 ; 1902, 24, 1109. See also Tolman : Ibid., 1904, 26, 826. 
haden and low grade linseed oil is not so pronounced as might be supposed. Lewkowitsch recommends the determination of the melting point of the phytosteryl acetate obtained from the oil. The crystals of phytosteryl acetate from pure linseed oil melt at $128^{\circ}-129^{\circ}$ (Bömer and Winter), while in the presence of cholesterol from fish oil much lower melting points are obtained. The method of Eisenschiml and Copthorne, as adopted by the Association of Official Agricultural Chemists, for the detection of fish oil in the presence of vegetable oils is as follows:

Dissolve in a test tube about 6 grams of the oil in 12 cc. of a mixture of equal parts of chloroform and glacial acetic acid. Add bromine drop by drop until a slight excess is indicated by the color, keeping the solution at about $20^{\circ} \mathrm{C}$. Allow to stand 15 minutes or more and then place the test tube in boiling water. If only vegetable oils are present, the solution will become perfectly clear, while fish oils will remain cloudy or contain a precipitate due to the presence of insoluble bromides.

This method is based on the fact that the bromides of the vegetable oils, although they may be precipitated abundantly in the cold, will be completely soluble in the mixture of chloroform and glacial acetic acid when heated as described in this test. Boiled linseed oil containing metallic driers cannot be tested by this method unless the metals are first removed.

This method of Eisenschiml and Copthorne is evidently an outgrowth of the "hexabromide" test of Hehner and Mitchell, the description of which follows.

\section{"Hexabromide" Test}

Hehner and Mitchell ${ }^{1}$ showed that linseed and fish oils differ from other oils in yielding considerable quantities of insoluble bromides when treated with bromine in ether solution. They applied the test directly to the oil as follows:

Dissolve 1 to 2 grams of oil in $40 \mathrm{cc}$. of ether acidulated with glacial acetic acid, cool the solution to $5^{\circ}$, and add bromine, drop by drop, until the solution is permanently colored brown.

${ }^{1}$ Analyst, 1898, 23, 310. Also Mitchell in Allen's Commercial Organic Analysis, 4th Ed., Vol. II, p. 28. 
After standing for at least 3 hours, preferably in an ice box, filter through a Gooch crucible, leaving most of the precipitate in the vessel in which it was formed, wash 4 times with ice-cold ether, dry the precipitate at $100^{\circ}$, and weigh.

Linseed oil yields 23 to 38 per cent (usually about 25 per cent) of the insoluble bromide, the amount increasing with the iodine number of the oil. Some of the fish oils yield equal or greater amounts; but tung, poppy, and walnut oils, and such seed oils as maize and cottonseed, yield almost none - according to the figures compiled by Lewkowitsch never over 2 per cent.

Mitchell regards the products precipitated in these tests as bromides of mixed glycerides containing one radicle of linolenic acid (or an isomeric acid), and states that the insoluble bromide from linseed oil contains about 56 per cent of bromine and melts at $143.5^{\circ}$ to $144^{\circ}$, whereas the corresponding bromides from marine animal oils decompose before melting so that even small amounts of such oils in linseed oil can be detected by this test.

Lewkowitsch recommends that the test be applied to the mixed fatty acids rather than to the oil itself. In the separation of the acids care must be taken to avoid oxidation by exposure to the air. 'The mixture of fatty acids from linseed oil yields 30 to 42 per cent of hexabromide, melting to a clear liquid at $175^{\circ}$ to $180^{\circ}$, whereas the corresponding products from fish, liver, and blubber oils do not melt at this temperature but become darker and are completely blackened at about $200^{\circ}$. Lewkowitsch states that this test is capable of showing the presence of 10 per cent of fish oil in linseed oil.

For further discussion of this test see the papers just cited and also Proctor: J. Soc. Chem. Ind., 25, 798 (1906).

\section{OILS ALTERED BY AGE OR OXIDATION}

It has been assumed in discussing the analytical "constants". that the oils under examination are fresh or have been kept under such conditions as to prevent any material alteration. Age alone probably has no appreciable effect upon the analytical properties of commercially pure fatty oils, but such oils when kept for a long time in contact with the air, for example, 
in partially filled or loosely stoppered vessels, take up atmospheric oxygen and gradually become considerably altered in those properties which are commonly regarded as "constants." This atmospheric oxidation naturally takes place much more rapidly with drying than with non-drying or semi-drying oils, and in open vessels than in those in which the oil is exposed to only a limited amount of air. It is probable that oils which have been thus altered are more frequently encountered in analytical work than has been supposed.

The influence of such oxidation upon the more important analytical properties is to increase the specific gravity, index of refraction, and temperature reaction with sulphuric acid, and to decrease the iodine number, the specific refractive power, ${ }^{1}$ and, in the case of olive oil, the viscosity of the soap solution. The acidity of the oil may increase at the same time, but this change does not always occur.

The following results were obtained upon oils intentionally exposed to the air, and while large as compared with the changes which should occur under ordinary conditions of laboratory storage, they do not represent the maximum change which may result from atmospheric exposure.

Table 19. - Effects of Exposure of Oils to Air

\begin{tabular}{|c|c|c|c|c|c|c|}
\hline Oil & & $\begin{array}{l}\text { Iodine } \\
\text { number }\end{array}$ & $\begin{array}{l}\text { Sp. Gr. } \\
\frac{15.5^{\circ}}{15.5^{\circ}}\end{array}$ & $\begin{array}{l}\text { Index of } \\
\text { refraction } \\
\text { at } 15.5^{\circ}\end{array}$ & $\begin{array}{l}\text { Specific } \\
\text { refractive } \\
\text { power }\end{array}$ & $\begin{array}{c}\text { Specific } \\
\text { tempera- } \\
\text { ture } \\
\text { reaction }\end{array}$ \\
\hline Olive oil before exposure & . . & 83.8 & 0.9165 & 1.4712 & 0.5141 & 100 \\
\hline Same after exposure . . . & - . & 77.3 & 0.9240 & 1.4722 & 0.5100 & $127^{2}$ \\
\hline Lard oil before exposure & - & 73.3 & 0.917 & 1.4697 & 0.5122 & 106 \\
\hline Same after exposure $\cdot{ }^{\circ}$ & - . & 56.2 & 0.943 & 1.4724 & 0.5010 & 141 \\
\hline Cottonseed oil before exposure & e & 105.2 & 0.923 & 1.4737 & 0.5132 & 171 \\
\hline Same after exposure . . . & . & 90.2 & 0.939 & 1.4779 & 0.5090 & $217^{2}$ \\
\hline Linseed oil before exposure & . & 177.1 & 0.934 & 1.4835 & 0.5177 & \\
\hline Same after exposure . . . & . & 136.9 & 0.969 & 1.4886 & 0.5042 & \\
\hline
\end{tabular}

1 Calculated by Landolt's formula $\frac{N-1}{D}$ (Ber., 1882, 15, 1031), in which $N$ is the index of refraction and $D$ is the specific gravity.

2 These numbers were determined earlier than the other data and presumably represent a lesser degree of change. 
Many other oils have been tested with similar results. It is evident that oils thus altered are very likely to be misjudged, especially if only one or two quantitative determinations are made. Thus if only the specific gravity and temperature reaction of the olive oil had been determined, the results would have been interpreted as indicating the presence of some seed oil. The iodine number of the linseed oil taken alone would indicate extensive adulteration with some oil of lower drying power. The results emphasize the importance of determining the iodine number and either the specific gravity or the index of refraction in all cases, and show the advantage of determining the temperature reaction, not as a substitute for the iodine number but for comparison with it. For a fuller discussion of this subject with the results obtained upon a number of other oils the reader is referred to two papers in the Journal of the American Chemical Society (July, 1903, and May, 1905).

As the result of this work it appears that the increase in specific gravity and the decrease in iodine number are almost exactly proportional to each other in non-drying and semidrying oils, so that in examining an altered oil belonging to either of these classes the original iodine number can be estimated by adding 0.8 to the number found on the exposed sample for each increase of 0.001 in the specific gravity. When the original specific gravity is not known, the calculation must be based upon the average specific gravity for oil of the species under examination. The error of this assumption can hardly be sufficient to affect the interpretation of the results.

The iodine numbers of exposed samples of linseed and fish oils cannot be corrected accurately by the rule given for semi-drying and non-drying oils, the number thus obtained being always too low. It has also been found that when linseed oil is thus changed by atmospheric oxidation the amount of insoluble bromide which it will yield in the hexabromide test is greatly reduced.

Commercial "blown" oils show greatly increased specific gravities and decreased iodine numbers; the changes appear to bear much the same relation to each other as in the oils which have been altered by exposure. 


\section{"UNKNOWN" OILS AND MIXTURES}

In the examination of an unknown oil the appearance, odor, and taste should be compared with those of typical oils of known purity. In testing transfer a drop of the oil by means of a glass rod to the back of the tongue and note both the first impression and the after taste. The odor may be observed not only cold, but also after heating a portion in a porcelain dish to $140^{\circ}-150^{\circ}$. Also after cooling sufficiently pour a few drops of the oil into the palm of one hand, rub with the other and smell again. These preliminary superficial observations and the determination of the iodine and saponification numbers and either the specific gravity or the index of refraction should locate the sample as one of a small group of oils, after which any special tests available for the detection of individual members of the group can be applied. The tests described in this and the preceding chapter taken in connection with the quantitative determinations mentioned enable the analyst, in the majority of cases, to identify the oil or, if a mixture, the principal constituent. In case of doubt one should not fail to consult the larger works, especially Lewkowitsch's Oils, Fats, and Waxes.

If a saponification number indicates that only fatty oil is present, but the relation of the specific gravity to the iodine number does not correspond to that ordinarily found in any pure oil, the determination of the specific temperature reaction and the acidity will usually show whether the discrepancy is to be attributed to oxidation or adulteration.

The relative commercial value will of course determine what oils can profitably be used as adulterants. Prices vary greatly in different markets, as well as with the degree to which the oils are refined, and are also likely to fluctuate from year to year so that no fixed order of commercial value can be given.

The list of oils in order of commercial value given by Gill and by Lewkowitsch show considerable variation, which doubtless is due largely to the differences between American and English markets. In each of the lists the highest-priced oils are given first. 
Gill. - Almond, castor, sesame, neatsfoot, rape, olive, sperm, whale, peanut (arachis), linseed, tallow, lard, fish, cottonseed, mineral, rosin oil.

Lewkowitsch. - Almond, sperm, olive, neatsfoot, lard, cod liver, arctic sperm, arachis, poppy, sesame, seal, rape, castor, cottonseed, maize, linseed, whale, fish, mineral, rosin oil.

In the examination of mixtures containing other than fatty oils, it may be necessary to separate the mixed fatty acids and examine this mixture in order to identify the fatty oils originally present. The "constants" of the mixed fatty acids of various oils, as well as of many oils and fats not mentioned in this work, are conveniently tabulated in Lewkowitsch's Laboratory Companion to the Fat and Oil Industries.

\section{REFERENCES}

I

Allen : Commercial Organic Analysis.

Church : Chemistry of Paints and Painting.

Envis : Linseed Oil and other Seed Oils.

FAhrion: Die Chemie der trocknenden Oele.

GiLL: Handbook of Oil Analysis.

Holley and LADD: Analysis of Mixed Paints, Color Pigments, and Varnishes.

Lewnowitsch : Chemical Technology and Analysis of the Oils, Fats, and

Waxes.

SAbIN : Technology of Paint and Varnish.

Scotт : White Paints and Painting Materials.

Tосн : Materials for Permanent Painting.

- : Technology of Mixed Paints.

II

1891. Ballantyne: (Oxidized Oils). J. Soc. Chem. Ind., 10, 29.

1892. Thompson and Ballantyne: (Same). J. Soc. Chem. Ind., 11, 506. 1898. Fahrion: (Same). Z. angew. Chem., 1898,781.

Hehner and Mitchell : ("Hexabromide"Test). Analyst, 23, 310.

1899. Gill and LaMB : American Linseed Oil. J. Am. Chem. Soc., 21, 29. McIlhiney: (Bromine Substitution Number). J. Am. Chem. Soc., 21, 1084.

1901. McIlhiney : Report on Linseed Oil and its Adulterants to the New York State Commissioner of Agriculture. (Reprinted in Sabin's Technology of Paint and Varnish, Chapter V.) 
1902. Lewkowitsch : (Oxidized Oils). Analyst, 27, 139.

McIlminey: Further notes on the Bromine Absorption of Oils. J. Am. Chem. Soc., 24, 1109.

1903. Dunlap and Schenk: Oxidation of Linseed Oil. J.Am. Chem. Soc., 25, 826 .

Sherman and Falk: Influence of Atmospheric Oxidation upon the Composition and Analytical Constants of Fatty Oils. J. Am. Chem. Soc., 25, 711.

SJollema : Linseed Oil. Z. Nahr. Genussm., 6, 631.

Urz: Poppyseed Oil. Chem Ztg., 27, 1176.

1904. Lewnowitsch : Linseed Oil. Analyst, 29, 2.

McCandless : Examination of Turpentine. J. Am. Chem. Soc., 26, 981.

1904-07. Reports and Discussions on Preservative Coatings for Iron and Steel. Proc. Am. Soc. Testing Materials, 4, 137 ; 5, 79; 6, 63; 7, 140.

1905. Languuir: Determination of Rosin in Shellac. J. Soc. Chem. Ind., 24, 12.

McGill: Examination of Turpentine. Bul. 79, Canadian Inland Revenue Laboratory.

RABY: Rotatory Power of Turpentine. Ann. de Chim. Anal., 10, 146 ; Analyst, 30, 210.

Sherman and Falk: Influence of Atmospheric Oxidation upon the Analytical Coristants of Fatty Oils. J. Am. Chem. Soc., 27, 605. Thompson: Proper Methods in Conducting Painting Tests. Proc. Am. Soc. Testing Materials, 5, 417.

Valenta: Examination of Turpentine. Chem. Ztg., 29, 807 ; Analyst, 30, 342.

1906. Beadle and Stevens: Analysis of Rosin Size. Chem. News, 93, 155 ; Chem. Eng., 4, 263.

Böнмe: Detection of Adulterants in Turpentine. Chem. Ztg., 30, 631.

Genther : Drying Process of Linseed Oil. Z Z angew. Chem., 1906 ; Chem. Abs., 1, 912 .

Gill: Determination of Rosin in Varnishes. J. Am. Chem. Soc., 28, 1723.

Holley: Turpentine and its Substitutes. 17th Ann. Rpt. North Dakota Agl. Expt. Station.

Levy : American Colophony. Ber., 39, 3043.

Proctor and Bennett: Examination of Marine Oils. J. Soc. Chem. Ind., 25, 798.

1907. Chessman: Priming Coats for Metal Surfaces-Linseed Oil vs.

Paint. Proc. Am. Soc. Testing Materials, 7, 479.

Clover: Philippine Wood Oils. Phil. J. Sci., 1, 191. 
1907. Committee Report on Shellac Analysis. J. Am. Chem. Soc., 29, 1221. Endemann: Testing Shellac for Purity. J. Frank. Inst., 164, 285. Hughes: Deleterious Ingredients in Paints (with Discussion). Proc. Am. Soc. Testing Materials, 7, 486.

Kress: Analytical Properties of some Pine Wood Oils. School of Mines Quarterly, 29, 46.

LAdD: Paint Legislation (with Discussion). Proc. Am. Soc. Testing Materials, 7, 523.

McGill: Examination of Turpentine. J. Soc. Chem. Ind., 26, 847. Perry: Physical Properties of Paint Films. Proc. Am. Soc. Testing Materials, 7, 511.

Pнокіn: (Oxidation and Polymerization Processes in Drying Oils). J. Russ. Phys. Chem. Soc., 39, 307, 308; Chem. Abs., 1, 1752, 1775.

RYAN and Marshall: Influence of Oxygen and Nitrogen, and Sunlight and Darkness on Olive Oil. Am.J. Pharm., 79, 308; Chen. Abs., 1, 2275.

Sмıтн: Physical Testing of Oil Varnishes (with Discussion). Proc. Am. Soc. Testing Materials, 7, 499.

Urz: Specific Gravity of Linseed Oil. Chem. Rev. Fett-Harz-Ind., 14, 137; Chem. Abs., 1, 2181.

1908. Coste: Examination of Turpentine and its Substitutes. Analyst, 33, 219.

FreY : Rapid Determination of Naphtha in Turpentine. J. Am. Chem. Soc., 30, 420.

Gill : Oxidation of Olive Oil. J. Am. Chem. Soc., 30, 874.

Lorentz: Unsaponifiable Matter of Linseed Oil. Chem. Ztg., 32, 819.

McIlniney : Analysis of Oil Varnishes. Eng. News, 60, 31; Chem. Abs., 2, 2630.

McIlniney: Method of Analyzing Shellac. J. Am. Chem. Soc., 30, 867.

Marcusson: Determination of Benzine in Turpentine. Chem. Rev. Fett-Harz-Ind., 17, 6; Chem. Abs., 4, 1541.

Richardon and Bowen: Analysis of Turpentine Oils. J. Soc. Chem. Ind., 27, 613.

Schultze: (Nature of Rosin Oil). Ann. Chem., 359, 129; Chem. Abs., 2, 1715.

1909. Committee Report on Preservative Coatings for Structural Materials. Proc. Am. Soc. Testing Materials, 9, 139.

Geer: Analysis of Turpentine by Fractional Distillation. Cir. 152, Forest Service, U. S. Dept. Agriculture.

Marcusson: Tủrpentine and Its Substitutes. Chem. Ztg., 33, 966, 978, 985; Chem. Abs., 4, 1236, 1542. 
PAUL: Turpentine and Its Adulterants. J. Ind. Eng. Chem., 1, 27.

White: Paints for Concrete - Their Need and Requirements (with

Discussion). Proc. Am. Soc. Testing Materials, 9, 526.

1910. Agrestini : Changes in Olive Oil kept for Over Two Centuries.

Staz. sper. agrar. ital., 43, 283 ; Chem. Abs., 4, 3147.

Erbner and Hue: Determination of Benzine in Turpentine. Chem. Ztg., 34, 643, 657 .

Ersenschiml and Copthorne: Detection of Fish Oils in Vegetable Oil. J. Ind. Eng. Chem., 2, 43.

Hepburs : Natural Changes Occurring in Fats and Oils. J. Frank. Inst., 168, 365, 421; 169, 23.

Kreikenbaum : Analytical Constants of Chinese Wood Oil. J. Ind. Eng. Chem., 2, 205.

Morrell: Testing Turpentine. J. Soc. Chem. Ind., 29, 241.

Singh: Analytical Constants of Shellac. J. Soc. Chem. Ind., 29, 1435.

VAubel: Analysis of Shellac. Chem. Ztg., 34, 991, 1008; Chem. Abs., 5, 1196.

Walker: Some Technical Methods of Testing Miscellaneous Supplies. U. S. Dept. Agriculture, Bur. Chem., Bul. 109, Revised.

1911. Chercheffsky: Methods of Testing Turpentine. Matières grasses,

3, 1925 ; Chem. Abs., 5, 1677.

Coste and NAsh : Turpentine Substitutes. Analyst, 36, 207.

Forrest: Characteristics of Creosote and Tar Oils Available for

Wood Preservation. J. Soc. Chem. Ind., 30, 193.

IxGLE: Linseed and Other Oils-Relations of "Constants" and

Effect of Heat. J. Soc. Chem. Ind., 30, 344.

Jensen: Examination of Linseed Oil. Pharm. J., 86, 839 ; Chem. Abs., 5, 2976.

LAvgmuin and White: The Analysis of Shellac. J. Soc. Chem. Ind., 30,786 .

Tocm : Fish Oil as a Paint Vehicle. J. Ind. Eng. Chem., 3, 627.

Veitch: Commercial Turpentines of the United States. J. Ind. Eng. Chem., 3, 521.

Vertch and Donk: Wood Turpentine; Its Production, Refining, Properties, and Uses. U. S. Dept. Agriculture, Bur. Chem., Bul. 144. 


\section{CHAPTER XI \\ Petroleum and Lubricating 0ils}

The petroleums from different localities vary considerably both in the hydrocarbons of which they are chiefly composed and in the amounts of nitrogen, sulphur, and oxygen compounds present. The Appalachian field, which includes the oil regions of Pennsylvania, New York, West Virginia, Kentucky, and southeastern Ohio, yields petroleum consisting chiefly of paraffin hydrocarbons - of which all the members of the series from $\mathrm{CH}_{4}$ to $\mathrm{C}_{16} \mathrm{H}_{34}$, as well as $\mathrm{C}_{25} \mathrm{H}_{52}, \mathrm{C}_{27} \mathrm{H}_{56}$, and $\mathrm{C}_{30} \mathrm{H}_{62}$, have been shown to be present-together with small amounts of the olefines and traces of the hydrocarbons of the benzene and napthene series. This is generally considered the best grade of petroleum produced in large quantities. It contains very little sulphur (between 0.05 and 0.1 per cent) practically no asphaltic matter and in refining gives good yields of gasoline, illuminating oils, and paraffin wax.

Oils from the middle western, Texas, and California fields now produced in much larger quantities than Pennsylvania petroleum, contain in general a somewhat lower proportion of the paraffin hydrocarbons, and larger percentages of the less desirable or undesirable constituents.

For descriptions of the composition of petroleum oils reference may be made to the works cited at the end of the chapter and particularly to a series of papers by Mabery in the "Proceedings of the American Academy of Arts and Sciences," Vol. 32, and subsequently.

\section{EXAMINATION OF CRUDE PETROLEUM}

An examination of crude petroleum may consist of a few simple tests or an elaborate investigation both by analytical 
methods and a laboratory imitation of actual refining processes, according to the importance of the case and the judgment and experience of the chemist. We shall consider here only a few factors of the superficial examination and fractional distillation.

A sample of petroleum as received at the laboratory may contain water, either naturally or because of having been put into a wet bottle. It should be allowed to stand so that any water may settle out as completely as possible before making any tests. If any water or sediment appear on standing, decant the oil and then pass it through a dry filter. In some cases it will be desirable to make a quantitative determination of the moisture and of the sediment.

Note the color and general appearance of the oil in a standard 4-oz. sample bottle. With experience the odor is also of much assistance in indicating the general character of the sample.

The density of the sample is usually taken with a delicate hydrometer reading either in specific gravity or degrees Baumé. In the oil trade the latter is more common, and the relation between specific gravity and Baumé density becomes a matter of importance.

In the past the different manufacturers of Baumé hydrometers have given many different values to the Baumé scale.1 Recently the U. S. Bureau of Standards has defined the value of the Baumé scale (for liquids lighter than water) in terms of specific gravity as follows :

$$
\text { Degrees Baumé }=\frac{140}{\text { Specific gravity } \frac{60^{\circ} \mathrm{F} .}{60^{\circ} \mathrm{F} .}}-130 .
$$

The same value was adopted by the Manufacturing Chemists' Association of the United States in 1903. Hydrometers purporting to read degrees Baumé should not be used unless they have been graduated in strict conformity with this scale.

In the absence of a sufficiently accurate and delicate hydrometer the specific gravity should be determined by means of a

${ }^{1}$ Chandler : The Baumé Hydrometers. Memoirs of the National Academy of Sciences, Vol. III (1881). 
pyknometer or a good Westphal balance. The results obtained may then be converted to Baumé (if desired) by means of the above equation.

In routine work it is customary to use a Baumé hydrometer with Fahrenheit thermometer attached and add or subtract $0.1^{\circ}$ from the Baumé reading for each degree Fahrenheit below or

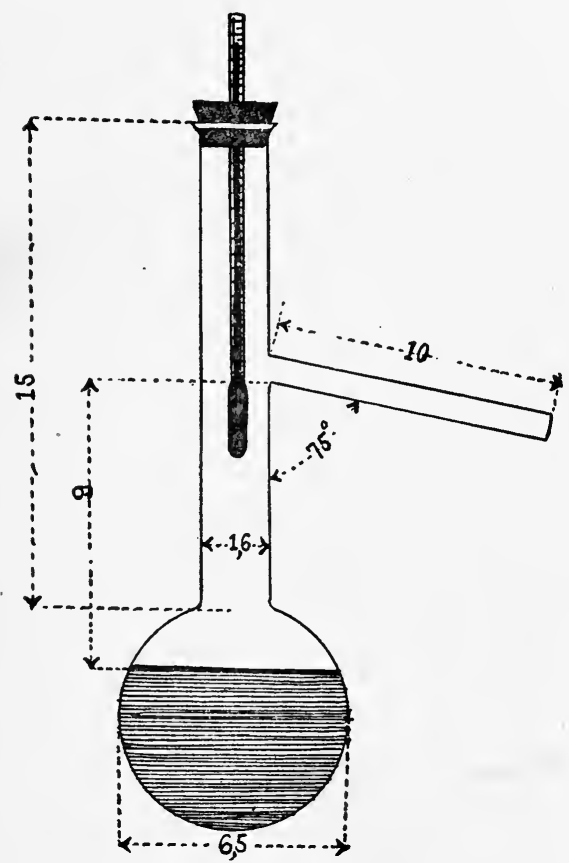

Fig. 13.-Diagram of Engler distillation flask. above $60^{\circ} .1$ When accuracy is desired, the density should be observed at the standard temperature so as to avoid the necessity for temperature corrections.

As a rough preliminary indication of the purposes for which the crude petroleum may be useful, it is often submitted to an Engler distillation test, in which $100 \mathrm{cc}$. of the oil are placed in a distilling flask of the size and shape specified by Engler (Fig. 13) and submitted to distillation, heating first over a wire gauze and then with a free flame with care to avoid drafts. The top of the thermometer bulb should be on a level with the bottom of the opening in the neck of the flask. Raise the temperature carefully (especially when in the neighborhood of $100^{\circ}$, as here drops of water may condense on the thermometer bulb and fall back upon the hot oil, producing small explosions which may endanger the experiment ${ }^{2}$ ) and at such a rate as to distill over as nearly as possible $2 \frac{1}{2}$ cc. per minute. When the ther-

1 Better results are obtained by the use of the correction table given in Tagliabue's Manual for Inspectors of Coal Oil.

2 Water should be removed in advance by settling as described above. 
mometer registers $150^{\circ} \mathrm{C}$., remove the flame and allow the temperature to fall $20^{\circ}$, and then heat again to $150^{\circ}$, cool again, and repeat as long as any distillate is obtained below $150^{\circ}$. Then raise the temperature and collect separately the second distillate, which is usually that obtained between $150^{\circ}$ and $200^{\circ}$, the distillation being conducted in the same way as before. Similarly collect and measure the distillates between $200^{\circ}$ and $250^{\circ}$, between $250^{\circ}$ and $300^{\circ}$; and finally all above $300^{\circ}$ until nothing but coke is left in the flask.

In order that the heavier portions may not undergo loss by hardening in the cold condenser tube, Low suggests that the water be drained from the condenser after the boiling point passes $200^{\circ}$.

Usually the three fractions collected between $150^{\circ}$ and $300^{\circ}$ are added together as an indication of the "illuminating oil," while that below $150^{\circ}$ is taken as an indication of the "naphtha," and that above $300^{\circ}$ the "lubricating oil," obtainable from the sample.

Gray's method for oils of the Pennsylvania type consists in distilling from 2 to 4 liters of the crude oil by means of outside heat and a current of steam, collecting the distillate in fractions each of which is 1 per cent (by volume) of the sample. The Baumé density of each fraction is observed as the distillation proceeds. All fractions reading over $57^{\circ}$ Baumé (lighter than 0.7487 specific gravity) are added together as "naphtha"; those between $57^{\circ}$ and $43^{\circ}$ B. (0.7487 to 0.8092 specific gravity) as "illuminating oils"; and all the distillate from this point until 18 per cent of the original oil remains in the still as "heavy distillate," the residue being "steam refined cylinder stock" unless the petroleum was of the asphaltic type.

Interpretation. - It is to be remembered that the competent petroleum expert uses these fractional distillations only as one factor in the detailed examination upon which to base a final opinion of the industrial possibilities of a crude petroleum. Much depends upon the character of the different fractions and their adaptability to further refining.

Petroleums are sometimes spoken of as having a "paraffin 
base" or an "asphalt base," according as the heavier hydrocarbons are solid paraffins or asphaltic substances. A pronounced example of either type may be recognized by the examination of the heavier fractions or residue obtained in a fractional distillation, but since the introduction of the middle western oils and those from northern Texas, there is no sharp dividing line between the paraffin and asphaltic base crude oils, many oils yielding both asphaltic material and paraffin wax. When the refining process is a destructive distillation, the asphaltic material may be broken up by "cracking" into fuel oil, lubricating oil, etc., and the paraffin wax recovered; but if the process is one of steam distillation, the residue being utilized for cylinder stock, the asphaltic matter is detrimental to the quality of the cylinder stock obtained.

The question sometimes arises whether a given sample is a genuine crude oil or a mixture of fractions of lesser commercial or refining value. In such cases it is usual to distill, collecting the distillate in fractions each one tenth (by volume) of the sample taken, and determine the density of each. A regular gradation of densities in the fractions is an indication of genuineness of the oil.

\section{EXAMINATION OF LUBRICATING OILS}

A thorough examination of lubricating oil involves: (1) the determination of the nature of the oil and, if it is a mixture, the proportion of each constituent; (2) tests to determine the efficiency and safety of the oil as a lubricant with special reference to the conditions of temperature, pressure, etc., to which it will be subjected in use. Among the more important properties of lubricating oils which can be measured in the laboratory are specific gravity, viscosity, acidity, or alkalinity, the temperature at which the oil solidifies, the flashing and burning points, asphaltic matter, and freedom from grit or other objectionable impurities. The usefulness of other determinations will depend upon the purposes for which the oil is intended. 


\section{Determination of Constituents}

Pure fatty and mineral oils are largely used as lubricants, both singly and mixed with each other in all proportions. Other substances are, however, often added to increase the viscosity of the oil, among the most common being "gelatin oils" containing aluminium oleate or other soaps. A better but more expensive means of increasing viscosity is to use castor oil or a "blown" oil.

In beginning the examination of a lubricating oil, note carefully any color, odor, turbidity, or fluorescence which may aid in identifying the oil or detecting foreign substances. The presence of soap is easily shown by burning a weighed portion of the oil, as refined fatty and mineral oils should not yield over 0.05 per cent of ash. Rosin oil can be detected by the Liebermann-Storch reaction.

Qualitative test for saponifiable oil is conveniently made by the Lux-Ruhemann method as follows: ${ }^{1}$ Put 3 to 4 cc. of the oil to be tested in a dry test tube, add a small piece of sodium hydroxide, or (better) metallic sodium, and heat in a paraffin bath for 15 minutes at $230^{\circ} \mathrm{C}$. in the case of pale-colored, or $250^{\circ} \mathrm{C}$. in the case of dark-colored or cylinder oils. On removing the tube from the bath and allowing it to cool, the presence of saponifiable oil is indicated by the partial or complete gelatinization of the contents of the tube or by the appearance of a soapy froth on the surface.

After making these preliminary observations the saponification number should be determined unless the sample is a pure mineral oil. In saponifying mixtures consisting largely of heavy mineral oil there is difficulty in securing sufficient contact between the sample and the alcoholic potash solution even though petroleum ether or gasoline be added. In such cases a Soxhlet extractor can be placed between the flask and the reflux condenser. ${ }^{2}$ The intermittent syphoning of the condensed solvent from the extractor into the saponification flask mixes

${ }^{1}$ J. Soc. Chem. Ind., 12, 470. Archbutt and Deeley : Lubrication and Lubricants, p. 209.

${ }^{2}$ Conradson: J. Am. Chem. Soc., 1904, 26, 672. 
the contents and facilitates saponification. In order to diminish the volume of solvent required and the interval between stirrings, the body of the extractor is partially filled with glass beads. Having found the saponification number (Chapter VIII), if the sample appears to be a mixture, the proportions of saponifiable and unsaponifiable matter are found either by separating and weighing the latter, or by estimating the former from the amount of fatty acids recovered from the soap solution after saponification.

\section{Determination of Unsaponifiable Oils}

Weigh 1 to 5 grams of oil (depending upon the saponification number and the method to be followed), saponify by heating with alcoholic potash on a water bath, ${ }^{1}$ evaporate off the alcohol, and separate the unsaponifiable matter by one of the following methods.

Method of Immiscible Solvents. - To the residue from the evaporation of alcohol add 75 cc. of water, stir thoroughly, transfer to a separatory funnel, add about an equal volume of petroleum ether or washed ethyl ether, close the funnel, shake vigorously, and allow to stand over night or until the aqueous and ethereal solutions separate completely. Draw off the aqueous layer into another separatory funnel; wash it again with ether and the ethereal layer again with water; repeat if necessary. Finally unite the ether solutions in a weighed flask, distill off the ether, and dry the unsaponifiable oil to constant weight in a boiling water oven.

If desired, the fatty acids can be recovered from the aqueous soap solution by adding an excess of mineral acid and shaking with ether or by separating the fatty acids as in soap analysis.

The principal objection to the separation by immiscible solvents is that emulsions frequently form in the separating funnel which remain even on standing for a day or more. The addition of 1 to $2 \mathrm{cc}$. of alcohol often helps to break the emulsion,

1 See also the method involving cold saponification given by Fahrion: $Z$. angew. Chem., 1898, 267. 
but if more alcohol is added it tends to carry soap into the ether layer. The separation of the solvents is also facilitated by chilling the funnel and twirling it gently, or, if the apparatus is available, by whirling in a centrifuge. Petroleum ether dissolves less soap than ethyl ether and gives less troublesome emulsions, but does not always extract the unsaponifiable matter completely.

Extraction of the Dry Soap. - To avoid the difficulties just noted the following modification of the method recommended by A. C. Wright ${ }^{1}$ may be used: Saponify 2 to 4 grams of oil, using 2 grams of caustic potash; after evaporating off the alcohol, add 3 grams of sodium bicarbonate and $10 \mathrm{cc}$. of pure methyl alcohol, stir well and evaporate, add 5 cc. more of methyl alcohol and 10 grams of precipitated chalk, mix well, dry on a water bath and then for a few minutes at $110^{\circ}$. Transfer the thoroughly dried mixture quickly to a Soxhlet extractor and extract the unsaponifiable matter with petroleum ether. Dry the extract to constant weight in a boiling water oven and weigh.

The mixture of calcium carbonate and soap from which the unsaponifiable matter has been extracted can be treated with hydrochloric acid to dissolve the carbonate and liberate the fatty acids, which can then be separated and examined further.

\section{Estimation and Identification of FAtTy Oils}

From the weight of fatty acid recovered as described above, the percentage of fatty oil can be calculated on the assumption that the oil yields 95 per cent of insoluble fatty acids. The result thus found serves as a check upon the determination of unsaponifiable oil.

If only fatty and mineral oils are present and the percentage of the former is small, it can be estimated with sufficient accuracy for most purposes from the saponification number, since the fatty oils which are likely to be present in mixed lubricants do not vary greatly in their saponification numbers. See table

1 Analysis of Oils and Allied Substances, p. 111. 
at end of Chapter VIII. If the fatty oil is identified, the average number for that species of oil should be used in estimating the percentage.

If the lubricant consists entirely of fatty oil with a known small amount of inert unsaponifiable matter, the usual methods for the identification of fatty oils can be employed. Otherwise the identification is based upon the examination of the separated fatty acids.

\section{VIscosity}

\section{Apparatus and Methods}

The viscosity of an oil can be determined either by measuring the resistance which it offers to the movement of a submerged solid, or by observing the rate at which it flows through an aperture under given conditions of temperature and pressure. Torsion viscosimeters, such as that of Doolittle, depend upon the first principle; but those depending upon the measurement of the rate of flow are much more generally used. Viscosimeters of this kind are made in a great variety of forms, for descriptions of which the reference books at the end of the chapter can be consulted. Among the viscosimeters most commonly used are those of Engler, Redwood, and Saybolt.

The Engler viscosimeter is probably more widely used the world over than any other form. It has long been regarded as the standard instrument in Germany, and has now been adopted in the United States government specifications, and by the American Society for Testing Materials.

It consists of a cylindrical reservoir with concave bottom, in the center of which is a capillary outlet. The reservoir is of brass, sometimes gold-lined, is surrounded by a water or oil jacket, and provided with a cover through which passes a thermometer for taking the temperature of the oil and a plug which closes the outlet. In testing an oil, $240 \mathrm{cc}$. are poured into the reservoir, and should fill it to the points of the studs which serve to indicate the correct leveling of the apparatus. The reservoir is then covered, the temperature regulated, and finally the plug is withdrawn, and the time required for the outflow of 
200 cc. is carefully noted. The time in seconds required by the oil divided by that required by water at $20^{\circ} \mathrm{C}$. is taken as the Engler viscosity number. Unless the oil is too viscous, or for some other reason a higher temperature is desired, it should be tested at $20^{\circ} \mathrm{C}$. In any case the temperature should be stated in reporting results. The instrument must be very carefully cleaned and dried before and after using, as any trace of oil around the outlet will interfere with the flow of water, and vice versa. Serious errors may also be caused by dust, grit, or scratches about the outlet. The time of flow of 200 cc. water from the Engler viscosimeter should be from 51 to 53 seconds. The "normal apparatus" is made according to strict specifications, and is carefully standardized by the German officials. Figure 14 shows a section of the apparatus in which $A$ represents the oil cylinder, $B$ the outer bath or jacket, $C$ the flask

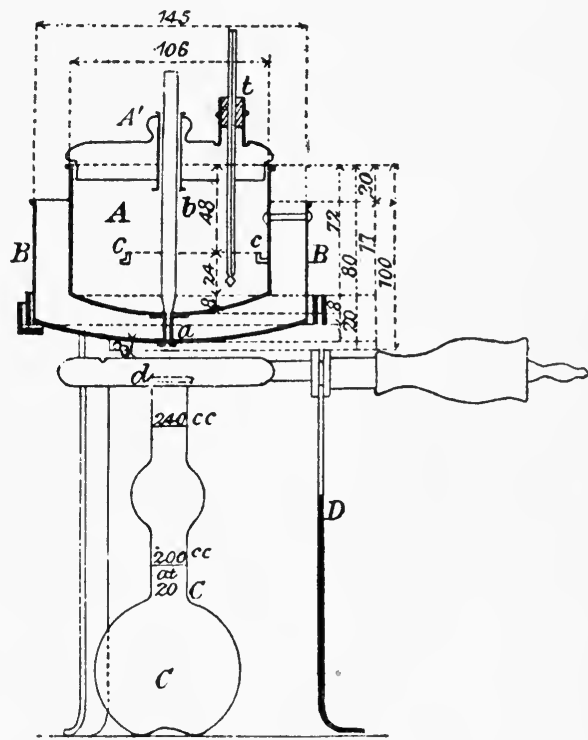

Fig. 14. - Diagram of Engler viscosimeter. From Lewkowitsch's Oils, Fats, and Waxes.

(Macmillan and Co.) marked at 200 and $240 \mathrm{cc}$. which serves both to measure the oil for the test and to receive it as it flows from the capillary, $b$ the plug, $t$ the thermometer, $D$ the tripod support, and $d$ the ring burner, which is used only when making tests at temperatures above that of the room. The correct dimensions of the apparatus are also indicated on this cut.

Redwood's viscosimeter, which has been largely used in Great Britain, consists of a cylinder about $4.7 \mathrm{~cm}$. in diameter and $8.7 \mathrm{~cm}$. high, having in the center of the bottom a cup-shaped 
agate jet which can be closed by means of a spherical plug. Inside the cylinder is a small fixed bracket of thick bent wire with an upturned point to indicate the height to which the oil , should extend at the beginning of the test. The apparatus is jacketed and provided with a closed side tube and a revolving stirrer so that determinations can be made at high temperatures if desired. The apparatus is intended to deliver 50 cc. of water at $15.5^{\circ}$ in 25.5 seconds, but as the rate of flow is influenced by many conditions, it must be determined by each observer for his own apparatus and method of working.

To use the apparatus at room temperature place it on a level support, insert the plug, and fill with the liquid to be tested until the surface comes exactly to the upturned point already mentioned. Place beneath the outlet a narrow-necked flask graduated at $50 \mathrm{cc}$., open the jet by lifting the ball valve, and observe the time required for $50 \mathrm{cc}$. to flow into the receiving flask. Whatever method is adopted for expressing the results, the report should always show the actual time of flow for the oil and for water and the temperature at which the test was made. The same precautions should be taken with this as with the Engler apparatus.

For a description of Saybolt's viscosimeter consult Gill's Oil Analysis.

\section{Significance of Results}

Since the object of lubricating with oil is to separate the moving surfaces by a fluid layer, it is important that the oil have sufficient viscosity or "body" to stay in place and keep the moving surfaces apart under the maximum pressure to which they will be subjected. The greater the pressure the more viscous the oil should be, but any viscosity beyond that which is necessary to keep the oil in place means an increase of fluid friction and consequent loss of power. The viscosity of the oil is, therefore, the most direct indication of its suitability as a lubricant under given conditions. For several reasons, however, the viscosity alone is not a conclusive measure of the lubricating power. The adhesion to solid surfaces which 
prevents the displacement of the oil by pressure is not always directly proportional to the true viscosity or internal friction. Oils vary greatly in the rate of change of viscosity with increasing temperature and pressure. The viscosity as measured by the rate of flow depends not only upon the internal friction of the oil, but also to some extent upon its adhesion to the sides of the outlet and upon the specific gravity. Hence, it is not to be assumed that any two oils having the same viscosity can be used interchangeably as lubricants. In order to duplicate an oil which has been found satisfactory in use, the kind of oil, the specific gravity, and the viscosity at least, should be specified. $\mathrm{V}$ iscosity is especially important in dealing with mineral oils because of the ease with which they can be varied in this respect, while any particular kind of fatty oil varies only within comparatively narrow limits. For a full theoretical discussion of viscosity and lubrication, the work of Archbutt and Deeley should be consulted. The reader must also be referred to this and other books and articles given below, for discussion of the many practical considerations affecting the selection of lubricating oils.

Mixed oils do not always show the viscosities which would be expected from the proportions and viscosities of the constituents of the mixture. In other words, viscosity is not an additive property in oil mixtures. In mixtures of oils whose viscosities are similar, the discrepancy between the estimated and observed viscosity of the mixture may not be apparent, but where oils of widely different viscosities are mixed, the discrepancy may be considerable.

Sherman, Gray, and Hammerschlag ${ }^{1}$ in a series of experiments with nine sets of mixtures found that the observed viscosity of the mixture was lower than the calculated value whether the mixture was that of two mineral oils, a mineral and a fatty oil, or a mineral oil and sperm oil; and in general, the greater the difference in viscosities between the oils mixed, the greater was the difference between the calculated and observed viscosity numbers of the mixtures. In a typical case a series was pre- 
pared, consisting of mixtures in tenths by weight of a high viscosity lubricating oil (" $\mathrm{H}$ ") and a low viscosity lubricating oil ("L"), both made from Pennsylvania petroleum. The pure oils and mixtures tested as follows:

Engler Viscosities at $20^{\circ} \mathrm{C}$.

\begin{tabular}{|c|c|c|c|c|c|c|c|}
\hline & & & & & Calculated & Found & Difference \\
\hline High viscosity oil ("H") . . . & . & & & & - & 25.56 & - \\
\hline 90 per cent " $\mathrm{H}$," 10 per cent " $\mathrm{L}$ " . & & & & & 23.41 & 20.04 & 3.37 \\
\hline 80 per cent " H," 20 per cent "L" & . & & & & 21.27 & 16.25 & 5.02 \\
\hline 70 per cent " $\mathrm{H}$, " 30 per cent " $\mathrm{L}$ " & . & & & & 19.12 & 13.37 & 5.75 \\
\hline 60 per cent " $\mathrm{H}$, ," 40 per cent " $\mathrm{L}$ " & . & & & & 16.97 & 10.90 & 6.07 \\
\hline 50 per cent “ H," 50 per cent "L" & . & & & & 14.83 & 9.04 & 5.79 \\
\hline 40 per cent " H," 60 per cent " L" & . & $\cdot$ & & & 12.68 & 7.69 & 4.99 \\
\hline 30 per cent " $\mathrm{H}$, " 70 per cent " $\mathrm{L}$ " & . & & & & 10.54 & 6.38 & 4.16 \\
\hline 20 per cent " $\mathrm{H}$," 80 per cent " L " & . & & & & 8.39 & 5.52 & 2.87 \\
\hline 10 per cent " $\mathrm{H}$," 90 per cent " $\mathrm{L}$ " & $\cdot$ & & & & 6.24 & 4.67 & 1.57 \\
\hline Low viscosity oil ("L") . . . . & . & $\cdot$ & $\bullet$ & & - & 4.10 & - \\
\hline
\end{tabular}

In every case the viscosity of the mixture was less than the value obtained by calculation from the percentages and viscosities of the constituents. The difference between the calculated

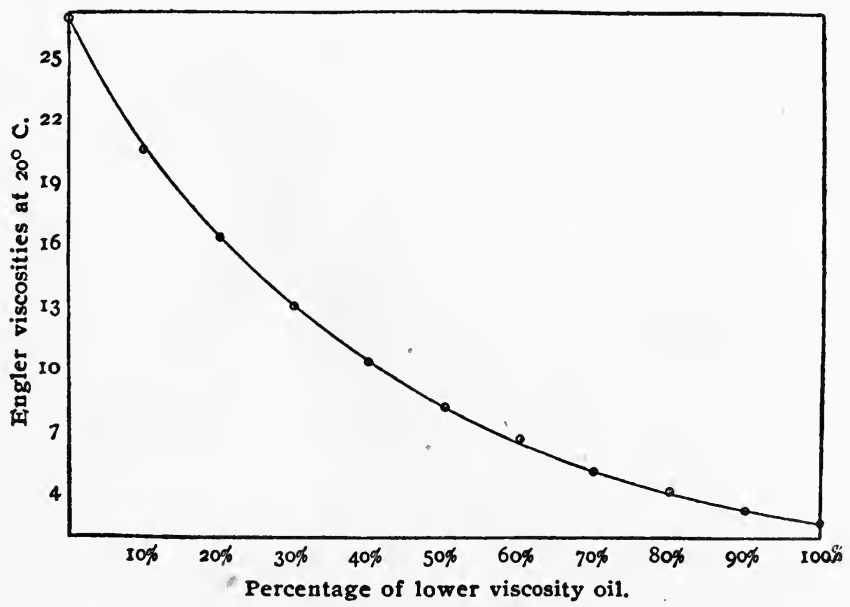

Fig. 15. - Viscosities of oil mixtures. 
and determined values increased with the increasing proportions of light oil in the mixture up to 40 per cent of light and 60 per cent of heavy oil ; with further increments of the light oil, the difference gradually decreased. The determined viscosities are plotted in Fig. 15 (the viscosities as ordinates, the percentages of " $\mathrm{L}$ " as abscissæ), and will be seen to form a very regular curve, dropping away from the calculated values somewhat more abruptly when the light oil is added to the heavy than when the heavy oil is added to light.

Subsequently Kessler and Mathiason ${ }^{1}$ obtained similar results.

\section{Acidity}

Weigh accurately 5 to 10 grams of oil in a 250-cc. Erlenmeyer flask, add 50 cc. of neutralized 85 per cent alcohol containing phenolphthalein as indicator, and titrate with standard sodium or potassium hydroxide, shaking vigorously after each addition until a permanent pink color is obtained. It is often necessary to allow the flask to stand after shaking until the oil separates from the alcohol solution before observing the color of the latter. ${ }^{2}$

To test for free mineral acid, shake 10-15 grams of oil with $100 \mathrm{cc}$. of warm water in a separatory funnel, allow to separate, draw off the water, filter through wet paper, cool, and add methyl orange. If mineral acid is found, shake the oil remaining in the funnel repeatedly with small portions of hot water until all mineral acid is extracted, filter as before, add the filtrate to the first portion containing methyl orange, and titrate very carefully with standard alkali. Concentrate the neutralized solution, test qualitatively to determine the nature of the mineral acid, and calculate the percentage. If the identification of the mineral acid is prevented by the presence of salts, calculate the mineral acidity as due to sulphuric acid. The acidity due

1 J. Ind. Eng. Chem., 3, 66.

2 For determining acidity in very dark colored fats the use as indicator of $10 \mathrm{cc}$. of a 2 per cent solution of "Alkali Blue II OLA" (Meister, Lucius and Brunig) in 99 per cent alcohol has been recommended by Freundlich : Oesterr. Chem. Ztg., 1901, 4, 441 ; Z. Nahr. Genussm., 1902, 5, 460. 
to organic acids, or the total acidity if only this is determined, is usually calculated as percentage of oleic acid. As much as 15 per cent of free oleic acid is sometimes allowed in lubricating oils. The best grades of lard oil do not contain over 1.5 per cent. Free mineral acids should be absent.

\section{Cold Test and Chilling Point or Cloud Test}

The "cold test" indicates the temperature at which the sample just ceases (or just begins) to flow ; the "chilling point" that at which the oil begins to become turbid or to show flocks or scales of solid. In either case the temperature required will be influenced by details of manipulation, so that an arbitrary method must be followed to obtain strictly comparable results. The directions below follow the procedure recommended by Gray.

Cold Test. - Pour about 25 cc. of oil into an ordinary bottle of about $100 \mathrm{cc}$. capacity and insert a stopper carrying a thermometer the bulb of which reaches just below the surface of the oil, cool the sample slowly to $50^{\circ} \mathrm{F}$. and then place in a freezing mixture of ice and salt. As the temperature falls every few degrees remove and tilt the bottle until the temperature is found where the oil just ceases to flow. This is called the "cold test" or "setting point." Cylinder and black oils which have not been treated either by acid or by filtration through fuller's earth may show abnormally low and irregular cold tests.

With oils having cold tests higher than $45^{\circ} \mathrm{F}$., it is customary to reverse the process, freezing the sample first and allowing it then to warm slowly until it just flows.

Chilling Point. - Usually it is only necessary to know whether the oil remains clear for a given number of minutes at a given temperature. Use the same bottle, amount of sample, and thermometer as for the cold test. Expose the liquid to cold, stirring with the thermometer, and hold at the required temperature for the specified time (usually ten minutes). If the oil remains transparent and free from flocks or scales, it meets the requirement as to chilling test. 
If it is required to find the chilling point, the procedure is similar; but the liquid after remaining clear as described is exposed to a temperature $3^{\circ}$ lower, allowed to stand with constant watching and occasional stirring with the thermometer until the oil is as cold as the bath, repeat this cooling until opacity or flocks or scales begin to show. The reading of the thermometer when this occurs shows the "chilling point" or "cloud test."

For further information on the cold test or setting point see the works of Archbutt and Deeley, Gill, Holde, Lewkowitsch, and Stillman.

\section{Flashing And Burning Points}

For the most accurate results closed testers such as are used in examining illuminating oils should be employed. Among these the Pensky-Martens apparatus is perhaps the best for this purpose. An illustrated description of this apparatus will be found in Lewkowitsch's Oils, Fats, and Waxes, 4th Ed. Vol. III. pp. 58-60.

For routine work in this country the "Cleveland Cup" tester is often used. This is a shallow, cylindrical, jacketed cup, open to the air and heated by a Bunsen flame. ${ }^{1}$ The test cup is filled to about $0.5 \mathrm{~cm}$. from the top and the thermometer is suspended in such a position that the bulb is entirely immersed in the oil at the center of the dish without touching the bottom. Heat by means of a Bunsen burner, and as the flashing point is approached, test at each second or third degree by slowly passing the test flame across the dish horizontally about $0.5 \mathrm{~cm}$. above the level of the oil and directly in front of the thermometer. Record the temperature at which the first flash is seen as the flashing point. Continue heating and testing in the same way until the liquid takes fire; note this temperature as the burning point. Remove the thermometer and blow out the flame or smother it by sliding a watch glass over the dish.

Notes. - The flashing and burning points must be determined in a place free from drafts. The heating should be so regulated

${ }^{1}$ In the absence of either of these forms of apparatus a porcelain dish, $4 \mathrm{~cm}$. deep and $4 \mathrm{~cm}$. in diameter, set deep in a sand bath, is sometimes used. 
that on approaching the flashing or burning point the rate of rise of temperature of the oil is not greater than $6^{\circ} \mathrm{C}$. per minute. Tests may then be made every half minute using a test flame not over $5 \mathrm{~mm}$. long. The test flame may be obtained from a narrow glass jet (similar to that used on a wash bottle) connected with the ordinary gas tubing, the flow of gas being regulated to give a flame of the size desired. Any variation of the conditions, either in size and form of dish, the rate of heating and testing, or the manner of applying the test flame may cause an appreciable discrepancy in the result.

In oil mixtures the flashing and burning points are not additive properties and cannot be predicted by simple interpolation. Sherman, Gray, and Hammerschlag ${ }^{1}$ found that in mixtures of mineral oils of different flashing and burning points, and of mineral oil with a fatty oil, or sperm oil, the flashing and burning points found were invariably lower than the figures which would be found by simple interpolation from the known properties and proportions of the oils in the mixture. It was also observed that when a high-test and a low-test oil were mixed in different proportions the discrepancy was greater in mixtures containing a high proportion of the high-test oil ; or, differently stated, the flashing and burning points were lowered by the presence of 25 per cent of the low-test oil to a greater extent than they were raised by the presence of 25 per cent of the high-test oil.

Kessler and Mathiason ${ }^{2}$ have also shown that the flashing and burning points are not additive properties in oil mixtures.

\section{Additional Determinations}

Additional tests and determinations are frequently required to show the suitability of the lubricant for the particular use intended. Friction tests on oil testing machines especially designed for the work are sometimes of great importance. A full discussion of such mechanical methods of testing will be found in Archbutt and Deeley's Lubrication and Lubricants.

1 J. Ind. Eng. Chem., 1, 13.

${ }^{2} J$. Ind. Eng. Chem., 3, 66. 
Loss by evaporation and tendency to "gum" are tested by heating a small amount of oil on a watch glass for several hours at the highest temperature to which it is likely to be subjected in use. The oil must not become sticky and the loss of weight should usually be less than 1 per cent. Suspended matter which may be invisible in a dark oil is detected by diluting the sample with gasoline or petroleum ether. Antifluorescents, added to destroy the fluorescence or "bloom" of mineral oils, can often be detected by boiling $1 \mathrm{cc}$. of the oil with $3 \mathrm{cc}$. of a 10 per cent solution of potassium hydroxide in alcohol. A red color indicates nitronaphthalene or nitrobenzene, which are the principal antifluorescents used. According to Holde, ${ }^{1}$ asphaltic matter can be approximately determined as follows: Dissolve 5 grams in 125 cc. of ether at $15^{\circ}$, add, drop by drop with constant shaking, 62.5 ce. of 96 per cent alcohol; after standing 5 hours at $15^{\circ}$, filter, wash with a mixture of alcohol and ether (1:2 by volume) until nothing more than traces of pitchlike substance is removed. Dissolve the residue in benzol, evaporate, dry one half hour at $105,^{\circ}$ and weigh.

For other tests and determinations and fuller discussions of most of those here given the reader is. referred to the works given for reference below.

\section{EXAMINATION OF LUBRICATING GREASES}

Lubricating greases are usually mixtures of soaps with fats, hydrocarbons, rosin, or tar, containing water and sometimes large amounts of mineral matter. On melting, the grease often separates into a soap solution and an oily layer. The soaps used in making such lubricants may contain salts of sodium, potassium, calcium, or heavy metals with either fatty or resin acids. Some greases consisting essentially of fats and hydrocarbons melt at temperatures to which they are subjected in use and may therefore be examined in the melted state by the methods used for lubricating oils. For most greases, however, it is necessary to adapt the analytical method to the nature of

${ }^{1}$ Mitt. Kgl. Techn. Versuchsanstalt, Berlin, 1902, 20, 253; Z. Nahr. Genussm., 1903, 6, 855. 
the mixture to be examined in each case, since the composition of these greases is too variable to allow the use of any fixed system of examination. It may often be necessary to resort to a combination of the methods used in the analysis of soaps, fats, and lubricating oils. For detailed information on the composition and testing of lubricating greases, see the reference books cited below and a review by Conradson: J. Am. Chem. Soc., 1904, 26, 705-712.

\section{REFERENCES}

I

Alder-Wright and Mitchell: Oils, Fats, Butters, and Waxes.

Allen: Commercial Organic Analysis:

Archiutt and Deeley: Lubrication and Lubricants.

DAvis: Friction and Lubrication.

Gill: Short Handbook of Oil Analysis.

Holde: Untersuchung der Schmiermittel.

Lewkowitsch : Oils, Fats, and Waxes.

LUNGE: Chemisch-technische Untersuchungsmethoden.

Post : Chemisch-technisehe Analyse.

Rakusin: Die Untersuchung des Erdöles und seine Producte.

REDWOOD: Petroleum and its Products.

Stillman: Engineering Chemistry.

Ubbelohde: Handbuch der Chemie und Technologie der Oele und Fette.

II

1904. EGER: Testing of Mineral Lubricating Oils. Z. angew Chem., 1904, 1577.

1905. Richardson and Hanson: Valuation of Lubricants with Special Reference to Cylinder Oils. J. Soc. Chem. Ind., 24, 315 .

1906. Gill: Apparatus for Testing Liability of Oils to produce Spontaneous Combustion. J. Soc. Chem. Ind., 26, 185.

Ross and Leather: Valuation of Oils for Gas-making. Analyst, 31, 284.

Tolman: Coöperative Work on the Cloud and Cold Test for 1906.

U. S. Dept. Agriculture, Bur. Chem., Bul. 105, p. 29.

VAlenta: Determination of Coal Tar Oils in Mineral and Rosin Oils. Chem. Ztg., 30, 266.

1907. Acheson: A New Lubricant. Defloculated Graphite. Eng. News, $53,127$. 
Charitschkow: Influence of Water on Flash-Point and Viscosity. Chem. Rev. Fett-Harz-Ind., 14, 225 : Chem. Abs., 2, 176.

Kissuing: Constants in Mineral Oil Analysis. Chem. Ztg., 31, 328.

Physikalisch-technische Reichsanstalt. Calibration of Engler Viscosimeters. $Z$. angew. Chem., 20, 832; $Z$. öffentl. Chem., 13, 204.

Schlicht and Halphen: Determination of Unsaponifiable Matter. Chem. Ztg., 31, 279.

Schrenber: Determination of Saponification Number in Lubricating Oils. J. Am. Chem. Soc., 29, 74.

Stolzenburg: Technical Examination of Lubricating Oils. Chem. Rev. Fett-Harz-Ind., 14, 239, 274 ; Chem. Abs., 2, 456.

UbBelohde: Improvements in Engler Viscosimeter. Chem. Ztg., 31, 38 .

1908. Holde: (Physical Behavior of Lubricants). Z. angew. Chem., 1908, 31, 2138.

Holde and Eickmann: Resinous Products in Mineral Oils. Petroleum, 1908, 3, 1077 ; Chem. Abs., 2, 1341.

Kissling: New Constants in Mineral Lubricating Oil Analysis. Chem. Ztg., 32, 938.

LEcocQ: Determination of Asphalt in Mineral Oils. Bull. Soc. Chim. Belg., 22, 81; Chem. Abs., 2, 1880.

Mabery and Mathews: Viscocity and Lubrication. J. Am. Chem. Soc., 30, 992 .

Review on Lubrication. Engineering, 85, 86; Chem. Abs., 2, 1342. U. S. Senate Document No. 469, 60th Congress, 1st Session. Plans for International Standards for Testing Mineral Oil Products.

1909. DAY and GilPIN : Changes in Crude Petroleum effected by Diffusion through Clay. J. Ind. Eng. Chem., 1, 449.

Grlletr: Analyses and Friction Tests of Lubricating Greases. J. Ind. Eng. Chem., 1, 351.

Hyde: Definition of Gasoline. J. Ind. Eng. Chem., 1, 377.

LADD: Experiments with Burning Oils. N. Dak. Agl. Expt. Sta., 18th Ann. Report, pp. 34-43; Chem. Abs., 3, 243.

Magruder: Rapid Method for the Determination of Sulphur in Crude Petroleum. Chem. Abs., 3, 115.

SAdTLER: (Methods of avoiding Emulsions in Extraction of Unsaponifiable Oil). J. Ind. Eng. Chem., 1, 479.

Sherman, Gray, and Hammerschlag: Comparison of Calculated and Determined Viscosity Numbers and Flashing and Burning Points in Oil Mixtures. J. Ind. Eng. Chem., 1, 13.

Stormer: Viscosimeter. J. Ind. Eng. Chem., 1, 317.

UbBelohde: Viscosity of Illuminating Oils and an Apparatus for its Determination. Petroleum, 1909, 4, 861; Chem. Abs., 3, 2376. 
1910. Conradson: Laboratory Tests of Lubricants - Interpretation of Analyses. J. Ind. Eng. Chem., 2, 171.

Goss: Oils and Lubricants. Modern Power, 1, No. 4; Chem. Abs., 5,379 .

Kissling: Examination of Crude Petroleum and of its Products. Petroleum, 1910, 5, 505; Chem. Abs., 4, 1365.

Kisslivg: Determination of Asphalt in Cylinder Oils. Chem. Rev. Fett-Harz-Ind., 17, 35; Chem. Abs., 4, 1367.

Mabery: Lubrication and Lubricants. J. Ind. Eng. Chem., 2, 115.

Meissner: Influence of Errors in the Dimensions of Engler's Viscosimeter. Chem. Rev. Fett-Harz-Ind., 17, 202; Chem. Abs., 4, 3148.

Roberts and Fraser: Estimation of Water in Petroleum. J. Soc. Chem. Ind., 29, 197.

Waters : Action of Sunlight and Air upon some Lubricating Oils. J. Ind. Eng. Chem., 2, 451.

1911. DAY: The Production of Petroleum in 1910. Published by U.S. Geological Survey.

Groschuff: Solubility of Water in Benzene, Petroleum, and Paraffin Oils. $\quad Z$. Electrochem., 17, 348; Chem. Abs., 5, 2550.

Kessler and Mathiason: On the Interpolation Method of Oil Analysis. J. Ind. Eng. Chem., 3, 66.

Loebell: Determination of Asphaltum Insoluble in a Mixture of Alcohol and Ether in Mineral Lubricating Oils. Petroleum, 6, 774; C'hem. Abs., 5, 3149.

WATERS: The Effect of Added Fatty and Other Oils upon the Carbonization of Mineral Lubricating Oils. J. Ind. Eng. Chem., 3, 812. 


\section{CHAPTER XII}

\section{Fuels}

THE purpose of this chapter is to outline the direct determination of the calorific value of solid and liquid fuels and then to consider the analytical determinations of most importance for the judgment of each of the chief types of fuel and especially the relation of the chemical composition to the calorific power.

\section{Determination of Calorific Power}

The heat of combustion or calorific power of a solid or liquid fuel is best determined by burning in oxygen in a bomb calorimeter according to the general method of Berthelot. The chief modifications of the Berthelot bomb in use in this country are those of Atwater, Emerson, and Mahler.

The Atwater apparatus has been fully described by Atwater and Snell in the Journal of the American Chemical Society for July, 1903. The original description of the Emerson calorimeter will be found in the Journal of Industrial and Engineering Chemistry for January, 1909, and the Mahler bomb is well described by Gill in his Gas and Fuel Analysis for Engineers. Since these descriptions as well as others referred to at the end of this chapter are readily accessible and full directions are usually furnished with the apparatus by the manufacturer, it is not so important here to discuss the exact details of manipulation, which depend to some extent upon the form of bomb calorimeter used, as to outline certain principles and precautions of general application.

The heat of combustion is determined by burning in the bomb in an atmosphere of oxygen a small amount of the sub- 
stance, usually enough to yield about 5 or 6 large calories, and measuring the rise of temperature of the known amount of water in which the bomb is immersed. In order to be able to determine the quantity of heat from the rise in temperature it is necessary to know not only the amount of water surrounding the bomb but also the heat capacity of the apparatus. There are four general methods of estimating this heat capacity :

(1) By calculation from the weights and specific heats of the materials of which the apparatus is composed.

(2) By some application of the "method of mixtures," such as placing the bomb in a known amount of water and after the temperature has become uniform adding a known amount of water of a different (known) temperature and noting the change of temperature of the system.

(3) By burning in the bomb under the conditions of an ordinary determination a weighed amount of substance whose heat of combustion is accurately known and noting the rise of temperature produced by this known amount of heat.

(4) By generating a known amount of heat in the bomb electrically and measuring the resulting rise of temperature.

The first method is not sufficiently accurate for final calibration of a bomb but is sometimes useful for a preliminary calculation of approximate heat capacity.

The second method is accurate only when carried out under conditions and with precautions so troublesome as to be practically prohibitive.

The third method is more accurate than the first or second, and is the one now generally used, but it involves the assumption that the heat of combustion of the "standard substance" is accurately known.

The fourth method ${ }^{1}$ is the most accurate but is hardly necessary except as an ultimate basis in the preparation of standard substances.

The Bureau of Standards recommends that calorimeters generally be calibrated by the third method using standard

1 Jaeger and Steinmehr: Ann. d. Phys., 21, 23-63 (1906) and Bulletin of the Bureau of Standards, Reprint No. 135. 
combustion samples, the heats of combustion of which have been carefully determined in calorimeters calibrated by electrical means.

The heat capacity of the apparatus is usually expressed as its "water equivalent" or "hydrothermal equivalent." Thus a "water equivalent" or " hydrothermal equivalent" of 408 would mean that the heat capacity of the apparatus was equal to that of 408 grams of water.

The specific heat of water changes somewhat with the temperature so that a slight error is introduced if a calorie be taken in one case as the heat capacity of water per degree centigrade at zero and in another case at room temperature. The Bureau of Standards adopts the heat capacity of water at $15^{\circ} \mathrm{C}$. as unity so that the calorie is defined as the heat capacity of one gram of water per degree centigrade at a temperature of $15^{\circ} \mathrm{C}$. and the B. T. U. as the heat capacity of one pound of water per degree Fahrenheit at a temperature of $60^{\circ} \mathrm{F}$. Fortunately it is not necessary in ordinary determinations of heat of combustion to change the basis of calculation with the temperature of working between $15^{\circ}$ and $25^{\circ} \mathrm{C}$., because the Bureau of Standards has found that the total heat capacity of an ordinary combustion calorimeter does not change appreciably with temperature between $15^{\circ}$ and $25^{\circ} \mathrm{C}$. This is due to the fact that the metal of the bomb and accessories has a positive temperature coefficient, while the water has a negative temperature coefficient throughout this range of temperature and the two nearly neutralize each other.

Standard Materials. - Among the substances which have been used in the standardization of combustion calorimeters are cane sugar, benzoic acid, naphthalene, glycocoll, hippuric acid, and camphor. The Bureau of Standards recommends the first three and supplies standard samples of these for this purpose.

Sucrose is not volatile nor strongly hygroscopic, but is rather difficult to ignite, sometimes fails to burn completely, and has a heat of combustion only about half as high as that of good coal. 
Benzoic acid is only slightly volatile, not very hygroscopic, burns readily, and has a heat of combustion about four-fifths that of ordinary coal.

Naphthalene is more volatile than benzoic acid, but is not hygroscopic, burns very readily, and has a heat of combustion a little higher than that of coal. The Bureau of Standards reports that the loss by sublimation from naphthalene pressed into pellets for combustion will hardly exceed 0.1 to 0.2 per cent in an hour, so that any error in standardization due to volatility of the naphthalene should be less than 0.1 per cent.

Although sugar has been most used in the past, the Bureau of Standards finds benzoic acid the most satisfactory substance for accurate standardization of bomb calorimeters.

Preparation of sample or charge. - Since the amount of mateterial used for a determination is usually not over a gram, the sample must be finely ground and thoroughly mixed. A portion of the mixed powdered sample is then pressed into a pellet or small briquet, which is weighed in the combustion crucible and then placed in the bomb. Pressing the charge into a pellet avoids the danger of portions being blown out of the crucible either when the oxygen is admitted to the bomb or during the rapid combustion which takes place when the substance is fired. In standardizing with napthalene the loss by volatilization is also much smaller from a pellet than from the same weight of loose material. Liquids, and anthracite coals which cannot be pressed into pellets, are conveniently burned in weighed hard "gelatin" capsules of determined calorific power. The objections sometimes offered to this method are probably due to the use of capsules of unsuitable character. The writer uses Parke-Davis capsules of "five-grain" size which weigh about one tenth gram each, have a heat of combustion of about 4480 calories per gram, are neither sticky nor hygroscopic, and burn readily, thus aiding the complete combustion of high-ash anthracite coals. Some prefer to burn anthracites in a loose condition and, if difficulty is experienced in obtaining complete combustion, to place beneath the anthracite a weighed amount of bituminous coal of known calorific 
power. Blakeley and Chance hold that the failure of high-ash coals to burn completely when placed directly in the combustion crucible is largely due to the cooling of that portion of the coal which is in contact with the metal floor of the crucible and may be avoided by placing a disk of asbestos beneath the coal.

Pasty solids and non-volatile liquids may be weighed and burned directly in the combustion crucibles. In the case of fatty oils it was found advantageous to add a little loose asbestos (previously ignited) to regulate the burning and prevent possible loss by spattering.

For a volatile liquid select a capsule having a body with a smooth edge and a snug-fitting cap, transfer the liquid from the sample bottle to the weighed capsule by means of a pipette or medicine dropper, close the capsule, stand it in the combustion crucible and weigh, then allow to stand about 5 minutes and weigh again to make sure that none of the sample is being lost by evaporation. Should it be found that the capsule permits loss by evaporation, it must be rejected and another prepared and tested in the same way.

Both in standardizing the bomb and in the testing of fuels the amount of the charge should depend upon its calorific power so that there may not be great differences in the amount of heat to be measured in the different determinations. When the charge has been prepared in the combustion crucible, it is placed in position, the fuse wire adjusted, the bomb closed and charged with oxygen.

The oxygen used for combustion must be the purest obtainable commercially, especially as regards freedom from combustible gases such as hydrogen, carbon monoxide, and hydrocarbons. Each cylinder of oxygen should be tested by determinations upon standard combustion samples such as are used in determining the heat capacity of the apparatus.

The bomb is charged with oxygen to a pressure of about 25 atmospheres, and, after being properly closed to prevent any escape of gas, is immersed in a weighed quantity of water, the terminals of the firing circuit adjusted, the covers placed in position, and the stirring and thermometric observations begun. 
The details of these operations vary with the different forms of apparatus and are explained for each in the descriptions already cited.

At the end of the "fore-period" in which the rate of change due to the temperature of the surroundings has been determined, the charge is ignited by closing the switch of the firing circuit so as to pass through the fuse wire a current sufficient to heat it to redness if platinum, or cause it to burn if iron, but of low potential so as to avoid danger of arcing with evolution of heat within the bomb.

Temperature measurements should be made with delicate differential thermometers of the Beckmann type which should be carefully calibrated, preferably by the Bureau of Standards or the Reichsanstalt. Accuracy requires that the proper corrections be determined and applied for errors of the scale, for the emergent stem, and for the amount of mercury removed from the bulb. The first, called "caliber correction," should be applicd to each reading; the second and third together are often called "thermometer correction," and this correction, which will depend upon the room temperature and the setting of the thermometer, is applied to the apparent rise of temperature observed during the combustion, to obtain the rise in true degrees.

The radiation correction is calculated from the readings taken before, during, and after the combustion, preferably by means of the Regnault-Pfaundler formula.

At the end of the after-period following a combustion the bomb is opened and a careful examination made for any evidences of incomplete combustion. Any portions of the fusewire which may remain unburned are weighed and deducted from the original weight. The bomb is rinsed out and the nitric acid which has been formed during the combustion is determined by titration. The heat of formation and solution of the nitric acid formed (230 calories per gram), as well as the heat of combustion of the iron fuse wire burned (1600 calories per gram), and of the gelatin capsule if used, must be allowed for in the calculation of the results. The full explanation of the calculation of results given by Atwater and Snell should be 
consulted. Fuels containing sulphur yield sulphuric acid on combustion in the bomb, so that a slight error is introduced if the total acidity of the bomb rinsings be calculated as nitric acid. To correct for this the usual subtraction for total acidity calculated as nitric acid may be made, then the sulphuric acid in the rinsings may be found by precipitation and the correction increased by 13 calories per gram for each per cent of sulphur in the fuel.1 This is based on the assumption that as fuel is ordinarily used the sulphur burns to sulphur dioxide.

The calorific powers as found in the bomb calorimeter represent somewhat more heat than is actually obtained from the fuel in ordinary use, not only because the combustion is complete, but especially because the water vapor produced in the combustion condenses in the bomb and the latent heat of this vapor is not lost as is usually the case when the fuel is burned in use. Occasionally an attempt is made to distinguish between "heat of combustion " and "calorific power," confining the latter term to the values obtained on subtracting from the heat of combustion the estimated heat of vaporization of the water. More often the heat of combustion is called the "calorific power (high value)" or simply "calorific power," and the term "calorific power (low value)" is used to designate the value obtained on deducting the latent heat of water vapor. In this chapter the "high values" are always given without the use of that term, or in other words the term calorific power is used as synonymous with heat of combustion.

\section{Chemical Composition and Calorific Power of Organic Compounds}

According to the usually accepted determinations, the heat of combustion of carbon is 8080 , and of hydrogen 34,500 , calories per gram. The heat of combustion of a compound consisting of carbon and hydrogen only is the sum of the heats of combustion of the carbon and hydrogen it contains minus the heat of formation of the compound, which is for hydrocarbons a rela-

1 Lord : U. S. Geological Survey, Professional Paper No. 48. 
tively small factor. In compounds containing oxygen the heat of formation is larger and the heat of combustion is proportionately less than the heat which would be obtained by burning the quantities of carbon and hydrogen present, for the obvious reason that these elements are already partially "oxidized" by the oxygen present in the molecule. From the heats of combustion of carbon and hydrogen one may readily calculate that a given weight of oxygen will cause a greater evolution of heat in burning hydrogen to water than in burning carbon to carbon dioxide. Hence in the case of a compound of carbon, hydrogen, and oxygen, the estimated calorific power will be lower if we assume that the oxygen in the molecule is to be considered as combined with hydrogen than if we assume that it is to be considered as combined with carbon.

Usually estimates of calorific power from ultimate analysis have been based on "Welter's rule," which assumes that the oxygen present is combined with hydrogen, or, that we shall offset the heat of formation if we deduct the quantity of heat which would be produced if the oxygen present combined with the hydrogen. On this assumption the heat of combustion of such a compound would be represented by the formula:

$$
X=8080 \mathrm{C}+34,500\left(\mathrm{H}-\frac{1}{8} \mathrm{O}\right) .
$$

Walker, in his Introduction to Physical Chemistry, pointed out that the values thus obtained are considerably too low in the cases of sugar and of butyric acid, and stated that " a better result may usually be obtained by subtracting the oxygen, not with the corresponding quantity of hydrogen, but with the corresponding quantity of carbon, and then estimating the heat of combustion of the elements in the residue." Putting this suggestion (which for convenience we may call "Walker's rule" as contrasted with "Welter's rule") in the form of an equation, we have,

$$
X=8080\left(\mathrm{C}-\frac{3}{8} \mathrm{O}\right)+34,500 \mathrm{H} .
$$

If, now, these two formulæ be applied to the various classes of pure organic compounds, and the results compared with the experimentally determined heats of combustion as compiled by 
Berthelot, or Vaubel, it will be found that in some cases the higher, in other cases the lower, of the calculated results approximates the true value, while for still other compounds the true value lies between them and at a distance from either. The relative values of these formulæe as applied to different classes of fuels is considered below.

In some cases fuel values are estimated from proximate, rather than ultimate, chemical composition. Thus, since the calorific power of pure alcohol and the relation of specific gravity to percentage strength of alcohol-water mixtures are both known, the specific gravity of any commercial alcohol which is essentially a mixture of pure alcohol and water will show the amount of alcohol present, and hence the calorific power of the liquid. In food analysis the fuel value is commonly estimated from the percentages of proteins, fats, and carbohydrates as has been fully explained in another volume. ${ }^{1}$

\section{Fuel Oils and Gasoline}

With appliances sufficiently well adapted to its properties, any petroleum oil can be burned with an evolution of heat much greater than that of any coal.

Since American petroleums are essentially mixtures of hydrocarbons and chiefly of the methane series, it was to be expected that the greater the specific gravity of the sample the greater would be the mean molecular weight and percentage of carbon and the less would be the percentage of hydrogen and the heat of combustion per gram. In general a specific gravity of

$0.7-0.75$ indicates about $11,500-11,300$ calories per gram;

0.75-0.8 indicates about 11,300-11,100 calories per gram ; $0.8-0.85$ indicates about $11,100-10,900$ calories per gram; 0.85-0.9 indicates about 10,900-10,700 calories per gram; $0.9-0.933$ indicates about 10,700-10,500 calories per gram.

More commonly in English-speaking countries the density of petroleum oils is stated in terms of the Baumé scale and the

1 Sherman : Chemistry of Food and Nutrition, Chapter IV. 
calorific power in British thermal units. 'The relations of the values are as follows:

calories per gram $\times \mathbf{1 . 8}=\mathrm{B}$. T. U. per pound ;

$$
\text { specific gravity }=\frac{140}{130+\mathrm{B}^{\circ}} \text {. }
$$

From the densities and calorific powers of 86 samples of American petroleum oils, 70 of which were examined by the writer and his associates at Columbia University, and 16 by Allen and Strong of the U. S. Geological Survey, is constructed the following table ${ }^{1}$ for estimating the calorific power from the Baumé density in commercially pure petroleum oils.

Table 20. - Approximate Calorific Powers, in British Thermal Units per Pound, of Petroleum Oils of $20^{\circ}$ to $67^{\circ}$ Baumé

\begin{tabular}{c|c||c|c||c|c}
\hline $\begin{array}{c}\text { Density } \\
\text { degrees } \\
\text { Baumé }\end{array}$ & $\begin{array}{c}\text { Calorific power } \\
\text { B. T. U. } \\
\text { per pound }\end{array}$ & $\begin{array}{c}\text { Density } \\
\text { degrees } \\
\text { Baumé }\end{array}$ & $\begin{array}{c}\text { Calorific power } \\
\text { B. T. U. } \\
\text { per pound }\end{array}$ & $\begin{array}{c}\text { Density } \\
\text { degrees } \\
\text { Baumé }\end{array}$ & $\begin{array}{c}\text { Calorific power } \\
\text { B. T. U. } \\
\text { per pound }\end{array}$ \\
\cline { 1 - 2 } 20 & 18930 & 36 & 19735 & 52 & 20220 \\
21 & 18990 & 37 & 19770 & 53 & 20245 \\
22 & 19050 & 38 & 19805 & 54 & 20270 \\
23 & 19110 & 39 & 19840 & 55 & 20290 \\
24 & 19170 & 40 & 19875 & 56 & 20310 \\
25 & 19225 & 41 & 19910 & 57 & 20330 \\
26 & 19280 & 42 & 19940 & 58 & 20350 \\
27 & 19335 & 43 & 19970 & 59 & 20370 \\
28 & 19390 & 44 & 20000 & 60 & 20390 \\
29 & 19445 & 45 & 20030 & 61 & 20410 \\
30 & 19495 & 46 & 20060 & 62 & 20430 \\
31 & 19545 & 47 & 20090 & 63 & 20450 \\
32 & 19590 & 48 & 20120 & 64 & 20470 \\
33 & 19630 & 49 & 20145 & 65 & 20490 \\
34 & 19665 & 50 & 20170 & 66 & 20510 \\
35 & 19700 & 51 & 20195 & 67 & 20530 \\
\hline \hline
\end{tabular}

The values given in the table were found by plotting the data of all the samples, drawing a smooth curve through the approximate mean results, and taking from this curve the calorific power (in the nearest multiple of 5 units) corresponding to each degree of density on the Baumé scale. On taking the calorific

${ }^{1}$ Revision of the work of Sherman and Kropff (J. Am. Chem. Soc., 30, 1626). 
powers from the table for each individual sample and comparing with the value determined in the bomb calorimeter, it was found that of 86 samples, ranging from gasoline to the heaviest crude oils, in only 1 case in 15 did the estimated value differ from that experimentally determined by as much as 1 per cent; in only 1 in 43 was the difference as much as 2 per cent; in no case was the difference as much as 3 per cent.

It is evident, therefore, that in commercially pure American petroleum oils the calorific power may be estimated from the density by means of the above table with a sufficient degree of accuracy for many practical purposes.

Since in gasoline engines, and in some of the machines using other fuel oils, the combustion is preceded by vaporization of the fuel, it is evident that volatility is an important factor in determining the adaptability of the gasoline or fuel oil to the engine in which it is to be used.

In general, the mechanical engineer judges the volatility from the density, but since commercial gasoline or other fuel oil is a mixture of numerous lighter and heavier hydrocarbons, "a definite constant density is not a guarantee that the composition may not change sufficiently to affect the action of the fuel in an engine" (Lucke and Woodward).

In order to determine the character of a sample in this respect, it may be submitted to distillation in an ordinary distilling flask, collecting the distillate in convenient fractions of the volume of the sample taken, and noting the temperature of distillation of each fraction by means of a thermometer so placed that the top of the mercury bulb is on a level with the bottom of the outlet in the neck of the distilling flask, so as to show the temperature of the vapors as they pass from the flask into. the condenser.

The "motor gasoline" used by Lucke and Woodward in their comparison of alcohol and gasoline as fuel for internal combustion engines in 1906-07 was examined in this manner, $150 \mathrm{cc}$. being distilled, and the distillate collected in fractions of $10 \mathrm{cc}$. each, with the following results: ${ }^{1}$

1 U. S. Dept. Agriculture, Office of Experiment Stations, Bul. 191, p. 23. 


\begin{tabular}{c|c||c|c}
\hline \hline Number of fraction & $\begin{array}{c}\text { Temperature of } \\
\text { distillation }{ }^{\circ} \mathrm{C} .\end{array}$ & Number of fraction & $\begin{array}{c}\text { Temperature of } \\
\text { distillation }{ }^{\circ} \mathrm{C} .\end{array}$ \\
\hline 1 & $46-60$ & 9 & $100-104$ \\
2 & $64-75$ & 10 & $104-108$ \\
3 & $75-80$ & 11 & $108-112$ \\
4 & 80 & 12 & $112-120$ \\
5 & $80-86$ & 13 & $120-126$ \\
6 & $86-92$ & 14 & $126-140$ \\
7 & $92-97$ & $15(5$ cc. $)$ & $140-155$ \\
8 & $97-100$ & Residue at $155^{\circ}$ C. $=5$ cc. or 3.3 pct. \\
\hline \hline
\end{tabular}

A sample purchased in New York City in 1908, and believed to be a representative specimen of satisfactory automobile gasoline, was examined by the writer with the following results:

(1) Distillation of 300 cc. in Fractions According to Volume

\begin{tabular}{c|c|c|c}
\hline Number of fraction & $\begin{array}{c}\text { Temperature of } \\
\text { distillation }{ }^{\circ} \mathrm{C} .\end{array}$ & Volume ce. & $\begin{array}{c}\text { Specific Gravity at } \\
15^{\circ} \mathrm{C} .\end{array}$ \\
\cline { 2 - 3 } 1 & $40-68$ & 30 & 0.669 \\
2 & $68-74$ & 30 & 0.678 \\
3 & $74-81$ & 30 & 0.692 \\
4 & $81-86$ & 30 & 0.704 \\
5 & $86-91$ & 30 & 0.712 \\
6 & $91-96$ & 30 & 0.720 \\
7 & $96-102$ & 30 & 0.727 \\
8 & $102-110$ & 30 & 0.734 \\
9 & $110-121$ & 30 & 0.741 \\
\multicolumn{2}{l}{ Residue at $121^{\circ} \mathrm{C}}$. & 30 & 0.756 \\
\hline \hline
\end{tabular}

(2) Distillation of 300 cc. in Fractions According to Temperature

\begin{tabular}{c|c|c|c|c}
\hline \hline \multirow{2}{*}{ Number of fraction } & \multirow{2}{*}{$\begin{array}{c}\text { Temperature of } \\
\text { distillation }\end{array}$} & \multicolumn{2}{|c|}{ Colume } & \multirow{2}{|c|}{$\begin{array}{c}\text { Specific gravity } \\
\text { at } 15^{\circ} \mathrm{C} .\end{array}$} \\
& $40-70$ & 40.5 & 13.5 & 0.670 \\
\cline { 3 - 4 } 2 & $70-80$ & 47.5 & 15.8 & 0.690 \\
3 & $80-90$ & 60. & 20. & 0.706 \\
4 & $90-100$ & 47.5 & 15.8 & 0.72. \\
5 & $100-110$ & 42.5 & 14.2 & 0.733 \\
6 & $110-120$ & 29.5 & 9.9 & 0.741 \\
7 & Residue at $120^{\circ} \mathrm{C}$. & 0.755 \\
\hline \hline
\end{tabular}


These data may be useful for purposes of comparison when examining commercial gasolines with reference to their utility as fuel for internal combustion engines.

Three samples of "fuel oil" purchased by gas manufacturers in New York City in 1905 and 1906, showed, when divided into fifths by fractional distillation, the following results :

Table 21. - Comparison of Fractions of Commercial "Fuel Oils'

\begin{tabular}{|c|c|c|c|c|c|c|c|c|c|c|c|}
\hline & & & & & & & & & \multirow{2}{*}{$\begin{array}{c}\text { Temperature of } \\
\text { Distillation } \\
\circ \mathrm{C} .\end{array}$} & \multicolumn{2}{|c|}{ Density at $15.5^{\circ} \mathrm{C} .\left(60^{\circ} \mathrm{F}.\right)$} \\
\hline & & & & & & & & & & $\begin{array}{l}\text { Specific } \\
\text { Gravity }\end{array}$ & $\begin{array}{l}\text { Degrees } \\
\text { Baumé }\end{array}$ \\
\hline \multicolumn{12}{|l|}{ Sample A. } \\
\hline 1st fraction & . & . & . & . & . & . & . & . & $165-257$ & 0.832 & 38.3 \\
\hline $2 \mathrm{~d}$ fraction & . & . & . & . & . & . & . & . & $257-290$ & 0.870 & 30.9 \\
\hline $3 d$ fraction & • & . & . & . & . & . & . & . & $290-318$ & 0.880 & 29.1 \\
\hline 4th fraction & . & . & . & . & . & . & . & . & $318-340$ & 0.886 & 28.0 \\
\hline Residue . & • & - & - & - & - & - & - & . & & 0.900 & 25.5 \\
\hline \multicolumn{12}{|l|}{ Sample B. } \\
\hline 1st fraetion & . & . & • & - & . & . & . & . & $138-243$ & 0.770 & 51.8 \\
\hline $2 d$ fraction & . & - & . & - & . & . & - & - & $243-288$ & 0.851 & 34.5 \\
\hline $3 d$ fraction & . & . & . & . & . & . & . & . & $288-327$ & 0.871 & 30.7 \\
\hline 4th fraction & • & . & . & . & . & . & . & . & $327-353$ & 0.883 & 28.5 \\
\hline Residue. . & - & . & . & - & . & . & - & $\cdot$ & & 0.906 & 24.5 \\
\hline \multicolumn{12}{|l|}{ Sample C. } \\
\hline 1st fraction & - & - & - & . & . & . & . & - & $160-260$ & 0.795 & 46.1 \\
\hline $2 \mathrm{~d}$ fraction & . & - & . & • & . & . & . & . & $260-286$ & 0.843 & 36.1 \\
\hline $3 d$ fraction & . & . & . & . & • & . & . & - & 286-305 & 0.853 & 34.1 \\
\hline 4th fraction & . & . & . & . & . & . & . & . & 305̃-330 & 0.861 & 32.6 \\
\hline Residue. . & . & . & . & . & . & . & . & . & & 0.881 & 28.9 \\
\hline
\end{tabular}

\section{Woods and Similar Fuels}

Wood, peat, spent tan, bagasse, and other similar materials used as fuel differ greatly in calorific power because of wide fluctuations in moisture and ash content and considerable differences in the nature of their organic constituents; for instance, some woods approximate cellulose, while others contain large amounts 
of resinous material of much higher calorific power. All the fuels of this general group are, however, characterized by high oxygen content and low heat of combustion as compared with good coal, and so in attempts to estimate the calorific power from the ultimate analysis the differences between the results obtained by the use of Welter's rule or Walker's suggestion as explained above are relatively larger for this group of fuels than for coals.

In order to ascertain which method of calculation is preferable and whether either yields accurate results, Sherman and Amend analyzed eight fuels of this type and compared the results obtained by each method of calculation with those actually determined by combustion in oxygen.

The results of ultimate chemical analysis, reduced to the basis of dry matter were as follows:

Table 22. - Ultimate Composition of Dry Matter of Wood, Etc.

\begin{tabular}{|c|c|c|c|c|c|c|}
\hline Sample & $\begin{array}{c}\text { Carbon } \\
\%\end{array}$ & $\begin{array}{c}\text { Hydrogen } \\
\%\end{array}$ & $\begin{array}{c}\text { Oxygen } \\
\%\end{array}$ & $\begin{array}{c}\text { Nitrogen } \\
\%\end{array}$ & $\begin{array}{c}\text { Sulphur } \\
\%\end{array}$ & $\begin{array}{l}\text { Ash } \\
\%\end{array}$ \\
\hline Chestnut wood chips & 50.28 & 5.58 & 43.21 & 0.10 & 0.03 & 0.80 \\
\hline Chestnut wood chips, leached & 50.09 & 5.65 & 43.33 & 0.10 & 0.02 & 0.81 \\
\hline Hemlock tan . . . . . . & 53.74 & 5.66 & 39.05 & 0.24 & 0.04 & 1.27 \\
\hline Hemlock tan, leached & 54.97 & 5.73 & 37.69 & 0.26 & 0.02 & 1.33 \\
\hline Oak tan, leached'. & 49.51 & 5.53 & 39.24 & 0.40 & 0.05 & 5.27 \\
\hline Bagasse . . . & 49.04 & 5.96 & 42.58 & -0.31 & 0.07 & 2.04 \\
\hline "Oil cake". & 48.20 & 6.47 & 38.02 & 4.24 & 0.25 & 2.82 \\
\hline Peat . . . & 57.30 & 4.66 & 19.26 & 1.13 & 0.77 & 16.88 \\
\hline
\end{tabular}

From these analyses the calorific power or heat of combustion was estimated (1) according to Dulong's formula

$$
X=8080 \mathrm{C}+34,500\left(\mathrm{H}-\frac{1}{8} \mathrm{O}\right)+2250 \mathrm{~S},
$$

based on Welter's rule of calculating the oxygen with the hydrogen, (2) according to Walker's suggestion of calculating the oxygen with the carbon. Using here the same calorific values for the elements as in Dulong's formula, we have the formula:

$$
X=8080\left(\mathrm{C}-\frac{3}{8} \mathrm{O}\right)+34,500 \mathrm{H}+2250 \mathrm{~S} \text {. }
$$


which must of course be applied to the percentages in the waterfree substance.

The results (in calories) obtained by these two formulæ along with those determined directly by means of the AtwaterMahler bomb calorimeter were as follows :

Table 23. - Estimated and Determined Calorific Powers

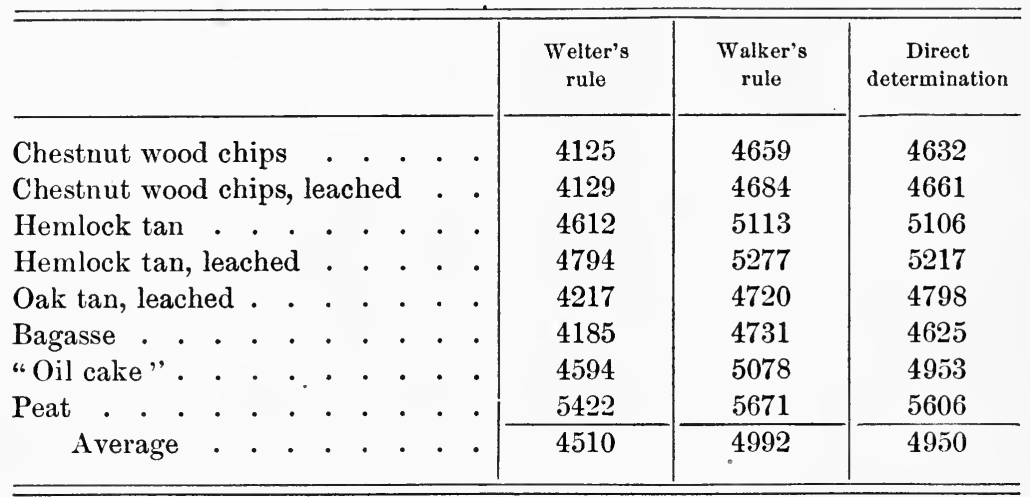

It will be seen that in the case of these woody fuels high in oxygen the calorific powers estimated by means of Dulong's formula based on Welter's rule are all much too low, the estimated values in terms of the actual ranging from 88.4 to 96.7 per cent and averaging 90.4 per cent, while the values estimated by a corresponding formula based on Walker's rule show a fair approximation to the values actually determined by the bomb calorimeter, the estimated values in terms of the determined ranging from 100.1 to 102.5 per cent and averaging 100.8 per cent.

The two methods of calculation were also applied to seven analyses of lignites taken from Bulletin 290 of the U. S. Geological Survey. In one of the seven cases the calorific power actually determined was lower than that found by Welter's rule; in one it was materially higher than that found by Walker's rule; in each of the other five cases and also in the average of all seven the results by Welter's rule were much too 
low and those calculated according to the suggestion of Walker were approximately correct.

The results indicate: (1) that too much reliance should not be placed upon estimates of calorific power from ultimate chemical composition, especially in fuels high in oxygen; (2) that Dulong's formula or any simular formula based on "Welter's rule" of calculating the oxygen with the hydrogen is likely to give results much below the truth; (3) that the higher results obtained by calculating the oxygen of the waterfree sample as combining with the carbon according to the suggestion of Walker are much more nearly correct, and in most cases show a fair approximation to the values determined directly.

CoAL

\section{Ultimate Composition and Calorific Power}

The data above given having shown that the calorific power of wood and similar fuels is related to the ultimate composition much more nearly according to Walker's than according to Welter's rule, a similar comparison of these two methods of calculating calorific power was made for coal. ${ }^{1}$ For this purpose the analyses and calorific powers of 67 coals examined by the U. S. Geological Survey and described in Professional Paper No. 48 were used. These data were determined in connection with the studies made by the Survey in its coaltesting plant at the St. Louis Exposition in 1904 and cover coals mined in 17 states and differing widely in composition and character.

A comparison of the calorific powers as calculated by Dulong's formula based on Welter's rule with those found by actual determination in a bomb calorimeter showed that the values calculated by this formula were below those found by the calorimeter in seven eighths of the cases and averaged 98.9 per cent of the determined values. In more than half of the cases the calculated differed from the determined values by

${ }^{1}$ Sherman, Bartlett, and Weatherless ; results not yet published. 
over 1 per cent; in 12 of the 67 cases or about 1 in 6 , by more than 2 per cent; in 4 cases or about 1 in 17 by more than 3 per cent, the greatest difference being 3.8 per cent.

Thus the error involved in the use of the Dulong formula based on Welter's rule was quite variable. The values thus calculated averaged $\mathbf{1 . 1}$ per cent too low, but it is evident that the calculated results of individual samples cannot be made accurate by raising them all by a corresponding percentage; for the extent of the discrepancy in each calculated value depends primarily upon the oxygen present in the sample, so that to increase all the calculated values by a fixed percentage would give calorific powers too high where the oxygen content was low and too low where the oxygen content was high. Better results were obtained by calculating the calorific powers in accordance with Walker's suggestion that the oxygen be figured as combining with the carbon rather than the hydrogen.

Calculating the calorific powers for the same 67 coals according to the formula,

$$
X=8080\left(\mathrm{C}-\frac{3}{8} \mathrm{O}\right)+34,500 \mathrm{H}+2250 \mathrm{~S}
$$

which must of course be applied to the percentages in the water-free coal, it was found that the calculated averaged 100.37 per cent of the determined values, and were about twice as often above the latter as below. In about two thirds of the cases the calculated and determined values agreed within 1 per cent, and in about one third of the cases differed by 1 per cent or more; 6 of the 67 cases, or about 1 in 11, differed by more than 2 per cent; none differed by as much as 3 per cent. Comparing the results obtained by the two methods of calculation it will be seen that the method of calculating the combined oxygen with the carbon instead of with the hydrogen gives a much better average result, a smaller proportion of cases in which the error exceeds a given margin (whether this be one or two per cent) and a smaller maximum error.

Of the formulie at present available for calculating the calorific power of coal from its ultimate analysis the one last given 
should therefore be used, but data thus calculated are much less reliable than those obtained by properly conducted determinations with an accurately standarized bomb calorimeter.

\section{Proximate Analysis of Coal}

The proximate analysis of coal consists in determining the moisture and ash, and separating the organic matter by an arbitrary heat treatment into volatile matter and fixed carbon. In order that results of different analysts may be comparable, a uniform method has been agreed upon as follows: ${ }^{1}$ The sample should be air-dried and ground to pass a 100 -mesh sieve.

Moisture. - Dry 1 gram of the fine-ground, air-dry sample in an open platinum or porcelain crucible in an oven at $104^{\circ}$ to $107^{\circ}$ C. for 1 hour; cool in a desiccator and weigh covered. The loss of weight is considered as moisture.

Volatile matter. - Heat one gram of the air-dry sample (or the portion which has been used for the determination of moisture) in a platinum crucible weighing 20-30 grams with a well-fitting cover over the full flame of a good Bunsen burner for 7 minutes, in a place free from drafts. The flame should be $25 \mathrm{~cm}$. high and the crucible supported on a platinum triangle so that the bottom of the crucible is 6 to $8 \mathrm{~cm}$. above the top of the burner and the entire crucible is surrounded by the hottest part of the flame. The upper surface of the cover should burn clean; any carbon on the under surface of the cover is weighed with the residue in the crucible. The loss in weight by this heating, corrected for moisture if necessary, is called the volatile combustible, or simply the volatile matter.

$A s h$. - Burn one gram of sample, or the residue from one of the above determinations, in a platinum crucible (open and inclined) first over a very low flame, then at a higher temperature, till free from carbon, and weigh the residue as ash.

${ }^{1}$ Report of Committee of American Chemical Society : J. Am. Chem. Soc.. 21, 1119. 
Fixed carbon is found by subtracting the sum of the percentages of moisture, volatile matter, and ash from 100.

The determination of sulphur, while not a part of the proximate analysis, is so often required in connection with it that the usual method may be outlined here. This is the modified Eschka method. One gram of the finely pulverized coal is mixed with $1^{\circ}$ gram of light magnesium oxide and 0.5 gram of dry sodium carbonate in a Meissen porcelain crucible (or platinum dish of about 75 cc. capacity) and heated with an alcohol lamp (or with a gas flame nearly free from sulphur, the dish being set in a smooth hole in an asbestos pad so that its contents are protected as much as possible from the products of combustion). The heat should be applied gently at first, especially with soft coals, and the mixture stirred frequently with a stout platinum wire. Gradually increase the heat until the bottom of the dish reaches low redness, and maintain at about this temperature with frequent stirring until all carbon is burned; then cool and transfer to a beaker with about $50 \mathrm{cc}$. water, add $15 \mathrm{cc}$. bromine water (saturated) and boil for at least 5 minutes to complete the oxidation of the sulphur compounds to sulphates; allow to settle, decant the solution through a filter, and boil the residue a second and a third time with about $30 \mathrm{cc}$. portions of water and then wash thoroughly with hot water. Acidify the filtrate with hydrochloric acid so as to have about $1 \mathrm{cc}$. of the latter in excess, boil to expel bromine, and precipitate the sulphates by means of barium chloride, observing the usual precautions in obtaining, washing, igniting, and weighing the barium sulphate. From the weight of the latter calculate the percentage of sulphur in the coal.

\section{Relation of Proximate Composition to Calorific Power}

The proximate analysis shows the amount, and something of the character, of the organic matter in a coal. The performance of such an analysis requires relatively little time and no special apparatus; the results thus readily obtained are undoubtedly often of value in helping to determine the adapta- 
bility of a coal to some particular purpose, and if the origin or general character of the coal is sufficiently well known, the proximate analysis may give a fair indication of its probable calorific power. Formulæ for estimating the calorific power of coal from its proximate analysis have been proposed, that of Goutal being most often quoted; but on the other hand, it has been found by experiments in the laboratories of the United States Geological Survey, that the volatile matter driven off by heat, as in the usual proximate analysis, consists to a considerable degree of inert gases, and that the proportion of these in the volatile matter "varies in different coal deposits, and makes it impossible to determine the heating value of the coal from the proximate analysis alone." 1

That there should be considerable differences in the calorific power of the volatile matter of different coals is obvious when one considers that the material volatilized from some bituminous coals is rich in hydrocarbons, while that from woody lignites contains a relatively large amount of water vapor.

In order to obtain definite data (1) on the average relation of calorific power to proximate composition, and (2) on the extent of the variations from the average relation to expected in individual samples or different types of coal, Sherman and Regester compiled and computed the results of analyses made in the laboratories of the United States Geological Survey, the Ohio Geological Survey, the West Virginia Geological Survey, and Columbia University covering in all 500 samples of coal from different parts of the United States, and believed to represent the principal American types of coal. In all cases the proximate composition had been determined by the method outlined above, and the calorific power by combustion in oxygen in a bomb calorimeter of standard type, such as the Atwater, Mahler, or Williams instrument. When the data of the 500 .samples were calculated to the basis of dry, ash-free material, and grouped according to percentage of volatile matter, it was found that the coals containing from 2 to 10 per

${ }^{1}$ U. S. Geological Survey, Búl. 339, p. 9. 
cent of volatile in the combustible matter averaged about $14,900 \mathrm{~B}$. T. U. per pound of combustible; beyond this the calorific power increased with the volatile matter until the latter reached about 17 per cent, after which it declined gradually with increasing proportion of volatile in the combustible matter, up to about 40 per cent, beyond which the average calorific power decreased more rapidly.

The average calorific powers for different percentages of volatile matter on the basis of dry, ash-free coal were then plotted, and the values estimated from this curve were compared with those found by the bomb calorimeter for each of the 500 coals.

The first hundred coals showed from 1.74 to 8.50 per cent of volatile matter in the dry, ash-free substance and a mean difference between the calculated and the determined values of 0.98 per cent; in the second hundred coals with 8.62 to 22.17 per cent volatile, the mean difference was 1.14 per cent; in the third hundred with 22.53 to 38.42 per cent volatile, the mean difference was 1.32 per cent; the next 160 coals showed 38.44 to 47.93 per cent volatile and a mean difference of 1.96 per cent ; while in the last 40 coals which contained 48.22 to 59.78 per cent volatile in the dry, ash-free substance the mean difference was 6.30 per cent.

It will be seen from the latter figure that among the coals having over 48 per cent of volatile matter in the dry, ash-free substance the probable variations are so great that a statement of the average relation of proximate analysis to calorific power cannot be made the basis of any general rule for the estimation of the latter value from the former. For coals in this region of proximate composition such relations if used at all should be worked out for each particular type or vein of coal.

On the other hand for coals containing up to 48 per cent of volatile matter in the dry, ash-free substance the proximate analysis gives some indication of the probable calorific power, the average relation being approximately as shown in the following table. 
Table 24.- Approximate Average Relation of Volatile Matter and Calorific Power in the Dry, Ash-free Substance of AMerican Coals

\begin{tabular}{c|c||c|c||c|c}
\hline \hline $\begin{array}{c}\text { Volatile matter } \\
\text { in dry, ash-free } \\
\text { substance } \\
\text { per cent }\end{array}$ & $\begin{array}{c}\text { British Thermal } \\
\text { Units per } \\
\text { pound of dry, } \\
\text { ash-free } \\
\text { substance }\end{array}$ & $\begin{array}{c}\text { Volatile matter } \\
\text { in dry, ash-free } \\
\text { substance } \\
\text { per cent }\end{array}$ & $\begin{array}{c}\text { British Thermal } \\
\text { Units per } \\
\text { pound of dry, } \\
\text { ash-free } \\
\text { substance }\end{array}$ & $\begin{array}{c}\text { Volatile matter } \\
\text { in dry, ash-free } \\
\text { substance } \\
\text { per cent }\end{array}$ & $\begin{array}{c}\text { British Thermal } \\
\text { Units per } \\
\text { pound of dry, } \\
\text { ash-free } \\
\text { substance }\end{array}$ \\
\cline { 1 - 3 } 0 & $14544^{1}$ & 17 & 15900 & 33 & 15500 \\
2 & 14900 & 18 & 15850 & 34 & 15500 \\
3 & 14900 & 19 & 15800 & 35 & 15500 \\
4 & 14900 & 20 & 15800 & 36 & 15500 \\
5 & 14900 & 21 & 15700 & 37 & 15300 \\
6 & 14900 & 22 & 15700 & 38 & 15100 \\
7 & 14900 & 23 & 15700 & 39 & 15100 \\
8 & 14900 & 24 & 15700 & 40 & 15100 \\
9 & 14900 & 25 & 15700 & 41 & 14600 \\
10 & 14900 & 26 & 15700 & 42 & 14600 \\
11 & 15050 & 27 & 15700 & 43 & 14600 \\
12 & 15200 & 28 & 15700 & 44 & 14600 \\
13 & 15350 & 29 & 15700 & 45 & 14500 \\
14 & 15500 & 30 & 15600 & 46 & 14400 \\
15 & 15650 & 31 & 15500 & 47 & 14300 \\
16 & 15800 & 32 & 15500 & 48 & 14200 \\
\hline
\end{tabular}

This table probably gives as good an idea as is now possible of the average relation of proximate composition to calorific power in American coals, but these average relations must not be given undue weight, because individual samples of coal may vary greatly from the average. An idea of the variations to be expected in individual cases may be obtained from the fact that among the 460 cases considered by Sherman and Regester which fall within the range of this table the calorific powers actually determined by combustion in oxygen differed from those calculated from the above table by 1.00 per cent or more in 233 cases or almost exactly one half of the total; by 2.00 per cent or more in 110 cases (about 1 in 4 ) ; by 3.00 per cent or more in 53 cases (about 1 in 8 ) ; by 4.00 per cent or more in 27 cases

1 Corresponding to 8080 calories per gram, the usually accepted value for carbon. 
(about 1 in 17); and by 5.00 per cent or more in 17 cases or about 1 case in 27 .

If this comparison were extended to the coals containing a larger amount of volatile matter, the proportion of cases showing serious discrepancies would of course be much greater. It is evident that in general the proximate analysis of a coal is of less value as an indication of its calorific power than is the ultimate analysis.

The results of either ultimate or proximate analysis become more significant when interpreted in the light of the foregoing data; but in cases in which an accurate knowledge of the calorific power of coal is required, one should accept only the results of direct determinations by a skilled observer using an accurately standardized bomb calorimeter.

\section{REFERENCES}

Berthelot: Thermochemie.

GiLL: Gas and Fuel Analysis for Engineers.

Hempel: Gasanalytische Methoden.

Jüptser: Heat Energy and Fuels.

Louguinine: Bestimmung der Verbrennungswarme.

LUNGE : Chemisch-technische Untersuchungsmethoden.

Ohio Geological Survey, Fourth Series, Bul. 9, Coal.

Poole : Calorific Power of Fuels.

Scheurer-Kestner: Pouvoir Calorifique des Combustibles.

Sorel: Carburetting and Combustion in Alcohol Engines (Transl. by Woodward and Preston).

Thomsen : Thermochemistry (Transl. by Burke).

U. S. Geological Survey, Professional Paper 48, and Bulletins 261, 290, 323, $329,339,341,362,378,382,392,428$.

VAUBfe: Quantitative Bestimmung organischer Verbindungen, Vol. I.

West Virginia Geological Survey, Vol. II, Coal Report, and Vol. II A, Supplementary Coal Report.

\section{II}

1877-78. Fraser: Classification of Coals. Trans. Am. Inst. Mining Engineers, 6, 430.

1889. Stohmanv : (Heats of Combustion of Various Organic Substances). $J$. prakt. Chem., 1889 et seq. 
1895. Noyes, McTaggart, and Craver: The Determination of the Heating Effects of Coals. J. Am. Chem. Soc., 17, 843.

1897. Kent : Calorific Power of American Coals. Trans. Am. Inst. Mining Engineers, Nov., 1897.

1899. Committee Report on Coal Analysis. J. Am. Chem. Soc., 21, 1116.

1902. Stoddart: Determination of Sulphur in Coal. J. Am. Chem. Soc., 24,852 .

1903. Atwater and Snell: Description of a Bomb Calorimeter and Method of its Use. J. Am. Chem. Soc., 25, 659.

1904. Sommermeier: Sulphur in Coal, Effect on Calorific Power. J. Am. Chem. Soc., 26, 555.

1905. Campbell: The Classification of Coals. Trans. Am. Inst. Mining Engineers, Sept., 1905.

Henderson: Heat of Combustion of Atoms and Molecules. $J$. Phys. Chem., 9, 40.

Richards, Henderson, and Forbes: The Elimination of Thermometric Lag and Accidental Loss of Heat in Calorimetry. Proc. Am. Acad. Arts and Sci., 41, 1.

Terry, Arnold, and Fisher: Molasses as Fuel. School of Mines Quarterly, 26, 283.

Thomsen: Heats of Combustion and Formation of Volatile Organic Compounds. Z. physik. Chem., 52, 343; J. Chem. Soc., 88, 1i, $571-574$.

1906. Aвbotr: Some Characteristics of Coal as Affecting Performance with Steam Boilers. J. Western Soc. Eng., 2, 529; Chem Abs., $\mathbf{1}, 98$.

PARr: The Classification of Coals. J. Am. Chem. Soc., 28, 1425.

- : Composition and Character of Illinois Coals. Bul. 3, Illinois State Geological Survey, 1906, 86 pp.; Chem. Abs., 1, 838.

Sommermeier : (Moisture and Volatile Combustible Matter, in Coals and Lignites). J. Am. Chem. Soc., 28, 1002, 1630.

1907. Bement : Analytical Data on American Coals. J. Soc. Chem. Ind., 26, 670 .

Benedict and Fletcher: Calorimetric Bomb Investigation. $J$. Am. Chem. Soc., 29, 739.

Envis: Efficiency of Fuel under Steam Boilers. Eng. Mag., 33, 413; Chem. Abs., 1, 2027.

Frankforter: Lignites of the Northwest. J. Am. Chem. Soc., 29, 1488.

Fries: Investigations in the Use of the Bomb Calorimeter. U. S.

Dept. Agriculture, Bureau of Animal Industry, Bul. 94.

Grout : The Composition of Coals. Econ. Geol., 2, 225 ; Chem. Abs., $1,1839$. 
1907. Holmes and Randall: Testing of Coals used by the United States

Government. Proc. Am. Soc. Testing Materials, 7, 537.

Junker Gas Calorimeter, new Automatic. Journal für Gasbeleuchtung, 50, 520; Chem. Abs., 1, 2632.

Richards, Henderson, and Frevert: Heat of Combustion by Adiabatic Method. Z. physik. Chem., 59, 532; Chem. Abs., 1, 2971.

Sy : Alcohol as a Fuel. J. Frank Inst., 163, 57.

Voorhees: Methods of Testing Coal. Proc. Am. Soc. Testing Materials, 7, 560 .

Woodwell: Purchase of Coal under Specifications. Proc. Am. Soc. Testing Materials, 7, 543.

1908. Ј Аков : (Calorimetry). Z $Z$. chem. Apparatenkunde, 2, 281, 313, 337, 369, 499, 533, 565, 597 ; Chem Abs., 2, 1803.

LorD: Coals of the United States. Chem. Eng., Sept. 1908; Chem. Abs., 2, 3278 .

Lyon and Carpenter: Peats of Indiana. J. Am. Chem. Soc., 30, 1307.

Nystrom: Peat and Lignite; their Manufacture and Uses in Europe. Canada Dept. Mines, Ottawa, 1908, 247 pp.

Parr and Hamilton: Weathering of Coal. Econ. Geol., 2, 693; Chem. Abs., 2, 650.

PARr and WheELer: Deterioration of Coal Samples. University of Illinois, Engineering Experiment Station, Bul. 17, p. 26.

Porter and Ovitz: The Nature of the Volatile Matter of Coal as Evolved under Different Conditions. J. Am. Chem. Soc., 30, 1486.

REDwood: Supply and Use of Mineral Oil. Engineering, 86, 118; Chem. Abs., 3, 372.

Woodwell : Commercial Results in the Purchase of Coal on Specifi-

cations. Proc. Am. Soc. Testing Materials, 8, 582.

1909. BaIley : Accuracy in Sampling Coal. J. Ind. Eng. Chem., $\mathbf{1}, 161$.

- : Calorimeter Standardization. J. Ind. Eng. Chem., 1, 328.

Brissuaid: Amount of Inert Volatile Matter in the Mineral Constituents of Coal. J. Ind. Eng. Chem., 1, 65.

Cox : Coal Calorimetry. Philippine J. Sci. (A), 4, 171; Chem. Abs., 3, 3006 .

Emerson: A New Bomb Calorimeter. J. Ind. Eng. Chem., 1, 17.

Fischer and Wrede: (Use of Platinum Resistance Thermometer in

Determining Heat of Combustion). Z. physik. Chem., 69, 218; Chem. Abs., 4, 537.

Fries: Methods and Standards in Bomb Calorimetry. U. S. Dept.

Agriculture, Bureau of Animal Industry, Bul. 124.

Langbein: Modified Bomb Calorimeter. Chem. Ztg., 33, 1055. 
1909. Lord : Coal Analysis. J. Ind. Eng. Chem., 1, 307.

PARr et al: Determination of Sulphur in Coal. J. Ind Eng. Chem., 1,689 .

Parr and Wheeler: The Ash of Coal and its Relation to Actual or Unit Coal Value. J. Ind. Eng. Chem., 1, 636.

Shimer: The Determination of the Volatile Combustible Matter in Coke and Anthracite. J. Ind. Eng. Chem., 1, 518.

1910. Bement : Influence of Oxygen on the Value of Coal. Science, 30, 922. Benedict and Higgins: An Adiabatic Calorimeter for Use with the Calorimetric Bomb. J. Am. Chem. Soc., 32, 461.

Burgess and Wheeler: Volatile Constituents of Coal. J. Chem. Soc., 97, 1917.

Fieldner and Davis: Some Variations in the Official Method for the Determination of Volatile Matter in Coal. J. Ind. Eng. Chem., 2, 304.

Huntley: Accuracy Obtainable in Fuel Calorimetry. J. Soc. Chem. Ind., 29, 917.

Myers: Tan Bark as a Boiler Fuel. School of Mines Quarterly, 31, 116.

PArr: A New Gas Calorimeter. J. Ind. Eng. Chem., 2, 337.

PARr and Barker: Occluded Gases in Coal. Univ. of Ill. Bul., 6, 32.

PArr and Wheeler: Unit Coal and the Composition of Coal Ash. Univ. of Ill. Bul., 6, 43; Chem. Abs., 4, 2199.

Porter and Ovitz: Losses in the Storage of Coal. J. Ind. Eng. Chem., $2,77$.

__ : Volatile Matter in Coal. U. S. Bur. Mines, Bul. 1.

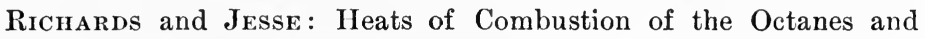
Xylenes. $J_{i}$ Am. Chem. Soc., 32, 268.

Walsh: Standard Gas Coal. Progressive Age, 28, 328; Chem. Abs., 4,2724 .

WeLD: Accuracy in Sampling Coal. J. Ind. Eng. Chem., 2, 426. (See also p. 543.)

White: (Temperature Measurements in Calorimeter Work). Physical Review, 31, 562.

1911. Allen : Specifications for the Purchase of Fuel Oil for the Government with Directions for Sampling Oil and Natural Gas. J. Ind. Eng. Chem., 3, 730.

Axonymous : Estimation of Moisture in Fuel Oil. Chem. Tech. Ztg., 1911 (6), 29, 47; Chem. Abs., 5, 2425.

Blakeley and Chance: Accurate Technical Estimation of the Calorific Power of Anthracite Coal. J. Ind. Eng. Chem., 3, 557. Burgess and Wheerer: The Volatile Constituents of Coal. J. Chem. Soc., 99, 649.

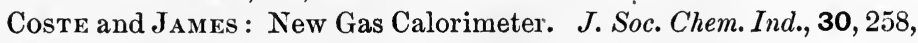


1911. Doane: The Purchase of Coal on the Efficiency Basis. Eng. Rec., 61, 502; Chem. Abs., 5, 584.

Holmes: The Sampling of Coal in the Mine. Technical Paper 1, U. S. Bur. Mines.

Kent and Allen : Formula for Purchase of Coal on Heat Unit Basis. Eng. News, 65, 109.

Parker: The Production of Coal in 1910. Published by U.S. Geol. Survey. (This includes a classified list (pp. 226-241) of the papers dealing with coal, coke, lignite, and peat contained in publications of the U. S. Geological Survey.)

Parr: The Determination of Volatile Matter in Coal. J. Ind. Eng. Chem., 3, 900.

Pope: Purchase of Coal by the Government under Specifications. U. S. Geol. Survey, Bur. Mines, Bul. 11.

U. S. Bureau of Standards. Circular No. 11, The Standardization of Bomb Calorimeters.

White: Recent Progress in Calorimetry. Met. Chem. Eng., 9, 202, $296,449$. 


\section{CHAPTER XIII}

\section{Soap and Glycerin}

\section{ANALYSIS OF COMMERCIAL SOAP}

THE determinations required in the examination of a commercial soap depend largely upon the purpose for which it is intended. The scheme outlined below is adaptable to almost all cases, as it can be easily extended to include additional determinations or shortened by omitting such steps as are unnecessary when only a partial analysis is required.

\section{Outline Scheme of Analysis ${ }^{1}$}

First Portion. Dry 2 to 5 grams of soap (I) ${ }^{2}$ and introduce into Soxhlet extractor, either in a thimble or supported by a firm plug of cotton, and extract with petroleum ether.

Solution. Evap- Residue. Allow petroleum ether to evaporate, transorate ether and fer the soap to a beaker, dissolve in hot water, and filter. weigh as unsaponified fat and unsaponifiable matter. (II.)

Solution. Decompose with a considerable excess of standard sulphuric (or hydrochloric) acid, separate aqueous solution from fatty layer. (III.)

Solution. Add Fatty Layer. methyl orange Dry and weigh as and titrate for fatty and resin total alkali. Test acids. (V.) for chlorides (or sulphates), sugar, and glycerin. (IV.)
Residue. Dry and weigh as insoluble matter.

Ignite and weigh as insoluble mineral matter. (X.)

1 Allen: Commercial Organic Analysis, Vol. II.

2 Numbers in parenthesis refer to sections which follow. 
Second Portion. Exhaust 2 to 5 grams of the fresh sample with carefully neutralized alcohol. (VI.)

Solution. Add Residue. Dry and weigh. (VIII.) Exhaust phenolphthalein thoroughly with boiling water.

and titrate for free caustic alkali or free fatty acid. (VII.)

\begin{tabular}{l|l|l}
$\begin{array}{l}\text { Solution. Divide into aliquot } \\
\text { parts. }\end{array}$ & $\begin{array}{l}\text { Residue. May be } \\
\text { examined instead } \\
\text { of residue from } \\
\text { Add methyl } \\
\text { orange and ti- } \\
\text { trate for alkali portion or } \\
\text { carbonate (and } \\
\text { borate, silicate, } \\
\text { if necessary de- } \\
\text { borate, silicate, } \\
\text { or aluminate if } \\
\text { aresent). (IX.) }\end{array}$ & $\begin{array}{l}\text { (IX.) } \\
\text { used for additional } \\
\text { tests or determina- } \\
\text { tions. (X.) }\end{array}$ \\
\hline
\end{tabular}

A cake of soap exposed to the air dries rapidly at the surface, forming a horny layer which to some extent prevents the evaporation of water from the interior. In sampling such a soap it is important to remember this variation in water content of different parts of the same cake. If the sample is to represent the individual cake as it existed at the time of commencing the analysis, a number of sections through the entire cake should be taken either by slicing or by means of a cork borer or a cheese or butter sampler. Often the purpose of the analysis is to show the composition of the sample as originally sold, in which case the dried surface should be rejected and the sample taken from the center of the cake. The sample for analysis should be reduced quickly to fine shavings and kept in a tightly stoppered bottle.

Details of Determinations Indicated in the Scheme

\section{Determination of Water}

If the soap is very hard and dry, it may be reduced to fine shavings and dried on a watchglass, heating first for some time at $40^{\circ}$ to $60^{\circ}$ and then finishing at $105^{\circ}$ to $110^{\circ}$. Very few soaps can be completely freed from moisture in this way, but some others may be sufficiently dried for the ether extraction, while the moisture determination is made on a separate sample 
as follows: Dissolve about two grams of soap in the minimum quantity of hot strong alcohol and evaporate on clean dry sand. Finish the drying at $110^{\circ}$ with frequent stirring, a small rod having been weighed with the dish. For approximate determination of moisture Allen recommends Smith's method: Heat 5 to 10 grams of finely divided soap in a large porcelain crucible on a sand bath over a small Bunsen flame. Stir continually with a small glass rod (weighed with the crucible) having a roughened end to facilitate breaking any lumps of soap which may be formed. Continue heating until there is no more evidence of water being expelled, then test by removing the burner and placing a cold glass at once over the crucible. If no moisture condenses on the glass, the soap is considered dry. With practice the drying can be finished in 20 to 30 minutes. Any burning of the soap is readily detected by the odor. The results are said to be reliable to 0.25 per cent.

\section{Petroleum Ether Extract}

Transfer the thoroughly dried soap to a paper "fat extraction thimble," plug tightly with fat-free cotton and treat in a Soxhlet extractor, ${ }^{1}$ heated on a safety water bath or electric heater, with petroleum ether of nearly constant boiling point. Regulate the heating so that the extractor fills to the siphoning point in ten or fifteen minutes and continue the extraction for four to six hours. Disconnect the extraction apparatus (observing that no free flame is near) as the solvent flows through the siphon to the flask; remove the thimble, reconnect the apparatus, and recover the solvent by placing a wide, short test-tube in the space previously occupied by the thimble, or by allowing the solvent to collect in this space and removing it before it reaches the top of the siphon. Having expelled nearly all of the petroleum ether, heat the flask containing the extract in a boiling water oven to constant weight.

The petroleum ether extract of a commercially pure soap

1 The parts of the Soxhlet extractor may be connected by ground glass joints, by mercury seal, or by corks covered with tinfoil. 
may contain unsaponified fat (or free fatty acids, which are now largely used in soap-making) as well as the " unsaponifiable matter" of the original soap grease. Hydrocarbons, phenols, and other substances soluble in petroleum ether may also be found in mixed and "medicated" soaps. Allen ${ }^{1}$ gives directions for the systematic examination of this extract, including the quantitative determination of phenol if present.

\section{Liberation of Fatty and Resin Acids.}

Decompose the water solution of the soap by adding a considerable excess (allow 5 to 7 cc. of normal acid for each gram of dry soap) of normal or half-normal sulphuric, nitric, or hydrochloric acid. Add the entire amount of acid at once, boil, stir thoroughly for some minutes, and keep the solution hot until the fatty acids collect at the surface, leaving the water solution nearly clear, then complete the separation as in the determination of insoluble acids in butter.

It is convenient to liberate the fatty acids in a tared beaker in which they can afterward be dried and weighed (V). If the fatty acids are liquid at ordinary temperature or form a cake too soft to be handled conveniently, a known weight of dry bleached beeswax or stearic acid may be added to the hot solution. The fatty acids become incorporated with the wax and on cooling a firm cake is obtained.

\section{Solution separated from Fatty Acids}

This solution contains, in the form of sulphate (or chloride if hydrochloric acid be used to decompose the soap), all the alkali originally present as soap, as carbonate (silicate or borate), or as hydroxide. On titrating this solution with alkali, using methyl orange as indicator, the amount of acid found to have been neutralized gives a measure of the total alkali of the soap. ${ }^{2}$

${ }^{1}$ Commercial Organic Analysis, Vol. II.

2 A quick determination of total alkali can also be made by burning a weighed portion of the soap to a white ash and determining the alkalinity of this ash, using methyl orange as indicator. 
Unless potash is known to be present, this total alkali is usually calculated as sodium oxide. The solution also contains any chlorides or other soluble salts, soluble fatty acids, glycerol, sugar, etc., which the soap may have contained. After titration the solution can be diluted to a known volume and separate portions taken for qualitative tests and quantitative determinations.

When sulphuric acid has been used to liberate the fatty acids, chlorides can be determined in a portion of the neutralized solution. If it is desired to determine both chloride and sulphate in this solution, the soap can be decomposed by means of standard nitric acid. It is usually more convenient to test for sulphates in the residue from the alcohol extraction as described below.

Soluble fatty acids will be found in this solution if the liberated acids of coconut or palm-nut oil soaps are washed with hot water, as is often recommended. When the fatty acids are separated cold and washed with cold water only, the amount dissolved can usually be neglected without appreciable error.

Sugar, if present, is detected in a part of this solution. After further treatment with acid to insure complete hydrolysis, the invert sugar is determined either volumetrically or gravimetrically (Chapter III). Sugar may also be determined by means of the polariscope, using a separate portion of the sample and precipitating the fatty acids as insoluble barium soaps. ${ }^{1}$

In the absence of sugar and other interfering substances, glycerol can be determined by treating a portion of the neutralized solution directly with sulphuric acid and standard dichromate as described beyond. Since the results thus found are often too high, because of the presence of organic impurities, Lewkowitsch recommends the following method: Decompose the water solution of the soap with sulphuric acid, separate the fatty acids, neutralize the filtrate with barium carbonate, evaporate to a sirup, and extract with a mixture of 3 parts 95 per cent alcohol and 1 part ether. The glycerol thus obtained can be determined by the acetin method after complete removal of alcohol.

1 Freyer: Oesterr. Chem. Ztg., 1900, 3, 25 ; Analyst, 1900, 25, 127. 


\section{Mixed Fatty and Resin Acids}

The mixture of acids liberated as already described (III) is dried to constant weight in a boiling water oven in a weighed flat-bottomed dish or beaker as in the determination of the insoluble acids in butter fat. The weight having been found, test a portion for resin acid by the Liebermann-Storch reaction as described under drying oils (Chapter $\mathrm{X}$ ). In the absence of resin acids it may be possible to show the nature of the fat from which the soap was made, by examining the mixed fatty acids according to the methods used in identifying fats and oils as described in Chapters VIII to XI, consulting special works such as those of Lewkowitsch or Benedikt-Ulzer for the "constants" which cannot be inferred from the properties of the corresponding fats. The separation of fatty and resin acids is best accomplished by Twitchell's method based upon the difference of behavior of these acids when exposed in alcoholic solution to the action of hydrochloric acid. By this treatment fatty acids are converted to ethyl esters, while resin acids remain practically unchanged. The method is carried out by Lewkowitsch ${ }^{1}$ as follows: Weigh 2 to 3 grams of the mixed acids in a flask, dissolve in 10 times their volume of absolute alcohol, immerse the flask in cold water, and pass a current of dry hydrochloric acid gas through the solution for an hour; then dilute the contents of the flask (which will have separated into two layers) with 5 times its volume of water and boil until the aqueous solution has become clear, the esters, with resin acids in solution, floating on top. Transfer the contents of the flask to a separating funnel by means of 50 cc. of petroleum ether (boiling below $80^{\circ}$ ); shake, allow to separate, draw off the acid solution, and wash the petroleum ether layer once with water. After the latter has separated completely and been removed, add a solution of 0.5 gram of potassium hydroxide and $5 \mathrm{cc}$. of alcohol in $50 \mathrm{cc}$. of water; shake, and allow to separate. The ethyl esters remain dissolved in the petroleum ether, while the resin acids are extracted by the dilute alkaline solution

1 Oils, Fats, and Waxes (4th Ed.), p. 501. 
forming soaps. Draw off the soap solution, wash the petroleum ether solution again with dilute alkali, unite the alkaline solutions; liberate the resin acids by means of hydrochloric acid, collect, dry, and weigh them as in the determination of liberated fatty acids.

The resin acids can be titrated after washing free from hydrochloric acid, instead of being separated and weighed. The volumetric method is more rapid than the gravimetric, but necessitates the assumption of a combining weight (346) for the resin acids, which is liable to considerable inaccuracy. According to Lewkowitsch, the results by the volumetric method are likely to be too high; those by the gravimetric method too low.

\section{Extraction with Alcohol}

Dry soap can be extracted with $9 \dot{5}$ per cent alcohol; for wet soap stronger alcohol should be used, so that after taking up the moisture of the sample it will still be too strong to dissolve an appreciable amount of carbonate. The alcohol to be used must first be very carefully neutralized, using phenolphthalein as indicator. In this neutralization there is danger of adding an excess of alkali unless it is remembered that the full pink color of the indicator will not appear in alcohol of this strength. If difficulty is experienced in detecting the neutral point, a small amount of the alcohol can be removed and mixed with an equal volume of boiling water to bring out the color of the indicator.

While the extraction of the soap with alcohol is often carried out in open vessels, filtering and washing in the ordinary way, it is usually more satisfactory to use the Soxhlet extractor. The soap can be put in a paper thimble as in the petroleum ether extraction, or between plugs of cotton in a glass tube with perforated bottom. In the latter case the progress of the extraction can be watched without disconnecting the apparatus. When the extraction is complete, the residue should be in powder form. If distinct pieces remain, these may contain soap which has been protected from the action 
of the alcohol by the formation of a layer of insoluble salts. In this case remove and crush the residue, replace, and extract again.

\section{Free Caustic Alkali or Fatty Acid}

To the alcoholic extract add a few drops of neutralized phenolphthalein solution. If the solution reacts alkaline, titrate with tenth-normal acid for caustic alkali; if acid, titrate with tenth-normal alkali for free acid. For a further discussion of this extract, including a rapid method for the partial analysis of soaps, see Allen.

\section{Residue Insoluble in Alcohol}

It is advisable to dry and weigh this residue so that the percentage of impurities not actually determined can be found by difference. A microscopic examination may also be of use in determining the subsequent treatment. Starch and gelatin if present could be separated from earbonate, borate, and sulphate by dissolving the latter salts in cold water; but silicate would probably be incompletely dissolved, and it is therefore better as a rule to extract with hot water and to use separate portions of the soap, if necessary, for the determination of starch and gelatin. In such a case extract the soap with alcohol and in the residue determine starch as described in Chapter V, or determine nitrogen by the Kjeldahl method and calculate the corresponding amount of gelatin, taking the nitrogen content of the latter as 17.9 per cent. ${ }^{1}$

\section{Carbonate, Silicate, Borate, and Aluminate}

Add to the water extract from the residue insoluble in alcohol an excess of standard acid and. boil to insure decomposition of the silicate. If it is important to distinguish quantitatively between carbonate and the other alkaline salts present, the carbonic acid given off during this boiling can be collected and weighed. Add methyl orange as indicator and

1 Richards and Gies: Am. J. Physiol., 1902, 7, 129. 
titrate with standard alkali to determine the total amount of alkali which was present as carbonate, silicate, borate, and aluminate.

To a portion of the solution add hydrochloric acid in excess, evaporate to small volume, and test for boric acid by means of turmeric paper; when dry, heat at $110^{\circ}$, take up with dilute hydrochloric acid, filter out, and determine silica, if present.

Other portions of the solution or the filtrate from silica can be used for the detection and determination of sulphates, aluminates, etc.

\section{Insoluble Matter}

This residue should be dried to constant weight at $100^{\circ}$, a portion examined microscopically and the remainder ignited and weighed. If over 1 per cent of insoluble mineral matter is found, it should be analyzed. Among the substances which may be found in this residue are oatmeal, bran, sawdust, clay, chalk, steatite, infusorial earth, pumice, sand, mineral pigments, etc.

\section{Calculation and Interpretation of Results}

In the case of hard soap, the results of the partial analysis usually required may be reported as follows :

Water ; unsaponified fat and unsaponifiable matter; fatty and resin anhydrides (97 per cent of the weight of free acids); sodium oxide combined as soap; sodium hydroxide; sodium carbonate; insoluble organic matter ; insoluble mineral matter. It is well to report also the total alkali in terms of sodium oxide.

The purpose for which a soap is intended must be known before an opinion as to its quality can safely be formed. In most cases the percentage of alkali combined as soap is the best measure of the amount of actual soap in the material, but for special purposes the presence or absence of other constituents is often of greater importance.

Toilet soaps should contain as little free alkali (either caustic or carbonate) as possible. Alder-Wright divided toilet soaps into three classes according to the proportion of free alkali to 
alkali combined as soap. The first class included those soaps which contained less than 2.5 per cent as much free as combined alkali; the second, those in which the percentage was 2.5 to 7.5 ; the third, those containing over 7.5 per cent as much free as combined alkali. In judging the quality of toilet soaps it is also important to consider the proportions and nature of all foreign matter, the amount of water, the hardness of the soap, and in some cases the origin must be sought by an examination of the fatty acids. The more expensive "transparent" toilet soaps may contain alcohol or glycerin; in cheaper grades a similar appearance is obtained by the addition of sugar.

Household soaps are made from cheaper and softer fats than those used for toilet soap. Alkali in the form of carbonate, silicate, or borate is not objectionable unless present in excessive amount. No appreciable amount of sugar or glycerol is likely to be present. Scouring soaps often contain large amounts of pulverized quartz, infusorial earth, etc., and are sometimes strongly alkaline with sodium carbonate or hydroxide.

For discussion of the adaptability of different types of soaps to specific uses see references at the end of the chapter.

\section{GLYCEROL}

Glycerol is a colorless, odorless, viscous liquid of sweet taste and neutral reaction, miscible in all proportions with water and with alcohol. It also dissolves in mixtures of alcohol and ether, but is only very sparingly soluble in pure ether ${ }^{1}$ and is practically insoluble in chloroform, carbon disulphide, and benzene. The specific gravity of pure glycerol at $15^{\circ}$ referred to water at the same temperature is variously stated at from 1.265 to 1.2677. Anhydrous glycerol boils at about $290^{\circ}$, but evaporates rapidly at lower temperatures $\left(160^{\circ}\right.$ or over), and the evaporation is greatly accelerated by the presence of a small amount of water. When kindled, glycerol burns with a blue flame and leaves no carbonaceous residue.

1 According to Lewkowitsch, one part of glycerol of $1.23 \mathrm{sp}$. gr. dissolves in about 500 parts of ether. 
These properties, together with the fact that it yields acrolein when heated with acid potassium sulphate, are usually sufficient for the identification of glycerol when in a fairly pure and concentrated state.

Glycerol is a good solvent for many substances, both organic and inorganic, and its presence often increases their solubility in aqueous and alcoholic solutions. This fact and the difficulty of distilling without loss make it troublesome to separate glycerol as a pure aqueous solution as is done in the determination of alcohol.

The percentage of glycerol in commercial glycerin is usually determined either by acetylating the glycerol and finding the amount of acetin by saponification (acetin method), or by quantitative oxidation of the glycerol by means of standard potassium dichromate (dichromate method). The acetin method requires that the glycerol be concentrated, and that other acetylizable substances if present shall be corrected for ; the dichromate method requires the removal of chlorides and all organic substances which would be oxidized by the dichromate treatment.

\section{Analysis of Crude Glycerin}

In recent years the increase in price of crude glycerin has resulted in the manufacture of glycerin from lower-grade fats than before, with the result that the product often contains impurities which behave so much like glycerol as to introduce serious discrepancies in the analytical determination of glycerol in crude glycerin. This led to the appointment of committees, both in this country and in Europe, to study methods of glycerin analysis. Representatives of these committees met as an international committee, which, after investigation, decided that the acetin method should be the basis on which glycerin should be bought and sold, but that the dichromate method, being more convenient for factory control, might continue to be used for some technical purposes in a properly standardized form. The methods recommended by the international committee for sampling and analysis of glycerin are as follows : ${ }^{1}$

${ }^{1}$ J. Ind. Eng. Chem., 3, 679-686. 


\section{Sampling}

The most satisfactory method available for sampling crude glycerin liable to contain suspended matter, or which is liable to deposit salt on settling, is to have the glycerin sampled by a sampler mutually approved by the buyer and seller as soon as possible after the glycerin is filled into drums, but in any case before any separation of salt has taken place. In such cases he shall sample with a sectional sampler (see appendix to original report), then seal the drums, brand them with a number for identification, and keep a record of the brand number. The presence of any visible salt or other suspended matter is to be noted by the sampler, and a report of the same made in his certificate, together' with the temperature of the glycerin. Each drum must be sampled. Glycerin which has deposited salt or other solid matter cannot be accurately sampled from the drums, but an approximate sample can be obtained by means of a sectional sampler which will allow a complete vertical section of the glycerin to be taken, including any deposit.

\section{Analysis}

1. Determination of Free Caustic Alkali. - Put 20 grams of the sample into a 100 -cc. flask, dilute with approximately 50 cc. of freshly boiled distilled water, add an excess of neutral barium chloride solution, 1 cc. of phenolphthalein solution, make up to the mark, and mix. Allow the precipitate to settle, draw off 50 cc. of the clear liquid, and titrate with normal acid. Calculate the percentage of caustic alkali as $\mathrm{Na}_{2} \mathrm{O}$.

2. Determination of Ash and Total Alkalinity. - Weigh 2-5 grams of the sample in a platinum dish, burn off the glycerin over a luminous Argand burner or other source of heat at a low temperature, to avoid volatilization and the formation of sulphides. When the mass is thoroughly charred, stir with hot water, filter, wash, and ignite the residue in the platinum dish. Return the filtrate and washings to the dish, evaporate the water, ignite carefully, avoiding fusion, and weigh the ash. Dissolve the ash in water and titrate total alkalinity, using as indi- 
cator methyl orange in a cold solution, or litmus, if the solution is boiled.

3. Determination of Alkali present as Carbonate. - Take 10 grams of the sample, dilute with $50 \mathrm{cc}$. water, add sufficient normal acid to neutralize the total alkali found at (2), boil under a reflux condenser for 15-20 minutes, wash down the condenser tube with water free from carbon dioxide, and then titrate the free acid in the solution with normal sodium hydroxide, using phenolphthalein as indicator. Calculate the percentage of $\mathrm{Na}_{2} \mathrm{O}$, deduct the $\mathrm{Na}_{2} \mathrm{O}$ found in (1). The difference is the alkali present as carbonate expressed in terms of $\mathrm{Na}_{2} \mathrm{O}$.

4. Alkali combined with Organic Acids. - The sum of the percentages of $\mathrm{Na}_{2} \mathrm{O}$ found at (1) and (3) deducted from the percentage found at (2) is a measure of the $\mathrm{Na}_{2} \mathrm{O}$ or other alkali combined with organic acids.

5. Determination of Acidity. - Take 10 grams of the sample, dilute with $50 \mathrm{cc}$. distilled water free from carbon dioxide, titrate with normal sodium hydroxide, using phenolphthalein as indicator, and express the result in terms of $\mathrm{Na}_{2} \mathrm{O}$ required to neutralize 100 grams.

6. Determination of Total Residue at $160^{\circ} \mathrm{C}$. - For this determination the crude glycerin should be slightly alkaline with sodium carbonate, not exceeding 0.2 per cent $\mathrm{Na}_{2} \mathrm{O}$ in order to prevent loss of organic acids. To avoid the formation of polyglycerols, this alkalinity must not be exceeded.

Ten grams of the sample are put in a 100-cc. flask, diluted with water, and the calculated quantity of normal hydrochloric acid or sodium carbonate added to give the required degree of alkalinity. The flask is filled to $100 \mathrm{cc}$., the contents mixed, and $10 \mathrm{cc}$. measured into a weighed flat-bottomed glass dish 2.5 inches in diameter and 0.5 inches deep. In the case of crude glycerins abnormally high in organic residue a smaller amount should be taken so that the organic residue shall not materially exceed 30-40 milligrams.

The dish is placed on a water bath (or on top of the oven kept at $160^{\circ}$ ) until most of the water has evaporated, then placed in an oven at $160^{\circ}$, leaving the door. of the oven open so as to have 
a temperature of $130^{\circ}-140^{\circ}$, until the glycerin, or most of it, has evaporated. When only a slight vapor is seen to come off, the dish is removed, allowed to cool, and 0.5 to $1 \mathrm{cc}$. of water added and by a rotary motion the residue brought wholly or nearly into solution. The dish is then allowed to stand on top of the oven until the water has evaporated and the residue is sufficiently dry to prevent spurting, when it is placed in the oven at $160^{\circ} \mathrm{C}$. The dish is then kept in the oven carefully maintained at $160^{\circ} \mathrm{C}$. for one hour, when it is removed, cooled, the residue treated with water, the water evaporated, and the residue subjected to a second baking of one hour, after which the dish is allowed to cool in a desiccator over sulphuric acid and weighed.

The treatment with water, etc., is repeated until a constant loss of 1 to 1.5 milligram per hour is obtained.

In the case of acid glycerin, a correction must be made for the alkali added, 1 cc. normal alkali representing an addition of 0.03 gram to the residue. In the case of alkaline glycerins a correction should be made for the acid added, by deducting the increase in weight due to the conversion of sodium hydroxide and sodium carbonate to sodium chloride. The corrected weight multiplied by 100 gives the percentage of total residue at $160^{\circ} \mathrm{C}$.

This residue is used for determination of the non-volatile acetylizable impurities as described under the acetin method below.

7. Organic Residues. - Subtract the ash from the total residue at $160^{\circ} \mathrm{C}$. Report as organic residue at $160^{\circ} \mathrm{C}$. (it should be noted that alkaline salts of fatty acids are converted into carbonates on ignition and that the carbon dioxide thus derived is not included in the organic residue).

\section{Glycerol by Acetin Method}

This process is the one agreed upon at a conference of delegates from the American, British, French, and German Committees, and has been confirmed by each of the above committees as giving results nearer to the truth than the dichromate method on crude glycerins in general. It is the process to be 
used (if applicable) whenever only one method is employed. On pure glycerins the results are identical with those obtained by the dichromate process. For the application of this method the crude glycerin should not contain over 60 per cent water.

Reagents. - $(A)$ Best acetic anhydride. - This should be carefully selected. A good sample must not require more than $0.1 \mathrm{cc}$. normal sodium hydroxide for saponification of the impurities in a blank test on 7.5 cc. Only a slight color should develop during digestion of the blank.

The anhydride may be tested for strength by the following method: Into a weighed stoppered vessel, containing 10 to 20 cc. of water, run about $2 \mathrm{cc}$. of the anhydride, replace the stopper, and weigh. Let stand, with occasional shaking, for several hours, to permit the hydrolysis of all the anhydride; then dilute to about 200 cc., add phenolphthalein, and titrate with normal sodium hydroxide. This gives the total acidity due to free acetic acid and acid formed from the anhydride. It is worthy of note that in the presence of much free anhydride a compound is formed with phenolphthalein, soluble in alkali and acetic acid, but insoluble in neutral solutions. If a turbidity is noticed toward the end of the neutralization, it is an indication that the anhydride is incompletely hydrolized, and inasmuch as the indicator is withdrawn from the solution, results may be incorrect.

Into a stoppered weighing bottle containing a known weight of recently distilled aniline (from 10 to 20 cc.) measure about $2 \mathrm{cc}$. of the sample, stopper, mix, cool, and weigh. Wash the contents into about $200 \mathrm{cc}$. of cold water and titrate the acidity as before. This yields the acidity due to the original preformed acetic acid plus one half the acid due to anhydride (the other half having formed acetanilide); subtract the second result from the first (both calculated to 100 grams) and double the result, obtaining the cubic centimeters of normal sodium hydroxide per 100 grams of the sample. Each cubic centimeter equals 0.0510 gram anhydride.

(B) Pure fused sodium acetate. - The purchased salt is again completely fused in a platinum, silica, or nickel dish, 
avoiding charring, powdered quickly and kept in a stoppered bottle or desiccator. It is important that the sodium acetate be anhydrous.

(C) A solution of sodium hydroxide for neutralizing, of about normal strength, free from carbonate. - This can be readily made by dissolving pure sodium hydroxide in its own weight of water (preferably free from carbon dioxide) and allowing to settle until clear or filtering through an asbestos or paper filter. The clear solution is diluted with water free from carbon dioxide for the strength required.

(D) Normal sodium hydroxide free from carbonate. - Prepared as above and carefully standardized. Some sodium hydroxide solutions show a marked diminution in strength after being boiled; such solutions should be rejected.

(E) Normal acid carefully standardized.

( $\left.\boldsymbol{F}^{\prime}\right)$ Phenolphthalein solution, a one half per cent solution in alcohol, neutralized.

Method.- In a narrow-mouthed flask (preferably round bottom), capacity about 120 cc., which has been thoroughly cleaned and dried, weigh accurately and as rapidly as possible 1.25 to 1.5 grams of the glycerin. A Grethan or Lunge pipette will be found convenient. Add about 3 grams of the anhydrous sodium acetate, then $7.5 \mathrm{cc}$. of the acetic anhydride, and connect the flask with an upright Liebig condenser. For convenience the inner tube of this condenser should not be over 50 $\mathrm{cm}$. long and 9 to $10 \mathrm{~mm}$. inside diameter. The flask is connected to the condenser by either a ground glass joint (preferably) or a rubber stopper. If a rubber stopper is used, it should have a preliminary treatment with hot acetic anhydride vapor. Heat the contents and keep just boiling for one hour, taking precautions to prevent the salts drying on the sides of the flask. Allow the flask to cool somewhat, and through the condenser tube add $50 \mathrm{cc}$. of distilled water, free from carbon dioxide, at a temperature of about $80^{\circ} \mathrm{C}$, taking care that the flask is not loosened from the condenser. The object of cooling is to avoid any sudden rush of vapors from the flask on adding water and to avoid breaking the flask. Time is saved 
by adding the water before the contents of the flask solidifies, but the contents may be allowed to solidify and the test proceeded with the next day without detriment, bearing in mind that the anhydride in excess is much more effectively hydrolized in hot than in cold water. The contents of the flask may be warmed to, but must not exceed, $80^{\circ} \mathrm{C}$., until the solution is complete except a few dark flocks representing organic impurities in the crude glycerin. By giving the flask a rotary motion, solution is more quickly effected.

Cool the flask and contents without loosening from the condenser. When quite cold wash down the inside of the condenser tube, detach the flask, wash off the stopper or ground glass connection into the flask, and filter the contents through an acid-washed filter into a Jena glass flask of about one liter capacity. Wash thoroughly with cold distilled water, free from carbon dioxide. Add 2 cc. of phenolphthalein solution $\left(F^{\prime}\right)$, then run in caustic soda solution $(C)$ or $(D)$ until a faint pinkish yellow color appears throughout the solution. This neutralization must be done most-carefully; the alkali should be run down the sides of the flask, the contents of which are kept rapidly swirling with occasional agitation or change of motion until the solution is nearly neutralized, as indicated by the slower disappearance of the color developed locally by the alkali running into the mixture. When this point is reached, the sides of the flask are washed down with carbon-dioxide-free water, and the alkali subsequently added drop by drop, mixing after each drop until the desired tint is obtained.

Now run in from a burette 50 cc. or a calculated excess of normal sodium hydroxide $(D)$ and note carefully the exact amount. Boil gently for fifteen minutes, the flask being fitted with a glass tube acting as a partial condenser. Cool as quickly as possible and titrate the excess of sodium hydroxide with normal acid $(E)$ until the pinkish yellow or chosen end point color just remains. ${ }^{1}$

1 A precipitate at this point is an indication of the presence of iron or aluminium and high results will be obtained unless a correction is made as described below. 
A further addition of the indicator at this point will cause an increase of the pink color; this must be neglected and the first end point taken.

From the amount of normal sodium hydroxide consumed, calculate the percentage of glycerol (including acetylizable impurities) after making correction for the blank test described below.

1 cc. normal sodium hydroxide corresponds to 0.03069 gram glycerol.

- The coefficient of expansion for normal solutions is 0.00033 per cubic centimeter for each degree C. A correction should be made on this account, if necessary.

Blank Test. - As the acetic anhydride and sodium acetate may contain impurities which affect the result, it is necessary to make a blank test, using the same quantities of acetic anhydride, sodium acetate, and water as in the analysis. It is not necessary to filter the solution of the melt in this case, but sufficient time must be allowed for the hydrolysis of the anhydride before proceeding with the neutralization. After neutralization it is not necessary to add more than $10 \mathrm{cc}$. of normal alkali $(D)$, as this represents the excess usually present after the saponification of the average soap lye crude glycerin. In determining the acid equivalent of the normal sodium hydroxide, however, the entire amount taken in the analysis, $50 \mathrm{cc}$, should be titrated after dilution with $300 \mathrm{cc}$. of water free from carbon dioxide and without boiling.

Determination of the Glycerol Value of the Acetylizable Impurities. - The total residue at $160^{\circ} \mathrm{C}$. is dissolved in one or two cubic centimeters of water, washed into the acetylizing flask, and evaporated to dryness. Then add anhydrous sodium acetate and acetic anhydride in the usual amounts and proceed as described in the regular analysis. After correcting for the blank calculate the result to glycerol.

Calculation of the Actual Glycerol Contents. - (1) Determine the apparent percentage of glycerol in the sample by the acetin process as described. The result will include acetylizable impurities, if any are present. 
(2) Determine the total residue at $160^{\circ} \mathrm{C}$.

(3) Determine the acetin value of this residue in terms of glycerol.

(4) Deduct the result found at (3) from the percentage obtained at (1) and report this corrected figure as glycerol. If volatile acetylizable impurities are present, these are included in this figure.

Trimethyleneglycol is more volatile than glycerol and can . therefore be concentrated by fractional distillation. An approximation to the quantity can be obtained from the difference between the results by the acetin and by the dichromate method on such distillates. The difference multiplied by $\mathbf{1 . 7 3 6}$ will give the glycol.

\section{Glycerol by Dichromate Method}

Reagents. - $(A)$ Pure potassium dichromate powdered and dried in air free from dust or organic vapors at $110^{\circ}$ to $120^{\circ} \mathrm{C}$. This is taken as the standard.

(B) Dilute dichromate solution. - 7.4564 grams of the above dichromate are dissolved in distilled water and the solution made up to one liter at $15.5^{\circ} \mathrm{C}$.

(C) Ferrous ammonium sulphate. - It is never safe to assume this salt to be constant in composition and it must be standardized against the dichromate as follows: Dissolve 3.7282 grams of dichromate $(A)$ in 50 cc. of water. Add 50 cc. of 50 per cent sulphuric acid (by volume) and to the cold undiluted solution add from a weighing bottle a moderate excess of the ferrous anmonium sulphate and titrate back with the dilute dichromate $(B)$. Calculate the value of the ferrous salt in terms of dichromate.

(D) Silver carbonate. - This is prepared as required for each test from $140 \mathrm{cc}$. of 0.5 per cent silver sulphate solution by precipitation with about 4.9 cc. normal sodium carbonate solution (a little less than the calculated quantity of sodium carbonate should be used, as an excess prevents rapid settling). Settle, decant, and wash once by decantation.

(E) Subacetate of lead. - Boil a 10 per cent solution of pure 
lead acetate with an excess of litharge for one hour, keeping the volume constant, and filter while hot. Disregard any precipitate which subsequently forms. Preserve out of contact with carbon dioxide.

(F) Potassium ferricyanide. - A very dilute freshly prepared solution containing about 0.1 per cent.

Method. - Weigh 20 grams of the glycerin, dilute to 250 cc., and take $25 \mathrm{cc}$. Add the silver carbonate, allow to stand with occasional agitation for about ten minutes, and add a slight excess (about 5 cc. in most cases) of the basic lead acetate $(E)$; allow to stand a few minutes, dilute with distilled water to $100 \mathrm{cc}$. and then add $0.15 \mathrm{cc}$. to compensate for the volume of the precipitate; mix thoroughly, filter through an air-dry filter into a suitable narrow-mouthed vessel, rejecting the first $10 \mathrm{cc}$, and return the filtrate, if not clear and bright. Test a portion of the filtrate with a little basic lead acetate, which should produce no further percipitate (in the great majority of cases 5 cc. are ample, but occasionally a crude glycerin will be found requiring more, and in this case another portion of 25 cc. of the dilute glycerin should be taken and purified with 6 cc. of the basic acetate). Care must be taken to avoid a marked excess of basic acetate.

Measure off $25^{\circ} \mathrm{cc}$. of the clear filtrate into a flask or beaker, previously cleaned with potassium dichromate and sulphuric acid. Add 12 drops of sulphuric acid ( 1 to 4 ) to precipitate the small excess of lead as sulphate. Add 3.7282 grams of the powdered potassium dichromate $(A)$. Rinse down the dichromate with $25 \mathrm{cc}$. of water and let stand with occasional shaking until all the dichromate is dissolved (no reduction will take place in the cold).

Now add 50 cc. of 50 per cent sulphuric acid (by volume) and immerse the vessel in boiling water for two hours and keep protected from dust and organic vapors, such as alcohol, until the titration is completed. Add from a weighing bottle a slight excess of the ferrous ammonium sulphate $(C)$, making spot tests on a porcelain plate with the potassium ferricyanide $\left(F^{\prime}\right)$. Titrate back with the dilute dichromate. From the 
amount of dichromate reduced, calculate the percentage of glycerol.

One gram glycerol $=7.4564$ grams dichromate

One gram dichromate $=0.13411$ gram glycerol .

The percentage of glycerol obtained above includes any oxidizable impurities present after purification. A correction for the non-volatile impurities may be made by running a dichromate test on the residue at $160^{\circ} \mathrm{C}$.

Notes. - (1) It is important that the concentration of acid in the oxidation mixture and the time of oxidation should be strictly adhered to.

(2) Before the dichromate is added to the glycerin solution it is essential that the slight excess of lead be precipitated with sulphuric acid as stipulated.

(3) For crude glycerins practically free from chlorides the quantity of silver carbonate may be reduced to one-fifth and the basic lead acetate to $0.5 \mathrm{cc}$.

(4) It is sometimes advisable to add a little potassium sulphate to insure a clear filtrate.

An appendix to the committee report from which the above methods are taken describes and illustrates a new form of sampling tube for taking samples of crude glycerin from drums.

\section{REFERENCES}

I

Alder-Wright and Mrtchell: Animal and Vegetable Fixed Oils, Fats, Butters, and Waxes.

Allen : Commercial Organic Analysis, Vol. II.

Benedikt-Ulzer: Analyse der Fette und Wachsarten.

Lamborn : Modern Soaps, Candles, and Glycerin.

Lew Kowrtscr : Chemical Technology and Analysis of the Oils, Fats, and Waxes.

\section{II}

1900. Devine: A Method of Determining Free Alkali in Soaps. J. Am. Chem. Soc., 22, 693.

Henriques and Mayer : (Determination of Total, Free, and Carbonated Alkali in Soaps). Z. angew. Chem., 1900, 785.

1902. Doane: The Disinfectant Properties of Washing Powders. Bul. 79, Maryland Agricultural Experiment Station. 
Friedrich: (Soap Analysis) 4 Bericht des Vereins gegen Verfälschung der Lebensmittel, etc., Chemnitz, 1902, 132; Z. Nahr. Genussm., 1903, 6, 851.

1903. Heller: (Significance of Soaps in Disinfectants). Arch. Hygiene, 47, 213.

1904. Heermans: Determination of Caustic and Carbonated Alkali in Soaps. Chem. Ztg., 28, 531 .

Martin : Determination of Glycerol in Soap. Moniteur Scientifique, [4], 17, 797.

VAN Slyke and Urner: The Composition of Commercial Soaps in Relation to Spraying. Bul. 257, New York State Agricultural Experiment Station.

1905. Devine: Determination of Rosin in Soaps. Chem. Eng., 1, 207.

Matrufws : The Effects of Alkaline Scouring Agents on the Strength of Woolen Yarns. J. Soc. Chem. Ind., 24, 659.

Rodet: Experiments on the Antiseptic Value of Common Soaps. Revue d'Hygiene, 27, 301.

1907. JACkson: Detergents and Bleaching Agents used in Laundry Work. J. Soc. Arts, 55, 1101, 1122 ; Chem. Abs., 2, 327.

1908. Bornemann : Fat, Soap, and Candle Industry in 1907. Chem. Ztg., 32, 741, 755 .

Fendler and Frank: Determination of Fatty Acid Content of Soaps. Z. angew. Chem., 22, 252.

Steiner : (Recent Development of the Soap Industry). Chem. Ztg., $32,445,458$.

1909. Dominikiewicz: New Method for Determination of Fatty Acids in Soap. Chem. Ztg., 33, 728 .

Sr : Mercury Seals in Fat Extraction Apparatus and a New Form of Flask. J. Ind. Eng. Chem., 1, 314.

1910. Coney and BACkus: The Coefficient of Expansion of Glycerin. $J$. Ind. Eng. Chem., 2,11.

1911. Committee Report on Glycerin Analysis. J. Ind. Eng. Chem., 3, 679. Hamilton: Soaps from Different Glycerides; their Germicidal and Insecticidal Values Alone and Associated with Active Agents. J. Ind. Eng. Chem., 3, 582. 


\section{CHAPTER XIV}

\section{Nitrogen, Sulphur, and Phosphorus}

\section{THE DETERMINATION OF NITROGEN}

THe well-known copper oxide method of Dumas, sometimes called the absolute method, has, the advantage of being applicable to all classes of nitrogen compounds. In the great majority of cases, however, it is equally accurate and much more convenient to use one of the methods based upon the conversion of nitrogen to ammonia and the determination of the latter by titration.

Before the introduction of the Kjeldahl process, the sodalime method of Will and Varrentrap was commonly used. In this method the finely ground substance is mixed with a large excess of soda lime and heated in a combustion tube, the ammonia given off being absorbed in standard acid. In order to insure the reduction of nitro-compounds or nitrates, this method was modified by the introduction of stannous sulphide (Goldberg); of sodium formate and sodium thiosulphate (Arnold); or of sodium thiosulphate and a mixture of equal parts sulphur and powdered sugar or charcoal (Ruffle). The soda-lime method has now been very generally superseded by the various modifications of the Kjeldahl process.

\section{The KJeldahl Method}

In this process the substance is decomposed by heating with strong sulphuric acid, usually with the addition of sone reagent which assists the decomposition either by raising the boiling point or by acting as a carrier of oxygen. When decomposition is complete, the nitrogen remains as ammonium sulphate in the sulphuric acid, the carbon and hydrogen of the substance hav- 
ing been oxidized and the products of oxidation boiled out of the solution. The oxidation takes place partly at the expense of the sulphuric acid, so that a considerable evolution of sulphur dioxide occurs, especially in the earlier stages of the process. After the completion of the digestion, the ammonia is liberated by means of fixed alkali and determined by distilling into standard acid. In the various modifications of the process, different reagents or combinations of reagents are used to hasten the decomposition of the organic matter by the sulphuric acid.

Kjeldahl originally directed 1 that the substance be heated with sulphuric acid, with or without the addition of phosphoric anhydride, until a clear solution is obtained; then potassium permanganate added in small portions to the hot solution until it remains permanently colored, the permanganate being added very cautiously on account of the danger of a loss of nitrogen if the reaction becomes too vigorous.

Willfarth ${ }^{2}$ introduced the use of mercuric oxide to facilitate the action of sulphuric acid, and stated that if the solution be boiled until colorless, neither phosphoric anhydride nor potassium permanganate need be used. In his earlier experiments Willfarth used copper instead of mercury. Arnold ${ }^{3}$ used both mercury and copper in addition to phosphoric anhydride.

Gunning ${ }^{4}$ used for the digestion a simple mixture of sulphuric acid with one third to one half its weight of potassium sulphate. Arnold and Wedemeyer ${ }^{5}$ modified the Gunning process by the use of mercury and copper in addition to the potassium sulphate. By this modification the time required to decompose the organic matter was greatly reduced and good results were obtained with a number of alkaloids and other compounds which had not readily yielded the whole of their nitrogen when treated by the methods previously used. Independently Dyer ${ }^{6}$ obtained equally good results on a wide range of organic com-

1 Z. anal. Chem., 1883, 22, 366.

2 Chem. Zentrbl., 1885, [3], 16, 17, 113.

${ }^{3}$ Archiv. der Pharm., [3], 24, 785 ; Z. anal. Chem., 26, 249.

4 Z. anal. Chem., 1889, 28, 188.

${ }^{5}$ Z. anal. Chem., 1892, 31, 525.

${ }^{6}$ J. Chem. Soc., 1895, 67, 811. 
pounds by the use of mercury and potassium sulphate without copper.

The Official Agricultural Chemists authorize ${ }^{1}$ the KjeldahlWillfarth and the Gunning methods for the analysis of foods, spices other than peppers, and fertilizers not containing nitrates. For peppers, to secure complete ammonification of the alkaloidal nitrogen, the Arnold-Wedemeyer modification of the Gunning method was provisionally adopted in 1902. In the experience of this laboratory, the Dyer modification has been found more rapid and slightly more accurate than either the Kjeldahl-Willfarth or Gunning method and fully as efficient in the case of alkaloids as is the Arnold-Wedemeyer method, while it has the advantage over the latter of requiring one less reagent and of yielding a colorless solution.

Applicability of the Kjeldahl Method. - The Gunning-ArnoldDyer modification, which for the reasons just mentioned is recommended for general use, is applicable to all classes of animal and vegetable substances, including such difficultly decomposable bases as betaine and pyridine and chinoline alkaloids, and to cyanides, ferrocyanides, and ferricyanides. It has also been tested $^{2}$ with good results on many other compounds, including acetanilid, sulphanilic acid, orthobenzoic sulphinid, aminobenzoic acid, benzamid, diaminophenol, naphthylamine, diphenylamine, diphenylthiourea, nitroso-dimethylaniline, indigotin, pyridine, and oxyphenyl methylpyrimidine.

Jodlbauer's moditication, ${ }^{3}$ devised for the determination of nitrogen in nitrates, was found by Dyer to be applicable to nitrocompounds, to azo-, hydrazo-, and amidoazo-benzene, to carbazol, and, with the addition of 1 or 2 grams of sugar, to hydroxylamine and oximes. Dyer did not obtain the whole of the nitrogen of hydrazine derivatives, but Dafert ${ }^{4}$ and Milbauer ${ }^{5}$ have published modifications which are said to give accurate results with this class of compounds.

${ }^{1}$ Bulletin 107, Revised, Bureau of Chemistry, U. S. Dept. Agriculture.

2 In some cases without the use of mercury. See in addition to the papers already cited, that of Gibson in $J$. Am. Chem. Soc., 1904, 26, 105.

${ }^{3}$ Chem. Zentrbl., 1886, 3, 17, 433.

${ }^{4}$ Landw. Versuchs-Sta., 1887, 34, 311.

${ }^{5}$ Z. anal. Chem., 1903, 42, 725 . 


\section{Gunning-Arnold-Dyer Modification}

Reagents. - Pure concentrated sulphuric acid. Mercury. Pure potassium sulphate. Potassium sulphide solution, 40 grams per liter. Saturated solution of sodium hydroxide (commercial). Granulated zinc or pumice stone. Paraffin. Solution of methyl orange, congo red, cochineal, lacmoid, or any other indicator suitable for titration in the presence of ammonium salts. Standard solutions of acid and alkali, preferably one fifth or one tenth normal.

Determination. - Weigh 0.5 to 5.0 grams sample, or so much as will probably yield from 50 to 75 milligrams of ammonia, and transfer to a pear-shaped Kjeldahl flask of 550 to 750 cc. capacity. Add 20 to 25 cc. concentrated sulphuric acid and about 0.7 gram of mercury. Place the flask in an inclined position (Fig. 16) and heat gently until the first vigorous frothing ceases, then raise the heat gradually until the liquid boils; remove the flame for a few minutes, add 10 to 12 grams of potassium sulphate, and boil. If the liquid is kept actually boiling, it will usually be clear and colorless

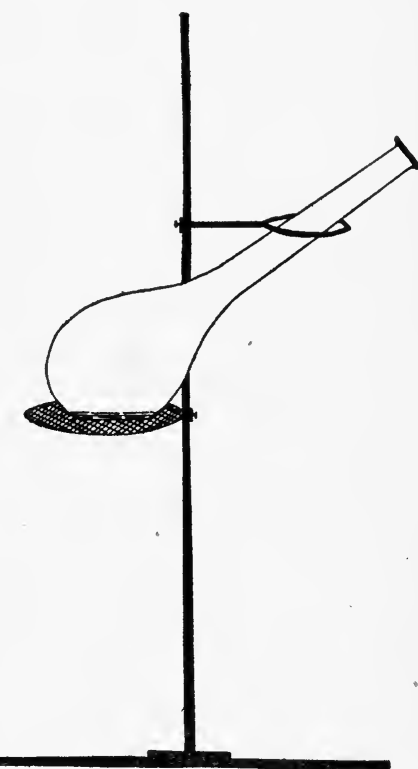

Fig. 16. - Kjeldahl digestion tlask in position on ordinary ring stand. within 30 minutes after the addition of the sulphate. Continue the boiling for at least 30 minutes after the solution becomes colorless or in any case for one hour from the time the potassium sulphate is added. If the sample contains alkaloids, the boiling should be continued for at least three hours in all and not less than two hours after the solution is colorless. When the digestion is finished, allow the flask to cool for 10 to 15 minutes or to $40^{\circ}-60^{\circ}$ (if allowed to become thoroughly cold 
the solution solidifies); then dilute carefully with 150 to 200 cc. of water ; allow to cool, add $25 \mathrm{cc}$. potassium sulphide solution, mix well, and then add 75 to $100 \mathrm{cc}$. (or enough to make the reaction strongly alkaline ${ }^{1}$ ) of a cold saturated solution of sodium hydroxide, pouring it carefully down the side of the flask, so that it does not mix immediately with the acid solution. Add a few pieces of granulated zinc or pumice stone to prevent bumping, and a piece of paraffin the size of a pea to diminish frothing ; connect the flask, preferably by means of a Hopkins distilling head (Fig. 17), with a condenser, the deliv-

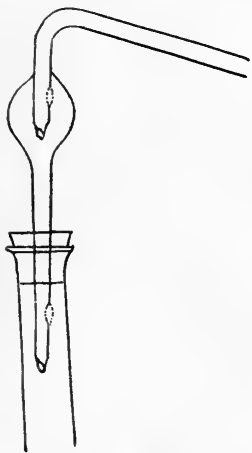

Fig. 17.-The Hopkins distilling head in position. ery tube of which dips into 50 ec. of tenth normal sulphuric or hydrochloric acid or its equivalent in the receiver; mix the contents of the flask by shaking, and distill until about one half of the liquid has passed into the receiver. Titrate the excess of acid in the receiver by means of standard alkali, using one of the indicators mentioned above.

The reagents used should be tested by making a blank determination with pure sugar or cellulose, carrying out all operations in exactly the same way as in a regular analysis.

Notes. - If in transferring the substance to the flask any particles or drops should lodge in the neck, they can be washed down by the sulphuric acid subsequently added. Dry samples can usually be weighed on a watch glass and brushed into the flask through a funnel having a wide stem which has been cut down to a length of about $1 \mathrm{~cm}$., or transferred by means of a narrow strip of glazed paper. It is often more convenient to weigh the sample on, or transfer to, a small piece of pure filter paper, fold the latter loosely over the weighed portion, and introduce the whole into the flask in such a way that the sample can be easily shaken free from the paper. The amount of cellulose thus introduced with the sample does not materially pro-

${ }^{1}$ Corallin (rosolic acid) may be used as indicator to show that the contents of the flask are alkaline before beginning the distillation. 
long the time required for digestion, while its reducing effect may aid in the ammonification of any firmly bound nitrogen present. Time should be allowed for thorough wetting of the sample by the sulphuric acid before heat is applied. During digestion a small funnel or balloon stopper may be placed loosely in the mouth of the flask to retard the evaporation of acid and guard against mechanical loss. The flask may rest upon a wire gauze, an iron plate, or a piece of asbestos having a circular hole about $4 \mathrm{~cm}$. in diameter which permits the free flame of the Bunsen burner to play upon the flask below the surface of the boiling liquid. The digestion should be conducted in a well-ventilated hood where the air is free from any considerable amount of ammonia. In order to hasten the decomposition of organic matter, Dakin ${ }^{1}$ and Milbauer (l.c.) recommend the use of potassium persulphate, while Krüger ${ }^{2}$ suggests the addition of potassium dichromate in excess of the amount necessary to oxidize all carbon and hydrogen present. The method here described is, however, sufficiently rapid and more convenient for ordinary work.

The precipitation of mercury as sulphide before rendering the solution alkaline is to prevent the formation of mercur-ammonium compounds which do not readily yield their ammonia on boiling with caustic alkali. An ordinary Liebig condenser can be used in this distillation, though block tin tubing is generally preferred as being more resistant to the action of steam and anmonia. Unless the condenser tube is straight and nearly vertical, it is safer to rinse it out with a little cold distilled water at the end of the distillation and add the rinsings to the distillate. The distillation as usually carried out requires from forty minutes to one hour. Only about one third of this time is actually required to expel the ammonia from the boiling solution, and if it is desired to hasten the operation, the steam can be conducted through tin tubing without condensation into a cooled receiver; or, as suggested by Benedict ${ }^{3}$ the water which cools the condenser may be drawn off after the solution

1.J. Soc. Chem. Ind., 1902, 21, 848.

2 Ber., 1894, 27, 609.

${ }^{3}$ J. Am. Chem. Soc., 1900, 22, 259. 
has been boiling 15 minutes, and the boiling continued until enough steam has passed through to carry all of the ammonia from the condenser tube into the receiver. Fixed alkali is preferable to ammonia for the final titration, since the latter reagent is liable to lose strength during use, probably through evaporation of ammonia from the tip of the burette and from the falling drop.

The reasons for preferring the method as above described to the original Kjeldahl-Willfarth or the Gunning method have been briefly outlined above. If either of the latter methods is used, the same details of manipulation may be followed except that the digestion should be continued for at least two hours after the solution becomes colorless and not less than three hours in all. Even with this longer time of boiling the results are often slightly low. For the results of a comparative study of the Kjeldahl-Willfarth, the Gunning, and the above described method with special reference to the time of boiling required see the Journal of the American Chemical Society for April and November, 1904.

\section{Method for Nitrates and Nitro-compounds}

The loss of nitric acid which might otherwise occur when sulphuric acid is poured upon a sample containing nitrates is avoided by having present some substance which is very easily nitrated, such as salicylic acid, benzoic acid, or phenol. The nitrogen having been thus fixed as a nitro-compound, the latter may be reduced by the addition of zine dust (Jodlbauer) or sodium thiosulphate (Forster), after which the determination is carried out in the usual manner.

Reagents. - Sulphuric-salicylic acid, prepared in advance by dissolving salicylic acid in pure concentrated sulphuric in the proportion of 2 grams of the former to $30 \mathrm{cc}$. of the latter. Zine dust, an impalpable powder; or crystallized sodium thiosulphate. Other reagents as for the method above described.

Determination. - To the weighed portion of substance in a Kjeldahl flask, add 30 ec. of the sulphuric-salicylic acid solution and shake until thoroughly mixed; then add 5 grams 
crystallized sodium thiosulphate, or add gradually with constant shaking 2 grams of zinc dust; warm gently until frothing subsides and then boil until thick white fumes cease to be given off. Allow to cool somewhat, add mercury and potassium sulphate, and complete the process as described above. Or the potassium sulphate may be omitted and the oxidation completed by means of permanganate as in the "modified Kjeldahl" method of the Official Agricultural Chemists.

Notes. - Thiosulphate is the more convenient reducing agent and can be safely used if the amount of nitric nitrogen is small. Zinc dust appears to be a safer reagent for samples rich in nitrates. In the presence of considerable amounts of chloride or of ammonium salts there is danger of a loss of nitrogen as oxide when the sample is treated with the sulphuric-salicylic acid mixture. In such cases the latter mixture should be thoroughly cooled before using and then poured as quickly as possible over the sample and the flask allowed to stand with occasional shaking for at least half an hour before the reducing agent is added. Ten minutes should be allowed for the reduction to take place before the solution is heated. With these precautions the loss of nitrogen will be reduced to an amount too small to be appreciable in ordinary work.

\section{THE DETERMINATION OF SULPHUR}

In the various methods employed for the determination of sulphur, organic matter is destroyed by oxidation and the resulting sulphuric acid is precipitated and weighed as barium sulphate in the usual way. The chief difficulty lies in securing complete oxidation without loss, since many substances contain sulphur in forms from which it is easily liberated as volatile compound.

\section{Comparative Outline of Methods}

The principal methods of bringing about the oxidation of the organic matter are:

1. By heating with nitric acid or an oxidizing acid mixture.

2. By burning in a combustion tube in a current of air, oxygen, or some oxidizing vapor. 
3. By heating in the presence of alkali and completing the reaction by the addition of an oxidizing agent.

4. By combustion in oxygen in a closed vessel.

Since the choice of a method depends on the nature of the sample to be examined and the apparatus at hand, no fixed rules can be laid down for this purpose. Several of the more important methods are therefore outlined, and three methods which are especially useful for plant and animal substances are given in full.

Of methods of the first group, that of Carius, which has already been mentioned in the previous chapter, is the most important. It is applicable to volatile as well as to non-volatile substances, but is somewhat troublesome and tedious, and determinations are frequently lost through breaking of the heated tubes, especially when much gas is produced by the oxidation of the carbon of the sample. For this reason only a small amount of any highly carbonaceous substance can be taken for analysis, and often the amount of sulphuric acid obtained is too small for satisfactory determination.

Several methods belonging to the second group have been proposed. Among the more important are: (a) The method of Brugelmann, ${ }^{1}$ in which the substance is burned in a current of oxygen, complete oxidation being insured by passing the mixture of gases over hot platinum, and absorbing the sulphuric acid in a column of soda lime. If the substance is poor in sulphur, several portions can be burned before dissolving out the lime and determining the sulphuric acid. (b) Mixter's modification ${ }^{2}$ of Sauer's method. This consists in burning the substance in a current of oxygen and passing the products of combustion into bromine water. (c) Clässon's method ${ }^{3}$ of burning in a current of nitric oxide. (d) Barlow's method, ${ }^{4}$ in which the substance is burned in a current of oxygen in a specially constructed tube and complete oxidation is insured by means of a second current of oxygen introduced laterally at

1 Z. anal. Chem., 1876, 15, 1, $175 ; 1877,16,1$.

${ }^{2}$ Am. Chem. J., 1880, 2, 396. $\quad{ }^{3}$ Ber., 1886, 19, 1910; 1887, 20, 3065.

${ }^{4}$ J. Am. Chem. Soc., 1904, 26, 341. 
a point between the boat and the absorbing column. Methods of this type, carefully carried out, insure complete oxidation, and several portions of a sample may be burned in succession so as to secure a larger amount of sulphuric acid for determination. They are, however, less rapid than the methods described below, which are usually quite as accurate.

Liebig destroyed organic matter and converted sulphur to sulphate by heating with a mixture of caustic alkali and alkaline nitrate, and methods of this type have been very generally used for plant and animal materials and other non-volatile substances. Of the many modifications of Liebig's method, the use of sodium peroxide as an oxidizing agent is most important. The peroxide may be used in connection with hydroxide as in Osborne's method, or the sample may be mixed at once with an excess of peroxide and ignited in a closed crucible ${ }^{1}$ or in the combustion chamber of the Parr calorimeter. ${ }^{2}$

Berthelot, in proposing the use of the oxygen calorimeter for the elementary analysis of organic materials, ${ }^{3}$ stated that on combustion with 25 atmospheres of oxygen the sulphur was completely oxidized and in the presence of moisture could be quantitatively recovered as sulphuric acid. This method has been found both convenient and accurate. Complete oxidation takes place instantaneously, and since the combustion chamber is hermetically sealed there is no possibility of a loss of volatile compounds. A small autoclave may be used for the combustion if a bomb calorimeter is not available. According to Hempel, ${ }^{4}$ the sample can be burned in a large glass bottle filled with oxygen at atmospheric pressure.

The nature and number of the samples to be analyzed will usually determine which of the above methods is to be pre-

${ }^{1}$ Sundstrom: J. Am. Chem. Soc., 1903, 25, 184. Pennock and Morton : Ibid., 25, 1265.

2 Parr: J. Am. Chem. Soc., 1900, 22, 646 ; 1904, 26, 1139. Konek: Z. angew. Chem., 1903, 516. Leclerc and Dubois: J. Am. Chem. Soc., 1904, 26, 1108.

${ }^{3}$ Compt. rend., 1892, 114, 318 ; 1899, 129, 1002. See also Hempel : Ber., 1897, 30, 202, and Langbein : Z. angew. Chem., 1900, 1227, 1259.

4 Z. angew. Chem., 1892, 393. See also Graeffe: Ibid., 1904, 616. 
ferred. For the majority of cases either Liebig's or Osborne's method, or if the facilities are available, Berthelot's method of combustion in compressed oxygen may be recommended.

\section{Liebig's Alkali Method}

In a large silver or nickel crucible mix about 8 grams potassium hydroxide and about 1 gram potassium nitrate. Fuse and stir with a silver or nickel spatula. Allow to cool and add 1 to 2 grams of the finely pulverized sample. Heat gently, and as soon as the mass softens, stir well so as to bring the whole of the sample into contact with the alkali before it is strongly heated, then gradually increase the heat, stirring frequently to keep down frothing, and continue heating until the mass becomes colorless. For samples which react violently when stirred into the alkali as above, a smaller proportion of nitrate should be used at the beginning, or the nitrate may be omitted at the start and added after the sample has been mixed with the fused hydroxide. If the substance is not quickly oxidized by the fusion mixture, small amounts of pulverized nitrate may be added from time to time and the heating continued with frequent stirring. The fusion will usually be perfectly colorless when the oxidation of organic matter is complete. After cooling dissolve the fusion in water in a beaker or casserole, acidulate with hydrochloric acid, and evaporate to dryness to expel nitric and nitrous acids and heat at $110^{\circ}$ to dehydrate silica if present. If much nitrate or silica was present, the evaporation with hydrochloric acid should be repeated. Dissolve the residue in cold water, adding a few drops of hydrochloric acid, filter if not perfectly clear, heat the solution to boiling, and precipitate at the boiling temperature by adding a solution of barium chloride, drop by drop with constant stirring, until in considerable excess. Boil 5 or 10 minutes longer and allow to stand in a hot place until the precipitate settles, leaving the supernatant liquid perfectly clear. Filter and wash the barium sulphate, first with water acidulated with hydrochloric acid, then with water till free from chlorides, and finally ignite and weigh with the usual precautions. 
Notes and Precautions. - The reagents should be tested by making a "blank" determination with sugar or cellulose. Do not reject this blank if no precipitate appears when the barium chloride is added, but allow the solution to stand over night to insure the separation of any small amount of barium sulphate present, then filter, wash, ignite, and weigh, and subtract the weight from that of the precipitate found in the determination. An apparently trifling amount of precipitate in the "blank" may weigh enough to appreciably affect the results. If the fusion is heated by an ordinary gas flame, it is likely to absorb sulphur compounds from the latter. To avoid this, use an alcohol flame for heating the fusions. It cannot be expected that the blank determination should show accurately the sulphur absorbed from the gas flame. Even when an alcohol flame is used and the sulphur obtained from the reagents is deducted, the results are sometimes too high. According to Keiser ${ }^{1}$ this is due chiefly to the fact that the fused alkali takes up some silver from the crucible, and, on acidifying with hydrochloric acid, the silver chloride formed is held in solution by the excess of potassium chloride present and is afterward carried down by the barium sulphate, increasing the weight of the precipitate. Keiser therefore recommends that the neutralized solution be cooled, diluted with water to about a liter, and allowed to stand. Any silver chloride present will then precipitate and may be filtered out before adding barium chloride.

A modification which is often useful consists in heating the substance with strong nitric acid until a considerable part of the organic matter has been oxidized, after which an excess of alkali is added and the mixture transferred to a crucible and carefully fused until oxidation is complete. Hammarsten ${ }^{2}$ found this method to give the same results as the methods of Liebig and of. Clässon when applied to casein, egg albumen, and gelatin.

\section{Osborne's Peroxide Method ${ }^{3}$}

Convert about 10 grams of sodium peroxide into hydroxide by adding to it, in a silver or nickel crucible, a slight excess of

${ }^{1}$ Am. Chem. J., 1883, 5, 207.

${ }^{2} Z$. physiol. Chem., 1885, 9, 273.

${ }^{3}$ J. Am. Chem. Soc., 1902, 24, 142. 
water and heating until the excess is boiled off. Allow the fusion to cool until pasty, then add one to two grams of the sample and stir it into the alkali as quickly as possible. Heat gently and stir well to keep down the frothing. When the first vigorous action is over, heat until the mass fuses and stir in small portions - of fresh peroxide until decomposition is complete and the fusion is practically colorless. Then allow to cool, dissolve the fusion, and acidulate with hydrochloric acid; boil to destroy any excess of peroxide and to expel chlorine, and complete the determination as in Liebig's method.

Notes. - Blank determinations must be made as in the case of the Liebig method, and the same precautions observed to prevent the absorption of sulphur compounds from gas flames or from the air of the laboratory. There is also the same danger of high results from contamination of the barium sulphate precipitate. If silica is absent, the acidulated solution need not be evaporated to dryness, since no nitrate is used in the fusion. For all such cases the method is considerably more rapid than that of Liebig, though requiring somewhat more careful attention during the fusion of the substance with the alkaline oxidizing mixture. This method has been extensively used during recent years in the analysis of proteins, food materials, and physiological products, and has lately been adopted by the Association of Official Agricultural Chemists. Several methods involving the use of sodium peroxide had previously been proposed, of which that of Asboth $^{1}$ is perhaps the best known. This consists in mixing the substance with 10 grams of dry sodium carbonate and 5 grams of peroxide in a nickel crucible and heating carefully at first and then more strongly until oxidation is complete. After dissolving the fusion in water the solution may be boiled with bromine to insure complete oxidation. The alkaline mixture here used being much less fusible than sodium or potassium hydroxide, the method should be used only for such substances as do not readily lose sulphur on heating. For such substances it offers the advantage of simplicity in manipulation.

A modified form of the sodium peroxide method is recom-

${ }^{1}$ Chem. Ztg., 1895, 19, 2040. 
mended by Neumann and Meinertz: Z. physiol. Chem., 1904, 43, 37. See also Dubois: J. Am. Chem. Soc., 1905, 27, 729.

\section{A. O. A. C. Method ${ }^{1}$}

Place from 1.5 to 2.5 grams of material in a nickel crucible of about $100 \mathrm{cc}$. capacity and moisten with approximately $2 \mathrm{cc}$. of water. Mix thoroughly, using a nickel or platinum rod. Add 5 grams of pure anhydrous sodium carbonate and mix. Add pure sodium peroxide, small amounts (approximately 0.50 gram) at a time, thoroughly mixing the charge after each addition. Continue adding the peroxide until the mixture becomes nearly dry and quite granular, requiring usually about 5 grams of peroxide. Place the crucible over a low alcohol flame (or other flame free from sulphur) and carefully heat with occasional stirring until contents are fused. (Should the material ignite, the determination is worthless.) After fusion remove the crucible, allow to cool somewhat, and cover the hardened mass with peroxide to a depth of about $0.5 \mathrm{~cm}$. Heat gradually, and finally with full flame until complete fusion takes place, rotating the crucible from time to time in order to bring any particles adhering to the sides into contact with the oxidizing material. Allow to remain over the lamp for ten minutes after fusion is complete. Cool somewhat; place warm crucible and contents in a 600-cc. beaker and carefully add about $100 \mathrm{cc}$. of water. After violent action has ceased, wash material out of crucible, make slightly acid with hydrochloric acid (adding small portions at a time), and complete the determination by precipitating with barium chloride in the usual manner.

\section{Berthelot's Compressed Oxygen Method}

Press into the form of a pellet a suitable amount of the substance (usually one to three grams), introduce into the bomb calorimeter, and burn in oxygen under a pressure of about 25 atmospheres in the same manner as in the determination of heat

1 Method adopted by the Association of Official Agricultural Chemists for determination of sulphur in plants. U. S. Dept. Agr., Bur. Chem., Bul. 107 (Revised), p. 23. 
of combustion. ${ }^{1}$ Screw into the exit tube of the bomb a coupling carrying a delivery tube of about $0.5 \mathrm{~mm}$. internal diameter and connect with a washing cylinder containing a little water. Carefully open the valve and allow the gas to bubble through the water until the contents of the bomb reach atmospheric pressure ; then disconnect, open the bomb, and rinse out all moisture which has condensed in the chamber, on the lining of the cover, or on the rods which support the combustion capsule. This must be done carefully, as the rinsings usually contain the greater part of the sulphur. In order to keep down the volume of the solution, the water from the washing cylinder may be used for the first rinsing. Dissolve any ash found in the combustion capsule in a little hydrochloric acid, remove silica, if necessary, and add the solution to that obtained by rinsing the bomb. Examine the lead gasket in the cover of the bomb, and if a slight film of sulphate is found, wash it into the main solution. ${ }^{2}$ Finally boil this solution down to the desired volume, neutralize any excessive amount of hydrochloric acid which may have been introduced with the solution of the ash, filter if necessary, and determine the sulphuric acid by precipitation with barium chloride as already described.

Notes. - This method, based mainly upon the suggestion of Berthelot and the subsequent work of Hempel, has been applied ${ }^{3}$ with very satisfactory results to a number of animal and vegetable substances as well as to synthetic organic compounds containing sulphur in forms not readily oxidized to sulphuric acid - e.g., benzyl sulphide and diphenyl-thiourea. So far as tested, no cases of incomplete combustion have been found. If this were suspected, the gas drawn off after the combustion should be led through bromine water or an alkaline bromine solution and the washings of the bomb added to this solution and boiled to insure oxidation of sulphites to sulphates. Unless the bomb is emptied gradually in some such way as has been

${ }^{1}$ Atwater and Snell : J. Am. Chem. Soc., 1903, 25, 659.

${ }^{2}$ Such minute quantities of lead sulphate as are ordinarily found will dissolve readily in the solution and will not interfere with the precipitation of sulphuric acid as barium sulphate.

${ }^{3}$ J. Am. Chem. Soc., 1902, 24, 1100. 
described, there is danger that some sulphuric acid may be carried away mechanically by the escaping gas. This is probably the principal cause of the low results occasionally reported. When properly carried out this method has several important advantages. Combustion takes place very rapidly and with no chance for the escape of volatile products. The products of combustion can be drawn off and examined as desired. The only reagents required in obtaining the sulphur as sulphuric acid are compressed oxygen and hydrochloric acid, both of which are easily obtained free from any appreciable amounts of sulphur. By eliminating the use of alkali, any danger of absorption of sulphur compounds from the air is avoided, and no considerable amount of foreign salts is introduced into the solution from which the sulphuric acid is to be precipitated.

\section{THE DETERMINATION OF PHOSPHORUS}

The oxidation of organic matter for the determination of phosphorus may be accomplished by any of the methods described in connection with the sulphur determination. It is of course unnecessary in this case to avoid the use of gas flames, and with most substances there is much less danger of loss by volatilization than in the case of sulphur. For these reasons and because of the care required in dissolving the fused residue of phosphates left in the ignition capsule, the compressed oxygen method has no such marked advantages in the determination of phosphorus as in that of sulphur.

\section{Alkali Method}

Liebig's method of oxidation, either with or without previous digestion with nitric acid or oxidation by peroxide, may be used, but the use of sodium carbonate, as in the following method, is generally more convenient.

Mix 1 to 3 grams substance with 6 to 7 grams dry sodium carbonate in a platinum dish and spread 3 or 4 grams of the carbonate over the mixture. Heat over a Bunsen burner, carefully at first until frothing ceases, then strongly until the mass fuses. To the fusion add small portions of pulverized potas- 
sium nitrate, stirring thoroughly with a platinum rod or spatula, until the mass is entirely colorless. The whole amount of nitrate required does not usually exceed 1 gram. After cooling, transfer the fusion to a beaker, dissolve in water, add an excess of nitric acid, and boil. Allow the solution to cool, neutralize with ammonia, add a few drops of nitric acid and then a moderate excess of molybdate solution. ${ }^{1}$ Digest at about $65^{\circ}$ for an hour with occasional shaking or stirring, filter, and wash with cold water or a cold solution of ammonium nitrate. Test the filtrate by adding more molybdate solution and digesting again at about $65^{\circ}$. Place the beaker used for the precipitation under the funnel, dissolve the precipitate through the paper by means of ammonia, and wash thoroughly with water, keeping the volume of the solution below $100 \mathrm{cc}$. Nearly neutralize with hydrochloric acid, cool thoroughly, and add a moderate excess of magnesia mixture, ${ }^{2}$ drop by drop, with constant stirring. After 15 minutes add $10 \mathrm{cc}$. of concentrated ammonia or its equivalent; let stand four to twenty-four hours ; filter (preferably on an asbestos felt in a Gooch crucible), wash with dilute ammonia ( 2.5 to 5 per cent) until practically free from chlorides, ignite, and weigh as magnesium pyrophosphate.

\section{ACID Method ${ }^{3}$}

To 1 to 3 grams substance in a Kjeldahl flask add 20 cc. concentrated sulphuric acid and 10 grams of ammonium nitrate, and heat carefully until frothing ceases, then allow to cool somewhat, add 10 grams more of ammonium nitrate, and heat to boiling; or treat the sample with concentrated or fuming nitric acid, shaking first in the cold then warming gently till vigorous action ceases, then add $10 \mathrm{cc}$. of sulphuric acid, 10

1 Of the usual molybdate solution containing about 5 per cent of molybdic acid, add at least $50 \mathrm{cc}$. for every decigram of phosphoric acid expected.

2 If the magnesia mixture is of the usual strength, containing about 5 per cent of magnesium chloride, add about $15 \mathrm{cc}$. for each decigram of phosphoric acid expected.

${ }^{3}$ Neumann : Dubois Reymond's Archiv. (physiol. Abth.), 1897, 552; 1900, 159 ; Z. physiol. Chem., 1900, 29, 146; 1902-3, 37, 115. Sherman : J. Am. Chem. Soc., 1902, 24, 1100. 
grams of ammonium nitrate, heat gently till frothing ceases, and then boil. Continue boiling until the liquid in the flask is concentrated to a volume not greater than the volume of sulphuric acid which has been used. At this volume the boiling point should be higher than that of sulphuric acid because of the ammonium sulphate in the solution. Boil at this higher temperature for about half an hour; then, if not colorless, cool somewhat, add nitric acid (concentrated or fuming) and boil again until the nitric acid is expelled and the low volume and high temperature are reached again, repeating as often as necessary until the liquid is colorless and shows no discoloration when heated for half an hour at low volume and high temperature as described. Cool the colorless solution, wash it out into a beaker, neutralize with ammonia, add 15 to 20 grams of ammonium nitrate, and determine phosphoric acid as in the preceding method, adding a somewhat greater excess of the molybdate reagent.

Notes. - This method has been found to give good results with a variety of foods and physiological products. The large amount of sulphates retards the formation of the phosphomolybdate precipitate, but by using liberal quantities of ammonium nitrate and molybdate reagent and allowing at least an hour for the precipitation, the whole of the phosphoric acid is obtained without difficulty. When the substance is first treated with fuming nitric acid, a smaller quantity of sulphuric acid may be used and the phosphomolybdate will then form somewhat more quickly.

In applying this method to liquids such as milk or urine, a suitable amount of sample ( $25 \mathrm{cc}$. milk or $50 \mathrm{cc}$. urine) is transferred to the flask, $20 \mathrm{cc}$. sulphuric acid added, and the mixture heated carefully as in the determination of nitrogen until the sample is well charred and the greater part of the water has been boiled off. Ammonium nitrate is then added and the determination is completed as above described.

\section{Magnesium Nitrate Method ${ }^{1}$}

Mix 2 grams of the dry substance (or a correspondingly larger amount if a liquid) with 5 cc. of a neutral 35 per cent solution

1 Adopted by the Association of Official Agricultural Chemists for the determination of phosphorus in plant substance. 
of magnesium nitrate ; dry and ignite; dissolve the residue in acid and determine phosphoric acid as usual.

This method is convenient but has not been so thoroughly tested on different organic forms of phosphorus as have the alkali and the acid methods described above.

\section{REFERENCES}

I

Aboerhalden: Handbuch der Biochemischen Arbeitsmethoden. I. Ephraim : Original-arbeiten über Analyse der Nahrungsmittel. Gattermann: Practical Methods of Organic Chemistry. Hoppe-Seyler : Physiologisch- und Pathologisch-Chemische Analyse. KöxIg: Chemie der Menschlichen Nahrungs und Genussmittel. III. LAssar-Conx: Arbeitsmethoden für Organisch-Chemische Laboratorien. LEA'́r : Food Inspection and Analysis.

Lincoln and Walton: Quantitative Analysis for Agricultural Students. MeYer: Analyse und Konstitutionsermittelung organischer Verbindungen. Monse: Exercises in Quantitative Chemistry.

Sudborough and James : Practical Organic Chemistry. U. S. Dept. Agriculture, Bureau of Chemistry, Bul. 107 (Revised). VAUBEL: Quantitative Bestimmung organischer Verbindungen. WEYL: Die Methoden der Organischen Chemie. I.

II

1895. Dyer: (Applicability of the Modified Kjeldahl Method). J. Chem. Soc., 67, 811.

1902. Sherman: The Determination of Sulphur and Phosphorus in Organic Materials. J. Am. Chem. Soc., 24, 1100.

1904. Girson: Study of the Kjeldahl Method. J. Am. Chem. Soc., 26, 105. Sherman, McLaughlin, and Osterberg: The Determination of Nitrogen in Food Materials and Physiological Products. J. Am. Chem. Soc., 26, 367.

Sherman and Falk: The Determination of Nitrogen in Organic Compounds. J. Am. Chem. Soc., 26, 1469.

1905. Duвors: Determination of Sulphur and Phosphoric Acid in Foods, Feces, and Urine. J. Am. Chem. Soc., 27, 729.

Flamand and Prager: Modification of Kjeldahl Method for Azocompounds, etc. Ber., 38, 558 .

Milbauer: Determination of Nitrogen in Hydrazones and Osazones by the Kjeldahl Method. Z. Zuckerind. Böhmen, 28, 338; Biochem. Centrbl., 1905, 469.

SADTLer: Inner Crucible Method for Determining Sulphur and Halogens in Organic Substances. J. Am. Chem. Soc., 27, 1188. 
1906. Folin: On Sulphate and Sulphur Determinations. J. Biol. Chem., $1,131$.

HAAs : Production of Methane as Source of Error in Nitrogen Determinations by the Absolute Method. Proc. Chem. Soc., 1906, 22, 81; Analyst, 31, 167.

1907. Gladdixg: Comparative Work on Nitrogen Estimations by the Kjeldahl and Gunning Methods and by a Combination of the Two Methods. U. S. Dept. Agriculture, Bur. Chem., Bul. 105. p. 85 .

1908. Dennstedt: Simultaneous Determination of Nitrogen and Sulphur with Carbon and Hydrogen. Ber., 41, 600, 2778.

Leavitt and LeClerc: Loss of Phosphorus in Ashing Cereals. J. Am. Chem. Soc., 30, 391, 617.

Richmond: Estimation of (Triazo) Nitrogen. Analyst, 33, 179.

Rupp: Determination of Sulphur by Carius Method. Chem. Ztg., 32, 984 ; Chem. Abs., 3, 296.

1909. Penny : Report on the Determination of Nitrogen. U. S. Dept. Agriculture, Bur. Chem., Bul. 122, p. 85.

Trowbridge: Determination of Phosphorus in Flesh. J. Ind. Eng. Chem., 1, 675 .

1910. Allen and Johnson: The Exact Determination of Sulphur in Soluble Sulphates. J. Am. Chem. Soc., 32, 588 .

Brown: Note on Kjeldahl Estimation of Nitrogen in Fatty Substances. Chem. News, 102, 51.

GrindLey and Ross: Determination of Inorganic and Organic Phosphorus in Meats. J. Biol. Chem., 8, 483.

HibBard : Notes on the Determination of Nitrogen by the Kjeldahl Method. J. Ind. Eng. Chem., 2, 463.

Jones and KelLogG: Report on Nitrogen Determinations. U. S. Dept. Agriculture, Bur. Chem., Bul. 132, p. 16.

Schreiber: Determination of Total Sulphur in Organic Matter. J. An. Chem. Soc., 32, 977.

Trowbridge and Stanley: Phosphorus in Flesh. J. Ind. Eng. Chem., 2, 212.

1911. Committee Report on Determination of Nitrogen. J. Ind. Eng. Chem., 3, 691.

VAN RIJN: (Note on Technique of Nitrogen Determinations). Pharm. Weekbl., 48, 27; Chem. Abs., 5, 2791.

Waters and Tutrle: The Determination of Total Sulphur in India Rubber. J. Ind. Eng. Chem., 3, 734.

Whitrier: Estimation of Inorganic Phosphorus in Animal Tissues. J. Ind. Eng. Chem., 3, 248. 


\section{CHAPTER XV}

\section{Proteins and Proteases}

THE proteins are nitrogen compounds ${ }^{1}$ of high molecular weight which consist, so far as is at present known, essentially of combinations of $\alpha$-amino acids and their derivatives. The classification and terminology of proteins recommended by a joint committee of the American Physiological Society and the Society of Biological Chemists is as follows :

I. Simple Proteins. - Protein substances which yield only $\alpha$-amino acids or their derivatives on hydrolysis.

(a) Albumins. - Simple proteins soluble in pure water and coagulable by heat.

(b) Globulins. - Simple proteins insoluble in pure water but soluble in neutral solutions of salts of strong bases with strong acids.

(c) Glutelins. - Simple proteins insoluble in all neutral solvents but readily soluble in very dilute acids and alkalies.

(d) Alcohol-soluble Proteins. - Simple proteins soluble in relatively strong alcohol (70-80 per cent), but insoluble in water, absolute alcohol, and other neutral solvents.

(e) Albuminoids. - Simple proteins which possess essentially the same chemical structure as the other proteins, but are characterized by great insolubility in all neutral solvents. These form the principal organic constituents of the skeletal structure of animals and also their external coverings and its appendages. This definition does not provide for gelatin, which is, however, an artificial derivative of collagen.

$(f)$ Histones. - Soluble in water and insoluble in very dilute ammonia and, in the absence of ammonium salts, insoluble even in an excess of ammonia; yield precipitates with solutions of

1 Most proteins contain between 15.5 and 18 per cent of nitrogen. On the assumption that the average is 16 per cent, the nitrogen content is often taken as a measure of the amount of protein present. 
other proteins and a coagulum on heating which is easily soluble in very dilute acids. On hydrolysis they yield a large number of amino acids, among which the basic ones predominate.

(g) Protamines. - Simpler polypeptids than the proteins included in the preceding groups. They are soluble in water, uncoagulable by heat, have the property of precipitating aqueous solutions of other proteins, possess strong basic properties and form stable salts with strong mineral acids. They yield comparatively few amino acids, among which the basic amino acids greatly predominate.

II. Conjugated Proteins. - Substances which contain the protein molecule united to some other molecule or molecules otherwise than as a salt.

(a) Nucleoproteins. - Compounds of one or more protein molecules with nucleic acid.

(b) Glycoproteins. - Compounds of the protein molecule with a substance or substances containing a carbohydrate group other than a nucleic acid.

(c) Phosphoproteins. - Compounds of the protein molecule with some, as yet undefined, phosphorus-containing substance other than a nucleic acid or lecithins.

(d) Hemoglobins. - Compounds of the protein molecule with hematin or some similar substance.

(e) Lecithoproteins. - Compounds of the protein molecule with lecithins (lecithans, phosphatids).

III. Derived Proteins. - 1. Primary Protein Derivatives. Derivatives of the protein molecule apparently formed through hydrolytic changes which involve only slight alterations of the protein molecule.

(a) Proteans. - Insoluble products which apparently result from the incipient action of water, very dilute acids, or enzymes.

(b) Metaproteins. - Products of the further action of acids and alkalies whereby the molecule is so far altered as to form products soluble in very weak acids and alkalies, but insoluble in neutral fluids. This group will thus include the familiar "acid proteins" and "alkali proteins," not the salts of proteins with acids. 
(c) Coagulated Proteins. - Insoluble products which result from (1) the action of heat on their solutions, or (2) the action of alcohols on the protein.

2. Secondary Protein Derivatives. - Products of the further hydrolytic cleavage of the protein molecule.

(a) Proteoses. - Soluble in water, uncoagulated by heat, and precipitated by saturating their solutions with ammonium sulphate or zinc sulphate.

(b) Peptones. - Soluble in water, uncoagulated by heat, but not precipitated by saturating their solutions with ammonium sulphate.

(c) Peptids. - Definitely characterized combinations of two or more amino acids, the carboxyl group of one being united with the amino group of the other, with the elimination of a molecule of water.

For descriptive accounts of the proteins reference must be made to some of the works cited at the end of the chapter. In ordinary analytical work some of the groups of proteins included in the above classification are seldom met in sufficient quantity to require separate consideration.

The groups of proteins of greatest interest to the analytical chemist are the following:

Albumins. - Soluble in water and dilute salt solutions; precipitated by adding sufficient alcohol or by saturating the aqueous solution with ammonium sulphate. The water solutions are coagulated by heating, commonly at $70^{\circ}-73^{\circ}$ (Halliburton), but sometimes much lower, leucosin coagulating at $52^{\circ}$ (Osborne).

Globulins. - Insoluble in pure water but soluble in dilute salt solutions. Precipitated by alcohol or by saturation with ammonium or magnesium sulphate; also precipitated from salt solutions on removing the salt by dialysis. Some of the vegetable globulins are distinctly crystalline and not coagulable by heating.

Alcohol-soluble Proteins. - These occur especially in the cereal grains. Gliadin, insoluble in water, dilute salt solution, or absolute alcohol, but soluble in 75 per cent alcohol is the best known member of this group. 
Albuminoids. - Some of these differ considerably from each other, but all are characterized by their resistance to reagents. Among the more important of the albuminoids are collagens which yield gelatins on boiling with water; keratins of skin, horn, hair, feathers, nails, etc.; elastin of connective tissue; skeletins, the nitrogenous compounds of the skeletal and related tissues of invertebrates, including the characteristic compounds of sponges and silk.

Gelatins (and collagens) are the only albuminoids which are likely to be met in connection with the true proteins in foods, etc., so that the other substances mentioned need not be considered in connection with the reactions given below.

Metaproteins. - By the action of acid or alkali upon native or coagulated protein the latter may be converted into a metaprotein, sometimes called an acid- or an alkali-albumin or albuminate. Both acid and alkali albuminates are nearly insoluble in water and dilute salt solution, but usually dissolve readily in water containing a very small amount of acid or alkali and are precipitated from such solutions by neutralization at ordinary temperature. The metaproteins or albuminates are precipitated from nearly neutral solutions by the general precipitants for soluble proteins (see below) but a very minute amount of acid or alkali is sufficient to prevent the coagulation of albuminate by boiling.

Proteoses and Peptones. - These are products derived from other proteins by digestion or by simple hydrolysis. They are soluble in water and not coagulated by boiling their aqueous solutions. No sharp line can be drawn either between proteoses and peptones or between peptones and the simpler nitrogen compounds which result from prolonged digestion. As the terms are generally used, peptones may be considered the final products of digestion or hydrolysis which are still proteins as judged by the biuret reaction (see below) and are precipitable by tannin (perhaps not always completely) or by addition of strong alcohol. Proteoses (albumoses) are intermediate products between metaproteins (albuminates) and peptones. In addition to the protein reactions shown by peptones, the proteoses are 
precipitated from aqueous solutions at ordinary temperatures by adding acetic acid and potassium ferrocyanide or by saturating the solution with zinc or ammonium sulphate.

The term peptone was formerly applied to all digestion products not coagulated by boiling and is still popularly used in the same sense, the best commercial "peptones" consisting largely of proteoses.

Coagulated Proteins. - This group includes proteins which have been coagulated by heating or by alcohol. The nature of the change which takes place in coagulation is not known. The coagulated proteins are insoluble in water, alcohol, salt solutions, or very dilute acids. They are dissolved and converted into metaproteins by stronger acids and alkalies, especially on warming.

Glycoproteins. - Glyco- or gluco-proteins are compounds of proteins with carbohydrates. They are practically insoluble in water, but easily soluble in very weak alkalies. On boiling with dilute mineral acids they yield considerable amounts of reducing sugars. Mucins and mucoids belong to this group.

Nucleoproteins. - These are compounds of proteins with nuclein or nucleic acid. Nucleoproteins are found especially in cell nuclei and are therefore particularly abundant in the highly nucleated cells of secreting organs such as the liver, pancreas, etc., and in the germs of seeds.

While all of the simple proteins are levorotatory, the nucleoproteins thus far studied ${ }^{1}$ have shown dextrorotation and the nucleic acid of the wheat embryo has been found to be strongly dextrorotatory. ${ }^{2}$

Phosphoproteins. - Casein of milk and vitellin of egg-yolk are the important members of this group. Methods for the separation of casein from the other proteins of milk are included in the references beyond.

Other Nitrogen Compounds. - Among the organic nitrogen compounds which occur with proteins in many animal or vegetable substances are the lecithins and related compounds, alkaloids, and the so-called "nitrogenous extractives," including amines, amids, and amino-acids. Ammonium salts and nitrates

1 Gamgee and Jones : Am. J. Physiol., 1903, 8, 447.

2 Osborne : Ibid., 1903, 9, 69. 
may occur in plant and animal tissues, but usually only in minute amounts. They may, however, be added to food materials as preservatives. In most natural food products the total amount of these simpler nitrogen compounds is small as compared with that of proteins, and it has become customary in food analysis to take the total nitrogen as a measure of the proteins present, so that the percentage of proteins as reported ordinarily means the percentage of nitrogen multiplied by 6.25 , this factor being based on the assumption that proteins contain approximately 16 per cent of nitrogen.

\section{ANALYTICAL REACTIONS OF PROTEINS}

Color tests and precipitation reactions of fairly general application to proteins are known, but no one of these tests or reactions is given exclusively by proteins, so that as a rule in order to establish the presence of protein beyond doubt, as many as possible of the tests should be applied. Particularly in examining solutions by means of color tests, one should in important cases not only test the solution but also obtain the protein from the solution by precipitation and perform the color reaction upon this precipitate. For this purpose it is usually best to precipitate by heat coagulation where possible and in other cases to precipitate by rendering the solution slightly acid with acetic acid and then adding a solution of potassium ferrocyanide which under these conditions gives a fine white precipitate with most of the proteins though not with peptones nor with gelatin. The precipitate thus obtained gives color reactions like those of the protein itself. (Winternitz: Z. physiol. Chem., 1892, 16, 439.)

\section{Color Reactions}

\section{The Biuret Reaction ${ }^{1}$}

On adding a very dilute solution of copper sulphate drop by drop to a protein solution strongly alkaline with sodium or

${ }^{1}$ Rose: Poggendorff's Annalen, 28, 132 (1833). Piotrowski: Sitzber. Akad. d. Wiss. Wien, math.-naturw. Classe, 24, 335 (1875). Wiedemann: Poggendor:ff's Annalen, 74, 67 (1849). Pickering: J. Physiol., 14, 347 (1893). Schiff, Ber., 29, 298 ; 30, 2449 ; Liebig's Annalen, 299, 236 ; 303, 183 ; 307, 231 ; 310, 37, 301 ; 319, 300. 
potassium hydroxide a rose-red to violet coloration appears. A more pronounced rose color is obtained with proteoses and peptones than with other proteins. This test is said to show 1 part of peptone in 100,000 of solution. For other proteins it is less delicate. To test for peptones in a solution containing other proteins, ${ }^{1}$ precipitate the latter by saturation with zinc sulphate, filter, to the filtrate add caustic soda until the zinc hydroxide first precipitated is completely dissolved, then add a few drops of a one per cent solution of copper sulphate. The reaction is given by many substances other than proteins ; according to Schiff ${ }^{2}$ by any compound containing two $\mathrm{CONH}_{2}$ groups united either directly by their carbon atoms or by means of a third carbon or a nitrogen atom. Examples of these three classes of compounds are oxamid, malonamid, and biuret.

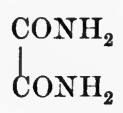

Oxamid

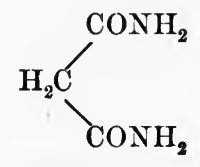

Malonamid

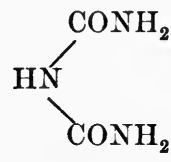

Biuret

For cases in which the biuret reaction is obtained when no - $\mathrm{CONH}_{2}$ groups appear to be present, as well as for a general discussion of the interpretation of the test in work upon di. gestion and synthetic products, see Mann's Chemistry of the Proteids, pp. 141-144.

According to Stokvis (Z. Biol., 1896, 34, 466) and Salkowski (Berl. klin. Wochenschr., 1897, No. 17) urobilin gives under the same treatment a color which cannot be distinguished from that of the biuret reaction.

Siegfried has also noted (Z. physiol. Chem., 35, 164) that the biuret reaction may be obscured by the presence of other bodies.

1 Neumeister : Z. Biol., 1890, 26, 324.

${ }^{2}$ Ber., 1896, 29, 298. 


\section{The Xanthoproteic Reaction ${ }^{1}$}

Proteins are colored yellow by nitric acid of 1.2 specific gravity or stronger. The color is intensified by heating and changes to orange or red on treatment with an excess of ammonia. According to Halliburton ${ }^{2}$ this reaction depends upon the presence of an oxybenzene nucleus.

Salkowski has proposed (Z. physiol. Chem., 12, 219; Vaubel, I, 381) to make use of this reaction for the approximate colorimetric estimation of peptones in solution.

\section{The Millon Reaction ${ }^{3}$}

On heating with Millon's reagent ${ }^{4}$ (a solution of mercuric nitrate containing nitrous acid) protein matter (including gelatin) gives a brick-red coloration which according to Nasse is also due to the presence of any oxybenzene nucleus in the protein molecule. For general description see Mann, p. 7, and for detailed discussions the papers of Vaubel ${ }^{5}$ and Nasse. ${ }^{6}$

\section{The Tryptophan Reaction 7}

When protein is treated first with glacial acetic acid and then with concentrated sulphuric a violet color usually appears. Hopkins and Cole ${ }^{8}$ found that the reaction occurred only when the acetic asid contained traces of glyoxylic acid and that a better reaction is obtained by mixing the protein with a little

${ }^{1}$ Fourcroy and Vauquelin : Ann. Chim., 56, 37. Fürth : Einwirkung von Salpetersäure auf Eiweisstoffe, Habilitationsschrift, Straussburg, 1899. Salkowski : Z. physiol. Chem., 12, 215 (1887). Rohde, Ibid., 44, 161 (1905).

${ }^{2}$ Schäfer's 'Textbook of Physiology, I, 47.

${ }^{3}$ Millon : Compt. rend., 28, 40 (1849).

${ }^{4}$ To prepare the reagent dissolve mercury in twice its weight of nitric acid, $1.42 \mathrm{sp}$. gr., and dilute the solution obtained with three times its volume of water. According to Nasse a better method is to use a solution of mercuric acetate containing a few milligrams of sodium or potassium nitrite, the solution having been recently acidulated with acetic acid.

5 Z. angew. Chem., 1900, 1125.

${ }^{6}$ Arch. ges. Physiol., (Pflüger), 1901, 83, 361.

${ }^{7}$ Adamkiewicz: Arch. ges. Physiol., 9, 156 (1874) and Ber., 8, 161 (1875).

${ }^{8}$ Proc. Royal Soc., 1901, 68, 21 ; J. Physiol., 27, 418 ; 29, 451. 
glyoxylic acid solution ${ }^{1}$ and afterward adding concentrated sulphuric acid. The color then appears at the line of contact of the liquids. This reaction, which is due to the presence of the tryptophan group in the protein molecule, is not given by gelatin. For other color reactions due to tryptophan, see Cole : J. Physiol., 1903, 30, 311.

Several other more or less characteristic color reactions of proteins may be found by consulting the references given at the end of the chapter.

\section{Protein Precipitants}

Heat coagulation and salting out processes have already been referred to in the general description of the proteins and have been made use of in classification.

Zinc sulphate is most used for salting-out in analytical work where the amount of protein precipitated is to be found by determining nitrogen in the precipitate.

Heavy metals have been considerably used as precipitants. Among these may be mentioned iron as chloride or acetate, copper as sulphate, acetate, or hydroxide, lead as neutral or basic acetate, mercury as chloride or as mercury-potassium-iodide, uranium as acetate.

Halogens form insoluble or sparingly soluble compounds with proteins, and bromine especially has been used as precipitant in analytical work.

Among the acids which are good precipitants for both proteins and alkaloids are phosphotungstic and phosphomolybdic acids, tannic acid, picric and picrolonic acids, trichlor-acetic acid.

Zinc sulphate. ${ }^{2}$ - In general on saturating a solution with zinc sulphate all proteins present except peptones are precipitated. This precipitation may therefore be used to separate peptones

1 Prepared as follows: Place a saturated solution of oxalic acid in a tall cylinder, add lumps of sodium amalgam (about 60 grams per liter of solution), allow to stand as long as hydrogen is evolved, then filter and dilute the solution with twice its volume of water.

${ }^{2}$ Bömer : Z. anal. Chem., 1895, 34, 562. Baumann and Bömer : Nahr.Zenussm., 1898, 1, 106. Zunz: Z. physiol. Chem., 1899, 27, 217. Van Slyke and Hart: Am. Chem. J., 1903, 29, 150. 
from other proteins. It is especially employed in the analysis of mixtures of proteins in connection with artificial digestion experiments. Here the proteins other than proteoses and peptones can usually be removed in other ways (for instance by neutralizing and heating the solution), the proteoses separated by saturating the solution with zinc sulphate, and the peptones determined in the filtrate.

- Ferric acetate is recommended by Allen ${ }^{1}$ as an efficient precipitant. A neutral solution of the reagent is added in excess and the liquid rapidly boiled, when all protein will be precipitated and can be determined from the amount of nitrogen found in the precipitate by the $\mathrm{Kjeldahl}$ method.

Copper ${ }^{2}$ is probably more generally used than any of the other heavy metals for the analytical precipitation of proteins, especially for the separation of protein from non-protein nitrogen in vegetable products. Moist cupric hydroxide (often called Stutzer's reagent) is most commonly used.

This method has been adopted by the Association of Official Agricultural Chemists in the following form:

Separation of Proteins from Amids, etc.

Preparation of Reagent. - Dissolve 100 grams of pure cupric sulphate in 5 liters of water, add 25 cc. of glycerol, and then a dilute solution of sodium hydroxide until the liquid is alkaline; filter; rub the precipitate up with water containing 5 cc. of glycerol per liter, and wash by decantation or filtration until the washings are no longer alkaline. Rub the precipitate up again in a mortar with water containing 10 per cent of glycerol, thus preparing a uniform gelatinous mass that can be measured out with a pipette. Determine the quantity of cupric hydroxide per cubic centimeter of this mixture.

Determination. - Place 0.7 gram of the substance in a beaker, add $100 \mathrm{cc}$. of water, heat to boiling, or, in the case of sub-

${ }^{1}$ Commercial Organic Analysis, IV (2), 38 (1898); and Vaubel: Bestimmung organischer Verbindungen, I, 227 (1902).

${ }^{2}$ Stutzer, J. Landw., 1880, 28, 103 ; 1881, 29, 473 ; Z. anal Chem., 1895, 34, 568. Mallet, U. S. Dept. Agriculture, Bur. Chem., Bul. 54. Fraps and Bizzell, J. Am. Chem. Soc., 22, 709. 
stances rich in starch, heat on the water bath ten minutes; add a quantity of cupric hydroxide mixture containing about 0.5 gram of the hydroxide, stir thoroughly, filter when cold, wash with cold water, and, without removing the precipitate from the filter, determine nitrogen by the Kjeldahl method, adding sufficient potassium sulphide solution to completely precipitate all copper and mercury. The filter papers used must be practically free from nitrogen. If the substance examined consists of seed of any kind, or residues of seeds, such as oil cake or anything else rich in alkaline phosphates, add a few cubic centimeters of a concentrated solution of alum just before adding the cupric hydroxide, and mix well by stirring. This serves to decompose the alkaline phosphates. If this be not done, cupric phosphate and free alkali may be formed, and the protein copper precipitate may be partially dissolved in the alkaline liquid.

This method has been considerably criticized, and to some extent replaced by precipitation with other reagents, especially phosphotungstic acid, but Fraps and Bizzell consider it still the best method available for vegetable materials. According to Schjerning's work it is likely to precipitate a considerable part of any purin body which may be present.

Lead $^{1}$ is an effective precipitant of proteins as shown by its successful use as a "clarifier" in preparing liquids containing sugar for polariscopic examination, for all proteins are optically active, and if not completely removed from the solution, would vitiate the polariscopic determination of sugar.

Mercuric chloride ${ }^{2}$ precipitates the proteins, including peptones, but is also liable to give precipitates with alkaloids and even with ammonium salts.

Uranium acetate precipitates proteins, and according to Schjerning's experiments ${ }^{3}$ does not precipitate the alkaloids or simpler nitrogen compounds likely to be met in analytical work, with the exception of piperazine.

${ }^{1}$ Hofmeister : Z. physiol. Chem., 1878, 2, 288.

${ }^{2}$ Kuhne : Z. Biol., 1885, 22, 423. Neumeister : Z. Biol., 1890, 26, 234. Siegfried: Z. physiol. Chem., 1902, 35, 164.

${ }^{3}$ Summarized by Vaubel: Bestimmung organischer Verbindungen, I, 224232. 
According to Schultz and Barbieri as quoted by Allen, the greatest source of error in the quantitative separation of proteins from simpler nitrogen compounds by means of reagents such as the above lies in the precipitation of more or less of the other nitrogen compounds along with the proteins. Hence they recommend that parallel precipitations be made with several different reagents. If then all the filtrates be proven free from protein by testing with acetic acid and potassium ferrocyanide, and the nitrogen be determined in each precipitate, the lowest result found is presumably the most nearly correct.

Bromine precipitation is sometimes employed for separation of proteins from simpler nitrogen compounds, such as the "extractives" of meat. The following example is from the methods of the Association of Official Agricultural Chemists:

Separation of Proteoses, Peptones, and Gelatin from Extractives

This is a combination of Bömer's ${ }^{1}$ method with that of Allen and Searle, ${ }^{2}$ as modified by Wiley. ${ }^{3}$

Evaporate the filtrate from the globulins to small volume, add 2 or 3 drops of $(1: 3)$ sulphuric acid, and saturate with powdered zinc sulphate. The excess of zine sulphate added should not be large, as otherwise serious "bumping" is likely to ensue. About 80 grams of the salt are required for each 50 cc. of liquid. Allow the coagulated proteins to settle, filter, and wash with a saturated solution of zinc sulphate.

Acidulate the filtrate from the zinc sulphate precipitate with 2 or 3 drops of strong hydrochloric acid, dilute with an equal volume of water, add about 2 cc. of liquid bromine, and shake the contents of the flask vigorously. (This can be most conveniently done in a Kjeldahl flask.) If the bromine be all taken up, add more until about 0.5 cc. of liquid bromine is left undissolved and the supernatant liquid thoroughly saturated. Allow the mixture to stand over night, decant the supernatant liquid through a filter paper, and wash with water, so directing the jet that the globule of bromine is stirred up and saturates the wash

\footnotetext{
1 Z. anal. Chem., 1895, 5, 562.

2 Analyst, 1897, 22, 258-263.

${ }^{8}$ U. S. Dept. Agriculture, Bur. Chem., Bul. 54.
} 
water. Return the filter paper and precipitate to the flask, add the zinc sulphate precipitate and filter paper containing it, and determine the nitrogen. The percentage of nitrogen so found, multiplied by 6.25 , gives the percentage of proteoses, peptones, and gelatin, including gelatin peptone.

Alkaloids, if present, are likely to be more or less completely precipitated by bromine along with the protein matter.

Phosphotungstic acid is an efficient precipitant of the proteins other than peptones. At ordinary temperatures simpler nitrogen compounds are also thrown down, but according to Mallet (Bul. 54, Div. Chem.) a satisfactory separation of ordinary proteins from the usual nitrogenous extractives, such as occur in meat extract, may be effected by precipitating at $90^{\circ}$ and washing with water at the same temperature. Fraps and Bizzell (J. Am. Chem. Soc., 22, 709) question the completeness of precipitation of proteins at temperatures above $60^{\circ}$. Alkaloids if present would be likely to be precipitated about as completely as the proteins.

Stutzer (Z. anal. Chem., 1895, 34, 568; 1896, 35, 493) and also Bondzynski (Landw. Jahrb. der Schweiz, 1894) used phosphotungstic acid as precipitant to separate proteins, including peptones, from amino-compounds. Van Slyke and Hart (Bul. 215, New York Agricultural Experiment Station, Geneva) consider that precipitation with this reagent in the cold gives a satisfactory separation of the proteins of cheese from monoamino compounds, but is liable to precipitate di-amino-compounds if these are present, arginin giving a precipitate with phosphotungstic acid which is soluble when hot, but separates out on cooling, while the lysin, histidin, and putrescine precipitates failed to redissolve completely even on boiling.

In using phosphotungstic acid for the determination of protein nitrogen in meat extract according to the suggestion of Mallet, the solution is first acidulated with acetic acid and boiled, filtered if necessary, and then treated with a slight excess of a solution of phosphotungstic acid in 2.5 per cent hydrochloric acid, heated to $90^{\circ}$, filtered hot, and the precipitate washed with water at about the same temperature. The re- 
sults thus obtained are usually slightly higher and somewhat more uniform than those obtained by precipitation with bromine.

Tannin ${ }^{1}$ precipitates proteins, including peptones, from solutions acidulated with acetic acid, the precipitation being favored by the presence of sodium chloride. According to Allen and also Halliburton, this precipitation, even in the case of peptones, is complete. Van Slyke and Hart, however, found that tannin precipitated much less of the -nitrogen compounds formed by peptic digestion of cheese than was precipitated by phosphotungstic acid; and after investigation they attributed this discrepancy mainly to incomplete precipitation of peptones by tannin. They concluded that phosphotungstic acid should be used to separate peptones from amino bodies when the amount of the latter is relatively small or when they consist largely of mono-amino-compounds ; while tannin should be preferred as a precipitant in those cases in which the amino-acids are present in relatively large amount as compared with peptones or when considerable amounts of di-amino-compounds are present.

Bigelow and Cook (J. Am. Chem. Soc., 1906, 28, 1485) as the result of a detailed study recommend, for the separation of proteoses and peptones from the amino bodies, the use of liberal amounts of tannin and salt at a temperature of approximately $12^{\circ} \mathrm{C}$. Their method is essentially as follows :

Dissolve 1 gram of the sample if a powder, 2 grams if a paste, or 10 grams if a concentrated solution, in a little cold water. in a 100-cc. flask, keeping the volume within $20 \mathrm{cc}$. [Or measure into the flask $20 \mathrm{cc}$. of the sample if a dilute solution.] Add $50 \mathrm{cc}$. of a solution containing 15 grams of sodium chloride, shake well, cool to approximately $12^{\circ}$, add $30 \mathrm{cc}$. of a 24 per cent tannin solution at the same temperature, dilute with cold water to $100 \mathrm{cc}$., shake thoroughly and allow to stand over night in an ice box at about $12^{\circ}$; then filter at the same temperature and determine nitrogen both in an aliquot part of

${ }^{1}$ Allen : Commercial Organic Analysis, Vol. IV (2d. ed.), p. 19. Schjerning : Z. anal. Chem., 1900, 39, 545. 
the filtrate and in a similar portion of the filtrate from a blank in which the reagents alone have been used. The corrected nitrogen of the filtrate represents that which was present as ammonia and amino compounds except that creatin if present is partly precipitated by the tannin-salt reagent. The amount of creatin thus thrown down may be found by estimating the amounts in the original solution and in the filtrate colorimetrically by the method of Folin, the excess of tannin being removed from the filtrate by means of barium.

Picric acid precipitates proteins very completely from solutions acidulated with acetic acid, but as the reagent contains nitrogen its use in quantitative analytical work is limited. Gelatin is precipitated when the picric acid is in excess, and milk or cream may be tested for gelatin by removing the milk proteins with acid mercuric nitrate and adding to the clear filtrate an excess of a saturated solution of picric acid, when gelatin, if present, yields a yellow precipitate.

Trichloracetic acid appears to be a convenient reagent for the separation of proteins under certain conditions, but has not been much used in analytical work. According to Halliburton ${ }^{1}$ the protein solution is mixed with an equal volume of a 10 per cent solution of trichloracetic acid, boiled and filtered hot, when the filtrate will contain the proteoses and peptones while the other proteins are precipitated. In the cold the proteoses are partially precipitated (Martin).

\section{Separation of Proteins from Simpler Nitrogen Com- POUNDS AND FROM EACH OTHER}

The separation of proteins from simpler nitrogen compounds is usually based upon the precipitation of the former by some one of the above reagents which will not precipitate the other nitrogen compounds believed to be present in the substance under examination. The choice of precipitant will therefore depend upon the nature of the substance from which the pro-

1 Schäfer's Physiology, I. 40-41, where the following references are given: Obermayer, Med. Jahrb., Wien, 1888, 375; Starling, J. Physiol., 14, 131 ; Martin, same, 15, 375; Halliburton, same, 17, 169, and J. Path. and Bact., 3, 295. See also Mendel and Blood, J. Biol. Chem., 8, 186, 189. 
tein is to be separated. Cupric hydroxide used as described above has been most commonly employed for vegetable materials, while precipitation with phosphotungstic acid, bromine or tannin and salt as described in the foregoing paragraphs has been more commonly used for animal substances. The methods of separating proteins from each other are based almost entirely upon differences in solubilities and coagulation or other precipitation reactions such as have been given in characterizing the groups already mentioned. As the reactions of the peptones approach those of some of the compounds of known structure, the separation of proteins from simpler compounds and from each other can best be studied as parts of the same problem. The methods available for these separations are so dependent upon the particular combination of compounds to be separated that any attempt to give detailed directions would be of less value than references to the original publications, a list of which will be found at the end of this chapter.

\section{PROTEASES OR PROTEOLYTIC ENZYMES}

For the determination of proteolytic powers of substances containing proteases (pepsin, trypsin, papain, etc.) many different methods have been used. Some of these may be grouped as follows :

1. The enzyme acts upon an insoluble protein and the rate at which the latter is digested into soluble products is observed (methods of Bidder and Schmidt, Grünhagen, Grützner, Mett, Palladin, U. S. Pharmacopœia).

2. The enzyme acts upon a solution or suspension of protein and the time required to carry the digestion to a definite stage, or the amount of protein remaining undigested at the end of a definite time, is determined (methods of Allen, Einhorn, Fuld and Levison, Gross, Jacoby-Solms, Robertson, Rose, Thomas and Weber, U. S. Pharmacopœia, Witte).

3. The enzyme acts upon protein or polypeptid, and the cleavage products are determined by chemical or physical methods (methods of Abderhalden, Allen, Hedin, Kober, Koelker, Levene and Rouiller, Schiff, Schütz, Sorenson, Volhard). 
4. The enzyme is allowed to act on a protein solution, and the progress of the digestion is measured by increase of electrical conductivity or decrease of turbidity or viscosity of the solution (methods of Bayliss, Hata, Liebermann, Spriggs).

Since the newer methods, such as the copper method of Kober and the optical method of Abderhalden and Koelker, are still being developed in detail, it seems best for the purposes of this work to describe fully only a few of the older methods which are now in more general use, after which will be given a chronological list of references which will guide the reader to the literature of the more recent methods.

\section{U. S. Pharmacoperia Method for Peptic Activity}

Mix 9 cc. of hydrochloric acid of 10 per cent strength by weight (1.049 sp. gr. at $25^{\circ} \mathrm{C}$.) with $291 \mathrm{cc}$. of distilled water and dissolve 0.100 gram of the pepsin to be tested in $150 \mathrm{cc}$. of the acid liquid. Immerse a hen's egg, which should be fresh, during 15 minutes in boiling water; remove the pellicle and all of the yolk; rub the white coagulated albumin through a clean No. 40 sieve. Reject the first portion that passes through the sieve, and place 10 grams of the succeeding portion in a widemouthed bottle of 100 cc. capacity. Add 20 cc. of the acid liquid and, with the aid of a glass rod tipped with cork or black rubber tubing, completely disintegrate the albumin; then rinse the rod with $15 \mathrm{cc}$. more of the acid liquid and add $5 \mathrm{cc}$. of the solution of pepsin. Cork the bottle securely, invert it three times, and place it in a water bath that has previously been regulated to maintain a temperature of $52^{\circ} \mathrm{C}$. Keep at this temperature for $2 \frac{1}{2}$ hours, shaking every 10 minutes by inverting the bottle once. Then remove it from the water bath, add 50 cc. of cold distilled water, transfer the mixture to a narrow graduated cylinder, and allow it to stand for half an hour. The deposit of undissolved albumin should not then measure more than $1 \mathrm{cc}$.

The relative proteolytic power of pepsin stronger or weaker than that just described may be determined by ascertaining through repeated trials the quantity of the above pepsin solu- 
tion required to digest, under the prescribed conditions, 10 grams of boiled and disintegrated egg albumin. Divide 15,000 by this quantity expressed in cubic centimeters to ascertain how many parts of egg albumin one part of pepsin will digest.

\section{U. S. Pharmacopgeia Test for Tryptic Activity of Pancreatin}

If 0.28 gram of pancreatin and 1.5 grams of sodium bicarbonate be added to $100 \mathrm{cc}$. of tepid water contained in a flask, and if $400 \mathrm{cc}$. of fresh cows' milk, which has been previously heated to $38^{\circ} \mathrm{C}$., be then added, and the temperature of the mixture maintained at this point for 30 minutes, the milk should be so completely peptonized that, if a small portion of it be transferred to a test tube and mixed with some nitric acid, no coagulation should occur.

If it be desired to compare the powers of two preparations, they may be tested side by side as above described and portions of each withdrawn and acidulated at frequent intervals. The rapidity with which the end point is reached will then give an indication of the comparative proteolytic power.

\section{Mett Method}

This method consists in allowing a solution of the proteolytic enzyme to act upon the ends of a column of coagulated egg albumin contained in a narrow glass tube and observing the rate at which the column is shortened by the digesting of the coagulum. While this cannot be considered a strictly quantitative method, it has obvious advantages as a means of demonstrating the presence or absence of proteolytic enzyme or any pronounced difference in proteolytic power between substances which it is desired to compare.

For this method obtain the white of a fresh egg, cut it thoroughly with scissors or stir it with an equal volume of water, and filter or strain through muslin or cheesecloth. The albumin thus prepared should be homogeneous, nearly clear, and entirely free from air bubbles. Fill some clean, dry capillary glass tubes of 1 to $2 \mathrm{~mm}$. diameter with the prepared albumin. 
This may be done either by drawing up the fluid into the tube as into a pipette or by lowering the tube into a column of the fluid. Great care must be taken to avoid air bubbles in filling the tubes. In order to coagulate uniformly the contents of a capillary tube, hold it (after filling with albumin) in the same manner as a pipette and with the finger over the upper end touch the lower end to the surface of the boiling water in a water bath until coagulation begins, then lay the tube horizontally in the boiling water; after 15 minutes immersion in boiling water, allow the tube of coagulated albumin to cool slowly, preferably under water, then remove and if the tube is not to be used at once, seal the ends by means of sealing wax or paraffin. For use cut the tube of coagulated albumin into sections about $2 \mathrm{~cm}$. in length (being careful to scratch the tube at right angles to its axis and to break it with all possible care to secure clean-cut square ends). Reject the end sections and any which contain air bubbles or in which the albumin has shrunken and does not completely fill the capillary. Immerse a perfect section of "Mett tube" thus prepared and selected in a measured volume of the liquid to be tested, with "blanks" alongside in which all of the conditions are the same except that the enzyme is omitted, and note the length of column digested out of the tube after standing 10 hours at $38^{\circ} \mathrm{C}$. or for such time and at such temperature as may best meet the requirements of the particular case.

When the test is applied to a liquid (e.g. to gastric juice) an equal volume of the same liquid, boiled to destroy the enzyme, may be used for the blank test.

In testing a solid, it should be dissolved in an acid, alkaline, or neutral medium according to the nature of the enzyme supposed to be present. For pepsin or trypsin the acidity or alkalinity prescribed respectively in the above methods from the U. S. Pharmácopœia may be used, and comparisons may be made with a sample of pepsin or trypsin whose power is known to approximate the requirement of the Pharmacopœia test.

See also the methods of expressing proteolytic power given by Rose and others in the journal articles cited below. 


\section{Allen Method Modified}

Dissolve 1 gram of dry powdered egg albumin in 20 cc. of lukewarm water in a 100-cc. flask, heat in a boiling water bath to coagulate the albumin (Note 1), and cool to $40^{\circ} \mathrm{C}$., add 0.1 gram of the sample of pepsin to be tested and 25 cc. of tenthnormal hydrochloric acid (Note 2) ; warm to $40^{\circ} \mathrm{C}$., and maintain at this temperature for 3 hours (Note 3 ); then add a volume of tenth-normal sodium carbonate solution exactly equivalent to the acid previously added (Note 4), mix thoroughly by shaking, and place the flask in a water bath at $90^{\circ} \mathrm{C}$. for 10 minutes to destroy the enzyme and coagulate any metaprotein (syntonin) which may have been dissolved by the acid and liberated when the latter was neutralized by the alkali. Cool, dilute with water to $100 \mathrm{cc}$, and filter through a dry paper, protecting the solution from unnecessary exposure to air so that it shall not change by evaporation. This filtrate (A) contains the proteoses, peptones, peptids and amino-acids which have been formed by digestive hydrolysis of the protein plus what existed in the pepsin added. Measure 25 cc. of filtrate A into a Kjeldahl flask and determine total nitrogen. To 50 cc. of filtrate A (representing one half of the solution), add powdered zinc sulphate in the cold with thorough stirring until the solution is saturated, allow to stand with occasional stirring for half an hour, filter through pure filter paper, and wash the precipitate with a saturated solution of zine sulphate. Transfer the precipitate with the paper to a Kjeldahl flask and determine nitrogen as a measure of the proteoses; acidulate the filtrate (B) with hydrochloric acid and precipitate with bromine as described above under determination of proteins by bromine precipitation. The nitrogen of the bromine precipitate serves as a measure of the amount of peptones ; the filtrate from the bromine precipitate (filtrate $\mathrm{C}$ ) contains the aminoacids and any peptids not precipitated by bromine. The amount of nitrogen in these forms may be found by difference. From the total nitrogen of proteoses, peptones, peptids, and amino-acids as found from an aliquot portion of filtrate $\mathbf{A}$ 
above, subtract the nitrogen of proteoses (zinc sulphate precipitate) and of peptones (bromine precipitate) ; the remainder is the nitrogen of peptids and amino-acids.

A blank test should be conducted, using a boiled solution of the pepsin, but in all other respects exactly as above described. Such a blank test will supply the combined corrections for the nitrogen of the pepsin and for the cleavage products which may possibly be produced by the action of the hydrochloric acid on the coagulated albumin.

For data illustrating the application of this method see Allen's Commercial Organic Analysis, Volume IV (2d ed.), pp. 357358.

Note 1. - The coagulated albumin should be in the form of finely divided flocks so as to be readily accessible to the enzyme. So far as the action of the enzyme on the albumin is concerned, it would seem better to leave the latter uncoagulated; but when this is done there may be difficulty in filtration at the end of the digestion period.

Note 2. - The addition of acid at this point is intended to give the reaction most favorable for the pepsin activity. If the enzyme under test works best in a neutral solution, the acid may be omitted; or in the case of trypsin, sodium carbonate may be added at this point to give the favorable degree of alkalinity.

Note 3. - While three hours is a suitable time for digestion in the case of commercial pepsin such as Allen was engaged in testing, it is obvious that a longer or a shorter period of digestion may be adopted when working with substances of much lower or much higher proteolytic power.

Note 4. - Compare Note 2. If no acid was added at the beginning of the digestion, no alkali should be added here. If alkali was added at the beginning of the digestion, an equivalent amount of acid must be added at the end. The object here is to restore the neutrality of the solution so that the subsequent heating and filtration will remove any dissolved but undigested protein along with the undissolved coagulated albumin. 


\section{OTHER Methods}

As indicated above, many other methods, some of them very promising, have been proposed for the measurement of proteolytic power. In research work especially, the various methods available should be carefully studied with a view to the selection of the one best adapted to the particular problem in hand. The references given at the end of this chapter will put the reader in touch with the literature of the subject.

\section{REFERENCES}

Abnerhalden: Biochemisches Handlexicon.

—_ : Handbuch der Biochemischen Arbeitsmethoden.

: Lehrbuch der Physiologische Chemie.

Allen: Commercial Organic Analysis.

Connherm: Chemie der Eiweisskörper.

Fisciner: Untersuchungen über Aminosäuren, Polypeptide, und Proteine. Frankel: Descriptive Biochemie.

Hammarsten: Physiological Chemistry (translated by Mandel).

Hawk: Practical Physiological Chemistry.

KöNıg: Chemie der Menschliche Nahrungs- und Genussmittel.

Manv: Chemistry of the Proteids.

Neuberg: Der Harn und Körperflussigkeiten.

Oppenheimer: Die Fermente und ihre Wirkung.

: Handbuch der Biochemie.

Osborne: Proteins of the Wheat Kernel.

: The Vegetable Proteins.

Plimmer: The Chemical Constitution of the Proteins.

Schaefer: Text-book of Physiology.

Sherman: Chemistry of Food and Nutrition.

II

\section{On Proteins}

(See also the references given in the text)

1880-86. Stutzer: Untersuchungen ueber die quantitative Bestimmung des Proteinstickstoffes und die Trennung der Proteinstoffe von anderen in Pflanzen vorkommenden Stickstoffverbindungen. Journ. f. Landwirthschaft, 28, 103; 29, 473 ; 34, 151. 
Schulze und Barbieri: Zur Bestimmung des Eiweisstoffe und der nichteiweissartigen Stickstoffverbiudungen in der Pflanzen. Landw. Vers. Stat., 26, 218.

1896. Teller: The Quantitative Separation of Wheat Proteids. Bul. 42, Ark. Agl. Expt. Sta., p. 81.

1897. Allen and Searle: Improved Method of determining Proteid and Gelatinoid Substances. Analyst, 22, 258.

1898. Baumann und Bömer: Ueber die Fallung der Albumosen durch Zinksulfat. Z. Nahr.-Genussm., 1, 106.

1899. Mallet : Analytical Methods for distinguishing between the Nitrogen of Proteids and that of the Simpler Amids or Amino-acils. Bul. 54, Div. Chem., U. S. Dept. Agriculture; Chem. News, 80, $117,168,179$.

Vivian: A Comparison of Reagents for Milk Proteids. 16th Ann. Rpt. Wis. Agricl. Expt. Sta., p. 179.

1900. Barnstein: Ueber eine Modifikation des von Ritthausen vorgeschlagenen Verfahrens der Eiweissbestimmung. Landw. Vers. Stat., 54, 327; Z. Nahr.-Genussm., 1901, 4, 688.

1894-1901. Schuerning : [A series of papers on the quantitative separation and precipitation of proteins]. $Z$. anal. Chem., 33, 263; 34, $135 ; 35,285 ; 36,643 ; 37,73,413 ; 39,545,633$.

1900. Fraps and Bizzell: Methods of determining Protein Nitrogen in Vegetable Matter. J. Am. Chem. Soc., 22, 709.

1901. Hart : Ueber die Quantitative Bestimmung der Spaltungsprodukte von Eiweisskörpern. Z. physiol. Chem., 33, 347.

1903. Тевв: The Precipitation of Proteins by Alcohol and Certain Other Reagents. J. Physiol., 30, 25.

VAN SLYKE and HART: Methods for the Estimation of the Proteolytic Compounds contained in Cheese and Milk. Bul. 215, New York Agl. Expt. Sta. ; Am. Chem. J., 29, 150.

Bigklow : Meat and Meat Products, U. S. Dept. Agriculture, Bur. Chem., Bul. 65, pp. 10, 17; Bul. 13, Part 10, p. 1396 ; Bul. 81, p. 104.

1904. Grindley: A Study of the Nitrogenous Constituents of Meats, U. S. Dept. Agriculture, Bur. Chem., Bul. 81, p. 110; J. Am. Chem. Soc., 26, 1086.

Haslam : Separation of Proteins. J. Physiol., 32, 267.

SNyder: The Determination of Gliadin in Wheat Flour by means of the Polariscope. J. Am. Chem. Soc., 26, 263.

1905. Chamberlain: Determination of Gliadin and Glutenin in Flour.

U. S. Dept. Agriculture, Bur. Chem., Bul. 81, p. 118; Circular 20, p. 14 .

Grindery and Enmett: The Chemistry of Flesh. J. Am. Chem. Soc., 27, 658 . 
1906. Bigelow and Соок: The Separation of Proteoses and Peptones from the Simpler Amino Bodies. J. Am. Chem. Soc., 28, 1485.

Chamberlain: Investigations on Properties of Wheat Proteins. J. Am. Chem. Soc., 28, 1657.

Mathewson: The Optical Rotation of Gliadin in Certain Organic Solvents. 'J. Am. Chem. Soc., 28, 1482.

1907. Acree: A Formaldehyde Color Test for Proteins. Am. Chem. J., 37, 604 .

Cook and Trescotr: A Modification of the Tannin-Salt Method. J. Am. Chem. Soc., 29, 605 .

1908. Bardach: A New Protein Reaction. Z. physiol. Chem., 54, 355.

Liebermand: (Formaldehyde Color Reaction for Protein). $Z$. Nahr.-Genussm., 16, 231.

Seamax and Gies: An Examination of Bardach's New Protein Test. Proc. Soc. Exp. Biol. Med., 5, 125; Chem. Abs., $2,2829$.

1910. Osbonve: Die Pflanzenproteine. Ergebnisse der Physiologie, 10, $47-215$.

WEYL : (Precipitation of proteins and some amino-acids by acetone).

$Z$. physiol. Chem., 65, 246. Also Ber., 43, 508.

1911. Mıско: (Examination of Meat Juices). Z. Nahr.-Genussm., 20, 537. Osborne and Guest: (Analysis of the Products of Hydrolysis of Proteins). J. Biol. Chem., 9, 333, 425.

VAN Slyke: The Analysis of Proteins by Determination of the Chemical Groups Characteristic of the Different Amino-acids. J. Biol. Chem., 10, 15.

\section{On Proteases}

1881. Roberts: Estimation of the Amylolytic and Proteolytic Activity of Pancreatic Extracts. Proc. Royal Soc., 32, 145.

1897. Allen: (Valuation of Pepsin). Pharm. J., 1897, 561; Z. anal. Chem., 42, 466.

1901. KrüGer: Quantitative Observations on Pepsin Action. Z. Biol., 1901, 41, 467; J. Chem. Soc., 1902, 82, ii, 33.

SAmoJloff: Mett's Method of estimating Peptic Activity. Arch. ges. Physiol. (Pflüger), 85, 86; J. Chem. Soc., 1901, 80, ii, 401.

Thomas and Weber: Quantitative Determination of Proteolytic Power. Centrbl. f. Stoffwechselversuche und Verdauungskrankheiten, 901, 2, 365; Z. Nahr.-Genussm., 1902, 5, 723.

1902. Peckelharing : On Pepsin. Z. physiol. Chem., 35, 8.

Spriggs: New Method of Determining Pepsin Activity. Z. physiol. Chem., 35, 465.

1903. Winogradow: Quantitative Experiments with Peptic Digestion. Z. Nahr.-Genussm., 6, 589. 
1905. Совв : Contribution to our Knowledge of the Action of Pepsin, with Special Reference to its Quantitative Estimation. Am. J. Physiol., 13, 448.

Loenleiv: Volhard's Titrimetric Method for Estimation of Pepsin and Trypsin. Beitr. chem. Physiol. Path., 7, 120.

1906. Levene and Rouiller: Estimation of Tryptophan in Protein Cleavage Products. J. Biol. Chem., 2, 481.

Robertson: (Hydrolysis of Casein by Trypsin). J. Biol. Chem., 2, 318.

1907. Abderhalden and Koelker : Employment of Optically Active Polypeptids as Proof of the Activity of Proteolytic Enzymes. $Z$. physiol. Chem., 51, 294.

Kütrner: The Volhard Method for Estimation of Pepsin. $Z$. physiol. Chem., 52, 63.

1908. Arrhenius: Law of Schütz and Reaction Velocities. Medd. Vetenskapsakademiems Nobelinst., 1, No. 9; Chem. Abs., 2, 2589.

EinhorN: Simplification of Jacoby-Solm Ricin Method of Pepsin Estimation. Berlin. klin. Woch., 45, 1567.

Fuld and Levison: Determination of Pepsin by Means of Edestin Test. Biochem. Z., 6, 473 .

Goodman: Ricin Method of Jacoby-Solms for the Quantitative Estimation of Pepsin. Am. J. Med. Sci., 136, 734.

Gross: Activity of Trypsin and a Simple Method for its Estimation. Arch. exp. Path. Pharm., 58, 157 ; Chem. Abs., 2, 1570.

MeYer: Is the Schütz Law for Peptic Digestion Invalid? Berlin. klin. Woch., 45, 1485.

Sorensen : (Estimation of Proteolytic Power by Titration of Aminoacids). Biochem. Z., 7, 45; Chem. Abs., 2, 1288.

Witte: (Modification of Jacoby-Solms Method). Berlin. klin.Woch., 1908, p. 643.

1909. Berg: Comparative Study of Digestibility of Different Proteins in Pepsin-Hydrochloric-Acid Solutions. Am. J. Physiol., 23, 420.

Dezani : Contribution to the Study of Pepsin. Atti della R. Accad. della Scienze di Torino, 45, 225.

Liebermane: New Method for Clinical Determination of Pepsin. Med. Klin., 1909, 1784.

1910. Blood: The Erepsin of the Cabbage (Brassica oleracea.) J. Biol. Chem., 8, 215.

Frank : Digestibility of White of Egg as Influenced by the Temperature at which it is Coagulated. J. Biol. Chem., 9, 463.

Hata : Estimation of Pepsin by the Clearing of Turbid Solutions of Egg Albumin. Biochem. $Z$., 23, 179.

Koelker: The Study of Enzymes by Means of Synthetical Polypeptids. J. Biol. Chem., 8, 145. 
Mendel and Blood: Some Peculiarities of the Proteolytic Activity of Papain. J. Biol. Chem., 8, 177.

Palladin: (Trypsin Activity and its Determination). Arch. ges. Physiol. (Pflüger), 134, 337; Chem. Abs., 5, 1615.

Rose: Modified Method for Clinical Estimation of Pepsin. Arch. Intern. Med., 5, 459; Chem. Abs., 4, 1980.

1911. Abderhalden and Steinbeck: Study of the Action of Pepsin. $Z$. physiol. Chem., 68, 293.

Amberg and Jones: On the Application of the Optical Method to a Study of the Enzymatic Decomposition of the Nucleic Acids. J. Biol. Chem., 10, 81.

Graber: Some Observations upon the Assay of Digestive Ferments. J. Ind. Eng. Chem., 3, 919.

Kober: A Method for the Study of Proteolytic Ferments. J. Biol. Chem., 10, 9.

Ramsay: Method of Determining the Tryptic Value of Pancreatin. J. Ind. Eng. Chem., 3, 822. 


\section{CHAPTER XVI}

\section{Grain Products}

In the routine analysis of vegetable foods and feeding-stuffs it is customary to determine moisture, fat, protein, fiber, and ash and to estimate the remaining substances as "carbohydrates by difference" or as "nitrogen-free extract." Often the separate determination of fiber is omitted, and this, as well as the sugars, starches, pentosans, etc., is included in the "carbohydrates by difference."

The present chapter will include the methods for such determinations, which are of fairly general application in food analysis, and also some special methods for the examination of grain products in particular.

\section{Preparation of Samples}

Samples for analysis should, if moist, be weighed into large flat-bottom dishes, dried until brittle (though not necessarily quite to constant weight) at a temperature not above that of the water oven, then allowed to stand exposed to the air until they neither gain or lose in weight, and the weight of the air-dry material recorded in percentage of the weight of the fresh substance. The air-dry material is then ground in a coffee or drug mill ${ }^{1}$ until all will pass through a sieve of one-half-millimeter mesh, or, if this is not feasible, through a sieve having round holes one millimeter in diameter.

Fineness of grinding is important, not only to secure sufficiently accurate portions for the separate determinations, but also to permit of efficient extraction in the determinations described below.

${ }^{1}$ In laboratories where many such samples are to be ground a special power mill is sometimes provided. 


\section{Determination of Moisture and Fat}

Dry 2 grams for 5 hours, or to constant weight, at the temperature of boiling water, if possible in a current of dry hydrogen or in vacuo. Consider the loss of weight as moisture. Extract the dried sample in a Soxhlet or continuous extractor, with anhydrous alcohol-free ether for sixteen hours. Dry the extract to constant weight in a boiling water oven. The ether extraction should be carried out at a distance from any free flame, the flask being heated by a safety water bath or, more conveniently, by an electric heater.

Notes. - The ether extract of vegetable substances often contains in addition to fat more or less of coloring matters and resinous substances, these being more readily soluble in ether containing fatty oils than in ether alone. In the cereal grains and especially in the milling products from which the outer layers of the grains have been separated the amount of such impurities is usually negligible. If the extract is made to percolate a layer of animal charcoal, practically pure fat is obtained. ${ }^{1}$

The reason for drying in a current of hydrogen rather than in air is that the oils of the cereal grains belong to the "semidrying" group and therefore absorb oxygen when exposed to air, especially at high temperature. This will of course increase the weight of the fat and make the apparent percentage of moisture too low. The partially oxidized oils are also apt to be incompletely extracted by ether. For a full discussion of the determination of water in foods and physiological preparations, see Benedict and Manning: Am. J. Physiol., 1905, 13, 309.

Fat may also fail of complete extraction, even when unchanged, by being occluded or mechanically inclosed in carbohydrate or protein material which is impervious to the ether.

Although ether extracts may be evaporated at the temperature of boiling water without loss of fat, such loss has been

1 Patterson: Am. Chem. J., 12, 261. 
found to occur in drying moist samples even in a current of hydrogen. That the loss in such cases is, in part at least, due to an actual volatilization of material may be shown by passing the current of hydrogen in which the sample is dried into strong sulphuric acid. This loss is probably due to the action of the escaping steam and may be practically avoided by drying at a lower temperature, preferable in a partial vacuum.

All three of the causes just mentioned tend toward a deficiency of fat in the analysis of cooked foods prepared from cereal products. Thus in a number of experiments on bread making ${ }^{1}$ the fat found by analysis of the dried bread was less than half of that contained in the materials used, and the iodine figure of the ether extract of the bread was only 60.4 as against 101.4 in that of the original flour, showing that a very considerable oxidation had taken place even in that portion of the fat which was still soluble in ether.

Berntrop's method for the determination of fat in breadstuffs is as follows: ${ }^{2}$ Mix 150 grams of fresh bread with 500 cc. of water, add $100 \mathrm{cc}$. of concentrated hydrochloric acid, and boil for two hours connected with a reflux condenser. ${ }^{3}$ Cool the resulting brown liquid to room temperature, filter with suction through a moistened fat-free paper, and wash with cold water. Dry the paper and residue for an hour at $100^{\circ}$ to $110^{\circ}$, remove the residue as completely as possible from the filter paper, and grind it with sand in a mortar. Cut up and add the filter paper, and transfer the dry mixture to a paper extraction thimble and treat with ether or petroleum ether in an extractor.

Dormeyer's method, ${ }^{4}$ designed originally for the determination of fat in animal tissues, has been adapted to vegetable foods by Beger. ${ }^{5}$ From 3 to 5 grams of substance are mixed with 480 cc. of water, 20 cc. of 25 per cent hy-

${ }^{1}$ Bul. 67, Office of Experiment Stations, U. S. Dept. Agriculture.

${ }^{2} Z$. angew. Chem., 1902, 121.

${ }^{3}$ In treating meal or flour, heat for an hour in a water bath and then boil fcr an hour with the reflux condenser attached.

${ }^{4}$ Arch. ges. Physiol. (Pflüger), 1895, 61, $341 ; 1896,65,90$.

¿ Chem. Ztg., 1902, 26, 112. 
drochloric acid, and 1 gram of fat-free pepsin. The mixture is kept at $37^{\circ}$ to $40^{\circ}$ for twenty-four hours, filtered with suction through a paper supported on a porcelain plate and covered with asbestos, and both the filtrate and the residue extracted with ether.

\section{Determination of Crude Fiber ${ }^{1}$}

Extract 2 grams of the substance with ordinary ether, or use the residue from the determination of the ether extract. To this residue, in a 500 -cc. flask, add 200 cc. of boiling 1.25 per cent sulphuric acid; connect the flask with an inverted condenser, the tube of which passes only a short distance beyond the rubber stopper into the flask. Boil at once, and continue the boiling for 30 minutes. A blast of air conducted into the flask may serve to reduce the frothing of the liquid. Filter, wash with boiling water till the washings are no longer acid; rinse the substance back into the same flask with $200 \mathrm{cc}$. of a boiling 1.25 per cent solution of sodium hydroxide, practically free from sodium carbonate; boil at once, and continue the boiling for 30 minutes in the same manner as directed above for the treatment with acid. Filter on a Gooch crucible, and wash with boiling water till the washings are neutral; dry at $110^{\circ}$; weigh; incinerate completely. The loss of weight is crude fiber.

The filter used for the first filtration may be linen, one of the forms of glass wool or asbestos filters, or any other form that secures clear and reasomably rapid filtration. The solutions of sulphuric acid and sodium hydroxide are to be made up of the specified strength, determined accurately by titration and not merely from specific gravity.

\section{Determination of Ash}

Char about 2 grams and burn to whiteness at the lowest possible red heat, preferably in a flat-bottomed platinum dish in a muffle.

1 Bul. 107, Revised, Bur. Chem., U. S. Dept. Agriculture. See also Thatcher : J. Am. Chem. Soc., 1902, 24, 1210 ; Browne : Ibid., 1904, 25, 315. 
If considerable quantities of phosphates are present, these may fuse over some of the carbon and render its combustion very slow. In such cases, extract the charred mass with a little hot acetic acid, set aside the solution till the char is burned, then evaporate it to dryness in the same dish and heat the residue to dull redness till the ash is white or nearly so. Samples containing added salt should be extracted with water before charring, and the determination finished as just described.

\section{Determination of Protein}

Determine total nitrogen by one of the modifications of the Kjeldahl method as described in Chapter XIV.

On the assumption that proteins in general contain approximately 16 per cent of nitrogen, it has been customary to multiply the percentage of nitrogen by 6.25 as an estimate of the percentage of protein.

The extended investigations by Osborne and his associates, ${ }^{1}$ and by Ritthausen, ${ }^{2}$ have shown that nearly all the cereal proteins contain over 16 per cent of nitrogen, so that the results obtained by multiplying the nitrogen by 6.25 are too high. The factors now regarded as most nearly correct are: for wheat, rye, and barley, $5.7^{3}$; for maize, oats, rice, and buckwheat, 6.00. The old factor 6.25 is, however, still frequently used for the sake of uniformity or for comparison with earlier work. In reporting results, therefore, the factor used should always be given.

\section{Separation of Wheat Proteins}

Nearly all the nitrogenous material in wheat, and especially in wheat flour, is in the form of proteins.

The proteins of wheat have been studied with great thorough-

${ }^{1}$ Reports Conn. Agl. Expt. Station, 1890 et seq. Much of the work has also appeared in J. Am. Chem. Soc., Am. Chem. J., Am. J. Physiol., or J. Biol. Chem.

2 Summarized in Landw. Vers. Stat., 1896, 47, 391.

3 The factor 5.68 has recently been proposed for wheat flour. 
ness by Osborne, who finds five forms and estimates the amount of each in average wheat as follows:

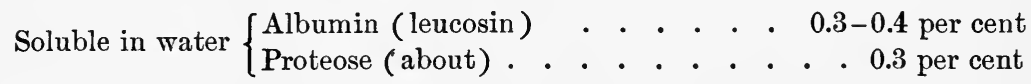

Soluble in ten per cent $\mathrm{NaCl}-$ Globulin (edestin) . . 0.6-0.7 per cent Soluble in 70 per cent alcohol_Gliadin (about). . . . . 4.25 per cent Insoluble in neutral solvents_Glutenin . . . . . 4.0-4.5 per cent

In fine flour the relative amount of gliadin is higher than in the whole grain, from one half to three fifths of the total nitrogen of fine flour being usually in the form of alcohol-soluble protein.

The gliadin and glutenin together make up the gluten, which to the bread maker is of greater importance than the other proteins of the flour. In addition to the percentage of gluten in the flour the proportion of gliadin in the gluten is important to its baking qualities.

Hence the protein separations of direct importance in establishing the commercial value of the flour are (1) to separate the water- and salt-soluble proteins from the gluten, (2) to determine the alcohol-soluble protein or gliadin. Knowing the amounts of these and the total protein present, the amount of glutenin may be found by difference.

On the basis of Osborne's studies, Chamberlain has developed the following methods for the routine determination of alcoholsoluble and salt-soluble proteins.

Determination of alcohol-soluble protein ("crude gliadin"). Treat 5 grams of the sample with $250 \mathrm{cc}$. of alcohol, 70 per cent by volume, for 24 hours, shaking every half hour during the first 8 hours; filter through a dry paper, determine nitrogen in 100 cc. of the filtrate, and multiply the result by 5.7 .

In making this determination of nitrogen the $100 \mathrm{cc}$. of solution may be transferred to a Kjeldahl flask, 3 cc. of sulphuric acid added, and the liquid boiled down to a small volume, after which the remainder of the acid is added and the determination completed as usual. This determination will usually give a result very slightly higher than the true amount of gliadin, since any amino-acids or amids present are likely to be dissolved by the alcohol. 
Determination of salt-soluble protein. - Treat 12 grams of the sample with 300 cc. of 5 per cent potassium sulphate as described under the determination of alcohol-soluble protein. Determine nitrogen in $100 \mathrm{cc}$. of the filtrate and multiply by 5.7 to estimate the salt-soluble proteins.

\section{Separation of Carbohydrates in Cereal Products}

Determination of Reducing Sugars, Sucrose, Dextrin, Starch, Pentosans, and Cellulose

The following scheme ${ }^{1}$ provides for each of the substances or groups mentioned and avoids the danger (inherent in any plan of making a number of independent determinations) of including the same substance as a constituent of more than one group.

Free the sample from fat by washing with ether.

Extract with boiling alcohol.

Solution A. - Evaporate the alcohol, dilute with water, and determine the reducing power of portions of the solution before and after hydrolysis. Calculate the reducing sugar and sucrose.

Residue A. - Extract with cold water.

Solution $B$. - Hydrolyze a portion and determine the resulting dextrose, calculate dextrin and soluble starch. In another portion, precipitate soluble starch by barium hydroxide, ${ }^{2}$ filter, and determine dextrin in the filtrate.

Residue B. - Boil with water and treat with malt extract or saliva, filter, and wash thoroughly.

Solution C. - Hydrolyze, determine resulting dextrose, and calculate starch.

Residue C. - Boil with 2 per cent hydrochloric or sulphuric acid until the maximum reducing power of the solution is reached. ${ }^{3}$

Solution D. - Determine reducing power in the same manner as for dextrose. Calculate as xylose, the reducing power of

1 Based on the following papers : Stone : J. Am. Chem. Soc., 1897, 19, 183. Sherman : Ibid., 1897, 19, 291. Browne and Beistle : Ibid., 1901, 23, 229.

2 Asboth: Chem. Ztg., 1889, 13, 591.

${ }^{3}$ For sulphuric acid this was found to be 4 to 6 hours. Stone prefers hydrochloric acid and states that the reaction is nearly complete in 1 hour. 
which is 1.03 times that of dextrose. ${ }^{1}$ From the pentose thus found calculate the percentage of pentosan. In the case of wheat it has been found 2 that the material ("hemicellulose") dissolved and hydrolyzed at this point is entirely pentosan. The same is probably true of the other cereals. The pentosan thus dissolved and hydrolyzed does not include necessarily the entire furfural-yielding substance of the cereal.

Residue D. - Boil for 30 minutes with 1 per cent sodium hydroxide, filter and wash, press out most of the water, and expose the moist fiber to chlorine gas for one hour. Wash with water, boil with a solution containing 2 per cent sodium sulphite and 0.2 per cent sodium hydroxide; filter, wash with warm water until the washings are neutral and colorless, then wash with strong alcohol, dry, and weigh. Deduct the ash which the residue contains and calculate the organic matter as cellulose. ${ }^{3}$

\section{Determination of Maltose, Dextrin, and Starch in Malted Cereal}

The absence of any considerable amount of dextrose or invert sugar must be shown by stirring some of the sample with about ten times its weight of water and testing the filtered extract by means of phenylhydrazine or Barfoed's solution as described in Chapter III. If no monosaccharide is present, the percentages of maltose, dextrin, and starch can be estimated as follows :

Mix 5 grams of sample with 125 cc. of cold water 4 in a 250 -cc. flask; allow to stand at room temperature for one hour, shaking frequently ; fill to the mark, shake, and filter through dry paper. Determine reducing power of one or more 25-cc. portions of

${ }^{1}$ Stone : Am. Chem. $J ., 1891$, 13, 82. Since the reducing power of arabinose does not differ greatly from that of xylose, this calculation would still be-nearly correct in case both pentoses were present.

2 J. Am. Chem. Soc., 1897, 19, 294.

3 This is the method of Cross and Bevan. For a comparison of this with other methods see J. Am. Chem. Soc., 1897, 19, 304.

${ }^{4}$ If the sample contains an active enzyme, some of the carbohydrate may be changed during this treatment with water. To prevent this a very dilute alkali solution, containing 0.02 per cent potassium hydroxide or an equivalent amount of sodium or ammonium hydroxide, may be used. Ling and Rendle; J. Inst. Brewing, 1904, 10, 238 ; Abs. J. Chem. Soc., 1904, 86, ii, 507. 
this filtrate by either Defren's or Allihn's method and calculate the amount of maltose. ${ }^{1}$ Measure $50 \mathrm{cc}$. of the same filtrate into a 100-cc. flask, add $5 \mathrm{cc}$. of hydrochloric acid of $1.125 \mathrm{sp}$. gr., and hydrolyze as in the determination of starch. Determine the resulting dextrose, deduct the amount due to maltose, and estimate the remainder as due to dextrin. Soluble starch if present would be counted as dextrin in this analysis.

Treat another portion of the original sample as described under the determination of starch, but without extracting the soluble carbohydrates. From the dextrose found, subtract that yielded by maltose and dextrin, and estimate the remainder as derived from starch.

The results require a slight correction on account of the presence of the insoluble residue when the solution is diluted to volume in the graduated flask. Although some details of the method are open to criticism, it gives results sufficiently exact for the purpose for which it is mainly used, which is to show whether the starch of the cereal has been largely changed to soluble products.

The same plan may be used in the examination of cereal foods prepared by parching or in other ways, provided only one reducing sugar is present in appreciable quantity. The amount of soluble carbohydrate in such preparations is usually too small for satisfactory determination by means of the polariscope.

\section{AcIdity}

Acidity in flour is objectionable both as an indication of deterioration and because it acts upon the gliadin, injuring the physical properties which are especially important in bread making.

To determine acidity, shake 10 grams of the dry sample with $100 \mathrm{cc}$. of cold water, filter, and titrate an aliquot part with tenth-normal sodium or potassium hydroxide, using phenolphthalein as indicator. In fine flour the acidity calculated as lactic acid should not exceed 0.10 per cent.

1 If Allihn's method is used, assume the reducing power of maltose to be 0.61 that of dextrose. 


\section{INTERPRETATION OF RESULTS}

\section{Official Definitions and Standards $\mathbf{1}$}

Grain is the fully matured, clean, sound, air-dry seed of wheat, maize, rice, oats, rye, buckwheat, barley, sorghum, millet, or spelt.

Meal is the sound product made by grinding grain.

Flour is the fine, sound product made by bolting wheat meal and contains not more than 13.5 per cent of moisture, not less than 1.25 per cent of nitrogen, not more than 1.0 per cent of ash, and not more than 0.50 per cent of fiber.

Graham flour is unbolted wheat meal.

Gluten flour is the product made from flour by the removal of starch and contains not less than 5.6 per cent of nitrogen and not more than 10 per cent of moisture.

Maize meal, corn meal, or Indian corn meal is meal made from sound maize grain and contains not more than 14 per cent of moisture, not less than 1.12 per cent of nitrogen, and not more than 1.6 per cent of ash.

Rice is the hulled and polished grain of Oryza sativa.

Oatmeal is meal made from hulled oats and contains not more than 8 per cent of moisture, nor more than 1.5 per cent of crude fiber, not less than 2.24 per cent of nitrogen, and not more than 2.2 per cent of ash.

Rye flour is the fine sound product made by bolting ryemeal and contains not more than $\mathbf{1 3 . 5}$ per cent of moisture, not less than 1.36 per cent of nitrogen, and not more than 1.25 per cent of ash.

Buckwheat flour is bolted buckwheat meal and contains not more than 12 per cent of moisture, not less than 1.28 per cent of nitrogen, and not more than 1.75 per cent of ash.

Where percentages are given in these standards they are, of course, intended to represent normal limits rather than averages or extreme limits.

1 Circular No. 19, Office of the Secretary, U. S. Dept. Agriculture. 


\section{Composition of Entire Grains}

Wiley $^{1}$ estimates the approximate composition of average typical American grains as follows:

Table 25. - Percentage Composition of Entire Grains (Wiley)

\begin{tabular}{|c|c|c|c|c|c|c|c|}
\hline & Barley & $\begin{array}{c}\text { Buck- } \\
\text { wheat }\end{array}$ & Maize & Oats & $\left|\begin{array}{c}\text { Rice } \\
\text { unhulled }\end{array}\right|$ & Rye & Wheat \\
\hline Moisture & 0.85 & 12.00 & 10.75 & 10.00 & 10.50 & 10.50 & 10.60 \\
\hline Protein (Nitrogen $\times 6.25$ ) & 11.00 & 10.75 & 10.00 & 12.00 & 7.50 & 12.25 & 12.25 \\
\hline Fat (Ether extract) . . & 2.25 & 2.00 & 4.25 & 4.50 & 1.60 & 1.50 & 1.75 \\
\hline Crude fiber . . . & 3.85 & 10.75 & 1.75 & 12.00 & 9.00 & 2.10 & 2.40 \\
\hline Ash . . . . . . . & 2.50 & 1.75 & 1.50 & 3.50 & 4.00 & 1.90 & 1.75 \\
\hline Carbohydrates (diff.) . & 69.45 & 62.75 & 71.75 & 58.00 & 67.40 & 71.75 & 71.25 \\
\hline
\end{tabular}

\section{Composition of Mill Products}

An extended study of the mill products of wheat, made by Teller at the Arkansas Experiment Station, 1894 to 1898, ${ }^{2}$ included a milling experiment in which the principal products of a long process ( 7 break) roller mill were analyzed with the following results :

Table 26. - Percentage Composition of Mill Products of Wheat (TELLER)

\begin{tabular}{|c|c|c|c|c|c|c|c|c|c|}
\hline & & & $\begin{array}{l}\text { Patent } \\
\text { Flour }\end{array}$ & $\begin{array}{c}\text { Straight } \\
\text { Flour }\end{array}$ & $\begin{array}{c}\text { Low } \\
\text { Grade } \\
\text { Flour }\end{array}$ & $\begin{array}{l}\text { Ship } \\
\text { Stuff }\end{array}$ & Bran & $\begin{array}{l}\text { Whole } \\
\text { Wheat }\end{array}$ & $\begin{array}{l}\text { Pure } \\
\text { Germ }\end{array}$ \\
\hline loisture & & - & 13.75 & 13.90 & 13.22 & 12.25 & 12.85 & & 6.8 \\
\hline Ash . . . & . & . & .33 & .47 & .90 & & 5.80 & 2.15 & 4.65 \\
\hline Crude fiber & . & • & .17 & .26 & .74 & 3.55 & 6.14 & 2.17 & 1.60 \\
\hline Fat . . & . & . & 1.05 & 1.25 & 1.70 & 4.80 & 5.20 & 2.15 & 14.38 \\
\hline Protein (Nitrogen & $\times 5$ & .7) & 9.69 & 10.37 & 12.88 & 16.36 & 15.56 & 12.31 & 36.00 \\
\hline Carbohydrates (dif & f.) & . $\cdot$ & 75.01 & 73.75 & 70.56 & 59.02 & 54.45 & 63.32 & 36.55 \\
\hline Total Nitrogen . & . & & & & 2.26 & & 2.73 & 2.16 & 6.34 \\
\hline Protein Nitrogen & & 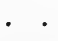 & 1.65 & 1.72 & 2.20 & 2.68 & 2.51 & 1.98 & \\
\hline Amid Nitrogen . & . & . & .05 & .10 & .06 & .19 & .22 & .18 & 3 \\
\hline
\end{tabular}

${ }^{1}$ Bul. 45, Bur. Chem., U. S. Dept. Agriculture.

2 Buls. 42 and 53, Ark. Expt. Station (Fayetteville, Ark.).

${ }^{3}$ The germ is richer in amid nitrogen than other parts of the wheat kernel. 
Under the system of milling now practiced in the Northwest a number of "streams" of flour are produced which are afterward united in different proportions to form the market grades of flour. Snyder has recently published ${ }^{1}$ the following analyses of the different "streams" as obtained in milling No. 1 Northern wheat by typical modern machinery.

Table 27. - Snyder's Analyses of Different Mill Products from One Sample of Wheat

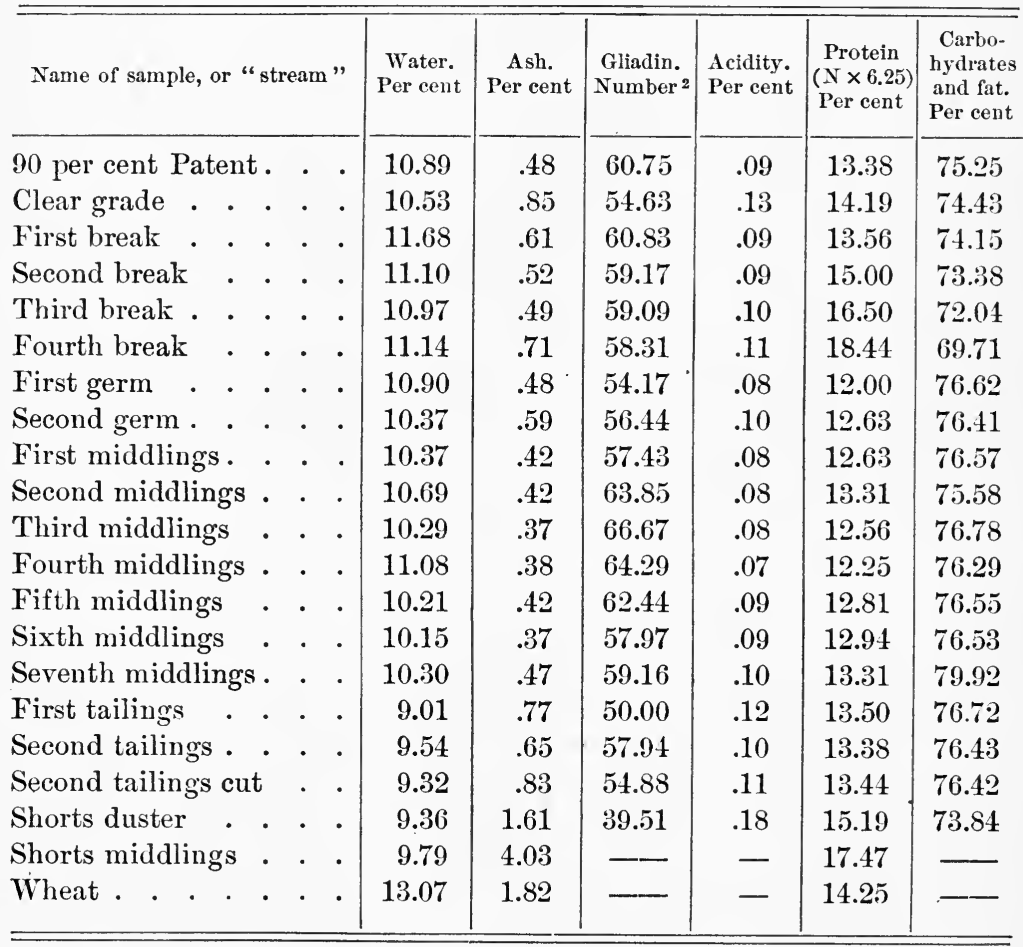

It will be seen that a sample of wheat containing 13.07 per cent moisture and 2.28 per cent nitrogen gave streams of flour containing from 9.01 to 11.68 per cent moisture, and from 1.92 to 2.95 per cent of nitrogen. The "gliadin number," or

1 Bul. 85, Minn. Agl. Expt. Station, St. Anthony Park, Minn., 1904.

2 Alcohol-soluble nitrogen in percentage of the total nitrogen. 
percentage of the total nitrogen existing in the form of alcoholsoluble proteins, varied from 39.51 to 66.67 . It is interesting to note that some of the streams of flour thus obtained from average wheat in the ordinary milling process contain considerably more nitrogen than is sometimes found in so-called gluten and diabetic flours obtained in the market.

For additional analyses and results of experiments upon the digestibility and nutritive value of cereal products see Bul. 13, Part 9, Bureau of Chemistry, and Buls. 28, 67, 85, 101, 126, and 143, Office of Experiment Stations, U. S. Department of Agriculture.

\section{REFERENCES}

I

Allen: Commercial Organic Analysis.

Atwater and Bryant: The Chemical Composition of American Food

Materials. Bul. 28, Revised, Office of Experiment Stations, U. S.

Dept. Agriculture.

$J_{A G O}$ : Science and Art of Breadmaking, Chemistry and Analyses of Wheat. : Technology of Breadmaking.

KöNIG: Chemie der Menschliche-Nahrungs- und Genussmittel.

LEACH: Food Inspection and Analysis.

Maurizio : Getreide, Mehl und Brot.

Osborne: Proteins of the Wheat Kernel.

SNYDER: Studies in Bread and Breadmaking, Buls. 67, 101, 126, Office of

Experiment Stations, U. S. Dept. Agriculture.

VogL: Die wichtigsten vegetabilischen Nahrungs- und Genussmittel.

WILEY : Foods and their Adulteration.

Winton: The Microscopy of Vegetable Foods.

\section{II}

1894. Osborne and Voorhees: Proteins of Wheat. J. Am. Chem. Soc., 16, 524.

1895. Wiley: Analyses of Cereals collected at the World's Columbian Exposition. U. S. Dept. Agriculture, Bur. Chem., Bul. 45.

1897. Osbonne: The Amount and Properties of the Proteins of the Maize Kernel. J. Am. Chem. Soc., 19, 525.

Sherman: The Insoluble Carbohydrates of Wheat. J. Am. Chem. Soc., 19, 291.

1898. Wiley et al.: Cerreals and Cereal Products. U. S. Dept. Agriculture, Bur. Chem., Bul. 13, Part IX. 
1899. Kraemer: An Examination of Commercial Flours. J. Am. Chem. Soc., 21, 650 .

1900. Guess: The Gluten Constituents of Wheat and Flour and their Relation to Bread-making Qualities. J. Am. Chem. Soc., 22, 263.

1904. SNyder: Wheat and Flour Investigations. Bul. 85, Minn. Agl. Expt. Station.

1905. Cochras: Estimation of Fat in Infants' and Invalids' Foods. $J$. Am. Chem. Soc., 27, 906.

Harper and Peter: Protein Content of the Wheat Kernel. Bul. 113, Kentucky Agricultural Experiment Station.

Hopkins, Sмith, and East: Breeding Corn. Bul. 100, Illinois Agricultural Experiment Station.

SNyder: Testing Wheat Flour for Commercial Purposes. J. Am. Chem. Soc., 27, 1068.

1906. Bremer: (Grading of Flour according to its Catalase Content). $Z$. Nahr.-Genussm., 11, 569.

Chamberlain: Investigation on the Properties of Wheat Proteins. J. Am. Chem. Soc., 28, 1657.

Norton: Crude Gluten. J. Am. Chem. Soc., 28, 8.

Winton: Diabetic Foods. Ann. Rept. Conn. Expt. Sta., 1906, p. 153.

1907. Alway and Gortner: The Detection of Bleached Flours. J. Am. Chem. Soc., 29, 1503.

Avery: A Contribution to the Chemistry of Bleached Flour. $J$. Am. Chem. Soc., 29, 571.

Harcourt : Breakfast Foods; their Chemical Composition, Digestibility, and Cost. J. Soc. Chem. Ind., 26, 240, and Ontario Dept. Agriculture, Bul. 162.

Shaw : Polariscopic Method for Determination of Gliadin. J. Am. Chem. Soc., 29, 1747.

Thatcher: A Comparison of Various Methods of estimating the Baking Qualities of Flour. J. Am. Chem. Soc., 29, 910.

Wood: Test for Strength of Wheat Flour. Nature, 75, 391; Chem. Abs., $\mathbf{1}, 1150$.

1908. BAKER and Hulton: Considerations affecting the Strength of Wheat Flours. J. Soc. Chem. Ind., 27, 368.

Mathewson: On the Analytical Estimation of Gliadin. $J . A m$. Chem. Soc., 30, 74.

1909. Herty: Rapid Determination of Oil in Cottonseed Products. $J$. Ind. Eng. Chem., $\mathbf{1}, 76$.

Holdfleiss and Wesslivg: Laboratory Experiments on the Determination of the Milling and Breadmaking Qualities of Wheat. J. Soc. Chem. Ind., 28, 808. 
1910. Polensine: The Determination of Fat in Foods. Arb. kais. Gesundheitsamte, 33, 563; Chem. Abs., 5, 325.

Willard : Changes in Weight of Stored Flour. Kansas State Board of Health, 7, 9; Chem. Abs., 5, 1636.

Winton: Diabetic Foods. British Food J., 12, 23 ; Chem. A.bs., 4, 1632.

1911. Greaves: Some Factors influencing the Quantitative Determination of Gliadin. J. Biol. Chem., 9, 271.

Hoagland: The Determination of Gliadin or Alcohol-soluble Protein in Wheat Flour. J. Ind. Eng. Chem., 3, 838. 


\section{CHAPTER XVII}

\section{Milk}

Cows' milk is concisely described as essentially an aqueous solution of milk sugar, albumin, and certain salts, holding in suspension globules of fat and in a state of semisolution casein together with mineral matter (Richmond). Small amounts of other compounds are also present, but need not be considered here.

Standard milk (whole milk) is defined ${ }^{1}$ as the lacteal secretion obtained by the complete milking of one or more healthy cows properly kept and fed, excluding that obtained within fifteen days before and five days after calving, and contains not less than 8.5 per cent of solids not fat, and not less than 3.25 per cent of milk fat.

These limits are considerably below the average and considerably above the lowest authentic figures which have been found. Average milk may be assumed to contain 12.9 to 13 per cent of total solids, made up of -

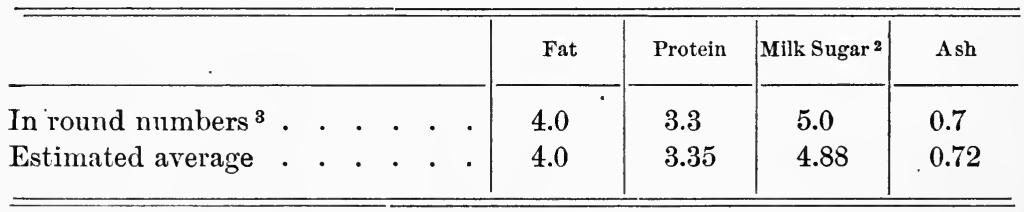

The protein content of average milk is, therefore, about onefourth of the total solids. In general the same relation holds in milk which is richer than the average. Each increase of 1

${ }^{1}$ Circular No. 19, Office of the Secretary, U. S. Dept. Agriculture.

2 The figures for milk sugar include the small amount of undetermined nonnitrogenous matter.

3 These are the figures used in most publications of the U. S. Dept. Agriculture. 
per cent in total solids thus involves on the average an increase of 0.25 per cent of proteins, the remaining 0.75 per cent being practically all fat. This increase in proteins and fat is usually accompanied by a slight increase in ash and decrease in milk sugar. The following average percentages illustrate these relations in rich milk:

\begin{tabular}{l|c|c|c|c|c}
\hline \hline & Total Solids & Fat & Protein & $\begin{array}{c}\text { Milk } \\
\text { Sugar }\end{array}$ & Ash \\
\hline $\begin{array}{c}\text { Average for 5 years; mixed even- } \\
\text { ing milk of } 400 \text { to } 500 \text { cows . . }\end{array}$ & 14.62 & 5.39 & 3.66 & 4.82 & 0.75 \\
$\begin{array}{c}\text { Average of } 13 \text { unusually rich } \\
\text { samples from individual cows }\end{array}$ & 18.03 & 7.76 & 4.68 & 4.76 & 0.83 \\
\hline \hline
\end{tabular}

The composition of milk of less than average richness cannot be so definitely stated. In some cases there is a deficiency of fat and protein with no decrease in milk sugar, while in other cases the reverse is true. Usually if an unadulterated milk is poor in fat, it will be found proportionately poor in protein; while if the fat is normal, the protein is usually also normal and the low percentage of solids not fat is due to a deficiency in milk sugar.

Several hundred American analyses, made before 1890, com. piled and averaged in ten groups arranged according to percentage of total solids, gave the following results : ${ }^{1}$

Table 28. - Cooke's Compilation of American Analysis of Milk

\begin{tabular}{c|c|c|c|c||c|c|c|c|c}
\hline \hline $\begin{array}{c}\text { Group } \\
\text { No. }\end{array}$ & $\begin{array}{c}\text { Total } \\
\text { solids. } \\
\text { Per cent }\end{array}$ & $\begin{array}{c}\text { Fat. } \\
\text { Per cent }\end{array}$ & $\begin{array}{c}\text { Proteins. } \\
\text { Per cent }\end{array}$ & $\begin{array}{c}\text { Sugar } \\
\text { and ash. } \\
\text { Per cent }\end{array}$ & $\begin{array}{c}\text { Group } \\
\text { No. }\end{array}$ & $\begin{array}{c}\text { Total } \\
\text { solids. } \\
\text { Per cent }\end{array}$ & $\begin{array}{c}\text { Fat. } \\
\text { Per cent }\end{array}$ & $\begin{array}{c}\text { Proteins. } \\
\text { Per cent }\end{array}$ & $\begin{array}{c}\text { Sugar } \\
\text { and ash. } \\
\text { Per cent }\end{array}$ \\
\hline 1 & 11.35 & 3.20 & 2.99 & 5.16 & 6 & 13.71 & 4.46 & 3.48 & 5.77 \\
2 & 11.77 & 3.36 & 3.03 & 5.38 & 7 & 14.25 & 4.87 & 3.65 & 5.73 \\
3 & 12.21 & 3.60 & 3.10 & 5.51 & 8 & 14.77 & 5.20 & 3.87 & 5.70 \\
4 & 12.75 & 3.82 & 3.29 & 5.64 & 9 & 15.17 & 5.47 & 4.07 & 5.63 \\
5 & 13.17 & 4.09 & 3.40 & 5.68 & 10 & 15.83 & 5.88 & 4.26 & 5.69 \\
\hline
\end{tabular}

${ }^{1}$ Cooke : Vermont Agricultural Experiment Station Report for 1890, p. 97. 
As a rule the percentage of milk sugar and ash is most nearly constant, that of fat is most variable, while the protein varies with the fat, but to a much smaller extent. The variations which may be regarded as usual, and the extreme variations which the writer has found authentically recorded, are as follows :

\begin{tabular}{l|c|c|c|c|c}
\hline & $\begin{array}{c}\text { Fat. } \\
\text { Per cent }\end{array}$ & $\begin{array}{c}\text { Solids not fat. } \\
\text { Per cent }\end{array}$ & $\begin{array}{c}\text { Proteins. } \\
\text { Per cent }\end{array}$ & $\begin{array}{c}\text { Milk sugar. } \\
\text { Per cent }\end{array}$ & $\begin{array}{c}\text { Ash. } \\
\text { Per cent }\end{array}$ \\
\hline $\begin{array}{l}\text { Usual variations . } \\
\text { Extreme variations 1 }\end{array}$ & $\begin{array}{c}3-6 \\
1.04-14.67\end{array}$ & $\begin{array}{c}8.5-9.5 \\
4.90-13.76\end{array}$ & $\begin{array}{c}3-4 \\
2.86-9.98\end{array}$ & $\begin{array}{c}4.6-5 \\
2.33-5.28\end{array}$ & $\begin{array}{c}0.7-0.78 \\
0.66-1.44\end{array}$ \\
\hline \hline
\end{tabular}

The extreme variations are of no practical value as a means of determining the limits within which milk shall be considered. unadulterated, partly because it is possible to practice "adulteration through the cow" (i.e. by selection, feeding, and manner of milking to obtain "genuine" milk much below the normal quality), but mainly because the milk which reaches market is practically always the mixed product of several cows so that individual variations have comparatively little effect.

There are many causes of variation in the composition of cows' milk. Only the most important can be given here. Other conditions being normal, the percentages of fat and proteins are higher in autumn and winter than in spring and summer; they also increase as the amount of milk decreases toward the end of each period of lactation. Milk drawn in the evening is generally 0.3 to 0.4 per cent richer in fat than that obtained in the morning, and at any one milking the last portions drawn are much richer than the first. The influence of a change of food upon the percentage composition of milk is usually only temporary. In general the peculiarities of breed ${ }^{2}$

1 Including only results obtained from apparently healthy cows, believed to have been milked regularly under normal conditions.

${ }^{2}$ For comparison of the milk of different breeds see Richmond's Dairy Chemistry, pp. 122-126, Report of the New York State Expt. Station for 1891 (abstracted in the Expt. Station Record, 4, 263), and Report of the Wisconsin Expt. Station for 1901, p. 85. 
and the qualities of individual animals are the most important factors in determining the richness of milk. Aside from all these conditions the milk of individual cows is subject to considerable fluctuation, especially in fat content. Thus the analyses of 60 monthly samples of the mixed milk of about 500 cows showed a variation of 0.89 in the percentage of fat, the greatest deviation from the average being 0.50 per cent. About one half of the determinations of fat in the milk of individual cows of the herd during the same period were more than 0.50 per cent and about one fifth were more than 1.0 per cent above or below the average. Milk representing the mixed product of several farms, such as is now commonly sold in large cities, should, therefore, be much more uniform in composition than that of a single cow or a small herd. ${ }^{1}$

\section{SAMPLING AND PRESERVATION OF SAMPLES}

If the lot of milk to be sampled is small it can be mixed by pouring from one vessel to another from two to ten times, according to the extent to which the cream has separated, and the portion for analysis dipped, or withdrawn by means of a pipette, from near the center. When the sample is too large to be handled in this way it should be transferred if necessary to a cylindrical can and sampled by means of a Scovell tube. ${ }^{2}$ In order to obtain a proper sample of a large lot of milk delivered in cans of the same diameter, it is only necessary to sample each can with the Scovell tube and mix the portions thus obtained.

The sample should be placed at once in a clean, dry, sterile bottle, tightly stoppered, and analyzed as soon as possible. Before withdrawing each portion for analysis the sample must be thoroughly mixed by pouring - not by shaking.

${ }^{1}$ Fuller discussions of the variations in the milk of individual cows and mixed milk of herds will be found in some of the reference books given at the end of the chapter, in the papers on the composition of milk published annually by Richmond in the Analyst, and in Hittcher's Gesammtbericht uber die Untersuchung der Milch, Berlin, 1899.

${ }^{2}$ Wiley's Agricultural Analysis, Vol. III., p. 470; Leach's Food Inspection and Analysis, $2 d$ ed., p. 131 . 'These sampling tubes are sold by dealers in dairy apparatus. 
If the analysis cannot be made at once or if the sample is to be kept for some time after the analysis, it must either be stored at a temperature near the freezing point or preserved by the addition of an antiseptic. Formaldehyde ${ }^{1}$ added, while the milk is still fresh, in the proportion of 1 : 1000 will preserve the sample for months without apparent change. This amount of formaldehyde has a scarcely perceptible influence upon the analytical results. If preservation for only a few days is required, a smaller amount of formaldehyde should be used, 1: 2000 to 1: 10,000 , according to the freshness of the milk.

\section{PRELIMINARY OR PARTIAL EXAMINATION}

\section{Determination of Specific Gravity}

The specific gravity of milk is usually between 1.029 and 1.034. Since cream is considerably lighter than milk, the specific gravity would be lowered by the addition of water or of cream, but cases in which genuine milk shows a low specific gravity as a result of high fat content are very rare. As already explained, high percentages of fat are normally accompanied by high percentages of proteins, so that in most cases the specific gravity is higher in rich than in poor milk. With practice the samples which are exceptions to this rule can usually be detected by noticing the apparent viscosity and opacity of the milk as it runs from the surface of the lower bulb of the lactometer. The specific gravity taken in connection with

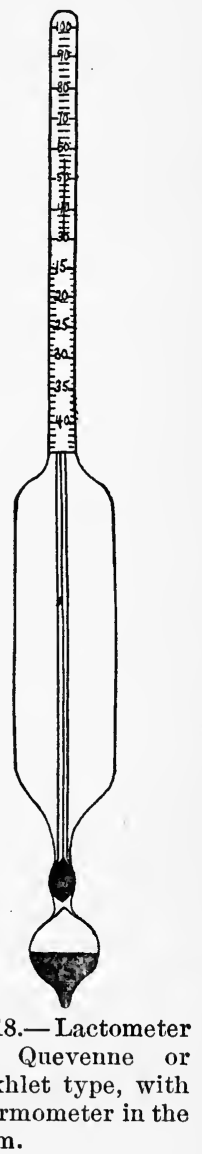
this appearance is much used as a preliminary test by milk inspectors and is recommended by Richmond as the best means of rapidly testing each lot of milk received by a large dairy.

1 Other preservatives are sometimes useful. See Richmond's Dairy Chemistry, p. 144; and Grelat: Chem. Abs., 1, 1588.

$2 \mathrm{~A}$ 
The Quevenne, Veith, and Soxhlet lactometers are hydrometers of sufficient range for use with milk and so graduated as to read the "excess gravity" over water taken as 1000 . Thus a milk of 1.0315 specific gravity gives a lactometer reading of $31.5^{\circ}$. These instruments are often made to include a Fahrenheit thermometer, the scale of the latter being on the same stem with the lactometer scale (Fig. 18). The lactometer reading should be taken between $50^{\circ}$ and $65^{\circ} \mathrm{F}$. and corrected for temperature by adding or subtracting $0.1^{\circ}$ for each degree $\mathrm{F}$. above or below 60. The New York Board of Health lactometer has a scale reading zero in pure water and 100 at 1.029 specific gravity.

\section{Volumetric Determination of Fat}

Babcock, in 1890, introduced the first satisfactory rapid method for the determination of fat in milk. On mixing milk with approximately an equal volume of strong sulphuric acid, the casein is dissolved while the fat remains unchanged and can be separated by centrifugal force. The test is performed in a bottle with a neck so graduated that the percentage of fat can be read off directly upon removing the bottle from the centrifuge.

Determination. - Measure 17.6 ce. of milk at $14^{\circ}$ to $18^{\circ}$ (about $55^{\circ}$ to $65^{\circ} \mathrm{F}$.) and introduce into the test bottle. Add 17.5 cc. of sulphuric acid of $1.82 \mathrm{sp}$. gr. (commercial concentrated acid is usually the right strength), allowing the acid to flow down the side of the bottle so as not to mix with the milk. When acid has been added to all of the bottles and everything is ready to start the whirling, mix the milk and acid quickly and thoroughly by shaking, and continue the shaking until the curd is in solution and the liquid has reached a permanent and uniform color. Then place an even number of the bottles in opposite pockets in the machine and whirl at the rate of 800 to 1200 revolutions per minute, according to the diameter of the wheel which carries the bottle. After whirling five minutes, fill with hot water to the shoulder of the bottle and whirl two minutes, then fill with hot water to near the top of the graduation in the neck and whirl again for two minutes. The per- 
centage of fat is now shown by the height of the column in the graduated neck of the bottle.

Notes. - The capacity of the graduated neck of the bottle from 0 to 10 is $2 \mathrm{cc}$. It is assumed that the $17.6 \mathrm{cc}$. of milk taken for the determination will weigh 10 times as much as 2 cc. of warm butter fat. It is important that the final readings be taken while the fat is still warm. On account of the unavoidable contraction of the fat while taking these readings it is customary to read from the bottom of the lower to the top of the upper meniscus. The result is usually within 0.2 per cent of that found by the gravimetric method. The column of fat should be of a clear yellow color throughout. If the acid used is too weak, flocks of undissolved casein are apt to be found in the lower part of the fat column; if too strong, the acid may char the fat. For a full discussion of the details of the test, with directions for applying it to other dairy products, see the work of Farrington and Woll. ${ }^{1}$

The most important modifications of the Babcock method are fully described in Richmond's Dairy Chemistry, pp. 174-192.

\section{Calculation of Solids from Specific Gravity AND FAT}

Many formulæ have been proposed by which to calculate the percentage of solids in milk from the percentage of fat and the specific gravity. The results thus obtained are sufficiently accurate for many technical purposes and often for routine inspection work which is not to be made the basis of legal action. Such formulæ may be found in many of the works referred to at the end of this chapter. They are necessarily based on the assumption that each per cent of fat causes a definite decrease, and each per cent of solids not fat a definite increase, in the specific gravity.

Since the solution densities of proteins, milk sugar, and milk ash differ considerably, ${ }^{2}$ any change in the relative

1 Testing Milk and Its Products. Madison, Wisconsin.

2 Allen : Commercial Organic Analysis, Vol. IV. (2d ed.), p. 166. 
proportions of these constituents must alter the solution density of the solids not fat and thus diminish the accuracy of this method.

One of the simplest of these formulæ is that of Richmond :

$$
\text { Total solids }=\frac{\text { Lactometer reading }}{4}+1.2 \text { fat }+0.14 \text {. }
$$

With samples which do not differ greatly from the average composition the results thus calculated are usually accurate within 0.25 per cent.

\section{DETERMINATION OF FAT, PROTEINS, MILK SUGAR, AND ASH}

\section{Total Solids AND Ash}

Into an accurately weighed flat-bottomed platinum dish introduce two to five grams (depending upon the size of the dish; see below) of the thoroughly mixed milk and weigh quickly to the nearest milligram. If this weighing cannot be accomplished within one minute, the dish should be covered with a weighed watch glass to retard evaporation. Place the open dish on a water bath or on top of the boiling water oven until nearly all of the water is expelled; dry to constant weight in a boiling water oven or an air bath kept constantly at $97^{\circ}$ to $100^{\circ}$. The residue is somewhat hydroscopic and must be weighed quickly upon removal from the desiccator in order to obtain the correct amount of total solids.

Ignite the dry solids in a muffle at $550^{\circ}$ to $600^{\circ}$, or, if this is not feasible, regulate a Bunsen burner to give a very small colorless flame and apply this carefully with constant attention so that no part of the dish is heated above the lowest possible redness. The ash should be white or very light gray. After obtaining the weight of the ash it may be used in testing for preservatives, as described below.

Notes on Total Solids. - When the same portion is not to be used for the determination of ash a platinum dish is not essential. Lead foil bottle caps are then very convenient, as they are easily numbered by scratching, quickly heated and cooled, and so 
cheap that each dish can be rejected after being used once. In order that a larger surface may be exposed, the dish may contain dry sand or other porous material and a small stirring rod weighed with the dish and used to stir the residue while drying. Unless absorbed upon porous material, no more than 0.5 gram of milk for each square centimeter of the area of the bottom of the dish should be taken for the determination.

The methods used in the Government Laboratory, London, for the determination of solids in sour or fermented milk and the estimation of the solids lost in fermentation have recently been described by Thorpe. ${ }^{1}$

Notes on Ash. - Normally about two-thirds of the ash of cows' milk is insoluble in hot water. The presence of a larger proportion of soluble ash may be due to the use of mineral preservatives or to the addition of salts to restore the density and ash content to milk which has been watered. Special tests for some of the mineral preservatives are given beyond. In important cases it may be necessary to analyze the ash to show whether it is of normal character. Richmond ${ }^{2}$ gives the following as the average composition of milk ash: calcium oxide, 20.27 per cent; magnesium oxide, 2.80 per cent; potassium oxide, 28.71 per cent; sodium oxide, 6.67 per cent; phosphoric anhydride, 29.33 per cent; chlorine, 14.00 per cent; carbonic anhydride, 0.97 per cent; sulphuric anhydride, trace ; ferric oxide, etc., 0.40 per cent. According to most other writers ${ }^{3}$ milk ash contains sulphates, but only traces of carbonates. A sample of ash from the mixed milk of about 500 cows examined by Thompson and the writer showed no appreciable amount of carbonates and only traces of sulphates. When the ash was prepared at known temperatures, there was no volatilization of chlorides below $650^{\circ}$, but even at $450^{\circ}$ to $500^{\circ}$ there was considerable loss of chlorine, due doubtless to the formation of acid products in the combustion of the organic constituents of the milk.

\section{J. Chem. Soc., 1905, 87, 206.}

2 Dairy Chemistry, p. 32.

${ }^{3}$ See tabulated analyses in Stohmann's Milch- und Molkereiproducte, p. 89. 


\section{Fat-Gravinetric Determination Adams' Paper Coil Method}

In this method the milk is dried on porous paper, the fat extracted by means of ether into a weighed flask, the ether evaporated, and the fat weighed.

Apparatus. - (1) Strips of thick absorbent fat-free paper about $55 \mathrm{~cm}$. long and $6.25 \mathrm{~cm}$. wide, ${ }^{1}$ each rolled into a loose coil and fastened by means of a piece of wire or fat-free thread. If difficulty is found in making a loose coil, two pieces of fat-free string may be laid lengthwise upon the paper strip before rolling it up. This, however, should not be necessary.

(2) A Soxhlet apparatus for ether extraction, the form having ground glass connections being recommended.

(3) A safety water bath or, preferably, an electric heater which can be easily regulated.

Determination. - Mix the milk thoroughly and absorb a known amount, about 5 grams, on the paper coil. The milk can be measured by means of a 5-cc. pipette and delivered directly upon the coil, but as milk is more viscous than water, an ordinary 5cc. pipette will deliver less than 5 cc. of milk, so that this method can be made accurate only by determining experimentally the amount of milk which the pipette actually delivers. A better method is to pour about 5 cc. into a very small beaker, weigh quickly to centigrams and at once absorb the milk by standing the coil in the beaker. The absorption can be hastened by inclining the beaker and rotating the coil. The last drops in the beaker must be carefully absorbed. Stand the coil upon the dry end and reweigh the beaker quickly to centigrams. If carried out rapidly, this method is considerably more accurate than measuring with a pipette. Dry the coil thoroughly in a boiling water oven, place in a Soxhlet extractor, and extract with anhydrous ether, using the electric heater or safety water bath and keeping the apparatus as far as possible from free flames. If

${ }^{1}$ Strips of paper especially prepared for this purpose are made by Schleicher and Schüll. If these are not available, the paper strips must be very carefully extracted before use. See Richmond's Dairy Chemistry, pp. 91-93. 
not more than 5 grams of milk is used and the extractor siphons at intervals of 10 to 15 minutes, the extraction need not be continued longer than three hours. At the end of extraction disconnect the apparatus, remove the coil, replace the extractor, recover nearly all the ether by allowing it to collect in the space formerly occupied by the coil, return the ether to its bottle, and heat the flask containing the fat in a boiling water oven until the weight is practically constant.

Notes. - As the milk is absorbed by the paper the greater part of the fat is left on or near the surface, so that it is very rapidly extracted by the ether. The coil must be thoroughly dried before extracting with ether. The drying can be hastened by pressing in the dry end of coil so that the inner layers of the wet end are made to project in the form of a cone. Such a coil will usually be dry after standing two to three hours in the boiling-water oven. On removing from the oven press back the projecting end of the coil and place it, milk end down, in the extractor; connect with the flask, pour in ether until it siphons into the flask, then enough more ether to cover about half the coil. This is sufficient to avoid any danger of the flask going dry during the extraction, if the heat is so regulated that no perceptible amount of ether escapes the reflux condenser. To dry the extract, leave it in the boiling water oven for three hours, allow to cool for one half hour, weigh, and then repeat, heating about one hour each time, until two successive weighings show a loss of less than one milligram. In laboratories where many determinations are made it is customary to dry the extract for a fixed length of time (usually five hours), which has been found by experience to be sufficient.

\section{Babcock Asbestos Method ${ }^{1}$}

Provide a hollow cylinder of perforated sheet metal, $60 \mathrm{~mm}$. long and $20 \mathrm{~mm}$. in diameter, closed $5 \mathrm{~mm}$. from one end by a disk of the same material. The perforations should be about $0.7 \mathrm{~mm}$. in diameter and about $0.7 \mathrm{~mm}$. apart. Fill loosely with from 1.5 to 2.5 grams of freshly ignited, woolly asbestos,

1 Bul. 107, Bur. Chem., U. S. Dept. Agriculture. 
free from fine and brittle material, cool in a desiccator, and weigh. Introduce a weighed quantity of milk (between 3 and 5 grams) and dry at $100^{\circ}$ to constant weight. This weight shows the percentage of total solids. Place the cylinder in an extractor and complete the determination of fat as described above.

This method avoids the possibility of having any ethersoluble matter in the porous substance used to absorb the milk. It is especially recommended for the determination of fat in cream which cannot be absorbed upon the paper coil without previous dilution.

\section{Proteins}

Formerly milk proteins were precipitated, or the milk evaporated to dryness, and the residue after washing with ether and dilute alcohol was dried, weighed, burned, and the ash deducted. On account of the difficulty of completely removing the sugar and fat, the results thus obtained were usually too high; so that in the older statements of the composition of milk (some of which are still often quoted) the proteins were usually overestimated.

Protein in milk is now calculated from the nitrogen content, multiplying the latter by the usual factor 6.25 or sometimes by a special factor, 6.33 or 6.37 , based on analyses of milk proteins showing less than 16 per cent of nitrogen.

To determine the total nitrogen in milk pour 5 to 10 grams of the sample into a small beaker, weigh quickly to centigrams, pour the milk carefully into a Kjeldahl flask, reweigh the beaker, and introduce 20 to 25 cc. of concentrated sulphuric acid into the flask in such a way as to wash down any milk which may have remained in the neck. Add 0.7 gram of mercury, heat gently over a very small flame until most of the water is expelled and no more frothing or spirting occurs, then increase the size of the flame and complete the determination as described in Chapter XIV.

Casein can be precipitated by acidulating the milk or by means of magnesium sulphate. Determination of nitrogen in the washed precipitate shows the amount of casein in the milk. Albumin can be precipitated by boiling the filtrate, and deter- 
mined in the same manner. Detailed directions for these determinations will be found in Bulletin 107, Bureau of Chemistry, U. S. Department of Agriculture.

\section{Milk Sugar or Lactose}

In most cases the direct determination of lactose is unnecessary, as the difference between the percentage of total solids and the sum of the percentages of fat, proteins, and ash should not differ from the true percentage of lactose by more than 0.1 to 0.2 per cent. When direct determination is desired, either the polariscopic method or one of the methods based upon the reduction of copper can be used. In the former case the proteins are precipitated and the solution clarified by means of mercuric nitrate or iodide; in the latter, by cupric hydroxide or acetic acid, alum, and aluminium hydroxide.

\section{Optical Determination ${ }^{1}$}

Place 65.8 grams of milk in each of two flasks, one graduated at 100 , the other at $200 \mathrm{cc}$. , to each add 4 cc. of mercuric nitrate solution, ${ }^{2}$ fill to the mark, shake, filter through dry paper, and polarize in a $200-\mathrm{mm}$. tube in the Schmidt and Haensch polariscope.

In each case the reading is too high on account of the volume occupied by the precipitate which contains the proteins and fat of the milk. This volume is twice as great in proportion in the 100 -cc. as in the 200 -cc. flask. The corrected reading and the volume occupied by the precipitate can, therefore, be calculated by the method of double dilution as in the following example: ${ }^{3}$

Weight of milk taken, $65.8 \mathrm{grams}$, or twice the "lactose normal" weight 4 for the Ventzke scale.

1 W.iley and Ewell : J. Am. Chem. Soc., 1896, 18, 428.

2 To prepare this solution dissolve mercury in twice its weight of nitric acid, 1.42 specific gravity, and add to the solution an equal volume of water; or prepare a solution of equal strength by dissolving solid mercuric nitrate in water acidulated with nitric acid.

${ }^{3}$ Compare Wiley's Agricultural Analysis, Vol. III, pp. 102, 278.

4 Calculated from the sucrose normal weight and the approximate specific rotatory powers of sucrose and lactose. These data and directions for the manipulation of the polariscope have been given in Chapters III and IV. 
Average reading from 100-cc. flask, 10.45 .

Average reading from 200-cc. flask, 5.075.

Then

$10.45-(5.075 \times 2)=0.30$ (half the error in the higher reading). $10.45-(0.30 \times 2)=9.85$ (corrected reading for 100-cc. flask). $9.85 \div 2=4.925$, corrected percentage of lactose.

The volume of the precipitate is calculated as follows : $10.45 \div 2=5.225$, apparent percentage of lactose ( 100 -cc. flask). Then $5.225: 4.925:: 100: x$.

$x=94.26$, the volume of solution in the 100-cc. flask. Hence the volume of the precipitate is $5.74 \mathrm{cc}$.

\section{Determination by Fehling Solution ${ }^{1}$}

Dilute 25 cc. of milk with 400 cc. of water in a 500 -cc. flask, add $10 \mathrm{cc}$. of the copper sulphate solution used in the Fehling method, mix and add 4.4 cc. of normal sodium or potassium hydroxide (or an equivalent amount of a weaker standard solution), fill to the mark, mix, and filter through dry paper. The filtrate must contain copper in order to insure the absence of any trace of free alkali. In this clear filtrate lactose can be determined by means of Fehling solution either by Defren's method as described in Chapter III., or by Soxhlet's method, Bul. 107, l. c. The milk is so greatly diluted in clarifying the solution that the volume of the precipitated proteins and fat can be neglected.

If lactose is to be determined volumetrically, the proteins can be precipitated and the solution clarified as described in Richards and Woodman's Air, Water, and Food.

\section{InTERPRETATION OF Results}

The principal adulterations affecting the percentages of nutrients in milk are the addition of water (sometimes containing dissolved solids) and the removal of cream. These

1 Bul. 107, loc. cit. 
adulterations are sometimes difficult to detect with certainty because genuine cows' milk varies considerably both in fat and in other solids. Since the percentage of fat is more variable than that of solids not fat, skimming is more difficult to detect than watering. If as much as one fourth of the fat were removed, the skimming would usually be indicated by the disturbance of the normal relation between the percentage of fat and that of proteins or of solids not fat; but the analysis cannot be said to prove the removal of cream unless it shows a lower percentage of fat than is ever found in genuine normal milk. Starting with average milk containing 4 per cent fat and 9 per cent solids not fat, one tenth of the fat could be removed by skimming and the resulting product containing 3.6 per cent fat could not be distinguished by analysis from genuine milk; while if the fat were reduced to 3.6 per cent by watering, the solids not fat would be reduced to 8.1 per cent, which is sufficiently below the normal to be detected without difficulty. Occasionally genuine milk contains even less than 8.0 per cent of solids not fat (the deficiency in most of these cases falling mainly upon the milk sugar), so that the limit of 8.5 for solids not fat might indicate watering where none had been practiced. Such errors are avoided by taking account of the proteins and ash. Milk should contain not less than 8.5 per cent of solids not fat, 3.0 per cent of proteins, 0.7 per cent of total ash, 0.5 per cent of ash insoluble in hot water. These four determinations, especially if supplemented by the refractometer examination of the serum as described below, will usually suffice to show whether the milk is genuine or has been watered with or without the addition of soluble solids.

In most cases it is not necessary to show conclusively whether milk has been skimmed or watered, but only whether it meets the requirements of a legal or trade standard. The principal standards in force in the United States in 1910 are given in the accompanying table, from the Twenty-seventh Annual Report, Bureau of Animal Industry, U. S. Department of Agriculture. 
Table 29.-United States and State Standards for Milk, 1910

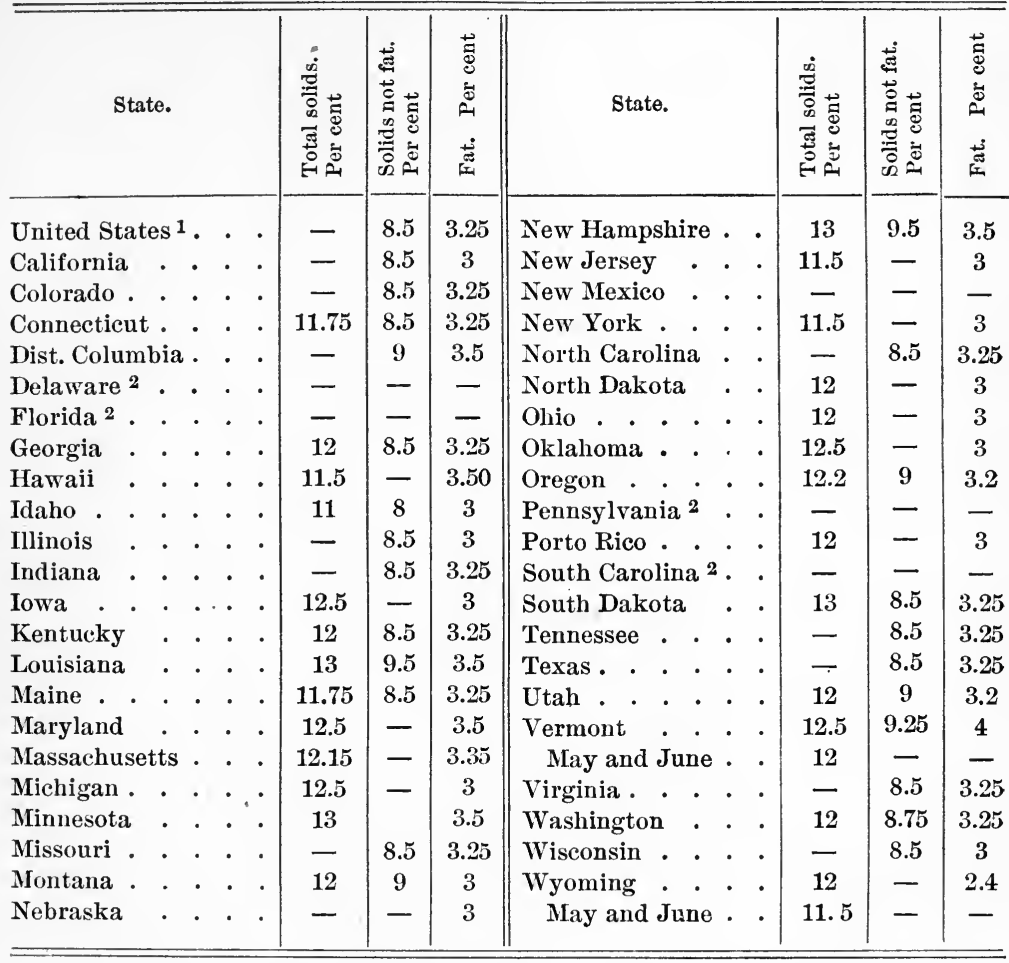

1 Standards of Purity of Food Products. United States Dept. Agriculture, Office of the Secretary, Circular 19.

2 Municipal control; no State standard.

\section{Examination of Milk Serum for AdDed Water}

In general the serum or whey obtained from milk under fixed conditions is believed to be much more uniform in properties than the milk itself, in which case watering will be more certainly detected by an examination of the whey than of the whole milk.

Woodman's method, adopted by Leach, for preparing the serum is as follows : To $100 \mathrm{cc}$. of milk at room temperature in a beaker, add 2 cc. of 25 per cent acetic acid, cover and heat in a water bath at $70^{\circ} \mathrm{C}$. for 20 minutes ; then place the beaker 
in ice water for 10 minutes, after which filter through a dry paper.

This should result in a clear filtrate (the serum or whey) which may be tested either for specific gravity or with the immersion refractometer. A specific gravity below 1.027 at $15^{\circ}$ C., or a reading of the refractometer below 39 at $20^{\circ} \mathrm{C}$., is a strong indication that the sample is watered.

The great advantage in rapidity and convenience of such a method over the determination of solids not fat commends it to inspection laboratories where many samples must be rapidly examined for adulteration, and in several such laboratories the refractometer reading of the serum is now taken as the chief criterion of watering. It should be noted, however, that the usual legal criterion is a minimum percentage of solids not fat.

According to data determined by Tice ${ }^{1}$ and by Leach ${ }^{2}$ it would appear that milk very poor in solids but free from added water may fall far below the usual legal minimum of 8.5 per cent solids not fat while yielding serum readings of 39 to 42 on the immersion refractometer scale. Hence if milk is declared watered only when both the percentage of solids not fat and the refractometer reading of the serum are below minimum limits, there will be much less danger of prosecutions for watering in cases of milk not watered but naturally poor in solids.

\section{Chemical Preservatives}

The chemical preservatives most likely to be used in milk are formaldehyde, hydrogen peroxide, boric acid or borax, and fluorides. Benzoates and salicylates may perhaps be used in rare instances. Methods for the detection and determination of these and other preservatives will be found in the next chapter.

Carbonate or bicarbonate is sometimes added to milk, not as a preservative properly so called, but as an adulterant to hide the fact that the milk has undergone acid fermentation, and so to give it a fraudulent appearance of freshness.

When milk contains the equivalent of 0.05 per cent of

${ }^{1}$ Report of the New Jersey State Board of Health, 1909, pp. 191-194.

${ }^{2}$ Leach : Food Inspection and Analysis, 2d ed., pp. 166-169. 
sodium carbonate, the ash obtained by direct ignition of the solids shows effervescence on addition of hydrochloric acid. Such effervescence is rarely if ever seen in the ash of pure milk, but since Richmond has found small amounts of carbonic acid in the ash of milk believed to have been pure, the presence of carbonate or bicarbonate should be confirmed by applying Schmidt's test, in which $10 \mathrm{cc}$. of milk are mixed with an equal volume of alcohol and a few drops of a 1 per cent solution of rosolic acid. The color is brownish yellow in pure milk but rose-red in milk containing carbonate or bicarbonate. A comparative test with pure milk should always be made. The reaction is nearly as delicate as the test for effervescence in the ash.

\section{REFERENCES}

\section{I}

Allen : Commercial Organic Analysis.

Chapin: Theory and Practice of Infant Feeding.

Cons: Bacteria in Milk and its Products.

Farrington and Woll : Testing Milk and its Products.

Fleischmann: Lehrbuch der Milchwirthschaft.

Grotenfelt : The Principles of Modern Dairy Practice.

KöNıG : Chemie der menschliche Nahrungs- und Genussmittel.

LEACH : Food Inspection and Analysis.

Richmond : Dairy Chemistry.

Rosevau : Milk in its Relation to Public Health.

RothsCHILD: Bibliographia Lactaria.

Russell: Dairy Bacteriology.

Sommerfeld: Handbuch der Milchkunde.

Stohmann: Milch- und Molkereiproducte.

Swithinbank and Newmax: Bacteriology of Milk.

U. S. Dept. Agriculture, Farmers' Bulletins 42 (Facts about Milk), 74 (Milk as Food).

VAn Slyke: Modern Methods of Testing Milk and Milk Products.

Winslow: Production and Handling of Clean Milk.

Wisconsin Agricultural Experiment Station, Bulletins and Reports.

II

1899. Richmond: The Composition of Milk and Milk Products. Analyst, 24, 197.

Woopman : On the Determination of Added Water in Milk. J.Am. Chem. Soc., 21, 503. 
1900. Richmond: The Composition of Milk and Milk Products. Analyst, $25,225$.

Whitaker: The Milk Supply of Boston and Other New England Cities. U. S. Dept. Agriculture, Bureau of Animal Industry, Bul. 26.

1901. Richmond : The Composition of Milk. Analyst, 26, 310.

1903. Richmond: The Composition of Milk. Analyst, 28, 289.

Sherman : On the Composition of Cow's Milk. J. Am. Chem. Soc., $25,132$.

1904. Leaci and Lythgoe: The Detection of Watered Milk. $J . A m$. Chem. Soc., 26, 1195.

1905. Richmond : 'The Composition and Analysis of Milk. Analyst, 30, 325. 1906. Frear : American Milk and Milk Standards. Proc. Assn. State and National Dairy and Food Departments, 1906, p. 172.

Leach: Report on Dairy Products (Refractometer Test for Watering). U. S. Dept. Agriculture, Bur. Chem., Bul. 105, p. 37.

Múller: Methylene Blue as a Test for the Freshness of Milk. Arch. Hyg., 56, 108; Analyst, 31, 299.

Rrchmond: Estimation of Fat in Homogenized Milk. Analyst, 31, $218,219-224$.

Richmond and Miller: Methods of Analysis of Milk Used by the Government Laboratory. Analyst, 31, 317.

RousseaU: Investigations on Sterilization of Milk by Means of Hydrogen Peroxide. Bull.soc.pharmacol., 13, 606 ; Chem. Abs., 1, 1592.

Seligmaxy: (Detection of Heated Milk). Z. angew. Chem., 1906, 1540.

Sherman: Seasonal Variations in the Composition of Cow's Milk. J. Am. Chem. Soc., 28, 1719.

1907. Ackermanv: Refractometric Detection of Added Water in Milk. Z. Nahr.-Genussm., 13, 186.

Anderson: The Detection of Cane Sugar in Milk and Cream. Analyst, 32, 87.

Baier and Neumans: Refractometer Examination of Milk. $Z$. Nahr.-Genussm., 13, 369.

Dubors : Analysis of Milk Chocolate. J. Am. Chem. Soc., 29, 556.

1907. Henkel: Acidity of Cows"Milk. Milchwirtsch. Zentrbl., 3, 340 ; Chem. Abs., 1, 2480.

How Ard: Analysis of Ice Cream. J. Am. Chem. Soc., 29, 1622.

Low: The Test for Formaldehyde in Milk by Leach's Modification of the Hydrochloric Acid and Ferric Chloride Test. J. Am. Chem. Soc., 29, 786.

1908. Baier and Neumans: Detection of Calcium Sucrate in Milk and Cream. Z. Nahr.-Genussm., 16, 51. 
Burr, Berberich and Lauterwald: Investigations of Milk Serum Milchwirtsch. Zentrbl., 4, 145, 210, 262 ; Chem. Abs., 2, 2961.

Frerichs: Detection of Calcium Sucrate in Milk and Cream. $Z$. Nahr.-Genussm., 16, 682.

HART: Centrifugal Method for Casein in Milk. Wisconsin Expt. Sta., Bul. 156 ; Chem. Abs., 2, 675.

MaI and Rothenfusser: Detection of Added Water in Milk by Means of the Refractometer. Z. Nahr.-Genussm., 16, 7.

1909. Fender and KunN: Determination of Dirt in Milk. Z. Nahr.Genussm., 17, 513.

Lythgoe and Nurenberg: A Comparison of Methods for the Preparation of Milk Serum. J. Ind. Eng. Chem., 1, 38.

Robertson: A Rapid Method of Determining the Percentage of Casein in Milk. J. Ind. Eng. Chem., 1, 723.

Rothenfusser: (Nitrate Test as Evidence of Added Water in Milk). Z. Nahr.-Genussm., 18, 353.

VAN Slyke and Bosworty: Volumetric Method for the Determination of Casein in Milk. J. Ind. Eng. Chem., 1, 768.

1910. Auzinger: (Test for Abnormal Milk). Milchwirtsch. Zentrbl., 5, 293, 352, 393, 430; Chem. Abs., 4, 619.

Eckles: Seasonal Variations in Percentages of Fat in Cows' Milk.

Milchwirtsch. Zentrbl., 5, 488; Chem. Abs., 4, 620.

HArt: A Volumetric Method for the Estimation of Casein in Milk. J. Biol. Chem., 6, 445.

Lythgoe and MARsh: The Relation Between Fat and Calcium in Cream. J. Ind. Eng. Chem., 2, 327.

Poetschкe: The Determination of Sodium Chloride in Milk. $J$. - Ind. Eng. Chem., 2, 210.

Rothenfusser: Detection of Cane Sugar and Calcium Sucrate in Milk and Cream. Z. Nahr.-Genussm., 19, 465.

Tillmans: (Determination and Significance of Nitrates in Milk). Z. Nahr.-Genussm., 20, 676.

1911. BACкE: Analysis of Sweetened Condensed Milk. Analyst, 36, 138. Bull: A Comparison Between the Refraction and the Specific Gravity of Milk Serum for the Detection of Added Water. J. Ind. Eng. Chem., 3, 44. 


\section{CHAPTER XVIII}

\section{Food Preservatives ${ }^{1}$}

\section{Formaldehyde ${ }^{2}$}

\section{Detection}

OF the many methods available for the detection of formaldehyde in milk and other foods only three of the best known and most delicate will be given here. For other methods see Bul. 107, Revised, Bureau of Chemistry, U. S. Department of Agriculture.

Sulphuric Acid Test. ${ }^{3}$ - Dilute 2 to 3 cc. of milk with an equal volume of water in a test tube, add carefully, so as not to mix the layers, from 3 to 5 cc. of concentrated commercial sulphuric acid or pure acid to which a trace of ferric salt has been added. If formaldehyde is present, a violet ring forms at the junction of the two liquids. The charring of the milk by the sulphuric acid makes it difficult to define the delicacy of the test. One part of formaldehyde in 100,000 of milk can be detected if the milk is fresh and the test is applied soon after adding the preservative.

Hydrochloric Acid Test. ${ }^{4}$ - Mix 10 cc. of milk and 10 cc. of concentrated hydrochloric acid containing about $2 \mathrm{mg}$. of ferric chloride and heat slowly nearly to boiling, rotating the mixture occasionally to insure solution of the curd. In the presence of

1 Trade names and analyses of many proprietary preservatives will be found in the Year-Book of the U. S. Department of Agriculture for 1900, Chapin's Theory and Practice of Infant Feeding, and the Zeitschrift für die Untersuchung der Nahrungs- und Genussmittel.

${ }^{2}$ See also the methods for detection and determination of formaldehyde given in Chapter II.

3 Hehner : Analyst, 1896, 21, 95.

${ }^{4}$ Leach : Ann. Rpts. Mass. State Board of Health, 1897, 558; 1899, 699; Food Inspection and Analysis, p. 140. See also Chapter II. 
formaldehyde a violet color develops, otherwise the solution slowly turns brown. The test is best performed in a porcelain casserole, and in case of doubt the violet color is made much more distinct by adding 50 to $75 \mathrm{cc}$. of water after having heated just below boiling for about a minute. The liquid must be observed carefully at the moment of dilution as the color brought out in this way fades very rapidly. This test is delicate to $1: 250,000$, but formaldehyde added to milk in such small quantities soon disappears. When added to the extent of $1: 50,000$ to $1: 100$,000 the presence of formaldehyde in the milk will be shown by this test for from 1 to 5 days. ${ }^{1}$ In testing sour or stale milk the brown color noted above will often obscure the reaction given by a small amount of formaldehyde until the solution is diluted with water, but at this point the violet color can be seen even though the milk may have been much charred by the acid.

Gallic Acid Test. - This test has been described in Chapter II. To apply it to milk or other liquid food acidulate $30 \mathrm{cc}$. with 2 cc. of normal sulphuric acid and distill. To the first 5 cc. of distillate add 0.2 to $0.3 \mathrm{cc}$. of a saturated solution of gallic acid in pure alcohol, incline the test tube, and pour in slowly 3 to $5 \mathrm{cc}$. of concentrated sulphuric acid. The presence of formaldehyde is shown by the characteristic blue ring described in detail in Chapter II. In the writer's experience this test is at least twice as delicate as either the sulphuric or the hydrochloric acid test. The latter would be sufficiently delicate for all practical purposes if milk samples could always be tested while fresh, but when small amounts of formaldehyde have been added one or two days previously, the gallic acid test may show the preservative where either of the other tests would fail. In a laboratory experiment, ${ }^{2}$ a sample of milk which originally contained 1:50,000 formaldehyde ceased to give any reaction by the hydrochloric acid and ferric chloride test after five days, but the distillate subsequently obtained from $30 \mathrm{cc}$. of this sample gave an unmistakable formaldehyde reaction when tested with gallic acid.

1 Rivas: University of Pennsylvania Medical Bulletin, 1904, 17, 175. Williams and Sherman: J. Am. Chem. Soc., 1905, 27, 1497.

2 Williams and Sherman: J. Am. Chem. Soc., 27, 1499. 
This gallic acid reaction may also be used very satisfactorily as a means of confirming any doubtful results obtained by either of the preceding tests, as there is little danger of interference due to charring or to the appearance of other colors.

\section{Determination}

Very small amounts of formaldehyde in milk can be determined by the following method, which is essentially that of Smith, ${ }^{1}$ except that a larger quantity of sample is used.

To 300 cc. of milk in a round-bottomed flask of about one liter capacity add 3 cc. of $(1: 3)$ sulphuric acid and some glass beads to prevent bumping, heat gradually to boiling, preferably by means of a small rose-top burner, and distill until the distillate measures $60 \mathrm{cc}$. Transfer this to a 100-cc. flask, add 10 cc. of standard potassium cyanide solution, approximately tenth-normal, and mix ; add a mixture of $\mathbf{1 5} \mathrm{cc}$. of tenth-normal silver nitrate and 6 to 8 drops of 50 per cent nitric acid, fill to the mark, shake, and filter through dry paper. Determine the excess of silver by the Volhard method and calculate the results as explained in Chapter II. under the description of the cyanide method as used for commercial solutions of formaldehyde. The precautions and the directions for standardizing there given should also be noted.

By this method from 32 to 39 per cent of the formaldehyde in the milk is recovered and determined in the distillate. Assuming that the amount recovered represents 35 per cent. of the quantity in the milk, the latter can be estimated with a probable error of about one tenth. Using this as a means of studying the disappearance of formaldehyde in milk, it was found in a typical experiment in which the proportion added was $1: 40,000$, that nearly three fourths of the preservative had disappeared after two days at room temperature. After four days no formaldehyde was shown by this method, but the violet color on dilution with water after heating with hydrochloric acid containing ferric chloride was unmistakable. The latter reaction could still be obtained after the mixture had stood for

1 J. Am. Chem. Soc., 1903, 25, 1036. 
two weeks. When formaldehyde is added to milk in large proportion, $1: 1000$ to $1: 10,000$, as in the preservation of samples for analysis or reference, the rate of disappearance is much slower.

\section{Hydrogen Peroxide}

\section{Detection}

Hydrogen peroxide in uncooked milk is easily detected by adding, to 10 to 15 cc. of the milk, 2 to 3 drops of a 2 per cent aqueous solution of paraphenylene diamine hydrochloride. In the presence of hydrogen peroxide a blue color appears either immediately upon shaking or after a few minutes, depending upon the amount present. The reaction depends upon the action of an oxidizing enzyme in the milk, and the condition of the milk, therefore, affects the delicacy of the test. According to Arnold and Mentzel ${ }^{1} 1$ part in 40,000 can be detected. Under ordinary conditions the delicacy is probably somewhat less than this. In comparative tests made immediately after adding the same amounts of peroxide to sweet milks 1 to 2 days old and to very sour curdled milks 3 to 4 days old, the former were found to give the reaction much more strongly than the latter. In practice, however, the preservative would be added while the milk was sweet and would probably disappear entirely before the occurrence of curdling. Milk which has been boiled can be tested after adding an equal volume of fresh milk known to be free from peroxide.

\section{Determination}

Chick, ${ }^{2}$ in an investigation of the germicidal properties and rate of disappearance of hydrogen peroxide in milk, used a method based upon the titration of the iodine liberated by the peroxide on adding potassium iodide and sulphuric acid.

$$
2 \mathrm{KI}+\mathrm{H}_{2} \mathrm{O}_{2}+\mathrm{H}_{2} \mathrm{SO}_{4}=\mathrm{K}_{2} \mathrm{SO}_{4}+\mathrm{I}_{2}+\mathrm{H}_{2} \mathrm{O} \text {. }
$$

Mettler ${ }^{3}$ has used the method with satisfactory results in the following modified form: To $40 \mathrm{cc}$. of water, 0.5 gram of

1 Z. Nahr.-Genussm., 1903, 6, 306.

${ }^{2}$ Centralblatt für Bacteriologie und Parasitenkunde, II. Abth., 1901, 1, 705.

3 Thesis for the degree of Bachelor of Science, Columbia University, 1905. 
potassium iodide, and 10 cc. of 12 per cent sulphuric acid in a glass-stoppered flask, add 10 ce. of the milk, stopper tight, and allow to stand in a cool, dark place for two and one half hours. In order to guard against any possible loss of iodine during this time, use a flask with flaring mouth as described in connection with the determination of the iodine number (Chapter VIII), and fill the gutter around the stopper with a solution of potassium iodide. Finally titrate the iodine which has been set free in the milk, using a fiftieth-normal solution of sodium thiosulphate. In this titration it is not necessary to use starch as indicator, since the disappearance of the yellow color produced by the action of the iodine upon the proteins affords a satisfactory end point. Test analyses gave results about 3 per cent too low, doubtless because of the absorption of iodine by the milk fat. This source of error can be avoided by curdling the milk with acid, filtering, and adding the iodide to a measured amount of filtrate; but this is considered inadvisable in view of the fact that the peroxide may be undergoing decomposition during the filtration.

\section{Boric Acid and Borates}

In routine milk analysis ${ }^{1}$ the ash obtained in the usual way is treated with two drops of dilute hydrochloric acid and about a cubic centimeter of water. A strip of turmeric paper is then placed in the dish, allowed to soak for a minute, removed, and allowed to dry in the air. A deep red color changing to green or blue when treated with dilute alkali shows the presence of boric acid. According to Leach this reaction is delicate to 1 part in 8000 . The well-known flame test with methyl alcohol is less delicate, but can be used in confirmation.

The methods adopted by the Association of Official Agricultural Chemists are as follows: ${ }^{2}$

\section{Qualitative Detection}

Render decidedly alkaline with lime water about 25 grams of the sample and evaporate to dryness on a water bath. Ignite the residue to destroy

1 Leach : Food Inspection and Analysis, 2d ed., p. 184.

2 U. S. Dept. Agriculture, Bur. Chem., Bul. 107, Revised. 
organic matter. Digest with about 15 cc. of water, add hydrochloric acid, drop by drop, until all is dissolved, and add $1 \mathrm{cc}$. in excess. Moisten a piece of delicate turmeric paper with the solution; if borax or boric acid is present, the paper on drying will acquire a peculiar red color, which is changed by ammonium hydroxide to a dark blue-green, but is restored by acid.

A preliminary test may be made by immersing a strip of turmeric paper in about $100 \mathrm{cc}$. of liquid foods, to which about $7 \mathrm{cc}$. of concentrated hydrochloric acid has been added. Solid and pasty foods may be heated with enough water to make them thoroughly fluid, hydrochloric acid added in about the proportion of 1 to 13 , and tested in the same manner.

\section{Quantitative Estimation}

Render 100 grams of the sample decidedly alkaline with sodium hydroxide and evaporate to dryness in a platinum dish. Ignite the residue thoroughly, heat with about 20 cc. of water, and add hydrochloric acid drop by drop, until all is dissolved. Transfer to a 100-cc. flask, the volume not being allowed to exceed 50 to $60 \mathrm{cc}$. Add 0.5 gram of calcium chloride and a few drops of phenolphthalein, then a ten per cent solution of caustic soda until a permanent slightly pink color is produced, and finally add $25 \mathrm{cc}$. of limewater. Make the volume up to $100 \mathrm{cc}$. Mix and filter through a dry filter. To $50 \mathrm{cc}$. of the filtrate add normal sulphuric acid until the pink color disappears, then methyl orange, and continue the addition of the acid until the yellow is just changed to pink. Boil to expel carbon dioxide. Add fifth-normal caustic soda until the liquid assumes the yellow tinge, excess of soda being avoided. Cool the solution, add a little phenolphthalein and an equal volume of glycerin. Titrate with standardized sodium hydroxide until a permanent pink color is produced.

One cubic centimeter of fifth-normal soda solution is equal to 0.0124 gram of crystallized boric acid.

Low ${ }^{1}$ proposes the following modification of the turmeric test. Ten grams of the sample (hashed meat for instance) are mixed with 5 cc. of half-normal solution of sodium carbonate, dried, and heated until volatile matter is completely driven off. The charred mass is powdered and treated with $10 \mathrm{cc}$. of water and 1 cc. of strong hydrochloric acid. After testing a small portion of the filtrate as usual, the remainder is placed in a shallow dish with a piece of turmeric paper and allowed to evaporate at $40^{\circ}-50^{\circ}$ in a desiccator, if necessary in a vacuum, when the usual color should be developed.

1 J. Am. Chem. Soc., 28, 807. 
This test is said to be much more delicate than drying on a steam bath. For full details and discussion of delicacy see the original paper. In this same paper, Low gives an improved quantitative method for the determination of boric acid in food, and data on the occurrence of boric acid in common salt and in laboratory apparatus and reagents.

\section{Fluorides}

If food containing a small amount of fluoride is burned to ash in the usual way, the fluorine is likely to be almost entirely lost. In the presence of a considerable excess of alkali this loss of fluorine does not occur.

To detect or determine fluorides, add 1 gram of sodium carbonate to $100 \mathrm{cc}$. of milk, ${ }^{1}$ evaporate, and burn to ash. If only qualitative results are required, examine for fluorides by the well-known etching test on glass. For a quantitative determination of the fluorine, leach the mixture of ash and sodium carbonate thoroughly with hot water, nearly neutralize with sulphuric acid, leaving the solution slightly alkaline, and then apply Rose's method as modified by Treadwell and Koch, $Z$. anal. Chem., 1904, 43, 469.

The details of the methods adopted by the Association of Official Agricultural Chemists are as follows : ${ }^{2}$

\section{Modified Method of Blarez}

Thoroughly mix the sample and heat $150 \mathrm{cc}$. to boiling (in the case of solid foods the filtrate prepared as directed under salicylic acid may be employed). Add to the boiling liquor 5 cc. of a 10 per cent solution of potassium sulphate and $10 \mathrm{cc}$. of a 10 per cent solution of barium acetate. Collect the precipitate in a compact mass (a centrifuge may be used advantageously) and wash upon a small filter. Transfer to a platinum crucible and ash.

Prepare a glass plate (preferably of the thin variety commonly used for lantern slide covers) as follows: First thoroughly clean, polish, and coat on one side by carefully dipping the plate while hot in a mixture of equal parts of Carnauiba wax and paraffin. Near the middle of the plate make a distinc-

1 Or equivalent amount of other food.

2 U. S. Dept. Agriculture, Bur. Chem., Bul. 107, Revised. 
tive mark through the wax with a sharp instrument, such as a pointed piece of wood or ivory, which will remove the wax and expose the glass without scratching the latter.

Add a few drops of concentrated sulphuric acid to the residue in the crucible and cover with the waxed plate, having the mark nearly over the center and making sure that the crucible is firmly embedded in the wax. Place in close contact with the top or unwaxed surface of the plate a cooling device, consisting of a glass tube considerably larger in diameter than the crucible, the bottom of the tube being covered tightly with a thin sheet of pure rubber. A constant stream of cold water is passed through the tube. Heat the crucible for an hour at as high a temperature as practicable without melting the wax (an electric stove gives the most satisfactory form of heat).

Remove the glass plate and indicate the location of the distinguishing mark on the unwaxed surface of the plate by means of gummed strips of paper, then melt off the wax by heat or a jet of steam, and thoroughly clean the glass with a soft cloth. If fluorine be present, a distinct etching will be apparent on the glass where it was exposed.

\section{Second Method}

If it is desired, the preceding method may be varied by mixing a small amount of precipitated silica with the precipitated calcium fluoride and applying the method given below for the detection of fluosilicates.

This method is of value in the presence of foods whose ash contains a considerable amount of silica, which unites with fluorine and forms fluosilicates. The sulphuric acid then liberates hydrofluosilicic acid, which would escape detection by the Blarez modified method.

\section{Fluoborates and Fluosilicates}

(Methods of the Association of Official Agricultural Chemists. U. S. Dept. Agriculture, Bur. Chem., Bul. 107, Revised.)

Make about 200 grams of the sample alkaline with lime water, evaporate to dryness, and incinerate. Extract the crude ash first obtained with water, to which sufficient acetic acid has been added to decompose carbonates, filter, burn the insoluble portion, extract with dilute acetic acid, and again filter. The insoluble portion now contains calcium silicate and fluoride, while the filtrate will contain all the boric acid present.

\section{First Method ${ }^{1}$}

Incinerate the filter containing the insoluble portion, mix with a little precipitated silica, and place, with the addition of 1 or $2 \mathrm{cc}$. of concentrated sulphuric acid, in a short test tube, which is attached to a small U-tube con-

1 Nivière and Hubert, Moniteur scientifique, 1895 [4], 9, 324. 
taining a few drops of water. Place the test tube in a beaker of water and keep it hot on the steam bath for from 30 to 40 minutes. If any fluoride be present, the silicon fluoride generated will be decomposed by the water in the U-tube and will form a gelatinous deposit on the walls of the tube.

Now test the filtrate as directed under boric acid. If both hydrofluoric and boric acids be present, it is probable that they are combined as borofluoride. If, however, silicon fluoride is detected and not boric acid, the operation is repeated without the introduction of the silica, in which case the formation of the silicon skeleton is conclusive evidence of the presence of fluosilicate.

\section{Second Method}

Incinerate the filter containing the insoluble portion in a platinum crucible, mix with a little precipitated silica, and add $1 \mathrm{cc}$. of concentrated sulphuric acid. Cover the crucible with a watch glass, to the under side of which a drop of water is suspended, and heat an hour at the temperature of $70^{\circ}$ to $80^{\circ} \mathrm{C} .1$ The silicon fluoride which is formed is decomposed by the water, leaving a gelatinous deposit of silica and etching a ring at the periphery of the drop of water. Test the filtrate for boric acid as described above.

\section{Sulphurous AcID}

The methods adopted by the Association of Official Agricultural Chemists are as follows :

\section{Qualitative Detection ${ }^{2}$}

To about 25 grams of the sample (with the addition of water, if necessary), placed in a 200-cc. Erlenmeyer flask, add some sulphur-free zinc and several cubic centimeters of hydrochloric acid. In the presence of sulphites hydrogen sulphide will be generated and may be tested for with lead paper. Traces of metallic sulphides are occasionally present in vegetables, and the above test will indicate sulphites. Hence positive results obtained by this method should be verified by the distillation method.

It is always advisable to make the quantitative determination of sulphites, owing to the danger that the test may be due to traces of sulphides. A trace is not to be considered sufficient indication of the presence of sulphur dioxide either as a bleaching agent or as a preservative.

\section{Quantitative Distillation Method ${ }^{3}$}

Distill from 20 to 100 grams of the sample (adding recently boiled water, if necessary) in a current of carbon dioxide, after the addition of about $5 \mathrm{cc}$.

1 The watch glass may be kept cool by means of a piece of ice.

2 U. S. Dept. Agriculture, Bur. Chem., Bul. 107, Revised.

3 Ibid., Bul. 137, p. 115. 
of a 20 per cent solution of glacial phosphoric acid, until $150 \mathrm{cc}$. have passed over. Collect the distillate in about $100 \mathrm{cc}$. of nearly saturated bromine water. Allow the end of the condenser to dip below the surface of the liquid in the receiver. The method and apparatus may be simplified without material loss in accuracy by omitting the current of carbon dioxide, adding $10 \mathrm{cc}$. of phosphoric acid instead of 5 cc., and dropping into the distilling flask a piece of sodium bicarbonate weighing not more than a gram, immediately before attaching the condenser. The carbon dioxide liberated is not sufficient to expel the air entirely from the apparatus, but will prevent oxidation to a large extent. When the distillation is finished, boil off the excess of bromine, dilute the solution to about 250 cc., add 5 cc. of hydrochloric acid ( 1 part of the concentrated acid to 3 of water), heat to boiling, and precipitate the sulphuric acid with a 10 per cent solution of barium chloride. Boil for a few minutes longer, allow to stand overnight in a warm place, filter on a weighed Gooch crucible, wash with hot water, ignite at a dull red heat, and weigh as barium sulphate.

Horne ${ }^{1}$ suggests that in distilling sulphurous acid it be passed through cadmium chloride solution to remove hydrogen sulphide before it reaches the bromine or iodine solution.

For other methods of detecting and determining sulphurous acid and sulphites, as well as for discussion and interpretation, see references at the end of this chapter.

\section{Salicylid Acid}

The method and precautionary notes of the Association of Official Agricultural Chemists are as follows: ${ }^{2}$

A small amount of salicylic acid occurs naturally in many fruits, and not more than 50 grams should be used for its qualitative detection in the examination of foods. A reaction obtained with this amount is due to added salicylic acid. The method described below is intended for the quantitative determination of salicylic acid. If only a qualitative determination be desired, many of the details may be omitted.

If the material be a solid or semisolid, macerate the sample in a mortar with water made slightly alkaline, and strain through a cotton bag or separate by means of a centrifuge. If preferred, macerate from 200 to 300 grams with about $400 \mathrm{cc}$. of water, and use aliquots of the filtrate for the determination of preservatives.

In quantitative work place the macerated mass in a graduated flask, make up to a definite volume with water, and shake from time to time until solu-

1 U. S. Dept. Agriculture, Bur. Chem., Bul. 105, p. 125.

2 Ibid., Bul. 107, Revised. 
tion is complete. Then strain as directed above and use an aliquot of the filtrate for extraction.

Extract in a separatory funnel $100 \mathrm{cc}$. of the sample or of the aqueous solution prepared from the sample as described above with a sufficient amount of sulphuric ether ${ }^{1}$ to prevent emulsion after the addition of 2 or 3 cc. of dilute (1-3) sulphuric acid. Separate the clear aqueous solution, and if any emulsion is present, give the separatory funnel a quick, vigorous shake, and allow to settle again. If the emulsion is not broken up in this way, it may be accomplished by means of a centrifuge, or by adding 10 or 15 ce. of low boiling point gasoline or petroleum ether, and shaking again.

Separate the clear, aqueous portion obtained from the emulsion and add it to the first aqueous portion separated. Then pour the ether into another separatory funnel, care being taken that none of the aqueous portion is left with the ether. Return the aqueous portion to the separatory funnel and again extract with ether, following the same procedure as before. Repeat this operation twice again, four separate extractions with ether being made in all.

In case of special difficulty in breaking up the emulsion in any of the

${ }^{1}$ If the nature of the substance is such that extraction with organic solvents is not practicable, as in the case of the presence of a large amount of fat, the salicylic acid may first be separated by distillation. In such cases acidify the macerated material with phosphoric acid and transfer to a distilling flask with a very short neck and wide mouth. An Erlenmeyer flask with inside diameter of mouth of $1 \frac{1}{4}$ inches is a good shape. The tube connecting the flask with condenser should be very short, with an inside diameter of not less than $\frac{3}{8}$ of an inch.

Conduct steam through a small tube passing through the stopper and dipping deeply into the material in the flask. The distillation of the salicylic acid is facilitated by submerging the distilling flask almost to the stopper in an oil bath and distilling with the temperature of the oil at from $120^{\circ}$ to $130^{\circ} \mathrm{C}$., or by adding about 20 grams of sodium chloride to the contents of the flask for each $100 \mathrm{cc}$. of the substance, to raise the boiling point. Care must be taken not to let the contents of the flask get too low, as the heat will decompose the organic matter.

Collect at least $600 \mathrm{cc}$. of the distillate and continue the distillation until the last $200 \mathrm{cc}$. gives no color on the addition of a drop of ferric solution. The distilling apparatus should in all cases be tested with known amounts of salicylic acid in order to determine the amount of distillate necessary to carry over a definite weight of salicylic acid.

It is sometimes practicable to determine the salicylic acid directly in the distillate by the colorimetric method with ferric chloride given above. If the mineral acid used in the distillation be carried over mechanically, however, the accuracy of the method is greatly impaired. Salicylic acid may be recovered from the distillate after making alkaline and evaporating, if desired, by extraction with ether and estimating colorimetrically as directed above. 
extractions, a small amount of ether may be allowed to remain with the aqueous portion rather than the reverse, as it is removed in successive extractions. Wash the combined ether extracts by shaking in a separatory funnel with one tenth their volume of water (using, however, not less than 20 cc. of water at each washing). Care must be taken at each washing to separate the aqueous portion completely from the ether, but none of the ether should be allowed to run into the wash water.

Distill slowly the greater part of the ether, transfer the remainder to a porcelain dish, and allow to evaporate spontaneously. Thoroughly dry in a vacuum desiccator ${ }^{1}$ over sulphuric acid, extract the dry residue with ten portions of 10 or 15 cc. each of carbon bisulphide or low boiling point petroleum ether, rubbing the contents of the dish with a glass rod or other suitable instrument and transferring the successive portions of solvent to a second porcelain dish. The extracted residue should finally be tested with a drop of ferric-alum solution, and if any reaction for salicylic acid be given it should be taken up in water, reextracted with ether, and the operation repeated. The gasoline extract is finally allowed to evaporate spontaneously.

Dissolve the residue in a small amount of hot water and dilute to a definite volume. Dilute aliquots of the solution and match, in Nessler tubes or with a colorimeter, the color obtained by adding a few drops of ferric chloride or ferric alum solution with that of a standard solution of salicylic acid containing about $1 \mathrm{mg}$. of salicylic acid in $50 \mathrm{cc}$. A 0.5 per cent solution of ferric chloride should be used, or a 2 per cent solution of ferric alum. ${ }^{2}$ In either case, and especially with ferric chlorid, an excess of reagent should be avoided, although an excess of $0.5 \mathrm{cc}$. of 2 per cent ferric alum solution may be added to $50 \mathrm{cc}$. of the solution of salicylic acid without impairing the results.

Salicylic acid may often be separated from fat extracted with the ether by washing the ether solution with dilute ammonium hydroxide. Then evaporate the aqueous liquid almost to dryness and test with ferric solution.

1 In examining a substance whose ether extract does not give a color or precipitate with ferric solution, the drying of the residue and its extraction with gasoline may be omitted. The residue may then be transferred by means of warm water directly from the distilling flask to the graduated flask, in which it is made up to a definite volume. Substances interfering with the ferric reaction may often be removed by precipitation with ferric chloride or lime.

2 This solution should be boiled until a precipitate appears, allowed to settle, and filtered. The acidity of the solution is slightly increased in this manner, but so precipitated it keeps clear for a considerable time, and the turbidity caused by its dilution with water is much less and does not appear for a much longer time than if the unboiled solution is employed. This turbidity is especially objectionable in the quantitative estimation of salicylic acid, as it interferes with the exact matching of the color. 
In the case of foods which yield to the gasoline solution of the ether residue a color that obscures the ferric chloride reaction (for example, tomatoes), the ether solution may be evaporated, the residue dried in a desiccator or in a current of dry air, sublimed, and collected on a watch glass cooled with ice. Then dissolve the sublimate in hot water and test with ferric alum.

The same difficulty may often be avoided, and in fact the extraction with gasoline of the dry residue from the ether extraction may sometimes be obviated, by precipitating before extraction with ferric chloride or calcium chloride, making alkaline, and filtering. By this means taunin is entirely separated from the product, and other substances whose color masks the salicylic acid reaction are often removed.

Delicacy of the Ferric Chloride Test. - Using fresh 1 per cent ferric chloride as reagent the test is dęlicate in our hands to a dilution of about $1: 400,000$ when applied to 10 cc. of solution, about $1: 750,000$ to $1: 1,000,000$ if $25 \mathrm{cc}$. of solution be tested. The violet color obtained with such small amounts of salicylic acid must be observed quickly, as it fades rapidly, passing through a rose-red color. A faint rose color may also be obtained on addition of ferric chloride to solutions containing salicylic acid in amounts too small to show violet reaction.

Interpretation. - The formation of a violet color with ferric chloride is a reaction by no means confined to salicylic acid. Mulliken's tables ${ }^{1}$ include many colorless compounds which give more or less distinctly violet reactions with ferric chloride, and some of these also resemble salicylic acid in solubilities and even in volatility. It is not safe to assume in testing foods that a constituent volatile with steam, soluble in ether, capable of sublimation and crystallization, and giving a violet reaction with ferric chloride, is necessarily salicylic acid.

Brand $^{2}$ found that an extract of caramel malt yielded a substance not salicylic acid which showed all of these properties. This substance he called " maltol."

The same or similar interfering substances have been found in dark beers ${ }^{3}$ and in solid foods consisting partly of baked cereal products. 4

1 Identification of Pure Organic Compounds.

${ }^{2} Z$. ges. Brauw., 15, 303 ; and Ber., 27, 806.

${ }^{3}$ Abraham : J. de Pharm. de Liege, 1898, 5, 173 ; Z. Nahr.-Genussm., 1, 157.

4 Backe: Ann. de falsifications, Nov., 1909. Sherman: J. Ind. Eng. Chem., 2, 24. Backe: Compt. rend., 150, $540 ; 151,78$. 
Among the tests for salicylic acid, other than the ferric chloride reaction, are the formation of the methyl ester or the nitro-compound, the reactions with bromine water and with Millons's reagent, and the Jorissen test.

The adoption by Mulliken of the methyl-ester and nitration tests for the identification of salicylic acid is sufficient evidence of their value for cases in which enough salicylic acid is involved to make them available; but these tests and also the test with bromine water seem not to be sufficiently delicate for the detection of very small amounts.

The Millon and Jorissen tests, however, are very delicate, and should be commonly used to confirm the findings of the official ferric chloride test.

\section{Test with Millon's Reagent ${ }^{1}$}

To 10 to 20 cc. of the final aqueous solution to be tested, add 2 drops of Millon's reagent (prepared as described in Chapter $\mathrm{XV}$ ), mix by shaking, and immerse in boiling water for 45 minutes unless a sufficient color develops in a shorter time. In the presence of salicylic acid a red or pink color is obtained.

By heating, if necessary, for as long as 45 minutes, this test is made so delicate that with practice and with blank tests for comparison no difficulty was found in detecting the presence of 1 part salicylic acid in 2,000,000 of water when 20 cc. were tested; when only 10 cc. were tested, the pinkish tint was barely perceptible at this dilution. Longer heating and variations in the amount of reagent added were tried without appreciably altering the result. The limit of delicacy of the test with Millon's reagent as here used seems, therefore, to be reached by heating in boiling water for 45 minutes and to lie at a dilution of about 1:2,000,000.

The Millon reaction also has the advantage over the ferric chloride test that the color produced even with very small amounts of salicylic acid shows no evidence of fading on standing overnight; but on account of the large number of sub-

${ }^{1}$ Sherman and Gross : J. Ind. Eng. Chem., 3, 492. 
stances which respond to the Millon reagent ${ }^{1}$ it seems unlikely that this reaction will prove as useful as that of Jorissen.

\section{Jorissen Test ${ }^{2}$}

This test in its original form is as follows:

To the solution to be tested add 4 or 5 drops of a 10 per cent solution of potassium (or sodium) nitrite, 4 or 5 drops of acetic acid, 1 drop of a 10 per cent solution of copper sulphate, and heat to boiling. In the presence of salicylic acid the solution turns reddish and with more than a very minute amount becomes blood-red. Jorissen found that phenol behaved in the same way, but benzoic acid did not. Abraham found that maltol does not give this reaction and recommended it as the most reliable test for salicylic acid.

By diminishing the amount of copper used in the above directions and prolonging the heating, this test can be made much more delicate than at first reported and considerably more delicate than the ferric chloride reaction. The longer heating is necessary to fully develop the characteristic color, at least when only very small amounts of salicylic acid are present, and the reduction in the amount of copper diminishes the slight green color due to the reagent which otherwise may interfere with the more delicate tests.

The modified Jorissen test as now used for very small amounts of salicylic acid is as follows: ${ }^{3}$ Bring the solution to be tested into a test tube, add 4-5 drops of 10 per cent sodium or potassium nitrite, 4-5 drops of 50 per cent acetic acid, and 1 drop of one per cent copper sulphate. Shake after addition of each reagent and finally place in a boiling water bath in such a position that the test liquid is completely immersed in the boiling water and allow to stand for 45 minutes, then

1 Vaubel: Z. angew. Chem., 1900, 1125. Nasse: Pflüger's Arehiv f. d. ges. Physiol., 83, 361 (1901). Mann: Physiological Histology, pp. 321-323, and Chemistry of the Proteids, p. 7.

2 Jorissen : Bulletins de l'Academie Royal des Sciences, etc., Belgique, 3d series, 3, 259. Sherman : J. Ind. Eng. Chem., 2, 24. Sherman and Gross: Ibid., 3, 492 .

${ }^{3}$ Sherman and Gross : J. Ind. Eng. Chem., 3, 492. 
remove, allow to cool, and examine against a white background, viewing the tube both vertically and horizontally and comparing with a blank test in which the same amounts of reagents have been added to pure water.

In this way, the presence of as little as 0.005 to 0.01 milligram of salicylic acid in pure water solution can be detected. Faint but perceptible reactions were obtained with 5 to $8 \mathrm{cc}$. of a solution of $1: 1,000,000$ and with 18 to $25 \mathrm{cc}$. of solutions of $1: 3,000,000$ to $1: 3,500,000$.

No advantage has been found in a brine bath over a water bath, in longer heating than 45 minutes, nor in varying the amounts of nitrite and acetic acid used. When larger amounts of salicylic acid are present, a drop of stronger copper sulphate solution may be used, up to a 10 per cent solution as originally recommended. Except with very small amounts of salicylic acid the red color of the Jorissen reaction develops quickly on heating, and the long immersion in the water bath then becomes unnecessary if only qualitative results are required.

A feature which will be of great importance in colorimetric estimations of small amounts of salicylic acid is that while the violet color of the ferric chloride test fades rapidly, the red color of the Jorissen test is quite stable. Even the faint colors obtained by long heating, where only very minute amounts of salicylic acid are involved, have shown no deterioration when allowed to stand overnight.

It may also be noted that the ferric chloride and Jorissen tests may be applied to the same portion of solution. After making the ferric chloride test the solution is cautiously diluted with water until the violet color just disappears and then very carefully submitted to the modified Jorissen test, when if salicylic acid is present a pink color will appear.

Maltol, isomaltol, orcin, arbutin, resorcin, phlorizin, and methyl-ethyl-aceto-acetate (all of which are among the substances giving blue, violet, or violet-red colors with ferric chloride) do not respond to the Jorissen reaction.

Phenol gives about the same color as salicylic acid in both the Millon and the Jorissen tests, but the limits of delicacy are 
quite different. Phenol can be detected by the Millon reaction to about $1: 2,000,000$. In the Jorissen test, phenol $1: 100,000$ gives practically the same color as salicylic acid $1: 1,000,000$.

Saligenin gives, in the Jorissen reaction, a red color at $1: 10,000$; a yellowish tint at $1: 100,000$; no . reaction at $1: 1,000,000$. The limit of delicacy for the ferric chloride reaction with saligenin lies between $1: 10,000$ and $1: 20,000$.

2-oxy-isophthalic acid gives the Jorissen reaction up to a dilution of $1: 100,000$ but is easily distinguished from salicylic acid in the color which it gives with ferric chloride.

\section{Benzoic Acid and Benzontes}

The methods of the Association of Official Agricultural Chemists ${ }^{1}$ are as follows :

\section{Qualitative Detection}

Separate benzoic acid as directed for salicylic acid. If benzoic acid be present in considerable quantity, it will crystallize from the evaporated ether in shining leaflets with characteristic odor on heating. Dissolve the residue in hot water, divide into two portions $(a)$ and $(b)$, and test by the following methods :

\section{(1) First Method}

Make portion (a) alkaline with ammonium hydroxide, expel the excess of ammonia by evaporation, take up the residue with water, and add a few drops of a neutral 0.5 per cent solution of ferric chloride. The presence of benzoic acid will be indicated by the formation of a brownish colored precipitate of ferric benzoate.

\section{(2) Second Method (Mohler's Method Modified)}

Add to the water solution (portion $b$ ), prepared as described above, from 1 to $3 \mathrm{cc}$. of third-normal sodium hydroxide and evaporate to dryness. To the residue, add 5 to 10 drops of concentrated sulphuric acid and a small crystal of potassium nitrate. Heat for 10 minutes in glycerol bath at $120^{\circ}$ to $130^{\circ} \mathrm{C}$., or for 20 minutes in a boiling water bath. This causes the formation of meta-di-nitro-benzoic acid. In no case must the temperature exceed $130^{\circ} \mathrm{C}$. After cooling, add $1 \mathrm{cc}$. of water, and make decidedly ammoniacal; boil the solution, to break up any ammonium nitrite which may have been formed. Cool and add a drop of fresh colorless ammonium sulphide, without allowing the layers to mix. A red-brown ring indicates benzoic acid. This is due to

${ }^{1}$ U. S. Dept. Agriculture, Bur. Chem., Bul. 107, Revised, p. 181; and Bul. 137, pp. 110-112, 113, 117-118. 
the formation of ammonium meta-di-amido-benzoic acid. On mixing, the color diffuses through the whole liquid; on heating it finally changes to greenish yellow, owing to the decomposition of the amido acid. This furnishes a means of distinguishing benzoic acid from salicylic or cinnamic acids. Both the latter form amido compounds, which are not destroyed by heating. The presence of phenolphthalein interferes with this test.

\section{Quantitative Estimation}

\section{General Method of Preparation}

Grind in a sausage machine, if solid or semisolid, thoroughly mix the sample, and transfer a convenient quantity (about 150 grams) to a 500-cc. graduated flask. Add enough pulverized sodium chloride to saturate the water in the sample, render alkaline with sodium hydroxide or milk of lime, and dilute to the mark with a saturated salt solution. Allow to stand for at least two hours with frequent shaking, and filter. If the sample contains large amounts of matter precipitable by salt solution, it is advisable to follow a method similar to that given under "Salt or dried fish." When alcohol is present, follow the method given under "Cider and similar products containing alcohol." Where large amounts of fats are present, it is well to make an alkaline extraction of the filtrate before proceeding as directed under Extraction and Titration. The following will illustrate the manner of applying the method to various classes of food products:

\section{Special Methods of Preparation}

Ketchup. - To 150 grams of the sample add 15 grams of pulverized sodium chloride and transfer the mixture to a 500-cc. graduated flask, using about $150 \mathrm{cc}$. of a saturated solution of sodium chloride for rinsing. Make slightly alkaline to litmus paper with strong sodium hydroxide and complete the dilution to $500 \mathrm{cc}$. with saturated salt solution. Allow to stand at least two hours with frequent shaking and then filter through a large folded filter. If any difficulty is experienced, the mixture may be centrifuged or squeezed through a muslin bag before filtering.

Jellies, Jams, Preserves and Marmalades. — Dissolve 150 grams of the sample in about $150 \mathrm{cc}$. of saturated salt solution and add 15 grams of pulverized sodium chloride. Render alkaline to litmus paper with milk of lime. Transfer to a 500-cc. graduated flask and dilute to the mark with saturated salt solution. Allow to stand at least two hours with frequent shaking, centrifuge if necessary, and filter through a large folded filter.

Cider and Similar Products containing Alcohol. - Render 250 cc. of the sample alkaline to litmus paper with sodium hydroxide and evaporate on the steam bath to about $100 \mathrm{cc}$. Transfer the sample to a 250-cc. flask, add 30 grams of pulverized sodium chloride, and shake until dissolved. Dilute to the 
original volume, $250 \mathrm{cc}$, with saturated salt solution, allow to stand at least two hours with frequent shaking, and filter through a folded filter.

Salt or Dried Fish. - Transfer 50 grams of the ground sample to a 500-cc. flask with water. Make slightly alkaline to litmus paper with strong sodium hydroxide and dilute to the mark with water. Allow to stand at least two hours with frequent shaking and then filter through a folded filter. Pipette accurately as large a portion of the filtrate as possible (at least $300 \mathrm{cc}$.) into a second 500-cc. flask. Add 30 grams of pulverized sodium chloride for each $100 \mathrm{cc}$. of solution. Shake until the salt has dissolved and dilute to the mark with saturated salt solution. Mix thoroughly and filter off the precipitated protein matter on a folded filter.

\section{Extraction and Titration}

Pipette a convenient portion of the filtrate (100 to 200 cc.), obtained as above, into a separatory funnel. Neutralize the solution to litmus paper with hydrochloric acid $(1: 3)$ and add an excess of $5 \mathrm{cc}$. of the same acid. In the case of salt fish a precipitation of protein matter usually occurs on acidifying, but the precipitate does not interfere with the extraction. Extract carefully with chloroform, using successive portions of $70,50,40$, and $30 \mathrm{cc}$. To avoid emulsion shake each time cautiously (vigorous shaking is not necessary). The chloroform layer usually separates readily at the bottom of the funnel after standing a few minutes. If any emulsion forms, it can be broken up by stirring the chloroform layer with a glass rod. If this is unsuccessful, the emulsified portion may be drawn off into a second funnel and given one or two sharp shakes from one end of the funnel to the other. If this also fails, the emulsion should be centrifuged for a few moments. As this is a progressive extraction great care must be taken to draw off as much of the clear chloroform solution as possible after each extraction, but under no circumstances must any of the emulsion be drawn off with the chloroform layer. If care is taken not to draw off any of the emulsion, it is unnecessary to wash the chloroform extract.

Transfer the combined chloroform extract to a porcelain dish, rinsing the container several times with a few cubic centimeters of chloroform, and evaporate to dryness at room temperature in a current of dry air. (See note.) Dry the residue overnight (or until no odor of acetic acid can be detected in case the product is a ketchup) in a sulphuric acid desiccator. Dissolve the residue of benzoic acid in neutral alcohol ( 30 to $50 \mathrm{cc}$.), add about one fourth this volume of water, a drop or two of phenolphthalein solution, and titrate with twentieth-normal sodium hydroxide; 1 cc. of twentieth-normal sodium hydroxide $=0.0072$ gram anhydrous sodium benzoate.

Note. - If a blast is convenient, it is preferable to evaporate the whole extract at room temperature. For this purpose the following simple apparatus may be used: A wide-mouth salt bottle is fitted with a cork; a glass tube extends through the center of the cork to the bottom of the bottle, and its 
upper end is attached to the blast by a rubber tube. As many other glass tubes as convenient are passed through the cork around the central tube. These terminate just inside the cork, and outside the cork are bent outward and downward at an angle of about $45^{\circ} \mathrm{C}$. The bottle is filled with calcium chlorid and by this means a current of dry air can be delivered to the dish containing the extract. In the absence of a blast an electric fan may be used for evaporating the extract.

If it is impracticable to evaporate the chloroform spontaneously or by means of a blast it may be transferred from the separatory funnel to a 300-cc. Erlenmeyer flask, rinsing the separatory funnel three times with 5 or $10 \mathrm{cc}$. of chloroform. Distill very carefully to about one-fifth the original volume, keeping the temperature down so that the chloroform comes over in drops, not in a steady stream. Then transfer the extract to a porcelain evaporating dish, rinsing the flask three times with 5 or $10 \mathrm{cc}$. portions of chloroform and evaporate to dryness spontaneously.

\section{SACCHARIN}

The usual method of testing for saccharin is to extract it by means of ether, then convert it into sodium salicylate by heating with sodium hydroxide, and finally apply the test for salicylate. The sweet taste of the saccharin serves as a preliminary test for its presence in the ether extract.

The recent ruling under the Federal Food and Drugs Act against the presence of saccharin in foods adds greatly to the significance of its presence or absence. The analyst therefore should not only follow the details of the official method with care in order to avoid confusing saccharin with " false saccharin" or salicylic acid, but should also consult the original papers on both saccharin and salicylic acid which are given in the references at the end of this chapter.

The qualitative method and precautionary notes of the Association of Official Agricultural Chemists are as follows :

Extract with ether (after maceration and exhaustion with water, if necessary), as described under salicylic acid. Allow the ether extract to evaporate spontaneously and note the taste of the residue. The presence of saccharin to the amount of $20 \mathrm{mg}$. per liter is indicated by a sweet taste. This may be confirmed by heating with sodium hydroxide, as described below, and detecting the salicylic acid formed thereby. Results by this method indicating the presence of a faint trace of saccharin in wines which did not contain it have been frequently obtained, owing to the presence in wine of so-called "false saccharin." 
Acidify 50 cc. of a liquid food (or the aqueous extract of 50 grams of a solid or semisolid, prepared as directed in the official method for salicylic acid as given above) and extract with ether. Test the extracted matter in the usual way for salicylic acid, return the gasoline extract to the dish containing the residue, dilute the whole to about $10 \mathrm{cc}$. volume, and add $2 \mathrm{cc}$. of sulphuric acid $(1: 3$.) Bring the solution to the boiling point and add a 5 per cent solution of potassium permanganate, drop by drop, to slight excess; partly cool the solution, dissolve in it a piece of sodium hydroxide, and filter the mixture into a silver dish (silver crucible lids are well adapted to the purpose); evaporate to dryness and heat for 20 minutes at $210^{\circ}$ to $215^{\circ} \mathrm{C}$. Dissolve the residue in water, acidify and extract with ether, evaporate the ether, and test the residue with two drops of a 2 per cent solution of ferric alum. By this method all the so-called false saccharin and the salicylic acid naturally present (also added salicylic acid when not present in too large amount) are destroyed, while $5 \mathrm{mg}$. of saccharin per liter is detected with certainty.

\section{BetA-NAPHTHOL ${ }^{1}$}

Extract 200 cc. of the sample (or of its aqueous extract prepared as on page 378 ) with $10 \mathrm{cc}$. of chloroform in a separatory funnel, add a few drops of alcoholic potash to the chloroform extract in a test tube, and place in a boiling water bath for two minutes. The presence of beta-naphthol is indicated by the formation of a deep blue color, which changes through green to yellow.

\section{Abrastol ${ }^{1}$ \\ (Calcium $a$-mono-sulphonate of $\beta$-naphthol)}

(a) Sinibaldi's Method ${ }^{2}$

Make 50 cc. of the sample alkaline with a few drops of ammonium hydroxide and extract with $10 \mathrm{cc}$. of amyl alcohol (ethyl alcohol is added if an emulsion is formed). Decant the amyl alcohol, filter if turbid, and evaporate to dryness. Add to the residue 2 cc. of a mixture of equal parts of strong nitric acid and water, heat on the water bath until half of the water is evaporated, and transfer to a test tube with the addition of $1 \mathrm{cc}$. of water. Add about 0.2 cc. of ferrous sulphate and an excess of ammonium hydroxide, drop by drop, with constant shaking. If the resultant precipitate is of a reddish color, dissolve it in a few drops of sulphuric acid, and add ferrous sulphate and ammonium hydroxide as before. As soon as a dark-colored or greenish precipitate has been obtained, introduce $5 \mathrm{cc}$. of alcohol, dissolve the precipitate in sulphuric acid, and shake the fluid well and filter. In

1 The methods given are those adopted by the Association of Official Agricultural Chemists. U. S. Dept. Agriculture, Bur. Chem., Bul. 107, Revised.

${ }^{2}$ Moniteur scientifique, 1893 (4), 7, 842. 
the absence of abrastol this method gives a colorless or light yellow liquid, while a red color is produced in the presence of 0.01 gram of abrastol.

\section{(b) Sanglé-Ferrière's Method ${ }^{1}$}

Boil $200 \mathrm{cc}$. of the sample with 8 cc. of concentrated hydrochloric acid for one hour in a flask with a reflux condenser attached. Abrastol is thus converted into beta-naphthol and is detected as directed above.

\section{SuCROL OR DULCIN ${ }^{2}$}

(para-phenetol carbamid)

\section{(a) Morpurgo's Method ${ }^{3}$}

Evaporate about $100 \mathrm{cc}$. of the sample (or of the aqueous extract prepared as directed on page 378) to a sirupy consistency after the addition of about 5 grams of lead carbonate, and extract the residue several times with alcohol of about 90 per cent; evaporate the alcohol extract to dryness; extract the residue with ether, and allow the ether to evaporate spontaneously in a porcelain dish. Add 2 or 3 drops each of phenol and concentrated sulphuric acid and heat for about 5 minutes on the water bath; cool; transfer to a test tube and pour ammonium hydroxide or sodium hydroxide over the surface with the least possible mixing. The presence of dulcin is indicated by formation of a blue zone at the plane of contact.

\section{(b) Jorissen's Method ${ }^{4}$}

Suspend the residue from the ether extract obtained as directed above in about 5 cc. of water; add from 2 to $4 \mathrm{cc}$. of an approximately 10 per cent solution of mercuric nitrate, and heat from 5 to 10 minutes on the water bath. In the presence of sucrol a violet-blue color is formed, which is changed to a deep violet by the addition of lead peroxide.

In the foregoing selection of methods for the detection of food preservatives, preference has been given to those which have been adopted by the Association of Official Agricultural Chemists, whose methods are usually accepted as standard in questions relating to adulteration of food. Other methods, however, should not be neglected. It is believed that the following references will put the reader in touch with the most important literature.

1 Comp. rend., 1893, 117, 796.

2 Method of the Association of Official Agricultural Chemists (loc. cit.).

${ }^{3}$ Z. anal. Chem., 1896, 35, 104.

4 Ibid., p. 628. 


\section{REFERENCES 1}

Allen: Commercial Organic Analysis.

Blyth: Foods, their Composition and Analysis.

LEACH : Food Inspection and Analysis.

Leffyann and Beam: Select Methods in Food Analysis.

U. S. Dept. Agriculture, Bur. Chem., Bul. 107, Revised. Methods of the Association of Official Agricultural Chemists.

\section{II}

1899. Beythien and Hempel: (Determination of Boric Acid and Borax in Meat Products). Z. Nahr.-Genussm., 2, 842.

1901. Pellet: Nature of the Substance giving the Ferric Chloride Reaction; Presence of Salicylic Acid in Pure Wines. Ann.chim. anal., 6, 328; J. Chem. Soc., 80, ii, 701.

Portes and Desmoulieres: Normal Occurrence of Salicylic Acid in Strawberries. Ann. chim. anal., 1901, 6, 401; Z. Nahr.Genussm., 1902, 5, 468.

1902. Arnold and Mentzel: Detection of Formaldehyde. Z. Nahr.Genussm., 5, 353.

Windisch : On the Question of the Occurrence of Salicylic Acid in Natural Wines. Z. Nahr.-Genussm., 5, 653.

1903. Arnold and Mentzel: Detection of Hydrogen Peroxide in Milk. Z. Nahr.-Genussm., 6, 305.

Grunhut: (Review of Methods for Determination of Boric Acid in Foods). $\quad Z$. anal. Chem., 42, 119.

Traphagen and Burke: Occurrence of Salicylic Acid in Fruits. J. Am. Chem. Soc., 25, 242.

Windisch: Natural Occurrence of Salicylic Acid in Strawberries and Raspberries. Z. Nahr.-Genussm., 6, 447.

1904. Allen and Tankard: The Determination of Boric Acid in Cider, Fruits, etc. Analyst, 29, 301.

Beytrien: (Detection of Sulphurous Acid in Foods.) Z. Nahr.Genussm., 8, 36.

Desmovlieres: Action of Ferric Chloride on Salicylic Acid, Methyl Salicylate, Hydrosalicylic Acid, and Some Other Phenol Derivatives. Ann. chim. anal., 8, 85; Z. Nahr.-Genussm., 7, 316.

Farnsteiner: Organically Combined Sulphurous Acid in Foods. Z. Nahr.-Genussm., 7, 449.

1 These references are to literature on the occurrence, detection, and determination of preservatives and do not include articles dealing primarily with their uses and effects. 
KenP: The Behavior of Sulphurous Acid in Foods. Z. Nahr.Genussm., 8, 53.

1905. Lefmann: Detection of Abrastol. Chem. Z $\mathrm{tg}$., 29, 1086.

MAson: The Occurrence of Benzoic Acid Naturally in Cranberries. J. Am. Chem. Soc., 27, 613.

Williams and Sherman: The Detection, Determination, and Rate of Disappearance of Formaldehyde in Milk. J. Am. Chem. Soc., 27, 1497.

1906. Acree: On the Detection of Formaldehyde in Milk. J. Biol. Chem., 2,145 .

Cribb and Arvaud: Detection of Boric Acid. Analyst, 31, 147.

Dubors: Estimation of Salicylic Acid. J. Am. Chem. Soc., 28, 1616.

Frabot: (Detection of Fluorides in Eggs). Ann. chim. anal., 11, $330 ;$ Analyst, 31, 362.

Holley: The Amount of Sodium Sulphite Recoverable from Food Products as a Basis for the Estimation of the Amount Originally Present. J. Am. Chem. Soc., 28, 993.

Horne: Determination of Sulphites in Sugar Products. U. S. Dept. Agriculture, Bur. Chem., Bul. 105.

Kastle: A Test for Saccharin. Public Health and Marine Hospital Service, Hygienic Laboratory, Bul. 26; Review of Amer. Chem. Research, 1906, 331.

Low: Boric acid; Its Detection and Determination in Large and Small Amounts. J. Am. Chem. Soc., 28, 807.

Mentzel: Determination of Sulphurous Acid in Meat. Z. Nahr.Genussm., 11, 320 .

Perrier: Occurrence of Formaldehyde in Foods. Compt. rend., 143, 600 ; Chem. Abs., 1, 205.

Shrewsbury: The Estimation of Preservatives in Milk. Analyst, 32,5 .

Trillat: Production of Formaldehyde in the Caramelization of Sugar. Bull. Soc. Chim., 35, 685; Analyst, 31, 410.

Woodman and Тацвот: The Etching Test for Small Amounts of Fluorides. J. Am. Chem. Soc., 28, 1437.

1907. Alexander: Determination of Sulphurous Acid in Gelatine. $J$. Am. Chem. Soc., 29, 783.

Azzarello: (On the Presence of Boric Acid in Genuine Wine). Gazz. chim. ilal., 36, ii, 575; Chem. Abs., 1, 1040.

Dubors: Determination of Salicylic Acid. J. Am. Chem. Soc., 29, 293.

RAMSEY: The Formation of Formaldehyde in Solutions of Cane Sugar and its Bearing on Hehner's Test for Formaldehyde in Saccharine Mixtures. J. Proc. Royal Soc. N. S. W., 41, 172; Chem. Abs., 3, 126. 
Richardson: Nitrates in Food. J. Am. Chem. Soc., 29, 1757.

Salomone: Test for Abrastol. Giorn. farm. chim., 55, 481 ; Chem. Abs., 1, 1447.

Scoville: Benzoic vs. Cinnamic Acid in Food Analysis. Am. J. Pharm., 79, 549 ; Chem. Abs., 2, 674.

Vitali: Detection of Salicylic Acid in Wine. J. Soc. Chem. Ind., 26, 269.

Winton and BAILeY: The Formation of Volatile Sulphur Compounds in Meat and their Influence on the Detection of Added Sulphites. J. Am. Chem. Soc., 29, 1499.

Woodman and Talbot: Fluorides in Malt Liquors. J. Am. Chem. Soc., 29, 1362.

1908. Carles: (Fluorides in Wines believed to be Pure). Ann. chim. anal., 13, 102 ; Chem. Abs., 2, 1994.

Feder: Detection of Hydrogen Peroxide in Milk. Z. Nahr.-Genussm., 15, 234.

Genersich : Detection and Estimation of Boric Acid, Salicylic Acid, and Benzoic Acid in Foods. $Z$. Nahr.-Genussm., 16, 210.

Gudeman: Determination of Sulphurous Acid in Foods. J. Ind. Eng. Chem., 1, 81.

Hopfgartwer : Reaction between Iron and Salicylic Acid. Monatsh. Chem., 29, 689 ; Chem. Abs., 3, 309.

Lavalle: Detection of Boric Acid in Foods by Means of Turmeric Paper. Chem. Ztg., 32, 816.

LA WALL and Bradshaw: The Quantitative Estimation of Benzoic Acid in Ketchup. Am. J. Pharm., 80, 171; Chem. Abs., 2, 1845.

Mannich and Preiss : Sensitive Test for Detection of Boric Acid in Foods. Chem. Ztg., 32, 314.

PAdé: Determination of Sulphurous Acid in Gelatine. Ann. chim. anal., 13, 299; Chem. Abs., 2, 3250.

Rothenfusser: The Detection of Hydrogen Peroxide, Formaldehyde, and Persulphates. $\quad Z$. Nahr.-Genussm., 16, 589.

Shrewsiury and KNAPP: Rapid Method for Detection and Estimation of Formaldehyde in Milk. Analyst, 34, 12.

Steiger: Estimation of Small Amounts of Fluorine. J. Am. Chem. Soc., 30, 219.

West : The Determination of Benzoic Acid in Tomato Ketchup and Other Food Products. J. Ind. Eng. Chem., 1, 190.

Wilkinson and Peters: Influence of Formaldehyde on the Detection of Hydrogen Peroxide in Milk. Z. Nahr.-Genussm., 16, 515.

Willard: Sulphurous Acid in Green Corn (and other vegetables). Bull. Kansas State Board of Health, 4, 216; Chem. Abs., 3, 87.

Woodman and Burwell: Detection of Formic Acid in Food. Tech. Quart., 21, 1. 
Zerban and Naquin: Determination of Sulphurous Acid in Molasses. U. S. Dept. Agriculture, Bur. Chem., Bul. 116.

1909. BAcke : (Source of Error in Salicylic Acid Test). Ann. falsifications, Nov., 1909.

Carlinfanti and Tuffi: Use and Detection of Fluorides in Tomato Conserves. Arch. farm. sper., 8, 377; Chem. Zentr., 1909, II, 1765.

Fischer and Gruenert: Detection of Benzoic Acid in Meat and Fats. Z. Nahr.-Genussm., 17, 721.

French Official Methods. Verh. kais. Gesundheitsamt., 32, 1292; Chem. Abs., 3, 554 .

Genth : Test for Saccharin in Foods and Beverages. Am.J. Pharm., 81, 536 ; Chem. Abs., 4, 352.

Hillyer: Method for determining Sodium Benzoate in Ketchups or Other Food Materials. J. Ind. Eng. Chem., 1, 538.

Jorgenson: Detection of Saccharin in Beer. Ann. falsifications, 2, 58.

LANGE : Sulphurous Acid in Gelatine. Arb. kais. Gesundheitsamte, 32, 144; Chem. Abs., 3, 2989.

Robrn: Test for Benzoic Acid in Fatty Substances. Ann. chim. anal., 13, 431; Chem. Abs., 3, 1938.

SchwARz and Weber: Quantitative Determination of Formic Acid in Fruit Sirups. Z. Nahr.-Genussm., 17, 194.

Testoni: Estimation of Saccharin in Foods. Z. Nahr.-Genussm., 18, 577 .

U. S. Dept. Agriculture, Bur. Chem., Bul. 116, 12.

Wauters : Detection of Saccharin. J. Soc. Chem. Ind., 28, 733.

1910. BACKE: (Substances mistaken for Salicylic Acid in Ferric Chloride Test). Compt. rend., 150, 540; 151, 78.

Bertainchand and Gauvry: Presence of Boron in Tunisian Wines. Ann. chim. anal., 15, 179; Chem. Abs., 4, 2179.

Bertrand and Agulhon: Determination of Boric Acid. Bul. soc. chim., 7, 90, 125; Chem. Abs., 4, 1439.

CAssel : Estimation of Salicylic Acid by.the Distillation of its Dilute Aqueous Solutions. Chem. News, 101, 289 ; Chem. Abs., 4, 2426. Collins: The Transfer of Boric Acid from Cattle Food to Cows'

Milk. Durham County Council Bul., 3, 21 ; Chem. Abs., 4, 1324. Comanduccr: Detection of Saccharin. Boll. chim. farm., 49, 791;

Chem. Zentrbl., 1910, II, 1951.

Dowzard: Detection and Determination of Sulphurous Acid in Lime Juice. Am. J. Pharm., 81, 561; Chem. Abs., 4, 1348.

Dugast : Presence of Boron in the Wines of Algeria. Compt. rend., 150, 839 ; Chem. Abs., 4, 2974.

Fischer and Greunert : Detection of Benzoic Acid in Meat and Fats. Z. Nahr.-Genussm., 20, 580. 
Greibel: Benzoic Acid in Cranberries. Z Z Nahr.-Genussm., 19, 241. Hubert : Disappearance of Sulphurous Acid (when added to wine). Ann. chim. anal., 14, 453; Chem. Abs., 4, 1080.

Kickton and Behrcke: Occurrence of Fluorine in Wines. $Z$. Nahr.-Genussm., 20, 193.

KüHN and RüHLE: Determination of Sulphurous Acid in Meats. Z. Nahr.-Genussm., 20, 10; Chem. Abs., 4, 2851.

PAwlowski: Detection of Saccharin in Beer. Z. ges. Brauw., 32, 281 ; Chem. Abs., 4, 948.

Pellet : Normal Presence of Salicylic Acid in Wines. Rev. soc. hyg. alim., 5, 806 ; Chem. Abs., 4, 1647.

Perrier: Presence of Formaldehyde in Certain Foods. Rev. soc. hyg. alim., 5, 804; Chem. Abs., 4, 1635.

Rosset : Detection of Fluoride in Foods. Ann. chim. anal., 14, 365 ; Chem. Abs., 4, 623.

Shermax: A Source of Error in the Examination of Foods for Salicylic Acid. J. Ind. Eng. Chem., 2, 24.

VoN DER HEIDE and JAKoB: The Detection of Benzoic, Cinnamic, and Salicylic Acids in Wine. Z. Nahr.-Genussm., 19, 137.

Von Fellenberg: Determination of Salicylic Acid in Preserves. Z. Nahr.-Genussm., 20, 63.

Yoder and TAggart: Occurrence of Formaldehyde in Sugar Cane Juice and Sugar-House Products. J. Ind. Eng. Chem., 2, 260.

1911. Fincke: Determination of Formic Acid in Foods. Z. Nahr.Genussm., 21, 1.

Folin and Flanders: Determination of Benzoic Acid. J. Am. Chem. Soc., 33, 1622.

Franzen and EgGer: Quantitative Determination of Formic Acid. J. prakt. Chem., 83, 323.

Lythgoe and Marsh: The Detection of Benzoic Acid in Coffee Extract. J. Ind. Eng. Chem., 3, 842.

Loock: Preservatives in Fruit Juices with Special Reference to the Detection of Formic Acid. $Z$. öffentl. Chem., 16, 350; Chem. Abs., 5. 537.

Polknske: Detection of Benzoic Acid in Food. Arb. kais. Gesundh., 38, 149 ; Chem. Abs., 5, 3860.

Polenske and Köpкe: Determination of Saltpeter in Meat. Arb. kais. Gesundh., 36, 291 ; Chem. Abs., 5, 1802.

Price and Ingersoll: Effects of Nitrates and Nitrites on the Turmeric Test for Boric Acid. U. S. Dept. Agr., Bur. Chem., Bul. 137, 115.

Sherman and Gross: The Detection of Salicylic Acid. J. Ind. Eng. Chem., 3, 492.

Vierhout: Quantitive Estimation of Salicylic Acid in Fruit Juices. Z. Nahr.-Genussm., 21, 664 . 



\section{SUBJECT INDEX}

Abbé refractometer, 165.

Abderhalden and Koelker's optical method for proteolytic enzymes, (ref.) 332.

Abraham's viscosity method, 183.

Abrastol, 389, 390, (ref.) 392-393.

Absolute alcohol, 2, 32, 33.

Acetanilid, determination in vanilla extract, (ref.) 49.

Acetate, 129, (ref.) 131-132.

Acetic acid, 129, (ref.) 131-132 ; determination in calcium acetate, 129131 ; in vinegar, 128.

Acetin method for glycerol, 279.

Acetone determination, 30 .

Acetylizable impurities in glycerin, 283.

Acetyl number, 144, 160.

Acid, arachidic, 134, 179, 180 ; behenic, 134 ; benzoic, 385,386 ; boric, 373 , 374 ; butyric, 133,195 ; capric, 133 , 195; caproic, 133,195 ; caprylic, $133 ; 195$; carnaubic, 134,139 ; cerotic, 135; clupanodonic, 137; dihydroxystearic, 137, 195; erucic 135; hypogæic, 135; isolinolenic, 137 ; lanoceric, 138; lauric, 134, 195 ; lignoceric, 134 ; linoleic, 136 ; linolenic, 137, 139; linusic, 137; melissic, 135; myristic, 134, 195; oleic, 135, 139, 195, 197 ; palmitic, 134,195 ; phycetoleic, 135 ; ricinoleic, 138; sativic, 137; stearic, 134, 195; sulphurous, 377. (See also Acids.)

Acid-albumin, 311.

Acidity, degrees of, 147.

Acidity of butter fat, 194; of glycerin, 278 ; of lubricating oil, 231 ; of milk, (ref.) 367.

Acid method for phosphorus, 304 .

Acid number, 147.

Acids, fatty, 133, 138, 139, 167, 196 ; fatty and resin, 271 ; in butter fat, 139, 195. (See also Acid.)

Acree's formaldehyde color reaction for proteins, (ref.) 331 .

Added water, detection of, in milk, 363, 364, (ref.) 366-368.

Addition reactions of aldehydes, 35 ; of formaldehyde, 44 .
Adiabatic calorimeter, (ref.) 264.

Adulterants in oils, 174-185, 199-202, 206-210, 213-217.

Albuminates, 311.

Albuminoids, 308, 311.

Albumins, 308, 310.

Alcohol, 2, 31-33; absolute, 2, 32, 33 ; aldehyde-free, 172 ; cetyl, 169 ; denatured, 4, 32, 33; density of solutions, $13,15,16$; detection of, $5,23,32,33$; determination of, $6-24$, $31-33$; fusel oil in, $28,32,33$; methyl alcohol in, 24, 32, 33 ; myricyl, 169; octodecyl, 169; references, 31-33; standards of purity, 29 ; U. S. P., $2,29$.

Alcohol-ether mixtures, analysis of, (ref.) 32 .

Alcohols, 1, 31-33, 169. (See also Alcohol.)

Alcohol-soluble proteins, 308, 310 .

Alcoholysis of fatty substances, (ref.) 172.

Aldehydes, 34, 47, 48, 49; detection of, 34 ; determination of in alcoholic liquors, (ref.) 32 . (See also Benzaldehyde, Formaldehyde, Vanillin.)

Alkali-albumin, 311.

Alkali method for phosphorus, 303; for sulphur, 298.

Alkalinity of vinegar ash, 123, 125, 126; 128.

Allein and Gaud reagent, 78.

Allen method for peptic activity, 327; for protein nitrogen, (ref.) 330 .

Allihn method for dextrose, 107.

Allihn's table for dextrose, 108.

Allspice, starch content of, 112.

Almond extract, determination of benzaldehyde in, 46,49 .

Almond oil, 171.

Almond shells, starch content of, 112.

Alumina cream, 89.

Aluminate in soap, 273.

Ammoniacal silver nitrate test for aldehydes, 34 .

Amyl alcohol, 28, 32, 33.

Amylases, 113-120, (ref.) 121-122.

Angular rotation, 83.

Apple vinegar, 123. 
Arabinosazone, 62, 64 .

Arabinose, 50, 54, 63, 85. (See also Pentosans, Pentoses.)

Arachidic acid, 134, 179, 180, (ref.) 199-201.

Arachis oil, 171, 179, 184, (ref.) 199-201.

Arnold-Wedemeyer method for nitrogen, 289.

Ash : in butter, 186; in coal, 256, 264; in grain products, 337 ; in milk, 356,357 ; in sugar, 97 ; in vinegar, 125.

Asphaltic matter in oils, 222, 235, 238.

Atwater calorimeter, 239.

Azo-compounds, determination of nitrogen in, (ref.) 306.

Balance, Westphal, 162.

Balling hydrometer ("spindle"), 100.

Bang method for reducing sugars, (ref.) 86.

Baobab oils, (ref.) 202.

Bardach's protein reaction, (ref.) 331.

Barfoed's method for reducing sugars, 76.

Barlow's method for sulphur, 296.

Bates polariscope, (ref.) 86.

Baudouin test, 181; influence of rancidity on, (ref.) 202.

Baumé hydrometer, 219.

Baumé scale, 219.

Beef tallow, 170.

Beeswax, 170, (ref.) 173.

Beets, determination of sugar in, 98 .

Behenic acid, 134.

Bellier method for arachidic acid, 181.

Benedict method for reducing sugars, (ref.) 86.

Benedict reagent, 78, (ref.) 86 .

Benzaldehyde, 46, 47, 49.

Benzene, water in, (ref.) 238.

Benzoate, detection and determination, 385, 386, (ref.) 393-395.

Benzoic acid, detection and determination of, 385, 386, (ref.) 391-395; occurrence of, in cranberries, (ref.) 392,395 ; standardization of calorimeter by means of, 242 ; titration of, 387 ; vs. cinnamic acid in food analysis, (ref.) 393.

Berthelot bomb calorimeter, 239.

Berthelot method for sulphur, 301 .

Beta-naphthol, 389.

Bicarbonate, detection of, in milk, 365 .

Bisulphite reaction of aldehydes, 35 .

Biuret reaction, 313 .

Blarez method for fluorides, 375 .

Blasdale's viscosity test, 183.

Boiling point, determination of, 24 .
Boiling point method for alcohol, 22 .

Bomb calorimeter, 239.

Borates, in food, 373, (ref.) 392-395; in soap, 273.

Boric acid, detection and determination, 373-375, (ref.) 391-395; natural occurrence in foods, 375, (ref.) 391395.

Boron in wines, (ref.) 394 .

Bran, starch content of, 112.

British thermal unit, 241.

Brix hydrometers ("spindles"), 100.

Bromine substitution number, 207.

Bromine water test for salicylic acid, 382.

Brugelmann method for sulphur, 296.

Buckwheat hulls, starch content of, 112.

Burning oils, (ref.) 236-237. (See also Illuminating oils.)

Burning point, 233.

Butter, 185; acid content of, (ref.) 200 ; analysis of, 186; detection of coconut fat, 198, (ref.) 202 ; Dutch, (ref.) 200,202 ; iodine number, (ref.) 200 ; keeping qualities of, (ref.) 200; renovated, (ref.) 200; standard, 185; substitutes, 197-202; synthetic color in, (ref.) 202; volatile acid content, 195-197, (ref.) 200; water content, 186-187, (ref.) 201-202.

Butter fat, analysis of, 188-194; composition of, 195-197; references, 199-202; relation of physical and chemical characters, 196 ; separation of acids in, (ref.) 139 ; variations in properties, 195.

Butyric acid, 133.

Butyrin, 146.

Butyro-refractometer, 165.

Cacao butter, 170.

Calcium acetate, 129.

Calorific power, 245; determination of, 239 ; estimated and determined in wood, etc., 253 ; of coal, 257-265; of fatty oils, 171; of fuel oils and gasoline, 247 ; of organic compounds, 245 ; of petroleum oils, 248 ; relation to chemical composition, 257, 260.

Calorimeter, adiabatic, (ref.) 264 ; Atwater, 239; Berthelot, 239; Emerson, 239; Junker, 263 ; Mahler, 239 ; radiation correction, 244 ; references, 261-265.

Calorimetry, 239-265.

Cane, determination of sucrose in, 98 .

Cane sugar, see Sucrose. 
Cane sugar factories, chemical control of, (ref.) 104.

Capric acid, 133, 195.

Caproic acid, 133, 195.

Caprylic acid, 133, 195.

Carbohydrates (general), 50-57; behavior on oxidation, 58; detection of, by Molisch reaction, 57; hydrolysis of, $60,94,106,109,110$; reactions with acids, 56 ; reactions with hydrazines, 61 ; rotation of polarized light, 78-85; separation by pure yeasts, (ref.) 104; separation in grain products, 340 ; specific rotatory powers, 82-85.

Carbonate, in glycerin, 278; in milk, 365 ; in soap, 273.

Carius method, (ref.) 307 .

Carnaüba wax, 170.

Carnaübic acid, 134, (ref.) 139.

Casein determination, (ref.) 368.

Casein test for formaldehyde, 39 .

Castor oil, 141, 142, 171.

Cellulose, 53, 55, 61 .

Cereals, determination of starch in, $106,110,112$, (ref.) 121; loss of phosphorus in ashing, (ref.) 307.

Cereals, see Grain products.

Cerotic acid, 135.

Cetyl alcohol, 169.

Chilling point, 232.

Chinese wood oil, 204, (ref.) 217.

Cholesterol, 169, (ref.) 200; separation from phytosterol, (ref.) 201.

Cider, composition of, (ref.) 132 ; determination of benzoic acid in, 386 .

Cider vinegar, 123-126, (ref.) 132.

Cinnamic acid, detection of, (ref.) $393,395$.

Citral, determination of, (ref.) 49.

Clarification in sugar analysis, 79, 89, 90, 92, (ref.) 105.

Clässon's method for sulphur, 296.

Clerget method for sucrose, 94 .

Cleveland cup tester, 233.

Cloud test, 232, (ref.) 236.

Cloves, starch content of, 112.

Clupanodonic acid, 137.

Coagulated proteins, 312 .

Coal, 254-265; accuracy in sampling, (ref.) 263; American, (ref.) 262; calorific power, 254-265; classification, (ref.) 261,262 ; composition in relation to calorific power, 254-260, (ref.) 262-265 ; deterioration of samples, (ref.) 263; gases occluded in, (ref.) 264 ; influence of oxygen in, 254, (ref.) 264 ; losses in storage, (ref.)
264 ; production of, in 1910, (ref.) 265 ; proximate analysis, 256 ; purchase on specifications, (ref.) 263-265; sampling, (ref.) 263, 265; standard, for gasmaking, (ref.) 264 ; sulphur determination, 257 ; volatile matter, 256, (ref.) 263-265; weathering of, (ref.) 263.

Coals of Illinois, (ref.) 262.

Coals of the United States, (ref.) 263.

Coal tar oils, determination in other oils, (ref.) 236.

Cocoa products, determination of starch in, (ref.) 121.

Cocoa shells, starch content of, 112 .

Coconut fat, detection in butter, 198; ethyl ester number, (ref.) 202.

Coconut oil, 141, 170; detection of, 198, (ref.) 201-202; of high iodine value, (ref.) 202. (See also Coconut fat.)

Codliver oil, 171, (ref.) 200-201.

Coefficient of purity, sce Quotient of purity.

Cold test, 232, (ref.) 236.

Collagens, 311.

Colophony, 170, (ref.) 215.

Color reactions of proteins, 313-316.

Colza oil, 171.

Combustion, heat of, 168, 239, 245, (ref.) 261-265.

Compensator, 81.

Compressed oxygen method for sulphur, 301.

Condensation reactions of aldehydes, $42,46,48$.

Condensed milk, analysis of sweetened, (ref.) 368 .

"Constants," analytical, of oils, fats, and waxes, 143, 170, 171, 176, 196.

Copak oil, 178.

Copper as protein precipitant, 317 .

Corn stover, starch content of, 112.

Cottonseed meal, influence of feeding, on fat, (ref.) 200.

Cottonseed oil, 171, 177, 178, 184, 208, (ref.) 199-202.

Cottonseed stearin, 170.

Coumarin, determination in vanilla extract, (ref.) 49 .

Cream, 267, 268.

Creosote, characteristics of, (ref.) 217.

Crismer's test, 194.

Crude fiber, 337.

Crude petroleum, 218, (ref.) 237. (See also Petroleum.)

Cyanide method for formaldehyde, 44 . 
Cylinder oils, (ref.) 236.

Cylinder stock, 221.

Defren's method for reducing sugars, 74 .

Degrees of acidity (fats), 147.

Denatured alcohol, 4.

Density: of alcohol solutions, 13, 15, 16 ; of sugar solutions, 100 ; of water, 17 .

Detergents, see Soap.

Dextrin, 53, 61, 85.

Dextrose, 51; detection, 62-68, 77 ; determination, 70, 74, 107 ; reducing power, $69,73,75$; rotating power, 84, 85, (ref.) 105.

Diabetic foods, (ref.) 348.

Diabetic sugar, 51. (See also Dextrose.)

Diastase, see Amylaises.

Diastase method for determination of starch, 110.

Diastatic power, 113-120, (ref.) 121122.

Dichromate method for alcohol, 23; for glycerol, 284.

Dichromate solution as light filter, 93.

Dinitrobenzoate test for alcohol, 5.

Dioxystearie acid, 137, 195.

Diphenyl hydrazine as reagent for sugars, 63.

Dirt in milk, (ref.) 368.

Disaccharides, 50, 51.

Distillation method for alcohol, 8 ; for gasoline, 249 ; for petroleum, 220.

Distilled vinegar, 124.

Drying oils, 203.

Dry lead clarification of sugar solutions, 90, (ref.) 104.

Dulcin, 390.

Dulong's formula, 252, 254.

Dyer method for nitrogen, 291.

Ebullioscope, 22.

Edestin, 339.

Edible oils and fats, 174.

Elaidin test, 136.

Elastins, 311.

Emerson calorimeter, 239, (ref.) 263.

Engler distillation test, 220.

Engler flask, 220.

Engler viscosimeter, 226, (ref.) 237, 238.

Enzymes, amylolytic, 113-120, (ref.) 121-122; proteolytic, 323-328, (ref.) $329,331-333$.

Erucic acid, 135.

Eschka method for sulphur, 257.

Essential oils, aldehydes and ketones in, (ref.) 48,49 .
Ester number, 147.

Esters, glyceryl, 143; in alcoholic liquors, (ref.) 32 ; in wood alcohol, 31; saponification data of, 146.

Ether-alcohol mixture, analysis of, (ref.) 32.

Ether extract, see Fat.

Ethyl ester number, (ref.) 202.

Evaporation test for oils, 235.

Factories, cane sugar, chemical control, (ref.) 104.

Fat, analysis, 143-217 ; determination, $268,335,354,358$. (See also Butter, Olive Oil, etc.)

Fats, 140-217; acetyl number, 160; alcohols of, 169 ; analytical methods, 143 ; analytical properties, 170,171 ; characteristics of animal, 169, (ref.) 172,173 ; classification, 141 ; "constants" of, $143,170-171$; edible, 174 ; heat of combustion, 168,171 ; index of refraction, $164,170,171$, $176,196,211$; iodine number, $148-157,170,171,176,197,211$; Maumené number, $157,171,176$, 211 ; melting point, 144, 167, 170; solubilities, 142 ; specific gravity, $162,170,171$.

Fatty acids, 133-139, 167. (See also Butter, Fats, etc.)

Fatty and resin acids in soap, 269.

Fatty oils in lubricants, 225.

Fehling's method for reducing sugars, 70 . Fehling's solution, 69, 70 .

Ferric acetate as protein precipitant, 317 .

Ferric chloride test for salieylic acid, 381 .

Fish liver oil, (ref.) 201.

Fish oil, 137, 139, 205, 208, 217.

Fixed carbon in coal, 257.

Flashing point, 233, (ref.) 237.

Flour ; analysis of, 334-340 ; composition of, 344-346; starch content of, 112.

Fluoborates, 376 .

Fluorides, 375, (ref.) 392-395.

Fluorine, see Fluorides.

Fluosilicates, 376 .

Folin's method for sulphur, (ref.) 307.

Food preservatives, 369-395.

Foods, see under names of individual articles of food.

Formaldehyde, 36 ; detection of, 38-40, 369-371, (ref.) 391-395; determination, 40-45, 48, 49, 371, (ref.) 392,393 ; influence of, on detection of hydrogen peroxide in milk, (ref.) 393; occurrence in foods, (ref.) $392-395$. 
Formic acid, detection and determination in foods, (ref.) 394-395.

Friction tests on lubricants, 234, (ref.) 237.

Fructose, see Levulose.

Fruit juices, (ref.) 395.

Fruit sugar, see Levulose.

Fuchsin test for aldehydes, 35.

Fuels, 239-265. (See also Coal, etc.)

Furfural, 32, 57.

Fusel oil, 28, 32, 33.

Galactans, 53, 59, 61 .

Galactosazone, 62, 64 .

Galactose, 51, 59, 62, 64, 84, 85 .

Gallic acid test for formaldehyde, 39 .

Gas-making, valuation of oils for, (ref.) 236.

Gasoline, 237, 247, 250.

Gelatin, 311.

Gerard reagent, 78 .

Gliadin, 339, (ref.) 346-348.

Globulins, 308, 310 .

Glucosazone, 62, 64-69.

Glucose, see Dextrose.

Glucose vinegar, 124.

Glutelins, 308.

Glutenin, 339.

Glycerides, 142-143. (See also Fats.)

Glycerin, 276-287. (See also Glycerol.)

Glycerol, 1, 140, 275, 283; determination by acetin method, 279 ; by dichromate method, 284; in soap, 270,287 ; significance in vinegar, (ref.) 132.

Glyceryl esters, 143.

Glycogen, 53, 54, 61, 85.

Glycoproteins, 309, 312.

Grain products, 334-348; analysis of, 334-342 ; composition, 344-346 ; definitions and standards, 343; digestibility and nutritive value, 346 ; references, 346-348.

Grain vinegar, 124.

Grape sugar, see Dextrose.

Grape vinegar, 123.

Graphite, deflocculated, (ref.) 236.

Gray's method for crude petroleum, 221.

Greases, lubricating, 235.

Gunning method for nitrogen, 289, 291.

Half-shade polariscope, 80,81 .

Halogens, as protein precipitants, 316, 319 ; determination in organic substances, (ref.) 306.

Halphen's reaction, 177, (ref.) 199-201.

Hanus method for iodine number, 153, 156.
Heat capacity of calorimeter, 240.

Heat coagulation of proteins, 316 .

Heat of combustion, 239-245; adiabatic method, (ref.) 263; of fats and fatty oils, 168, 171; of fuels, 245265 ; of other organic substances, (ref.) 261.

Heavy distillate, 221.

Heavy metals as protein precipitants, 316.

Hehner number, 144, 148, 191.

Hemicellulose, 61.

Hemoglobins, 309.

Hemp oil, 171.

Herzfeld's method for reducing sugars, 96.

Hexabromide test, 209, 214.

Histones, 308.

Homogenized milk, (ref.) 367 .

Honey, (ref.) 105.

Hopkins distilling head, 292.

Hübl number, 148, 156, 170, 171.

Hydrazine reactions of sugars, 61 .

Hydrazones, determination of nitrogen in, (ref.) 306.

Hydrochloric-acid-casein test for formaldehyde, 39 .

Hydrogen peroxide, detection and determination, 372, (ref.) 391-393; method for formaldehyde, 41.

Hydrolysis of carbohydrates, 60 .

Hydrometer, Baume, 219.

Hydrothermal equivalent of calorimeter, 241.

Hydroxy acids, 137, 138, 161.

Hypogæic acid, 135.

Ice cream, analysis of, (ref.) 367 .

Illuminating oils, 218, 221, (ref.) 236238.

Immersion refractometer, 17-21, 32, 33.

Immiscible solvents, 224.

Imported sugars and molasses, testing of, (ref.) 104.

Index of refraction, 17-22, 164-166. (See also under Fats.)

International methods, for oil testing, (ref.) 237 ; for sugar analysis, 91.

Invertase method for sucrose, (ref.) 105.

Invert sugar, 51,85 .

Iodimetric method for formaldehyde, 40.

Iodine number, 148-157. (See also Fats.)

Iodoform test for alcohol, 5 .

Isolinolenic acid, 137. 
Japan wax, 170.

Jodlbauer method for nitrogen, 290.

Jorissen method for sucrol or dulcin, 390.

Jorissen reaction for salicylic acid, 382 . Junker gas calorimeter, (ref.) 263.

Kapok oil, 178, 202.

Kendall method for reducing sugars, (ref.) 86 .

Keratins, 311.

Kjeldahl method for nitrogen, 288295, 306-307.

Kjeldahl-Wilfarth method, 289.

Koettstorfer number, 144.

Lactobiose, see Lactose.

Lactometer, 353.

Lactosazone, 62, 64.

Lactose, $51,59,61,64$; determination, $59,70,74,361$; hydrolysis of, 52 , 104 ; rotating power, 84 , 85 ; separation from maltose, (ref.) 103 ; solubility, 54,55 .

Lanoceric acid, 138.

Lard, 170, (ref.) 199-202 ; effect of feed upon properties, (ref.) 202.

Lard oil, 141, 171, 182, 184.

Laurent polariscope, 80.

Lauric acid, 134, 195.

"Lead number" of maple products, (ref.) 104 .

Lecithoproteins, 309.

Legler's method for formaldehyde, 43.

Lemon oils and extracts, determination of citral, (ref.) 49.

Leucosin, 339.

Levulinic acid reaction, 58.

Levulose, 51, 84, 85, 103, 105.

Lieben's test for alcohol, 5 .

Liebermann-Storch reaction, 207.

Liebig's method for phosphorus, 303; for sulphur, 296, 297.

Light filter, 93.

Lignites, 253, 262.

Lignoceric acid, 134.

Linoleic acid, 136.

Linolein, 146.

Linolenic acid, 137, 139.

Linolenin, 146.

Linseed meal, starch content of, 112.

Linseed oil, 141, 171, 203, 205, (ref.) $215-216$; vs. paint as priming coat, (ref.) 215 .

Lintner's method for diastatic power, $115,121$.

Lintner's scale, 115, 116.

Linusic acid, 137.
Low's test for boric acid, 374 .

Lubricants, 218, 222-235, (ref.) 236-238.

Lubricating greases, see Lubricants.

Lubricating oils, see Lubricants.

Lux-Ruhemann test for saponifiable oil, 223.

Mace, starch content of, 112 .

Magnesium nitrate method for phosphorus, 305.

Mahler calorimeter, 239.

Maize oil, 171, 182, 184, 199, 200, 204, 208.

Malic acid in vinegar, 125.

Malt extracts, 114,115 .

Maltosazone, 62, 64 .

Maltose, 52, 60, 70, 74, 84-86, 104-105.

Malt sugar, see Maltose.

Malt vinegar, 123.

Mannose, 51, 85.

Maple sugar, (ref.) 104.

Maple sirup, (ref.) 104.

Margarine, 197, 202.

Marine oils, (ref.) 215. (See also Fish oils.)

Maumené number, 144, 157, 159. (See also Fats.)

Meat, determination of starch in, 112 . Melissic acid, 135.

Melting points, 6, 40, 64, 134-135, $144,167,170,180,192,193,202$.

Menhaden oil, 171, 205.

Metaformaldehyde, 37 .

Metaproteins, 309, 311.

Methyl alcohol, 24-26, 30-33.

Methylene blue test for freshness of milk, (ref.) 367.

Methylene-di- $\beta$-Naphthol test for formaldehyde, 40.

Methyl ester test for salicylic acid, 382 .

Methylphenylhydrazine as reagent for sugars, 63.

Mett's method for proteolytic enzymes, $325,331-332$.

Milk, 349-368; analysis of, 352-368; composition of, 349-352, 366-368; condensed, (ref.) 368; detection of added water, $363,364,366-368$; of cane sugar and calcium sucrate, (ref.) $367-368$; of heated, 367 ; of preservatives in, 365 (see also Food preservatives); determination of dirt, (ref.) 368; homogenized, (ref.) 367 ; preservation of, 352, 366, 367 ; sampling, 352 ; serum, 363, 366368 ; standards, $349,364,367$; watered, $363,366-368$.

Milk chocolate, (ref.) 104, 105, 367. 
Milk sugar, see Lactose.

Milk supply, (ref.) 366-368.

Millon reaction, 315,382 .

Mineral oil, 171, 206, 238, 263. (See also Lubricants, Petroleum.)

Mixed oils, viscosity of, 229.

Moisture determination, in butter, 186 ; in grain products, 335 ; in milk, 356 ; in sugar, 97.

Molasses, (ref.) 103-105; as fuel, (ref.) 262 .

Molisch reaction for carbohydrates, 57 .

Monosaccharides, 50.

Morley's alcohol table, (ref.) 3.

Morpurgo's method for sucrol or dulcin, 390.

Mucic acid method, 59.

Multirotation, 79, 86.

Mulliken's test for alcohol, 5 .

Munson and Walker method for reducing sugars, (ref.) 85 .

Mustard oil, 171.

Mutarotation, 79, 86.

Muter's method for fatty acids, 136, 138.

Mutton tallow, 170.

Myricyl alcohol, 169.

Myristic acid, 134, 195.

Naphtha, 221.

Naphthalene, 242.

Neatsfoot oil, 171.

Neumann's method for phosphorus, 304 .

New method for diastatic power, 117120.

New scale for diastatic power, 117-118.

New York Board of Health lactometer, 354.

New York Sugar Trade Laboratory, (ref.) 105.

Nitrates, in food, (ref.) 393 ; in milk, (ref.) 368 ; nitrogen in presence of, 294.

Nitric acid test for oils, 178.

Nitrocompounds, 294.

Nitrogen determination, 288-295, (ref.) 306-307; error due to - methane, (ref.) 307 .

Nitrogen-free extract, 334 .

Nitrogenous extractives, 312 .

Nucleoproteins, 309, 312.

Nutmeg, starch content of, 112.

Oatmeal, definition and standard, 343; starch content of, 112. (For methods of analysis, see Grain products.)

Octodecyl alcohol, 169.

Oil, almond, 171; arachis, 171, 179,
184, (ref.) 199-202; baobab, (ref.) 199-202; castor, 142, 171; coconut, 170,198 , (ref.) 199-202; codliver, 171, (ref.) 199-202; copac, 178; corn, 171,182 , (ref.) 199-202 ; cottonseed, 171, 177, 208, (ref.) 199202 ; fish, 17, 208 ; fish-liver, 171, (ref.) 199-202; groups, 141: hemp, 171; Japanese sardine, 205; kapok, 178, (ref.) 202 ; lard, 171, 182, 184; linseed, 171,203 , (ref.) 214-217; maize, $171,182,184,204,208$, (ref.) 199202 ; menhaden, 171, 205, 208; mineral, 171, 206, 218-238; mixtures, 159, 213, 229; mustard, 171 ; oleo, 171, 197; olive, 171, 174185, (ref.) 199-202; palm, (ref.) 199-202 ; peanut, see Arachis ; poppyseed, 171, 182, 184, 204; rapeseed, 171, 184, (ref.) 199-202; rosin, 171, 207; salad, see Olive; seal, 171; sesame, 171, 181, 184, (ref.) 199202 ; soy bean, 204; sperm, 171; sunflower, 171; tung, 171, 204; walnut, 204. (See also Oils.)

Oil analysis, 140-185, 199-238.

Oil coatings, preservative, for iron and steel, (ref.) 215.

Oils, acetyl number, 160 ; adulterants, 213 (see also under individual oils); altered by age or oxidation, 210 ; analysis (see Oil analysis) ; analytical properties, table, 170-171; blown, 168; bromine absorption, (ref.) 215 ; classification, 141 ; commercial value, 214; committee report on method of analysis, (ref.) 173 ; drying, 141, 203-217 ; edible, 174-202 ; fatty, 140-185, 199-217, 223-226 ; fish, 139,205 ; identification of, 213 ; index of refraction, $164,170-$ 171 ; iodine number, $148-157,170$ 172 ; lubricating, 222-238; Maumené number, 157, 171; " new constant," (ref.) 173; non-drying, 141; olive, from different countries, 175176 ; oxidized, 210 , (ref.) 214-215 ; prices, 214 ; refractometer readings, $164,170-171,172,176,211$; salad, 172,174 , (see also Oil, olive) ; semidrying, 141; specific gravity, 162, 171 ; "unknown," 213; unsaponifiable matter, 169, 173; vegetable, drying, 203 ; viscosity, 168, 226231, (ref.) 236-238.

Oleic acid, 135, 195, 197 ; manufacture and examination of commercial, (ref.) 139. 
Olein, 146.

Oleomargarine, 197.

Oleo oil, 170, 197.

Olive oil, 141, 171, 174-185, (ref.) 199$202 ; 216$.

Optical activity, of carbohydrates, 7885 ; of osazones, $63-64$; of sugars, 88-85; of vinegars, 125.

Optical method for proteolytic enzymes, (ref.) $332,333$.

Organic compounds, composition and calorific power, 245 .

Osazones, 62-69, 306.

Osborne's method for sulphur, 299.

Ost reagent, 78 .

Ostwald pyknometer, 11.

Oxidation, of alcohols, 23; of carbohydrates, 58 ; of oils, 211.

Oxygen calorimeter, 168.

Oxygen method for sulphur, 301 .

Oxymethylene, 37.

Paint, 203-205, 216, 217.

Palmitic acid, 134, 173, 195.

Palmitin, 146, 173.

Palm oil, 170, 200.

Parabrombenzylhydrazide as reagent for sugars, 63 .

Paraffin, 170.

"Paraffin base" petroleum, 221.

Paraffin oils, water in, (ref.) 233.

Paraformaldehyde, 37.

Paraphenetol carbamid, 390.

Pavy's reagent, 78.

Peanut oil, 171, 179.

Peat, (ref.) 263.

Pensky-Martens oil tester, 233.

Pentosans, 54-58, 61.

Pentoses, 50, 57, 58.

Pepper, starch content of, 112.

Pepsin, 324, (ref.) 331-333.

Peptids, 310.

Peptones, 310, 311.

Petroleum, 218-238, 247-251.

Petroleum oils, calorific powers, 248.

Petroleum production, (ref.) 238.

Phenylhydrazine method for benzaldehyde, 46.

Phosphoproteins, 309, 312.

Phosphorus determination, 303-307.

Phosphotungstic acid as protein precipitant, 320.

Phycetoleic acid, 135.

Phytosterol, 162, 172, 183, 194, (ref.) 200-201.

Phytosterylacetate test, 183, 194, (ref.) 200-201.

Picric acid as protein precipitant, 322.
Pine wood oils, (ref.) 216.

Platinum resistance thermometer, (ref.) 263.

Polariscope, 80-94.

Polariscopic methods, 79-105.

Polarization, relation to sugar content, 94.

Polarized light, 78.

Polenske method, 199, (ref.) 202.

Polysaccharides, 50, 52.

Poppyseed oil, 171, 182, 184, 204, 215.

Potassium cyanide method for formaldehyde, 44.

Potatoes, determination of starch in, 106, 112, (ref.) 121.

Preservative coatings for structural materials, (ref.) 216.

Preservatives for food, see Food preservatives.

Proof spirit, 3, 4 .

Protamines, 309.

Proteans, 309.

Proteases, 323, 329, 331-333.

Proteins, 308-323, 329-331 ; coagulated, 310 ; conjugated, 309 ; derived, 309 ; in grain products, 338 ; in milk, 369 ; simple, 308.

Proteolytic enzymes, 323-329, 331333.

Proteolytic power, 324-329, 331-333.

Proteoses, 310, 311.

Pulfrich refractometer, 165.

Purity of sugar solutions, 100.

Pyknometer, 11, 164.

Quartz compensator, 81 .

Quartz wedges, 81 .

Quevenne lactometer, 353, 354.

Quotient of purity, 100.

Radiation correction, 244.

Rafinose, 50, 52, 59, 61, 85, 104.

Rancidity of butter fat, 194.

Rapeseed oil, 141, 171, 184, 202.

Raw sugar, 87, 104.

Rectified spirit, 4 .

Reducing sugars, 69, 85, 86, 96.

Redwood viscosimeter, 227.

Refraction, index of, 17-19, 32-33, 144, $-164,166,170-171,172,176,211$.

Refractometer, $\quad 17-19, \quad 32-33, \quad 165$, 364.

Refractometric methods, 17-19, 32-33, $165,364,367-368$.

Regnault-Pfaundler cooling correction, 244.

Regulations of international commission on sugar analysis, 91 . 
Reichert-Meissl (Reichert-Wollny) number, 144, 148, 188, 196, 200-202.

Renard-Tolman method for arachidic acid, 179.

Resin acids in soap, 271, 287.

Resin and resin oil in linseed oil, 207.

Resinous products in mineral oils, (ref.) 237.

Resorcin test for formaldehyde, 38 .

Ricinoleic acid, 138.

Ricinolein, 146.

Rose method for fluorides, 375 .

Rosin, 170, 207; in shellac, (ref.) 215; in soaps, 271 , (ref.) 287 ; in varnishes, (ref.) 215 ; size, (ref.) 215.

Rosin oil, 171, 207.

Rotation and rotating power, 78-86.

Rotation dispersion, 83.

Rubber, determination of sulphur in, (ref.) 307.

Saccharimeter, 81, 82; observations, unification of, 104.

Saccharin, detection, 388, (ref.) 391395: estimation in foods, 394 .

Saccharose, see Sucrose.

Salad oil, 174-185, (ref.) 199-202.

Salicylic acid, 378-385, (ref.) 391-395 ; action of ferric chloride on, (ref.) 391 ; bromine water test, 382 ; detection, 378-385, (ref.) 393, 395; determination, 378-384 ; (ref.) 391-394 ; ferric chloride test, delicacy, interpretation, 381 ; in pure wines, (ref.) 391,395 ; in strawberries, (ref.) 391 ; in fruits, (ref.) 391; Jorissen test, 382-383; methyl ester test, 382 ; Millon's reagent test, 383 ; reaction with iron, (ref.) 393; substances mistaken for, in ferric chloride test, 381 , (ref.) $394,395$.

Saliva method for determination of starch, 110.

Saltpeter, determination in meat, (ref.) 395.

Sanglé Ferrières method for abrastol, 390.

Saponifiable oil, test for, in lubricating oil, 223.

Saponification, data on pure esters, 146; equivalent, 146 ; number, 144 ; number of butter fat, 191, 196; number (table), 170-171.

Sardine oil, Japanese, 205.

Sativic acid, 137.

Sauer's method for sulphur, 296.

Sausages, determination of starch in, 112.

Seal oil, 171.
Sesame, meal, influence of feeding, on fat, (ref.) 200; oil, 171, 181, 184, (ref.) 199-202.

Shellac analysis, (ref.) 216, 217.

Sidersky reagent, 78 .

Silicate in soap, 273.

Sinibaldi's method for abrastol, 389 .

Sitosterol, 172.

Skeletins, 311.

Smith and Menzies' method for determination of boiling point, 24 .

Soap, 266-275, (ref.) 286-287 ; aluminate in, 273 ; borate in, 273 ; carbonate in, 273 ; chlorides, 271 ; commercial, analysis, 266; determination of water, 267 ; extraction with alcohol, 272; fatty acid content, (ref.) 287; fatty and resin acids, 269,271 ; free alkali or acid, 273 ; glycerol in, 270, (ref.) 287 ; industry, recent development, (ref.) 287; insoluble matter, 274; petroleum ether extract, 268; residue insoluble in alcohol, 273.

Soaps, antiseptic value, (ref.) 287; commercial, composition, (ref.) 287 ; free alkali, (ref.) 286 ; from different glycerides, (ref.) 287; germicidal and insecticidal values, (ref.) 287 ; rosin in, 287; silicate, 273 ; soluble fatty acids, 270; significance in disinfectants, (ref.) 287; total, free, and carbonated alkali, (ref.) 286, 287 ; sugar in, 270.

Soldani reagent, 78 .

Solidifying points of fats, 167.

Solids, of milk, calculation of, 355 ; of milk, determination of, 356 ; of vinegar, 124.

Solution densities of sugars, (ref.) 105.

Sorensen's method for proteolytic power, (ref.) 332.

Soxhlet lactometer, 353-354.

Soy bean oil, 204.

Specific gravity, 11, 112, 133, 134, 162, $170-171,176,185,191,196,353$, 365.

Specific refractive power, 211.

Specific rotating (rotatory) power, 82 , 83.

Specific temperature reaction, 157 ; of olive oils (table), 176.

Spermaceti, 142, 170.

Sperm oil, 142, 171.

Spices, starch content of, 112.

Spirit vinegar, 124.

Spontaneous combustion, test for oils, (ref.) 236. 
Sprigg's method for peptic activity, (ref.) 331.

Standard materials for calorimeter, 241.

Standards, alcohol, 2, 4, 29, 30; butter, 185; grain products, 343 ; milk, 364 ; olive oil, 177; vinegar, 123.

Starch, 52, 61; soluble, 54, 55, 56; content of foods and spices, 112; determination of, 106-113, (ref.) 121 ; determination in cocoa products, (ref.) 121; determination in potatoes, (ref.) 121; hydrolysis of, by acid, 106; hydrolysis of, by enzymes, 110, 113-120, (ref.) 121122 ; rotating power, 85 ; sugar, 51.

Steam-refined cylinder stock, 221.

Stearic acid, 134, 195; glycerides of, (ref.) 173.

Stearin, cottonseed, 170; manufacture, methods, (ref.) 173; saponification number, 146.

Straw, starch content of, 112.

Stutzer method for protein nitrogen, (ref.) 329 ; reagent, 317 .

Sucrol, 390.

Sucrose, 51; Clerget method for, 94 ; combustion, 241; determination by use of invertase, (ref.) 105; determination in beets and cane, 98 ; determination in condensed milk, (ref.) 103; determination in milk, (ref.) 367,368 ; determination in molasses, (ref.) 105 ; determination in presence of commercial glucose (ref.) 103; hydrolysis, 60; hydrolysis, (ref.) 104 ; rotating power, 84,85 ; solubility in alcohol, 55 .

Sugar, see also Sucrose; analysis, 87; beet, optically active non-sugars of, (ref.) 105; beets, analysis of, 98, (ref.) 104, 105; beets, see also Beets; cane, determination of sucrose in, 98 ; in soap, 270 ; manufacture control, (ref.) 104, 105 ; mixtures, analysis of, 101, 102; mixtures, analysis of, (ref.), 85, 86; periodicals devoted to, 103 ; solutions, density, and purity of, 100 ; vinegar, 124, 126, 127.

Sugars, 50-105; behavior on oxidation, 58; toward caustic alkalies, (ref.). 86 ; toward Fehling's solution, 69, (ref.) 86 ; toward hydrazines, 61; estimation by means of refractometer, 101, (ref.) 104, 105; identification, 101; oxidation of, (ref.) 86 ; rotatory powers of, 84,85 ; "unknown," 101. (See also Carbohydrates.)
Sulphite method for benzaldehyde, 46 ; sodium, recoverable from food, (ref.) 392. (See also Sulphites.)

Sulphites, determination in foods, (ref.) 393 ; in gelatin, (ref.) 392, 393, 394 ; in green corn, (ref.) 393 ; in lime juice, (ref.) 394; in meat, (ref.) 392,395 ; in molasses, (ref.) 394 ; in sugar products, (ref.) 392. (See also Sulphurous acid.)

Sulphur, 295-303; compounds, volatile in meat and influence on detection of added sulphites, (ref.) 393 ; determination, 295-303, (ref.) 306-307 ; determination in petroleum, 237; in coal, $257,262,264$.

Sulphurous acid, 377 ; behavior in foods, (ref.) 392 ; detection in foods, (ref.) 391; detection, 377 ; disappearance when added to wine, (ref.) 395 ; organically combined in foods, (ref.) 391 ; quantitative estimation, 377.

Sunflower oil, 171.

Suspended matter in oils, 235.

Suzzi, notes on Maumené number, 160

Tallow, beef, 170; detection of lard, (ref.) 201 ; group, 141 ; mutton, 170.

Tan bark as boiler fuel, (ref.) 264 .

Tannin, as protein precipitant, 321.

Tar oils, (ref.) 217.

Temperature coefficients in polarization of raw sugars, (ref.) 104.

Thomas and Weber's method for proteolytic power, (ref.) 331.

Titer test, 144, 167.

Tocher's test, 182.

Tollen's aldehyde reagent, 34,35 .

Total solids, determination in milk, 356 .

Treasury Dept. methods of sugar analysis, (ref.) 104.

Triazo nitrogen, determination of, (ref.) 307.

Trichloracetic acid, as protein precipitant, 322.

Triglycerides, in butter fat, 195 ; mixed, 143 ; simple, 143.

Trioxy methylene, 37 .

Trisaccharide, $50,52$.

Tryptic activity, 325 .

Tryptophan reaction, 315 .

Tung oil, 171, 204.

Turmeric test for boric acid or borates, 374 ; Low's modification, 374.

Turpentine, (ref.) 215-216; adulterants, (ref.) 215,217 ; benzene in, (ref.) 216,217 ; commercial, of United States, (ref.) 217 ; naphtha in, (ref.) 
216 ; oils, analysis, (ref.) 216 ; sub-। stitutes, (ref.) 215-217; wood, production, etc., (ref.) 217 .

Twitchell's Method for fatty acids, 271 .

Unification, of methods for diastatic power, 120 ; of reducing sugar methods, (ref.) 85 ; of saccharimeter observations, (ref.) 104.

Unsaponifiable matter of fats and waxes, 169, (ref.) 173, 237.

Unsaponifiable oils, determination of, in lubricants, 224.

Vanilla extract, analysis of, (ref.) 49.

Vanillin, determination, 48, 49.

Van Slyke's method for analysis of proteins, (ref.) 331.

Vegetable drying oils, 203.

Ventzke scale, 82,88 .

Vinegar, 123-132, (ref.) 131, 132; analyses of typical, 126 ; determination of source, 124 ; glycerol content of, (ref.) 132 ; identification of, 124; methods of analysis, 127; standards, 123, 132.

Viscosimeter, 226.

Viscosity, 168, 226, (ref.) 236-238; of fats, 144; of illuminating oils, (ref.) 237 ; numbers, 184 ; test, Abraham's modification, 183; test, Blasdale's, 183.

Volatile matter, average relation to calorific power (table), 260; in coke and anthracite, (ref.) 264; of coal, 256.

Volhard's method for proteolytic power, (ref.) 332 .

Walker's rule, 246.

Walnut, oil, 204; shells, starch content of, 112.

Washing powders, disinfectant properties, (ref.) 286.
Water determination, see Moisture.

Water, equivalent of calorimeter, 241 ; density of, 17 ; in butter, 186.

Watering of milk, detection of, 363,364 .

Wax, bees, 170 ; carnaüba, 170, 173 ; Japan, 170.

Waxes, alcohols of, 169 ; analytical properties (table), 170-171; classification, 141; general methods, 140; unsaponifiable matter of, 169.

Welman's reaction, (ref.) 201.

Welter's rule, 246.

Westphal balance, 162.

Whale oil, $142,171$.

Wheat, see Grain products.

Whisky, (ref.) 32,33 .

Wijs method and solution, 152, 156, (ref.) 173.

Wiley's method for melting point, 192, 202.

Wilfarth method for nitrogen, 389 .

Wine vinegar, 123.

Wohlgemuth's method for diastatic power, 116, (ref.) 122; scale, 117, (ref.) 122.

Wood, calorific power, 253; oil, Chinese, 204; oils, Philippine, (ref.), 215; ultimate composition, 252; and similar fuels, 251.

Wood alcohol, requirements for use in denaturing, 30 .

Wright's factors for specific gravity calculations, 164.

Xanthoproteic reaction, 315 .

Xylosazone, 62, 63, 64.

Xylose, 63, 85.

Yeasts, pure, separation of carbohydrates by, 104.

Zeiss refractometer, 165.

Zero-point of polariscope, $80,81,87$.

Zinc sulphate, as protein precipitant, 316 . 
4 
$7 \mathrm{HE}$ following pages contain advertisements of Macmillan books by the same author or on kindred subjects. 



\title{
Chemistry of Food and Nutrition
}

\author{
By HENRY C. SHERMAN, Ph.D. \\ Professor in Columbia University
}

Cloth, I2mo, viii +355 pages, $\$ 1.50$ net

The purpose of this volume is to present the principles of the chemistry of food and nutrition with special reference to the food requirements of man and the considerations which should underlie our judgment of the nutritive values of food. The food is here considered chiefly in its nutritive relations. It is hoped that the more detailed description of individual foods and the chemical and legal control of the food industry may be treated in a companion volume later.

The present work is the outgrowth of several years' experience in teaching the subject to collegiate and technical students who have represented a considerable diversity of previous training and points of view, and, while published primarily to meet the needs of the author's classes, it is hoped that it may also be of service to students and teachers elsewhere and to general readers whose main interests may lie in other fields, but who appreciate the importance of food and nutrition as factors in hygiene and preventive medicine.

While neither the size nor the purpose of this book would permit an historical or technically critical treatment, a limited number of historical investigations and controverted views have been mentioned in order to give an idea of the nature and validity of the evidence on which our present beliefs are based, and in some cases to put the reader on his guard against theories which, while now outgrown, are still sometimes encountered.

\section{THE MACMILLAN COMPANY}

Publishers

64-66 Fifth Avenue

New York 


\title{
Introduction to General Science
}

\author{
By PERCY E. ROWELL, B.Sc.
}

\section{Cloth I2mo 302 pages $\$ .75$ net}

The increasing demand for a thorough, comprehensible, and practical text-book on the subject is met by Rowell's "Introduction to General Science," the distinctive features of which are :

I. The book gives a broad, adequate view of the general field of science. The elements of physics and chemistry receive particular emphasis, but biology, physiography, and several phases of applied science are duly considered.

2. Despite the necessity for treating various sciences, the book is remarkable for its continuity of thought. The interrelation of scientific facts is constantly and clearly pointed out.

3. The treatment is clear-cut, brisk, and thorough, the material well chosen and up to date, while the general workmanship of the book renders it attractive and serviceable.

4. There are over 90 laboratory exercises in the book, a number of them entirely new, and all of them illustrative of the text.

5. Reference reading is encouraged, and lists of carefully selected standard reference books are suggested. Those teachers, however, who do not care to take up detailed work of this sort may give a satisfactory course by using in connection with the book United States Government bulletins alone, to which definite reference is made in the lists of reference readings throughout the book.

\section{TEACHERS}

call the book "thorough," "practical," "comprehensive," "clear," "satisfactory," "a great aid to the science teacher."

Mr. N. P. Lawson of Taunton, Washington, writes :

"I have examined Rowell's 'Introduction to General Science' quite carefully. It wears. The more I use it the better I like it. There is room left for the teacher. He doesn't have to crowd himself in. This book can hardly fail to innoculate any one with the bulletin habit, in which I am a firm believer. In its encouragement of wide reading and first-hand acquaintance with books through the excellent references given, this compact little volume is an education in itself. If I were with your company I should wish to push this book. I believe in it."

\section{THE MACMILIAN COMPANY}

\section{Publishers 64-66 Fifth Avenue New York}





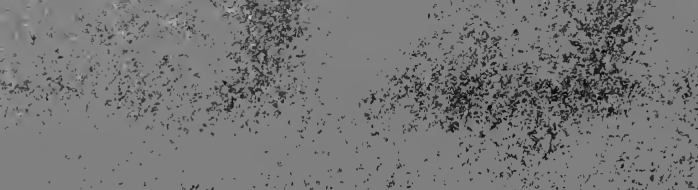

$\because 2$

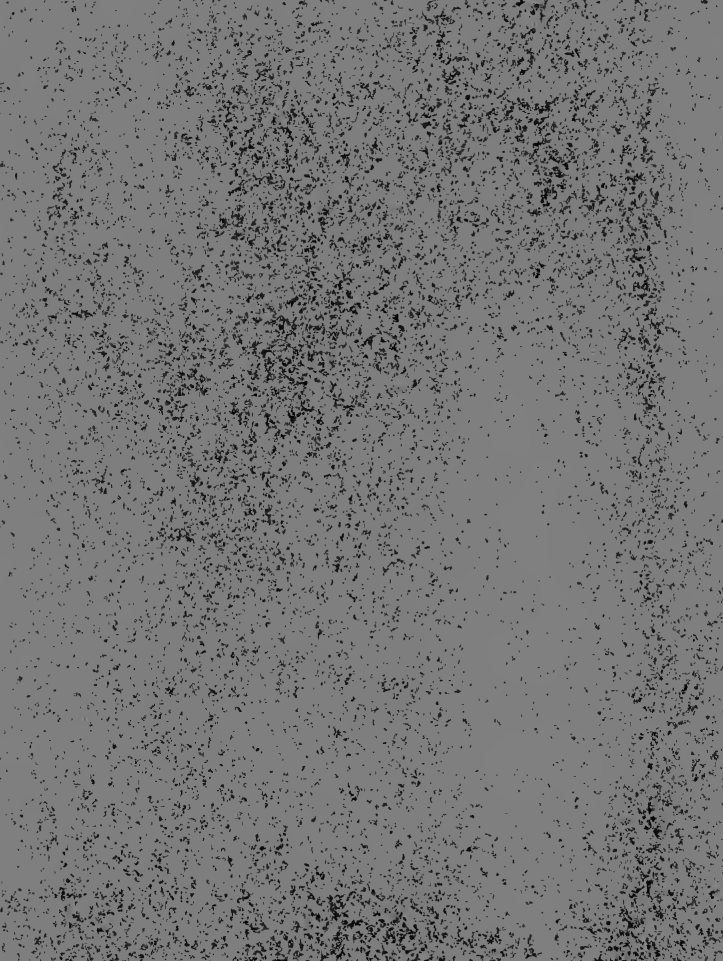

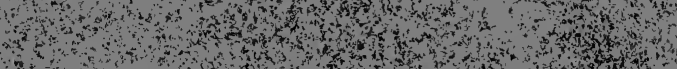

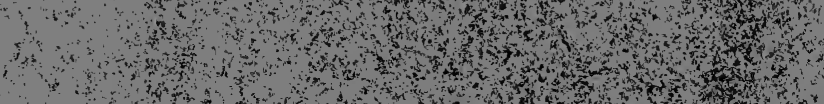
a du

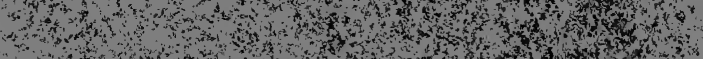

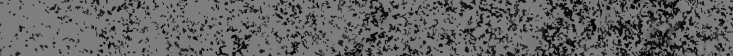

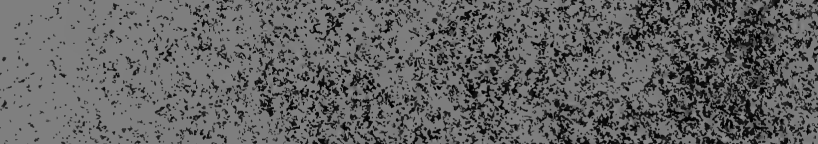

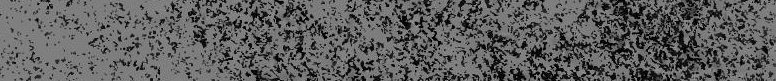

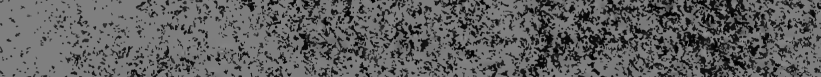

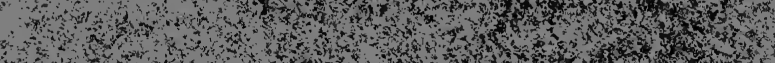

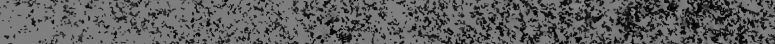

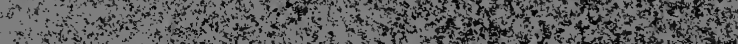

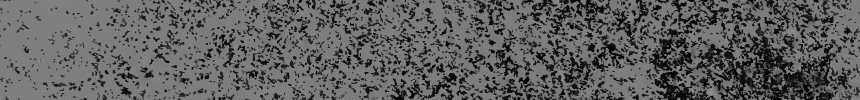

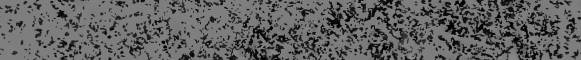

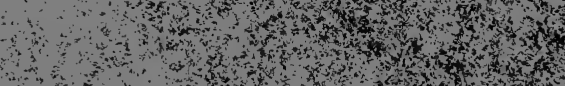

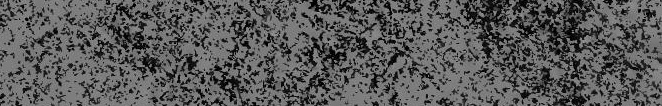

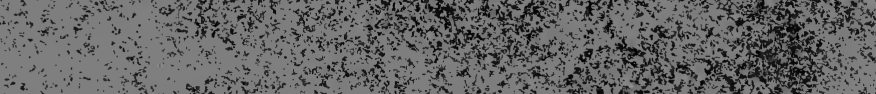

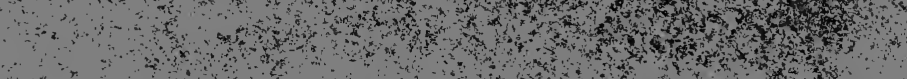

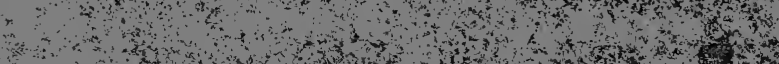




\section{UNIVERSITY OF GALIFORNIA LIBRARY BERKELEY}

Return to desk from which borrowed.

This book is DUE on the last date stamped below.

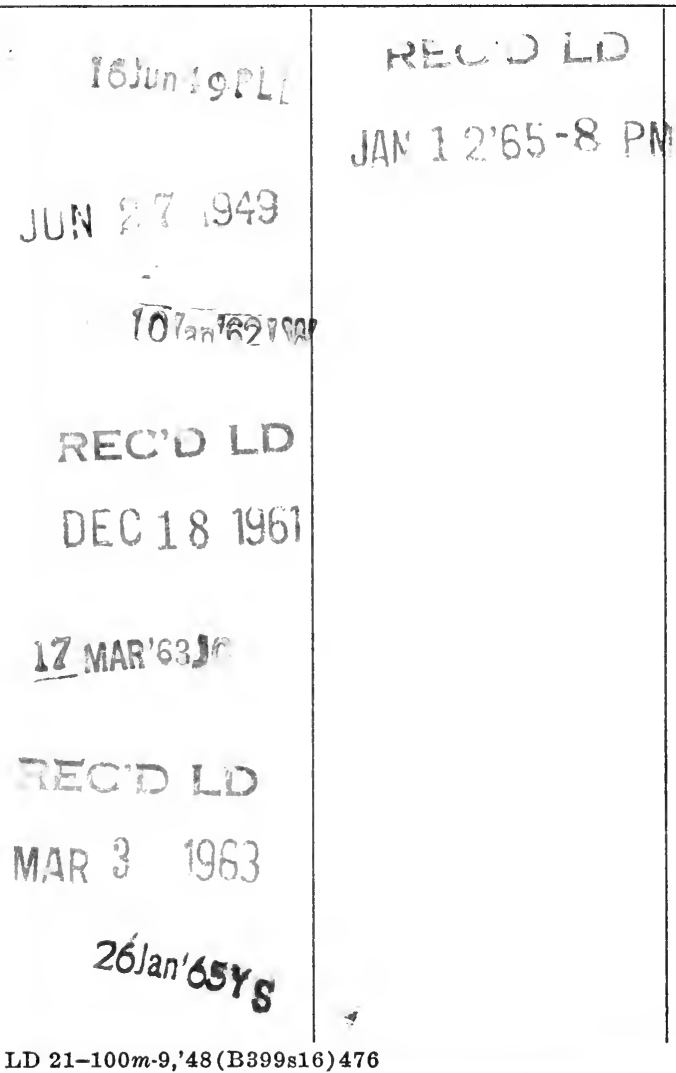




\section{YC 21642}

$$
258+9+2
$$




\section{UNIVERSITY OF CALIFORNIA LIBRARY BERKELEY}

Return to desk from which borrowed.

This book is DUE on the last date stamped below.

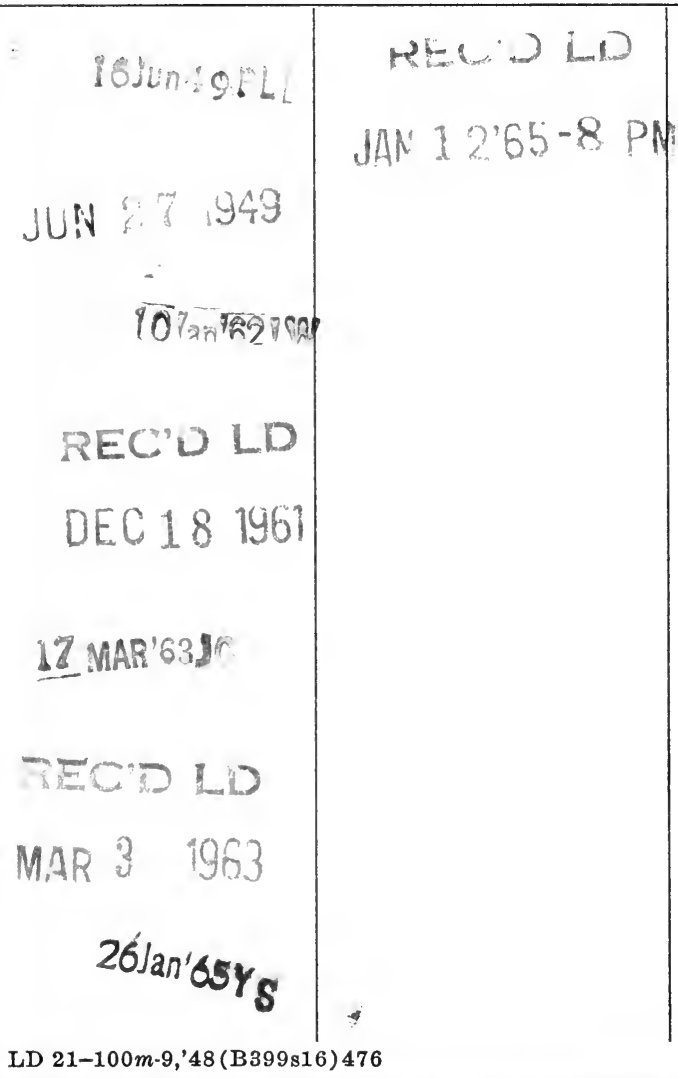




\section{YC 21642}

$$
\begin{array}{r}
2507 x+47 \\
55 \\
012
\end{array}
$$




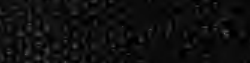

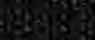

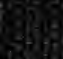

atsons

of

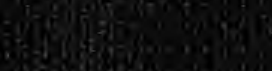

30 - 350 .

(150)

The

구의

ingos

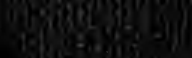

- ing foris?

जाำ

3.

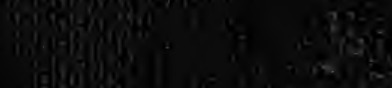

$3 x^{3} 80$

(1) 1134

6inas

, 019,908

is

क日⿱

30stio

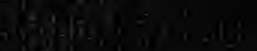

8.

$5 \times 3.25$ 